\section{Lunge und Beatmung}

\section{NIV, Weaning, Monitoring}

Early re-intubation after cardiac operations: impact of nasal continuous positive airway pressure (nCPAP) or non-invasive positive pressure ventilation (NPPV)

\section{U. Boeken, P. Feindt, J. Litmathe, M. Kurt, E. Gams}

Thorax- und Kardiovaskularchirurgie, Chir. Klinik, Heinrich-Heine-Universität, Düsseldorf

Objective: There is an increasing incidence of respiratory failure after primary postoperative extubation in cardiosurgical patients. We wanted to study whether nCPAP may improve pulmonary oxygen transfer and may avoid reintubation after cardiac surgery. Additionally, we compared this protocol to a non-invasive positive pressure ventilation (NPPV). Methods: For a period of 3 years we analyzed all patients that were extubated within 12 hours after cardiac surgery, and in whom pulmonary oxygen transfer $\left(\mathrm{PaO}_{2} /\right.$ $\mathrm{FIO}_{2}$ ) deteriorated without hypercapnia so that all these patients met predefined criteria for reintubation. There were three groups of patients: $A=$ immediate reintubation $(n=125), B=n C P A P-$ treatment with intermittent mask CPAP $(n=264), C=N P P V$ $(n=36)$. Results: $25 \%$ of group B- and $19.5 \%$ of group C-patients were also intubated after a period of CPAP or NPPV. All other patients of groups $\mathrm{B}$ and $\mathrm{C}$ could be weaned from these devices (B: $33.3 \pm 5.6$ hours; C: $25.2 \pm 4.2 \mathrm{~h} ; \mathrm{p}<0.05)$ and were well oxygenated by face mask at ambient pressure $\left(\mathrm{PaO}_{2} / \mathrm{FIO}_{2}: \mathrm{B}: 156 \pm 16, \mathrm{C}\right.$ : $160 \pm 16)$. In group A we found a higher mortality (8\%) than in group B (4.2\%) and group C (5.6\%). Stay on ICU and in-hospitalstay were significantly prolonged in group $\mathrm{A}$. The incidence of pulmonary infections (A: $22.4 \%$, B: $10.2 \%$, C: $11.1 \%, \mathrm{p}<0.05)$ and the need for catecholamines were significantly increased in group A, whereas nCPAP-patients significantly more often suffered from an impaired sternal wound healing (A: 4.8\%, B: 8.7\%, p<0.05). Conclusion: We conclude that reintubation after cardiac operations should be avoided since nCPAP and NPPV are safe and effective to improve arterial oxygenation in the majority of patients with nonhypercapnic oxygenation failure. However, it is of great importance to take special care of sternal wound complications in these patients

Effektivität der Hi0 $\times \mathbf{8 0}$-Sauerstoffmaske unter Routineeinsatzbedingungen

J. Dörrstein ${ }^{1}$, J. Hinkelbein ${ }^{1}$, E. Glaser ${ }^{2}$, T. Frietsch ${ }^{1}$

${ }^{1}$ Universitätsklinik für Anästhesiologie und Operative Intensivmedizin, Universitätsklinikum Mannheim, ${ }^{2}$ Viasys Healthcare $\mathrm{GmbH}$, Hoechberg

Einleitung: Bei respiratorischer Insuffizienz ist die adäquate Oxygenierung essentiell, hängt aber von der Effektivität der genutzten Sauerstoffmaske ab. Eine frühere Studie wies signifikante Vorteile der $\mathrm{HiO} \times 80$-Maske im Vergleich $\mathrm{zu}$ einer gewöhnlichen (Standard-)Sauerstoffmaske bei gesunden Probanden nach [1]. Ziel der vorliegenden Studie war die Überprüfung der Abhängigkeit der inspiratorischen Sauerstoffkonzentration $\left(\mathrm{FiO}_{2}\right)$ vom eingestellten Sauerstoff-Flow bei Patienten mit eingeschränkter Spontanatmung als Maß für die Effektivität der jeweiligen Sauerstoffmaske. Material und Methoden: Bei Patienten mit eingeschränkter Spontanatmung aufgrund einer hochsitzenden Spinalanästhesie über dem Niveau von Th 9 wurde intraoperativ in randomisierter Reihenfolge die $\mathrm{FiO}_{2}$ mit einem $\mathrm{O}_{2}$-Sensor (Draeger Medical, Lübeck) in Abhängigkeit vom Sauerstoff-Flow gemessen. Analysiert wurden Flow-Werte von $1,2,3, \ldots, 12 \mathrm{~L} \cdot \mathrm{min}^{-1}$ mit einer gewöhnlichen
Sauerstoffmaske (Intersurgical Ltd., Berkshire, UK) und mit der $\mathrm{HiO} \times 80$ Maske (Viasys Healthcare $\mathrm{GmbH}$, Hoechberg). Zur statistischen Analyse wurden Statistika ${ }^{\circledR}$ und der t-Test verwendet, $\mathrm{p}<0,05$ galt als signifikant. Ergebnisse: Insgesamt wurden $n=18$ Patienten $(8 \mathrm{~m}, 10 \mathrm{w})$ mit einem Alter von $69 \pm 9$ Jahren und einem Body-Mass-Index von 27,2 $\pm 3,2 \mathrm{~kg} \cdot \mathrm{m}-2$ untersucht. Mittlere Höhe der Spinalanästhesie war Th 7 . Bei einem Flow von 1 $\mathrm{L} \cdot \mathrm{min}^{-1}$ erreichten sowohl die Standardmaske, als auch die $\mathrm{HiO} \times 80$-Maske vergleichbare Werte $(24 \pm 3 \%$ vs. $27 \pm 5 \%$, n.s. $) . \mathrm{Ab}$ einem Flow von $2 \mathrm{~L} \cdot \mathrm{min}^{-1}$ war zwischen beiden Gruppen immer ein statistisch signifikanter Unterschied in der $\mathrm{FiO}_{2}$ bei gleichem Sauerstoff-Fluss nachweisbar $(\mathrm{p}<0,05)$. Beim maximal untersuchten Flow von $12 \mathrm{~L} \cdot \mathrm{min}^{-1}$ war der Unterschied am stärksten ausgeprägt: Mit der Standardmaske wurde eine $\mathrm{FiO}_{2}$ von $40 \pm 12 \%$ erreicht, mit der $\mathrm{HiO} \times 80$-Maske eine $\mathrm{FiO}_{2}$ von $67 \pm 16 \%(\mathrm{p}<0,05)$. Schlussfolgerung: Selbst bei Patienten mit eingeschränkter Spontanatmung erlaubt die $\mathrm{HiO} \times 80$-Maske eine effektivere Nutzung des Sauerstoff-Flow, da mit gleichen Einstellungen eine höhere $\mathrm{FiO}_{2}$ wie mit einer gewöhnlichen Sauerstoffmaske erreicht werden kann. Entsprechend scheinen eine Einsparung von Sauerstoff und damit eine Kostenersparnis plausibel.

Literatur: [1] Hinkelbein et al: Aviat Space Environ Med 2006; 77(5):540-544

Weaning-Versagen bei zwei Intensivpatienten ist mit 015 Veränderungen vom Myosinsubtypen und mit gestörter Funktion der Muskelmembran assoziiert

R. Nowak, M. Winterhalter, S. Piepenbrock, N. Rahe-Meyer Medizinische Hochschule Hannover, Zentrum Anästhesiologie

In den vergangenen Jahren wurde deutlich, dass charakteristische Krankheitsbilder des neuromuskulären Systems die Ursache fortschreitender Paresen bei Intensivpatienten sind. Mit einem neuentwickelten Diagnosegerät, wurden elektrophysiologische und mechanische Muskelfunktionen bei zwei Intensivpatienten mit Weaning-Versagen untersucht. Muskelbiopsien lieferten Informationen über den Zustand der kontraktilen Elemente ihres Muskelsystems. Methodik: Nach Genehmigung durch die Ethikkommission der MHH wurde bei zwei erwachsenen Intensivpatienten nach durchgemachter Sepsis mit klinischen Zeichen einer neuromuskulären Ermüdung und konsekutiv erfolgloser Entwöhnung vom Respirator die Untersuchung durchgeführt (durchschnittliche Beatmungsdauer 55 Tage). Der N. ulnaris wurde mit Einzelreizen, Doppelreizen und Tetanusreizen bis $100 \mathrm{~Hz}$ supramaximal stimuliert. Bestimmt wurden Nervenleitgeschwindigkeit, Refraktärzeit, optimaler Reizabstand und maximale Superposition, ferner Muskelsummenaktionspotential und Kraft bei Einzelreiz, Doppelreizen und tetanischen Reizen. Vergleichswerte wurden bei Gesunden erhoben. Muskelbiobsien wurden vom Musculus quadrizeps femoris entnommen. Die Trennung von Tropomyosin (TM) sowie der Myosinsubtypen - leichten (MLC) und schweren Myosinketten (MHC) - erfolgte durch Gelelektrophorese (Sodium-dodecyl-sulfat-Polyacrylamid-Gel-Elektrophorese und zweidimensionale Gelelektrophorese). Ergebnisse: Die Messungen zeigen Werte für eine intakte Neuronmyelinisierung und motorische Endplatten unserer Patienten. Es zeigten sich pathologische Werte für Kraft unter allen genannten Stimulationsformen sowie eine verlängerte Refraktärzeit und eine Verlängerung optimaler Reizabstand beim Erreichen der maximaler Superposition. Muskelbiobsien: Patient Nr. 1: $35 \%$ MHC I und 23\% MHC II a und 42\% MHC IIb. Patient Nr. 2: $10 \%$ MHC I und II a und 90\% MHC II b. Bei beiden Muskelproben fanden wir weder MLC noch TM vom langsamen Typ. Alle MLC und TM waren vom schnellen Typ. Schlussfolgerung: Die elektrophysiologischen und mechanographischen Messungen zeigen eine Funktionsstörung der Myozyten im Bereich der Membran, der kontraktilen Elemente bzw. der Kopplung dieser beiden Strukturen. Die Patienten bildeten hauptsächlich die anaeroben, schnell zuckenden, schnell ermüdenden MHC IIb Isomere aus; dieses Muster beobachtet man bei Patienten 2 Jahre nach Rückenmarks- 
verletzungen [1]. Da keine langsamen MLC und TM gefunden wurden muss angenommen werden, dass alle MHC I funktionslos waren. Unter diesen Bedingungen kann der Muskel zwar eine Kontraktion ausüben, er ermüdet jedoch schnell. Eine Funktionsstörung der Membran, sowie der kontraktilen Elemente deuten auf eine Myopathie hin, die ein Weaning-Versagen erklärt.

Literatur: [1] Mohr T et al: Spinal Cord 1997; 35:1-16

Veränderung der Refraktärzeit der Muskelmembran bei kritisch kranken mit Weaning-Versagen

R. Nowak ${ }^{1}$, M. Winterhalter ${ }^{1}$, M. Leuwer ${ }^{2}$, S. Piepenbrock ${ }^{1}$, N. Rahe-Meyer ${ }^{1}$

${ }^{1}$ Medizinische Hochschule Hannover, Zentrum Anästhesiologie

${ }^{2}$ University of Liverpool, Department of Anesthesiology

Sekundäres Atemversagen bei septischen Patienten wird nach heutigen Erkenntnissen einer spezifischen Erkrankung des Nervenund Muskelsystems zugeschrieben [1]. Mit einem neuentwickelten Diagnosegerät, wurden elektrophysiologische und mechanische Muskelfunktionen bei Intensivpatienten mit Weaning-Versagen untersucht. Methodik: Nach Genehmigung der Ethikkommission wurden 21 Messserien bei zwei erwachsenen Intensivpatienten nach Lebertransplantation und postoperativer Sepsis durchgeführt. Die Patienten zeigten klinische Zeichen muskulärer Ermüdung; Entwöhnungsversuche vom Respirator blieben erfolglos. Der N. ulnaris wurde mit Einzelreizen, Doppelreizen und Tetanusreizen bis $100 \mathrm{~Hz}$ supramaximal stimuliert. Bestimmt wurden Nervenleitgeschwindigkeit, Refraktärzeit, Muskelsummenaktionspotential (MSAP) und Kraft bei Einzelreiz, Doppelreizen (maximale Superposition und optimaler Reizabstand) und Tetanusreizen. Zum Vergleich wurden 40 Messserien an zehn gesunden Probanden durchgeführt. Ergebnisse: Normalwerte für Nervenleitgeschwindigkeit und Ermüdung (tetanic fade) sprechen für intakte Neuronmyelinisierung und motorische Endplatten unserer $\mathrm{Pa}$ tienten. Die Kraft der Einzelkontraktion war erniedrigt (6,4 \pm 4 vs. $10 \pm 2 \mathrm{~N}$ bei Gesunden). Die tetanische Kraft war im Durchschnitt um mehr als $60 \%$ erniedrigt. Die Kraft bei maximaler Superposition war vermindert (Kraft im Verhältnis zur Einzelkontraktion: $169 \pm 28$ vs. $310 \pm 20 \%$ bei Gesunden) und der optimale Reizabstand war bei dem Pat. um ein vielfaches verlängert. Die absolute Refraktärzeit war verlängert $(2 \pm 0,3$ vs. $0,98 \pm 0,06 \mathrm{~ms}$ bei $\mathrm{Ge}$ sunden). Mit klinischer Erholung der Muskelfunktion und abgeschlossener Entwöhnung vom Respirator der Patienten (nach 21 bzw. 90 Tagen Beatmung) normalisierten sich die Messwerte für Refraktärzeit vollständig. Die Werte für Kraft erholten sich verzögert. Schlussfolgerung: Veränderungen in der Refraktärzeit sprechen für Veränderungen der Membranfunktionen der Myozyten. Mit Kraftmessungen bei Doppelreizung im Bereich der maximalen Superposition ließ sich ein Defekt des kontraktilen Apparates nachweisen. Es ließ sich also eine Muskelfunktionsstörung aufdecken und im Verlauf gelang es, die Erholung der Muskeltätigkeit messtechnisch zu erfassen. Hierbei scheint sich die Refraktärzeit - also die Funktion der Muskelmembran - am schnellsten zu erholen. Die Kraftwerte erholten sich verzögert, möglicherweise bedingt durch eine Versteifung des serienelastischen Apparates, was an einer verzögerten Erholung des optimalen Reizabstandes und der maximalen Superposition zu sehen war. Die Messung der Refraktärzeit gelang in der vorliegenden Untersuchung sowohl mechanomyographisch als auch elektrophysiologisch (computergestützte Analyse des MSAP). Die mechanographischen Messungen auf der Intensivstation waren weniger störanfällig als die elektrophysiologischen Untersuchungen.

Literatur: [1] Hund E: J Neurol. 2001 Nov; 248(11):929-934
Quantifizierung PEEP-induzierter Verschiebungen der regionalen Lungenventilation mittels elektrischer Impedanztomographie

I. Frerichs, G. Schmitz, S. Pulletz, D. Schädler, G. Zick, J. Scholz, N. Weiler

Klinik für Anästhesiologie und Operative Intensivmedizin Universitätsklinikum Schleswig-Holstein, Campus Kiel

Einleitung: Eine bettseitige Überwachung der regionalen Lungenventilation könnte bei beatmeten Intensivpatienten zur Optimierung der Beatmungstherapie und zur Minimierung der unerwünschten Nebenwirkungen beitragen. Z.Zt. steht kein medizinisches Monitoring-Verfahren zur Verfügung, das in dieser Anwendung klinisch verwendet werden kann. Ein neues bildgebendes Verfahren, die elektrische Impedanztomographie (EIT), hat das Potential diese Art der Beatmungsüberwachung zu gewährleisten $[1,2]$. Die Voraussetzung für einen routinemäßigen klinischen Einsatz ist jedoch die Entwicklung geeigneter Auswertungsverfahren, die relevante Parameter zur Charakterisierung der regionalen Ventilation generieren. Das Ziel unserer Studie war die Entwicklung einer Methode zur Quantifizierung topographischer Änderungen der Ventilationsverteilung im Thoraxquerschnitt mittels EIT. Methoden: Die Untersuchungen wurden an 8 Schweinen $(39 \pm 2 \mathrm{~kg}$; mittl. Körpergewicht $\pm S D)$ nach Genehmigung durch die zuständige Tierschutzkommission durchgeführt. Die Tiere wurden anästhesiert und volumenkontrolliert in Rückenlage mit einem Atemhubvolumen von $435 \pm 30 \mathrm{ml}$ beatmet. EIT-Messungen wurden mit dem Goe-MF II EIT-Gerät (Viasys Healthcare, Höchberg, Deutschland) bei wiederholter Änderung des positiven end-exspiratorischen Drucks (PEEP) von $0 \mathrm{cmH}_{2} \mathrm{O}$ auf $10 \mathrm{cmH}_{2} \mathrm{O}$ durchgeführt. Die regionale Lungenventilation wurde in der rechten und linken Thoraxhälfte, sowie in insgesamt 64 Regionen bestimmt, die in der ventrodorsalen Richtung symmetrisch auf die rechte und linke Thoraxhälfte aufgeteilt waren. Aus den Daten wurden ventrodorsale Profile der Ventilationsverteilung in der rechten und linken Thoraxhälfte generiert und zur Quantifizierung der Ventilationsverschiebungen zwischen den unabhängigen und abhängigen Lungenregionen der Schwerpunkt der Ventilation in Relation zum Thoraxdurchmesser bestimmt. Ergebnisse: Der Anteil der Ventilation in der rechten Thoraxhälfte an der Gesamtventilation im Thoraxquerschnitt betrug $0,54 \pm 0,04$. Nach der ersten PEEP-Erhöhung $(0,52 \pm 0,04)$, sowie nach der erneuten PEEPReduktion $(0,53 \pm 0,04)$ und Erhöhung $(0,52 \pm 0,04)$ wurden keine signifikanten Änderungen festgestellt. Die erste PEEP-Erhöhung führte zu einer Umverteilung der Ventilation zu Gunsten der dorsalen Lungenregionen mit Verschiebung des Schwerpunktes der Ventilation von $45 \pm 3 \%$ zu $49 \pm 3 \%$ des Thoraxdurchmessers in der rechten und von $47 \pm 2 \%$ zu $50 \pm 2 \%$ in der linken Thoraxhälfte. Die wiederholten Messungen nach erneuter PEEP-Senkung und Erhöhung zeigten eine gute Reproduzierbarkeit der Ergebnisse mit identischen Mustern der Ventilationsverteilung wie bei den initialen Messungen. Schlussfolgerung: Die vorgestellte Auswertung der EIT-Daten mit korrekter Wiedergabe der untersuchten beatmungsbedingten Ventilationsumverteilung eignet sich zur Quantifizierung der Topographie der regionalen Ventilation.

Literatur: [1] Frerichs: Physiol Meas 2000; 21:1-21. [2] Brown: J Med Eng Technol 2003; 27:97-108

Sicherheit und Effektivität eines neuen Systems zur automatischen Beatmung und Entwöhnung von der künstlichen Beatmung

D. Schädler, I. Frerichs, G. Schmitz, S. Pulletz, G. Zick, J. Scholz, N. Weiler

Klinik für Anästhesiologie und Operative Intensivmedizin

Universitätsklinikum Schleswig-Holstein, Campus Kiel

Einleitung: Wissensbasierte Systeme zur automatischen Entwöhnung von der Beatmung können den Entwöhnungsprozess durch Verkürzung der Entwöhnungszeit, der Beatmungszeit und der 
Länge des Aufenthaltes auf der Intensivstation verbessern [1]. Allerdings ist momentan kein System in der Lage alle Beatmungsparameter automatisch $\mathrm{zu}$ steuern. Das Evita-Weaning-System (EWS) ist ein wissensbasiertes System zur vollautomatischen Beatmung und Entwöhnung von Patienten von der Beatmung. Vier Aspekte der künstlichen Beatmung (alveoläre Ventilation, Oxygenierungsleistung, Spontanaktivität, Beatmungsaufwand) werden durch ein neu entwickeltes Scoresystems analysiert und mit dem Ziel der Reduktion des Beatmungsaufwandes durch das Expertensystem optimiert. Ziel dieser Untersuchung war es, die Sicherheit und die Effektivität des EWS zu untersuchen und zu überprüfen. Methodik: Nach Zustimmung durch die lokale Ethikkommission und schriftlicher Einwilligung konnten 19 hämodynamisch stabile Patienten in diese Anwendungsbeobachtung eingeschlossen werden. Das EWS wurde auf einem Standard-PC implementiert und steuerte einen modifizierten Evita-4-Respirator (Dräger Medical, Lübeck) im BIPAP/ASB-Modus. Die Beatmungseinstellungen des Stationsarztes wurden mit den Einstellungen des EWS 10 Minuten nach Initiierung der automatischen Beatmung und am Ende der Studienperiode verglichen (ANOVA für wiederkehrende Messungen, $\mathrm{p}<0,05)$. Ergebnisse: Die Einstellungen zu Beginn der automatischen Beatmung unterschieden sich außer einer Reduktion der inspiratorischen Sauerstoffkonzentration statistisch nicht signifikant von den Einstellungen des Stationsarztes. Alle Patienten wurden durch das EWS innerhalb von $37 \pm 17$ Minuten von einer kontrollierten Beatmung in eine assistierte Spontanatmung überführt werden. Nach einer mittleren Beatmungszeit von $173 \pm 53$ Minuten konnte am Ende der Studienperiode eine signifikante Reduktion der inspiratorischen Sauerstoffkonzentration, der maschinellen Atemfrequenz, der Beatmungsdrücke, des Atemzugvolumens und des Scores für den Beatmungsaufwand festgestellt werden. Der Oxygenierungsindex der Patienten, das Atemminutenvolumen und der PEEP zeigten über die gesamte Studienperiode keine signifikanten Änderungen. Die exspiratorische $\mathrm{CO}_{2}$-Konzentration und die spontane Atemfrequenz waren zu Studienende statistisch signifikant höher als die jeweiligen Ausgangswerte. 15 der 19 Patienten konnten am Ende der Studie erfolgreich extubiert werden. Schlussfolgerungen: Das EWS ist in der Lage in einem heterogenen Patientenkollektiv sichere Beatmungseinstellungen zu treffen und kann den Beatmungsaufwand reduzieren. Randomisierte, kontrollierte Studien sind notwendig, um die Effizienz dieses neuen Systems zu überprüfen.

Literatur: [1] Lellouche F et al: Intensive Care Med 2004, 30:S69

\section{Supportive endotracheale Jetventilation} bei spontanatmenden Intensivpatienten

\section{T. Bingold, H. Wissing, S. Klösel, C. Wullstein* ${ }^{*}$ B. Zwißler, \\ C. Byhahn}

Kliniken für Anästhesiologie und Intensivmedizin und ${ }^{\star}$ Allgemeinchirurgie Klinikum der J.-W.-Goethe-Universität Frankfurt am Main

Hintergrund: Patienten nach großen abdominellen oder thorakalen Eingriffe haben ein erhöhtes Risiko für postoperative pulmonale Komplikation, z.B. durch Atelektasen, Ödem oder Pneumonie. Die nicht-invasive CPAP-Beatmung (NIV) ist oftmals effektiv, um eine Reintubation zu vermeiden. Kontraindikationen für die NIV umfassen jedoch z. B. frische ösophageale Anastomosen oder Anastomoseninsuffizienzen in diesem Bereich. Wir berichten über zwei Patienten, bei denen alternativ zur NIV eine Jetbeatmung angewendet wurde. Fall 1: Ein 76-jähriger Patient wurde am 8. Tag nach Ösophagusresektion und Magenhochzug bei tracheoösophagealer Fistel in Allgemeinanästhesie und Jetventilation mit einem $8 \mathrm{~cm}$ langen Trachealstent versorgt. Postoperativ erfolgte die Beatmung zunächst über eine Larynxmaske (LMA), die jedoch am 5 . Tag wegen blutender Druckulzera im Pharynx entfernt werden musste. Da eine NIV des sedierten, aber im PPS-Modus spontanatmenden Patienten kontraindiziert war, erfolgte die bronchosko- pische Platzierung eines nasotrachealen Jetkatheters, dessen Spitze im Stent zum Liegen kam. Die Spontanatmung wurde für 10 Tage mit einem kontinuierlichen Jet $\left(\mathrm{FiO}_{2} \quad 0,8\right.$; Frequenz: $150 / \mathrm{min}$; Druck: 1,5 bar) unterstützt und führte zu einer adäquaten Oxygenierung. Dennoch verstarb der Patient letztlich im septischen Schock. Fall 2: Bei einem am 1. postoperativen Tag nach Ösophagusresektion extubierten 64-jährigen Patienten kam es durch Würgen zu einer Dislokation der Magensonde. Eine endoskopische Replatzierung wurde zum Schutz der Anastomose nicht vorgenommen, so dass am 2. Tag bei respiratorischer Verschlechterung im Rahmen einer Pneumonie die NIV kontraindiziert war. Wiederum erfolgte die bronchoskopisch gesteuerte Einlage eines nasotrachealen Jetkatheters. Der nicht sedierte und im Zimmer mobilisierte Patient wurde für 12 Tage kontinuierlich mit einer $\mathrm{FiO}_{2}$ von 0,6 , einer Frequenz von 170/min und einem Abstrahldruck von 1,5 bar jetunterstützt. In den ersten 5 Tagen war zusätzlich eine tägliche Bronchoskopie zur Bronchialsekretentfernung erforderlich. Der Patient konnte am 16. postoperativen Tag auf die Normalstation verlegt werden. Diskussion: Die beiden Fälle zeigen die Effizienz der unterstützenden Jetventilation bei respiratorisch insuffizienten Patienten mit Kontraindikationen für eine NIV. Im ersten Fall konnte keine endotracheale Intubation durchgeführt werden, da der Tubuscuff aufgrund der Länge des Stents innerhalb dessen zum Liegen gekommen wäre und den Stent beschädigt hätte. Aufgrund der pharyngealen Ulzerationen war die erneute Anwendung einer LMA ebenfalls nicht möglich. Im anderen Fall wäre die Reintubation die einzige Alternative gewesen. Jetkatheter und -beatmung wurden von dem wachen, kooperativen und mobilisierten Patienten hervorragend toleriert. Aufgrund dieser Daten halten wir die supportive Jetventilation für eine wertvolle Alternative im Atemwegsmaganement von respiratorisch insuffizienten Intensivpatienten mit Kontraindikationen zur NIV.

Einfluss von rhDnase auf die Beatmungsdauer

bei Intensivpatienten - Ergebnisse der Interimsanalyse der LUFIT-Studie

N. Deschner ${ }^{1}$, R. Vonthein ${ }^{2}$, W. Brehm ${ }^{3}$, J. Riethmueller ${ }^{3}$

${ }^{1}$ Klinik für Anästhesiologie und Intensivmedizin, ${ }^{2}$ Institut für

Medizinische Biometrie, ${ }^{3}$ Klinik für Kinder- und Jugendmedizin

Hintergrund: rhDNase (Dornase alfa, PulmozymeÒ) fördert die Rheologie des Trachealsekrets durch Spaltung der DNA in abgestorbenen neutrophilen Granulozyten. Sie ist ein etablierter, wirksamer Bestandteil der Behandlung der Cystischen Fibrose [1]. Bei pädiatrischen Patienten konnte nach kardiochirurgischen Eingriffen eine Reduktion der Beatmungsdauer durch rhDNase nachgewiesen werden [2]. Das Ziel dieser Studie war zu untersuchen, ob rhDNase in der Lage ist, die Beatmungsdauer von erwachsenen, beatmeten Patienten zu reduzieren. Methode: Nach Zustimmung durch die örtlichen Ethik-Kommissionen führten wir eine doppelblinde, randomisierte, placebokontrollierte, multizentrische nationale Studie durch. Zwei Strata, mit bzw. ohne vorangegangene Operation, wurden unterschieden. Die Patienten erhielten zweimal täglich 2,5 ml rhDNase oder NaCl-Placebo endotracheal appliziert. Ergebnis: Einflussfaktoren wie Geschlecht, Gewicht, Nikotinanamnese und Häufigkeit chronischer Vorerkrankungen waren in beiden Armen in beiden Strata gleich verteilt. Bei nicht-operativen Patienten konnten in der rhDNase-Gruppe 44 Patienten analysiert werden, bei denen die mediane Beatmungsdauer 5,2 Tage betrug. In der Placebo-Gruppe wurden 41 Patienten analysiert. Hier dauerte die Beatmung im Median 13 Tage. Bei operativen Patienten konnten in der rhDNase-Gruppe 47 Patienten analysiert werden, bei denen die Beatmung im Median 15,7 Tage andauerte. In der Placebo-Gruppe wurden 39 Patienten analysiert mit einer medianen Beatmungsdauer von 10,8 Tagen. Eine Detailanalye im anteilig größten Subkollektiv der neurochirurgischen Patienten in einem Zentrum ergab Nachoperationen bei 12 von 16 Patienten in der rhDNase-Gruppe und bei nur 4 von 15 Patienten in der NaClGruppe. Die kontinuierliche Sedierung erfolgte in der rhDNase- 
Gruppe für 11,7 Tage, in der NaCl-Gruppe für 7 Tage. Die Beatmungsdauer nach der letzten Operation betrug dagegen unter rhDNase 5,25 Tage, unter $\mathrm{NaCl}$ 7,3 Tage. Schlussfolgerungen: Diese Zwischenauswertung weist darauf hin, dass rhDNase in der Lage ist, bei nicht-operativen beatmeten Intensivpatienten die Beatmungsdauer zu verkürzen. Dieses Ergebnis steht im Einklang mit den Befunden bei post-kardiochirurgischen pädiatrischen Patienten [2]. Bei operativen erwachsenen Patienten lässt sich dieser Befund nicht nachvollziehen. Es ist möglich, dass vor allem in der Gruppe der neurochirurgischen Patienten durch die Randomisierung nicht kontrollierte Faktoren wie z. B. längere Sedierungsdauer und häufigere Nachoperationen Einfluss auf das Ergebnis dieser Zwischenauswertung haben.

Literatur: [1] Fuchs et al: N Engl J Med 1994; 331:637-642. [2] Riethmueller J et al: Pediatr Pulmonol 2005; 41:61-66

\section{Entwicklung einer computergestützten-protokoll- basierten Entwöhnungssoftware (WineS) zur standardisierten Entwöhnung des Patienten von der maschinellen Beatmung}

\section{S. Terbeck ${ }^{1}$, P. Herrmann ${ }^{3}$, Ph. Heermann ${ }^{2}$, U. Scheid ${ }^{1}$, R. Kuhlen ${ }^{1}$, M. Quintel ${ }^{2}$ Operative Intensivmedizin Universitätsklinikum Aachen ${ }^{1}$ und Göttingen ${ }^{2}$, Technologie Institut Medizin GmbH Göttingen ${ }^{3}$ der Georg-August-Universität Göttingen}

Hintergrund: Auch wenn die Reduktion der Beatmungsdauer durch Einsatz einer protokollbasierten Entwöhnung evident ist [1], wird sie in der klinischen Routine kaum angewendet. Eine Implementierung eines solchen Protokolls in die Software konventioneller Beatmungsgeräte könnte zu einer besseren klinischen Handhabung führen. Der von uns entwickelte computergestützte Algorithmus bietet über Logikabfragen eine Entscheidungshilfe und könnte zu einer Prozessoptimierung und Qualitätssteigerung in der Intensivmedizin beitragen. Methodik: Ein auf internationalen Richtlinien basierendes Entwöhnungsprotokoll [1] wurde als Grundlage zur Entwicklung einer interaktiven Software erstellt. Neben Abfrage- und Dialogfunktionalitäten wurden Routinen zur Online Datenerfassung, Speicherung und Visualisierung der respiratorischen Parameter des Beatmungsgerätes mit Hilfe einer grafischen Sprache („G“, LabVIEW, National Instruments, Texas, USA) programmiert. Das Programm wurde auf einem Touchscreen PC (I-Panel, GE Healthcare, Betriebssystem Windows XP Prof.) installiert und an das Beatmungsgerät (Centiva5/plus) angeschlossen. Es erfasst alle respiratorischen Parameter online aus dem Beatmungsgerät, wobei speziell definiert nach $\mathrm{FiO}_{2}$ und PEEP-Werten Logikschleifen zugeführt werden. Sind definierte Grenzwerte erreicht, generiert die Weaningsoftware Dialogfenster und Abfrageformulare auf die der behandelnde Arzt reagieren muss. Bei jeder negativen Antwort muss eine Begründung plus Eingabe zur Wiederholung erfolgen. Wurden alle Fragen positiv beantwortet, ist der Patient zur Durchführung eines Spontanatmungsversuches (SBT) bereit. Während des SBT wird der rapid shallow breathing index $(\mathrm{RSBI}=\mathrm{f} 2 / \mathrm{MV})$ kontinuierlich registriert. War der SBT erfolgreich, werden Abfragen und Hinweise erzeugt, die den Weg bis zur erfolgreichen Extubation weiterhin unterstützen. Ergebnisse: Nach einer Evaluierungsphase am Lungensimulator (ATL 01, TIM GmbH, Göttingen) wurde die Software für den Einsatz am Patienten optimiert. Das Team der Intensivstation zeigte eine deutlich höhere Motivation bei der Anwendung der Entwöhnungssoftware verglichen zum handschriftlichen Protokoll. Die Software ist bedienerfreundlich und aufgrund eines optischen Anzeigesystems können Patienten, die bereit für eine Entwöhnung sind, leichter identifiziert werden. Die ersten Patienten wurden bereits erfolgreich anhand des computerbasierten Protokolls von der maschinellen Beatmung entwöhnt. Schlussfolgerung: Die Entwöhnungssoftware ist eine praktikable Möglichkeit einen protokollbasierten Algorithmus in der klinischen Routine anzuwenden. Ob die Software zu einer Verkürzung der Beatmungsdauer führt, wird eine derzeit durchgeführte Pilotstudie zur Anwendung des computergestützten versus handschriftlichen Entwöhnungsprotokoll zeigen. Ein nächster Schritt wäre die Integration der Funktionalitäten in ein Patientendaten-Managementsystem (PDMS) oder direkt in ein Beatmungsgerät.

Literatur: [1] MacIntyre NR et al:. Chest 2001; 120:375-395.

Das Tracheotomie-Endoskop für Dilatations-

tracheotomien nach KLEMM - Ein neues Verfahren zur Percutanen Dilatativen Tracheotomie

in der Intensivmedizin

\section{E. Klemm, A. Nowak, K.F. Rothe}

HNO-Klinik, Klinik für Anästhesiologie und Intensivtherapie, Städtisches Klinikum Dresden-Friedrichstadt

Einleitung: Zahlreiche Beobachtungen und zahlreiche Veröffentlichungen $\mathrm{zu}$ schweren peri-interventionellen Komplikationen (Knorpelfrakturen, Tracheahinterwandverletzungen, Blutungen, falscher Punktionsort, Trachealstenosen) der Perkutanen Dilatationstracheotomie (PDT) lassen die Forderung nach einem verbesserten Management der PDT erkennen [1, 3-5, 7, 8]. Vor diesem Hintergrund erfolgte die Entwicklung des Tracheotomie-Endoskopes (TED). Methode: Die Methode beruht auf dem Prinzip der Beatmungslaryngoskopie mittels eines starren Rohres nach FABIAN und BRANDT [2,6]. Das TED [7] wurde speziell den Erfordernissen der PDT mit folgender Zielsetzung angepasst: Schutz der Tracheahinterwand vor Verletzungen, starker Diaphanoskopieeffekt durch anteflektierten Leuchtstab, Beatmung des Patienten über das Endoskoprohr oder als Jetbeatmung über integrierte proximale oder distale Jet-Injektoren, separater $\mathrm{ETCO}_{2}$-Messkanal, gute Sicht auch bei Blutungen, leistungsstarke Absaugung, Elektrokoagulation, endoskopische Sofortkorrektur dislozierter Knorpelfrakturen, Verwendung des Endoskopes für alle gängigen PDTVerfahren

- Kupplung für Bildmonitoring,

- Sterilisierbarkeit aller Endoskop-Teile.

Ergebnis: Das TED wurde im Jahr 2005 in die klinische Praxis eingeführt. Medizinische Erfordernisse wurden technisch umgesetzt. Schlussfolgerung: Die ersten Erkenntnisse einer Pilotuntersuchung bestätigen die Erwartung, dass mit der Anwendung des TED eine Risikoreduktion bei der PDT zu erreichen ist. Interdisziplinäre Studien sind erforderlich und werden folgen.

Literatur: [1] Ambesh SP, Pandey CK, Srivatava S, Agarwal A, Singh DK: Percutaneous Tracheostomy with Single Dilatation Technique: A prospektive randomized Comparison of Ciaglia Blue Rhino versus Griggs Dilatating Forceps. Anaesth Analg 95:17391745 (2002). [2] Brandt RH: Barbiturat-Relaxansnarkose bei Beatmungsendoskopien. HNO 13: 343-345 (1965). [3] Delank KW, Schmäl F, Stoll W: HNO- ärztliche Erfahrungen mit traumatischen Läsionen der Tracheahinterwand. Laryngo-Rhino-Otol 81:299304 (2002). [4] Dost PH, Koeser K: Komplikationen der dilatativen Punktionstracheotomien in deutschen Hals-, Nasen-, Ohren-Abteilungen. Laryngo- Rhino-Otol 78:81-85 (1999). [5] Fabian G, Tzschoppe A, Wiedemann A: Direkte Laryngoskopie in RelaxansBarbiturat-Narkose. HNO 12:173-174 (1964). [6] Klemm E: Tracheotomie-Endoskop für Dilatationstracheotomien (TED) Verlag Endo-Press, Tuttlingen, ISBN: 3-89756-113-1 (2006). [7] Oeken J, Adam H, Bootz F: Translaryngeale Tracheotomie (TLT) nach Fantoni mit starrer endoskopischer Kontrolle. HNO 50:638-643 (2002). [8] Stein M: Untersuchungen zur perkutanen Dilatationstracheotomie und Vergleiche mit der konventionellen Tracheotomie. Dissertation Universität Leipzig 1997 
Klinische Validierung der funktionellen elektrischen Impedanztomographie (f-ElT)

\section{S. Pulletz, G. Schmitz, D. Schädler, G. Zick, J. Scholz, I. Frerichs,} N. Weiler

Klinik für Anästhesiologie und Operative Intensivmedizin Universitätsklinikum Schleswig-Holstein Campus Kiel

Einleitung: Inadäquate Ventilatoreinstellungen können während künstlicher Beatmung das Lungengewebe schädigen. Mit einer bettseitigen Online-Überwachung der regionalen Lungenfunktion könnte die Beatmungstherapie den regionalen Lungenverhältnissen angepasst werden. Von besonderem Interesse ist hierbei die Differenzierung von atelektatischem und überdehntem Lungengewebe. Mit der funktionellen elektrischen Impedanztomographie (EIT) steht eine neue Technik zur Verfügung, die eine strahlenfreie nicht-invasive kontinuierliche Überwachung der regionalen Lungenfunktion ermöglicht $[1,2]$. Ziel dieser Studie war die Evaluation der EIT zur Darstellung von unterschiedlichen Volumenverschiebungen an kontrolliert beatmeten Patienten. Die Ein-Lungen-Ventilation ermöglichte eine getrennte Ventilation mit definierten Tidalvolumina. Methoden: In die Studie wurden zehn lungengesunde Patienten (Alter: $60 \pm 10$, Mittelwert \pm Standardabweichung) mit Indikation zur Ein-Lungen-Beatmung bei elektivem Thoraxeingriff eingeschlossen. Die Untersuchungen erfolgten mit dem Goe MF-II EIT-Gerät (Viasys Healthcare, Höchberg, Deutschland). Sechzehn selbstklebende Elektroden (3M Red Dot 2239, 3M Health Care, Borken, Deutschland) wurden zum rotierenden Einspeisen von geringem elektrischen Strom $(5 \mathrm{mArms}, 50 \mathrm{k} \mathrm{Hz})$ und zur Spannungsmessung transversal um den Thorax geklebt. Die EIT-Daten wurden mit einer Aufzeichnungsrate von 13 Bildern/s über einen Zeitraum von einer Minute erhoben. Die Daten wurden präoperativ während der Beatmung von beiden Lungen bzw. rechter und linker Lunge erhoben. Bei beidseitiger Lungenbeatmung wurden die Patienten mit einem Tidalvolumen von $800 \mathrm{ml}$ und bei einseitigem mit $400 \mathrm{ml}$ beatmet. Ergebnisse: Die ventilationsbedingten Impedanzänderungen, die im ganzen Thoraxquerschnitt während einseitiger Beatmung der rechten und linken Lunge ermittelt wurden, unterschieden sich nicht signifikant voneinander und entsprachen $47,6 \pm 5,6 \%$ bzw. $48,5 \pm 7,8 \%$ der Impedanzänderungen bei beidseitiger Beatmung. Während Ein-Lungen-Ventilation war eine Trennung zwischen beatmetem und nicht beatmetem Lungengewebe gut möglich, da $92,2 \pm 3,9 \%$ bzw. $90,2 \pm 4,1 \%$ der ventilationsbedingten Impedanzänderungen auf der beatmeten Thoraxseite bei Ventilation der rechten bzw. linken Lunge auftraten. Diskussion: Mit der EIT steht eine sensitive Methode zur Überwachung topographischer Verteilung der Ventilation zur Verfügung. Globale Änderungen des Tidalvolumens werden von der EIT bei bilateraler und unilateraler Beatmung adäquat dargestellt. Die Trennung von ventilierten und nicht ventilierten Arealen während Ein-Lungen-Ventilation ist möglich.

Literatur: [1] Frerichs et al: J Appl Physiol 2002; 93:660-666. [2] Victorino et al: Am J Respir Crit Care Med 2004; 169:791-800

\section{Einfluss von inspiratorischer Sauerstoffkonzentration und positivem end-exspiratorischen Druck \\ auf die regionale Lungenventilation \\ und das Lungenvolumen ermittelt \\ durch elektrische Impedanztomographie \\ G. Schmitz, S. Pulletz, D. Schädler, G. Zick, J. Scholz, N. Weiler, I. Frerichs \\ Klinik für Anästhesiologie und Operative Intensivmedizin Universitätsklinikum Schleswig-Holstein, Campus Kiel}

Einleitung: Atelektasenbildung mit erhöhtem pulmonalem Shuntfluss aufgrund inadäquater Beatmungseinstellungen stellt einen wesentlichen Faktor für Gasaustauschstörungen unter mechanischer Beatmung dar. Während eine hohe inspiratorische Sauerstoffkonzentration $\left(\mathrm{FIO}_{2}\right)$ mit einer vermehrten Atelektasenbildung assoziiert ist, verbessert die Anwendung eines positiv end- exspiratorischen Drucks (PEEP) den Gasaustausch durch Rekrutierung nicht ventilierter Areale [1]. Ziel dieser Studie war die Untersuchung des Einflusses unterschiedlicher $\mathrm{FIO}_{2}$ - und PEEPEinstellungen auf die regionale Lungenventilation bzw. das regionale Lungenvolumen mittels elektrischer Impedanztomographie (EIT). Methoden: Acht anästhesierte Schweine $(39 \pm 4 \mathrm{~kg}$, Mittelwert \pm Standardabweichung) wurden in Rückenlage volumen-kontrolliert mit konstantem Atemhubvolumen $(394 \pm 72 \mathrm{ml})$ und einer Frequenz von 20 Atemzügen/min beatmet. $\mathrm{FIO}_{2}$ wurde von 0,21 auf 1,0 und zurück auf 0,21 eingestellt. Bei jeder $\mathrm{FIO}_{2}$ wurden die Tiere für $15 \mathrm{~min}$ ohne und anschließend für $5 \mathrm{~min}$ mit einem PEEP von $5 \mathrm{cmH}_{2} \mathrm{O}$ ventiliert. Die EIT-Messungen (Goe MF II, Viasys Healthcare, Höchberg, Germany) wurden jeweils am Ende einer Periode durchgeführt, wobei die Änderungen der regionalen Lungenventilation bzw. des end-exspiratorischen Lungenvolumens über entsprechende Änderungen der tidalen bzw. end-exspiratorischen Impedanz bestimmt wurden. Für die Auswertung wurden die Lungen in vier ventro-dorsal ausgerichtete Regionen unterteilt. Ergebnisse: Die regionale Lungenventilation wurde durch Anwendung von $5 \mathrm{cmH}_{2} \mathrm{O}$ PEEP in beiden ventralen Regionen signifikant reduziert und in beiden dorsalen Regionen erhöht. Diese Effekte traten bei allen aufeinander folgenden $\mathrm{FIO}_{2}$-Einstellungen auf. In der untersten Region kam es ohne PEEP nur aufgrund unterschiedlicher $\mathrm{FIO}_{2}$ zu einer Minderung der Ventilation. Das regionale Lungenvolumen wurde in den oberen drei Regionen durch den PEEP signifikant erhöht. In der dorsalen Region und bei $\mathrm{FIO}_{2}$ von 0,21 wurde das Lungenvolumen durch PEEP leicht erhöht, während es bei einer $\mathrm{FIO}_{2}$ von 1,0 konstant blieb. Darüber hinaus verminderte sich das regionale Volumen bei identischen PEEPEinstellungen mit der Zeit. Dieser Effekt war in allen Regionen, v.a. aber in der dorsalen Region sichtbar. Schlussfolgerung: Die EIT stellt ein geeignetes, nicht-invasives Verfahren für die Darstellung PEEP-induzierter Änderungen der regionalen Ventilation und des Volumens dar. Die Anwendung von PEEP resultiert in einer Umverteilung der Ventilation von ventral nach dorsal unabhängig von $\mathrm{FIO}_{2}$. Obwohl die tidalen Änderungen des regionalen Lungenvolumens in der dorsalen Region durch den PEEP gesteigert werden konnten, wurde der end-exspiratorische Luftgehalt bei einer $\mathrm{FIO}_{2}$ von 0,21 nur wenig und bei 1,0 nicht beeinflusst. Ein PEEP von $5 \mathrm{cmH}_{2} \mathrm{O}$ könnte daher gerade in der abhängigen Lunge und bei einer $\mathrm{FIO}_{2}$ von 1,0 nicht ausreichen, einen Alveolarkollaps zu verhindern.

Literatur: [1] Magnusson, Spahn: Br J Anaesth 2003; 91:61-72

Mittlere Größenunterschiede der Lufträume der Lunge 180 zwischen Inspiration und Exspiration gemessen mit diffusions-gewichteter 19-Fluor-Magnetresonanztomographie (19-F-dMRT)

A. Scholz, U. Wolf, W. G. Schreiber

Klinik für Anästhesiologie, Klinik und Poliklinik für diagnostische und interventionelle Radiologie der Johannes-GutenbergUniversität Mainz

Der durch diffusions-gewichteter Magnetresonanztomographie (dMRT) erfassbare scheinbare Diffusionskoeffizient (ADC: apparent diffusion coefficient) ermöglicht eine Aussage über die Größe kleinster gasgefüllter Strukturen, wie sie in der Lunge zu finden sind. Mit hochpolarisierten Kontrastgasen wie 3-Helium und 129-Xenon konnten mit dieser Methode bereits pathologisch vergrößerte Lufträume des Lungenemphysems nachgewiesen werden. Das Ziel der vorliegenden Studie war es, mit 19-F-dMRT des Gases Octafluorcyclopropan (C4F8) zu untersuchen, ob die Erweiterung von Lunge und Bronchialsystem durch tiefe Inspiration gegenüber der Exspiration zu einer signifikanten ADC-Erhöhung in der Lunge führt. Fünf anästhesierte Schweine (Deutsches Landschwein, 21,4 kg, Standardabweichung (SD) $3 \mathrm{~kg}$ ) wurden mit einem Gasgemisch aus 30\% $\mathrm{O}_{2}$ und 70\% C4F8 beatmet. Die diffusionsgewichteten 19-F-dMRT-Messungen wurden in Exspirationsstellung und nach einem Liter Inspiration jeweils in Atemanhalte- 
technik durchgeführt. Die Aufnahmen erfolgten mittels „fast low angle shot"-Sequenz in koronarer Projektion in einem 1,5 TeslaTomographen (Magnetom Vision, Siemens Medical Solutions, Erlangen) und einer birdcage-Spule (Rapid Biomedical, Würzburg). MRT-Einstellungen betrugen: Repetitionszeit 16 und $16,6 \mathrm{~ms}$, Echozeit 8,7 und $8,1 \mathrm{~ms}$, Flipwinkel $40 \mathrm{Grad}$, Rohdatenmatrix $64 \times 128$, effektives Messfeld $400 \times 400 \mathrm{~mm}$, b-Wert 49,84 und $30,40 \mathrm{~s} / \mathrm{cm} / \mathrm{cm}$. Es ergaben sich Messzeiten von 57 und 59 Sekunden. Die verwertbaren Einzelversuche $(n=9)$ wurden mittels gepaarten t-Test auf signifikante Unterschiede statistisch untersucht. Das Signifikanzniveau wurde auf 0,05 festgelegt. Das arithmetische Mittel des ADC betrug in Exspiration 0,0129 (SD 0,0028) und in Inspiration 0,0154 (SD 0,0029) $\mathrm{cm} \times \mathrm{cm} / \mathrm{s}$, die Änderung war statistisch signifikant $(\mathrm{p}=0,006)$. Dieses Ergebnis zeigt, dass die 19-F-dMRT eine vom eingeatmeten Gasvolumen abhängige Änderung der „Porengröße“ der Lunge prinzipiell erfassen kann. Denkbare Anwendungsgebiete dieser Methode sind einerseits die Diagnostik regionaler emphysematischer Änderungen und andererseits das experimentelle Monitoring der Größe der Gasräume in der Lunge im Sinne von Überblähung und Atelektase. Allerdings wird mit dieser Methode ein lokaler Mittelwert aller Gasräume der Lunge (aus Atemwegen, Acini und Alveolen) erfasst.

\section{ARDS, ALI}

Protective role of ecto-apyrase (CD39) and ecto-5'-nucleotidase (CD73) in acute lung injury

T. Eckle, L. Füllbier, M. Wehrmann, H. K. Eltzschig

Department of Anesthesiology and Intensive Care Medicine, Tübingen, Institute für Pathology, Universitätsklinikum Tübingen

Acute lung injury (ALI) significantly contributes to morbidity and mortality of critical illness. Due to its anti-inflammatory and barrier-protective properties, we examined the role of extracellular adenosine generation via ecto-apyrase (CD39) and ecto-5'-nucleotidase (CD73) during acute lung injury induced by mechanical ventilation. Following our initial observation that murine CD39/ 73 are induced by mechanical ventilation, we detected pressureand time-dose-dependent increases in albumin leakage pulmonary edema, and neutrophil-accumulation in cd39-/-mice during ventilation. Similarly, pharmacological inhibition or targeted gene-deletion of CD73 was associated with dramatically increased symptom severity of ventilator-induced ALI. Reconstitution of cd39-/-/ cd73-/-mice with soluble apyrase $/ 5^{\prime}$-nucleotidase, respectively, almost completely reversed such increases. Moreover, ALI was significantly attenuated and survival improved after i.p.-treatment of wildtype-mice with apyrase or 5'-nucleotidase. Taken together, these data reveal a previously unrecognized role for CD39/73 in lung protection during mechanical ventilation and suggest treatment with their soluble compounds as novel therapeutic strategy for ALI.

Vergleich der Effekte von inhalativem Iloprost und Stickstoffmonoxid auf das extravaskuläre Lungenwasser unter Volumensubstitution im Modell des akuten Lungenschadens im Schaf

C. Hucklenbruch, H.D. Stubbe, H. van Aken, C. Berger, M. Lange, F. Hinder

Klinik und Poliklinik für Anästhesiologie

und operative Intensivmedizin Universitätsklinikum Münster

Fragestellung: Inhalativ appliziertes Stickstoffmonoxid (iNO) ist ein selektiver pulmonaler Vasodilatator und hat sich in der Therapie des pulmonalen Hypertonus etabliert. Aufgrund des Mangels an positiven Outcome-Daten, Toxizitätsrisiken und der Gefahr eines Rebound-Phänomens nach abruptem Absetzen der Therapie besteht zunehmendes Interesse an alternativen Substanzen. $\mathrm{Zu}$ diesen gehört das Prostazyklin-Derivat Iloprost, das aufgrund seiner deutlich längeren Halbwertszeit diskontinuierlich appliziert werden kann. Wir konnten zeigen, dass iNO im Schafmodell des Sepsis-assoziierten Lungenschadens nicht nur den Pulmonaldruck senkte, sondern auch das begleitende Lungenödem signifikant reduzierte. In der hier vorgestellten Studie wurde die Wirkung von iNO mit Iloprost im Schafmodell des Ölsäure-induzierten Lungenschadens verglichen. Methodik: In 18 anästhesierten, mechanisch ventilierten Schafen wurde ein akuter Lungenschaden durch zentralvenöse Gabe von Ölsäure $(0,1 \mathrm{ml} / \mathrm{kg})$ induziert. Alle Tiere wurden während des neunstündigen Messzeitraums positiv flüssigkeitsbilanziert $(5 \mathrm{ml} / \mathrm{kg} / \mathrm{h})$. Der Atemluft der Tiere der Gruppe I (iNO) wurden nach Induktion des Lungenschadens kontinuierlich 20 ppm NO zugefügt, die Tiere der Gruppe II (Iloprost) erhielten zweistündlich $40 \mu \mathrm{g}$ Iloprost (in einem Vorversuch zur Dosisfindung ermittelt) über einen Ultraschallvernebler. Gruppe III (Kontrolle) erhielt inhalatives $\mathrm{NaCl}$ 0,9\%. Die Quantifizierung des Lungenödems erfolgte mit Hilfe der Thermodilutionsmethode (PiCCOSystem). Ergebnisse: Die Ölsäureinfusion induzierte in allen Tieren einen ausgeprägten Lungenschaden mit signifikantem Anstieg des mittleren pulmonalarteriellen Druckes (MPAD) (Gruppe I: $+55 \pm 6 \%$; Gruppe II: $+45 \pm 7 \%$; Gruppe III: $+41 \pm 13 \%)$ und des extravaskulären Lungenwasserindex (EVLWI) $(+92 \pm 9 \% ;+116 \pm 23 \%$; $+81 \pm 9 \%$ ) (Daten zum Zeitpunkt T0, 0,5 h nach Ende der Ölsäureinfusion). Inhaliertes NO senkte den pulmonalen Gefäßwiderstand (PVRI) $(-52 \pm 13 \%)$, MPAD $(-34 \pm 2 \%)$ und effektiven pulmonalen Kapillardruck (Pceff) $(-28 \pm 2 \%)$ signifikant. Dieser Effekt war mit einem signifikanten Abfall des extravaskulären Lungenwassers assoziiert $(-18 \pm 4 \%)$. Sowohl unter Iloprost-Inhalation als auch in der Kontrollgruppe zeigten sich keine signifikanten Veränderungen von PVRI, MPAD, Pceff sowie EVLWI (Daten jeweils 8 Stunden nach Therapiebeginn, bezogen auf T0). Schlussfolgerungen: Im hier untersuchten Modell führte die Iloprost-Inhalation nicht zu einer mit inhaliertem NO vergleichbaren Senkung von pulmonalem Gefäßwiderstand, MPAD und extravaskulärem Lungenwasser. Dies steht in Kontrast zu anderen Untersuchungen zur Effektivität von inhalativem Iloprost zur Behandlung der pulmonalen Hypertonie. Die im Vergleich zu NO sehr gute Wasserlöslichkeit von Iloprost bedingt möglicherweise die fehlende Wirkung des Iloprosts durch dessen Lösung im, klinisch gesehenen, alveolären Lungenödem.

Die Auswirkung uniaxialer mechanischer Dehnung auf die Expression von E-Selectin in humanen umbilikalen Endothelzellen (HUVEC)

K. Suchodolski ${ }^{1}$, C.T. Mierke ${ }^{2}$, B. Reischl ${ }^{2}$, M. Hedwig-Geissing ${ }^{1}$, J. Schüttler ${ }^{1}$, B. Fabry ${ }^{2}$, W.H. Goldmann ${ }^{2}$ ${ }^{1}$ Anästhesiologische Klinik, ${ }^{2}$ Institut für medizinische Physik, Friedrich-Alexander-Universität Erlangen-Nürnberg

Hintergrund: Ventilatorinduzierter Lungenschaden (VILS) entsteht als Folge aggressiver Beatmung (Barotrauma, Volutrauma) sowie einer durch Entzündungsmediatoren bedingten Leukozyteninfiltration des Lungenparenchyms. E-Selectin, welches an der Oberfläche des Endothels exprimiert wird, ist für das Anhaften und den Durchtritt von Leukozyten durch die Basalmembran mitverantwortlich. Der Mechanismus der E-Selectin-Expression sowie der Einfluss von E-Selectin auf die Entstehung und den Verlauf des VILS sind weitgehend ungeklärt. Ziel dieser Studie war es, den Einfluss von mechanischem Dehnungsreiz auf die Expression von E-Selectin auf der Oberfläche von HUVEC-Zellen zu untersuchen. Methoden: Humane Endothelzellen aus der Nabelschnur (HUVEC) wurden auf mit $5 \mu \mathrm{g} / \mathrm{ml}$ Kollagen beschichteten elastischen Silikonmembranen als Monolayer kultiviert. In der StretchGruppe wurden die Zellen mit dem STREX ST-140-04 Stretcher (B-Bridge, Sunnyvale, CA, USA) über einen Zeitraum von 6 bzw. $24 \mathrm{~h}$ bei $37^{\circ} \mathrm{C}, 5 \% \mathrm{CO}_{2}$ mit einer Dehnungsamplitude von $30 \%$ und einer Frequenz von $11 \mathrm{~Hz}$ uniaxial gedehnt. Als negative 
Kontrollgruppe wurden die Zellen identisch zur Stretch-Gruppe kultiviert, jedoch keiner mechanischen Belastung ausgesetzt. Als positive Kontrollgruppe, für den Nachweis einer E-Selectin-Expression wurden die Zellen mit $10 \mathrm{ng}$ TNF $\alpha$ für 6 bzw. 24 h stimuliert. Die E-Selectin-Expression der HUVEC-Zellen wurde mittels Immounofluoreszenz mit dem Durchflusszytometer FACSCalibur (Becton Dickinson, Heidelberg, Germany) gemessen. Morphologische Veränderungen der Zellstruktur und deren Ausrichtung wurden im zeitlichen Verlauf mikroskopisch erfasst. Ergebnisse: Die E-Selectin-Expression in der mit TNF $\alpha$ stimulierten Gruppe war nach $6 \mathrm{~h}$ (MW \pm SD) mit $80,4 \pm 3,1 \%$ und nach $24 \mathrm{~h}$ mit $50,6 \pm 4,3 \%$ signifikant höher $(\mathrm{p}>0,05)$ verglichen mit der negativen Kontrollgruppe: $3,4 \pm 0,9 \%$ nach $6 \mathrm{~h}$ und $3 \pm 0,1 \%$ nach $24 \mathrm{~h}$ sowie der Stretch-Gruppe: $4,2 \pm 1,3 \%$ nach $6 \mathrm{~h}$ und $3,8 \pm 0,2 \%$ nach $24 \mathrm{~h}$. Es waren keine morphologischen Veränderungen der Zellstruktur und deren Ausrichtung nach 6 bzw. $24 \mathrm{~h}$ uniaxialer Dehnung erkennbar. Zusammenfassung: Weder $6 \mathrm{~h}$ noch $24 \mathrm{~h}$ mechanischer uniaxialer Dehnung (Dehnungsamplitude 30\%, Frequenz $11 \mathrm{~Hz}$ ) führte zur einer erhöhten Expression von E-Selectin auf der Oberfläche von HUVEC-Zellen, noch zu deren morphologischen Veränderungen. Diese Daten sprechen gegen die Hypothese, dass die erhöhte Leukozyteninfiltration nach Baro- bzw- Volutrauma bei Beatmungspatienten durch eine dehnungsinduzierte E-Selektin-Expression von Endothelzellen vermittelt wird.

Respiratorisches Versagen nach Metalldampfinhalation

\section{Held, B. Jany}

Abteilung Innere Medizin, Missionsärztliche Klinik Würzburg

Wir berichten über einen Patienten mit akutem respiratorischen Versagen nach beruflicher Metalldampfinhalation: In unserer Notfallambulanz stellte sich ein bis dahin gesunder Patient mit schwerer respiratorischer Partialinsuffizienz vor. Der 42-Jährige litt außer an Husten, Ruhedyspnoe und Fieber auch an Kopfschmerzen, Erbrechen und Diarrhoe. Im weiteren Verlauf entwickelte sich zusätzlich eine Polyurie. Sowohl konventionell radiologisch als auch computertomographisch stellten sich ausgedehnte konsolidiert wirkende beidseitige pulmonale Infiltrate dar. Die Serumentzündungsparameter waren nur diskret erhöht. Bei initial unklarer Ursache wurde zunächst sowohl eine breite Antibiotikatherapie als auch eine systemische Korticosteroidtherapie eingeleitet. Eine durchgeführte bronchoalveoläre Lavage ergab keine spezifischen Ergebnisse. Es entwickelte sich eine respiratorische Globalinsuffizienz, die eine dreitägige invasive Beatmung erforderte. Unter der maschinellen Ventilation mit zunächst hohen Beatmungsdrücken entwickelte sich ein Pneumothorax. Die erweiterte Anamnese hatte ergeben, dass der Patient wenige Tage vor Krankheitsbeginn eine berufliche Tätigkeit aufgenommen hatte, bei der er verflüssigte Chrom-, Nickel- und Aluminium-Legierungen mittels Sprühpistole auf Oberflächen auftrug. Bereits 8 Monate zuvor hatte er bei einer ersten Exposition ähnliche Symptome in milderer Form entwickelt. Nachdem die Erregersuche negativ blieb wurde die Antibiose nach drei Tagen wieder beendet. Auch die Immunserologien waren negativ. Im Urin fanden sich erhöhte Konzentrationen von Nickel und Chrom. Unter Fortführung der Steroidtherapie kam es rasch und nachhaltig zur Stabilisierung des klinischen Zustandes und zur Rückbildung der pulmonalen Infiltrate. Zur histologischen Sicherung der Diagnose wurde thorakoskopisch ein Lungenkeilresektat gewonnen: Es ergab sich die Diagnose einer kryptogenen organisierenden Pneumonie. Die Steroidtherapie wurde längere Zeit fortgeführt. Die Lungenfunktionsparameter besserten sich im Verlauf kontinuierlich. Schlussfolgerung: Bei akutem respiratorischen Versagen muss auch eine Metalldampfinhalation als Ursache in Erwägung gezogen werden und nach dem histologischen Bild einer kryptogen organisierenden Pneumonie gesucht werden.
Acute lung injury: postconditioning with a volatile anaesthetic

B. Beck-Schimmer ${ }^{1,2}$, T. Yue ${ }^{1,2}$, B. Roth Z'graggen ${ }^{2}$, L. Reyes ${ }^{2}$, C. Booy ${ }^{2}$, M. Steurer ${ }^{1,2}$, D.R. Spahn ${ }^{3}$, E.R. Schmid

${ }^{1}$ Institute of Anaesthesiology, University of Zurich;

${ }^{2}$ Institute of Physiology, University of Zurich;

${ }^{3}$ Department of Anaesthesiology, University of Lausanne

Background: Acute lung injury is a common complication in intensive care patients. Different treatment methods proved to be of rather limited efficacy and lethality remains high. Alveolar epithelial cells (AEC) have been shown to play an important role in the inflammatory response in acute lung injury [1]. Endotoxin-induced injury is a very useful experimental in vitro and in vivo model closely resembling acute lung injury and acute respiratory distress syndrome in humans. Preconditioning with an anaesthetic followed by lipopolysaccharide (LPS) stimulation is promising as recently shown [2]. However, previous knowledge of the onset of the injury is required to provide effective protection. For this reason, intervention with a volatile anaesthetic soon after the insult (postconditioning), might be an even more efficacious strategy to protect an organ against an injury. Most of the postconditioning studies performed thus far have focused on myocardial ischemia-reperfusion damage. Other organs such as the lung and different types of injury including the endotoxin-induced injury have not yet been determined with regard to volatile anaesthetics and postconditioning. Therefore, we examined possible anti-inflammatory effects of the volatile anaesthetic sevoflurane on LPS-stimulated AEC in vitro, mimicking a ,postconditioning situation. Methods: Rat AEC were stimulated with LPS for $2 \mathrm{~h}$, followed by a co-exposure to a $\mathrm{CO}_{2}$ /air mixture with sevoflurane $2.2 \mathrm{Vol} \%$ (for controls only $\mathrm{CO}_{2} /$ air) for another 4 to $24 \mathrm{~h}$. Cytokine-induced neutrophil chemoattractant-1 (CINC-1), monocyte chemoattractant protein-1 (MCP-1), and intercellular adhesion molecule-1 (ICAM-1) were analyzed, and functional assays were performed. Results: A significant biphasic reduction of these inflammatory mediators in LPS-stimulated AEC, exposed to sevoflurane ( $4 \mathrm{~h}$ and $12 \mathrm{~h}$ of postconditioning), was found. Treatment with sevoflurane significantly attenuated protein expression of CINC-1, MCP-1, and ICAM-1 (11-100\%, p <0.05). Chemotaxis was reduced by $48-76 \%(p<0.05)$, neutrophil adherence by $71 \%$ $(\mathrm{p}<0.0001)$, and neutrophil-induced AEC killing by $16 \%$ $(\mathrm{p}<0.0005)$. Conclusions: The data presented in this study provide strong evidence that anaesthetic postconditioning with sevoflurane mediates cytoprotection in the respiratory compartment in acute lung injury by reducing the expression of proinflammatory mediators and in consequence attenuating neutrophil recruitment and effector-target cell interaction. Well-designed in vivo studies, followed by clinical studies are needed to transfer these results of basic research into clinical practice.

References: [1] Beck-Schimmer et al: Eur Respir J 2002; 19: 1142-5110. [2] Reutershan J et al: Anesthesiology 2006; 104:511517

ARDS bei Wegenerschen Granulomatose - Fallbericht

B. Gottschlich, G. Höffken, M. Ragaller, I. Tenner

Klinik für Anästhesiologie und Intensivtherapie;

Medizinische Klinik I

Das Akute Lungenversagen ARDS ist häufig die Folge von Pneumonie, Aspiration, oder Sepsis. In seltenen Fällen kann jedoch auch eine autoimmunologische Erkrankung ursächlich verantwortlich sein. Wir berichten über die Diagnostik und Therapie eines ARDS in Folge einer isolierten pulmonalen Wegenerschen Granulomatose. Kasuistik Eine 36-jährige Patientin $(168 \mathrm{~cm}$; $58 \mathrm{~kg})$ wurde mit akuter Dyspnoe und Zyanose $\left(\mathrm{SpO}_{2}: 60 \%\right)$ aufgenommen. Das Thoraxröntgenbild zeigte ausgedehnte, flächenhafte Infiltrationen beidseits. Es wurde initial die Diagnose ARDS 
bei ambulant erworbener Pneumonie gestellt. Unter druckkontrollierter Beatmung (AZV 6 ml/kgKG; PEEP 14 mbar, Pmax 26 mbar, Af 24/min, $\mathrm{FiO}_{2}$ 0,7), Sedierung, Lagerung und Antibiose konnte der Gasaustausch stabilisiert werden $\left(\mathrm{paO}_{2} \quad 11,8 \mathrm{kPa} ; \mathrm{paCO}_{2}\right.$ $6,7 \mathrm{kPa} ; \mathrm{pH}$ 7,38). Laborchemisch ergab sich kein eindeutiger Hinweis auf eine Infektion (Leukozyten 8,04 Gpt/l; PCT 0,20 $\mu \mathrm{g} / \mathrm{l}$; CRP $115 \mathrm{mg} / \mathrm{l})$. Weder im Bronchialsekret noch in den Blutkulturen konnten Keime nachgewiesen werden, Untersuchungen auf Chlamydien, Mykoplasmen und Legionellen blieben ebenfalls negativ. Rezidivierende Blutungen aus dem Bronchialsystem gaben den Hinweis auf eine pulmonale Manifestation einer Vaskulitis. Die immunologische Diagnostik ergab: cANCA mit 1:1280, sowie die Proteinase-3 mit 148,30 U/ml stark erhöht. Die Kombination von erhöhten cANCA sowie erhöhter PR-3 erhärtete die Diagnose einer Wegenerschen Granulomatose. Neben der allgemeinen Intensivtherapie wurde eine Cyclophosphamid-Pulstherapie mit $500 \mathrm{mg} / \mathrm{m}^{2}$ KOF $(830 \mathrm{mg}$ ) kombiniert mit einer Kortikoidtherapie (Methylprednisolon $500 \mathrm{mg}$ ) durchgeführt. Die Patientin konnte am 7. Tag extubiert, am 12. Behandlungstag auf Normalstation verlegt und am 19. Tag in die ambulante Weiterbehandlung entlassen werden. Diskussion: Für die erfolgreiche Therapie eines ARDS mit schwerer Hypoxie ist, neben einer adäquaten Beatmung die Klärung der Genese des ARDS essentiell. Im vorliegenden Fall musste bei pulmonalen Hämorrhagien differentialdiagnostisch eine Vaskulitis verifiziert werden. Die Bestimmung von cANCA und der Proteinase 3 (PR-3) erlauben innerhalb von $24 \mathrm{~h}$ bei hoher Spezifität und Sensitivität die Sicherung oder den Ausschluss einer Vaskulitis. Im vorliegenden Fall war die Kombination von erhöhten CANCA und erhöhter PR-3 richtungweisend für eine Wegenersche Granulomatose. Die Durchführung einer aggressiven immunsuppressiven Therapie beim beatmeten Intensivpatienten erfordert eine breite antibiotische Abschirmung und die rasche Entwöhnung von der Beatmung. Schlussfolgerung: Eine Wegenersche Granulomatose kann sich isoliert an der Lunge manifestieren und $\mathrm{zu}$ einem ARDS führen. Mit Hilfe der Bestimmung von cANCA und Proteinase-3 kann die Diagnose rasch und sicher gestellt werden. Während der aggressiven, immunsuppressiven Therapie sind eine ausreichende antibiotische Abschirmung und ein engmaschiges infektiologisches Monitoring erforderlich.

Mobilisation beatmeter Patienten in $60^{\circ}$-Position - eine Pilot-Studie -

\section{A. Schink, B. Wind, C. Boerner, D. Dueck}

Zentrale Intensiv- und Notfallmedizin (ZIN)

Klinikum der Universität zu Köln

Einführung: Im akuten respiratorischen Versagen führt bei beatmeten Patienten ein Wechsel von der Rücken- zur Bauchlage zu einer Verbesserung der Oxygenation. Allerdings führen zahlreiche Kontraindikationen zu einer Limitierung des Verfahrens und eine echte Verbesserung des Outcomes der Patienten konnte bisher nicht gezeigt werden. Andererseits konnte nachgewiesen werden, dass eine $40-45^{\circ}$-Lagerung von Patienten im ARDS den Gasaustausch signifikant verbessert und die Rate an nosokomialen Pneumonien senkt. Ziel dieser Pilot-Studie war es, erstmals Daten zur Effektivität einer Mobilisation von Beatmungspatienten in einer beinahe stehenden $60^{\circ}$-Position zu erheben. Methodik: An $9 \mathrm{~Pa}-$ tienten, die mindestens $48 \mathrm{~h}$ beatmet waren, wurden als BaselineMessung in Rückenlage folgende kardiovaskuläre und pulmonale Parameter erhoben: Herzfrequenz (HF), mittlerer arterieller Druck (MAP), zentralvenöser Druck (ZVD), Herzzeitvolumen (HZV), systemischer vaskulärer Widerstand (SVR), Atemminutenvolumen (AMV), Compliance (C), funktionelle Residualkapazität (FRC), $\mathrm{paO}_{2}$ und $\mathrm{paCO}_{2}$. Daraufhin wurde der Patienten in einen multifunktionalen Mobilisationsstuhl (Fa. Arjo International AG, Basel, Schweiz) in eine stehende $60^{\circ}$-Position gebracht. Die anschließende Erfassung der o.g. Parameter erfolgte zu weiteren 5 Messzeitpunkten: (1) unmittelbar nach Erreichen der $60^{\circ}$-Position (2) nach $1 \mathrm{~h}$ in $60^{\circ}$-Position (3) nach $2 \mathrm{~h}$ in $60^{\circ}$-Position (4) unmittel- bar nach Erreichen der Ausgangslage (5) $1 \mathrm{~h}$ nach Erreichen der Ausgangslage. Sämtliche Messungen wurden bei einer $\mathrm{FiO}_{2}=1,0$ durchgeführt. Resultate: Wir fanden im Mittel eine 50\%ige Erhöhung der FRC in der $60^{\circ}$-Position $(1,2$ vs. 1,9 l) sowie eine ca. 10\%ige Erhöhung nach $1 \mathrm{~h}$ in der Ausgangslage. Die Compliance war in Ausgangsposition um ca. $20 \%$ erhöht $(50$ vs. $68 \mathrm{ml} /$ $\mathrm{mmHg}$ ). Das AMV bei paCO $=40 \mathrm{mmHg}$ sank von $10,1 \mathrm{l} / \mathrm{min}$ auf $8,2 \mathrm{l} / \mathrm{min}$. Der $\mathrm{paO}_{2}$ war $1 \mathrm{~h}$ nach Erreichen der Ausgangslage erhöht ( 334 vs. $396 \mathrm{mmHg}$ ). Die kardiovaskulären Parameter verhielten sich wie folgt: der MAP stieg nach $2 \mathrm{~h}$ in $60^{\circ}$-Position von 75 auf $89 \mathrm{mmHg}$, die HF von 77 auf 94/min. Der ZVD sank nach $1 \mathrm{~h}$ von 15,7 auf 9,7 mmHg. Das HZV war im Stehen kaum verändert $(6,5$ vs. $6,91 / \mathrm{min})$ und der SVR war von 720 auf $916 \mathrm{dyn} \cdot \mathrm{s}^{-1} \cdot \mathrm{cm}^{-5}$ angestiegen. Diskussion: Wir fanden eine verbesserte Oxygenierung sowie eine reduzierte Totraumventilation. Die Complianceerhöhung weist auf eine Verbesserung der Lungenmechanik hin. Der diskrete Anstieg der FRC deutet an, dass Lungenareale rekrutiert werden konnten. Die Lagerung der Patienten in stehender $60^{\circ}$-Position wurde vom kardiovaskulären System erstaunlich gut kompensiert. In keinem der Fälle musste die Lagerungsmaßnahme abgebrochen werden. Die Verbesserung der pulmonalen Parameter bei stabilen Kreislaufverhältnissen lässt die aufrechte Positionierung von beatmeten Patienten als mögliche Lagerungsalternative im Rahmen der mechanischen Beatmung kritisch kranker Patienten erscheinen.

Therapie eines schweren ARDS bei einem Schädel-Hirn177 traumatisierten Patienten - Ein Fallbericht

\section{F. Bach, R. Borgstedt, R. Grünzig, G. Braun, F. Mertzlufft}

Klinik für Anästhesiologie und Intensivmedizin,

Evangelisches Krankenhaus Bielefeld; 33617 Bielefeld - Bethel

Hintergrund: Die Prognose des Schädel-Hirn-Traumas (SHT) wird durch Art und Umfang des Primärtraumas sowie durch Vermeiden und Minimieren von Sekundärschäden bestimmt. Therapeutisches Ziel ist die Optimierung von Homöostase-Parametern, insbesondere sind Normoxie und Normokapnie eine Voraussetzung zur Optimierung von Perfusion und Energiestoffwechsel im ZNS. Fallbericht: Wir berichten von einem 13-jährigen Patienten, der mit einem schweren, isolierten SHT (GCS 5) nach Verkehrsunfall hypotherm $\left(35,9^{\circ} \mathrm{C}\right)$ in die Klinik eingeliefert wurde. Nach initialer Stabilisierung und Diagnostik wurden die Ausräumung eines akuten epiduralen Hämatoms und eine Dekompressionskraniektomie durchgeführt. Postoperativ wurde eine therapeutische Hypothermie mit der Zieltemperatur von $32,0^{\circ} \mathrm{C}$ über 5 Tage eingeleitet und der Patient bei zunächst unauffälligem pulmonalen Gasaustausch druckkontrolliert (BIPAP ${ }^{\circledR}$, EvitaXL) beatmet. Auf dem Boden einer beatmungsassoziierten Pneumonie bot sich am 5. postoperativen Tag das Bild eines septischen Schocks und eines akuten Lungenversagens (ARDS). Additiv zu der an aktuellen Leitlinien ausgerichteten Sepsistherapie [1], einer organprotektiven Beatmung [2] sowie kinetischen Therapie (Rotorest-Bett) wurde bei hyperkapnischen und hypoxischen Blutgaswerten ein „arteriovenöses extrakorporales Assist-Device“ (Nova-Lung ${ }^{\circledR}$ ) installiert. Darunter normalisierten sich zunächst die $\mathrm{paCO}_{2}$-Werte bei jedoch fortbestehender arterieller Hypoxie (Oxygenierungs-Indexmin: 38,1). Als Ultima ratio wurde mit einer Hochfrequenz-Oszillations-Ventilation (HFOV, Sensormedics 3100B) begonnen (Frequenz: $9 \mathrm{~Hz}$, Mittlerer Druck: $35 \mathrm{mbar}, \mathrm{FiO}_{2}: 1.0$ ). Unter diesem Regime verbesserten sich Gasaustausch und systemische Entzündungsparameter kontinuierlich, so dass nach drei Tagen unter erneut konventioneller Beatmung ein Weaning-Prozess eingeleitet werden konnte. Letztlich konnte der Patient 27 Tage nach Aufnahme auf die Intensivstation mit stabilen Vitalparametern, spontan atmend und mit insgesamt gutem neurologischen Outcome zur Rehabilitation verlegt werden. Diskussion: Der klinische Verlauf dieses Patienten zeigt die besondere Problematik der zum Teil kontroversen Ansätze zur Therapie eines ARDS einerseits und eines SHT andererseits. An Hand dieses Einzelfalles konnte gezeigt 
werden, dass durch kombinierten Einsatz eines extrakorporalen Assist-Device und einer HFOV in Verbindung mit einer standardisierten Sepsistherapie eine absolut kritische Situation beherrscht werden konnte. Aufgrund fehlender zerebraler Druckmessung (Dekompressionskraniektomie) konnten keine Daten bezüglich des Verlaufes von Hirndruck und zerebralem Perfusionsdruck unter einem Beatmungsregime mit hohem Beatmungsdruck erhoben werden. Allerdings spricht das gute neurologische Outcome des Patienten gegen diesbezügliche nachhaltige Limitierungen.

Literatur: [1] Dellinger RP et al: Crit Care Med 2004; 32:858873. [2] ARDS-Network: N Engl J Med 2000; 342:1301-1308

Einfluss von inhalativem Stickstoffmonoxid (iNO) 200 und assistierten Spontanatmungsverfahren auf die 0xygenierung beim Lungenversagen

\section{S. Terbeck, R. Dembinski, N. Hochhausen, R. Bensberg,}

\section{F. Stadermann, R. Kuhlen}

Operative Intensivmedizin des Universitätsklinikums der RWTH Aachen

Hintergrund: Der klinische Nutzen von inhalativem Stickstoffmonoxid (iNO) als selektiver pulmonaler Vasodilatator ist gut belegt und wird als Rescue-Therapie bei schwerster Hypoxämie von Patienten mit akutem Lungenversagen (ARDS) angewendet. Neben einer Optimierung des Ventilations-Perfusions-Verhältnisses kann der Gasaustausch verbessert werden, ohne dass bisher eine Senkung der Letalität von ARDS-Patienten gezeigt werden konnte. Zudem kann eine Beatmungstherapie mit erhaltener Spontanatmung auf die Verteilung von Ventilation und Perfusion von Vorteil sein. Unklar ist bisher, welches der zur Verfügung stehenden Spontanatmungsverfahren den größten Benefit bringt. Ziel dieser Studie ist, die Evaluierung der additiven Effekte der pulmonalen Vasodilatation mittels iNO in Kombination mit verschiedenen Spontanatmungsverfahren tierexperimentell beim Lungenversagen zu untersuchen. Methodik: Das akute Lungenversagen (ALI) wurde mittels einer standardisierten Methode (repetitive bronchoalveoläre Lavage mit physiologischer Kochsalzlösung) am Tiermodell induziert. Wir verglichen an 24 Schweinen mit ALI, randomisiert in 4 Gruppen, 4 verschiedene Spontanatmungsverfahren, Assist-Control (ACV), Pressure Support (PS), Biphasic Positive Airway Pressure (BIPAP), Volume Assured Pressure Support (VAPS), mit einem kontrollierten Beatmungsverfahren (Controlled Mechanical Ventilation, CMV) in Kombination mit und ohne iNO. Nach einstündiger Beatmung in jeder Gruppe (Spontanatmungsverfahren und CMV) wurde anschließend für weitere 15 Minuten iNO $(10 \mathrm{ppm})$ appliziert. Wir untersuchten das Ventilations-PerfusionsVerhältnis (VA/Q) bei jedem Beatmungsmodus jeweils mit und ohne iNO. Die Gasaustauschfunktion der Lunge wurde bei jeder Messung mit der Blutgasanalyse und der multiplen Inertgas-Eliminationstechnik (MIGET) analysiert. Ergebnisse: In jeder Gruppe erhöht iNO die Perfusion von Lungenbezirken mit normaler VA/Q Verteilung und reduziert den Shunt während assistierter und kontrollierter Beatmung. Wohingegen während der reinen, assistierten Spontanatmungsverfahren eine signifikante Verbesserung des VA/Q-Verhältnis nur während PS und BIPAP zu erkennen ist. Die Optimierung des VA/Q-Verhältnisses bei der Anwendung dieser beiden Modi (PS und BIPAP) kombiniert mit iNO verglichen mit CMV plus iNO zeigte untereinander keine statistische Signifikanz. Schlussfolgerung: Das Ventilations-PerfusionsVerhältnis kann sowohl mit iNO als auch mit assistierter Spontanatmung bei PS und BiPAP verbessert werden. Durch die Kombination (iNO plus PS, iNO plus BIPAP) konnte in dieser Studie keine positiven additiven Effekte auf den Gasaustausch gezeigt werden, möglicherweise aufgrund eines bereits zuvor durch die Spontanatmung reduzierten intrapulmonalen Shunts.
Inhalatives NO-therapeutische Option

des akuten posttraumatischen Lungenversagens?

C. Schmidt

Städtisches Klinikum Dessau/Universitätsklinikum

Gießen-Marburg

Einleitung: Das Ventilations-Perfusions-Missverhältnis mit Ausbildung intrapulmonaler Rechts-Links-Shunts stellt einen wesentlichen Pathomechanismus des akuten Lungenversagens im Rahmen eines Polytrauma oder als Folge einer Lungenkontusion dar. Der infolge des Shuntvolumens steigende pulmonalarterielle Druck kann die weitere Ausbildung eines Permeabilitätsödems mit zunehmender Surfactantstörung begünstigen. Neben den evidenzbasierten Therapieoptionen des akuten Lungenversagens wie lungenprotektiver Beatmung, permissiver Hyperkapnie, Beatmung mit PEEP und Lagerungstherapie stehen heute supportive Therapieoptionen zur Verfügung, zu denen neben speziellen Beatmungsformen auch der Einsatz von inhalativem NO oder Prostacyclinen gehört. Material und Methoden: An ausgewählten Patientenbeispielen mit schwerem, posttraumatischen Lungenversagen (Horovitz-Oxygenierungsquotienten $<100$ bei $\mathrm{FiO}_{2}=1,0$ ) und fehlender Besserung des Lungenversagens unter Ausnutzung der evidenzbasierten Therapieoptionen (protektive Beatmung, permissive Hyperkapnie, PEEP und Lagerungstherapie) wurde supportiv inhalatives NO in einer Konzentration von $10 \mathrm{ppm}$ eingesetzt. Neben der Auswirkung auf die Oxygenierung wurden die Auswirkungen auf Hämodynamik, extravaskuläres Lungenwasser (EVLW) und kapillären Permeabilitätsindex sowie die Veränderungen der Compliance untersucht. Ergebnisse: In 2 ausgewählten Patientenbeispielen mit akutem, posttraumatischen Lungenversagen infolge stumpfem Thoraxtrauma mit Lungenkontusion und bestehendem hämodynamischen Monitoring setzten wir bei persistierender Hypoxämie (Horovitz $<100$ bei $\mathrm{FiO}_{2}=1,0$ ) inhalatives $\mathrm{NO}$ in einer Konzentration von $10 \mathrm{ppm}$ supportiv $\mathrm{zu}$ den evidenzbasierten Therapieoptionen im Rahmen einer diskontinuierlichen Applikation ein. Im beobachteten Applikationszeitraum von jeweils 36 Stunden konnte eine rasche Besserung der arteriellen Oxygenierung mit einem Anstieg des Horovitz-Quotienten jeweils von $<100$ auf $>250$ beobachtet werden. Die Parameter Herzindex, systemischer Widerstand und arterieller Blutdruck blieben unverändert. In beiden Fällen konnte eine Abnahme des kapillären Permeabilitätsindex und des extravaskulären Lungenwassers (EVLW) beobachtet werden, parallel dazu bestand eine Besserung der Compliance. Schlussfolgerung: Der Einsatz von inhalativem NO ist beim schweren und gegenüber den evidenzbasierten Therapieverfahren therapierefraktärem posttraumatischen Lungenversagen ein supportives Therapieverfahren, mit welchem im Rahmen einer Early-goal-directed therapy sekundäre Traumafolgen durch Hypoxämie vermieden werden können. Neben einer deutlichen Verbesserung der Oxygenierung durch Verminderung des pulmonalen Shuntvolumens besteht ein weiterer Vorteil in der Verminderung des Permeabilitätsödems durch Senkung des pulmonalarteriellen Druckes und damit in der Verminderung eines sekundären Surfactantschadens, was sich in einer Stabilisierung der Compliance und einem durch Rückgang des EVLW und der kapillären Permeabilität zeigt.

Hochfrequenzoszillation versus konventionelle lungenprotektive Beatmung im Großtier ARDS-Modell

R.M. Muellenbach, M. Kredel, H. Said, B. Klosterhalfen *, M. Schmidt ${ }^{*}$, B. Zollhöfer, H. Wilckens, C. Wunder, N. Roewer, J. Brederlau

Klinik und Poliklinik für Anästhesiologie und ${ }^{*}$ Abt. für Pneumologie, Julius-Maximilians-Universität, Würzburg; ${ }^{\star}$ Institut für Pathologie, Krankenhaus Düren

Fragestellung: Eine Maximalvariante der lungenprotektiven Beatmung könnte die Hochfrequenzoszillation (HFOV) darstellen. In Kleintierstudien konnte damit eine Reduktion der beatmungsasso- 
ziierten Lungenschädigung (VILI) nachgewiesen werden [1]. Wir untersuchten im Großtier-ARDS-Modell, wie sich Gasaustausch, inflammatorische Zytokinfreisetzung und Histopathologie im Langzeitvergleich von HFOV und konventioneller lungenprotektiver Beatmung (PCV) unterscheiden. Material und Methoden: Mit Genehmigung der Bezirksregierung wurde bei 16 Schweinen $(55,2+3,9 \mathrm{~kg})$ durch wiederholte $\mathrm{NaCl}$-Lavage ein akutes Lungenversagen (Ali) induziert. Nach Randomisierung in zwei Gruppen von je 8 Tieren (Gruppe 1: PCV; $\mathrm{Vt}=6 \mathrm{ml} / \mathrm{kg} \mathrm{KG;} \mathrm{f}=30-40 / \mathrm{min}$; Gruppe 2: HFOV; Amplitude $=60-80 \mathrm{cmH}_{2} \mathrm{O} ; \mathrm{f}=360 / \mathrm{min}$ ) wurde ein Rekrutierungsmanöver $\left(50 \mathrm{cmH}_{2} \mathrm{O}\right.$ über $60 \mathrm{Sek}$.) bei einer $\mathrm{FiO}_{2}$ von 1,0 durchgeführt. Danach wurde der Atemwegmitteldruck (mPaw) schrittweise erhöht, solange dies von einer $\mathrm{PaO}_{2}$-Steigerung begleitet war. Dann wurden die $\mathrm{FiO}_{2}$ bis auf 0,4 und der mPaw schrittweise bis zum Ziel- $\mathrm{PaO}_{2}$ von $90-110 \mathrm{mmHg}$ abgesenkt. Gasaustausch und Hämodynamik wurden kontinuierlich über den 24-stündigen Versuch gemessen. Die Konzentrationen von TNF- $\alpha$, IL-1- $\beta$, IL- 6 und IL-10 in der bronchoalveolären Lavage (BAL) wurden zu den Zeitpunkten 0 und $24 \mathrm{~h}$ bestimmt. Die mRNA-Expression der Zytokine im Lungengewebe wurde außerdem am Versuchsende mittels semiquantitativer RT-PCR gemessen. Die Lungenschnitte wurden histologisch begutachtet. Statistik: Two-way ANOVA und Mann-Whitney Rank Sum Test $(\mathrm{p}<0,05)$. Ergebnisse: Der Oxygenierungsindex $(\mathrm{OI})$ war in beiden Gruppen nach Rekrutierung signifikant gegenüber Ali und nach 6 und $18 \mathrm{~h}$ signifikant in Gruppe 2 gegenüber Gruppe 1 verbessert (6 h: PCV 7,6 $\pm 3,4$ vs. HFOV 2,7 $\pm 1,4,18$ h: PCV 7,1 $\pm 2,1$ vs. HFOV $3,3 \pm 0,7 ; p<0,05)$. Der mPaw war in Gruppe 2 nach 6-24 h gegenüber Gruppe 1 und Ali signifikant erniedrigt. Die Konzentration von TNF- $\alpha$, IL-1- $\beta$ und IL- 6 in der BAL war am Versuchsende in beiden Gruppen erhöht, ohne signifikante Unterschiede zwischen den Gruppen. Histopathologisch zeigte sich eine signifikante Erniedrigung der Inflammationsparameter in der HFOV-Gruppe $(p<0,05)$. Es ließen sich jedoch keine signifikanten Unterschiede im Gesamtschädigungsscore nachweisen. Die mRNA-Expression von IL-1- $\beta$ im Lungengewebe war signifikant in der HFOV-Gruppe reduziert $(\mathrm{p}<0,05)$. Schlussfolgerungen: Wir konnten durch Rekrutierung eine anhaltende Verbesserung der Oxygenierung in beiden Gruppen erreichen. In der HFOV-Gruppe kam es histologisch zu einer signifikanten Erniedrigung der Inflammationsparameter sowie zu einer Reduktion der mRNA-Expression von Il-1- $\beta$. Erniedrigte mPaw's sowie kleinere Tidalvolumina während der HFOV könnten im Großtier-ARDS-Modell zu einer weiteren VILI-Reduktion führen.

Literatur: [1] Imai Y et al: Comparison of lung protection strategies using conventional and high-frequency oscillatory ventilation. J Appl Physiol 2001; 91:1836-1844

Das toxische Inhalationstrauma bei Brandverletzten

230 im Vergleich zur militär-historisch beschriebenen Wirkung von Lungenkampfstoffen - eine Fallstudie

\section{R. Wahba, Ludwig, Sieber, Göller, E. Kollig}

Klinik für Unfallchirurgie/Wiederherstellungschirurgie/

Handchirurgie/Verbrennungsmedizin Bundeswehrzentralkranken-

haus Koblenz

Einleitung: Das toxische Inhalationstrauma (IHT) trat mit dem Einsatz chemischer Lungenkampfstoffe (Phosgen) im 1. Weltkrieg in den Blick der Medizin. Eine kausale Therapie, die das Überleben der Soldaten sicherte, existierte nicht. Es wurden Aderlässen bis zur künstlichen Beatmung mit $\mathrm{O}_{2}$ über Maske versucht. Der Tod trat aber sofort, nach 3-4 Tagen oder maximal 4 Wochen ein. Pathologisch zeigte sich eine massive Zerstörung des Bronchialund Alveolargewebes mit Lungenödem, bei längerem Überleben Hämorrhagien und Emphysembildung und später das Bild einer Bronchiolitis Obliterans. Heute tritt das toxische IHT bei Bränden mit Kunststoffverbrennung auf. 20-35\% aller Brandverletzten erleiden ein IHT (Letalität 60\%). Es kommt zur Mucosaschädigung in Trachea und Bronchien unter Ödembildung mit Exsudation in den Alveolarraum, Atelektasen- und Emphysembildung (bis zu $48 \mathrm{~h}$ ). Es folgen massive Zelluntergänge (bis $72 \mathrm{~h}$ ) und eine Bindegewebsproliferation ( $a b$ dem 4. Tag). Nur maschinelle Beatmung oder ECMO bleiben als Therapie. Fallreport: Beim Löschen einer brennenden Kunststoffduschkabine inhalierte ein 36-jähriger Mann die freiwerdenden Gase. Etwa $3 \mathrm{~h}$ später im BWZK war der Patient orientiert, kreislaufstabil und atmete schwerfällig spontan unter $8 \mathrm{llO}_{2}$ über Maske bei peripherer $\mathrm{O}^{2}$-Sättigung von $96 \%$. Ohne $\mathrm{O}_{2}$-Maske wurde er panisch. $5 \%$ der Körperoberfläche wiesen tropfenartige Verbrennungen $3^{\circ}$ auf. Bei feuchten Rasselgeräuschen über allen Lungenabschnitten lieferte die erste arterielle $\mathrm{BGA}$ einen $\mathrm{PO}^{2}$ von 68 mbar. Es erfolgte die Intubation und lungenprotektive Beatmung mit hohem PEEP. Die Bronchoskopie bestätigte ein $2^{\circ}-3^{\circ}$ IHT mit ischämischer-ödematöser Mucosa, Hämorrhagien und Nekrosen. Unter intensivmediznischer Maximaltherapie mit Wechsellagerung kam es im Verlauf zu keiner wesentlichen Besserung der Lungenfunktion. Eine Extubation war zu keinem Zeitpunkt möglich. Bei Pneumonie (3fach Antibiose) kam es am 30. Tag zum Nierenversagen, so dass eine CVVHDF durchgeführt wurde. Trotz chemischer und manueller Reanimation kam es am 41. Behandlungstag zum Exitus letalis. Die Obduktion zeigte unbelüftete hochgradig flüssigkeitsreiche Lungen im Sinne eines protrahierten respiratorischen Distress-Syndroms. Histologisch ergab sich eine Schocklunge $\mathrm{III}^{\circ}$ mit Bronchiolitis Obliterans, Hämorrhagien und disseminierten intravasalen Thromben (Lungengewicht $4025 \mathrm{~g}$ ). Todesursache war ein rechtsführendes Herz-Kreislauf-Versagen bei akuter Leberdystrophie. Ergebnis: Das toxische IHT bei Wohnungsbränden mit Kunststoffverbrennungen ist mit einer inhalativen Lungenschädigung durch militärische Kampfgase wie Phosgen zu vergleichen. Die klinischen und pathologisch-histologischen Befunde zeigen bei beiden Krankheitsbildern ähnliches. Die bereits durch Aschoff (1916), Koch (1921) und Richter (1941) beschriebenen Veränderungen können im Rahmen dieser Fallstudie bestätigt werden. Trotz moderner Intensivmedizin- und Beatmungstherapiekonzepte ist die Prognose auch heute noch infaust.

Hämodynamik und Gasaustausch beim Einsatz

einer pumpenlosen, arteriovenösen, extrakorporalen

097 Lungenunterstützung während Reanimation im Tiermodell

G. Zick, D. Schädler, G. Schmitz, I. Frerichs, S. Pulletz, J. Scholz, N. Weiler

Klinik für Anästhesiologie und Operative Intensivmedizin, Universitätsklinikum Schleswig-Holstein

Einleitung: Ein pumpenloses, arteriovenöses System zur extrakorporalen Lungenunterstützung (Interventional Lung Assist, ILA, Novalung, Hechingen, Germany) kann bei Patienten mit ARDS zu einer lungenprotektiven Beatmungsstrategie beitragen. Ziel dieser Studie war es, die Effekte dieses Systems auf Hämodynamik und Gasaustausch unter den Bedingungen einer Reanimation zu untersuchen und zu klären, ob das Klemmen des Systems dabei vorteilhaft ist. Methodik: Die Untersuchung wurde als kontrollierte randomisierte Studie nach Zustimmung durch die Tierschutzkommission an 12 anästhesierten Schweinen durchgeführt. Das ILA-System wurde an einen $15 \mathrm{~F}$ Katheter in der A. femoralis und einen $17 \mathrm{~F}$ Katheter in der V. femoralis angeschlossen. Ein akutes Lungenversagen wurde durch wiederholte bronchoalveoläre Lavage herbeigeführt bis der arterielle Sauerstoffpartialdruck (Pao2) von unter 100 Torr bei Beatmung mit $100 \% \mathrm{O}_{2}$ für mindestens $30 \mathrm{Mi}$ nuten erreicht wurde. Mittels eines transvenösen Schrittmachers wurde Kammerflimmern ausgelöst und sofort mit manuellen Thoraxkompressionen begonnen, die 30 Minuten lang durchgeführt wurden. Bei 6 Tieren wurde das ILA-System offen gelassen, bei den anderen 6 Tieren sofort geklemmt. Das Monitoring wurde mit PA-Katheter, kontinuierlicher Blutgasmessung und einer Flussmessung im ILA-Schlauchsystem durchgeführt. Ergebnisse: Bei keinem der 12 Tiere in beiden Gruppen konnte nach der 
Reanimation ein eigener Kreislauf wiederhergestellt werden. Die arteriellen, zentralvenösen und pulmonalarteriellen Blutdruckwerte unter Reanimation waren in beiden Gruppen vergleichbar. Die Pao2-Werte waren in der Gruppe mit offenem ILA-System höher, die Paco2-Werte niedriger (nach $20 \mathrm{~min}$ : Pao2 $191 \pm 140 \mathrm{mmHg}$ vs. $57 \pm 14 \mathrm{mmHg}, \mathrm{Paco} 229 \pm 20 \mathrm{mmHg}$ vs. $80 \pm 25 \mathrm{mmHg}$ ). Bei 5 der 6 Tiere mit offenem System trat eine Flussumkehr im ILASystem auf (im Median nach $20 \mathrm{~min}$ ). Schlussfolgerungen: Bei vergleichbaren Blutdruckwerten in beiden Gruppen wurden $\mathrm{PaO}_{2}$ und $\mathrm{PacO}_{2}$ durch das ILA-System günstig beeinflusst. Beim Eintreten einer Flussumkehr war dieser Effekt nicht mehr vorhanden. Das ILA-System scheint im Tiermodell keine nachteiligen Auswirkungen bei einer Reanimation zu haben. Spätestens beim Auftreten einer Flussumkehr im ILA-System sollte das System aber geklemmt werden.

\section{Kardiologische Intensivmedizin}

\section{Herzinfarkt, kardiogener Schock, Herzinsuffizienz, Reanimation}

\section{Zeitnahes modernes Management bei Herzinfarktbehandlung}

\section{U. Walter, C. Lange, Dr. H. Thiele}

Klinik für Innere Medizin/Kardiologie Herzzentrum Leipzig GmbH

Modernes zeitnahes Management des Herzinfarktes erfordert die ständige Optimierung der Ablauforganisation bei Patienten mit akutem ST-Hebungs-Infarkt. Im November 2003 durchgeführte Vergleiche zwischen drei Leipziger Zentren die an der Versorgung von Patienten mit akutem Herzinfarkt teilnehmen, zeigten sehr unterschiedliche Zeiten von der Alarmierung des Notarztes bis zur Herzkatheteruntersuchung. Zur Verbesserung der Versorgung der Patienten mit einem STEMI (ST-Strecken-Hebungsinfarkt) wurden im Herzzentrum Leipzig alle räumlichen und zeitlichen Komponenten erfasst, die zur Verkürzung des Zeitfensters beitragen können. Voraussetzung für die Ablaufoptimierung ist eine frühzeitige Ankündigung des Patienten durch den Notarzt oder das zu verlegende Krankenhaus. Als Fazit der Erfassung werden die für eine percutane Intervention nicht relevanten Tätigkeiten, wie zwischenzeitiges Monitoring auf der Intensivstation oder in der Notaufnahme, erneutes Ableiten eines 12-Kanal-EKG auf Papier, Blutentnahmen zur Enzymbestimmung, zeitaufwendige Erklärungen und nicht sofort benötigte Untersuchungen - wie das transthorakale Echo - erst nach der Herzkatheteruntersuchung durchgeführt. Wenn ein freier Herzkatheterplatz zur Verfügung steht wird in Ausnahmefällen auch mit der Transportliege des Notdienstes direkt in den Kathetersaal gefahren, um dem hochakuten Patienten ein zusätzliches Umbetten zu ersparen. Erklärtes und angestrebtes Ziel war: Senken der "Door-to-Balloon-Zeit“ (D-t-B) vom Eintreffen im Krankenhaus bis zur Entfaltung des Ballons im Herzkatheterlabor auf deutlich weniger als 30 Minuten bei angekündigten Patienten und $<60$ Minuten bei unangekündigten Patienten. Um im verkürzten Zeitfenster alle für die Betreuung des Patienten erforderlichen administrativen, medizinischen und pflegerischen Tätigkeiten zu realisieren, wurde das Management verändert, es wurde ein standardisiertes Ablaufschema (Protokoll) erstellt und dieses wird bis heute genutzt. Das erarbeitete Formular sichert das Nachvollziehen der zeitlichen Abläufe, die Vollständigkeit der Datenerfassung und der Untersuchungen, dient gleichzeitig der Qualitätskontrolle und liefert zusätzlich Daten für wissenschaftliche Untersuchungen. Ergebnisse: Die D-t-B-Zeiten von 2001-2003 für angekündigte Pat. mit STEMI 67 \pm 21 min, für unangekündigte Pat. $95 \pm 37$ min. Nach Einführung des STEMIProtokolls wurden die D-t-B signifikant reduziert. Die Umstellung der gesamten Arbeitsabläufe und eine hohe Motivation beim Pflegepersonal brachten sehr schnell signifikante Ergebnisse. Die durchschnittliche „Door-to-balloon-Zeit“ für angekündigte Infarktpatienten lagen bei $25 \pm 7 \mathrm{~min}$. Um eine gleich bleibend gute, zeitnahe Versorgung zu erreichen, müssen die ermittelten Zeiten regelmäßig ausgewertet und stark abweichende Ergebnisse problembezogen hinterfragt/diskutiert werden. Die Einbeziehung möglichst vieler am Ablauf beteiligter Pflegekräfte hat sich bewährt.

Critical role of the 5 '-ectonucleotidase (CD73) and the adenosine A2B receptor in cardiac ischemic preconditioning

T. Eckle, A. Grenz, D. Köhler, M. Faigle, M. Wehrmann, J. Schneermann, L.F. Thompson, H. Osswald, H.K. Eltzschig Department of Anesthesiology and Intensive Care Medicine, Institute of Parmacology und Pathology, Universitätsklinikum Tübingen

Cardiac ischemic preconditioning (IP) has been described as the strongest form of in vivo protection during acute myocardial infarction (AMI). Recent studies have shown that increased nucleotide phosphohydrolysis (CD73 conversion of AMP to adenosine) and nucleoside signalling via adenosine receptors may be critical for the cardioprotective effects of IP. However, such studies have been limited to pharmacological approaches or indirect lines of evidence. To further identify the contribution of extracellular generated adenosine by CD73 and the following adenosine signalling for IP, our aim was to study IP in mice with genetic disruption of CD73 and of the four adenosine receptors (A1, A2a, A2b, A3). After the development of a novel murine model of IP we first investigated the relative mRNA expressional levels of CD73. After IP CD73 mRNA was upregulated in the preconditioned myocardium. Immunhistochemistry and enzyme activity measurements showed induction of CD73 protein and functional enzyme after IP as well. Pharmacological inhibition of CD73 abolished the infarct size limiting effect of IP. Investigation of cd73-/- mice showed significantly bigger infarct sizes than in littermate controls and the infarct size limiting effect of IP was not seen. Adenosine infusion in cd73-/- mice could partly reconstitute the protective effect of IP. After having shown that extracellular generated adenosine by CD73 was crucial for the cardioprotective effects of IP we investigated the relative mRNA expressional levels of the adenosine receptors. After IP the Ado A2b mRNA was strongly upregulated in the preconditioned myocardium. Following analysis of the adenosine receptor deficient mice showed that infarct size reduction due to IP could be seen in the AdoR A1-/-, AdoR A2a-/- and AdoR A3-/- -mice but not in the AdoR A2b-/- -mice. Immunhistochemistry and western blot analysis of the $\mathrm{A} 2 \mathrm{~b}$ receptor showed induction after IP according to mRNA results. Specific inhibition of the A2b receptor with PSB 1115 abolished the infarct size limiting effect. Thus our data reveal for the first time direct in vivo evidence that $\mathrm{CD} 73$ and the $\mathrm{A} 2 \mathrm{~b}$ receptor are required for IP in the heart. The A2b receptor will allow the use of novel specific $\mathrm{A} 2 \mathrm{~b}$ agonists in the treatment of acute myocardial infarction. 
Serumkonzentration von Neuronenspezifischer Enolase (NSE) und S 100 Protein (S 100 b) bei Patienten mit akutem Herzinfarkt

\section{R. Pfeifer ${ }^{1}$, A. Börner ${ }^{2}$, T. Deufel ${ }^{2}$, H.R. Figulla ${ }^{1}$ \\ ${ }^{1}$ Klinik für Innere Medizin I und ${ }^{2}$ Institut für klinische Chemie und Laboratoriumsdiagnostik, Klinikum der Friedrich-Schiller- Universität Jena}

Hintergrund: Eine der häufigsten Ursachen eines Herzstillstandes ist ein akuter Myokardinfarkt. Wenn nach erfolgreicher Reanimation die Patienten komatös bleiben werden die Neuroproteine NSE und $S 100$ b häufig zur Beurteilung der zerebralen Prognose herangezogen. In der Literatur wurden extrazerebrale Vorkommen beider Marker beschrieben. Es war daher naheliegend zu untersuchen, ob die Serumspiegel von NSE und S $100 \mathrm{~b}$ bei Patienten mit akutem Myokardinfarkt ansteigen. Methode: Wir untersuchten bei 43 Patienten (Pat.) mit akutem Myokardinfarkt, 13 Frauen, 30 Männer, mittleres Alter 64,6 Jahre, den Serumkonzentrationsverlauf von NSE und S $100 \mathrm{~b}$ über $48 \mathrm{~h}$ nach Aufnahme auf die Intensivstation. Alle Patienten mussten erhöhte Werte für TNI und CK aufweisen. Ausgeschlossen wurden Patienten mit kardiogenem Schock, Notwendigkeit einer mechanischen Kreislaufunterstützung, Reanimation und zerebralen Erkrankungen innerhalb der letzten 6 Monate vor dem Herzinfarkt. Die Bestimmung von NSE und S $100 \mathrm{~b}$ erfolgten parallel zu den üblichen Kontrollen der Creatinkinase (CK) auf dem LIAISON Analyser der Fa. Sangtec Medical, Schweden. Ergebnisse: Alle 43 Pat. überlebten mit einem GOS von 5. Kein Pat. entwickelte im Verlauf klinische Zeichen einer zerebralen Komplikation. Der SAPS-II-Score lag im Mittel bei 25,4 (8-32) Punkten, die maximale TNI-Konzentration der ersten $24 \mathrm{~h}$ bei 52,33 (1,42-454) ng/ml. In 63,8\% aller Proben fanden wir NSE-Konzentrationen oberhalb des cut-off-Wertes von $12,5 \mathrm{ng} / \mathrm{ml}$ und in $26,5 \%>25 \mathrm{ng} / \mathrm{ml}$, (Maximum $54,8 \mathrm{ng} / \mathrm{ml}$ ). $31,8 \%$ aller Serumproben wiesen eine S $100 \mathrm{~b}$-Konzentration oberhalb des cutoff-Wertes von $0,15 \mu \mathrm{g} / \mathrm{l}$ auf und in $14,5 \%$ der Proben fanden wir S 100 b-Konzentrationen $>0,2 \mu \mathrm{g} / \mathrm{l}$ (Maximum $0,31 \mu \mathrm{g} / \mathrm{l}$ ). Bei beiden Markern fanden wir Serumkonzentrationen, die in der Literatur beschriebene Grenzwerte zur Differenzierung der Prognose der hypoxischen Hirnschädigung nach Reanimation überschritten. Während die NSE-Konzentrationen nach $24 \mathrm{~h}$ nahezu gleichsinnig zum Serumkonzentrationsverlauf der CK abfielen, blieben die S 100 b-Konzentrationen erhöht. Diskussion und Schlussfolgerung: Aufgrund fehlender klinischer Hinweise auf akute zerebrale Läsionen während des Beobachtungszeitraumes sowie dem Fehlen jeglichen Schockgeschehens gehen wir von einer extrazerebralen Freisetzung von NSE und S $100 \mathrm{~b}$ aus ischämischem Myokardgewebe aus. Dieses Ergebnis steht im Widerspruch zu bisherigen Publikationen bei denen weder NSE noch S $100 \mathrm{~b}$ in Myozyten gefunden wurde (Haimoto $\mathrm{H}$ et al 1985, 1987; Zimmer DB et al 1995; Kanefuso et al 1981). Kiewitz et al. (2000) fanden zwar S 100A1 im Herzmuskel aber kein S 100 b. Bei Pat. mit akutem Myokardinfarkt ist mit einer Freisetzung von NSE und S $100 \mathrm{~b}$ aus ischämischem Myokard zu rechnen. Das kann bei Pat., die im Rahmen eines Myokardinfarktes reanimationspflichtig wurden, zu einer Fehleinschätzung des Ausmaßes der Hirnschädigung führen, wenn NSE und $S 100 \mathrm{~b}$ zur Prognoseabschätzung verwendet werden.
Influence of Intraaortic Balloon Pump Counterpulsation on Transesophageal Echocardiography Determinants of Diastolic Function

P. Rosenberger, M. Nowak, S. G. Memtsoudis, J.K. Tuli, H.K. Eltzschig, S.K. Shernan Brigham and Women's Hospital/Harvard Medical School Boston MA, USA und Department of Anesthesiology and Intensive Care Medicine, Tübingen University Hospital, Eberhard-Karls-University, Tübingen

Introduction: Intraaortic balloon pump counterpulsation (IABP) is often used in patients with acute coronary syndrome (ACS) to relieve refractory symptoms of ischemia. Although IABP is known to facilitate systolic cardiac performance by optimizing coronary perfusion and decreasing cardiac work, the impact of IABP on improving diastolic dysfunction (DD) associated with ACS has not been thoroughly investigated. Therefore, we investigated the influence of IABP on measures of DD determined by intraoperative transesophageal echocardiography (TEE) in patients undergoing coronary artery bypass grafting (CABG). Methods: Intraoperative TEE was performed following induction of general anesthesia, in 12 patients undergoing CABG, in whom an IABP was placed preoperatively for refractory ischemia. After full heparinization, the IABP was paused to evaluate cardiac and valve function without IABP support, and compared to values obtained immediately prior on full IABP support $(1: 1)$. Measures of DD including transmitral Doppler inflow velocities (E/A), deceleration time (Dt) and propagation velocity $(\mathrm{Vp})$, as well as mitral annular tissue Doppler velocities $\left(\mathrm{E}^{\prime} / \mathrm{A}^{\prime}\right)$, isovolumic relaxation time (IVRT), and pulmonary venous Doppler velocities (S/D) were acquired. Off-line analysis was performed postoperatively by two anesthesiologists certified in perioperative TEE. For statistical analysis Wilcoxon Sign-Rank test was employed, and a $p<0.05$ was considered significant. Results: All patients (mean age $71 \pm 11 \mathrm{yrs})$ were hemodynamically stable and did not require inotropic support during TEE examination. Mitral inflow E/A ratio, $\mathrm{Dt}$, IVRT and S/D ratio were not significantly different between full and no IABP support, although trends in these measures were consistent with improved left ventricular relaxation during IABP. However, statistically significant increases in mitral annular tissue Doppler E'/A' $(0.74$ vs. $0.64 ; \mathrm{p}<0.05)$ and $\mathrm{Vp}(44 \mathrm{~cm} / \mathrm{s}$ vs. $35 \mathrm{~cm} /$ $\mathrm{s} ; \mathrm{p}<0.01$ ) did suggest improved diastolic function during full IABP support. Conclusion: A significant influence of IABP support on load-dependent intraoperative TEE determinants of DD could not be demonstrated in this study population. However, improvement in left ventricular relaxation as suggested by increases in typically less load-dependent measures including $\mathrm{E}^{\prime} / \mathrm{A}^{\prime}$ and $\mathrm{Vp}$, were consistent with improved diastolic function during IABP. Thus, in addition to the known favourable influence of IABP on systolic cardiac function, IABP may also optimize diastolic function in patients with ACS.

Endotheliale Progenitorzellen im kardiogenen Schock

H.-J. Busch, J. Sunkomat, T. Schwab, B. Engert, C. Bode Albert-Ludwigs-Universität Freiburg, Abteilung für Kardiologie und Angiologie

In aktuellen Untersuchungen konnte gezeigt werden, dass im Rahmen kardiovaskulärer Ereignisse die Höhe zirkulierender endothelialer Progenitorzellen mit dem Outcome assoziiert ist. Ein bedeutendes kardiovaskuläres Ereignis stellt der kardiogene Schock mit einer Mortalität von über $50 \%$ dar. Mit dieser Untersuchung soll überprüft werden, inwieweit Patienten mit einem kardiogenen Schock eine veränderte Freisetzung von CD34-, AC133- und KDRpositiven Zellen aus dem Knochenmark aufweisen und diese mit dem kardiovaskulären Outcome assoziiert ist. Es wurden 3 Gruppen mit insgesamt 17 Patienten untersucht. Hiervon acht Patienten mit einem kardiogenen Schock, 4 Patienten mit einem septi- 
schen Schock sowie 5 gesunde Probanden. Mononucleäre Zellen wurden aus $9 \mathrm{ml}$ EDTA isoliert und mittels Durchflusszytometrie weiter differenziert. Die kardiale Pumpfunktion wurde echokardiographisch und hämodynamischen bestimmt. Vier der acht $\mathrm{Pa}$ tienten mit einem kardiogenen Schock verstarben während des Aufenthaltes. Die durchschnittliche linksventrikuläre Ejektionsfraktion betrug $31 \%( \pm 15 \%)$ bei Patienten mit einem kardiogenen Schock vs. $60 \%( \pm 5 \%)$ bei gesunden Kontrollen, $p<0,01$. Patienten im kardiogenen Schock zeigten gegenüber gesunden Probanden einen signifikant höheren Anteil zirkulierender CD34AC133positiver Zellen $(0,8 \pm 0,5 \%$ vs. $0,1 \pm 0,1 \%, p=0,01)$. Auch ergab sich ein Trend für einen höheren Anteil von zirkulierenden endothelialen Progenitorzellen im kardiogenen Schock verglichen mit Patienten im septischen Schock $(0,8 \% \pm 0,5 \%$ vs. $0,3 \pm 0,3 \%$, $\mathrm{p}=0,07)$. Bei Patienten, welche verstarben konnte durchschnittlich einen höheren Anteil zirkulierender Progenitorzellen nachgewiesen werden verglichen mit Patienten, welche den kardiogenen Schock überlebten $(1 \pm 0,5 \%$ vs. $0,5 \pm 0,4 \%)$. Zusammenfassend scheint eine erhöhte Freisetzung von Progenitorzellen im kardiogenen Schock vorzukommen. Inwieweit sich das Ausmaß der Freisetzung auf das Outcome auswirkt müssen weitere Untersuchungen klären.

\section{Mikrozirkulation bei Patienten mit Assisstsystemen im kardiogenen Schock}

\section{A.A. Krack, B.K. Pethig, C.M. Ferrari, D. R. Pfeifer, \\ E. H.-R. Figulla}

Klinik für Innere Medizin I (Kardiologie, Angiologie, Pneumologie, Internistische Intensivmedizin), Friedrich-Schiller-Universität Jena

Hintergrund: Sowohl beim septischen als auch beim kardiogenen Schock besteht eine Einschränkung der Mikrozirkulation. Beim septischen Schock wurde eine Korrelation zwischen Mortalität und Reduktion der Kapillardurchblutung gezeigt. Wir untersuchten, ob der Einsatz verschiedener kardialer Assisstsysteme die Mikrozirkulation verbessern konnte. Methoden: Zehn Patienten (8 Männer, 2 Frauen) im kardiogenen Schock wurden untersucht. Neben der Versorgung durch eine Akut-PCI sowie mit Dobutamin (und bei Bedarf mit einem Vasopressor) erhielten alle Patienten ein kardiales Assisstsystem (2 perkutane HLM, 1 Impella-Pumpe, 7 IABP). Die Patienten wurden mit einem HZV-Monitoring versehen. Bei den Patienten wurde die Mikrozirkulation innerhalb von 24 Stunden nach Beginn des Schocks durch sublinguale $\mathrm{Ka}$ pillarmikroskopie (MicroScan, Fa. Microvisionmedical, Niederlande) dargestellt. Es wurde eine Ausgangsmessung an drei verschiedenen Orten durchgeführt und anschließend das Assisstsystem für wenige Sekunden pausiert. Nach erneuter dreifacher Messung wurde das System wieder angestellt. Die Messungen wurden anschließen gemittelt, es wurde ein Perfusionsindex errechnet und die Unterschiede zwischen Beginn und Messzeitpunkt errechnet. Ergebnisse: Bei sieben Patienten ließ sich kein relevanter Unterschied in den Phasen von ein- oder ausgeschaltetem Assisstsystem feststellen (Perfusionsindex „ein“ und "aus“ jeweils im Mittel 2,75). Bei drei Patienten war eine Verbesserung durch die kardiale Unterstützung nachweisbar (Perfusionsindex „ein“ im Mittel 2,75; „aus“ im Mittel 2,25). Bei Analyse wiesen alle sieben erstgenannten Patienten Zeichen eines SIRS auf und hatten in der HZV-Messung einen deutlich erniedrigten systemvaskulären Widerstand im Vergleich zu den anderen drei Patienten gezeigt (im Mittel 485 dyn vs. 923 dyn). Schlussfolgerung: Kardiale Assisstsysteme verbessern das herzminutenvolumen von Schockpatienten, jedoch nicht in jedem Fall die Mikrozirkulation. Insbesondere bei gleichzeitigem Vorliegen eines SIRS in Verbindung mit einer peripheren Hyperzirkulation zeigte sich bei einer kleinen Patientenzahl keine Verbesserung der Kapillardurchblutung. Dies kann für die künftige Untersuchungen bedeutsam werden, wenn Mikrozirkulationsmessungen vermehrt für die Therapiesteuerung bei Schockpatienten verwendet werden.
Levosimendan: Effizienz und Sicherheit bei Patienten mit schwerer endgradiger chronischer Herzinsuffizienz

C. Berndt, B. Lamp, A. Dorszewski, H. K. Schmidt, K. P. Mellwig, R. Körfer, D. Horstkotte

Kardiologische Klinik, Herz- und Diabeteszentrum NRW, Ruhr-Universität Bochum, Bad Oeynhausen

Einleitung: Die schwere chronischer Herzinsuffizienz (CHF) führt $\mathrm{zu}$ häufigen Krankenhausaufenthalten, verursacht hohe Kosten und hat eine schlechte Prognose. Levosimendan (L), ein neuer Calcium-Sensitizer, führt zu einer deutlichen Verbesserung der hämodynamischen Situation von Patienten mit akuter Dekompensation einer schweren CHF. Ziel unserer Studie war, die Sicherheit und Effizienz von L bei Patienten im low output als Folge einer ischämischen Kardiomyopathie, dilatativen Kardiomyopathie (DCM) oder von Klappenvitien zu prüfen. Methode: 42 Patienten (Pat.) mit schwerer CHF (Alter 64,7 \pm 13 Jahre, männlich: $n=37$, KHK: $\mathrm{n}=26$, DCM: $\mathrm{n}=9$, Vitien: $\mathrm{n}=5$, andere: $\mathrm{n}=2$, EF: $27,2 \pm 6 \%, \mathrm{VO}_{2} \max : 10,0 \pm 3,5 \mathrm{l} / \mathrm{kg} / \mathrm{KG} / \mathrm{min}$., NYHA $3,0 \pm 0,4, \mathrm{CI}$ $1,7 \pm 0,31$, SVR $1797 \pm 131 \mathrm{dyn} \cdot \mathrm{s} / \mathrm{cm}^{-5}$, BNP: $1735 \pm 423 \mathrm{pg} / \mathrm{ml}$ ) erhielten L. Der Aufsättigungsdosis von $12 \mu \mathrm{g} / \mathrm{kg}$ i.v. über $10 \mathrm{~min}$ folgte eine kontinuierliche Infusion mit von $0,05 \mu \mathrm{g} / \mathrm{kg} / \mathrm{min}$ über 24 Stunden. Alle Pat. standen unter einer CONSENSUS-Medikation, 21 Pat. erhielten zusätzlich Katecholamine. Kontinuierlich wurden Blutdruck, EKG, Cardiac Index (CI), systemvaskulärer Widerstand (SVR), Elektrolyte, Retentionsparameter sowie Bilanz dokumentiert. Resultate: 38 Pat. tolerierten L ohne Nebenwirkungen. In 4 Fällen musste die Levosimendan-Infusion aufgrund einer ausgeprägten Hypotension beendet werden. Andere Nebenwirkungen traten nicht auf. Bei allen 38 Pat. bestand akut eine signifikante klinische Verbesserung der NYHA-Klasse auf 2,8 $\pm 0,7$ $(\mathrm{p}<0,05)$. Signifikante Verbesserungen zeigten der CI auf $2,49 \pm 0,7,(\mathrm{p}<0,05)$, der SVR auf $1160 \pm 140 \mathrm{dyn} \cdot \mathrm{s} / \mathrm{cm}^{-5}(\mathrm{p}<0,05)$ und das BNP auf $949 \pm 202 \mathrm{pg} / \mathrm{ml}(\mathrm{p}<0,05)$. Nicht signifikant war der Anstieg der EF auf 30,0 $\pm 5,3 \%$. Zusammenfassung: Der positiv inotrope Effekt von L stellt sich schnell ein und bedingt eine signifikante klinische sowie hämodynamische Verbesserung der Pat. mit schwerer CHF. Die Applikation von L ist sicher, ein invasives Monitoring ist nicht zwingend erforderlich. Somit scheint L eine vielversprechende Ergänzung in der Therapie der endgradigen CHF zu sein.

Effekt einer parenteralen Glutaminsubstitution auf Out003 come und Klinikverweildauer bei schwerer kardiopulmonaler Insuffizienz

N. Scheffold ${ }^{1}$, I. Dinkel ${ }^{1}$, R. Kilian ${ }^{2}$, R. Maitra ${ }^{1}$, C. Sucker $^{1}$, J. Cyran

${ }^{1}$ Medizinische Klinik I/Kardiologie und ${ }^{2}$ Klinikapotheke, SLK Klinikum Heilbronn, Klinikum am Gesundbrunnen

Hintergrund: Mehrere klinische Studien konnten bei überwiegend chirurgischen Intensivpatienten mit sehr heterogenen Erkrankungen den positiven Effekt einer Glutaminsubstitution auf Krankenhausverweildauer und Mortalität nachweisen. Ziel dieser doppelblinden, randomisierten Pilotstudie war es, den Effekt einer parenteralen Glutaminsubstitution auf Langzeitmortalität und Klinikverweildauer bei internistischen Intensivpatienten mit schweren kardiopulmonalen Erkrankungen zu untersuchen. Methoden: 32 Patienten (23 Männer, 9 Frauen, mittleres Alter: 63 Jahre) mit schwerer intensivpflichtiger kardiopulmonaler Erkrankung (mittlerer Apache-Score 23) wurden über mindestens 5 Tage parenteral ernährt. In der Glutamingruppe wurden $0,3 \mathrm{~g} / \mathrm{kg}$ L-Alanyl-L-Glutamin substituiert (0,2 g Glutamin/kg/d). Die Kontrollgruppe wurde isokalorisch und isonitrogen ernährt. Neben der Krankenhausverweildauer wurde die Sterblichkeit auf der Intensivstation (ICU) sowie 6 Monate nach Entlassung untersucht. Ergebnisse: Unerwünschte Nebenwirkungen traten unter parenteraler Glutaminsubstitution nicht auf. Auf der ICU starb 1 Patient aus der Glutamingruppe und 5 Patienten in der Kontrollgruppe. Die Gesamt- 
mortalität nach 6 Monaten war in der Glutamingruppe mit 25\% (4 Patienten) geringer als in der Kontrollgruppe mit 37,5\% (6 Patienten). Die Hälfte der Todesfälle in der Kontrollgruppe ereignete sich jedoch während mindestens 10-tägiger, glutaminfreier parenteraler Ernährung. Die mittlere Klinikverweildauer der Glutamin-

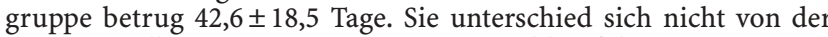
der Kontrollgruppe (39,3 \pm 17 Tagen). Schlussfolgerung: Die parenterale Glutaminsubstitution $(0,2 \mathrm{~g} / \mathrm{kg} / \mathrm{d})$ erwies sich als sicher. Bei Patienten mit schwerer kardiopulmonaler Insuffizienz war die Sterblichkeit auf der ICU unter Glutaminsubstitution deutlich geringer was im Trend auch nach 6 Monaten noch nachweisbar war. Eine Verkürzung der Krankenhausliegezeit wurde nicht erzielt. Die Daten legen nahe, dass auch bei internistischen Intensivpatienten mit schwerer kardiopulmonaler Erkrankung das Outcome durch eine parenterale Glutaminsupplementierung gebessert wird.

\section{Hämodynamische Effekte des $\mathrm{Ca}^{2+}$-Sensitizers}

Levosimendan bei Patienten im kardiogenen Schock

048 nach chirurgischer Koronar-Revaskularisation

\section{B. Korbmacher, U. Sunderdiek, E. Gams, J.D. Schipke}

Klinik f. Thorax- und Kardiovaskuläre Chirurgie, Heinrich-Heine-Universität Düsseldorf

Hintergrund: In die vorliegende Studie wurden 24 konsekutive $\mathrm{Pa}$ tienten im kardiogenen Schock eingeschlossen: 16 nach isolierter Koronar-Revaskularisations-Operation, bei 6 Patienten erfolgte zusätzlich ein Aortenklappenersatz, bei 2 weiteren zusätzlich ein Mitralklappenersatz. Ziel der vorliegenden Studie war die Evaluierung der Effekte der $\mathrm{Ca}^{2+}$-Sensitizers Levosimendan (LS) zusätzlich zu hochdosierter Katecholamin- und PDE III-Hemmer-Therapie. Patienten und Methoden: Die Einschlusskriterien für die LSTherapie (kontinuierliche Infusion von $0,2 \mathrm{mg} / \mathrm{min} / \mathrm{kg}$; ohne Bolus) waren: Cardiac Index (CI) $<2,01 / \mathrm{m}^{2} / \mathrm{min}$; PCWP im Durchschnitt $>15 \mathrm{mmHg}$ und eine Medikation mit $>0,5-1,0 \mathrm{mg} / \mathrm{min} / \mathrm{kg}$ Adrenalin; $0,5-1,0 \mathrm{mg} / \mathrm{min} / \mathrm{kg}$ Noradrenalin und $0,5 \mathrm{mg} / \mathrm{min} / \mathrm{kg}$ Milrinon. An bedeutsamen präoperativen Risikofaktoren lagen in 12 Fällen $(=50 \%)$ eine Niereninsuffizienz, in $16(66 \%)$ ein Diabetes, in $16(66 \%)$ ein Zustand nach Myokardinfarkt vor. Die LVEjektionsfraktion war bei $13(54 \%)>30 \%$, bei $11(46 \%)<30 \%$. Perioperative Daten: EKZ-Dauer: $128 \pm 11 \mathrm{~min}$; Ischämie-Zeit: $62 \pm 14$ min; IABP: 14 (6 präoperativ). Die hämodynamischen Werte wurden vor, $1 \mathrm{~h}, 8 \mathrm{~h}$ und $24 \mathrm{~h}$ nach LS-Gabe mittels SwanGanz-Katheter ermittelt. Ergebnisse: Während der LS-Infusion verbesserten sich die hämodynamischen Parameter signifikant: $\mathrm{CI}$ von $1,7 \pm 0,3$ auf $2,7 \pm 0,8 \mathrm{l} / \mathrm{m}^{2} / \mathrm{min}$, Schlagvolumen von $35 \pm 10$ auf $53 \pm 11 \mathrm{ml}$, PCWP fiel von $22 \pm 9$ auf $18 \pm 8 \mathrm{mmHg}$, systemischer Widerstand von $1150 \pm 550 \mathrm{dyn} \cdot \mathrm{s} \cdot \mathrm{cm}^{-5}$ auf $850 \pm 450 \mathrm{dyn} \cdot \mathrm{s} \cdot \mathrm{cm}^{-5}$; die Katecholamindosis konnte bei den Pat. nach isolierter Revaskularisations-Op. signifikant reduziert werden; 5 Pat. waren NonResponder (hiervon 4 Kombinations-Eingriffe). Insgesamt verstarben 11 Pat. Schlussfolgerungen: Der $\mathrm{Ca}^{2+}$-Sensitizer LS ist auch im Falle hochdosierter Katecholamin- und PDE-III-Hemmer-Therapie eine zusätzlich wirkungsvolle inodilatorische Substanz bei Patienten im kardiogenen Low-Cardiac-Output-Syndrom nach aorto-koronarer Bypass-Operation. Die positive Wirkung scheint bei Patienten mit Kombinations-Eingriff abgeschwächt. Zur weiteren Validierung einer systematischen LS-Therapie wäre eine prospektiv randomisierte Studie an Postkardiotomie-Patienten erforderlich.
Peripartale Kardiomyopathie (PPCM): die (un)bekannte maternale Erkrankung in der Schwangerschaft

H. V. Groesdonk ${ }^{1}$, W. Doblanzki ${ }^{2}$, U. Doblanski ${ }^{3}$, C. Galm $^{3}$, C.-M. Muth ${ }^{2}$

${ }^{1}$ Universität Leipzig, Herzzentrum, Abtl. Anästhesiologie und Intensivmedizin II, ${ }^{2}$ Universitätsklinikum Ulm,

Abtl. Anästhesiologie und Intensivmedizin, ${ }^{3}$ Universitätsklinikum Ulm, Abtl. Kinderkardiologie

Fragestellung: Die peripartale Kardiomyopathie (PPCM) ist eine seltene, oft unbekannte/unerkannte mütterliche Komplikation in der Spätschwangerschaft bzw. bis 5 Monate post partum und ist gekennzeichnet durch eine akute Einschränkung der linksventrikulären Pumpfunktion. Anhand des vorliegenden Fallberichtes einer 17-jährigen Patientin, die nach primärer Sectio caesaria das klinische Bild einer akuten Herzinsuffizienz bot und intensivmedizinisch versorgt werden musste, soll auf das Problem der PPCM nochmals hingewiesen und der derzeit verfügbare Wissensstand in einer Übersicht dargestellt werden. Material und Methoden: Der beschriebene Fall wurde in einer interdisziplinären Konferenz aufgearbeitet und der aktuelle Wissensstand durch eine umfassende Literaturrecherche („Medline/Pubmed“) ermittelt. Ergebnisse: Erstmalig dokumentiert wurde ein Zusammenhang zwischen Schwangerschaft und dilatativer Kardiomyopathie 1870 von Virchow und Ritchie. Angaben zur Inzidenz der PPCM reichen weit auseinander und werden realistisch mit 1:3000-4000 Geburten beziffert. Als Risikofaktoren werden spät gebärende Patientinnen (>30 Jahre), vorangegangene Gestosen, Hypertonie, Zwillingsschwangerschaften und schwarze Rasse beschrieben. Die Äthiologie ist weitestgehend unbekannt wobei eine infektiös-entzündliche Genese angenommen wird. Das klinische Bild der PPCM ist das klassische Bild der dekompensierten Herzinsuffizienz. Eine früh gestellte Diagnose ist entscheidend für das korrekte intensivmedizinische Management und wird in erster Linie durch die Echokardiographie gestellt. Eine spezifische Therapie der PPCM existiert nicht, umfasst aber die Behandlungsstrategien der akuten Herzinsuffizienz. Als ultima ratio steht die Herztransplantation zur Verfügung. Die Prognose hängt nach Literaturangaben wesentlich von der Erholung der linksventrikulären Funktion innerhalb der ersten 6 Monate nach Krankheitsbeginn ab. Schlussfolgerung: Das seltene Krankheitsbild der peripartalen Kardiomyopathie sollte dem, in der Geburtshilfe tätigen, Anästhesisten bzw. dem peripartal tätigen Intensivmediziner bekannt sein. Differenzialdiagnostisch sollte dieses Krankheitsbild bei Zeichen einer globalen Herzinsuffizienz im letzten Schwangerschaftsmonat bis zu 5 Monaten post partum mit in die Überlegungen einbezogen werden. Entscheidend für das mütterliche „outcome“ ist eine frühzeitige Diagnosestellung mittels Echokardiographie und eine aggressive Therapie der Herzinsuffizienz, die sich nicht von der anderer Insuffizienzformen unterscheidet.

Effiziente Oberflächenkühlung nach Reanimation mit einem neuen externen Kühlsystem

N. Scheffold, J. Graf, M. Obergföll, J. Cyran

Medizinische Klinik I, Schwerpunkt Kardiologie, SLK Kliniken Heilbronn, Klinikum Am Gesundbrunnen

Hintergrund: Die „International Liaison Committee on Resuscitation" (ILCOR) empfiehlt die milde Hypothermie zur Neuroprotektion nach kardiopulmonaler Reanimation (Klasse-I-Empfehlung). Entsprechende Kühlsysteme stehen zur Verfügung, sind jedoch meist sehr kostenintensiv und deshalb nicht verbreitet. Nach Abschluss einer Pilotstudie und technischer Modifikation stellen wir erstmals ein effizientes externes Kühlsystem vor. Methoden: 17 Patienten (7 Frauen, 10 Männer, mittleres Alter 72 Jahre) wurden nach kardiopulmonaler Reanimation infolge Herzkreislaufstillstand für 24 Stunden auf $32-34{ }^{\circ} \mathrm{C}$ abgekühlt. Als Kühlsystem wurde ein modifizierter Hico Variotherm 541 mit Kompressortechnik (Hirtz \& Co, Köln) eingesetzt. Zwei Kühldecken wurden 
in Sandwichtechnik an den Patienten angelegt. Die Temperaturregulation erfolgte über eine Anpassung der Kühlwassertemperatur unter kontinuierlicher Messung der Blasentemperatur. Ergebnisse: Nach technischer Modifizierung des HicoVariotherm 541 war erstmals eine maximale Absenkung der Kühlwassertemperatur auf $5^{\circ} \mathrm{C}$ möglich. Hierdurch konnte die Zieltemperatur bei allen Patienten nach duchschnittlich $120 \mathrm{~min}$ (60-240 min) ohne weitere externe Kühlmaßnahmen erreicht werden. Hautirritationen oder störende Kondensationsphänomene an Kühlschläuchen traten nicht auf. $\mathrm{Zu}$ einer Hyperkaliämie $>6 \mathrm{mmol} / \mathrm{l} \mathrm{kam}$ es bei einem Patienten, eine interventionspflichtige Bradykardie wurde nicht dokumentiert. Schlussfolgerung: Nach technischer Modifikation des HicoVariotherm 541 ist es gelungen, die milde Hypothermie nach dem Prinzip der Oberflächenkühlung rasch und stabil gemäß den Empfehlungen der ILCOR umzusetzen. Damit steht dem Intensivmediziner eine kostengünstige Alternative gegenüber den derzeit auf dem Markt befindlichen teuren und invasiven Systemen zur Verfügung.

\section{Induzierte milde Hypothermie} nach Herz-Kreislaufstillstand

Eine neue kombinierte Kühlmethode zum schnellstmöglichen Erreichen der Zieltemperatur

H.-J. Busch, T. Schwab, K. Fink, C. Bode

Albert-Ludwigs-Universität Freiburg, Abteilung für Kardiologie und Angiologie, Freiburg im Breisgau

Klinische und experimentelle Untersuchungen konnten zeigen, dass nach erfolgreicher kardiopulmonaler Reanimation die Induktion einer milden Hypothermie neuroprotektiv wirkt. Ein wesentlicher Faktor für den Kühlerfolg stellt der Zeitraum bis zum Erreichen der Zieltemperatur dar. Bei Patienten mit einem primär kardialen Ereignis, welche initial über das Herzkatheterlabor versorgt werden, geht hiermit wertvolle Zeit verloren. Wir berichten über eine kombinierte Kühlmethode, bei welcher die Patienten unmittelbar nach Aufnahme im Herzkatheterlabor mittels $4{ }^{\circ} \mathrm{C}$ kalten Infusionen und anschließend nach Ankunft auf der Intensivstation mit einem konventionellen Kühlsystem versorgt wurden im Vergleich zu Patienten ohne primärer Kühlung im Herzkatheterlabor. Die häufigsten Angaben zu Kühlzeiten in der Literatur beziehen sich auf die Temperatur bei Aufnahme auf der Intensivstation. Es wurden insgesamt 23 Patienten nach kardiopulmonaler Reanimation untersucht. Zieltemperatur war eine zentrale Temperatur (Harnblasentemperatur) von $33-34^{\circ}$. Die durchschnittliche Temperatur bei Aufnahme war bei beiden Gruppen gleich $(35,6 \pm 0,8$ vs. $35,4 \pm 0,9)$. Die mittlere Kühldauer zum Erreichen der Zieltemperatur nach Aufnahme war bei der kombinierten Kühlmethode signifikant kürzer ( 5 h $30 \mathrm{~min} \pm 1 \mathrm{~h} 45 \mathrm{~min}$ vs. $12 \mathrm{~h}$ 15 min \pm 6 h 45 min, $p<0,05)$. Ebenso war der Zeitraum zum Erreichen der Zieltemperatur nach Aufnahme auf der Intensivstation bei der kombinierten Kühlmethoden signifikant kürzer $(2 \mathrm{~h}$ $45 \mathrm{~min} \pm 1 \mathrm{~h} 45 \mathrm{~min}$ vs. $7 \mathrm{~h} \pm 7 \mathrm{~h} 45 \mathrm{~min}, \mathrm{p}<0,05)$. Es traten nach Infusion $4{ }^{\circ} \mathrm{C}$ kalter Infusionslösungen keine vermehrten Nebenwirkungen auf. Zusammenfassend ist die kombinierte Kühlmethode mit einer initialen Kühlung von $4{ }^{\circ} \mathrm{C}$ kalten Infusionslösungen und anschließender konventioneller Kühlung ein sicheres und sehr effektives prästationäres Kühlverfahren zur Durchführung einer milden Hypothermie. Mit diesem Verfahren kann die Zieltemperatur ohne das Auftreten von Nebenwirkungen signifikant schneller erreichen werden. Da die Kühlung schon vor der Intensivaufnahme begonnen wird, kann die kostenintensive und pflegeaufwendige Kühlphase deutlich verkürzt werden.
Signifikant erhöhte Serum-D-Dimer-Werte in Patienten nach prolongierter Reanimation

H.-J. Busch, T. Schwab, A. Geibel, C. Bode

Albert-Ludwigs-Universität Freiburg, Abteilung für Kardiologie und Angiologie, Freiburg im Breisgau

In klinischen und experimentellen Untersuchungen konnte gezeigt werden, dass es bei Patienten nach kardiopulmonaler Wiederbelebung zu einer Aktivierung des Gerinnungssystems kommt. Inwieweit es sich hierbei um eine Aktivierung im Sinne der pathophysiologischen Ursache oder aber um Auswirkung einer vorhandenen Mikrozirkulationsstörung während und nach kardiopulmonalen Wiederbelebung handelt und inwieweit diese einen limitierenden bzw. prognostischer Faktor darstellt ist bis jetzt noch ungeklärt. In dieser retrospektiven Untersuchung wurde das Ausmaß der Gerinnungsaktivierung in Patienten nach temporärer und prolongierter Wiederbelebung anhand der D-Dimer-Wert-Erhöhung bestimmt. Im Zeitraum vom 01. 10. 2004-31. 03. 2005 konnten retrospektiv 43 Patienten nach erfolgreicher Wiederbelebung eingeschlossen werden. Ausgeschlossen wurden Patienten mit schon initial deutlich erhöhten Entzündungswerten, Nachweis einer Lungenembolie und Patienten mit aktiven Tumorleiden. Unmittelbar nach Aufnahme auf unsere Intensivstation wurden die D-Dimere im Rahmen der Standardblutentnahmen bestimmt. Hierbei zeigten sich in allen Patienten signifikant erhöhte SerumD-Dimer Werte nach Reanimation. Nach prolongierter Reanimation (Wiederherstellung eines suffizienten Kreislaufes nicht innerhalb der ersten $30 \mathrm{~min}$ ) waren die D-Dimere signifikant gegenüber Patienten, bei welchen es gelang innerhalb der ersten $30 \mathrm{Mi}$ nuten einen suffizienten Kreislauf herzustellen, erhöht $(3328 \mu \mathrm{g} / \mathrm{l}$ vs. $663 \mu \mathrm{g} / \mathrm{l} \mathrm{p}<0,0001)$. Die Dauer des Herz-Kreislaufstillstandes korrelierte mit der Aktivierung des Gerinnungssystems (Spearman-Rang: $r=0,8 ; p<0,0001)$. Unsere Daten zeigen eine zeitabhängige, signifikante Gerinnungsaktivierung nach kardiopulmonaler Wiederbelebung. Weitere Untersuchungen müssen analysieren inwieweit diese Veränderungen konsekutiv zur Unterstützung und Unterhaltung der Reperfusionsschäden nach kardiopulmonaler Reanimation beitragen und somit das Outcome und den klinischen Verlauf dieser Patienten bestimmen können.

\section{Rhythmusstörungen, Monitoring, Gerinnungsmanagement}

Atrial Fibrillation in a General Intensive Care Unit

I. D. Welters ${ }^{1,2}$, R. Shankar ${ }^{2}, M$. Leuwer ${ }^{1}$

${ }^{1}$ Academic Unit of Critical Care Medicine, University of Liverpool and ${ }^{2}$ Intensive Care Unit, Royal Liverpool University Hospital, Liverpool, UK

Objective: Although atrial fibrillation (AF) is the most commonly sustained arrhythmia in the general population, little is known so far about its occurrence in critically ill patients [1]. The aim of this study was to evaluate the incidence of atrial fibrillation (AF) and to identify possible risk factors in patients admitted to a General Intensive Care Unit. Material and Methods: We performed a retrospective study during a three months period (October 2005December 2005) in a General Intensive Care Unit (ICU) of a University Hospital in the United Kingdom. 110 patients admitted to the ICU were screened for development of AF analysing daily management charts and case notes. Results: Out of 110 patients screened, 29 developed AF (26.3\%). Mean age of the patients was 71.8 ( \pm 9.2$)$ years. APACHE II score was $21.3( \pm 6.3)$, with a mortality rate of $24.1 \%$. Electrolytes were normal in $85 \%$ of patients with $\mathrm{K}+$ being in the range of $3.4-4.3$ and $\mathrm{Mg}^{++}$in the range of $0.7-0.8 \mathrm{mmol} / \mathrm{l}$. The patients were admitted under the following teams: abdominal surgical $(40.9 \%)$, pulmonary $(22.7 \%)$, vascular 
(9.1\%), cardiac $(9.1 \%)$, renal/urology $(13.6 \%)$ and infectious disease $(4.5 \%)$. The main cardiovascular risk factors encountered in our study were hypertension $(71.4 \%)$ and coronary artery disease (48.3\%). 22 of the patients developing AF were septic (75.9\%), 24 $(82.8 \%)$ needed inotropic support. Other cardiac risk factors, such as diabetes mellitus and obesity were present in $17.2 \%$ of patients with AF. Two patients had a previous history of AF and one patient suffered from congenital heart disease. $18(62.1 \%)$ patients went back into sinus rhythm. 19 patients $(65.5 \%)$ received amiodarone, five $(17.2 \%)$ digoxin, four $(13.8 \%) \beta$-blockers and one patient $(3.6 \%)$ was cardioverted electrically. Troponin $\mathrm{T}$ was determined in 13 patients and elevated in $10(34.5 \%)$ patients. Echocardiography was performed in $10(34.5 \%)$ of our patients. 7 patients had a valve pathology and reduced left ventricular function was found in 5 of the patients. Only in 1 patient echocardiography findings were reported as normal. Conclusions: AF was most frequently associated with increased age as well as a history of hypertension and/or ischaemic heart disease. Patients on inotropic support or with sepsis were frequently affected. Echocardiography revealed valve pathology and/or reduced left ventricular function in $31 \%$ of intensive care patients developing AF and may be a useful tool to identify contributing pathologies.

References: [1] Heinz G. Atrial fibrillation in the intensive care unit. Intensive Care Med 2006; 32:345-348. [2] Seguin P et al: Incidence and risk factors of atrial fibrillation in a surgical intensive care unit: Crit Care Med 2004; 32(3):722-726. [3] ACC/AHA /ESC guidelines for the management of patients with atrial fibrillation: Circulation 2001; 104:2118-2150

Supraventrikuläre und ventrikuläre Arrhythmien in Schwangerschaft und Stillzeit

J. Vormbrock, Y. Saklaoui ${ }^{2}$, H.-J. Trappe

Medizinische Klinik II (Kardiologie und Angiologie), Ruhr-

Universität Bochum, ${ }^{2}$ Frauenklinik, Ruhr-Universität Bochum

Hintergrund: Bislang ist nur wenig über Art, Häufigkeit, Symptome und Manifestationszeitpunkt von Herzrhythmusstörungen während der Schwangerschaft und Stillzeit bekannt. Diese Kenntnis ist allerdings bedeutsam zur Prognoseeinschätzung und der resultierenden Therapie sowie zur Indikationsstellung zu intensivmedizinischen Maßnahmen. Nicht oder falsch behandelte Herzrhythmusstörungen können besonders bei herzkranken Schwangeren schwerwiegende Folgen für Mutter und Kind haben. Ziel unserer Untersuchung ist die systematische Erfassung von Art, Häufigkeit, Manifestationszeitpunkt und Symptomen von Herzrhythmusstörungen in der Schwangerschaft und Stillzeit. Methodik: 59 Patientinnen (Pt) (Alter 27,6+6,7 Jahre) in unterschiedlichen Stadien der Schwangerschaft bzw. post partum wurden mittels EKG, Langzeit-EKG und Echokardiographie untersucht. Herzfrequenz, Herzrhythmus und Morphologie des Herzen wurden analysiert. Ergebnisse: Alle Pt zeigten im Langzeit-EKG einen durchgehenden Sinusrhythmus. Bei einer Pt $(1,7 \%)$ zeigte sich intermittierend ein AV-Block $\mathrm{II}^{\circ}$, Typ Mobitz. $38 \mathrm{Pt}(64,4 \%)$ waren normfrequent, $21 \mathrm{Pt}(35,6 \%)$ wiesen eine Sinustachykardie auf. In 27 Fällen $(45,8 \%)$ zeigten sich keine, bei $32 \mathrm{Pt}(54,2 \%)$ vereinzelt supraventrikuläre (SV) Extrasystolen im Langzeit-EKG. Bei $25 \mathrm{Pt}$ $(42,4 \%)$ konnten keine, in 31 Fällen $(52,5 \%)$ vereinzelt, bei $3 \mathrm{Pt}$ $(5,1 \%)$ häufige ventrikuläre Extrasystolen festgestellt werden. SVCouplets konnten bei 6 Pt (10,2\%), SV-Triplets bei 2 Pt $(3,4 \%)$ nachgewiesen werden. Alle Pt hatten eine gute linksventrikuläre Funktion mit einer EF von 68,8+5,7 (normfrequente $\mathrm{Pt}$ ) bzw. 64,4+6,5\%. (tachykarde Pt). Zusammenfassung: Während der Schwangerschaft und Stillzeit kommt es häufig zu Sinustachykardien, vereinzelt zu supraventrikulären und ventrikulären Extrasystolen, welche allerdings selten symptomatisch sind. Eine intensivmedizinische Behandlung war bei keiner Pt notwendig.
Manifestationszeitpunkt kardialer Arrhythmien während der Schwangerschaft

J. Vormbrock, Y. Saklaoui ${ }^{2}$, H.-J. Trappe

Medizinische Klinik II (Kardiologie und Angiologie),

Ruhr-Universität Bochum, ${ }^{2}$ Frauenklinik, Ruhr-Universität Bochum

Hintergrund: Über den genauen Zeitpunkt des Auftretens von Herzrhythmusstörungen während der Schwangerschaft und Stillzeit ist bisher nur wenig bekannt. Diese Kenntnis ist jedoch von Bedeutung bei der Einschätzung, ob und wann eine intensivmedizinische Behandlung von Schwangeren indiziert ist. Ziel unserer Untersuchung ist, die Art und den Manifestationszeitpunkt von Herzrhythmusstörungen in den einzelnen Stadien der Schwangerschaft und Stillzeit systematisch zu erfassen. Methodik: Bei 59 Patientinnen (Pt) (Alter 27,6+6,7 Jahre) wurden EKG, LangzeitEKG und Echokardiographie in unterschiedlichen Stadien der Schwangerschaft (SW) bzw. post partal (pp) durchgeführt. $2 \mathrm{Pt}$ $(3,4 \%)$ waren im 1 . Trimenon (Gruppe G1), 13 Pt (22,0\%) im 2. Trimenon (Gruppe G2) und $39 \mathrm{Pt}(66,1 \%)$ im 3. Trimenon (Gruppe G3). $5 \mathrm{Pt}(8,4 \%)$ wurden pp untersucht (Gruppe G4). Ergebnisse: Bei allen Pt lag ein Sinusrhythmus vor, bei 1 Pt (1,7\%) (G4) kam es zu einem intermittierenden AV-Block $\mathrm{II}^{\circ}$, Typ Mobitz. Sinustachykardien (ST), supraventrikulären Extrasystolen (SVES), vereinzelte (a) ventrikuläre Extrasystolen (VES) waren in allen Stadien der SW eher häufig, vermehrte (b) VES, SV-Couplets und Triplets dagegen eher selten.

\begin{tabular}{llrll} 
& G1 & G2 & G3 & G4 \\
& $\mathbf{n = 2}$ & n=13 & n=39 & $\mathbf{n = 5}$ \\
\hline ST & $/$ & $4(30,8 \%)$ & $16(41,0 \%)$ & $1(20 \%)$ \\
SVES & $2(100 \%)$ & $10(76,9 \%)$ & $17(43,6 \%)$ & $3(60 \%)$ \\
VESa & $1(50 \%)$ & $7(53,8 \%)$ & $22(56,4 \%)$ & $1(20 \%)$ \\
VESb & $/$ & $/$ & $2(5,1 \%)$ & $1(20 \%)$ \\
SV-Couplets & $/$ & $1(7,7 \%)$ & $4(10,3 \%)$ & $1(20 \%)$ \\
SV-Triplets & $/$ & $/$ & $2(5,1 \%)$ & $/$ \\
\hline
\end{tabular}

Zusammenfassung: ST, SVES und VES sind während der SW relativ häufig, komplexe Rhythmusstörungen dagegen nicht. Die Häufigkeit von ST nimmt mit fortschreitender SW- Dauer zu. Dennoch war eine antiarrhythmische oder intensivmedizinische Behandlung bei keiner Pt notwendig.

Beurteilung von Vorlast und Flüssigkeits-Haushalt 021

bei Intensivpatienten: Klinik vs. ZVD vs.

hämodynamisches Monitoring mittels PicCO

W. Huber, S. Ringmaier, A. Umgelter, W. Reindl, K. Wagner, S. von Delius, T. Weber, A. Weber, M. Franzen, R.M. Schmid Klinikum Rechts der Isar; II. Med. Klinik; Technische Universität München

Hintergrund: Die Bedarfs-adaptierte Volumenzufuhr ist eine der wesentlichen Therapie-Grundlagen bei Intensivpatienten. Zur Einschätzung des Volumenstatus stehen klinische Symptome wie Halsvenen-Füllung (intravasaler Raum), Beinödeme (Interstitium), Ascites und Pleuraerguss („dritter" Raum), einfache Funktionstests (Trendelenburg-Manöver) sowie hämodynamische Parameter wie ZVD, PAWP und der mittels PiCCO-System ermittelte Intrathorakale Blutvolumen-Index (ITBI) zur Verfügung. Letzterer hat sich in neueren Untersuchungen als valider Parameter der VorlastEinschätzung und „volume-responsiveness“ etabliert. Die klinische Einschätzung ist bei Krankheitsbildern wie der globalen Herzinsuffizienz mit gleichsinniger Beeinflussung der o.g. Kompartimente verlässlich. Bei anderen Krankheitsbildern wie Sepsis, Leberzirrhose und Pankreatitis ist die Interpretation der klinischen Symptome durch z.T. gegensinnige Volumenveränderungen schwierig. Auch Druck-basierte Vorlastparameter wie der ZVD sind hier nur z.T. aussagekräftig. Ziele: Ziel unserer Studie war es 
daher, bei hämodynamisch instabilen Intensivpatienten den Stellenwert der klinischen Vorlast-Diagnostik und des ZVD mit dem ITBI zu vergleichen. Methoden: Bei 60 Patienten einer internistischen Intensivstation wurden zunächst der Volumenstatus in den o.g. Kompartimenten von Arzt (A) und nicht auf der Station tätigem Untersucher (U) bewertet und anschließend mit ZVD und den Hämodynamik-Parametern der PiCCO-Messung (Pulsion; München) korreliert. Ergebnisse: Patientencharakteristika: $n=60$ ( 27 w, 33 m; u. a. 18 Pat. mit Zirrhose; 9 Patienten mit Pankreatitis; 16 Pat. mit Sepsis); Alter 64,9 $\pm 14,2$ Jahre; APACHE-II-Score $24,3 \pm 8,1$. Das Ausmaß der Beinödeme korrelierte signifikant mit dem ZVD $(\mathrm{r}=0,325 ; \mathrm{p}=0,023)$ und negativ mit dem ITBI $(\mathrm{r}=-0,371 ; \quad \mathrm{p}=0,009)$. ZVD und ITBI korrelierten nicht $(r=-0,169 ; p=0,198)$. Der extravasculäre Lungenwasser-Index korrelierte mit dem auskultatorischen Ausmaß feuchter pulmonaler Rasselgeräusche $(r=0,374 ; p=0,003)$ und dem ITBI $(r=0,538$; $\mathrm{p}<0,001)$, nicht mit ZVD ( $\mathrm{r}=-0,176 ; \mathrm{p}=0,178)$. Schlussfolgerungen: 1. Die Einschätzung des Volumenstatus bei Intensiv-Patienten ist komplex. 2. Beinödeme und ZVD spiegeln dabei den Volumenstatus interstitiell bzw. vor dem rechten Herzen. Beide Kompartimente stehen allerdings als „unmittelbare“ Vorlast nicht zur Verfügung. Das Ausmaß der Beinödeme korrelierte mit dem ITBI negativ. 3. Aus dem Vorhandensein von Ödemen und hohem ZVD auf einen intravasculären Volumen-Überschuss und die Gefahr eines Lungenödems zu schließen, kann bei diesem Patientengut fatale Folgen haben. Daher muss bei diesen Patienten eine differenzierte Bewertung des Volumenstatus in allen Kompartimenten erfolgen, ggf. unter Einsatz moderner Hämodynamik-Systeme und/ oder der Echokardiographie.

Dihydralazin - nicht aber Glyceroltrinitrat steigert die mikrovaskuläre Schleimhaut-0xygenation bei anästhesierten Hunden.

L.A. Schwarte, I. Schwartges, A. Fournell, T. W.L. Scheeren, O. Picker

Klinik für Anästhesiologie, Universitätsklinikum Düsseldorf

Fragestellung: Eine adäquate mikrovaskuläre Oxygenation der gastrointestinalen Schleimhaut ist wesentlich für die Aufrechterhaltung einer intakten Schleimhautbarriere [1], welche bedeutsam scheint für die Prävention von Sepsis und Multiorganversagen [2]. Ungeklärt ist bisher der Effekt von Vasodilatatoren auf die mikrovaskuläre Schleimhaut-Oxygenation. Wir haben daher den Effekt der intensivmedizinisch relevanten Vasodilatatoren Dihydralazin und Glyceroltrinitrat auf die mikrovaskuläre MagenschleimhautOxygenation untersucht. Methoden: Chronisch instrumentierte Hunde $(\sim 30 \mathrm{~kg}, \mathrm{n}=12$ Experimente, mit Genehmigung der Bezirksregierung) wurden anästhesiert (Propofol $15 \mathrm{mg} / \mathrm{kg} / \mathrm{h}$ ) und mechanisch beatmet $\left(\mathrm{FiO}_{2}\right.$ 0,3; etCO $\left.35 \mathrm{mmHg}\right)$. Gemessen wurden auf systemischer Ebene der arterielle Mitteldruck (MAD), der systemische Sauerstofftransport $\left(\mathrm{DO}_{2}\right.$, aus arteriellem $\mathrm{O}_{2}$-Gehalt und dem Herz-Minuten-Volumen (HMV)) und auf regionaler Ebene die mikrovaskuläre Hämoglobin-Oxygenation der Magenschleimhaut $\left(\mu \mathrm{HbO}_{2}\right.$, Gewebespektrophotometrie, EMPHO-II [3]). Nach einer Stabilisierungsphase und Messung der Ausgangswerte wurden randomisiert äquihypotensive Dosierungen von Dihydralazin oder Gyceroltrinitrat infundiert. Statisktik: Fisher's PLSD sowie gepaarter $t$-Test, $p<0,05$. In äquihypotensiver Dosierung (MAD fiel von $\sim 80 \mathrm{mmHg}$ auf $\sim 65 \mathrm{mmHg}$ in beiden Gruppen, kein Unterschied zwischen den Gruppen) steigerte nur Dihydralazin die $\mu \mathrm{HbO}_{2}$ (von $64 \pm 2$ auf $70 \pm 2 \%, \mathrm{p}<0,05$ ), wohingegen Glyceroltrinitrat keinen signifikanten Effekt hatte (von $63 \pm 2$ auf $61 \pm 3 \%)$. Auf systemischer Ebene steigerte Dihydralazin den $\mathrm{O}_{2}$-Transport $\left(\mathrm{DO}_{2}\right)$ von $15 \pm 1$ auf $26 \pm 2 \mathrm{ml} / \mathrm{kg} / \mathrm{min}(\mathrm{p}<0,05)$, wobei Glyceroltrinitrat auch hier ohne signifikanten Effekt blieb (von $14 \pm 1$ auf $16 \pm 2 \mathrm{ml} / \mathrm{kg} / \mathrm{min}$ ). Dihydralazin steigerte hierbei deutlich das HMV (von $82 \pm 2$ auf $129 \pm 7 \mathrm{ml} / \mathrm{kg} / \mathrm{min}, \mathrm{p}<0,05$ ), wohingegen Glyceroltrinitrat ohne signifikanten Effekt blieb (von $87 \pm 4$ auf $97 \pm 10 \mathrm{ml} / \mathrm{kg} / \mathrm{min}$ ). Die arteriellen $\mathrm{pH}$ - (jeweils $\sim 7,37$ ) und
Laktatwerte (jeweils $\sim 1,0 \mathrm{mmol} / \mathrm{l}$ ) blieben in beiden Gruppen stabil. Wenn diese Ergebnisse auf die Klinik übertragen werden können, so ist Dihydralazin geeignet, die mikrovaskuläre Schleimhaut-Oxygenation zu steigern, nicht hingegen eine äquihypotensive Dosierung von Nitroglyzerin. So könnte Dihydralazin die Aufrechterhaltung der Schleimhautbarriere bei gefährdeten Patienten unterstützen.

Literatur: [1] Sato N, Kawano S, Kamada T et al: Dig Dis Sci 1986; 31:35-41. [2] Pastores SM, Katz DP, Kvetan V Am J Gastroenterol 1996; 91:1697-1710. [3] Kuchenreuther S, Adler J, Schutz W et al: J Clin Monit 1996; 12:211-224

Anaesthetics induce differential changes in haemodynamic entropy

M. Stephan, L. Schwarte, I. Schwartges, O. Picker

Department of Anaesthesiology University Clinic of Duesseldorf

Introduction: Cardiovascular dynamics in health and disease appear to reflect complex regulatory mechanisms [1], which can be described by analysing haemodynamic entropy. Decreases in entropy have been associated with critical pathologies [2]. We studied the impact of four anaesthetics on haemodynamic entropy. Methods: Anaesthetized and mechanically ventilated foxhounds $\left(\mathrm{FiO}_{2} 0.3\right.$, etCO $\left.35 \mathrm{mmHg}\right)$ received 2 doses of either a volatile anaesthetic (desflurane [DES] or sevoflurane [SEV], each at 1 and $2 \mathrm{MAC}$ ) or an intravenous anaesthetic (propofol [PRO] at 10 and 20 mgkg-1h-1 or methohexital [MET] at 20 and $40 \mathrm{mg} \mathrm{kg}^{-1} \mathrm{~h}^{-1}$ ). Systolic arterial pressure (SAP) and heart rate (HR) time series (720 cardiac cycles) were analysed using a multiscale entropy algorithm as previously described (vector length 1 to 2, tolerance 0.15 , scale 1 to 4 ) [3]. Statistics: Means \pm SEM, ANOVA (Bonferroni), $\mathrm{p}<0.05$. Results: SAP entropy was significantly higher with PRO than with other anaesthetics. Between DES, SEV and MET entropy of SAP did not differ. With entropy of HR there were no intergroup differences. Conclusion: Anaesthetics have complex influence on haemodynamic entropy. These effects seem to be substance- but not dose-dependent with PRO preserving SAP entropy on the highest level. Thus, in patients, the choice of anaesthetic agents may be one factor differentially influencing haemodynamic entropy.

References: [1] Glass L: Synchronization and rhythmic processes in physiology. Nature 2001; 410:277-284. [2] Lake DE, Richman JS, Griffin MP, Moorman JR: Sample entropy analysis of neonatal heart rate variability. Am J Physiol Regul Integr Comp Physiol 2002; 283:R789-797. [3] Costa M, Goldberger AL, Peng CK: Multiscale entropy analysis of complex physiologic time series. Phys Rev Lett 2002; 89:068102

Hämodynamik und intraabdomineller Druck (IAD)

während Ösophagogastroduodenoskopie (ÖGD)

auf der Intensivstation: Vorläufige Ergebnisse einer prospektiven Beobachtungsstudie

\section{P. Thies, S. von Delius, R. M. Schmid, W. Huber}

II. Medizinische Klinik und Poliklinik, Klinikum rechts der Isar, Technische Universität München

Hintergrund: Die ÖGD ist ein Standardverfahren zur Diagnostik und Therapie gastrointestinaler Erkrankungen auf der Intensivstation. Es wurde untersucht, ob es über eine Erhöhung des IAD (in Folge der Luftinsufflation im Rahmen der Endoskopie) zu hämodynamischen Veränderungen kommt, die ursächlich für (wenn auch seltene) kardiovaskuläre Komplikationen sein könnten. Daneben wären auch Reaktionen auf Vagusreize, sowie Stressreaktionen denkbar. Patienten und Methoden: Bei kritisch kranken Patienten mit installiertem PiCCO (pulse contour cardiac output)-System und Blasendauerkatheter wurden vor (t0), während (t1) und nach ( $\mathrm{t} 3)$ einer ÖGD-Thermodilutionsmessungen zur Erfassung des Herzindex (HI), intrathorakalen Blutvolumenindex (ITBVI) und 
vaskulären Widerstandsindex (SVRI) durchgeführt. Parallel wurden Herzfrequenz (HR), mittlerer arterieller Blutdruck (MAP) und Änderungen des Blasendruckes (zur Bestimmung des IAD) erfasst. Ergebnisse: Es wurden 37 Messreihen bei 29 Patienten (mittleres Alte$\mathrm{r} \pm$ Standardabweichung: $62 \pm 11$ Jahre) durchgeführt. Der mittlere Apache-II-Score lag bei $21 \pm 8$. Wir beobachteten einen mäßigen Anstieg des HI $\left[1 / \mathrm{min} / \mathrm{m}^{2}\right] \quad(\mathrm{t} 0: \quad 3,91 \pm 0,96 ; \quad \mathrm{t} 1: \quad 4,03 \pm 1,00$ t3: $4,19 \pm 1,05)$, ITBVI $\left[\mathrm{ml} / \mathrm{m}^{2}\right] \quad(\mathrm{t} 0: 943 \pm 205 ; \mathrm{t} 1: 944 \pm 163$; t3: $981 \pm 213)$ und HR $\left[\mathrm{min}^{-1}\right](\mathrm{t} 0: 86 \pm 15 ; \mathrm{t} 1: 88 \pm 15$; $\mathrm{t} 3: 90 \pm 16)$, sowie einen deutlichen Anstieg des SVRI $\left[\right.$ dyn $\cdot \mathrm{s} \cdot \mathrm{cm}^{-5} \cdot \mathrm{m}^{2}$ ] (t0: $1417 \pm 563 ; \mathrm{t} 1: 1566 \pm 564 ; \mathrm{t} 3: 1564 \pm 1016)$, MAP [mmHg] (t0: $78 \pm 17 ; \mathrm{t} 1: 87 \pm 19 ; \mathrm{t} 3: 85 \pm 16)$ und IAD [ $\left.\mathrm{cmH}_{2} \mathrm{O}\right]$ (t0: $16 \pm 7$; t1: $21 \pm 10$; $\mathrm{t}$ : $18 \pm 10$ ). Schlussfolgerung: Bei kritisch kranken Patienten kommt es während einer ÖGD zu einem ausgeprägten, aber schnell reversiblen Anstieg des IAD. Hinsichtlich der hämodynamischen Parameter wurde insbesondere ein Anstieg von MAP und SVRI (möglicherweise als Folgen einer Stressreaktion) beobachtet.

\section{Verlässlichkeit der kontinuierlichen Messung des Herzindex durch arterielle Pulskonturanalyse mittels PiCCO-System bei kritisch kranken Patienten \\ M. Franzen, A. Umgelter, S. von Delius, A. Weber, \\ J. Reichenberger, M. Dohmen, W. Huber, R. M. Schmid \\ II. Medizinische Klinik und Poliklinik, Technische Universität München}

Hintergrund: Ein verlässliches kontinuierliches hämodynamisches Monitoring ist bei kritisch kranken Patienten für ein effektives Volumenmanagment und den adäquaten Einsatz vasoaktiver Substanzen unerlässlich. Das PiCCO-System ermöglicht die kontinuierliche Messung des Herzindex (CI) mittels der arteriellen Pulskonturanalyse. Zur Kalibrierung nutzt das System die transpulmonale Thermodilutionsmethode. Ziel unserer Untersuchung war es, die Verlässlichkeit der kontinuierlichen Messung des CI mit der arteriellen Pulskonturanalyse (CIpc) mittels PiCCO-System im Vergleich mit dem bei der regelmäßig durchgeführten Kalibrierung durch transpulmonale Thermodilution ermittelten Herzindex (CItd) zu prüfen. Patienten und Methoden: Die Untersuchung erfolgte an 10 kritisch kranken Patienten (8 männlich, 2 weiblich, Alter 37-84 Jahre, im Mittel 64,1 $\pm 13,0$ ) mittels PiCCO-Monitoring. 5 Patienten hatten ein septisches Kreislaufversagen, 3 ein hepatorenales Syndrom, und 2 eine dekompensierte Herzinsuffizienz. Insgesamt wurden 140 Messungen ausgewertet, wobei jeweils zunächst der CIpc erfasst und im Anschluss eine dreifache Messung des CItd durchgeführt wurde. Dabei erfolgte gleichzeitig eine Neukalibrierung des Pulskonturalgorithmus des PiCCO-Systems. Pro Patient wurden im Mittel 14 $\pm 9,4$ Messungen in einem zeitlichen Abstand von im Mittel 12 h 54 min \pm 7 h 47 min durchgeführt. Ergebnisse: Der Vergleich von CIpc und CItd erbrachte einen Korrelationskoeffizienten von 0,84 mit einem p-Wert von 0,02. In der Bland-Altman-Analyse lag der CIpc im Mittel $0,14 \mathrm{l} / \mathrm{min} / \mathrm{m}^{2}$ niedriger als der CItd. Die Standardabweichung betrug $0,72 \mathrm{l} / \mathrm{min} / \mathrm{m}^{2}$. Ein Zusammenhang zwischen zeitlichem Abstand der Neukalibrierung und Differenz von CIpc und CItd konnte nicht ermittelt werden $(r=-0,03 ; p=0,13)$. Schlussfolgerung: Das PiCCO-System ermöglicht mittels der arteriellen Pulskonturanalyse ein zuverlässiges kontinuierliches Monitoring des CI bei kritisch kranken Patienten. In unserer Untersuchung konnten wir auch bei längeren Zeitintervallen zwischen zwei Kalibrierungen mittels der transpulmonalen Thermodilution keine $\mathrm{Zu}$ nahme der Abweichung zwischen CIpc und CItd feststellen. Da die Kalibrierung mit relativ geringem Aufwand durchzuführen ist und zusätzliche Daten zur Abschätzung des intrathorakalen Blutvolumens und des extravaskulären Lungenwassers liefert, ist eine 12-stündliche Durchführung sinnvoll.
Erfolgreiche Thrombolyse von Transitemboli mit und ohne Inkarzeration im Foramen ovale

H. Langenfeld, M. Spindler, J. Wagner, G. Burrows

Medizinische Klinik und Poliklinik I der Universität Würzburg

Patienten, bei denen echokardiographisch im rechten Herzen oder eingeklemmt im Foramen ovale, ein Transitembolus (sog. TypA-Embolus) sichtbar ist, haben unter reiner Antikoagulation eine schlechte Prognose und versterben häufig im Verlauf an einer fulminanten Lungenembolie. Die chirugische Thrombektomie ist ebenfalls mit einer hohen Letalität verbunden. Wir beschreiben 6 Fälle aus den letzten Jahren, bei denen eine Fibrinolyse mit 50-100 mg rt-PA als Bolus innerhalb kurzer Zeit den Embolus auflösen konnte. Bei einem Patienten mit Zustand nach einer Skrotalhernien-OP war bereits nach 20 Minuten ein großer Embolus im rechten Vorhof und Ventrikel ohne Zeichen einer Lungenembolie lysiert (100 mg rt-PA). Eine 81-jährige und eine 62-jährige Patientin mit im offenen Foramen ovale inkarzeriertem Embolus wurden mit 50, bzw. $100 \mathrm{mg}$ rt-PA behandelt, wobei nach 1 Stunde die Emboli vollständig lysiert waren. Die 81-Jährige zeigte nach 3 Tagen einen Verschluss der Arteria femoralis mit erfolgreicher chirurgischer Thrombektomie. Die 62-Jährige entwickelte 10 Stunden nach der Fibrinolyse ein Subduralhämatom, welches nach Einbringung eines Vena-Cava-Filters erfolgreich neurochirurgisch entlastet wurde. Bei einer 84-Jährigen mit einer WeberC-Fraktur war die Behandlung (100 mg rt-PA) ohne Nebenwirkungen erst nach 2 Tagen erfolgreich. Ein 46-Jähriger mit einem großen Embolus im rechten Ventrikel entwickelte 3 Minuten nach einem Bolus von $100 \mathrm{mg}$ rt-PA eine fulminante Lungenembolie, die sich jedoch nach 30 Minuten deutlich besserte und nach einem Tag nicht mehr nachweisbar war. Ein 66-Jähriger zeigte 9 Tage nach Pouch-OP mit Neoblase im Rahmen einer HIT II eine submassive Lungenembolie mit im rechten Herzen sichtbarem Embolus. Dieser war 12 Stunden nach Gabe von $100 \mathrm{mg}$ rt-PA verschwunden. Die thrombolytische Therapie mit einem Bolus von $50-100 \mathrm{mg}$ rt-PA ist eine äußerst erfolgreiche Therapie bei Transitemboli mit und ohne Inkarzeration im offenen Foramen ovale und sollte die Therapie der ersten Wahl sein. Wegen möglicher Komplikationen sollte diese nur auf einer Intensivstation erfolgen.

Kombinationstherapie mit Argatroban und gleichzeitiger intraarterieller Thrombolysetherapie mit Urokinase bei einem Patienten mit HIT-II-induzierter akuter Beinischämie

\section{J. Beyer, S. Gehrisch, J. Leonhard, M. Weise, S. Schellong}

Universitätsklinikum „C.G.Carus" der Technischen Universität Dresden, Fetscherstr. 74, 01307 Dresden

Hintergrund: Wir berichten über eine Erstanwendung von Argatroban bei gleichzeitiger intraarterieller Lysetherapie mit Urokinase bei einem Patienten mit manifester HIT-II und akuter Extremitätenischämie. Patienten und Methoden: Ein 68-jähriger Patient wurde wegen akuter arterieller Verschlüsse der rechten Beckenachse und der distalen Unterschenkelarterien links vorgestellt. Bei neu aufgetretenem Vorhofflimmern war der Patient wenige Tage zuvor auf eine Therapie mit Nadroparin 19000 aXa/d eingestellt worden. Bei Aufnahme bestand eine Thrombopenie (Abfall von $130 \mathrm{Gpt} / \mathrm{l}$ eine Woche zuvor auf $37 \mathrm{Gpt} / \mathrm{ml}$ ) und HIT-II-Antikörper (HPF4- und HIPA-Ak) konnten nachgewiesen werden. Eine Vorexposition mit Heparin war nicht zu eruieren. Die rechte Beckenachse konnte operativ revaskularisiert werden, wobei sich ein white clot fand. Bei kritischer Beinischämie des distalen Unterschenkels und Fußes links und angiografischem Komplettverschluss aller Arterien ab dem distalen Unterschenkelswurde bei fehlender operativer Revaskularisationsoption die Indikation zur Lysetherapie gestellt. Diese erfolgte über $48 \mathrm{~h}$ mit Urokinase (50000 bis $80000 \mathrm{IE} / \mathrm{h}$ ) über einen intraarteriellen Lysekatheter sowie mit Argatroban $5-6 \mathrm{mg} / \mathrm{h}(1,5-1,8 \mu \mathrm{g} / \mathrm{kg} / \mathrm{min})$ über die Kathe- 
terschleuse. Die Dosisanpassung von Argatroban erfolgte durch engmaschige Gerinnungsanalysen anhand der Thrombinzeit (Ziel 50-80 s bei normal 15-20 s). Ergebnisse: Unter Argatroban zeigte sich ein Anstieg der Argatrobanspiegel, der aPTT, der Thrombinzeit und der clotting time sowie ein Abfall der Prothrombinfragmente. Trotz hoher lokaler Dosierungen führte die Urokinasetherapie nur zu einem geringen Anstieg der Fibrinspaltprodukte und der D-Dimere; im Thrombelastogramm zeigte keine relevante systemische Hyperfibrinolyse. Entsprechend der mehrmals täglichen Gerinnungskontrollen inkl. Thrombelastogramm erfolgten Dosisanpassungen, Blutungskomplikationen traten nicht auf. Nach $\mathrm{Ab}$ schluss der Lysetherapie zeigte sich eine deutliche klinische Besserung der Perfusionssituation. Die Nachbehandlung des Patienten bestand in einer Antikoagulation mit Fondaparinux 7,5 mg/d sc und überlappender oraler Antikogulation mit Phenprocoumon. Schlussfolgerungen: Die Anwendung von Argatroban als Antikoagulans während einer intraarteriellen Urokinasetherapie ist möglich. Eine intensivmedizinische Überwachung mit engmaschiger Kontrolle der Gerinnungsparameter inklusive der Thrombinzeit ist zur Therapiesteuerung notwendig. Die Kombination aus intraarterieller Thrombolysetherapie und Antikoagulation mit $\mathrm{Ar}$ gatroban kann bei einzelnen Patienten mit HIT-II eine Therapieoption zur Rekanalisation peripherer Gefäßverschlüsse darstellen.

\section{Die Metforminlaktazidose - eine eigene Entität?}

\section{S. Friesecke, P. Abel, S. Runge}

Klinik für Innere Medizin B (Kardiologie, Pneumologie, Internistische Intensivmedizin) Klinikum der Universität Greifswald

Hintergrund: Metformin ist das Medikament der Wahl in der oralen Therapie übergewichtiger Typ-II-Diabetiker. Die wichtigste Komplikation ist die Metformin-assoziierte Laktazidose (MALA). Die meisten Publikationen hierzu stellen die pathogenetische Bedeutung des Metformins für die Entstehung der Laktazidose in Frage und postulieren eine koexistente akute Gewebshypoxie. Wir haben in 2 Jahren 6 Pat. mit MALA behandelt, bei denen ein toxisch-letaler Metforminspiegel nachgewiesen und ein Schock septischer, kardiogener oder hypovolämischer Genese und eine regionale Ischämie ausgeschlossen wurde. Ziel und Methoden: Um zu zeigen, dass sich die MALA von den Lakatazidosen anderer Genese unterscheidet, untersuchten wir retrospektiv alle Fälle, die 2004/2005 mit einem Laktat $>5 \mathrm{mval} / \mathrm{l}$ auf unsere internistische ITS aufgenommen wurden. Ergebnisse: Bei MALA zeigt sich ein signifikant tieferer $\mathrm{pH}$ Wert $(6,74 \pm 0,14 ; p<0,001)$ und ein signifikant höheres Laktat $(20,3 \mathrm{mval} / \mathrm{l} \pm 5,9 \mathrm{p}=0,001)$, verglichen $\mathrm{zu}$ den Lakatazidosen anderer Genese $(7,25 \pm 0,18$; bzw 11,2 $\pm 6,6)$. Die Signifikanz bleibt bestehen im Vergleich mit jeder einzelnen Erkrankung (n/pH:Mittelwert/Laktat:Mittelwert): Postreanimation $(24 / 7,23 / 12,0)$, Sepsis $(24 / 7,30 / 9,4)$, kardiogener Schock $(21 / 7,24 / 10,7)$, Mesenterialischämie $(5 / 7,38 / 9,7)$, sonstige $(8 / 7,23 / 11,8)$. Die Mortalität war hingegen nicht unterschiedlich. Schlussfolgerung: Die MALA unterscheidet sich signifikant in $\mathrm{pH}$-Abweichung und Laktat von allen anderen Ursachen einer Laktazidose. Somit muss die MALA als eigene Entität gewertet werden. Wir empfehlen, bei schwerster metabolischer Azidose auch nach Metformin-Einnahme zu fahnden, da mit intensiver Therapie, v.a. sofortigem Nierenersatzverfahren die Prognose besser ist, als bei einem $\mathrm{pH}<7 \mathrm{zu}$ erwarten.

\section{Herzchirurgische Intensivmedizin}

Early extubation and fast track management in elderly 002 coronary bypass surgery patients

\section{U. Boeken, P. Feindt, J. Litmathe, E. Gams}

Thorax- und Kardiovaskularchirurgie, Chir. Klinik, Heinrich-Heine-Universität, Düsseldorf

Objective: In cardiac surgery shorter hospital stays and decreased costs can be reached by early extubation and fast-track management protocols. The feasibility of such protocols in elderly patients is still discussed controversially. Methods: We analyzed 600 consecutive patients undergoing CABG. 372 patients (62\%) underwent early extubation, defined as extubation within 6 hours postoperatively (group e). In the early extubated group elderly patients of at least 70 years $(n=150$, mean age 75.1 years $)$ were compared with younger patients $(n=222,61.4$ years $)$. Results: The mean length of stay (LOS) in hospital for all patients extubated within 6 hours was 9.1 days vs 13.3 days for patients who underwent later extubation $(p<0.01)$. The hospital mortality rate was comparable between the older and the younger group of patients $(2.34 \%$ vs $2.19 \%)$. Reintubation rate was negligible in both groups of patients. Older patients had a significantly higher incidence of postoperative atrial fibrillation. We found a shorter LOS in hospital among the younger patients $(8.5 \pm 1.1$ vs $10 \pm 1.4$ days, $\mathrm{p}<0.05$ ), although the LOS on ICU was similar (old: $31 \pm 6$ vs. $27 \pm 4$ hours, $\mathrm{p}>0.05)$. In group e we could differ between immediate extubation (before ICU) and extubation in the following 6 hours. The immediate extubation could be correlated to the most uncomplicated postoperative courses among all early extubated patients. Conclusions: The results confirm the possibility of early extubation among elderly patients undergoing CABG procedures. Although they have more comorbid conditions, a significant number of these patients can be extubated early, with the consequence of a shortened LOS in hospital.

CABG-procedures in patients with pretreatment with the GPIIb/IIla-receptor antagonist tirofiban (Aggrastat ${ }^{\circledR}$ ): Modification of perioperative management?

U. Boeken, P. Feindt, J. Litmathe, M. Kurt, E. Gams Thorax- und Kardiovaskularchirurgie, Chir. Klinik, Heinrich-Heine-Universität, Düsseldorf

Objective: Due to an increasing number of patients with preoperative administration of tirofiban, a modification of perioperative management is important to reduce the incidence of postoperative bleeding complications.

Methods: We retrospectively analyzed all CABG-patients which received tirofiban preoperatively between $1 / 2002$ and $6 / 2005$ $(n=232) .3$ groups of patients regarding the perioperative administration of antifibrinolytic drugs were compared: group $A=$ controls $(n=70)$, group $B=$ aprotinin $(n=110)$, group $C=$ tranexamic acid $(n=52)$ Furthermore we could divide the patients depending on the time when tirofiban was stopped $(<2$ hours, $2-6$ hours, $>6$ hours preoperatively). For the preoperative use of further anticoagulatory drugs we could not find significant differences between the groups. Results: The postoperative blood loss was significantly higher in all tirofiban-patients (A-C) compared to a group of CABG-patients without tirofiban $(810 \pm 540 \mathrm{ml}$ vs $430 \pm 280 \mathrm{ml}, \mathrm{p}<0.05)$. The best results concerning blood loss, transfusion of red cell concentrates (rcc), fresh frozen plasma (ffp) and incidence of resternotomy could be found in patients with aprotinin (blood loos: A: $1150 \pm 560 \mathrm{ml}, \mathrm{B}: 630 \pm 305 \mathrm{ml}, \mathrm{C}$ : $910 \pm 480 \mathrm{ml}, \mathrm{p}<0.05$; rcc: A: $820 \pm 520 \mathrm{ml}, \mathrm{B}: 440 \pm 210 \mathrm{ml}$, C: $690 \pm 420 \mathrm{ml}, \mathrm{p}<0.05$; ffp: A: $600 \pm 290 \mathrm{ml}, \mathrm{B}: 300 \pm 180 \mathrm{ml}, \mathrm{C}:$ $590 \pm 450 \mathrm{ml}, \mathrm{p}<0.05$; resternotomy: A: $10 \%$, B: $5.45 \%$, C: $7.69 \%$, $\mathrm{p}<0.05)$. A further significant improvement could be seen in pa- 
tients who received platelets, intraoperative hemofiltration and in which tirofiban was stopped $>4$ hours preoperatively. The incidence of perioperative myocardial infarctions was not significantly different between the groups. Conclusions: The preoperative treatment with the GPI.

Pentoxifylline reduziert die Zeit der Intensivtherapie bei herzchirurgischen Patienten

H. Heinze, M. Heringlake, L. Bahlmann, C. Rosemann, M. Misfeld *, W. Eichler

Kliniken für Anästhesiologie und Herzchirurgie*

Universität zu Lübeck

Fragestellung: Die Pathogenese des Organdysfunktionssyndrom nach cardio-pulmonalem Bypass (CPB) ist sehr komplex. Hierbei spielt die Entzündungsreaktion eine entscheidende Rolle. Pentoxifylline (PTX), ein nicht-spezifischer Phosphodiesteraseinhibitor, besitzt anti-inflammatorische und rheologische Eigenschaften und könnte daher sinnvoll bei herzchirurgischen Patienten nach CPB eingesetzt werden. Wir untersuchten in dieser prospektiven, randomisierten, plazebokontrollierten Studie die Auswirkungen einer Einzelgabe von $300 \mathrm{mg}$ PTX vor CPB. Material und Methoden: Nach Zustimmung der örtlichen Ethikkommission und schriftlicher Einwilligung, untersuchten wir 39 Patienten, welche sich einer Aorto-coronaren Bypassoperation unterzogen. 20 Patienten erhielten vor Einleitung der Anästhesie $300 \mathrm{mg}$ PTX intravenös, 19 Patienten erhielten Kochsalz als Plazebo. Hämodynamische Parameter, Lungenfunktionsparameter und Plasmaspiegel von Tumornekrosefaktor $\alpha(\mathrm{TNF} \alpha)$ wurden nach Einleitung der Anästhesie (T1), nach Beeindigung des CPB (T2), eine Stunde nach Aufnahme auf die Intensivstation (T3) und am Morgen des ersten postoperativen Tages bestimmt. Zusätzlich wurden Outcomeparameter erhoben. Ergebnisse: PTX-behandelte Patienten zeigten niedrigere systolische und mittlere pulmonalarterielle Druckwerte, sowie niedrigere TNF $a$-Werte. Andere Hämodynamik- und Lungenfunktionsparameter unterschieden sich nicht zwischen den Gruppen. Im Trend wurden die Patienten in der PTX-Gruppe früher extubiert. Der Aufenthalt auf der Intensivstation in Kombination mit der Intermediate Care Station war in der Plazebogruppe signifikant länger. Schlussfolgerung: Auch eine Einmalgabe von PTX vor $\mathrm{CPB}$ reduziert TNF $\alpha$-Werte, ein Effekt, welcher schon mit höheren und häufigeren Gaben gezeigt werden konnte. PTX könnte die Beatmungszeit reduzieren und verkürzt die Zeit der intensivmedizinischen Therapie.

Vergleich einer postoperativen Analgosedierung mit Propofol-Ketamin S versus Propofol-Piritramid 024 nach koronarchirurgischer Bypassoperation bezüglich Hämodynamik, Patientenzufriedenheit, Aufwach- und Traumverhalten

\section{S. N. Piper, K.D. Röhm, T.A. Schöllhorn, C. Ochmann, J. Boldt, S. W. Suttner \\ Klinik für Anästhesiologie und Operative Intensivmedizin, Klinikum Ludwigshafen}

Fragestellung: Die Opioidgabe ist ein fester Bestandteil der postoperativen Analgosedierung auf der Intensivstation (ICU). Bei geringen kardiovaskulären Nebenwirkungen und hoher analgetischer Potenz, zeichnen sie sich aber auch durch Atemdepression, Übelkeit und Erbrechen, Muskelrigidität und verminderte Motilität des Gastrointestinaltraktes aus. Ketanest $S$ ist ein potentes Analgetikum, das den Halluzinogenen nahe steht und eine dissoziative Anästhesie hervorruft. Es induziert eine sympathoadrenerge Reaktion. Unter Monogabe wurden Alpträume und Halluzinationen beschrieben. Ziel dieser Arbeit sollte es sein, eine postop. Analgosedierung mit Propofol-Ketamin S vs. Propofol-Piritramid hinsichtlich Hämodynamik, Patientenzufriedenheit, Aufwachund Traumverhalten, nach koronarer Bypass-OP $\mathrm{zu}$ evaluieren.
Methode: Prospektive, randomisierte Doppelblindstudie. Nach Zustimmung der Ethikkommission wurden 42 Patienten zur elektiven, koronarchirurgischen Bypass-OP in die Studie eingeschlossen. Nach Ankunft auf der ICU wurden Patienten der Gruppe A $(\mathrm{n}=21)$ mit Propofol $(1-3 \mathrm{mg} / \mathrm{kg} / \mathrm{h})$ und Ketamin $(1-4 \mathrm{mg} / \mathrm{kg} / \mathrm{h})$ oder mit Propofol $(1-3 \mathrm{mg} / \mathrm{kg} / \mathrm{h})$ und Piritramid $(7,5 \mathrm{mg}$ iv. als Bolus nach Bedarf; Gruppe B; $\mathrm{n}=21$ ) analgosediert. Das Aufwachverhalten wurde durch die Extubationszeit (Zeit von Ende Sedierung bis Extubation) und den Zeitpunkt des 1. Augenöffnens objektiviert. Das Monitoring erfolgte mit invasiver Blutdruckmessung und Pulmonaliskatheter. Zu den Messzeitpunkten ICU-Aufnahme (T0), 2 Std. (T1) und 24 Std. (T2) postoperativ wurden MAP, HR, HZV, PAMP, PCWP, SVR und PVR dokumentiert. Die Patientenzufriedenheit wurde 2 und 24 Stunden nach Extubation mittels visueller Analogskala ermittelt. Die Patienten wurden nach Träumen und Traumqualität befragt, und die Inzidenz von PONV und Shivering wurde dokumentiert. Ergebnisse: Es bestanden zu keiner Zeit signifikante Unterschiede bezüglich aller hämodynamischen Parameter. Die Extubationszeiten (Gruppe A: 258 \pm 109 vs. B: $257 \pm 140 \mathrm{~min}$ ) und die Patientenzufriedenheit (2 Std.: Gruppe A: Median: 9 Range: $6-10$ vs. B: Median: 9 Range: 7-10; 24 Std.: Gruppe A: Median: 9 Range: $7-10$ vs. B: Median: 9 Range: 7-10) unterschieden sich nicht signifikant. Patienten der Gruppe A $(82 \pm 51 \mathrm{~min})$ öffneten signifikant schneller die Augen als Patienten der Gruppe B (156 $\pm 110 \mathrm{~min})$. Patienten der Gruppe A träumten signifikant häufiger (2 Std.: A: $67 \%$ vs. B: $28 \%$; 24 Std.: A: $38 \%$ vs. B: $9 \%) .3$ Patienten der Gruppe A (14\%) und 2 Patienten (9\%) der Gruppe B hatte Angstträume. Patienten der Gruppe A litten signifikant seltener an PONV (5 vs. 33\%) und Shivering (5 vs. 43\%). Schlussfolgerungen: Wir fanden keine Unterschiede der Extubationszeit und der Patientenzufriedenkeit. Insgesamt träumten Patienten der Ketamingruppe signifikant häufiger, es traten aber nicht mehr unangenehme Traumereignisse auf. Eine Alteration der Hämodynamik war unter Ketamin nicht nachweisbar. Patienten der Ketamingruppe litten signifikant seltener unter PONV und Shivering.

Die serielle Erfassung des SOFA-Score in der Herzchirurgie

\section{A. Kroener, K. Hekmat, H.-J. Geissler, T. Wahlers}

Klinik für Herz- und Thoraxchirurgie, Universitätsklinik Köln

Einleitung: Evaluierung der seriellen Erfassung des SOFA- (Sequential Organ Failure Assessment) Score zur Pädiktion der Letalität in der herzchirurgischen Intensivmedizin. Patienten und Methoden: Tägliche prospektive Erfassung aller herzchirugischer $\mathrm{Pa}-$ tienten ( $>18$ Jahre, $\mathrm{n}=1444$, mittleres Alter 65,4 Jahre) vom 01.01. 03 bis 31.08 .04 mit einem Aufenthalt von mehr als 12 Stunden auf der ICU und 24 stündliche Erfassung des SOFA sowie 48 und 96 stündlicher Berechnung des Delta-SOFA. Die Kalibrierung des Scores wurde mit dem Hosmer-Lemeshow goodness-offit Test und die Diskriminierungsfähigkeit mit der ROC-Analyse getestet. Ergebnisse: Der SOFA-Score korreliert nach herzchirurgischer Operation gut mit der Letalität. In der binär logistischen Regression kann in den ersten 6 Tagen eine hohe Kalibrationsstärke nachgewiesen werden, lediglich am 1 . postoperativen Tag konnte ein signifikanter Unterschied zwischen vorhergesagter und beobachteter Letalität gefunden werden. Die ROC-Analyse zeigte eine hohe Diskriminierung in den ersten 96 Stunden (Tag 0: 0,81, $\mathrm{p}<0,0005$; Tag 1: 0,91, p <0,0005; Tag 2: 0,88, p<0,0005; Tag 3: $0,88, p<0,0005)$, die höchste Diskriminierung der seriellen Erfassung ergabt der Delta SOFA $48(0,85, \mathrm{p}<0,0005$ bei $\mathrm{n}=650)$ und Delta SOFA $96(0,85, \mathrm{p}<0,0005$ bei $\mathrm{n}=262)$. Die Letalität stieg erwartungsgemäß mit ansteigendem Delta SOFA 48 (Delta SOFA $48<0$ 6,3\%; Delta SOFA 48=0 17,2\%; Delta SOFA $>0$ 23,1\%). In allen hierauffolgenden Untergruppen des Delta SOFA 96 zeigte sich jedoch eine drastische Steigerung der Letalität bei ansteigenden Delta SOFA 96 (Delta SOFA 96>0 33,3-44,4\%). Der Max-SOFAund Mean-SOFA-Score wiesen bei einer guten Diskriminierung ei- 
ne unzureichende Kalibration auf. Schlussfolgerung: Der SOFAScore hat in der direkt postoperativen Phase durch fehlende Einbeziehung von speziellen herzchirugischen Therapiefomen (Assist devices) und spezieller kurzfristiger und zügig behebbaren Problemen nach Einsatz der Herz-Lungen-Maschine (z.B. $\mathrm{pO}_{2}$-Abfall durch Atelektasen) schwächen im prädiktivem Wert. Die serielle Erfassung der Prognose wird durch den Delta-SOFA nach 48 und 96 Stunden hingegen gut unterstützt und das Letalitätsrisiko adäquat dargestellt.

\section{Postoperative Nachblutungen -} ein diagnostisches und therapeutisches Problem

\section{G. Spalding}

Abteilung für Kardiochirurgie, Evangelisch-Freikirchliches Krankenhaus und Herzzentrum Brandenburg in Bernau

Postoperative Nachblutungen in der Kardio- und Thoraxchirurgie stellen oftmals eine Herausforderung dar. Handelt es sich um die Folgen einer intraoperativen Verdünnungskoagulopathie oder sind die Verluste allein chirurgischer Genese? Die klassische Labordiagnostik dauert für schnell zu treffende Entscheidungen zu lange und spiegelt dann nicht mehr die aktuelle Situation wider. Die Rotationsthrombelastometrie (ROTEM) kann als bed side Diagnostikum frühzeitig wertvolle Hinweise zur Beurteilung einer vorhandenen plasmatischen Gerinnungsstörung geben. Anhand von zwei Kasuistiken, sowohl einem thoraxchirurgischen Eingriff als auch einer aortokoronaren Revakularisationsoperation, sollte der Stellenwert der ROTEM-Analyse in der Diagnostik und Therapie bei vermehrten postoperativen Drainageverlusten demonstriert werden. Die Substitution bei einem nachweislichen Defizit von Gerinnungsfaktoren erfolgte sowohl mit FFP als auch mit Einzelfaktoren. Dabei zeigte sich eine eindeutige Überlegenheit der Einzelfaktorenapplikation gegenüber der Therapie mit FFP hinsichtlich zeitlicher Verfügbarkeit, Volumenbelastung und Wirksamkeit, sowie des nicht zu vernachlässigenden Infektionsrisikos. Selbst bei eindeutig nachweisbarer chirurgischer Blutung ist es unerlässlich, eine suffiziente Therapie der Verlust- und Verdünnungskoagulopathie durchzuführen. Durch ein frühzeitiges effizientes Gerinnungsmanagement lassen sich sowohl mögliche Komplikationen postoperativer Nachblutungen als auch der Verbrauch an Blutprodukten besser kontrollieren und damit insgesamt reduzieren.

Perioperative hämodynamische Effekte des Angiotensin I-Converting Enzym Insertion/Deletion Polymorphismus bei koronarer Bypassoperation

\section{A.F. Popov, B.J. Hinz* C.O.J. Liakopoulos, D.J.D. Schmitto,}

E.H. Dörge, F. F. A. Schöndube

Thorax-, Herz- und Gefäßchirurgie Universitätsklinikum Göttingen

*Anästhesiologie, Rettungs- und Intensivmedizin,

Universitätsklinikum Göttingen

Hintergrund: Der Insertion/Deletion Polymorphismus des Angiotensin Converting Enzym (ACE) zeigt drei Genotypen (II, ID, $\mathrm{DD})$ und weist bei D-Allel-Träger nach koronaren Bypassoperationen (ACB) mit Herz-Lungenmaschine (HLM) nach zwei Jahren Assoziationen zu Mortalität und kardialen Morbiditäten auf [1]. Über einen Einfluss in der direkten perioperativen Phase ist bislang nichts bekannt. Wir vermuten, dass der Polymorphismus in der perioperative Phase einen Effekt hat und untersuchten deshalb $\mathrm{zu}$ verschiedenen Zeitpunkten den Einfluss des Polymorphismus auf hämodynamische Veränderungen bei ACB mit HLM. Methoden: Einhundertzehn Patienten mit geplanter ACB wurden in einer klinisch prospektiven Studie eingeschlossen. Perioperative hämodynamische Messungen wurden mittels Pulmonaliskatheter (Thermodilution-Technik) vor und nach HLM, sowie postoperativ nach $4 \mathrm{~h}, 9 \mathrm{~h}$ und $19 \mathrm{~h}$ durchgeführt. Neben der systemischen Hämodynamik wurde der Herzindex (CI), systemische und pulmo- nale Gefäßwiderstand (SVRI/PVRI), pulmonalarterieller Mitteldruck (PAP) und die postoperative Katcholaminunterstützung gemessen. Der ACE-Polymorphismus wurde aus präoperativ entnommenen Blutproben der Patienten mit der Polymerase Kettenreaktion bestimmt. Patientengruppen wurden entsprechend ihres Genotyps in zwei Gruppen (I/I-Gruppe, I/D + DD-Gruppe) eingeteilt. Alle Ergebnisse sind als Mittelwert und Standardabweichung angegeben. Die Unterschiede zwischen den Gruppen wurden mittels T-Test auf einem Signifikanzniveau von $p<0.05$ untersucht. Ergebnisse: Es zeigte sich eine Verteilung für den Polymorphismus von $18 \%$ (II), 57\% (I/D) und 25\% (DD). Die untersuchten 110 Patienten unterschieden sich nicht hinsichtlich Alter (II 66 \pm 6 , ID/DD $66 \pm 8$ Jahre), Body-mass-index (II 28 2 , ID/DD $29 \pm 5 \mathrm{~kg} / \mathrm{m}^{2}$ ), Geschlecht (II 16/4, ID/DD $63 / 27 \mathrm{~m} / \mathrm{w}$ ) und Euroscore (II $3,1 \pm 1,9$, ID/DD $3,5 \pm 2,1$ ). Es zeigte sich kein Unterschied in der Mortalität. In der II-Gruppe starben 2 von $20 \mathrm{~Pa}-$ tienten und in der ID/DD-Gruppe 2 von 88 Patienten. Vor HLM war der PVRI in der ID/DD-Gruppe gegenüber der II-Gruppe signifikant erhöht (II: $227 \pm 121$, ID/DD $297 \pm 169 \mathrm{dyn} \cdot \mathrm{s} \cdot \mathrm{m}^{2} \cdot \mathrm{cm}^{-5}$ ). Sowohl direkt nach Sternumverschluss als auch vier Stunden nach HLM zeigten sich hierfür keine signifikanten Veränderungen. Neun Stunden nach HLM ist eine Tendenz für den PVRI (II: $247 \pm 134, \mathrm{ID} / \mathrm{DD} 290 \pm 117 \mathrm{dyn} \cdot \mathrm{s} \cdot \mathrm{m}^{2} \cdot \mathrm{cm}^{-5}$ ) und ein signifikanter Unterschied beim PAP (II: $19 \pm 6$, ID/DD: $23 \pm 8$ ) zu beobachten gewesen. 19 Stunden nach HLM zeigten sich keine signifikanten Unterschiede in der Hämodynamik. Schlussfolgerung: Das D-Allel des ACE-Genpolymorphismus hat keinen signifikanten Einfluss auf die systemische Hämodynamik und Frühmortalität in Patienten mit ACB und HLM. Transiente Veränderungen der pulmonalen Hämodynamik waren mit dem D-Allel assoziiert und könnten auf einer reduzierten Aktivität von ACE nach HLM basieren [2].

Literatur: [1] Völzke H et al: Chest 2002; 122:31-36. [2] Gorin AB, Liebler J: Am Rev Respir Dis 1986; 134:79-84

Korrelation von Procalcitonin und C-reaktivem Protein 100 als inflammatorischer Marker nach Bypass-Operationen

\section{T. Chavez, D. Diaz, I. Ennker, D. Petrowski,}

\section{Osthoff, L. Vöhringer, J. Ennker}

Herzchirugie, Herzzentrum Lahr/Baden

Fragestellung: Inflammatorische Prozesse nach Bypass-Chirugie, Thorakotomie und Manipulation des Herzens bzw. Herzstillstands verursachen erhebliche postoperative Komplikationen. Eine Möglichkeit zur Erfassung des Schweregrades der inflammatorischen Ereignisse ist die Kontrolle des CRP und Procalcitonin-Wertes. Methoden/Ergebnisse: Bei 120 Patienten, die sich einer BypassOperation unterzogen haben (75 OPCAP/45 HLM), wurden CRP, PCT, Leukozyten, Blutzucker, Temperatur, Kreatinin, CK und CKMB präoperativ sowie an sechs folgenden postoperativen Tagen bis zur Entlassung gemessen. Die maximale Konzentration des CRP-Wertes in beiden Gruppen wurde am zweiten und dritten postoperativen Tag gemessen, allerdings war der maximale Wert in der Gruppe der Patienten mit HLM höher $(p<0,0001)$. Die Anzahl der Leukozyten korreliert nicht mit dem CRP bzw. PCT Wert. Präoperativ erhöhte Kreatinin-Werte haben keinen signifikanten Einfluss auf die erste postoperative Phase. Zusammenfassung: Der CRP Wert zeigt am zweiten bis dritten postoperativen Tag sein Maximum (solange keine begleitende bakteriell/virale Entzündung vorhanden ist). Der Procalcitonin-Wert steigt erst am fünften bis sechsten Tag an. Patienten, die präoperativ bereits erhöhte Procalcitonin-Werte haben, zeigen einen protrahierten postoperativen Verlauf. In dieser Gruppe ist die gesamte Prognose schlechter als bei Patienten mit einem normalen ProcalcitoninWertes. Für eine Prognose und Einschätzung des Schweregrades eines inflammatorischen Prozesses eignet sich der CRP-Wert besser, der Procalcitonin-Wert ist besser für die Einschätzung einer bakteriellen/viralen Entzündung. 
Prediction of mortality and organ dysfunction in icu cardiac surgical patients

\section{K. Hekmat ${ }^{1}$, A. Kroener ${ }^{2}$, U. Fischer ${ }^{2}$, H-J. Geissler ${ }^{2}$,} U. Mehlhorn ${ }^{2}$, A. Hannekum ${ }^{1}$

${ }^{1}$ Herzchirurgie, Universität Ulm, ${ }^{2}$ Herz- und Thoraxchirurgie, Universität zu Köln

Background: The purpose of this study was to develop and validate a specific postoperative score in intensive care unit (ICU) cardiac surgical patients for assessment of organ dysfunction and survival. Methods: This prospective study consisted of all consecutive adult patients admitted after cardiac surgery to our ICU over a period of 5.5 years. Evaluation of variables was performed using the first year patients who stayed in ICU for at least 24 hours. The reproducibility was then tested in two validation sets using all patients. Performance was assessed with the Hosmer-Lemeshow (HL) goodness-of-fit test and receiver operating characteristic (ROC) curves and compared with the APACHE II and MODS. Results: A total of 6007 patients were admitted to the ICU after cardiac surgery. Mean HL-values for the new score were 5.8 (APACHE II, 11.3; MODS, 9.7) for the construction set, 7.2 (APACHE II, 8.0; MODS, 4.5) for the validation set I and 4.9 for the validation set II. The mean area under the ROC curve was 0.91 (APACHE II, 0.86; MODS, 0.84) for the new score in the construction set, 0.88 (APACHE II, 0.84; MODS, 0.84) in the validation set I and 0.92 in the validation set II. Conclusion: Our new ten-variable risk index performs very well, with calibration and discrimination very high, better than general severity systems, and it is an appropriate tool for daily risk stratification in ICU cardiac surgery patients. Thus, it may serve as an Expert System for diagnosing organ failure, decision making, resource evaluation, and predicting mortality in ICU cardiac surgical patients.

\section{Descending Thoracic Aorta Atheroma Increases \\ the Risk of Postoperative Renal Dysfunction \\ in Cardiac Surgical Patients with Intraaortic \\ Balloon Pump Counterpulsation \\ M. Nowak, S. K. Shernan, J.D. Rawn, P.S. Shekar, J.K. Tuli, K. Unertl, H.K. ltzschig, P. Rosenberger}

Brigham and Women's Hospital/Harvard Medical School Boston

MA, USA und Department of Anesthesiology and Intensive Care Medicine, Tübingen University Hospital, Eberhard-Karls-University, Tübingen, Germany

Introduction: Intraaortic balloon pump counterpulsation (IABP) is often used to treat perioperative signs and symptoms of refractory myocardial ischemia or low cardiac output in cardiac surgical patients. The use of IABP has been linked to an increased incidence of perioperative renal dysfunction (RD). Several investigations have speculated whether the development of renal $\mathrm{RD}$ in cardiac surgical patients with an IABP, could be associated with emboli from descending thoracic aortic (DTA) atheroma. Therefore, we investigated the possible correlation between the degree of DTA atheroma assessed by intraoperative transesophageal echocardiography (TEE) and postoperative RD in cardiac surgical patients in whom an IABP had been placed. Methods: The patient population consisted of 227 who underwent cardiac surgery between 2001 and 2005, and in whom an IABP had been placed either pre- or intraoperatively. Patients with preoperative RD (creatinine $>1.5 \mathrm{mg} / \mathrm{dl}$ ) or poor TEE imaging of the DTA were excluded. The patients were matched to controls according to age, gender, type of surgery, preoperative ejection fraction and grade of DTA atheroma. Postoperative RD was defined as an increase of creatinine $>2$ or 2 times the most recent preoperative creatinine level according to criteria from the Society of Thoracic Surgery. Patients were classified into four Groups according to grade of DTA atheroma: I) none; II) intimal thickening; III) plaque protruding less than $<5 \mathrm{~mm}$, and IV) plaque protruding $>5 \mathrm{~mm}$ or mobile. Statistical analysis was performed using multivariable lo- gistic regression analysis and Chi-Square test. Results: Demographic data did not differ between IABP and control patients. There was an equal distribution in the type of surgical procedure between Groups I-IV. CPB time (Group I: 136 vs. 159 min; Group II: 128 vs. $146 \mathrm{~min}$; Group III: 149 vs. $173 \mathrm{~min}$; Group IV: 190 vs. $160 \mathrm{~min}$ ) and cross clamp time (Group I 85 vs. 100 min; Group II: 92 vs. $95 \mathrm{~min}$; Group III: 105 vs. $105 \mathrm{~min}$; Group IV: 124 vs. $116 \mathrm{~min}$ ) were not significantly different. The incidence of RD in the IABP patients vs. controls by Group were as follows: I: 6.4 vs $3.2 \%$; II: 7.1 vs $2.0 \%$; III: 11.4 vs $7.4 \%$; IV: 21 vs $3.5 \%$ ( $<<0.05$ for all groups). Conclusion: Perioperative IABP is associated with a higher incidence of RD in cardiac surgical patients. DTA calcification alone is not an independent risk factor for RD. However, cardiac surgical patients with DTA atheroma who require IABP, have an even greater risk for postoperative RD.

Einfluss der Saugerblutseparation während koronarchirurgischer Eingriffe mit HLM auf die Ausschüttung inflammatorischer Zytokine

A.A. Peivandi, S. Sperling, N. Kayhan, D. Peetz ${ }^{*}$ M. Dahm, C.H. Vahl

Klinik und Poliklinik der Herz-, Thorax- und Gefäßchirurgie ${ }^{*}$ Institut für Klinische Chemie

Fragestellung: Um die Gabe homologer Blutprodukte in der Herzchirurgie zu minimieren, werden verschiedene Maßnahmen zur Wiederverwertung von Eigenblut angewendet. Hierunter fällt die routinemäßige Retransfusion des im Kardiotomiereservoir innerhalb der Operation gesammelten mediastinalen Blutes (Saugerblut). Verschiedene Studien konnten zeigen, dass dieses Blut extrem hohe Konzentrationen an Zytokinen und anderen Mediatoren enthält. Diese Substanzen können möglicherweise zu einer klinisch relevanten nicht selten auftretenden SIRS sowie Organdysfunktionen führen. In einer prospektiven klinischen Studie wurde der Einfluss der Saugerblutseparation auf die Ausschüttung inflammatorischer Zytokine untersucht. Material und Methodik: In die Studie eingeschlossen wurden 2 Gruppen von insgesamt 102 Patienten mit koronarer Herzkrankheit, die sich einer elektiven Koronarrevaskularisation unterzogen. In Gruppe I $(n=52$, Alter: $65,2 \pm 8,3$ Jahren, $13 \mathrm{w} / 39 \mathrm{~m}$ ) wurde Saugerblut in einem Kardiotomiereservoir gesammelt, gewaschen und anschließend als autologer Erythrozytenkonzentrat retransfundiert. In Gruppe II $(\mathrm{n}=50$, Alter: 63,1 $\pm 9,4$ Jahren, $14 \mathrm{w} / 36 \mathrm{~m})$ wurde das Saugerblut ohne Separationsvorgang gefiltert dem Herzlungenmaschinenkreislauf wieder zugeführt. Bei allen Patienten wurden Enzymverläufe der pro- und antiinflammatorischen Zytokine IL-6, IL-8, IL-10, TNF- $\alpha$ sowie C-reaktives Protein (CRP) und Leukozytenzahl engmaschig prä-, intra- und postoperativ bestimmt. Ergebnisse: In beiden Gruppen war IL-6 im Serum präoperativ nicht nachweisbar bzw. im Normbereich und stieg intra- und postoperativ signifikant an. Dieser Anstieg war in der Gruppe II jedoch $\mathrm{zu}$ allen untersuchten Zeiten statistisch signifikant höher als in der Gruppe I. IL-6 erreichte seinen medianen Gipfel in der Gruppe I schon nach $2 \mathrm{~h}(62,79 \mathrm{pg} / \mathrm{ml},[\mathrm{Q} 1=43,15, \mathrm{Q} 3=79,38]$ und in der Gruppe II erst nach $4 \mathrm{~h} \quad(55,94 \mathrm{pg} / \mathrm{ml}, \quad[\mathrm{Q} 1=39,23$, $\mathrm{Q} 3=110,02]$. Für IL-8 konnten weder signifikante Unterschiede in den einzelnen Gruppen, noch ein bedeutender Anstieg der Enzymkonzentration nachgewiesen werden. In der Gruppe II wurden $\mathrm{zu}$ entsprechenden Zeiten höhere Konzentrationen von TNF- $\alpha$ erreicht. Diese Ergebnisse waren allerdings nicht statistisch signifikant. Als antiinflammatorisches Zytokin war IL-10 nur in der Gruppe I in messbaren Konzentrationen nachweisbar. Die Peaks für die Konzentration von CRP in beiden Gruppen waren 48 Stunden postoperativ. Signifikante Unterschiede zwischen den beiden Gruppen gab es allerdings nicht. Schlussfolgerung: Die Unterschiede im postoperativen Anstieg der Enzymkonzentrationen von IL-6 und TNF- $\alpha$ nach Transfusion von gewaschenem und ungewaschenem Saugerblut weisen auf die Effektivität der Saugerblutseparation hin. Ob diese Ergebnisse hinsichtlich der Reduktion ei- 
nes SIRS klinisch relevant sind und damit die Saugerblutseparation als Routineverfahren eingesetzt werden sollte, muss in zukünftigen Untersuchungen näher geklärt werden.

\section{Änderungen in Laktat, gemischtvenöser Sauerstoffsättigung und Indozyaningrün- Plasmaverschwinderate bei Patienten nach herzchirurgischem Eingriff}

O. Thümer, A. Holland, C. Schelenz, S. G. Sakka

Klinik für Anästhesiologie und Intensivtherapie,

Friedrich-Schiller-Universität Jena

Einleitung: Parameter der globalen und regionalen Durchblutung werden zur Steuerung der Therapie bei kritisch kranken Patienten empfohlen. In dieser klinischen Studie untersuchten wir daher, ob und inwieweit Änderungen in regionalen Parametern anhand globaler Parameter und untereinander abgeschätzt werden können. Wir betrachteten die Änderungen im Herzindex, der gemischtvenösen Sauerstoffsättigung $\left(\mathrm{SvO}_{2}\right)$, dem Serumlaktat, der gastralen Durchblutung (Tonometrie) und der Indozyaningrün-Plasmaverschwinderate (ICG-PDR) als Parameter der Leberfunktion/-perfusion. Patienten und Methoden: Mit Zustimmung durch die lokale Ethikkommission und schriftlichem Einverständnis wurden 52 Patienten (39 männlich, 13 weiblich, Alter $65 \pm 10$ Jahre) eingeschlossen. Alle Patienten wurden auf Grund klinischer Indikation mittels Pulmonalarterienkatheter und invasiver Blutdruckmessung überwacht. Serumlaktat und $\mathrm{SvO}_{2}$ wurden mit Hilfe eines kommerziellen Blutgasanalysegerätes bestimmt. Die ICG-PDR wurde mit Hilfe eines transkutanen Systems gemessen und der gastrale Kohlendioxidpartialdruck $\left(\mathrm{PRCO}_{2}\right)$ anhand der Lufttonometrie. Alle Messwerte wurden postoperativ unmittelbar nach Aufnahme auf der Intensivstation und 2 Stunden später erfasst. Für die statistische Auswertung wurde ein nicht-parametrischer Test für verbundene Stichproben und eine lineare Regressionsanalyse benutzt. Ein $\mathrm{p}<0,05$ wurde als statistisch signifikant betrachtet. Ergebnisse: Herzindex $\left(2,8 \pm 0,8\right.$ vs. $\left.3,2 \pm 0,7 \mathrm{l} / \mathrm{min} / \mathrm{m}^{2}\right)$, Serumlaktat $(1,9 \pm 1,2$ vs. $2,4 \pm 1,6 \mathrm{mmol} / \mathrm{l})$, und $\mathrm{PRCO}_{2}(5,2 \pm 1,0$ vs. $5,6 \pm 1,1 \mathrm{kPa})$ stiegen im Untersuchungszeitraum signifikant an. Hingegen blieben die $\mathrm{SvO}_{2} \quad(66 \pm 7$ vs. $68 \pm 6 \%)$ und die ICG-PDR $(21,4 \pm 6,9$ vs. $21,8 \pm 7,4 \% / \mathrm{min})$ unverändert. Der zentrale Venendruck ( $8 \pm 3$ vs. $8 \pm 4 \mathrm{mmHg})$ und der Hämoglobingehalt $(6,2 \pm 0,9$ vs. $6,0 \pm$ $0,8 \mathrm{mmol} / \mathrm{l})$ waren konstant. Die Körpertemperatur $(36,0 \pm 0,8$ vs. $36,6 \pm 0,7^{\circ} \mathrm{C}$ ) und die Dosierungen für Noradrenalin (im Mittel 0,04 vs. $0,02 \mu \mathrm{g} / \mathrm{kg} / \mathrm{min}$ ) und Adrenalin (im Mittel 0,02 vs. $0,03 \mu \mathrm{g} / \mathrm{kg} /$ min) waren jedoch signifikant unterschiedlich. Die Änderungen zwischen $\mathrm{SvO}_{2}$ und Herzindex korrelierten moderat $(\mathrm{r}=0,43$, $\mathrm{p}<0,001)$. Hingegen ließ sich keine Korrelation nachweisen zwischen den Änderungen von Herzindex und ICG-PDR $(r=0,07$, $p=0,62)$ bzw. Herzindex und Serumlaktat $(r=0,01, p=0,91)$. Die Korrelation zwischen den Änderungen in Herzindex und $\mathrm{PRCO}_{2}$ betrug $r=0,37(p=0,006)$. Es fanden sich keine Beziehungen zwischen den Sauerstofftransport- bzw. regionalen Durchblutungsvariablen. Die jeweiligen Korrelationskoeffizienten betrugen: $r=0,06(p=0,65)$ für deltaICG-PDR/deltaSvO $, r=0,009(\mathrm{p}=0,95)$ für deltaICG-PDR/ deltaLaktat und $\mathrm{r}=0,24(\mathrm{p}=0,08)$ für deltaSvO ${ }_{2} /$ deltaLaktat. Schlussfolgerung: Änderungen in Serumlaktat, $\mathrm{SvO}_{2}$, ICG-PDR und $\mathrm{PRCO}_{2}$ korrelieren nicht unbedingt mit Änderungen im Herzindex und zeigten keine Korrelation untereinander, so dass von den Änderungen eines dieser Parameter nicht auf die eines jeweils anderen zurückgeschlossen werden kann.
Inhalative iloprost for weaning from cardiopulmonary

199 bypass support in patients with right heart failure during implantation of the novel axial flow left ventricular assist device system HeartMate II

M. Winterhalter ${ }^{*}$ S. Fischer, R. Tessmann, A. Goerler, S. Piepenbrock*, MD, A. Haverich, MD, M. Strueber, MD ${ }^{*}$ Zentrum Anästhesiologie, Abteilung für Thorax-Herzund Gefässchirurgie; Medizinische Hochschule Hannover

Background: Ventricular assist devices (VAD) are increasingly implanted into patients with medication-refractory hart failure NYHA Class IV, who are at risk of imminent death. In patients with severe pulmonary hypertension left (L)VAD implantation may not be reasonable, because these patients are at high risk for acute right ventricular (RV) failure after implantation and may, therefore, not be weanable from cardio-pulmonary bypass (CPB). Thus, in LVAD implantation sufficient RV function is essential to avoid temporary mechanical RV support or even biventricular (B)VAD implantation. Case reports: Here, we report two cases with severe acute intraoperative RV failure following implantation of the novel axial flow LVAD HeartMate II and the successful use of inhaled iloprost for weaning from CPB. During weaning from $\mathrm{CPB}$ an iloprost inhalation system was attached to the ventilatory circuit for 4 minutes by using a novel ultrasonic atomizer (Schill multisonic ${ }^{\circledR}$, Schill GmbH, Probstzella, Germany). Patient 1: A 32year-old male patient presented with recurrent left heart failure due to dilative cardiomyopathy. Echocardiography showed a dilated left ventricle (LVEF: 16\%). The mean pulmonary artery pressure was $28 \mathrm{mmHg}$. Due to rapid deterioration a HeartMate II LVAD was implanted. Post-implantation a gradual reduction of extracorporeal circulation was attempted and the LVAD was turned on at $6.500 \mathrm{rpm}$. However, RV failure developed despite high doses of epinephrine and dobutamine. The additional administration of $28 \mathrm{ppm}$ of inhaled Nitric oxide (NO) did not improve RV function. Further weaning from CBP was impossible. To possibly avoid RV bypass, $20 \mu \mathrm{g}$ of aerolized iloprost were administered. Immediately, an improvement of RV contraction was observed. Pulmonary artery pressures (PAP) immediately decreased from $36 \mathrm{mmHg}$ (mean) to $30 \mathrm{mmHg}$ (mean) after iloprost inhalation. After stabilization, the patient could be weaned from CPB. Patient 2: A Heart Mate II was implanted into a 29 year-old male patient with decompensated heart failure NYHA class IV due to dilative cardiomyopathy. Echocardiography showed a severely dilated heart with reduced ventricular function (LVEF: 15\%). After HeartMate II implantation, an attempt was made to wean the patient from CPB. However, RV failure developed despite high doses of epinephrine and dobutamine. Immediate inhalation of iloprost $(20 \mu \mathrm{g})$ achieved an improvement of RV function. PAPmean values immediately decreased from $30 \mathrm{mmHg}$ (mean) to $22 \mathrm{mmHg}$ (mean) after iloprost inhalation. Then, the patient could be gradually weaned from bypass. Conclusion: Tue to improvement of RV function mechanical RV support or even BVAD implantation could be avoided. Inhaled iloprost is a promising alternative to inhalative NO and may have the potential to avoid or treat acute RV failure after LVAD implantation.

Use of ROTEM ${ }^{\circledR}$ thromboelastometry to monitor peri-operative haemostasis in patients with on-pump cardiac surgery

W. Lösche, M. Reinhöfer ${ }^{1}$, C. Macholdt, U. Franke ${ }^{2}$, M. Brauer, D. Barz ${ }^{1}$, K. Reinhart, G. Marx

Klinik für Anästhesiologie und Intensivtherapie, ${ }^{1}$ Institut für Transfusionsmedizin und ${ }^{2}$ Klinik für Herz- und Thoraxchirurgie, Universitätsklinikum Jena

On-pump cardiac surgery is frequently associated with intra- and post-operative bleeding. ROTEM that is done ex vivo in whole blood samples reflects more closely than classical coagulation measurements the in vivo situation of haemostasis, in particular 
the contribution of fibrinogen and platelets to clot formation. After approval by the ethical committee and informed consensus 200 elective cardiac surgery patients (bypass $49 \%$, valve replacement $19 \%$, others $32 \%$ ) with a minimum on-pump time of $45 \mathrm{~min}$ were enrolled. Samples of citrated blood were obtained immediately before anaesthesia (P1), immediately after the end of the surgery (P2) and $14-18 \mathrm{~h}$ post-operatively (P3). In 50 patients samples were also obtained before and during cardiopulmonary bypass (CPB). ROTEM (Pentapharm Munich) was done according to the manufacturer's instruction. On-pump surgery resulted in a significant impairment of haemostasis as indicated in a prolongation of clotting time (CT) and clot formation time (CFT) as well as a decrease in the clot formation rate (CFR) and maximum clot firmness (MCF) at P2 with more frequent pathological values when coagulation was activated via the intrinsic pathway (INTEM) as compared to the extrinsic pathway (EXTEM). Most of the parameters returned to the basal values at time P3. Intra-operatively, CT displayes a transient maximum when patients had been prepared for $\mathrm{CPB}$ and remained afterwards at a nearly constant level, whereas CRT increased and CFR and MCF decreased with time. In $17 \%$ of the patients a reduced MCF $(<9 \mathrm{~mm})$ in FIBTEM at P2 may indicate a need for fibrinogen substitution. An ex vivo haemostatic activity at $\mathrm{P} 2$ below the normal range was associated with enhanced post-operative blood loss (median $=545 \mathrm{ml}$ ). Surprisingly, increased but not decreased ex vivo haemostatic activity at P1 (top versus bottom quartile) was associated with an enhanced need of intra-operative platelet transfusion or plasma product infusion. High haemostatic activity at P1 (upper quartile), including high platelet counts and fibrinogen levels, were associated with high leukocyte count and CRP levels. To prove whether an enhanced post-operative blood loss might be due to an insufficient heparin neutralisation by protamine, we measured HEPTEM in those patients showing prolonged CT in INTEM. Heparin effects were observed in $37 \%$ of patients with high and in $24 \%$ with low blood loss indicating that insufficient heparin neutralisation may not predict excessive bleeding. The data demonstrate that ROTEM is a valuable tool to detect impaired peri-operative haemostasis in cardiac surgery on-pump patients and may be able to predict an increased risk for post-operative bleeding. In contrast intra-operative bleeding risk is more likely associated with high rather than low ex vivo haemostatic activity. This paradox finding might be eventually caused by an acute phase reaction affecting ROTEM values and a long on-pump time that is known to increase bleeding risk.

\section{Chirurgische Intensivmedizin}

\section{Chirurgische Intensivmedizin I}

\section{Untersuchung zur Zeitdauer der erfolgreichen Platzierung von Jejunalsonden ohne technische Hilfsmittel für die enterale Ernährung von Intensivpatienten \\ S. Schroeder, W. Raabe, B. Bein ${ }^{*}$, T. von Spiegel}

Klinik für Anästhesiologie und Intensivmedizin, Westküstenklinikum Heide ${ }^{*}$ Klinik für Anästhesiologie und Intensivmedizin, Universitätsklinikum Kiel

Die enterale Ernährung postoperativer Intensivpatienten wurde lange Zeit wegen einer schwer vorhersehbaren Einschränkung der Verdauungsfunktion als nicht oder kaum durchführbar erachtet. Die Atonie des Magens kann unter Umständen Tage andauern, während das Jejunum seine Resorptionsfähigkeit innerhalb von
12-24 Stunden wiedererlangen kann. Im Rahmen dieser Untersuchung wurden nach Erhalt des Ethikvotums und der Einwilligung der Patienten in die Untersuchung zwei Sonden der Firmen Cook (Tiger Tube) und Pfrimmer Nutricia (Bengmark Sonde) hinsichtlich der Zeitdauer der erfolgreichen Platzierung ohne technische Hilfsmittel geprüft. Für beide Sonden wird geworben, dass sie nicht invasiv durch eine geringe Restperistaltik über den Magen in das Jejunum wandern können. Die Auswahl der entsprechenden Sonden erfolgte nach dem Zufallsprinzip. Es wurden insgesamt 16 Patienten einer operativen Intensivstation für den Tiger Tube und 12 Patienten für die Bengmark Sonde ausgewählt, die trotz Gastrokinetika und Oberkörperhochlage bei 4-stündlichen Transportkontrollen im Rahmen der enteralen Ernährung einen Reflux von mehr als $200 \mathrm{ml}$ aufwiesen. Eine Sonde galt als korrekt platziert, wenn sie innerhalb von 72 Stunden am Ziel angekommen war, verifiziert durch ein entsprechendes Röntgenbild. Dabei wurden bei 14 von 16 Patienten mit einem Tiger Tube im Median nach 18,5 Stunden (Spannweite 2-68) eine korrekte Platzierung nachgewiesen. Im Vergleich war die Platzierung der Bengmark Sonde nur in 2 von 12 Fällen mit einer Zeitdauer im Median von 36,5 Stunden (Spannweite 28-45) erfolgreich. Damit scheint die Anwendung des Tiger Tube hinsichtlich der Dauer der erfolgreichen Platzierung ohne technische Hilfsmittel Erfolgsversprechender zu sein.

Massive Blutung während Colektomie 013

bei Colitis ulcerosa Therapie mit Faktor XIII-Konzentrat

L. Heuer, K. von den Veert, M. Kruse, D. Blumenberg Institut für Anästhesiologie - Operative Intensivstation Klinikum Osnabrück GmbH

Einleitung: Der Gerinnungsfaktor XIII (FXIII) wird von Thrombin aktiviert und vernetzt Fibrin zu einem stabilen Netzwerk. Ein FXIII-Mangel kann einerseits mit intra- und postoperativen Blutungen einhergehen, andererseits aber auch zu Wundheilungsstörungen führen. Kasuistik: Im Folgenden wird ein Fallbeispiel einer FXIII-Mangel-Blutung bei entzündlicher Darmerkrankung dargestellt, bei dem die Nichtverfügbarkeit ausreichender Mengen von FXIII-Konzentrat zu einer dramatischen Zunahme der Blutungsneigung und zur Entwicklung erster Anzeichen einer Verdünnungs- und Verbrauchskoagulopathie geführt hat, die erst durch entsprechende Substitutionsmaßnahmen beherrschbar war. Bei dem 40-jährigen Patienten $(178 \mathrm{~cm}, 65 \mathrm{~kg})$ mit hochaktiver Colitis ulcerosa (Prednisolon, Cyklosporin) wurden ursprünglich eine Coecalfistel (CF) sowie ein Sigma-AP angelegt. Nebendiagnose: Frische Thrombose der V. femoralis nach Druckverband i.R. einer Angiographie. Im Verlauf kam es zu einer Hb-relevanten (Hb 7,9 g/dl) Blutung über die CF (Globaltests, Fibrinogen, Thrombozyten im Normbereich, FXIII 58\%). Innerhalb von $24 \mathrm{~h}$ wurden 10 Erythrozytenkonzentrate (EK), 2 FFP, 2 g Fibrinogen und 5000 I.E. FXIII substituiert. Zunächst sistierte die Blutung. Über Nacht trat aber eine erneute Blutung auf (Hb 6,5 g/dl). Die Endoskopie ergab eine diffuse Blutung im gesamten Colonrahmen (Gerinnungsstatus normal, FXIII 79\%). Es wurden 10 EK, 3 FFP und $2 \mathrm{~g}$ Fibrinogen in $24 \mathrm{~h}$ verabreicht. Bei persistierender Blutung wurde der Entschluss zur Colektomie getroffen. Intraoperativ zeigte sich zunächst eine adäquate Hämostase, dann aber eine zunehmende diffuse Blutungsneigung ( $\mathrm{Hb} 4,3 \mathrm{~g} / \mathrm{dl}$ ) aus der großen Wundfläche. Perioperativ wurden 12 EK, 10 FFP, 2 Thrombozytapheresekonzentrate (TK), $6 \mathrm{~g}$ Fibrinogen, $0,3 \mu \mathrm{g} / \mathrm{kg}$ DDAVP und $1 \mathrm{~g}$ Tranexamsäure verabreicht (Hb post-OP $7,1 \mathrm{~g} / \mathrm{dl}->2 \mathrm{EK})$. Ein FXIII-Konzentrat war perioperativ nicht verfügbar. Während $12 \mathrm{~h}$ postoperativ bestand weiterhin eine Blutungsneigung auf niedrigerem Niveau ( $\mathrm{Hb} 7,9 \mathrm{~g} / \mathrm{dl}$, Thrombozyten $47 \mathrm{G} / \mathrm{l}$, Quick 55\%, PTT $47 \mathrm{~s}$, Fibrinogen $131 \mathrm{~g} / \mathrm{l}$, FXIII $29 \%$, AT $31 \%$ ). Es wurden 3000 IE AT, 4 EK und 2 TK verabreicht. Erst nach Infusion von 2500 IE FXIII sistierte die Blutung. Der Hb-Wert lag auch an den Folgetagen stabil um 9,5 g/dl, FXIII um 95\%. Diskussion: Ursache 
der Blutung war neben der Entzündung im Darmtrakt ein FXIIIMangel, der zwar anfangs substituiert wurde, aus logistischen Gründen intraoperativ aber nicht adäquat therapiert werden konnte. Erst durch die erneute Applikation konnte eine definitive Blutstillung erreicht werden. Der bei entzündlichen Darmerkrankungen nicht seltene FXIII-Mangel (Verlust, vermehrter Abbau) kann über die Screeningteste Quick und PTT nicht erfasst werden, er muss immer gesondert bestimmt werden. Die Mindestspiegel an FXIII dürften postoperativ bei großen Wundflächen und ausgedehnten diffusen Blutungen (Verlust, geringe Syntheserate) im unteren Normalbereich liegen.

Therapie einer postoperativen Blutung auf Grund eines 046 erworbenen Faktor-XIII-Mangels bei Hüftprothesenwechsel

H. Schöchl ${ }^{1}$ L. Rodewald ${ }^{2}$

${ }^{1}$ Abteilung für Anästhesie, Unfallkrankenhaus Salzburg,

${ }^{2} \mathrm{ZLB}$ Behring GmbH, Hattersheim

Einleitung: Der Gerinnungsfaktor XIII (FXIII) vernetzt das bei Gerinnungsprozessen gebildete Fibrin zu einem mechanisch stabilen dreidimensionalen Netzwerk, das zusammen mit der thrombozytären Gerinnung die definitive Blutstillung bewirkt. FXIIIMangel ist einerseits mit Blutungen verknüpft, wobei die Blutungsneigung sich bei operativen Eingriffen häufiger nicht schon intraoperativ, sondern erst postoperativ, in der Regel einige Stunden nach OP-Ende klinisch manifestiert. Die nachfolgende $\mathrm{Ka}$ suistik beschreibt ein Fallbeispiel einer FXIII-Mangelblutung, bei der die ROTEM-Diagnostik den FXIII-Mangel aufgedeckt und durch entsprechende Substitution mit einem FXIII-Konzentrat (Fibrogammin HS, ZLB Behring Marburg) eine Revisions-OP verhindert hat. Patientencharakteristik: 62-jähriger Patient $(72 \mathrm{~kg}$ $\mathrm{KG}, 175 \mathrm{~cm}$ ) mit Alkohol- und Nikotinabusus; insgesamt reduzierter Allgemeinzustand; Leber: Bilirubin ges.: $0,26 \mathrm{mg} / \mathrm{dl}$, Transaminasen: normal, CHE: normal Niere: Kreatinin: 0,44mg/dl, Harnstoff: $17 \mathrm{mg} / \mathrm{dl}$. Hüftendprothesenwechsel aufgrund einer luxierten Hüftendoprothese links. Am 4. postoperativen Tag kam es zu einer Hb-relevanten schweren Nachblutung. Wegen des von 9,7 g\% auf 7,4 g\% abgesunkenen $\mathrm{Hb}$ werden $3 \mathrm{EK}$ transfundiert. Für den nächsten Tag wurde von chirurgischer Seite eine Revisions-OP geplant. Ein mögliches von Willebrand-Syndrom konnte ausgeschlossen werden: vWF AG: 193,6\%; Ristocetin-Cofaktor: $190 \%$. In der ROTEM-Analyse fand sich ein maximaler Lyseindex von $38 \%$ im EXTEM. Da dieser auch im Aprotinintest unverändert hoch lag und somit eine Hyperfibrinolyse ausgeschlossen werden konnte, wurde ein FXIII-Mangel vermutet und durch einen Labortest bestätigt (FXIII: 58\%). Therapie und weiterer Verlauf: Nach Gabe von 1.250 E Fibrogammin ${ }^{\circledR}$ HS (FXIII-Konzentrat) sistierte die Blutung ohne weitere Maßnahmen. Der maximale Lyseindex im EXTEM normalisierte sich auf einen Wert von 15\% (Abb. 2). Eine Applikation von Erythrozytenkonzentraten war ebenso wenig notwendig wie eine operative Revision zum Aufsuchen der Blutungsquelle. Zusammenfassung: Der Patient war bereits zur Revisions-OP vorgesehen, als eine durchgeführte ROTEM-Gerinnungsanalyse erste Hinweise auf einen FXIII-Mangel gab, der durch die konventionelle Labordiagnostik bestätigt wurde (FXIII-Aktivität: $58 \%)$. Die Substitution von 1.250 E Fibrogammin ${ }^{\circledR}$ HS (FXIIIKonzentrat) führte $\mathrm{zu}$ einer sofortigen und dauerhaften Blutstillung, so dass auf die Revision verzichtet werden konnte. Da der FXIII durch die gängige Gerinnungsanalytik (Quick, PTT, Thrombozytenzahl, Fibrinogen) nicht erfasst wird, sollte bei unklaren postoperativen Blutungen differentialdiagnostisch immer an einen FXIII-Mangel gedacht werden.
Bilateral ischaemia following cannulation of the radial arteries

\section{B. L. Hübner, S. Schröder ${ }^{\star}$}

University Hospital Maastricht, the Netherlands

*Charité Universitätsmedizin, Campus Mitte, Berlin, Germany

Description: A 65-year-old male patient was admitted to the Intensive Care Unit following surgical drainage of a pelvic abcess. The patient had a history of a recurrent rectal carcinoma and had received local radiotherapy. During surgery, his hemodynamic condition worsened, and treatment with high doses of Norepinephrin (up to $1 \mathrm{mcg} / \mathrm{kg} / \mathrm{min}$ ) was started in the operating theater. Blood pressure was monitored by use of an arterial catheter in the right radial artery. Immediately following the patient's arrival in the ICU, it was noted that the first three fingers of his right hand were ischaemic and showed a dark blue to black discoloration. The involved cannule was removed, and the abnormalities disappeared within half an hour. Another arterial cannule then was inserted in the patient's left radial artery. Two days later, this hand became ischaemic- too, and, as with his right hand, the first three fingers were noted to be discolored. Following removal of the cannule, the symptoms again rapidly and completely disappeared. Because the groin was inaccessable for cannulation, due to the proximity of the operation field, the right brachial artery was cannulated thereafter, and no adverse events were noted from this procedure. Discussion: It is well known that arterial cannulation may result in arterial occlusion and ischaemia of the arterial flow tract. Current medical literature suggests, that in the majority of instances, these occlusions are temporary and fully disappear after catheter removal. Still, some case reports describe permanent injury to fingers or the entire hand. Arterial vascularisation of the hand usually consists of a collateral system involving the radial and ulnar arteries. In the patient described here, this system may not have been complete, which resulted in ischaemia. It is unknown, when ischaemia has developed in one hand following cannulation, how often the other hand will behave the same. This case report is the first published description of the occurrence of ischaemia in one single individual on both sides. Anatomical studies describe many variants in the arterial system; however, little attention has been paid to symmetry within individuals. The usefulness of additional tests prior to cannulation, like the (modified) Allen's test or Doppler measurements, is still a subject of discussion. Most clinicians consider the radial artery to be the preferred site for cannulation, because of the low risk of complications. Conclusion: Ischaemia of the fingers is a rare and usually transient complication of arterial cannulation. When ischaemia happens in one hand, the risk of ischaemia upon cannulation of the other hand is unknown. The radial artery remains the site of choice for arterial cannulation. Adhering to correct indications for cannulation, and frequent inspections of the cannulated extremity both are essential to avoiding complications.

Einfluss strukturierter Triglyceride (Structolipid ${ }^{\circledR}$ ) auf die hepatozelluläre Integrität. Ein Vergleich zu einer MCT-LCT-MISCHUNG (Lipofundin 20\% ${ }^{\circledR}$ ) und einer reinen LCT-Emulsion (Lipovenös 20\% ${ }^{\circledR}$ ) bei postoperativen chirurgischen Patienten

S. N. Piper, K. D. Röhm, B. Odermatt, T. A. Schöllhorn, J. Boldt, I. Schade, S. W. Suttner

Klinik für Anästhesiologie und Operative Intensivmedizin, Klinikum Ludwigshafen

Fragestellung: Im Rahmen der totalen parenteralen Ernährung (TPN) in der postoperativen Phase wird häufig in Folge eines Postaggressionsstoffwechsels die Lipoproteinlipase gehemmt, so dass konsekutiv eine Hypertriglyceridämie auftritt. Während die Transaminasen GOT und GPT in hoher Konzentration in den periportalen und in niedriger Konzentration in den zentrolobulären Hepatozyten $\mathrm{zu}$ finden sind, ist die Alpha-Glutathione-S-Trans- 
ferase (Alpha-GST) gleichmäßig verteilt. Aufgrund ihrer schnellen Freisetzung nach Schädigung und wegen ihrer kurzen Halbwertszeit stellt die Alpha-GST einen fast idealen Marker zur Erfassung akuter Veränderungen der hepatozellulären Funktion dar. In der vorliegenden Studie sollte nun unter anderem durch Bestimmung der Alpha-GST überprüft werden, ob die Gabe von strukturierten Lipiden im Vergleich zu herkömmlichen Fettlösungen $\mathrm{zu}$ einer Verbesserung der hepatozellulären Integrität führt. Methode: Prospektive, randomisierte Studie. Nach Zustimmung der Ethikkommission wurden 45 Patienten, die sich einem chirurgischen Eingriff unterzogen hatten und postoperativ einer parenteralen Ernährung bedurften, in die klinische Studie eingeschlossen. Die Nichtproteinkalorien wurden $\mathrm{zu} 60 \%$ als Glukose und $\mathrm{zu} 40 \%$ als Fettemulsion verabreicht. Die Gesamtkalorienzahl pro Tag betrug $25 \mathrm{kcal} / \mathrm{kg}$ KG. Das Sedierungsregime war standardisiert, Propofol kam nicht zur Anwendung. Es wurden drei Gruppen unterschieden: Gruppe A $(n=15)$ erhielt strukturierte Fette (Structolipid $\left.20 \%{ }^{\circledR}\right)$, Gruppe B $(n=15)$ eine MCT-LCT-Mischung (Lipofundin $\left.20 \%{ }^{\circledR}\right)$ und Gruppe C $(n=15)$ eine reine LCT-Emulsion (Lipovenös $20 \%{ }^{\circledR}$ ). Die Fettgabe erfolgte über 5 Tage. Die Bestimmung der Transaminasen GPT und GPT, sowie der Alpha-GST erfolgte $\mathrm{zu}$ folgenden Messzeitpunkten: Vor Beginn der parenteralen Fettgabe (T0), am 1. Tag, (T1), am 2. Tag, (T2), am 3. Tag, (T3) und am 5. Tag (T4) nach Beginn der Fettapplikation. Eine Signifikanz wurde für $(\mathrm{p}<0,05)$ angegeben. Ergebnisse: $\mathrm{Zu}$ keinem Messzeitpunkt waren zwischen den Gruppen signifikante Unterschiede bezüglich GPT- oder GOT-Konzentrationen nachweisbar. Die Alpha-GST-Spiegel im Serum differierten am 1. und 2. Tag unter parenteraler Fettapplikation nicht signifikant. Am 3. und 5. Tag waren die Alpha-GST-Konzentrationen der Gruppe A (T3: 1,9 $\pm 1,8$ T4: $3,2 \pm 2,7 \mu \mathrm{g} / \mathrm{L}$ ) signifikant niedriger als in Gruppe B (T3: 9,4 $\pm 9,9 ;$ T4: $14,6 \pm 19,5 \mu \mathrm{g} / \mathrm{L})$ und Gruppe C (T3: $14,2 \pm 20,8$; T4: $22,4 \pm 39,3 \mu \mathrm{g} / \mathrm{L})$. Schlussfolgerungen: Die Gabe strukturierter Fettlösung führte im Rahmen einer postoperativen TPN am 3. und 5. Tag der Ernährung im Vergleich zur Applikation von MCT-LCT-Mischungen oder reinen LCT-Lösungen, zu signifikant niedrigeren Alpha-GST-Spiegeln, während die Transaminasen sich nicht signifikant unterschieden. Die niedrigeren Alpha-GST-Spiegeln weisen auf eine geringere Alteration der hepatozellulären Integrität unter Gabe strukturierter Fette im Vergleich zu MCT-LCTMischungen oder LCT-Lösungen hin.

Langzeitüberleben geriatrischer Patienten

039 nach chirurgischer Intensivtherapie

\section{P. K. Baier, P. Ober, U.T. Hopt, S. Utzolino}

Chirurgische Universitätsklinik Freiburg, Abt. Allgmeinund Viszeralchirurgie mit Poliklinik

Hintergrund: Bei geriatrischen Patienten wird die Intensivtherapie aufgrund der erhöhten Letalität und Morbidität sowie der altersbedingt eingeschränkten Prognose häufig infrage gestellt. Nur die Möglichkeit eines Langzeitüberlebens mit guter Lebensqualität nach Beendigung der Intensivbehandlung kann diese rechtfertigen. In dieser Studie wird nach Faktoren gesucht, die das Langzeitüberleben von chirurgischen Intensivpatienten beeinflussen. Methode: In einer retrospektiven Analyse wurden 314 Patienten über 75 Jahre untersucht, die 2001-2004 mehr als 48 Stunden auf der unserer chirurgischen Intensivstation behandelt wurden. Die Patienten wurden im Wesentlichen wegen viszeralchirurgischer oder traumatologischer Erkrankungen behandelt. In Überlebensanalysen wurden Faktoren ermittelt, die das Überleben nach Entlassung von der Intensivstation beeinflussen. Ergebnisse: Die 314 Patienten mit einem mittleren Alter von 80,9 Jahren waren im Mittel 8,6 Tage auf der Intensivstation, 52 (16,5\%) davon verstarben auf der Intensivstation. Das mittlere Überleben nach Entlassung von der Intensivstation betrug 24,5 Monate, nach 5 Jahren lebten noch $40 \%$ der Patienten. Der APACHE-II-Score bei Aufnahme war im Mittel 15,5 (6-38), der mediane Tages-Core-TISS 10 -Score lag bei 3,9 (0-26). Mit der Überlebenszeit korrelierten invers der APACHE $(p<0,001)$ und TISS Score $(p<0,001)$ sowie die Dauer der Intensivbehandlung $(\mathrm{p}<0,001)$, nicht jedoch das Alter $(p=0,54)$. Die Überlebenszeit wurde von Vorerkrankungen wie Herzinsuffizienz größer NYHA II $(p=0,023)$, Niereninsuffizienz $(p<0,001)$, neurologischen Vorerkrankungen $(p<0,001)$ und Lungenerkrankungen $(p<0,001)$ signifikant beeinflusst, nicht jedoch von Malignomen $(p=0,342)$ KHK $(p=0,3)$, pAVK $(p=0,29)$, Diabetes $(p=0,85)$ oder psychiatrischen Vorerkrankungen $(p=0,73)$. Ob der Patient wegen traumatologischer oder viszeralchirurgischer Erkrankungen behandelt wurde, hatte ebenso keinen Einfluss auf das Überleben wie Infektionserkrankungen $(p=0,42)$, Alkoholabusus $(p=0,57)$, endokrinologische $(p=0,89)$, gastrointestinale $(p=0,616)$ oder urologische Vorerkrankungen $(\mathrm{p}=0,16)$. Negativ auf das Überleben wirkten sich nosokomiale Infektionen $(p=0,035)$, Pneumonien $(p<0,001)$, akutes Nierenversagen $(p<0,001)$, Sepsis $(p<0,001)$, Beatmungspflicht $(p<0,001)$, kardiopulmonale Reanimation $(\mathrm{p}<0,001)$ und die Notwendigkeit der Katecholamin- $(p<0,001)$ oder Dialysetherapie $(p<0,001)$ aus. Keinen Einfluss hatten Operationen $(p=0,55)$, Transfusionen $(p=0,12)$ und das Auftreten eines Durchgangssyndroms $(p=0,67)$. Schlussfolgerung: Bei geriatrischen Patienten ist ein Langzeitüberleben nach Intensivtherapie wegen chirurgischer Erkrankungen möglich. Intensivtherapie darf nicht allein wegen hohen Alters vorenthalten werden. Prognostisch relevant sind APACHE und TISS Score, sowie vorbestehende Lungen- Herz- oder Niereninsuffizienz. Komplikationen während des Intensivaufenthaltes mindern das Überleben signifikant.

Intra- und postoperatives Immunmonitoring nach single-shot Antibiose in der kolorektalen Chirurgie

E. Schröpfer, H. B. Reith ${ }^{\star}$ A. Thiede und Th. Meyer

Chirurgische Universitätsklinik und Polikinik,

Bayerische Julius-Maximilians-Universität Würzburg und

*Chirurgische Klinik II, Klinikum Konstanz, Deutschland

Einleitung: Aufgrund des steigenden Patientenalters als auch die zunehmenden Bedeutung multiresistenter Erreger kommt der Prophylaxe von bakteriellen Infektionen, gerade im operativen Sektor, mehr und mehr Bedeutung zu. Neben dem Erreger ist jedoch auch die Freisetzung bakterieller Lipopolisacchardie (wie z.B. Endotoxin) bei der Wahl des Antibiotikums wichtig. Vor allem bei einer einmaligen Verabreichung eines hochpotenten Antibiotikums ist dieser Faktor ausschlaggebend. Ziel der vorliegenden Studie war es daher, Zytokinverschiebungen und Endotoxinwerte im prä-, inter- und postoperativen Verlauf zu bestimmen. Desweiteren sollten die Wirkstoffe Ertapenem (Invanz ${ }^{\circledR}$ ) und Moxifloxacin $\left(\right.$ Avalox $\left.{ }^{\circledR}\right)$ auf ihre Anwendbarkeit in der kolorektalen Chirurgie untersucht werden. Material und Methoden: In eine genehmigte prospektive Studie wurden bisher 11 Patienten (Durchschnittsalter: 70,89 Jahre) eingeschlossen, die an einem Tumor des Rektums oder des rekto-sigmoidalen Übergangs therapiert wurden. Ausschlusskriterien waren stattgehabte Entzündungen, Immunsupression, Metastasierung oder eine Vorbehandlung mit einem anderen Antibiotikum. Pro Patient wurde über einen Zeitraum von 3 Tagen insgesamt 9 Blutproben entnommen. Neben der Bestimmung der Leukozytenzahl sowie des CRP, wurden die Werte für Endotoxin, IL-6, IL-8, IL-10, PCT, Neopterin und TNF $a$ bestimmt. Ergebnisse: Endotoxin erreichte Maximalwerte von $3,45 \mathrm{EU} / \mathrm{ml}$ (Avalox ${ }^{\circledR}$ ) und 2,47 EU/ml (Invanz ${ }^{\circledR}$ ). Am dritten post-operativen Tag erreichte das Endotoxin bei beiden Antibiotika Werte, die mit dem präoprativem Tag vergleichbar waren. Die IL-6 Messungen ergaben bei allen Patienten einen Anstieg, wobei sie unter Invanz ${ }^{\circledR}$-Medikation signifikant höher waren. Gleiches zeigte sich für die Bestimmung der Werte von Neopterin und TNFa. Lediglich beim PCT lagen die Invanz ${ }^{\circledR}$-Werte unter denen der Patienten, die mit Avalox ${ }^{\circledR}$ behandelt worden waren. Neopterin zeigte als einziger Wert kein Anstieg, sondern ein Abfall der Werte $4 \mathrm{~h}$ nach Antibiose, dann aber einen Anstieg der Werte von diesem Minimum aus zum ersten postoperativem Tag. Beim IL-8 waren 
die deutlichsten Unterschiede zwischen den beiden o.g. Antibiotika zu verzeichnen. Während die Avalox ${ }^{\circledR}$-Patienten hier keine Werte über $14,28 \mathrm{pg} / \mathrm{ml}$ erreichten, zeigten die Invanz ${ }^{\circledR}$-Patienten Maximalwerte von $134,8 \mathrm{pg} / \mathrm{ml}$ am ersten postoperativem Tag. Schlussfolgerung: Im Rahmen dieser ersten Vorstudie zeigte sich, dass für Endotoxin als Früherkennungsparameter die differenzierte Auswahl des applizierten Antibiotikums eine entscheidende Rolle spielt. Mit dem hier dokumentierten Modell, lässt sich die Kinetik der pro- und kontrainflammatorischen Serum-Parametern der ersten drei postoperativen Tage zeigen, in denen weder bildgebende Verfahren, noch das Drainagesekret immer richtungweisende Informationen über mögliche Komplikationen geben können.

Volumenersatztherapie an Intensivstationen:

Resultat einer österreichweiten Umfrage

K.-H. Smolle ${ }^{1}$, E. Schatz ${ }^{2}$, H. Gombotz ${ }^{2}$

${ }^{1}$ Medizinische Universitätsklinik Graz, ${ }^{2}$ Abteilung für Anästhesio-

logie und Intensivmedizin, Allgemeines KH Linz, Austria

Die Debatte über die optimale Wahl einer Volumentherapie hält nach wie vor unvermindert an. Um diese Frage neuerlich aufzugreifen wurden den anästhesiologischen und internistischen Intensivstationen (ICU) in Österreich Fragebögen zugesandt. Ergebnis: Die Responserate war etwa 50\% (104 Fragebögen wurden vollständig zurückgesandt und ausgewertet $74 \%$ anästhesiologische und $26 \%$ internistische ICU). $63,5 \%$ der Krankenhäuser lagen mit der Bettenzahl zwischen 250-500 Betten, 33,4\% hatten $>500$ Betten und 18,3\%>1000 Betten. 11,5\% der auswertbaren Daten kamen von Universitätskliniken. Die Zahl der Intensivbetten $<1075 \%), 10-20(21,2 \%)$ und $>20(1,9 \%)$. Standards hinsichtlich der Volumentherapie existieren nur in $18 \%$. In einer der relevanten Fragen ob Kristalloide (KR) oder Kolloide (KO) als primäre Volumenersatztherapie verwendet werden, wurden die KR mit häufig in $69 \%$ (davon $9 \%$ hypertone Lösungen) und immer in $20 \%$, gegenüber den KO (64\% häufig und immer in $5 \%$ ) klar präferenziert. Auffallend gering in den Angaben war der Bedarf an Gelatine mit 8\%, Dextrane 2\% und Albumin 3\%. Bezüglich Effektivität der Lösungen meinten $78 \%$ der Befragten, dass KO den KR überlegen sei, allerdings waren $43 \%$ der Meinung KR wären, wenn in entsprechender Menge verabreicht, den KO im Effekt vergleichbar. Bei den Kolloiden waren die auf Stärke basierenden Lösungen eindeutig die der ersten Wahl. Bei Betrachtung spezieller Anwendungsgebiete wie der Abdominal-und Gefäßchirurgie, der Gynäkologie sowie der Orthopädie zeigt sich ein ausgewogenes Verhältnis der Verwendung von KR und $\mathrm{KO}$ etwa jeweils von $35-45 \%$. An neurochirurgischen ICU und bei Patienten nach Organtransplantationen, vor allem aber in der Pädiatrie und bei Verbrennungen wurden bevorzugt KR Lösungen verwendet. Im Gegensatz dazu kamen in der Traumatologie leicht bevorzugt KO zur Anwendung. Bei bestehenden Organdysfunktionen (Herz, Niere, Lunge, Leber) und auch bei septischen Zustandsbildern kommen häufiger KR als KO zum Einsatz, bei Hypovolämien sprechen die Angaben allerdings dafür, dass die behandelnden Ärzte vom stärkeren Volumseffekt der KO überzeugt sind. Auch bei aktiven Blutungen kommen neukonzipierte KO-Lösungen gehäuft zum Einsatz. Wie vorhin erwähnt, wird Albumin als $\mathrm{KO}$ nur in ganz geringem Ausmaß verwendet, trotzdem möchte man beim Leberversagen oder bei Verbrennungen, so zeigen die Daten, weiterhin nicht darauf verzichten. Mehr als 50\% der Befragten bestätigten eine Veränderung der Strategie in der Volumentherapie in den letzten 5 Jahren. So wurden in 32\% Dextrane als KO durch Stärkelösungen ersetzt. Allgemein hat die Verwendung von KO deutlich zugenommen. Nach wie vor von untergeordneter Bedeutung bleiben Gelatine und Albumin. Als Gründe für diesen Strategiewechsel gaben die Befragten neu publizierte Daten (81\%), Expertenmeinungen (52\%), Guidelines (25\%) und ökonomische Gründe $(30 \%)$ an.
Inhalative Sedierung intensivmedizinischer Patienten

mit Sevofluran - Erste Erfahrungen mit dem

Anästhesiegasrezirkulierungssystem $\mathrm{AnaConDa}{ }^{\circledR}$

\section{J. Soukup, K. Schwalb, M. Bomplitz, J. Radke}

Universitätsklinikum (AöR) Medizinische Fakultät

der Martin-Luther-Universität Halle-Wittenberg Klinik

für Anästhesie und Operative Intensivmedizin

Das neue Anästhesiegasrezirkulierungssystem AnaConDa ${ }^{\circledR}$ ermöglicht den Einsatz volatiler Anästhetika im Rahmen der Langzeitsedierung intensivmedizinischer Patienten. Wir berichten über die ersten Erfahrungen mit Sevofluran ${ }^{\circledR}$. Methode: Die Applikation von Sevofluran (Sevorane ${ }^{\circledR}$, Abbott) erfolgte kontinuierlich über einen Perfusor in einen Miniaturverdampfer (AnaConDa ${ }^{\circledR}$, SedanaMedical, Sweden), welcher an Stelle des üblichen Beatmungsfilters in das Beatmungsschlauchsystem integriert wurde. Die inund exspiratorische Anästhesiegaskonzentration wurde via externen Gasmonitor gemessen. Zur Restgasfiltration wurden spezielle Filter verwendet (Novasorb ${ }^{\circledR}$, Fa. NovaMed). Zur Analgesie erhielten die Patienten Remifentanil $0,1-0,3 \mu \mathrm{g} / \mathrm{kg} / \mathrm{min}$. Die Steuerung der Analgosedierungstiefe erfolgte mit dem Ziel RAMSAY 4. Ergänzt wurde das klinische Monitoring bei Verfügbarkeit durch den Bispectral-Index (BIS-Module, Aspect-Medical Systems Inc.). Alle Patienten wurden druckkontrolliert beatmet (AZV: $4-6 \mathrm{ml} / \mathrm{kg}$, AF20/min, PEEP 12 mbar). Entsprechend dem stationsinternen Standard wurde das System zunächst bei Patienten mit zu erwartender Langzeitsedierung oder unzureichender intravenöser Analgosedierung eingesetzt. Die Datengewinnung erfolgte retrospektiv durch Analyse der elektronischen Patientenakte im Patientendatenmanagementsystem (ICM, F. Dräger, Lübeck). Die Ergebnisse werden als Median mit Angabe der Minimal- und Maximalwerte dargestellt. Ergebnisse: Bisher konnten insgesamt 23 intensivmedizinische Patienten im Alter von 51,2 $\pm 19,5$ Jahren mit einer durchschnittlichen Anwendungsdauer von 94,9 $\pm 56,9 \mathrm{~h} /$ Patient bei einer bisherigen Gesamtanwendungszeit von 2183,1 Stunden analysiert werden. Für das Erreichen der angestrebten Sedierungstiefe (RAMSAY 4) waren $6,1 \pm 1,9 \mathrm{ml} / \mathrm{h}$ Sevofluran notwendig. Darunter lag die endexspiratorische Sevoflurankonzentration zwischen $0,5-1,1$ Vol\%. Nach Beendigung der Sevofluranapplikation konnten 12 Patienten nach 13,6 $\pm 6,4$ min je nach medizinischer Zielstellung entweder adäquat neurologisch beurteilt oder in die Spontanatmung überführt und extubiert werden. Bei zwei weiteren Patienten gelang dies erst nach 50 min (Sedierungsdauer $130,8 \mathrm{~h}$ ) bzw. $106 \mathrm{~min}$ (Sedierungsdauer $173 \mathrm{~h}$ ). Bei den verbleibenden 9 Patienten erfolgte eine Änderung der Sedierungskonzeptes infolge einer Anpassung an die vorliegende klinische Situation. Laborchemisch konnten keine klinisch relevanten Veränderungen beobachtet werden. Eine eigene Kalkulation der Medikamentenkosten ohne Berücksichtigung weiterer Materialkosten ergab für Sevofluran ${ }^{\circledR}(6 \mathrm{ml} / \mathrm{h})$ ca. $75,00 €$ und für Isofluran $(3 \mathrm{ml} / \mathrm{h}) 12,00 €$ (im Vergleich Propofol $400 \mathrm{mg} / \mathrm{h}$ ca. 48,00€). Schlussfolgerung: Die Anwendung von Sevofluran im Rahmen der Analgosedierung auf der ITS ist mit dem Anästhesiegasrezirkulierungssystem Ana$\mathrm{ConDa}^{\circledR}$ sicher möglich, gestattet unseren Erfahrungen nach in Kombination mit Remifentanil eine gute Steuerung der Analgosedierungstiefe mit schnellen Aufwachphasen.

Morbidity and mortality rates following 126 major hepatectomy

K. Rellos, M. Rizos, D. Fotakis, E. Dimitriadou, A. Raftopoulou, A. Michalopoulos

Henry Dunant Hospital, Intensive Care Unit, Athens, Greece

Objektive of this prospective study was to examine morbidity and mortality in patients undergoing major hepatectomy. PatientsMethods: We examined all adult patients who underwent major hepatectomy. We recorded dempgraphics, type of surgery, red blood cell units transfused, plasma units transfused, liver function indices during the early postoperative period, postoperative com- 
plications, duration of mechanical ventilation, length of ICU and hospital stay, and outcome. Results: Sixty-seven patients of mean $( \pm \mathrm{SD})$ age $62.4( \pm 8.2)$ years old underwent major hepatectomy during a three year period. The majority of them were males $(82.1 \%)$. Sixty-five percent had metastatic cancer. The patients received 3.1(+1.7) red blood cell units and $4.9(+2.6)$ plasma units in the operating room and the ICU. Seventeen $(25.3 \%)$ patients developed acute renal failure. During the early postoperative period, mean maximum values of serum bilirubin, SGOT and SGPT were $5.2(+4.3) \mathrm{mg} / \mathrm{dl}, 532(+375) \mathrm{U} / \mathrm{lt}$ and $559(+387) \mathrm{U} / \mathrm{lt}$, respectively. Mean duration of mechanical ventilation was $14.5(+6)$ days, respectively. Hospital mortality was $2.9 \%$. Conclusions: Patients undergoing major hepatectomy presented with low morbidity and mortality rates.

\section{Chirurgische Intensivmedizin II}

\author{
Desufflation nach Laparoskopie - \\ eine verkannte Gefahr
}

S. Richter, C. Matthes, T. Huckstadt, T. Ploenes, F. Schier Klinik für Kinderchirurgie Universitätsklinik Mainz

Hintergrund: Laparoskopische Operationen werden unter erhöhtem intraabdominellen Druck durchgeführt. Aufgrund von Zeitmangel in der täglichen Operationsroutine versuchen sowohl Chirurgen als auch Anästhesisten, die Operationszeit so kurz wie möglich zu halten. Daher erfolgt die abschließende Desufflation des Abdomens meist sehr schnell, und mögliche Folgen werden vernachlässigt. Ziel: Es soll an einem Tiermodell überprüft werden, welche kardiopulmonalen Auswirkungen ein schneller abdomineller Druckablass zur Folge hat. Methode: In einer laparoskopischen Studie an 20 neugeborenen Schweinen (mittleres Gewicht $6,2 \mathrm{~kg}$ ) wurde der intraabdominelle Druck schrittweise auf $24 \mathrm{mmHg}$ angehoben. Das Kapnoperitoneum wurde insgesamt für 90 Minuten aufrechterhalten. Anschließend erfolgte die schnelle Desufflation über einen $5 \mathrm{~mm}$-Trokar. Die Tiere wurden intramuskulär analgosediert. Die Narkose wurde intravenös mit Pentobarbital, Fentanyl und Pancuronium fortgeführt. Vor Anlage des Kapnoperitoneums bestand Normokapnie ( $\mathrm{paCO}_{2} 35-40 \mathrm{mmHg}$ ). Ventiliert wurden alle Tiere bei einem $\mathrm{FiO}_{2}$ von 1,0 mit einer Inspirationsdauer von $0,5 \mathrm{~s}$, einer Exspirationszeit von 2,5 s und einem PEEP von $4 \mathrm{cmH}_{2} \mathrm{O}$ am Babylog 1 (Dräger). Messparameter waren Herzfrequenz (HF), mittlerer arterieller Blutdruck (MAP) in der Arteria carotis, mittlerer pulmonalarterieller Druck (PAP), mittlerer zentralvenöser Druck (ZVD) in der Vena cava superior und der endtidale Kohlendioxidpartialdruck $\left(\mathrm{etCO}_{2}\right)$ (Tierversuchsnummer 1.5 177-07/051-6). Ergebnisse: 3 von 20 Schweinen starben nach schneller Desufflation. Nach einem initialen Anstieg der Herzfrequenz ( Median von 116 auf $127 / \mathrm{min}$ ) folgten eine Minute nach der Desufflation Änderungen im Median für MAP von 69 auf $61 \mathrm{mmHg}$, für PAP von 22 auf $24 \mathrm{mmHg}$, ZVD von 4 auf $3 \mathrm{mmHg}$ und für etCO $\mathrm{C}_{2}$ von 37 auf $38 \mathrm{mmHg}$. Bei drei Tieren resultierte hieraus eine kardiopulmonale Dekompensation. Die überlebenden Tiere erreichten nach drei Minuten wieder die Ausgangswerte. Schlussfolgerung: Schnelle Desufflation nach längeren laparoskopischen Eingriffen kann fatale kardiopulmonale Auswirkungen zur Folge haben. Prophylaktisch ist vor Narkosebeginn eine Hypovolämie dringend auszugleichen. Das kardiorespiratorische Monitoring ist während der Desufflationsphase von besonderer Bedeutung. Weitere Studien müssen durchgeführt werden, um die Abhängigkeit der kardiopulmonalen Reaktionen von weiteren Einflussfaktoren wie Höhe und Dauer des intraabdominellen Druckes zu evaluieren.

\section{Druckverteilung im Abdomen unter Laparoskopie}

S. Richter, C. Matthes, T. Huckstadt, T. Ploenes, F. Schier Klinik für Kinderchirurgie Universitätsklinik Mainz

Hintergrund und Ziele: Der intraabdominelle Druck ist ein wichtiger Messparameter in der Intensivmedizin. Er wird oft mittels eines suprapubischen Katheters überwacht, um ein mögliches abdominelles Kompartmentsyndrom frühzeitig zu erkennen. Diese Studie wurde durchgeführt, um die intraabdominelle Druckverteilung unter Laparoskopie zu evaluieren. Material und Methode: Vierzehn Ferkel im Alter von 2 bis 3 Wochen mit einem Gewicht von 5,0 bis $7,3 \mathrm{~kg}$ dienten als Säuglingsmodell (Tierversuchsnummer 1.5 177-07/051-6). Die Tiere wurden intramuskulär analgosediert. Die Narkose wurde intravenös mit Pentobarbital, Fentanyl und Pancuronium fortgeführt. Ventiliert wurden alle Tiere bei einem $\mathrm{FiO}_{2}$ von 1,0 mit einer Inspirationsdauer von $0,5 \mathrm{~s}$, einer Exspirationszeit von $2,5 \mathrm{~s}$ und einem PEEP von $4 \mathrm{~cm} \mathrm{H}_{2} \mathrm{O}$ am Baby$\log 1$ (Dräger). Bei Untersuchungsbeginn bestand Normokapnie, die durch Anpassung des Beatmungsdruckes beibehalten wurde. Codman-ICP-Sonden, zur Messung des intrakraniellen Parenchymdruckes bestimmt, wurden laparoskopisch in der Leber, der Blase und der Bauchhöhle platziert. Ein weiterer Sensor wurde transöophageal in den Magen eingebracht. Zentralvenöse Katheter wurden in die Venae cavae gelegt. Nach der Erhebung der Basiswerte wurde ein Kapnoperitoneum angelegt und in $6 \mathrm{mmHg}$ Schritten auf $24 \mathrm{mmHg}$ angehoben. Jede Druckstufe wurde für 20 Minuten erhalten und die Werte registriert. Ergebnisse: Der voreingestellte Druck am Insufflator und der gemessene intraabdominelle Druck zeigten keinen signifikanten Unterschied. Die intravesikalen Drücke waren proportional $\mathrm{zu}$ den intraabdominellen, gastralen und hepatischen Messungen. Bei steigendem intraabdominellen Druck von 0 auf $24 \mathrm{mmHg}$ erhöhten sich die Mittelwerte linear für die Blase von 4 auf $26 \mathrm{mmHg}$, sowie für den Magen und die Leber von 3 auf $25 \mathrm{mmHg}$. Der Druck in der Vena cava superior blieb unverändert, jedoch erhöhte sich der Druck in der Vena cava inferior von 2 auf $24 \mathrm{mmHg}$, ebenfalls proportional $\mathrm{zu}$ den intraabdominellen Drücken. Schlussfolgerung: Unter laparoskopischen Bedingungen ist die Druckverteilung homogen. Codman-ICP-Sonden stellen eine überzeugende und aufgrund ihrer Größe auch bei Neugeborenen komfortable Möglichkeit dar, intraabdominelle Drücke zu registrieren. Der Bauchdruck kann einfach und nicht-invasiv über eine gastrale, transösophageal eingebrachte Sonde registriert werden. Dies stellt eine Alternative zur Blasendruckmessung dar, die in der heutigen Intensivmedizin standardmäßig als Referenz für den intraabdominellen Druck gilt.

Gasembolien während Laparoskopie Luft versus Kohlendioxid

C. Matthes, S. Richter, T. Huckstadt, T. Ploenes, F. Schier Klinik für Kinderchirurgie Universitätsklinik Mainz

Hintergrund und Ziele: Gefäßverletzungen durch die Veres-Nadel sind eine seltene, aber schwerwiegende Komplikation, die während der Laparoskopie zu Gasembolien führen kann. Zurzeit ist noch ungeklärt, in welchem Ausmaß sich Kohlendioxid- von Luftembolien unterscheiden. Hieraus ergeben sich folgende Fragestellungen: Wie wirkt sich eine kontinuierliche Luftembolie aus? Wie wirkt sich eine kontinuierliche Kohlendioxidembolie aus? Was passiert bei einer größeren Bolusgabe von Kohlendioxid? Methode: In einer tierexperimentellen Studie (Tierversuchsnummer 1.5 177-07/051-6) wurde bei 15 Schweinen im Alter von 2 bis 3 Wochen (Gewicht im Median 6,5 kg) das Abdomen auf $10 \mathrm{mmHg}$ insuffliert und kontinuierlich Luft $(n=7)$ oder $\mathrm{CO}_{2}(n=7)$ in die Vena cava inferior für 30 Minuten appliziert. Die Luft wurde mit $0,2 \mathrm{ml} / \mathrm{kg} \mathrm{KG} / \mathrm{min}$ injiziert. Bei der Vermutung einer besseren Verträglichkeit von Kohlendioxid wurde dessen Applikationsrate um $150 \%$ auf $0,5 \mathrm{ml} / \mathrm{kg} \mathrm{KG} / \mathrm{min}$ gesteigert. Ein Ferkel erhielt nach der kontinuierlichen Gabe von $0,4 \mathrm{ml} / \mathrm{kg} \mathrm{KG} / \mathrm{min}$ Kohlendioxid zusätzlich einen einmaligen Bolus von $20 \mathrm{ml}$ Kohlendioxid. Die Tiere 
wurden intramuskulär analgosediert. Die Narkose wurde intravenös mit Pentobarbital, Fentanyl und Pancuronium fortgeführt. Bei Untersuchungsbeginn bestand Normokapnie $\mathrm{paCO}_{2}$ $35-40 \mathrm{mmHg}$ ). Ventiliert wurden alle Tiere bei einem $\mathrm{FiO}_{2}$ von 1,0 mit einer Inspirationsdauer von $0,5 \mathrm{~s}$, einer Exspirationszeit von $2,5 \mathrm{~s}$ und einem PEEP von $4 \mathrm{cmH}_{2} \mathrm{O}$ am Babylog 1 (Dräger) Die Beatmung blieb nach Versuchsbeginn unverändert. Messparameter waren Herzfrequenz (HF), mittlerer arterieller Blutdruck (MAP) in der Arteria carotis, mittlerer pulmonalarterieller Druck (PAP), mittlerer zentralvenöser Druck (ZVD) in der Vena cava superior und der endtidale Kohlendioxidpartialdruck (et$\mathrm{CO}_{2}$ ). Nach Registrierung der Ausgangswerte erfolgten weitere Messungen direkt bei Gasinjektionsende sowie eine Stunde später. Ergebnisse: Die Tiere der Luftemboliegruppe waren nach $22 \mathrm{Mi}-$ nuten verstorben; dies entspricht einem kumulativen Gasvolumen von $2,7-3 \mathrm{ml} / \mathrm{kg} \mathrm{KG}$. Alle Tiere überlebten die Kohlendioxidembolie. Im PAP sowie im endtidalen und arteriellen Kohlendioxidpartialdruck war ein Anstieg um jeweils 84, 31 und 29\% im Median $\mathrm{zu}$ verzeichnen. Die restlichen Werte blieben im physiologischen Bereich. Nach Bolusinjektion kam es zusätzlich zu einem Anstieg des PAP um $65 \%$ und der HF um 35\% und zu einem Abfall des et $\mathrm{CO}_{2}$ um $49 \%$ und des MAP um 52\%. Alle Veränderungen erreichten nach 90 Minuten Erholungsphase wieder die Ausgangswerte. Schlussfolgerung: Geringe Mengen von injizierter Luft können tödlich sein. Kleine kontinuierliche Gaben von Kohlendioxid führen nicht $\mathrm{zu}$ einem Verschluss der Lungenstrombahn. Nur unter Bolusgabe höherer Volumina kommt es bei Kohlendioxidinjektion $\mathrm{zu}$ embolietypischen Reaktionen. Um einen tödlichen Verlauf bei Gefäßverletzungen zu vermeiden, sollten Luftverunreinigungen des Insufflationssystemes vermieden werden.

\section{Rationales Konzept zur Differentialdiagnostik und Therapie abdomineller Thrombembolien und Thrombozytopenien bei kritisch Kranken: Fallberichte und Literaturübersicht}

D. Tittelbach-Helmrich, M. Kaffarnik, U.T. Hopt, S. Utzolino Chirurgische Universitätsklinik Freiburg, Abt. Allgmein- und Viszeralchirurgie mit Poliklinik

Hintergrund: Thrombembolien intraabdomineller Gefäße sind typischerweise eine Erkrankung älterer Patienten mit Vorhofflimmern oder fortgeschrittener Gefäßsklerose. Bei Patienten außerhalb dieser Risikogruppe müssen angeborene oder erworbene $\mathrm{Ge}$ rinnungsstörungen ausgeschlossen werden. Besteht gleichzeitig eine Thrombozytopenie oder tritt diese im Verlauf der intensivmedizinischen Behandlung auf, muss eine Heparin-induzierte Thrombozytopenie (HIT) in die Diagnostik mit eingeschlossen werden. Die Differenzierung zwischen HIT und anderen unspezifischen Ursachen (z.B. Transfusion von Blutprodukten) und Infektionen (SIRS/Sepsis) ist nicht immer eindeutig. Methode: Wir stellen vier Patienten mit einem Durchschnittsalter von 44 Jahren vor, die aufgrund einer abdominellen Thrombose in unserer Klinik aufgenommen wurden und entweder bereits bei Aufnahme oder im Verlauf eine Thrombozytopenie entwickelten. Keiner der Patienten hatte eine Vorerkrankung, die ursächlich für die Thrombose oder Thrombozytopenie in Frage kam. Alle Patienten durchliefen eine umfangreiche Gerinnungs- und immunologische Diagnostik. Bei einem Patienten konnten Heparin-Antikörper festgestellt werden. Ein Patient war initial Antikörper-negativ, im Verlauf kam es zu einer Konversion. In diesem Fall wurde der Heparin-induzierte Plättchen-Aktivierungs-Assay (HIPA-Test) unspezifisch gehemmt und war somit nicht aussagekräftig. Eine Patientin hatte einen negativen Heparin-Antikörpertest, aber positive Phospholipid-Antikörper (IgM). Die vierte Patientin war Heparin-Antikörper-negativ und zeigte auch in der weiteren Diagnostik keine wegweisenden Ergebnisse. Basierend auf den Fallvorstellungen und nach ausführlicher Literaturdurchsicht stellen wir ein rationales Konzept zur Diagnostik vor, das die Differenzierung zwischen verschiedenen Ursachen der Thromboseentstehung erlaubt. Mit- einbezogen wird die Differentialdiagnostik der Thrombozytopenie, die bei etwa 20\% der kritisch kranken Patienten auf einer Intensivstation auftritt. Daraus resultierend beschreiben wir ein Therapiekonzept der Antikoagulation bei Thrombosen im Zusammenhang mit einer Thrombozytopenie, das einen Leitfaden für den Kliniker darstellen soll. Schlussfolgerung: Bei Auftreten einer Thrombozytopenie im Zusammenhang mit einer Thrombose bei kritisch kranken Patienten sollte eine umfangreiche Diagnostik durchgeführt werden. Die Heparin-Antikörper-Diagnostik ist nicht ausreichend sensitiv und spezifisch. Auch der Bestätigungstest (HIPA) kann uneindeutig ausfallen. Ist die Thromboseneigung und Thrombozytopenie nicht klinisch, immunologisch oder genetisch erklärt, sollte frühzeitig, auch bei primär negativer HeparinAntikörper-Diagnostik auf ein Heparin-Ersatzpräparat umgestellt werden. Bei geringer Wahrscheinlichkeit einer HIT als Ursache der Thrombozytopenie (z.B. im Rahmen einer Sepsis) kann bis zum Erhalt der Testergebnisse abgewartet werden.

Die Immunonutritionslösung Intestamin beeinträchtigt die Dünndarmperistaltik und verstärkt die motilitätshemmende Wirkung von Sedativa. Untersuchungen am Meerschweinchendünndarm in vitro

\section{R. Weis, M. K. Herbert, F. Czapek*, P. Holzer *}

Klinik u. Poliklinik für Anästhesiologie, Universität Würzburg

*Pharmakologisches Institut, Medizinische Universität Graz, Austria

Fragestellung: Der Benefit enteraler Ernährung und von Immunonutritionslösungen auf die immunologische Funktion und die Integrität des Darms wurde in vielen Untersuchungen gezeigt. Hingegen ist unklar, welche Wirkung Nutritionslösungen auf die Darmmotilität haben. Die Hemmung der Darmmotilität beim Intensivpatienten ist ein häufiges und bisweilen schwerwiegendes Problem. In der vorliegenden Studie wird an einem In-vitro-Peristaltikmodell die Wirkung einer enteralen Standarddiät (Fresubin Original) und einer Immunonutritionslösung (Intestamin) auf die Dünndarmmotilität untersucht. Desweiteren interessierte, ob diese Nutritionslösungen einen Einfluss auf die motilitätshemmende Wirkung der Sedativa Midazolam und Propofol haben. Methodik: Dünndarmsegmente des Meerschweinchens von etwa $10 \mathrm{~cm}$ Länge wurden im Organbad kontinuierlich mit Tyrode-Lösung gegen einen Druck von 400 Pascal perfundiert. Dabei wird ab einer konstanten Schwelle des intraluminalen Drucks (peristaltic pressure threshold, PPT) Peristaltik ausgelöst und der Darminhalt ausgeworfen. Unter dem Einfluss einer inhibitorischen Substanz steigt die PPT an oder es ist überhaupt keine Peristaltik mehr auslösbar. Nach einer Kontrollphase wurden die Darmsegmente endoluminal über $60 \mathrm{~min}$ entweder weiter mit Tyrode-Lösung, Fresubin Original oder Intestamin jeweils in einer Verdünnung (mit Tyrode-Lösung) von 1:10 und 1:3 perfundiert. Danach wurde den Darmsegmenten extraserosal $10 \mu \mathrm{M}$ Midazolam oder $50 \mu \mathrm{M}$ Propofol in das Organbad zupipettiert. Untersucht wurden je 6 bzw. 8 Darmsegmente von 6 bzw. 8 verschiedenen Meerschweinchen, jedes Darmsegment wurde nur mit einer Substanz behandelt. Die Signifikanzprüfung erfolgte auf dem Niveau von $\mathrm{p}<0,05$ (Kolmogorov-Smirnov-Test, ANOVA, Tukey post hoc Test). Ergebnisse: Tyrode-Lösung ist ohne Wirkung auf die PPT und Fresubin Original bewirkt eine geringfügige Steigerung der Effizienz der Kontraktionen, hat aber keinen Effekt auf die PPT. Durch Intestamin hingegen steigt die PPT konzentrationsabhängig signifikant an und bei 5 Segmenten kam es durch die Verdünnung von 1:3 bzw. 2 Segmenten bei 1:10 zur völligen Hemmung der Peristaltik (hier wurde kein Sedativum mehr hinzugegeben). Der Anstieg der PPT durch $10 \mu \mathrm{M}$ Midazolam und $50 \mu \mathrm{M}$ Propofol wurde durch Intestamin konzentrationsabhängig verstärkt, wohingegen Fresubin Original keinen derartigen Effekt hat. Schlussfolgerungen: Diese In-vitro-Untersuchungen zeigen erstmals, dass die Immunonutritionslösung Intestamin per se die Dünndarmmotilität konzentrationsabhängig beeinträchtigt und zudem die moti- 
litätshemmende Wirkung der in der Intensivmedizin routinemäBig eingesetzten Sedativa Midazolam und Propofol verstärkt. Die enterale Standarddiät Fresubin Original hat keine hemmende Wirkung auf die Dünndarmperistaltik.

\section{Verbrennungsverletzungen - Besonderheiten \\ des geriatrischen Patienten auf einer Intensiveinheit für Schwerbrandverletzte}

\section{F. Siemers, J. A. Lohmeyer, R. Battermann, M. Kaun,}

H.-G. Machens, P. Mailänder

Universitätsklinikum Schleswig Holstein, Campus Lübeck

Intensiveinheit für Brandverletzte

Einleitung: Geriatrische Patienten stellen hinsichtlich Pathogenese, Pathophysiologie und Therapeutischem Procedere eine gesonderte Gruppe in der Behandlung Schwerbrandverletzter dar. Altersabhängige Veränderungen der Haut, steigende Komorbiditäten und verminderte Reaktionsfähigkeit im Rahmen des Traumas führen im Allgemeinen zu schwerwiegenderen Verläufen. Methoden: Im Zeitraum von 1990 bis 2001 wurden auf der Intensiveinheit für Schwerbrandverletzte am Universitätsklinikum SchleswigHolstein, Campus Lübeck 53 Patienten mit einem Lebensalter von mehr als 60 Jahren stationär behandelt. Das Durchschnittsalter der 27 Frauen und 26 Männer betrug 76 Jahre (60-102 Jahre), im Mittel waren 33,2\% der Körperoberfläche (5-95\%) betroffen, 25 Patienten wiesen ein bronchoskopisch gesichertes Inhalationstrauma auf. 77,4\% erlitten Verbrennungen ${ }^{\circ}$ III. Bei Aufnahme wurde ein durchschnittlicher ABSI-Score von 12 errechnet. Als relevante Begleiterkrankungen fanden sich koronare Herzerkrankung $(\mathrm{n}=32)$, Herzinsuffizienz NYHA III-VI $(\mathrm{n}=27)$, arterieller Hypertonus $(\mathrm{n}=25)$, COPD $(\mathrm{n}=11)$, Adipositas mit BMI $>30 \quad(\mathrm{n}=14)$ und chronischer Alkoholabusus $(n=9)$. Ergebnisse: Bei $(22 / 52)$ Patienten wurde eine Frühnekrektomie (0-4 Tage nach Unfall) durchgeführt, 13 dieser Patienten überlebten im Verlauf. Bei 17 Patienten konnte der Ersteingriff erst zu einem späteren Zeitpunkt erfolgen, 14 Schwerbrandverletzte wurden aufgrund früher Letalität nicht operiert. 26 Patienten (49\%) entwickelten im Verlauf eine Pneumonie. Die Gesamtmorbidität betrug 26 von 53. Diskussion: Wir sahen im Vergleich zu unserem Patientenkollektiv unter 60 Lebensjahren eine deutliche Zunahme an Komorbiditäten, aber auch schwerwiegendere Verläufe der Verbrennungstraumata. Verbrennungstiefe, verbrannte Körperoberfläche und Inhalationstraumata nehmen im Alter signifikant zu. Die häufige kardiopulmonale Instabilität lässt eine angestrebte frühe operative Versorgung vielfach nicht zu. Brandverletzungen beim älteren Menschen stellen eine schwerwiegende Erkrankung dar, die eine frühzeitige Verlegung auf eine Intensiveinheit für Schwerbrandverletzte erforderlich macht.

Endovaskuläre Therapie verbessert die Prognose des rupturierten Bauchaortenaneurysmas

M. Brandt, T. Jahnke*, N. Haake, J. Marxsen, K. Graw, J. Cremer, S. Müller-Hülsbeck*

Klinik für Herz- und Gefäßchirurgie, ${ }^{\star}$ Klinik für Radiologie, Universitätsklinikum Schleswig-Holstein, Campus Kiel

Die offene Operation des rupturierten Bauchaortenaneurysmas ist trotz moderner Intensivtherapie immer noch mit einer hohen Letalität behaftet. Ziel dieser Studie war es, den Einfluss der Einführung der endovaskulären Therapie in Notfallsituationen auf die Ergebnisse zu untersuchen. Wir analysierten retrospektiv 39 konsekutive Patienten, die wegen eines rupturierten infrarenalen Aortenaneurysmas zwischen 2001 und 2004 behandelt wurden. Die Patienten wurden in zwei Gruppen eingeteilt: 15 Patienten, die zwischen 2001 und 2002 offen operiert wurden (Gruppe I) und 24 Patienten, die nach Einführung der endovaskulären Therapie zwischen 2003 und 2004 entweder offen $(n=13 ; 54 \%)$ oder endovaskulär $(\mathrm{n}=11 ; 46 \%)$ therapiert wurden (Gruppe II). Alter, Geschlecht und Aneurysmagröße unterschieden sich nicht zwischen den Gruppen. Die 30-Tage-Letalität lag bei 53\% in Gruppe I und $8 \%$ in Gruppe II $(\mathrm{p}=0,003)$. Die mediane Operationszeit war kürzer bei der endovaskulären Versorgung. Die Dauer des Intensivstations- und Krankenhausaufenthaltes war mit 22,0 29,6 und $29,7 \pm 33,8$ Tagen in Gruppe I deutlich länger als mit 5,6 $\pm 4,4$ und $16,1 \pm 10,9$ Tagen in Gruppe II $(\mathrm{p} \leq 0,03)$. Ebenfalls war in Gruppe I die Anzahl der transfundierten Erythrozytenkonzentrate signifikant niedriger. In der Gruppe II waren 11 Patienten aufgrund des Aneurysmahalses $(n=5)$, der Iliacalarterien $(n=3)$ oder aus beiden Gründen $(n=3)$ nicht für ein endovaskuläres Therapieverfahren geeignet. Nach Einführung der endovaskulären Therapie auch in Notfallsituationen wurden $46 \%$ der Patienten endovaskulär therapiert. Weitere $8 \%$ wären ebenfalls für ein endovaskuläres Verfahren geeignet gewesen. Die Einführung der endovaskulären Therapie hat in unser Klinik zu einer deutlichen Verbesserung der Ergebnisse bei Patienten mit rupturierten infrarenalem Bauchaortenaneurysma geführt.

Die Aktivierung nikotinerger Acetylcholinrezeptoren 212 durch Nikotin und Rezeptorsubunit-selektive Agonisten fördert nicht die Dünndarmperistaltik. Untersuchungen am Meerschweinchendünndarm in vitro

M. K. Herbert, M. Schubring, N. Roewer, P. Holzer *

Klinik u. Poliklinik für Anästhesiologie, Universität Würzburg *Pharmakologisches Institut, Medizinische Universität Graz, Austria

Fragestellung: Die Hemmung der Darmmotilität beim Intensivpatienten kann schwerwiegende Folgen nach sich ziehen, z.B. durch unzureichende enterale Ernährung den Verlust der Darmmukosabarriere und im ungünstigen Fall die Entwicklung von SIRS, Sepsis oder Multiorganversagen. Da für die Behandlung der Darmatonie wenige und zudem oft nur unzureichend wirksame Properistaltika zur Verfügung stehen, stellt sich die Frage, ob Nikotin oder Rezeptorsubunit-selektive Agonisten hier eine therapeutische Option darstellen. Denn vom Zigarettenrauchen ist bekannt, dass es beim Menschen die Darmtätigkeit und Defäkation fördert. Methodik: Dünndarmsegmente des Meerschweinchens von etwa $10 \mathrm{~cm}$ Länge wurden im Organbad kontinuierlich mit TyrodeLösung gegen einen Druck von 400 Pascal perfundiert. Dabei wird ab einer konstanten Schwelle des intraluminalen Drucks (peristaltic pressure threshold, PPT) Peristaltik ausgelöst und der Darminhalt ausgeworfen. Unter dem Einfluss einer inhibitorischen Substanz steigt die PPT an oder es ist überhaupt keine Peristaltik mehr auslösbar. Nach einer Kontrollphase wurde den Darmsegmenten das Lösungsmittel (Tyrode-Lösung) oder Nikotin (nicotine hydrogen tartrate) in den Konzentrationen von 0,01-10 $\mu \mathrm{M}$ extraserosal dem Organbad zugegeben. Des Weiteren wurden die hoch Subunit-selektiven Agonisten für nikotinerge Acetylcholinrezeptoren (nACh-Rs) RJR 2403 fumarate ( $\alpha 4 \beta 2-n A C h-R$, 1-30 $\mu \mathrm{M})$ und PNU $282987(\alpha 7-\mathrm{nACh}-\mathrm{R}, 0,1-10 \mu \mathrm{M})$ sowie die selektiven Antagonisten Mecamylamine ( $\alpha 4 \beta 2-\mathrm{nACh}-\mathrm{R}, 0,01-0 \mu \mathrm{M})$ und Methylcacotinine $(\alpha 7-\mathrm{nACh}-\mathrm{R}, 0,1-10 \mu \mathrm{M})$ appliziert. Weiterhin wurden Darmsegmente vor der Gabe von $3 \mu \mathrm{M}$ Nikotin zunächst mit $0,5 \mu \mathrm{M}$ Naloxon (Opioidrezeptorantagonist) oder $0,5 \mu \mathrm{M}$ Apamin (Blocker von small conductance Ca2+-activated $\mathrm{K}^{+}$-channels) vorbehandelt. Ergebnisse: Nikotin hebt konzentrationsabhängig die PPT zur Auslösung peristaltischer Kontraktionen an, nach $10 \mu \mathrm{M}$ Nikotin war die Motilität in allen getesteten Segmenten $(n=8)$ komplett gehemmt. Die hoch Subunit-selektiven Agonisten RJR 2403 fumarate ( $\alpha 4 \beta 2$-nACh-R) und PNU 282987 $(\alpha 7-\mathrm{nACh})$ hatten keine Wirkung auf die Darmmotilität. Hingegen hemmten die selektiven Antagonisten Mecamylamine und Methylcacotinine konzentrationsabhängig die Peristaltik. Die Vorbehandlung mit Naloxon und Apamin verhinderte die Hemmwirkung des Nikotins. Schlussfolgerungen: Diese in vitro-Untersuchungen zeigen, dass Nikotin und die hoch Subunit-selektiven Agonisten für nACh-Rs RJR 2403 fumarate und PNU 282987 keine stimulatorische Wirkung auf die Dünndarmperistaltik haben, im Gegenteil, 
dass Nikotin die Motilität konzentrationsabhängig hemmt und somit keine Therapieoption darstellt. Die Antagonisierungsversuche bestätigen aber, dass $a 4 \beta 2$-nACh-R und $\alpha 7-n A C h-R$ im enterischen Nervensystem vorhanden sind und zeigen zum anderen einen komplexen Hemmmechanismus des Nikotins über Aktivierung sowohl inhibitorischer opioiderger als auch nicht-adrenerg nicht-cholinerger (NANC) Neurone.

\section{Onkologische Patienten auf der Intensivstation}

223

\section{K. Rellos, M. Rizos, Z. Mastora, P. Malamos, A. Raftopoulou, V. Boursinos, A. Michalopoulos \\ Henry Dunant Hospital, Intensivmedizin, Athen, Griechenland}

Einleitung: In den letzten 10 Jahren sind in unserem Land der Bedarf an Intensivbetten sowie die Inzidenz der onkologischen $\mathrm{Pa}$ tienten(Pat) sehr angestiegen. Die Aufnahme dieser Pat auf der Intensivstation (IPS) ist eine umstrittene Frage. Das Ziel unsere Studie war die Charakteristika dieser Pat, sowie die Morbidität, die Dauer der Behandlung und das Outcome zu untersuchen. $\mathrm{Pa}$ tienten und Methoden. Nach Genehmigung durch die Ethikkommission wurden für die Gesamtdauer des Intensivaufenthaltes alle onkologischen Pat, während des Zeitraumes (Januar 2002-Dezember 2004), eingetragen und untersucht. Aus der Studie Wurden die Pat ausgeschlossen, die direkt postoperativ nach Tumorexploration auf der IPS kamen. Ergebnisse: Es wurden 92 erwachsene Pat studiert. Es waren 61 Männer mit Durchschnittsalter 60,6 Jahre und hatten bei der Aufnahme auf der IPS ein Durchschnittlichen APACHE Score II von 17.6. Der primär sitzende Tumor war in der Lunge $(n=28)$, Eierstöcke $(n=6)$, Dickdarm $(n=6)$, Mamma $(n=5)$, Pankreas $(n=3)$, Prostata $(n=3)$, Harnblase $(n=3)$, Hirn $(n=3)$, Haut $(n=3)$. Magen $(n=2)$, Niere $(n=2)$, Rhinopharynx $(n=1)$, Hoden $(n=1)$ und Oesophagus $(n=1) .21$ Patienten hatten hämatologische maligne Erkrankungen und Sarkome. Metastasen waren bei 62 Pat entdeckt. Der Grund der akut Aufnahme auf der IPS war hauptsächlich akute Respiratorische Insuffizienz wegen Pneumonie $(n=41)$, Lungenembolie $(n=5)$, Infektion/Sepsis $(n=27)$, Tamponade $(n=4)$, Koma $(n=5)$, Synkope Anfall $(n=3)$ und akutes Abdomen $(n=3)$. Davon wurden 59 Pat beatmet. Die Durchschnittliche Beatmungsdauer und Aufenthaltsdauer auf der IPS waren 4,3 und 5,6 Tage entsprechend. Die Mortalität bei diesen lag bei $41,3 \%$ und war unabhängig vom Alter. $70,4 \%$ der septischen Pat starben auf der IPS. Die höchste Letalität wiesen die Patienten auf, die in der Anamnese Mamma Karzinom (80\%) und Eierstock Neoplasien hatten. Schlussfolgerung: 1. Es wurde eine hohe Letalitätsrate bei den onkologischen Patienten, im Vergleich zu den üblichen IPS-Patienten, beobachtet. 2 . Die höchste Mortalität hatten die septischen Patienten. Und 3. obwohl diese Patienten-Gruppe eine hohe Mortalitätsrate aufweist, darf man Sie nicht von der Intensivstations-Behandlung ausschließen.

\section{Volumenmanagement mittels PicCO-Monitoring \\ bei lungenchirurgischen Eingriffen -}

Ergebnisse einer prospektiven Validierungsstudie

\section{A. Falthauser, A. Thomas}

Abt. für Anästhesie und Intensivmedizin, Bundeswehrzentralkrankenhaus Koblenz

Hintergrund: In einer prospektiven Untersuchung soll überprüft werden, ob intrathorakales Blutvolumen (ITBI) und extravaskuläres Lungenwasser(EVLWI) bei thoraxchirurgische Patienten perioperativ valide Steuerungsgrößen sind und sich damit die bei diesem Patientenklientel besonders wichtige Volumen- und Katecholamintherapie optimieren lässt. Methode: Zwischenergebnis einer prospektiven klinischen Studie an geplant 30 thoraxchirurgischen (Lob- oder Pneumonektomie) ASA-III-Patienten. Bei standardisierter TIVA mit Remifentanil und Propofol wurden Volumenstatus, Katecholamintherapie mit Noradrenalin (NA) und Urinaus- scheidung (U/h) bei 20 Patienten zu verschiedenen Zeitpunkten der Operation miteinander verglichen und mittels Fishers exact t-test $(\mathrm{p}<0,05)$ und ANOVA analysiert. Zu definierten Zeitpunkten nach Einleitung (1), vor (2), während (3) und unmittelbar nach (4) der Einlungenventilation (ELV) sowie unmittelbar nach Extubation und alle $8 \mathrm{~h}$ postoperativ erfolgte die Bestimmung von HZV, SVV, EVLW und ITBV aus den Mittelwerten von drei transthorakalarteriellen Thermodilutionen (TDa). Ergebnisse: in Klammern $\pm 2 S D$, unter PEEP+6:

\begin{tabular}{lllllll} 
& $\begin{array}{l}\text { MAP } \\
(\mathbf{m m H g})\end{array}$ & $\begin{array}{l}\text { ZVD } \\
(\mathbf{m m H g})\end{array}$ & $\begin{array}{l}\text { CI } \\
(\mathbf{( I / q m})\end{array}$ & $\begin{array}{l}\text { SVI } \\
(\mathbf{m l} / \mathbf{q m})\end{array}$ & $\begin{array}{l}\text { ITBI } \\
(\mathbf{m l} / \mathbf{q m})\end{array}$ & $\begin{array}{l}\text { EVLWI } \\
(\mathbf{m l} / \mathbf{k g})\end{array}$ \\
\hline Vor ELV (2) & $67( \pm 18,2)$ & $11( \pm 3,2)$ & $3,2( \pm 1,1)$ & $34( \pm 12)$ & $890( \pm 133)$ & $7,4( \pm 1,3)$ \\
Während ELV (3) & $62( \pm 23,1)$ & $14( \pm 5,3)$ & $2,7( \pm 1,3)$ & $27( \pm 13,2)$ & $753( \pm 154)$ & $6,7( \pm 1,2)$ \\
nach ELW (4) & $71( \pm 16,4)$ & $12( \pm 5,8)$ & $2,9( \pm 0,9)$ & $33( \pm 12,5)$ & $813( \pm 122)$ & $7,6( \pm 1,5)$ \\
Aufn. ITS (5) & $74( \pm 17,5)$ & $14( \pm 6,1)$ & $3,1( \pm 1,3)$ & $35( \pm 13,89)$ & $955( \pm 176)$ & $8,9( \pm 1,9)$ \\
p-value & n.s. & n.s. & n.s. & $>0,05$ & $>0,05$ & n.s. \\
\hline
\end{tabular}

Diskussion: Die Bestimmung von ITBV und EVLW lieferte über den gesamten Zeitraum valide und reproduzierbare Ergebnisse. Die Abweichungen innerhalb der Thermodilutionsmessungen eines Messpunktes waren unter den Bedingungen der ELV nicht signifikant höher als außerhalb der ELV (ANOVA). Die Änderung der Vorlast ITBI ist ebenso signifikant wie die Änderung des Schlagvolumens. Daher kann man schlussfolgern, dass sich die volumetrischen Daten ITBI und EVLWI aus der transthorakalarteriellen Thermodilution für die Steuerung der Volumensubstitution eignen. Es muss allerdings darauf geachtet werden, dass durch den Verlust von Lungengewebe und pulmonalarterieller Strombahn sich auch die Normwerte für ITBI und EVLWI um den Teil des Resektates verringern. Eine entsprechende Normwerttabelle lässt sich aus dem vorliegenden Datenmaterial noch nicht sicher ableiten, ist aber eines der Ziele der Studie.

\section{Ethik, Ökonomie und allgemeine Aspekte}

Wie beurteilen die Angehörigen das Gespräch mit der Bitte um die Organspende?

\section{Mauer, S. Moos, M. Schmid, A.B. Blaess}

Deutsche Stiftung Organtransplantation Region Mitte, Mainz

Hintergrund: Die Betreuung der Angehörigen nach Feststellung des Hirntodes ist von entscheidender Bedeutung für die Zustimmung zur Organspende. Ziel der Untersuchung ist die kontinuierliche Evaluation der Qualität der Gesprächsführung zur Verbesserung der Begleitung trauernder Angehöriger im Umfeld der postmortalen Organspende. Das Angehörigenprojekt der Region Mitte (Hessen, Rheinland-Pfalz, Saarland) ist eine erste flächendeckende Initiative über 5 Jahre, die Betreuung von Angehörigen über das Einwilligungsgespräch hinaus auszudehnen. Methodik: Befragung der Angehörigen, die im Jahr 2004 einer Organspende in der Region Mitte zustimmten, mit einem standardisierten Fragebogen 8-12 Monate nach der Organspende. Ergebnisse: Im Untersuchungszeitraum kamen 73 Fragebögen zur Auswertung. In $49,3 \% \quad(\mathrm{~N}=46)$ wurde die Organspende vom Stations-/Oberarzt angesprochen, aber in $18 \%(\mathrm{~N}=13)$ wurde von den Angehörigen selbst das Gespräch initiiert. In $11 \%(\mathrm{~N}=8)$ sprach der DSO-Koordinator die Angehörigen erstmals auf die Organspende an, in der überwiegenden Anzahl der Fälle $(\mathrm{N}=52)$ wurde der Koordinator zum Gespräch hinzugezogen. 90\% $(\mathrm{N}=66)$ der Befragten fühlten sich in ihrer Entscheidung nicht unter Druck gesetzt. Die Mehrzahl der Angehörigen bewertete die zurückliegende Ent- 
scheidung zur Organspende positiv: $93 \%(\mathrm{~N}=68)$ würden erneut zustimmen, $6 \%(\mathrm{~N}=4)$ sind sich nicht sicher, nur eine Familie bereute die Entscheidung zur Organspende. 88\% $(\mathrm{N}=64)$ der Angehörigen gaben an, dass die Organspende die Zeit der Trauer nicht erschwerte, in 29\% $(\mathrm{N}=21)$ erleichterte die Organspende sogar die Zeit der Trauer. In der Hälfte der Fälle $(\mathrm{N}=36)$ wurde den Angehörigen eine Abschiednahme nach der Organspende angeboten. Diskussion: Die Ergebnisse zeigen, dass die Familien die Entscheidung für die Organspende nach einem Jahr positiv beurteilen und, dass in der überwiegenden Zahl der Fälle eine stabile Entscheidung für die Organspende möglich war. Das Projekt liefert wichtige Erkenntnisse für Ärzte und Pflegende in den Krankenhäusern zur Verbesserung der Begleitung trauernder Angehöriger im Umfeld der postmortalen Organspende und ermutigen, die Frage nach der Organspende in allen Fällen des festgestellten Hirntodes zu stellen. Langfristig kann das Öffentlichmachen der positiven Erfahrungen von Angehörigen die Organspendebereitschaft in Deutschland weiter erhöhen.

Erhebung des Ist-Zustandes zur Patienteneinwilligung bei klinischen Studien und Forschungsdatenbanken mit nichteinwilligungsfähigen erwachsenen Patienten

\section{U. Corvinus, K. Weismüller, U. Harnischmacher, P. Ihle,}

E. Sellge, R. Röhrig

Klinik für Anästhesiologie, Intensivmedizin, Schmerztherapie

Universitätsklinikum Gießen u. Marburg - Standort Gießen

Einleitung: Eine Voraussetzung für die Durchführung klinischer Studien und die Verarbeitung studienbezogener, personengebundener Daten in Datenbanken ist das Einverständnis des Patienten. Dieses Einverständnis muss vom Patienten eigenverantwortlich im Sinne des „informed consent“ nach eingehender Aufklärung gegeben werden können und vom Arzt dokumentiert werden $[1,2]$. In einem Projekt der Telematikplattform für Medizinische Forschungsnetze e.V. wurde für einwilligungsfähige Patienten eine Modelllösung für Patienteneinwilligungserklärungen unter Berücksichtigung datenschutzrechtlicher Aspekte erarbeitet und veröffentlicht [3]. Die Erforschung vieler Krankheitsbilder, insbesondere in der Intensiv- und Notfallmedizin, erfordert jedoch den Einschluss nichteinwilligungsfähiger erwachsener Patienten. Die Einbeziehung nichteinwilligungsfähiger erwachsener Patienten in klinische Studien und Datenbanken ist durch eine Reihe von Gesetzen und Richtlinien wie z.B. das Arzneimittelgesetz \$\$ 40-42, die GCP-Verordnung, die überarbeitete Deklaration von Helsinki und das Bundesdatenschutzgesetz $\$ \$ 13$ und 28 sowie die verschiedenen Landesdatenschutzgesetze geregelt. Bei deren Umsetzung entstehen dennoch häufig Auslegungsschwierigkeiten und Unsicherheiten. Das Ziel der Arbeit ist die Durchführung einer Bestandsaufnahme des aktuellen Status beim Einschluss nichteinwilligungsfähiger erwachsener Patienten in Forschungsvorhaben und der Verarbeitung ihrer Daten in Forschungsdatenbanken. Die Intention ist, die bestehende Modelllösung für Patienteneinwilligungserklärungen der TMF zu erweitern. Methodik: Mittels Literaturrecherche wurden Schlüsselfaktoren herausgearbeitet, die im Zusammenhang mit dem Einschluss nichteinwilligungsfähiger erwachsener Patienten in Studien und Datenverarbeitung stehen. Diese stellen die Basis eines Erhebungsbogens dar und sind im Folgenden aufgeführt:

- Berücksichtigung des Patientenwillens

- Wahl und Befugnis der stellvertretend entscheidenden Personen

- Einfluss von akuten Situationen (z. B. Schlaganfall, Polytrauma)

- Reaktion auf veränderte Einwilligungsunfähigkeit des Patienten

- Einfluss der Risiko-Nutzen-Abwägung

- gesonderte Anforderungen an den Datenschutz

Nach der Durchführung von Pre-Tests erfolgte der Versand der Erhebungsbögen an die Ethikkommissionen der Landesärztekammern und Länder und der Universitätsklinik, an die Datenschutzbeauftragten des Bundes und der Länder und die Mitgliedsver- bände der TMF (KKS, Forschungsnetze). Ergebnisse und Diskussion: Die Ist-Erhebung war zu dem Zeitpunkt der Einreichung noch nicht abgeschlossen. Sponsor: Telematikplattform für Medizinische Forschungsnetze e.V.

Literatur: [1] Höfling $W$ et al: Zur Forschung an Nichteinwilligungsfähigen. MedR 1999; 12:540-546. [2] Fröhlich U: Forschung wider Willen? Springer, Berlin 1999. [3] Harnischmacher U et al: Ergebnisbericht für das Projekt DS 3.8 der Telematikplattform für medizinische Forschungsnetze (TMF), Köln April 2003

\section{SexGender im Blick der Intensivmedizin}

\section{Lj. Verner, A. Vo $\beta^{\star}$}

Zentrum Anästhesiologie, ${ }^{*}$ Abt. Geschichte, Ethik und Philosophie der Medizin. Medizinische Hochschule Hannover

Die Realität der Intensivmedizin stellt nicht nur den Mythos vom „starken Geschlecht" in Frage, sondern auch die klinische Geschlechterforschung vor eine große Herausforderung. Zweigeschlechtlichkeit als Leitdifferenz berührt dabei sowohl das biologische (sex) wie das soziale Geschlecht (gender), deren tatsächliche Bedeutung für das intensivmedizinische Management bislang unzureichend berücksichtigt wird. Neben einem immunologischen Geschlechtsdimorphismus und einer geschlechtsdifferenten Pharmakodynamik (PD) und Pharmakokinetik (PK) finden sich Unterschiede hinsichtlich der Inzidenz der Aufnahme auf der Intensivstation, diagnostischer und therapeutischer Interventionen, Inzidenz von Sepsis und Multiorganversagen sowie dem Outcome. Nur ein Teil der gefundenen Unterschiede erklärt sich jedoch aus physiologischen/biochemischen geschlechtsabhängigen und -spezifischen Faktoren. So konzentriert sich die Interpretation bei gefundenen Geschlechtsdifferenzen in PK- und PD-Studien vor dem Hintergrund einer somatischen Zweigeschlechtlichkeit als Konstanz auf intrinsische Faktoren, während extrinsische Faktoren wie Applikationsart der Medikamente und Dosisregime, d.h. die Interaktion von ärztlichem Handeln (doing doctor) mit dem $\mathrm{Pa}-$ tienten, teilweise oder sogar hauptsächlich für die gefundenen Unterschiede verantwortlich sind. Als Folge gesellschaftlich wie klinisch geprägter Geschlechtsnormierungen (Gender-Norm-Hypothese) treten geschlechtsdependente Versorgungsungleichheiten auf horizontaler wie vertikaler Ebene auf. Dieses als "doing gender" bezeichnete Herstellen von Geschlecht durch Normierung wie Interaktion kann sich auf den gesamten Behandlungsprozess auswirken, d.h. zu geschlechtsspezifischen Unter- wie zu Überversorgungen führen (gender gap). Die Visualisierung des von uns entwickelten Modells eines sex-based wie gender-bezogenen $\mathrm{Zu}$ gangs zu Patienten und Patientinnen der Intensivmedizin soll die verschiedenen Facetten einer geschlechtssensiblen Forschung und Praxis in ihrem Innovationspotenzial verdeutlichen. Ziel des Modellprojekts zur Implementierung systematischer Geschlechterforschung in die Intensivmedizin ist die Optimierung intensivmedizinischer Versorgung durch: Überwindung von Übergeneralisierung und Überbewertung des sozialen wie des biologischen $\mathrm{Ge}-$ schlechts, Detektierung sozial konstruierter somatischer $\mathrm{Ge}$ schlechterdifferenzen durch kritische Sexforschung sowie Transfer, Modifikation und Weiterentwicklung der Methoden der Geschlechterforschung zur Analyse und Interpretation empirischer Daten sowohl inter- wie intrageschlechtlicher Differenzen.

Literatur: [1] Chen, ML: Confounding factors for sex differences in pharmacokinetics and pharmacodynamics: Focus on dosing regimen, dosage form, and formulation, Clin Pharmacol Ther 2005; 78:322-329. [2] Verner Lj, Voß A: Geschlechterforschung - Einführung und anästhesiologische Aspekte, Anästhesiol Intensivmed Notfallmed Schmerzther 2005; 40:191-198 
Ablauf von Therapiebegrenzungsentscheiden:

\section{Eine systematische Fallserie auf einer Intensivstation}

B. Meyer-Zehnder ${ }^{1,2}$, H. Pargger ${ }^{2}$, S. Reiter-Theil ${ }^{1}$

${ }^{1}$ Institut für Angewandte Ethik und Medizinethik, Universität

Basel, ${ }^{2}$ Departement Anästhesie, Operative Intensivbehandlung,

Universitätsspital Basel

Fragestellung: Das Thema Therapiebegrenzung wird seit mehreren Jahren stark diskutiert. In verschiedenen Ländern wurden Studien zur Häufigkeit von Therapiebegrenzungsentscheiden durchgeführt. Noch kaum untersucht wurde folgende Fragestellung: Wie wird die Therapiebegrenzung praktisch umgesetzt? Gibt es einen Ablauf, der regelmäßig beobachtet werden kann? Wie wird sie begründet, und welche Rolle spielt der Patientenwille? Methode: Auf einer chirurgischen Intensivstation wurden 10 Fälle prospektiv im Detail dokumentiert, bei denen eine Therapie entweder nicht begonnen (Therapieunterlassung) oder eine bereits begonnene Therapie abgebrochen (Therapieabbruch) wurde. Alle Krankengeschichten wurden darauf untersucht, in welcher Reihenfolge und $\mathrm{zu}$ welchem Zeitpunkt Therapien begonnen bzw. begrenzt wurden. Resultate: Die Entscheidung, mindestens einen Teil der kardiopulmonalen Reanimation nicht durchzuführen, steht bei allen 10 Fällen am Anfang des Ablaufes einer Therapiebegrenzung. Danach lässt sich ein Muster ablesen. Bei infauster Prognose werden Therapien gleichzeitig abgebrochen. Ist die Prognose aber noch unklar, werden zuerst Therapien unterlassen. Wenn sich die Situation klärt, folgt ein Therapieabbruch. Neue Therapien werden, trotz bereits stattgefundener Therapiebegrenzung, begonnen (z.B. Hämodialyse). Als Grund für die Begrenzung wird in 4 Fällen eine infauste Prognose angegeben. Ein $\mathrm{zu}$ erwartendes schlechtes neurologisches Outcome und ein mutmaßlicher Patientenwille, der gegen ein Fortführen der Therapie spricht, sind in weiteren 4 Fällen der Grund. Der direkte Patientenwille (PW) kann in keinem Fall erhoben werden. In drei Fällen wird auch der mutmaßliche PW nicht ermittelt, weil er wegen einer infausten Prognose als nicht relevant beurteilt wird. In 4 anderen Fällen hingegen, bei denen ein schlechtes neurologisches Outcome mit hoher Pflegebedürftigkeit zu erwarten ist, wird der mutmaßliche Wille des Patienten über die Angehörigen sorgfältig ermittelt und als sehr wichtig angesehen. Eine Patientenverfügung liegt nur in einem Fall vor, sie wird aber erst einige Tage nach Aufnahme vorgelegt. In 4 Fällen wird beobachtet, dass der eigentliche mutmaßliche PW von den Wünschen der Angehörigen nicht angemessen unterschieden wird. Schlussfolgerung: Der Ablauf der Therapiebegrenzung folgt einem Muster, das jeweils an den individuellen Patienten angepasst wird. In den untersuchten Fällen spielt der mutmaßliche Patientenwille vor allem dann eine Rolle, wenn mit einem schlechten neurologischen Outcome zu rechnen ist. Die Ermittlung des mutmaßlichen Patientenwillens sollte mit großer Sorgfalt geschehen. Nach einer Patientenverfügung wird bisher nicht routinemäßig gefragt.

Das ethische Dilemma nach primär erfolgreicher, präklinischer Reanimation eines Tumorpatienten mit Patientenverfügung

\section{T. Birkholz, M. Göhring, J. Schmidt, S. Albrecht, M. Marsch} Anästhesiologische Klinik, Universitätsklinikum Erlangen

Einleitung: Die Entscheidung zur präklinischen Reanimation ist zumeist durch mangelnde Kenntnis über die Erkrankungen des Patienten und die Umstände des Kreislaufstillstandes gekennzeichnet. Dies kann die Reanimation eines Patienten mit vorbestehend infauster Prognose zur Folge haben $(1,2)$. Kasuistik: Wir stellen den den Fall eines Tumorpatienten vor, der nach asphyktischem Kreislaufstillstand im häuslichen Umfeld und bei begonnener $\mathrm{Ba}-$ sisreanimation durch die Angehörigen vom Notarzt primär erfolgreich reanimiert wurde. Nach Gabe von $1 \mathrm{mg}$ Adrenalin konnte ein ROSC beobachtet werden. Zeitgleich berichteten die anwesenden Angehörigen - Ehefrau und Tochter -, dass ein T4-Adenokar- zinom der Lunge vorbestehe. Sie gaben sich beide als Ärzte zu erkennen und berichteten von einer Patientenverfügung und einer Vorsorgevollmacht. Kraft dieser Vollmacht verlangt die bevollmächtigte Ehefrau einen Abbruch der Maßnahmen und verweigert die Zustimmung zur Intubation bei noch nicht intubiertem Patienten. Der anwesende Notarzt konsultierte daraufhin den diensthabenden Facharzt der den Notarztdienst organisierenden Klinik. Dieser riet zu einer Verifizierung der gemachten Angaben durch Einsichtnahme der Arztbriefe, der Patientenverfügung und der Vorsorgevollmacht. Nach Authentifizierung dieser Angaben wurde in Anlehnung an den innerklinischen Standard (Erlanger Empfehlungen zur Patientenautonomie am Lebensende, 3) auf eine Intubation verzichtet und die Maskenbeatmung eingestellt. Der schnappatmende Patient verstarb innerhalb weniger Minuten exzitationslos im Beisein seiner Angehörigen. Schlussfolgerung: Bei einerseits mangelndem Informationsstand und emotional motiviertem Handeln der Angehörigen und andererseits erfolgtem ROSC eröffnete sich zunächst ein ethisches Dilemma für den anwesenden Notarzt. Medizinisch wies der Patient durch die stattgehabte Asphyxie und den Kreislaufstillstand unbestimmter Dauer eine eher ungünstige Prognose auf (2). In Anbetracht des zugrundeliegenden Tumorleidens musste bei Fortführung der Maßnahmen von einer zukünftig schlechten Lebensqualität ausgegangen werden $(2,4)$. Im Konsens mit den Angehörigen wurde der in der Patientenverfügung erklärte Wille des Patienten respektiert und der durch die Bevollmächtigte geforderten Einstellung aller weiterer Maßnahmen entsprochen. Es konnte ein Versterben im häuslichen Umfeld ermöglicht werden. Die Tatsache, dass eine eindeutige Vorausverfügung vorlag, konnte die Widersprüchlichkeit der initialen Einsatzsituation im Sinne des Patienten auflösen. Hilfreich war zudem die Orientierung an bestehenden Handlungsalgorithmen und zeigt die Notwendigkeit der Implementierung von Algorithmen zur Therapie am Lebensende. Literatur:

Literatur: [1] Horsted et al: Resuscitation 2004; 63:287-293.

[2] Ewer et al: Cancer 2001; 92:1905-1912.

[3] www.gesch.med.uni-erlangen.de/eth/projekte/Klin_eth/ ethik_ordner/ethikordner_leitlinien.htm.

[4] Reynolds et al: Thorac Surg Clin. 2005; 15:469-480

Angst, Befindlichkeit und Depression: Vergleich zwischen tracheotomierten und intubierten Patienten einer Intensivstation

P. Saur ${ }^{1}$, K. Lipka ${ }^{1}$, S. Gatzert ${ }^{2}$, D. Kettler ${ }^{2}$

${ }^{1}$ Regionales Zentrum für Anästhesie, Schmerztherapie, Rettungsund Intensivmedizin, Sana Kliniken Lübeck und Ostholstein GmbH ${ }^{2}$ Zentrum Anästhesie, Rettungs- und Intensivmedizin,

Georg-August-Universität Göttingen

Diese Studie untersuchte erstmals prospektiv, ob sich die Angst, Befindlichkeit und depressive Verstimmung zwischen intubierten und tracheotomierten Patienten einer interdisziplinären Intensivstation unterscheidet. Methoden: 17 intubierte Patienten (7 Frauen und 10 Männer) und 9 tracheotomierte Patienten (3 Frauen und 6 Männer) einer interdisziplinären Intensivstation nahmen nach Genehmigung der lokalen Ethik-Kommission an der Untersuchung teil. Die Angst und depressive Verstimmung wurde mittels der Anxiety and Depression Scale (HADS), die Befindlichkeit anhand der Befindlichkeits-Skala BL nach Von Zerssen, die Vigilanz durch die Glasgow Coma Scale und die Schwere der Erkrankung nach dem Simplified Acute Physiology Score (SAPS II) erhoben. Ergebnisse: Alter, Body Mass Index, Vigilanz, SAPS II, Angst und die depressive Verstimmung unterschieden sich nicht zwischen den intubierten und tracheotomierten Patienten. Der mittlere HADS-A der intubierten Patienten betrug 9,8 $(\mathrm{SD}=4,7)$ und $10(\mathrm{SD}=3,6)$ bei den tracheotomierten Patienten. 47,1\% der intubierten Patienten und $44,4 \%$ der tracheotomierten Patienten wiesen eine pathologische HADS-A mit einem Cut-off von mehr als 10 Punkten auf. Der mittlere HADS-D der intubierten Patienten betrug 10,9 $(\mathrm{SD}=6,1)$ und $8,7(\mathrm{SD}=4,5)$ bei den tracheotomierten Patienten. 
$52,9 \%$ der intubierten Patienten und 44,4\% der tracheotomierten Patienten wiesen einen pathologisch erhöhten HADS-D mit einem Cut-off von mehr als 8 Punkten auf. HADS-A und HADS-D unterschieden sich nicht zwischen den intubierten und tracheotomierten Patienten. Die globale Auswertung der Beschwerdeliste nach Von Zerssen ergab keine Unterschiede zwischen den Gruppen. Kloßgefühl, Enge und Würgen im Hals, subjektive Kurzatmigkeit sowie innere Unruhe wurden in beiden Gruppen gleich stark angegeben. Lediglich gaben die intubierten Patienten signifikant $(\mathrm{p}<0,05)$ stärkere Schluckbeschwerden an als die tracheotomierten Patienten. Schlussfolgerung: Angst und depressive Verstimmung, gemessen anhand der Hospital Anxiety and Depression Scale HADS sowie die Befindlichkeit nach der Beschwerdeliste nach Von Zerssen ergaben keine Unterschiede zwischen intubierten und tracheotomierten Patienten, waren aber pathologisch verändert. Intubierte Patienten gaben signifikant stärkere Schluckbeschwerden an als die tracheotomierten Patienten. Diese Ergebnisse sind interessant, zumal die tracheotomierten Patienten zum Zeitpunkt der Befragung länger beatmet waren als die intubierten Patienten.

Gesundheitsökonomische Untersuchung zur Kostenermittelung auf einer interdisziplinär, operativen Intensivstation eines Universitätsklinikums

L. Mehlhorn, B. Gottschlich, S. Weigel, T. Koch, M. Wiese*, M. Ragaller

*Westsächsische Hochschule Zwickau (FH) Fachbereich Gesundheits- und Pflegewissenschaften

Fragestellung: Intensivmedizinische Leistungen werden im DRGSystem unzureichend abgebildet und sind einer innerbetrieblichen Leistungsverrechnung bislang nicht zugänglich. Ziel der Untersuchung ist die Ermittlung der tatsächlich entstandenen patientenbezogenen Kosten während der intensivmedizinischen Behandlung zur Vorbereitung einer innerbetrieblichen Leistungsverrechnung. Methodik: Die Untersuchung wurde auf 2 typische Patientengruppen fokussiert: 1 . Gruppe der postoperativen Intensivpatienten mit einer Liegedauer $>24 \mathrm{~h}(\mathrm{n}=50), 2$. Gruppe der Sepsispatienten $(n=25)$. In einer retrospektiven Datenerhebung im 1 . und 2. Quartal 2005 wurden für jeden Patienten der Untersuchungsgruppe alle Kosten erfasst, die während seines Aufenthaltes auf der Intensivstation (ITS) täglich entstanden. In Anlehnung an das Handbuch „Kalkulation von Fallkosten“ des Instituts für Entgeltsystem im Krankenhaus (InEK) erfolgte der Aufbau der Kostenkalkulation nach Kostenartengruppen. Die Personalkosten des Ärztlichen-, Pflege- und Medizinisch-Technischen Dienstes wurden Top-down über den Verteilungsschlüssel „Anzahl Behandlungstage“ $(4,554)$ auf den Patienten pro Tag verteilt. Die täglich verabreichten Arzneimittel und die dazugehörigen Kosten konnten Bottom-up für jeden Patienten durch EDV-Abfragen ermittelt werden. Die Sachkosten des Medizinischen Bedarfs (Verbands-/Heilmittel usw.) wurden Top-down über den Verteilungsschlüssel Behandlungstage auf die Patienten umgelegt. Durch Kooperation mit den Fremdleistung erbringenden Institutionen (Mikrobiologie, $\mathrm{Pa}$ thologie usw.) konnten die Kosten die je Tag und Patient hierfür erbracht wurden eruiert werden. Die Personal- und Sachkosten der medizinischen Infrastruktur wurden über einen Verteilungsschlüssel umgelegt. Zusätzlich wurden pro Patient und Behandlungstag sonstige Verwaltungs- und Personalkosten und ein Basiskostensatz zugeschlagen. Ergebnisse: Im Beobachtungszeitraum werden jedem Patienten pro Behandlungstag $144 €$ für die Personalkosten des ärztlichen Dienstes, 336 für den Pflegedienst und $23 €$ für den Medizinisch-Technischen Dienst zugerechnet. An Sachkosten sind jedem Patienten $64 €$ zuzurechnen. Die Kosten für Arzneimittel und Leistungen von fremden Institutionen variieren täglich, da die tatsächlichen IST-Kosten erfasst wurden. Für die medizinische Infrastruktur, Verwaltungsbedarf und Basiskosten werden insgesamt pro Patient und Behandlungstag $140 € \mathrm{zu}-$ geschlagen. Insgesamt ergeben sich bei den postoperativen Patien- ten Durchschnittskosten von $837 €$ und bei den Sepsispatienten von $1162 €$ pro Behandlungstag. Schlussfolgerung: Die Ergebnisse belegen, dass eine große Spannweite zwischen den Kosten verschiedener Patientengruppen existiert. Für eine innerbetriebliche Leitungsverrechnung ist es daher nicht sinnvoll eine Pauschale anzusetzen, sondern es sollte eine Differenzierung nach verschiedenen Krankheitsbildern erfolgen. Hierfür werden weitere Kostenkalkulationen für verschiedene Patientengruppen erforderlich sein.

Penetration of the English language in science: 127 the case of a German national interdisciplinary critical care conference

K. Rellos, E. Fabritsi, F. Chalvatzoglou, M. Rizos, A. Michalopoulos, M. Falagas

Henry Dunant Hospital, Intensive Care Unit, Athens, Greece

Objektive: The English language has undoubtedly benefited significantly from the new era, in gradually penetrating into other cultures in many fields such as music, arts, education, and more recently science. Methods: We assessed the used of the English language in the published abstracts of a major German national conference, namely the German Interdisciplinary Conference of Intensive Care Medicine and Emergency Medicine (DIVI). The abstract books off all seven DIVI conferences, organized between 1991 and 2004, were reviewed to count the number of abstracts presented at each conference as well as the subsets of abstracts presented in English. All abstracts presented in English were reviewed further to identify the country of origin of the authors. These abstracts were categorized into three groups. The first group included those abstracts presented solely by investigators based in German universities, hospitals or institutions. Abstracts that were the result of collaboration between investigators from Germany ant other countries were included in a second category, and abstracts presented by investigators from countries other than Germany were classified a third group. Results: We show that the total number of abstracts presented in the seven DIVI conferences, as well as the proportion of abstracts presented in English. No abstract was presented in English during the first, second and third DIVI conferences. However, there was a gradual increase in the proportion of abstracts presented in English during the subsequent DIVI conferences (from $1.6 \%$ to $25.1 \%$ ). Of the total of 98 abstracts presented in English during the last four DIVI conferences, 86 were written by solely German investigators, on the basis of the information provided. Three abstracts were the result of collaboration between investigators from Germany and other countries (Austria, Israel and the USA). The remaining nine abstracts were presented by investigators from countries other than Germany (Poland (4), UK (2), Austria (1), Slovakia (1) and the USA (1). Conclusions: We found that about one-quarter of abstracts presented at German medical conference were written in English, indicating a significant penetration of the English language into a German national multidisciplinary conference. Our findings suggest that researchers around the world should engage in an open and unbiased discussion about the future of languages in science. 
Nutzen von Patienten-Daten-Management-Systemen bei der Durchführung von klinischen Studien

K. Weismüller, C. Katzer, M. Meier ${ }^{2}$, R. Röhrig, S. Little, T. Menges, T. Chakraborty ${ }^{1}$, G. Hempelmann

Klinik für Anästhesiologie, Intensivmedizin, Schmerztherapie,

${ }^{1}$ Institut für Mikrobiologie und Virologie,

${ }^{2}$ Abteilung für klinische und administrative Datenverarbeitung Universitätsklinikum Gießen und Marburg, Standort Gießen

Einleitung: Die Durchführung klinischer Studien ist mit einem hohen, meist redundanten Dokumentationsaufwand verbunden. Mit der zunehmenden Verbreitung von Patienten-Daten-Management-Systemen (PDMS) entsteht der Bedarf nach einer Unterstützung klinischer Studien. Das Ziel dieser Arbeit war die Entwicklung und Evaluation studienunterstützender Funktionen mit einem PDMS. Methodik: Basis des Projektes war das PDMS ICUdata (Fa. IMESO $\mathrm{GmbH}$ ), welches auf den Intensivstationen des Universitätsklinkums Gießen etabliert ist $[1,2]$. Die Standard Operating Procedures für den Einschluss klinischer Daten und die Probenentnahmen sind im Thearpie-Assistenten des PDMS integriert und werden über die graphische Benutzeroberfläche (GUI) des PDMS für den Studienarzt sichtbar gemacht. Anhand dieser Informationen werden die Probenentnahmen durchgeführt und die klinischen Daten und Befunde auf ihre Studienrelevanz überprüft. Relevante Daten werden über das GUI durch die Markierung mit Attributen (Flags) validiert und das monitoring dokumentiert. Der Export der so validierten klinischen Daten in die Forschungsdatenbank wurde auf Datenbankebene implementiert. Zusätzlich zu den klinischen Daten werden die für die Qualitätssicherung notwendigen Metadaten erfasst. Use-Case: Das vorgestellte Konzept wurde in den verschiedenen Studienarmen des "Gießen Research Center in Infectious Diseases“ (GRID), welches sich Schwerpunktmäßig mit den molekularbiologischen Grundlagen und der Entwicklung prognostischer Modelle der Sepsis beschäftigt bei guter Anwenderzufriedenheit etabliert. Schlussfolgerung: Die Erfassung von Daten gemäß den Regeln für Good Clinical Practice erfolgt normalerweise manuell in ein papierbasiertes Studienprotokoll. Durch die Einführung von eTrials, bei denen der Studienarzt die studienrelevanten Parameter direkt in der Studiensoftware, z.B. über einer Web-Portal erfasst, konnte ein Arbeitsschritt und damit eine Quelle für Übertragungsfehler verringert werden [3, 4]. PDMS können nach dem eTrial-Modell von Kuchinke et. al [4] für die Bereiche Patienten-Rekrutierung und Data-Capturing eingesetzt werden und damit die Datenerfassung und das Monitoring der Studiendaten weiter vereinfachen da manuelle Übertragungsschritte entfallen. Von einer Verbesserung der Datenqualität ist auszugehen, muss aber noch untersucht werden.

Literatur: [1] Michel A: Design principles of a clinical information system for intensive care units (ICU Data). Stud Health Technol Inform 2000; 77:921-924. [2] Röhrig R et al: Patientenzentrierte Online-Dokumentation. Dtsch Ärztebl 2001; 98:A2240-2244. [3] Kuchinke W et al: Elektronische klinische Studien - „eTrials“ werden zur Routine. Dtsch Ärztebl 2003; 100:A3081-3084. [4] Kuchinke W et al: Einsatz des CDISC-Standards für die vernetzte klinische Forschung in der Telematikplattform für medizinische Forschungsnetze (TMF eV) in Jäckel (Hrsg) Telemedizinführer Deutschland 2006:338-345

\section{Abbildung von LEP Version 3 mit einem Patienten- Daten-Management-System in der Intensivmedizin \\ M. Müller, A. Luh, L. Schleifenbaum, D. Wosilat, R. Mader, P. Eckhart, R. Röhrig \\ Universitätsklinikum Gießen und Marburg, Standort Gießen}

Einleitung: Durch die Einführung der G-DRG und dem damit verbundenenden Kostendruck in der Klinikfinanzierung entsteht ein Bedarf nach Instrumenten zu Personalsteuerung in der Intensivpflege, und damit der korrekten Abbildung des Pflegeaufwandes [1]. Neben der PPR findet neben den klassischen Scoring-Syste- men wie TISS oder NEMS zunehmend die kommerzielle Methode LEP (LEP AG, Schweiz) Verbreitung [2]. In LEP Version 2 werden Pflegeleistungen in definierten Variablen zusammengefasst und mit Pflegeminuten hinterlegt. In der Version 3 werden die Pflegeleistungen in einer zusätzlichen Ebene durch Interventionen differenziert. Ziel der Arbeit war die Untersuchung, wie die Methode LEP V3 in einer Beta-Version auf einer bestehenden Intensivdokumentation mit einem Patienten-Daten-Management-System (PDMS) abgebildet werden kann. Methodik: Die Relevanz und Vollständigkeit des Kataloges wurde von 5 Pflegedienstleistungen anhand der Pflegeleistungen auf Ihren Intensivstationen, die Abbildbarkeit von den Systembetreuern gegen die Parametrierung der Pflegedokumentation des PDMS (ICUdata, Firma IMESO $\mathrm{GmbH})$ geprüft. Ergebnisse: Zwischen 500 (71\%) und 537 (76\%) der 708 Interventionen stellen Pflegeleistungen auf den verschiedenen Stationen dar. 70 Pflegeleistungen waren nicht oder nicht ausreichend differenziert berücksichtigt. $36(5,1 \%)$ Interventionen können aus der Medikamentendokumentation, 33 (4,7\%) aus der ärztlichen und $25(3,5 \%)$ aus der automatischen Datenübernahme generiert werden. Die 614 Interventionen die über die pflegerische Verlaufsdokumentation abzubilden sind, können 328 (46\%) eindeutig (1:1) abgebildet werden und 168 (24\%) haben eine höhere Differentierung im PDMS (1:n) und können damit aus der Routinedokumentation abgeleitet werden. Bei 118 (17\%) der Interventionen ist eine eindeutige Ableitung aus den Items des PDMS ohne weitere Differentierung nicht möglich ( $\mathrm{n}: 1$, unscharfe Abbildung). Ursache ist meist, dass eine Pflegeleistung in dem Katalog mehrfach mit verschiedenen Motivationen aufgeführt ist. Schlussfolgerungen: Die Pflegeleistungen in der Intensivmedizin werden durch die Methode LEP V3 gut abgebildet. Bei den Interventionen mit einer unscharfen Abbildung sollten Leistung und Motivation getrennt werden. Wie gut die Pflegeleistung gemessen wird, ist ein einer weiteren Studie zu untersuchen.

Literatur: [1] Vermeiden von Fehlentwicklungen durch korrekte Abbildung des Pflegeaufwands im G-DRG-System;

http:// www.deutscher-pflegerat.de/pdfs/

DRG-Weiterentwicklung_060320.pdf.

[2] Brügger U: 2002; www.LEP.AG

Unterschiede zwischen Männern und Frauen

in der Internistischen Intensivmedizin

M. W. Prull, B. Henze, C. Perings, H.J. Trappe

Medizinische Klinik II, Marienhospital Herne, Ruhr-Universität Bochum, Herne, Deutschland

Einleitung: Frauen haben bei kardiovaskulären Erkrankungen und nach kardiochirurgischen Eingriffen eine schlechtere Prognose als Männer. Insbesondere scheinen Beatmungsdauer und invasive Diagnostik zu Ungunsten von Frauen auszufallen. Wir sind im Folgenden der Frage nachgegangen, ob es geschlechtsspezifische Unterschiede hinsichtlich Alter, Schwere der Erkrankung, Aufenthaltsdauer auf der Intensivstation und im Krankenhaus und hinsichtlich des Ausmaßes therapeutischer Bemühungen auf einer Medizinischen Intensivstation existieren. Methode: Es wurden die Daten der ersten $485 \mathrm{Pt}$ aus 2003 einer Medizinischen 9-BettenIntensivstation einer Universitätsklinik retrospektiv ausgewertet und Alter, Gesamtaufenthalt, Intensivaufenthalt, Schweregrad der Erkrankung (APACHE-II-Score) sowie der therapeutischen Aufwand (TISS-28-Score) am Aufnahme- und Entlasstag erfasst. Als Bestandteil des TISS-28-Scores wurden die Beatmungstage invasiv vs. nicht-invasiv differenziert. Mittelwerte \pm Standardabweichung (SD), Student's t-test, Signifikanzniveau $p<0,05$. Ergebnisse: Von $485 \mathrm{Pt}$ waren $300 \mathrm{M}$ (61,9\%). Signifikanter Unterschied im Alter $(\mathrm{p}<0,001)$. Ansonsten $\mathrm{p}=\mathrm{ns}$ für Alter (M 64,1+14,5 Jahre, F 67,2+14,3 Jahre), KH-Dauer (M 15,5+12,8 Tage, F 18,8+22,7 Tage), ITV-Dauer (M 4,9+8,2 Tage, F $6,4+19$ Tage), APACHE II (M $11,8+9,3$, F $12,7+10,4)$, TISS-28 Aufn. (M 20,4+13,3, F $21+14,8$ ), TISS-28 Entl. (M 14,4+13,3, F 14,4+9,8), inv. Beatmung (M 2,0+8,5 Tage, F 3,3+19,8 Tage), NIV (M 1,7+2,8 Tage, 
F 1,7+2,2 Tage). Zusammenfassung: Es gibt einen signifikanten Altersunterschied zwischen M und F. Ein Grund könnte darin liegen, dass F zum Zeitpunkt der Aufnahme einen (tentendziell) höheren APACHE-II-Score haben als M. F scheinen später als M qualifizierte ärztliche Hilfe in Anspruch zu nehmen. F sind schwerer erkrankt als $\mathrm{M}$. Obwohl F länger invasiv beatmet werden, zeigt der therapeutische Aufwand während des Aufenthaltes auf der Intensivstation hingegen keinen geschlechtsspezifischen Unterschied.

\section{Wissenschaftliches Kongresssekretariat DIVI 2006 -} eine Analyse von Referentendemographie und -interaktion mit dem Sekretariat

K. Schick, K. W. Jauch, J.N. Hoffmann Chirurgische Klinik, Klinikum Großhadern, München

Einleitung: Traditionell wird das wissenschaftliche Sekretariat der DIVI durch Mitarbeiter des jeweiligen Kongresspräsidenten gestellt. Die folgende Analyse beschäftigt sich mit der fachbezogenen Verteilung von Referenten und Vorsitzenden und deren Interaktion mit dem Kongresssekretariat. Methodik: Prospektiv wurde im Rahmen der Kongressvorbereitung erfasst: Medizinische Fachrichtung, akademischer Grad, Geschlecht, Staatsangehörigkeit, PLZ der Arbeitsstätte, Zeitpunkt und Inhalt der Rückmeldung nach Einladung. Ergebnisse: Zur DIVI 2006 wurden insgesamt 597 Referenten und Vorsitzende nach Vorschlag durch das wissenschaftliche Komitee eingeladen. Davon waren 552 Männer $(92,5 \%)$ und $45(7,5 \%)$ Frauen. Die Einladung von $>90 \%$ der Referenten erfolgte von 04.11.2005 bis 10.11.2005 (also über ein Jahr vor Kongressbeginn) per Post mit kompletter Sitzungs- und Terminübersicht bei vorhandener Saalplanung. Insgesamt wurden 335 Professoren (56\%), 103 Privatdozenten (17,2\%), 137 Doktoren $(22,9 \%)$ und 15 Referenten $(2,5 \%)$ ohne akademischen Titel eingeladen, sowie ein Diplom-Chemiker und ein Pastor. Aus den medizinischen Fachbereichen wurden 34\% Anästhesisten, 31\% Internisten, $14 \%$ Neurologen und Neurochirurgen, 11\% Chirurgen, 5\% Pädiater, 3\% Psychiater und 1\% Gynäkologen und Radiologen angefragt. Es gingen insgesamt 43 Absagen (7,2\%) ein, wobei von 3 Referenten das Sekretariat auch nach mehrmaliger Kontaktaufnahme keine Antwort erhielt. Die Absagen waren zwischen Frauen und Männern ähnlich verteilt ebenso wie zwischen den akademischen Graden. Professoren antworteten signifikant schneller als Doktoren $(28 \pm 3$ Tage vs. $50 \pm 7$ Tage; $\mathrm{p}<0,01)$, wohingegen Privatdozenten eine Mittelstellung einnahmen ( $41 \pm 6$ Tage). 44 Referenten (7\%) wurden aus dem Ausland eingeladen (Absagequote $15 \%)$. Aus den neuen Bundesländern wurden $16 \%$ der Referenten und Vorsitzenden rekrutiert. Schlussfolgerungen: Auch in diesem Jahr war es möglich, eine hohe Zahl an Referenten für die DIVI $2006 \mathrm{zu}$ berücksichtigen, wobei weniger als 10\% Frauen waren. Mehr als zwei Drittel der Referenten waren zum Zeitpunkt der Einladung habilitiert. Trotz eingeschränkter Möglichkeiten der Kostenerstattung für Referenten und Vorsitzende war eine extrem niedrige Absagequote $(<10 \%) \mathrm{zu}$ verzeichnen. Die Referenten zeichneten sich insgesamt durch eine zügige Interaktion mit dem Kongresssekretariat aus, wobei Professoren signifikant schneller reagierten als Doktoren. Eventuell könnte in der Zukunft der Frauenanteil, der Anteil an ausländischen Referenten und der Anteil von Referenten aus den neuen Bundesländern noch gesteigert werden.

\section{Intensivstationsmanagement}

Validierung von neuen Alarmalgorithmen in der Intensivmedizin

S. Siebig ${ }^{1}$, U. Gather ${ }^{2}$, M. Imhoff ${ }^{2,3}$, S. Kuhls ${ }^{2}$, J. Langgartner ${ }^{1}$, C.E. Wrede

${ }^{1}$ Klinik und Poliklinik für Innere Medizin I, Universität Regensburg, Deutschland, ${ }^{2}$ FB 475 des Fachbereichs Statistik, Universität Dortmund, Deutschland, ${ }^{3}$ Abteilung für Medizinische Informatik, Biometrie und Epidemiologie, Ruhr-Universität Bochum, Deutschland

Hintergrund: Die Mehrzahl aller Alarme, die von derzeit auf dem Markt befindlichen Monitoringsystemen generiert werden, weisen bei hoher Sensitivität auch eine hohe Inzidenz falsch positiver Alarme auf. In Studien zeigte sich, dass bei einer Fehlalarmrate von $90 \%$ nur auf $10 \%$ aller Alarme reagiert wurde, die aber nicht notwendigerweise mit den $10 \%$ der zu Recht ausgelösten Alarmen korrespondierten. Dies schränkte die klinische Wertigkeit einer hohen Sensitivität des Alarmsystems stark ein. Im Rahmen dieser Studie wird untersucht, ob neu entwickelte Alarmalgorithmen die häufig auftretenden falsch positiven Alarme im intensivmedizinischen Monitoring reduzieren können, ohne dabei die Sensitivität der bislang verwendeten Schwellenwert-Alarme zu unterbieten. Methoden: Klinisch annotierte Datensätze werden mittels Videoaufzeichnungen von Intensivpatienten erstellt, welche zum einen hinsichtlich ihrer Alarmhäufigkeit sowie der klinischen Alarmrelevanz im bisherigen Monitoringsystem ausgewertet werden. Anhand von diesem Datenmaterial findet anschließend eine Simulation von neuen Alarmalgorithmen statt, denen statistische Zeitreihenanalysen mit Online-Signalextraktion und Erkennungsverfahren für Ausreißer, Trends und Niveauänderungen zugrunde liegen. Ergebnisse: In den bislang durchgeführten Arbeiten wurde zunächst die Extraktion von Monitor-Messwerten im 1-SekundenTakt und von Alarmen sowie Alarmgrenzen der Bettplatz-Monitore etabliert. Weiterhin wurde eine kontinuierliche Videoüberwachung und -Aufzeichnung ausgewählter Bettplätze der Intensivstation eingerichtet. Erste klinische Annotationen zeigten eine hohe Rate falsch positiver Alarme, welche durch die eingesetzten Alarmalgorithmen reduziert werden konnten. Diskussion: Im Gegensatz $\mathrm{zu}$ vorangegangenen Studien anderer Arbeitsgruppen führt die klinische Annotation der Alarme anhand von Videoaufzeichnungen $\mathrm{zu}$ einer Reduktion eines Beobachtungs-Bias und ermöglicht erstmals eine kontinuierliche 24-stündige Auswertung. Die so erhobenen Datensätze sollen zur weiteren Verbesserung der Alarmalgorithmen führen. Mittelfristig ist im Rahmen eines Transferprojektes der DFG in Kooperation mit der Firma Draeger Monitoringsysteme eine Implementation dieser Algorithmen in ein ,intelligentes“ Monitorsystem geplant, welches über eine Reduktion der Alarmhäufigkeit bei erhaltener hoher Sensitivität zu einer Verbesserung der Patientensicherheit führen soll.

Liegedauer, Pflegeaufwand und Fallkosten nach Prozessoptimierung (P0) auf einer Intensivstation

K. Müller ${ }^{1}$, P. K. Schädlich ${ }^{2}$ J. G. Brecht ${ }^{2}$

${ }^{1}$ Intensivstation, Sana-Krankenhaus Rügen GmbH, Bergen;

${ }^{2}$ InForMed GmbH - Outcomes Research and Health Economics

Hintergrund: Auf der 10-Betten-Intensivstation (ITS) des SanaKrankenhauses Rügen haben im Jahr 2002 folgende PO-Maßnahmen stattgefunden: Optimierung diagnostischer und therapeutischer Verfahren, Vereinheitlichung von Medizinprodukten, Ausweitung der Nierenersatzverfahren und Anwendung eines einheitlichen Beatmungs- und Analgosedierungskonzeptes mit ausschließlichem Einsatz von Remifentanil/Propofol (RP) anstelle von Fentanyl/Midazolam (FM). Ziel der Studie war die Untersuchung vom Einfluss dieser Maßnahmen auf Liegedauer, Pflegeaufwand und Fallkosten auf der ITS. Methoden: Im Rahmen einer retro- 
spektiven Analyse von Daten zu Falleigenschaften bei Aufnahme, Liegetagen und Behandlungskosten aus der Routinedokumentation der ITS wurden zwei Zeiträume verglichen: Jahr 2000 und 2001 zusammen vor PO und Jahre 2003 und 2004 zusammen nach PO. Fallbezogene Behandlungskosten setzten sich aus Personalkosten, Laborkosten und Kosten des medizinischen Bedarfs jeweils in Preisen von 2004 zusammen. Als Signifikanzniveau für Unterschiede zwischen den Zeiträumen wurde ein p-Wert von kleiner 0,05 verwendet. Ergebnisse: Vor PO wurden insgesamt 1704 Fälle behandelt, nach PO waren es 2044 Fälle. Es gab keine signifikanten Unterschiede bei Alter, Geschlecht und Krankheitsschwere der Fälle, beim Verhältnis von Intensivbehandlung $\mathrm{zu}$ Überwachung, bei Beatmungstagen sowie bei fallbezogener Mortalität. Hingegen sank die durchschnittliche Liegedauer von 4,5 Tagen vor PO signifikant auf 3,8 Tage nach PO. Der Pflegeaufwand (simplified Therapeutic Intervention Scoring System, TISS-28) sank ebenfalls signifikant. Die mediane Summe der TISS28 -Punkte pro Fall betrug 49 vor und 40 nach PO. Die durchschnittlichen Fallkosten sanken signifikant von 2435 Euro vor auf 1815 Euro nach PO, obwohl die Kosten des Dialysebedarfs durch Ausweitung der Nierenersatzverfahren nach PO 2,9-mal so hoch und die Kosten des Narkose- und sonstigen OP-Bedarfs durch ausschließlichen Einsatz von RP nach PO 2,1-mal so hoch waren. In der Subgruppe der Fälle mit Langzeitbeatmung (vier Tage oder länger) gab es signifikante Unterschiede bei den Falleigenschaften. So waren die 135 Fälle nach PO signifikant älter (Median 67 vs. 63 Jahre) und schwerer krank (medianer Acute Physiology and Chronic Health Evaluation II Score 21 vs. 18) als die 129 Fälle vor PO. Jedoch gab es keine signifikanten Unterschiede bei Pflegeaufwand und Liegedauer nach vs. vor PO: Die mediane Summe der TISS-28-Punkte betrug 498 vs. 495, und die mediane Liegedauer betrug jeweils 14 Tage. Schlussfolgerung: Die PO-Maßnahmen haben insgesamt zu einer signifikanten Reduktion der Liegedauer um 16\%, des Pflegeaufwands um 18\% und der Fallkosten um 25\% geführt. Bei Einbettung in eine Gesamtstrategie zur Prozessoptimierung kann der ausschließliche Einsatz von RP zur Analgosedierung mechanisch beatmeter Patienten zu Einsparungen auf der ITS insgesamt führen.

\section{Management von Motilitätsstörungen}

und enteraler Ernährung bei kritisch kranken Patienten

- Eine Umfrage an deutschen Intensivstationen

\section{K.D. Röhm, T. Schöllhorn, J. Boldt, M. Wolf, M. Papsdorf,}

S. N. Piper

Klinikum Ludwigshafen, Klinik für Anästhesiologie und Operative Intensivmedizin, Ludwigshafen

Einleitung: Gastrointestinale Motilitätstörungen treten aufgrund von Zirkulationsstörungen im Splanchnikusgebiet bei kritisch kranken Patienten gehäuft auf. Ursachen sind Volumenverschiebungen, der Einsatz vasoaktiver Substanzen und Opiate sowie chirurgische Manipulationen im Abdomen. Klinisch imponieren Bilder von Erbrechen und paralytischem Ileus, daneben können Komplikationen wie Mikroaspirationen und Pneumonien bis zur Sepsis und Multiorganversagen durch bakteriellen Translokation auftreten. Die Durchführung einer frühen enteralen Ernährung (EN) zur Erhaltung der gastrointestinalen Integrität stellt ein primäres Therapiekonzept bei diesen Patienten dar. Ziel dieser Umfrage sollte die Erfassung des aktuellen Standes zu Management von Motilitätsstörungen und praktischer Durchführung der EN in Deutschland sein. Methodik: 1493 Fragebögen wurden an deutsche Intensivstationen im September 2005 verschickt. Die Antworten wurden über 2 Monaten gesammelt, die Datenanalyse erfolgte mittels eines Datenbankprogramms (Access 2003). Ergebnisse: Insgesamt wurden 593 Fragebögen (Rücklaufquote 41\%) beantwortet, davon $63 \%$ von Anästhesisten und $17 \%$ von Internisten. Die Ernährung des kritisch kranken Patienten wurde in $70 \%$ als kombiniertes enteral- parenterales Regime begonnen. Ernährungsprotokolle wurden in $44 \%$ benutzt. Der Einsatz der frühen EN wurde von 58\% durchgeführt, wobei initial mit Ernährungsvolumina von $250-500 \mathrm{ml}$ pro Tag (66\%) begonnen und um $200-400 \mathrm{ml}$ pro Tag gesteigert wurde (55\%). EN wurde meist über Magensonden (76\%) via kontinuierlichem Pumpensystem (72\%) mit kurzen Unterbrechungen von $<4 \mathrm{~h}(86 \%)$ appliziert. Abbruchkriterien der EN waren Erbrechen (82\%), Reflux von 500-1000ml (44\%) und Diarrhö (30\%). Standardmäßig wird hochmolekulare, nährstoffdefinierte Sondenkost $(86 \%)$ verwendet, Speziallösungen für Diabetes, Nieren- oder Leberinsuffizienz werden sehr selten eingesetzt, Immunonutrition hat keinen Stellenwert im Bereich der EN von kritisch Kranken. Routinemäßig setzen $79 \%$ eine Ulkusprophylaxe bei kritisch Kranken ein. Prokinetische Medikamente werden standardmäßig in $39 \%$ verabreicht, darunter insbesondere Metoclopramid, Laxantien und Neostigmin. Weitere Therapieoptionen stellen darüber hinaus die Verabreichung von Klistieren (96\%), Gastrografin (72\%), die Kolonmassage (39\%) und die Epiduralanästhesie (24\%) dar. Zusammenfassung: Diese Umfrage kann aufgrund der hohen Rücklaufquote als repräsentativ für die praktische Durchführung der EN und Probleme der Darmmotilität an deutschen Intensivstationen angesehen werden. Das Konzept der frühen EN stellt einen festen Bestandteil der Intensivtherapie dar. Die EN erfolgt bereits an vielen Häusern protokollgestützt, zumeist mit nährstoffdefinierten Lösungen über Magensonde in einer Dosierung von $250-500 \mathrm{ml}$ pro Tag. Der Einsatz einer Ulkusprophylaxe ist weit verbreitet, prokinetische Medikamente werden neben weiteren Therapieoptionen regelhaft eingesetzt.

Ärztliche Personalbedarfsermittlung in der Intensivmedizin

D. A. Vagts

Universität Rostock, Klinik und Poliklinik für Anästhesiologie und Intensivtherapie

Die Intensivmedizin ist einer der kostenintensivsten aber auch kostensensibelsten Bereiche eines Krankenhauses: 3-5\% aller stationären Patienten werden intensivmedizinisch behandelt, und die hierfür benötigten finanziellen Ressourcen betragen 13-15\% des Krankenhausbudgets. Davon sind dann nochmals ca. 20\% für das ärztliche Personalbudget anzusetzen. Allerdings ist die Intensivmedizin mit ihrem finanziellen Bedarf für Personal- und Verbrauchsmittelkosten mit Einführung der Abrechnung nach German Diagnosis Related Groups (G-DRG) bisher nur unzureichend abgebildet. Insbesondere für die ärztliche Personalbedarfsberechnung bestehen bisher keine befriedigenden Konzepte, die sowohl einer ökonomischen als auch einer medizinisch qualitativen $\mathrm{Be}$ trachtung gerecht werden. Unter den neuen G-DRG-Bedingungen zielen auf der einen Seite die Überlegungen der Personalbedarfsberechnung von Seiten der Krankenhausträger nun auf den Kostenaufwand im Personalbereich, um den Erlösen realistische Kostengrößen gegenüberstellen zu können. Auf der anderen Seite stehen einer vom Kostenträger anvisierten Reduktion der Personalkosten und damit einer Reduktion von Personalzahlen bzw. von qualifiziertem („teurem“) Personal die Qualitätssicherung und das Qualitätsmanagement (strukturierter Qualitätsbericht) gegenüber. Damit bleibt die Personalbedarfsermittlung nicht nur ein Instrument der klassischen Personalverwaltung, sondern sie wird zu einem Instrument des Controlling und der Qualitätssicherung. Betrachtet man die Personalbedarfsermittlung vor dem Hintergrund sozial- und arbeitsrechtlicher Rahmenbedingungen, medizinisch qualitativer Notwendigkeiten, sowie räumlich-logistischer und organisatorischer Bedingungen sowie der Zusammensetzung des Patientengutes, so zeigt sich, dass die herkömmlichen Instrumente der Personalbedarfsermittlung wie Arbeitsplatzmethode, Anhaltsund Kennzahlen (Jahresbesetzung: 1 Arzt pro 2 ITS Betten) oder Minutenwerte den heutigen Bedürfnissen nicht mehr gerecht werden. Berechnungen für den Personalbedarf auf dem Boden der g-DRG-Personalanteile, des Basisfallwertes und der Relativgewichte sind für jede Intensivstation individuell möglich, haben aber 
den Nachteil, dass sie erst retrospektiv das Personalbudget exakt festlegen können. Bei annährend konstantem Patientenzufluss ist diese Methode aber ein hilfreiches Instrument zur Steuerung. Von den derzeit publizierten Instrumenten zur Personalbedarfsermittlung bilden die Anhaltszahlen des DKI (Plücker 2004), nach denen ein Arzt auf der Intensivstation 100-125 Fälle pro Jahr behandeln muss, den Arbeitsaufwand, der sich durch zunehmendes Alter und Krankheitsschwere der Patienten und Dokumentationsbedarf, erweiterte diagnostische Methoden, sowie Ausbildung etc. ergibt, am besten ab.

\section{Aufgabenstruktur von Oberärzten}

in der Intensivmedizin - Ärzte oder Manager?

\section{B. C. Kother, D. A. Vagts}

Universität Rostock, Klinik und Poliklinik für Anästhesiologie und Intensivtherapie

Oberärzte in der Intensivmedizin versorgen heute nicht mehr nur Patienten medizinisch, sondern durch Aufgabenverschiebungen, -verdichtungen und -zuwächse, sowie vermehrte Kommunikation hat sich ihr Aufgabenspektrum stark verändert. Zunehmend müssen sie Management- und immer weniger ärztliche Aufgaben wahrnehmen. Diese Studie untersuchte die Struktur der täglichen Arbeitsbelastung von intensivmedizinisch tätigen Oberärzten an einem Universitätsklinikum. Die Daten wurden im Rahmen einer observativen Studie auf einer universitären interdisziplinären Intensiveinheit erhoben. Der Dienst habende Oberarzt wurde zwei Wochen von 6:00 Uhr bis Dienstende begleitet. Jede Aktion, ihre Dauer, jede Störung wurden aufgezeichnet und der Kategorie „Kommunikation“ oder „Aufgabe“ zugeordnet. Die deskriptive Statistik erfolgte mittels Medianen, Mittelwerten sowie Mini- und Maximalwerten. Es wurden 2 Oberärzte beobachtet. Die tägliche Arbeitszeit betrug netto $10 \mathrm{~h} 18 \mathrm{~min}$ bis $14 \mathrm{~h} 07 \mathrm{~min}$ bei geplanten Arbeitszeiten von $8 \mathrm{~h} 30 \mathrm{~min}$ pro Tag. Davon fielen auf Forschungstätigkeit 1-3 h/d. Während eines Arbeitstages wurden im Mittel 191 Aktionen ausgeführt, die sich auf 47\% Aufgaben und $53 \%$ Kommunikation aufteilten. 21,3 Aufgaben/h dauerten $4 \mathrm{~min} 28 \mathrm{sec}$ pro Aufgabe (Min $7 \mathrm{~s}$, Max $1 \mathrm{~h} 8 \mathrm{~min}$ ). Der Median der Aufgabendauer war $37 \mathrm{~s}$. Ungeplante Unterbrechungen passierten 85/Tag. 1/3 der Arbeitszeit war durch direkten Patientenkontakt bestimmt, 40\% waren notwendig für Dokumentation, DRGs etc. 1-2 $\mathrm{h}$ wurde durch Kommunikation im eigenen Ärzteteam, $1 \mathrm{~h}$ für Visiten mit mitbehandelnden Ärzten anderer Disziplinen verbraucht. Weniger als $10 \%$ der Arbeitszeit verfiel auf private Kommunikation, Kaffee- und Mittagspause, Toilettengänge etc. Die Aufgaben als Oberarzt einer universitären Intensivstation implizieren verschiedene Rollen, die durch die konzentrierte und verdichtete Arbeitsbelastung widergespiegelt werden. Dies zeigt sich insbesondere an der Häufigkeit sehr kurzer und ungeplanter Tätigkeiten mit der Notwendigkeit kurzfristiger Entscheidungen. Unsere Ergebnisse zeigen, dass die oberärztliche Arbeitsstruktur auf der untersuchten Intensivtherapiestation der Arbeitsstruktur eines Managers sehr ähnelt. Der Oberarzt in der Intensivmedizin hat verschiedene Rollen: Er repräsentiert seine Station und die Abteilung/Klinik nach außen, er ist verantwortlich für die Arbeitsleistung seiner Mitarbeiter und er ist die Verbindungsperson $\mathrm{zu}$ mitbehandelnden Kollegen. Informell muss er zudem beobachten, informieren, entscheiden, personelle und materielle Ressourcen verteilen und seine Mitarbeiter unterstützen. Die Verantwortlichkeit und die Arbeitsbelastung und -struktur entspricht damit voll und ganz denen eines Managers in der freien Wirtschaft - mit Ausnahme des Gehalts. Diese Arbeitsstruktur muss deshalb u. a. bei der Planung von Personal für eine Intensivtherapiestation mit einer zusätzlichen Stelle berücksichtigt werden, spielt aber leider bisher aus „Kostengründen“ nur eine untergeordnete Rolle.
Personalstruktur und Arbeitsbelastung auf deutschen Intensivstationen vorläufige Ergebnisse einer Umfrage

D. A. Vagts, E. Uhlig

Universität Rostock, Klinik und Poliklinik für Anästhesiologie und Intensivtherapie

Der ökonomische Druck, der durch die Einführung der German Diagnosis Related Groups und unter den Kostensteigerungen im Gesundheitssystem auf die Krankenhäuser ausgeübt wird, führt zu einer primären Reduktion von Personal. Gerade für die Intensivmedizin konnte aber in zahlreichen Studien der letzten Jahre gezeigt werden, dass eine ungenügende ärztliche Personalausstattung $\mathrm{zu}$ einer Reduktion der Behandlungsqualität und $\mathrm{zu}$ einer Erhöhung der Gesamtkosten führt. Unsere Umfrage (anonymer Fragebogen) sollte den Ist-Stand der ärztlichen Personalausstattung und der Arbeitsbelastung auf deutschen Intensivtherapiestationen ermitteln und zu Bettenzahlen, behandelten Patienten, strukturellem Arbeitsaufwand (Transporte, Dokumentation, etc.) und, soweit möglich zur Krankheitsschwere der behandelten Patienten korrelieren. Alle angegebenen Werte sind Mittelwerte. Bisher nahmen 200 Intensivtherapiestationen an der Umfrage teil (15\% Universität, 15\% Maximal-, 25\% Schwerpunkt-, 45\% Regelversorgung), die Träger waren zu 30\% kirchlich, 55\% öffentlich rechtlich und $15 \%$ privat. $60 \%$ der Krankenhäuser besitzen nur eine Intensivstation, 20\% 2 oder 3 Stationen, 20\% mehr als 3 Stationen. $66 \%$ der Stationen haben 8-14 Betten, die sich zu $80 \%$ auf 1- und 2-Bett-Zimmer verteilen. Die Arbeitsleistung beträgt 105 Patienten pro Bett pro Jahr oder 244 Patienten pro Arzt pro Jahr, wobei $50 \%$ der Stationen bis zu 50\% Intermediate Care Patienten mitversorgen. Die Verweildauer aller Stationen beträgt 3,3 Tage, die der Universitätsklinika 3,8 Tage. Außerhalb der Station finden 800 Prozeduren (CT, MRT, Röntgen, etc.) statt, für die eine reine Transportzeit von 20 min benötigt wird. Pro Monat ein Student auf jeder Station ausgebildet, in den Universitätskliniken sogar bis $\mathrm{zu}$ 4. Die ärztliche Besetzung setzt sich aus 1,5 Oberärzten, 1,7 spezialisierte Fachärzten, 2,6 Fachärzten, 1,8 Jungassistenten und 2,9 Altassistenten zusammen. Im Frühdienst sind 2,4, im Spätdienst 1,5, im Nachtdienst 1,1 Ärzte im Schichtsystem anwesend. Der Zeitaufwand für die Visiten beträgt früh $48 \mathrm{~min}$, spät 35 min und nachts $15 \mathrm{~min}$. Die Aufwendung für die körperliche Untersuchung bei Aufnahme beträgt $19 \mathrm{~min}$, in der Routine $11 \mathrm{~min}$, für Kurvendokumentation bei Aufnahme 21 in der Routine 18 min, für DRG-Dokumentation 25 min pro Patient und Tag. Abschlussdokumentation und Arztbriefe kosten 38 min. Ein PDMSSystem ist in 33\%, eine Dokumentationsassistenz in $16 \%$ und eine Sekretärin in $33 \%$ vorhanden. Diese Daten belegen, dass die herkömmlichen Anhaltszahlen für die ärztliche Personalbedarfsermittlung mit 1 Arzt auf 2 ITS-Betten nicht mehr adäquat den Personalbedarf auf deutschen Intensivtherapiestationen widerspiegeln. Hier sind insbesondere die Stationen mit sehr hohem Intensivpatientenanteil nachteilig betroffen, also vorwiegend universitäre Stationen. Die vorhandenen Daten werden noch auf regionale Unterschiede, Unterschiede zwischen den Krankenhausträgern und dezidierter auf Unterschiede zwischen den Versorgungsstufen an.

Einführung von Patienten-Daten-Managment-Systemen erhöht die Zufriedenheit mit der Intensivdokumentation

M. Euler, L. Quinzio, B. Quinzio, R. Röhrig, D. Brammen, M. Specht, H. Burchardi, A. Junger Marienhospital Brühl

Einleitung: Mit der Einführung der DRGs, Verpflichtung zur Qualitätssicherung nach SGB V und der Einführung des Kerndatensatz Intensivmedizin sind die Ansprüche an die Dokumentation in der Intensivmedizin gestiegen. Obwohl es bereits seit den 70er Jahren EDV-Lösungen zur Intensivdokumentation mit PatientenDaten-Management-Systemen (PDMS) gibt [1], haben die Systeme 
noch nicht die erwartete Verbreitung gefunden [2, 3]. Ziel dieser Untersuchung war eine IST-Analyse zur Verbreitung und Akzep$\tan z$ von PDMS in der deutschen Intensivmedizin. Methodik: Der Erhebungsbogen wurde von einem Expertenteam entworfen und durch einen Pre-Test in Zusammenarbeit mit der IAG PDMS der DIVI überprüft. Auf der Jahrestagung der DIVI 2004 wurden insgesamt 3400 Erhebungsbögen an Teilnehmer ausgeteilt. Ergebnisse: Der Rücklauf betrug 247 (7,3\%) Bögen. $158(64,0 \%)$ gaben an eine rein manuelle Dokumentation (MD) durchzuführen. 27 $(10,9 \%)$ setzten eine computerassistierte (CAD) Dokumentation ein und $51(20,7 \%)$ gaben an ein PDMS zu verwenden. Bei der MD-Gruppe äußerten 42 (26,6\%), bei der CAD-Gruppe 8 (29,6\%) und bei der PDMS- Gruppe $42(82,4 \%)$ zufrieden mit ihrer Intensivdokumentation zu sein. Die Gruppe mit PDMS unterschied sich signifikant $(\mathrm{p}<0,01)$ von den beiden anderen Gruppen. Gründe für die Unzufriedenheit mit der manuellen oder computerassistierten Dokumentation waren der zu hohe Zeitaufwand, die Mehrfachdokumentation sowie die mangelnde Auswertbarkeit. 90,8\% der Ärzte und $90,6 \%$ des Pflegepersonals halten die Einführung eines PDMS für sinnvoll, von Ärzten und Pflegern in leitender Position waren es $94,3 \%$, bei DRG-Beauftragten $95,0 \%$. Die drei am häufigsten genannten Argumente für die Einführung eines PDMS waren die Verbesserung der Auswertungsmöglichkeiten, der Dokumentationsqualität und der geringere Dokumentationsaufwand. Als Hauptgründe gegen ein PDMS wurden die hohen Investitionskosten, mangelnde Ressourcen in der Abteilung und ein unübersichtliches Marktangebot angegeben. Schlussfolgerungen: Da PDM-Systeme die Zufriedenheit mit der Dokumentation in der Intensivmedizin erhöhen, scheint die Leistungsfähigkeit der Systeme nicht der Grund für die geringe Verbreitung zu sein. Bedingt durch die Höhe der Investitionskosten ist die Verbreitung von Informationstechnologien häufig an den Druck gesetzlicher Veränderungen gebunden [4]. Damit könnte die Einführung der Komplexbehandlung Intensivmedizin sowie der erhöhte Aufwand für MDK-Anfragen [5] die Nachfrage erhöhen. Für die Industrie und die Fachgesellschaften gilt es durch Standards und Kompetenzvermittlung die Investitionskosten und -risiken zu senken.

Literatur: [1] Schillings H: Methods Inf Med. 1978; 173-176. [2] Seiver A: Crit Care Clin 2000; 601-621. [3] Martich GD: J Intensive Care Med 154-163. [4] Ammenwerth E: IT-Projektmanagement 2005. [5] Brand J: Deutsches Ärzteblatt 2004 A-1241/B-1023/ C-991

Vergleichende prospektive randomisierte Studie zum Verordnungsverhalten einer kalkulierten Antibiotikatherapie - herkömmlich vs. computergestützt H. Beuteführ, B. Hartmann, R. Röhrig, E. Niczko, R. Füssle ${ }^{1}$, A. Junger

Klinik für Anästhesiologie, Intensivmedizin, Schmerztherapie und ${ }^{1}$ Institut für Medizinische Mikrobiologie; Universitätsklinikum Gießen und Marburg, Standort Gießennische Mikrobiologie

Hintergrund: Eine initial inadäquate empirische Antibiotikatherapie bei Intensivpatienten wird mit verantwortlich gemacht für eine erhöhte Letalität [1-3]. Durch den Einsatz von entscheidungsunterstützenden Computersystemen könnte die adäquate Verordnung einer Antibiotikatherapie verbessert werden [4,5]. Ziel dieser vergleichenden prospektiv randomisierten Studie war es, den Einfluss eines computergestützten regelbasierten Therapieassistent für die kalkulierte Antibiotikatherapie („Antibiotika-Wizard“) auf das Verordnungsverhalten von intensivmedizinisch tätigen Ärzten $\mathrm{zu}$ überprüfen. $\mathrm{Zu}$ diesem Zweck sollten die bisher praktizierte Entscheidungsfindung und die Therapieverordnung mit Hilfe der im klinischen Alltag zur Verfügung stehenden Informationsmöglichkeiten mit dem über den „Antibiotika-Wizard“ verglichen werden. Methodik: Insgesamt 40 intensivmedizinisch tätige Ärzte sollten nach einer randomisierten Zuteilung der Verordnungsmethode („konventionell“: unter Zuhilfenahme der herkömmlichen Hilfsmittel, wie beispielsweise Lehrbuch, Rote Liste ${ }^{\circledR}$, Inter- net vs. „Antibiotika-Wizard“) eine kalkulierte Antibiotikatherapie für zwei exemplarische Krankheitsfälle verordnen. Die Studie wurde mit jedem Arzt einzeln im Arztzimmer des jeweiligen Arbeitsplatzes durchgeführt, wo sämtliche Hilfsmittel der klinischen Routine zur Verfügung standen. Zum Zeitpunkt der Untersuchung war der Therapieassistent mindestens sechs Monate im Betrieb. Für die Bewertung einer adäquaten Antibiotikatherapie wurde der Wirkstoff bzw. die Wirkstoffkombination, die Dosierung, das Dosierungsintervall und die Berücksichtigung von Begleiterkrankungen beurteilt. Die erhobenen Daten wurden mit dem Chi-QuadratTest auf einen signifikanten Unterschied $(\mathrm{p}<0,05)$ zwischen den beiden Verordnungsverfahren geprüft. Ergebnisse: Nach der Randomisierung wurde zu jedem Studienpatient jeweils 20-mal eine kalkulierte Antibiotikatherapie „konventionell“ bzw. mit dem „Antibiotika-Wizard“ verordnet. Bei der konventionellen Verordnungsmethode zogen 16 Ärzte ein Lehrbuch und 14 die Rote Liste ${ }^{\circledR}$ zu Rate (vier verwendeten beides), 14 Ärzte benutzten keines der beiden Hilfsmittel. Das Internet, schriftlich fixierte Richtlinien oder Arzneimittelblätter wurden nicht verwendet. Über den Therapieassistenten wurde signifikant häufiger eine richtige kalkulierte Antibiotikatherapie verordnet. Die Begleiterkrankungen wurden signifikant häufiger berücksichtigt und die ausgewählten Therapeutika richtig dosiert. Schlussfolgerung: Mit der Entwicklung des Therapieassistenten wurde ein Werkzeug geschaffen, das zu einer Verbesserung des Verordnungsverhaltens beitragen kann. Es bedarf jedoch noch weiterer Verbesserungen sowie eine konstante Aktualisierung und Weiterentwicklung ist notwendig.

Literatur: [1] Kollef MH: Am J Med 2003; 115:582-584. [2] Kollef MH: Clin Infect Dis 31, 2000; Suppl4:S131-S138. [3] Harbarth S: Am J Med 2003; 115:529-535. [4] Kawamoto K: BMJ 2005; 330:765-772. [5] Garg AX: JAMA 2005; 293:1223-1238

Prospektive klinische Anwendungsuntersuchung zur computergestützten Verordnung einer kalkulierten Antibiotikatherapie bei Intensivpatienten

E. Niczko, B. Hartmann, R. Röhrig, S. Böttger, J. Klasen, R. Füssle*, A. Junger

Klinik für Anästhesiologie, Intensivmedizin, Schmerztherapie und * Institut für Medizinische Mikrobiologie; Universitätsklinikum Gießen und Marburg, Standort Gießennische Mikrobiologie Universität Hintergrund: Bei Intensivpatienten ist das Auftreten von Infektionen und deren rechtzeitige und adäquate Behandlung u.a. durch eine initiale kalkulierte Antibiotikatherapie maßgeblich für das weitere Outcome [1-3]. Durch den Einsatz von entscheidungsunterstützenden Computersystemen könnte die initiale kalkulierte Antibiotikatherapie verbessert werden [4]. Bei der Entwicklung solcher Systeme wurde jedoch festgestellt, dass sie unter Laborbedingungen relativ gut ihren Anforderungen gerecht werden, jedoch in der geplanten Anwendung scheitern. Ziel dieser prospektiven klinischen Anwendungsstudie war es zu überprüfen, ob der in ein bestehendes PDMS integrierte regelbasierte Therapieassistent zur Unterstützung bei der Verordnung einer kalkulierten Antibiotikatherapie den Anforderungen in der klinischen Routine gerecht wird. Methodik: Die Erhebung erfolgte auf einer chirurgischen Intensivstation (14 Beatmungsplätzen, jährlich etwa 1500 Patienten, darunter ca. 1/3 länger als 3 Tage). Zweimal wöchentlich findet eine mikrobiologische Visite statt. Für diese Studie wurde diese Visite ein halbes Jahr vor der Einführung des Therapieassistenten (P I) und nachher (P II) regelmäßig begleitet und standardisiert dokumentiert. Für die vergleichenden Untersuchungen wurden nur die Patienten eingeschlossen, bei denen mindestens eine Visite dokumentiert wurde. Die Antibiotikatherapie wurde detailliert erfasst und in Hauptzielgröße "adäquat“ oder „,inadäquat" beurteilt. Sämtliche weitere Daten (z.B. Alter, Geschlecht, Liegedauer, Beatmungsdauer, Intensivsterblichkeit, SAPS II und SOFA) wurden aus den Datenbanken des PDMS extrahiert. Mit dem Mann-Whitney-U- bzw. dem Chi-Quadrat-Test wurde auf signifikante Unterschiede $(\mathrm{p}<0,05)$ zwischen den beiden Perioden 
geprüft. Ergebnisse: Die Untersuchung umfasste 142 (P I) bzw. 272 (P II) Behandlungstage mit insgesamt 43 (P I) bzw. 113 (P II) dokumentierten Visiten; darunter 35 in P II, bei denen über den "Antibiotika-Wizard“ mindestens einmal eine Antibiotikatherapie angeordnet wurde. Alter, Geschlecht, SAPS-II- und SOFA-Score bei Aufnahme waren nicht unterschiedlich; die Intensivsterblichkeit war in P I höher gegenüber den Patienten, die mit dem Therapieassistenten eine Antibiotikatherapie verordnet bekamen. Die kalkulierte Antibiotikatherapie wurde in P II signifikant häufiger als adäquat bewertet und war aus der Sicht der behandelnden Ärzte erfolgreicher. Schlussfolgerung: Die vorgestellten Ergebnisse sind die ersten Resultate einer ersten Version eines regelbasierten Therapieassistenten. Da ein wissensbasiertes System einer ständigen Überprüfung und Weiterentwicklung unterliegt, sollten bei der Überarbeitung einer neuen Version die gewonnenen Erkenntnisse aus dem Einsatz in der klinischen Praxis berücksichtigt werden.

Literatur: [1] Kollef MH: Am J Med 2003; 115:582-584. [2] Kollef MH: Clin Infect Dis 31, 2000; Suppl4:S131-S138. [3] Harbarth S: Am J Med 2003; 115:529-535. [4] Kawamoto K: BMJ 2005; 330:765-772

IT gestützte Erfassung und Berechnung der Intensivmedizinischen Komplexbehandlung (IMKB) am Deutschen Herzzentrum Berlin (DHZB)

\section{Regitz, M. Werners, M. Mechnich}

\section{Controlling, Deutsches Herzzentrum Berlin}

Die IMKB wurde 2005 im OPS-Katalog (8-980) als Scoringsystem zur Beurteilung des Betreuungsaufwandes von Patienten auf Intensivstationen (IPS) aufgenommen. Ab 2006 ist die IMKB auch vergütungsrelevant. Sie beinhaltet insgesamt 23 Parameter: SAPS II ohne Glascow Coma Scale (z. B. Herzfrequenz, $\mathrm{PaO}_{2}-\mathrm{FiO}_{2}$ Quotient, Harnstoffwerte oder Körpertemperatur), TISS-28 (z. B. Nierenersatzverfahren oder Beatmung), das Alter, den Aufnahmestatus und chronische Leiden. Gewertet wird jeweils der Wert des Parameters, der die höchste Punktzahl ergibt. Während die Erfassung der Daten auf der IPS durch Pflegekräfte, Ärzte oder MTA's auf Papierkurven, Patienten-Daten-Management-Systeme (PDMS) und Krankenhausinformationssysteme (KIS) im Normalfall unproblematisch ist, stellt die Berechnung der Punkte die Beteiligten vor logistische und zeitliche Probleme. Die Berechnung, die manuell pro Parameter im Durchschnitt bis zu einer Minute betragen kann, stellt bei einer IPS von 20 Betten $(20$ Patienten $* 23 \mathrm{~Pa}$ rameter) mit 5-6 Stunden Berechnungszeit eine nicht unwesentliche Zeitkomponente dar. Was bei dem Parameter "Herzfrequenz“ noch relativ unkompliziert ist, wird bei dem " $\mathrm{PaO}_{2} / \mathrm{FiO}_{2}$ Quotienten" schon deutlich aufwendiger. Besonders arbeitsintensiv wird es bei dem Parameter "Flüssigkeitsersatz in hohen Mengen ( $>5$ Liter/24 h)“, da unterschiedliche Einfuhrmengen und -arten addiert werden müssen. Da im DHZB die Patientenkurven über ein PDMS geführt werden, sind alle Parameter in Datenbanken vorhanden und müssen „nur“ ausgewertet werden. In Zusammenarbeit mit der Firma INTERSYSTEMS ${ }^{\circledR}$ aus Darmstadt wurde ein Projekt gestartet, das auf der Integrationsplattform ENSEMBLE ${ }^{\circledR}$ basiert. ENSEMBLE ${ }^{\circledR}$ bekommt von dem KIS die Aufnahme- bzw. Verlegungsdaten der Patienten über ein HL7-Interface in Echtzeit geliefert und entscheidet, ob der Patienten auf einer IMKB relevanten Station liegt und die Kriterien (Alter über 14 Jahre, Liegezeit $>24$ Stunden) erfüllt. Dabei werden überlappende 24-Stunden-Intervalle des ersten Tages berücksichtigt. Sind die Kriterien erfüllt, holt sich ENSEMBLE ${ }^{\circledR}$ alle relevanten Daten per SQL-Query aus dem PDMS und bewertet die Daten auf Grundlage der hinterlegten Businesslogiken aus. In diesen Businesslogiken sind für jeden Parameter, die von DIMDI vorgegebenen Regelwerke hinterlegt. Über ein Webinterface kann sich der Mitarbeiter auf der IPS eine tägliche Stationsliste aufrufen und alle auf der Station liegenden Patienten, mit den schon ausgerechneten Punktwerten, sehen. Ein Klick auf „speichern“ und ENSEMBLE ${ }^{\circledR}$ speichert die Punktwerte des Patienten zur Weiterverarbeitung im das KIS.

\section{Qualitätsmanagement}

Einfluss eines optimierten Notaufnahmekonzeptes auf Akzeptanz und Einweisungshäufigkeit durch den Notarzt und Rettungsdienst

U. Füssel, W. Jakob, B. Vatankhah, A. Fürst, A. Janzen, J. Winkler, U. Bogdahn

Klinik und Poliklinik für Neurologie der Universität Regensburg im Bezirksklinikum Regensburg

Fragestellung: Ist eine Steigerung der Einweisungszahlen, insbesondere in den erlösrelevanten DRGs (u. a. B70 A, B70B, B83) in eine neurologische Notaufnahme ohne zusätzliche personelle und materielle Kapazitäten möglich? Ausgangsposition: Die Klinik und Poliklinik für Neurologie des Universitätsklinikums Regensburg befindet sich getrennt vom Hauptklinikum auf einem separaten Gelände. Diese Lage bedingt einen erhöhten logistischen Aufwand bei der Akutversorgung und dem Management von neurologischen Notfallpatienten. Es existiert ein zweites Krankenhaus der höchsten Versorgungsstufe mit Neurologischer Klinik im Stadtgebiet. Nach Abschluss von Modernisierungsarbeiten im Mai 2004 wurde ein neues Konzept für die neurologische Notaufnahme entwickelt und schrittweise eingeführt. Zielstellung war die Steigerung der Zuweisungszahlen, insbesondere in den DRG-relevanten Gruppen im Stroke-Bereich (u. a. B70A, B70B, B83) ohne zusätzliche personelle oder materielle Kapazitäten. Datenerhebung und Zielgrößen: Der Erfolg des Konzeptes wurde durch eine Analyse der Einweisungszahlen vom 1.1. bis 15.10.2004 (altes Konzept) und 1.1. bis 15.10.2005 (neues Konzept) sowie durch eine Umfrage unter dem Rettungsdienstpersonal sowie eine Erfassung der Einflussfaktoren im personellen und kommunikativen Bereich dokumentiert. Ergebnisse: Der Zufriedenheitsgrad des einweisenden Rettungsdienstpersonals stieg von 4,8 auf 1,7 (Schulnotenprinzip). $88 \%$ aller Befragten beziehen unsere Einrichtung häufiger in Einweisungsüberlegungen ein. Insbesondere im sensiblen und zeitkritischen Kollektiv der Patienten mit der Verdachtdiagnose „cerebrale Ischämie im Lysezeitfenster" konnte eine deutliche Steigerung der Einweisungszahlen und eine Verdreifachung der durchgeführten Lysetherapien im Vergleich der Untersuchungszeiträume 2004 und 2005 erreicht werden. Fazit: Auch bei unveränderten Ressourcen lässt sich durch eine Optimierung von Prozessen in der Notaufnahme eine erlösrelevante Steigerung der Zuweisungs- und Aufnahmezahlen erreichen

Aus Fehlern lernen?

A. Uhl

$B G U$ - Hamburg

Einleitung: In der Entwicklung des Menschen gibt es neben Versuch und Irrtum auch eine lange Reihe von Erfahrungen aus Fehlern. Das Lernen aus Fehlern ist also keine Erfindung der Neuzeit, sondern basiert auf die Menschheitsgeschichte schlechthin. Im Medizin und also auch im Anästhesiebereich treten Fehler auf, die unterschiedliche Auswirkungen haben können. Öffentlich gemachte Fehler mit gravierenden Folgen rufen immer ein besonderes Echo hervor. Was können wir nun tun um Fehler zu minimieren oder zu vermeiden? Aus Fehlern lernen! Methode: Im eigenen Arbeitsbereich wird ein Fehlererfassungssystem etabliert, das dem Mitarbeiter die Möglichkeit gibt entweder anonymisiert einen Fehlerbogen auszufüllen oder im Gespräch mit einer definierten Person das Dokument zu bearbeiten. Nach Abschluss wird dieser Fehlerbericht abgeheftet und das Protokoll im Rahmen einer Mitarbeiterbesprechung anonymisiert diskutiert. Ergebnis: Die Akzeptanz der Hälfte der Mitarbeiter war nach kurzer Zeit erreicht.Die meisten Fehler wurden offen diskutiert. Die Protokolle sind offen zugänglich und geben die Möglichkeit eigene Fehler zu vergleichen. Nach einer zunächst unstrukturierten Sammlung 
wird nun nach Fehlerursachen abgelegt. Es dominieren die Gerätefehler, Bedienungsfehler vor Fehlern in der Anwendung von anderen Medizinprodukten und defekten Medizinprodukten. Fehler durch unzureichende Ausbildung und fehlende Kompetenz rangieren am Ende der Skala. Die Diskussion in der Gruppe öffnet den Blick und hat einen Lerneffekt für erfahrene wie unerfahrene Mitarbeiter. Das Zugeben von Fehlern muss erlernt und praktiziert werden. Ein Denkfehler in der Kette, dass der, der einen Fehler zugibt, mit Repressalien rechnen muss, konnte wiederlegt werden. Auch hier kann aus dem Denkfehler gelernt werden. Schussfolgerung: Aus Fehlern lernen? Ja! Hilfreich ist das Angebot eines anonymisierten Erfassungssytemes da hier Angstbarrieren beseitigt werden. Fehlerreporte dienen der Steigerung der Qualität des Arbeitsbereiches. Fehlerreporte dienen der Patientensicherheit.

Patientennahe Qualitätssicherung bei der maschinellen Autotransfusion

\section{A. Uhl \\ BG-Unfallkrankenhaus - Hamburg}

Einleitung: Bei der maschinellen Autotransfusion(MAT) wird aus Wundblut ein gewaschenes Erythrocytenkonzentrat hergestellt. Dies geschieht Patientennah, meist im OP aber auch im AufwachIntensivbereich oder im Schockraum/Notaufnahme. Die maschinelle Autotransfusion sollte ausschließlich von geschultem Personal standardisiert nach einem Indikationskatalog durchgeführt werden. In der Klinik sollte eine Transfusionsordnung nach den Vorgaben des Transfusionsgesetzes Anwendung finden. Um sicher $\mathrm{zu}$ sein, ein hochwertiges Produkt hergestellt $\mathrm{zu}$ haben muss eine Qualitätskontrolle vor Transfusion erfolgen. Methode: Wir führen ein Dokumentationssystem das neben Personen und Maschinendaten auch auch eine Laborkontrolle erfasst. Aus dem Retransfusionsbeutel wird eine Probe zur Bestimmung des Hämoglobingehaltes und des Hämatokrit entnommen. Die Probe wird vor Ort mit einem Blutgasanalysegerät durchgeführt. Die Mitarbeiter sind geschult, die Einsätze werden erfasst. Es muss darauf geachtet werden, dass alle Mitarbeiter regelhaft maschinelle Autotransfusionen durchführen. Eine zeitnahe Auswertung der Dokumentation ist als Steuerung hilfreich. Alle erfassten Daten werden der Transfusionskommision vorgelegt und diskutiert. Ergebnis: Die konsequente Anwendung der MAT führt zu einem guten Trainingsstand der Mitarbeiter. Die Dokumentation der Maßnahme schärft die Aufmerksamkeit und ermöglicht eine vernünftige Auswertung. Die Laborkontrolle lässt Fehler in der Anwendung aber auch Fehlfunktionen der Geräte erkennen. Handlungsanweisungen für alle Maßnahmen sind schriftlich hinterlegt. Die durchschnittlichen gemessenen Hämoglobinwerte unseres Retransfusionsblutes betragen $22 \mathrm{~g} / \mathrm{dl}$. Schlussfolgerung: Mit einfachen Maßnahmen, Schulung, Dokumentation, Laborkontrolle lässt sich Qualitätssicherung bei der MAT unmittelbar durch den Anwender betreiben.

\section{Ungenügende Ausbildung von Rettungsassistenten für den Intensivtransport - Ergebnisse einer Umfrage \\ P. Gloger, B. Hossfeld, M. Helm \\ Abt. Anästhesiologie \& Intensivmedizin, Bundeswehrkrankenhaus Ulm}

Fragestellung: Der Interhospitaltransfer von Intensivpatienten ist eine rettungsdienstliche Aufgabe. Die Durchführung erfolgt daher in der Regel durch Notärzte und Rettungsassistenten. Dabei ist das Transportmanagemnent vor allem von beatmeten Patienten ein komplexer Vorgang. Unter anderem gilt es differenzierte Beatmungsformen $\mathrm{zu}$ übernehmen und fortzuführen, Katecholamintherapien aufrechtzuerhalten und invasives Monitoring zu überwachen. Darüber hinaus müssen alle wesentlichen Informationen bezüglich Anamnese, Diagnosen und Verlauf des Patienten erfasst und weitergegeben werden. Während den Notärzten im Gegensatz zu den Rettungsassistenten mit dem Curriculum Intensivtransport der DIVI für dieses spezielle Aufgabenfeld eine zusätzliche Ausbildung angeboten wird, stellt sich die Frage nach dem Ausbildungsbedarf beim nicht-ärztlichen Rettungsdienstpersonal. Methodik: Um den Bedarf einer Intensivtransportausbildung für Rettungsdienstpersonal $\mathrm{zu}$ erfassen, wurde ein Fragebogen an Notärzte, Rettungsassistenten und Pflegepersonal auf Intensivstationen verschickt. Ergebnisse: Insgesamt konnten 1033 Fragebögen ausgewertet werden. Die Ergebnisse bestätigen die Annahme, dass die überwiegende Mehrheit der Intensivtransporte mit Rettungsassistenten als Assistenzpersonal durchgeführt wird (97\%). 93\% der Rettungsassistenten denken, dass eine zusätzliche Ausbildung ihrerseits die Transportqualität steigern könnte, aber lediglich 33\% bestätigen die Möglichkeit zur Teilnahme an derartigen Fortbildungsangeboten. Nur jeder zweiten Pflegekraft ist die Qualifikation der Rettungsassistenten bekannt und nur bei 30\% der Pflegekräfte konnte das nicht-ärztliche Rettungsdienstpersonal bei Übernahme und Übergabe von Intensivpatienten den Umgang mit Respirator und invasiven Monitoring beherrschen. Fazit: Eine Erweiterung der intensivmedzinischen Aus- und Weiterbildung des nicht-ärztlichen Rettungsdienstpersonals könnte zur Optimierung der Transportqualität und Transportsicherheit im Interhospitaltransfer beitragen.

Unterschiedliche Adsorbtion von Insulin an Kathetermaterialien auf der Intensivstation: Polyethylen vs. Polyurethan - möglicher Grund für Hypoglykämien bei intensivierter Insulintherapie? S. C. Ley ${ }^{1}$ J. Ammann ${ }^{1}$ C. Herder ${ }^{2}$ M. Hartmann ${ }^{3}$, D. Kindgen-Milles ${ }^{1}$

${ }^{1}$ Klinik für Anästhesiologie am Universitätsklinikum Düsseldorf

${ }^{2}$ Deutsches Diabetes Zentrum DDZ

${ }^{3}$ Klinik für Anästhesiologie und Intensivmed. Uniklinik Essen

Hintergrund: Patienten (Pat.) auf chirurgischen Intensivstationen profitieren von einer intensivierten Insulintherapie. Die Einstellung des Blutzuckers (BZ) auf Werte zwischen 80 und $110 \mathrm{mg} / \mathrm{dl}$ birgt jedoch die Gefahr der Hypoglykämie, welche sich negativ auf die Morbidität und Mortalität auswirken kann. In diesem Kontext könnte die Adsorption von Insulin an Plastikmaterialien bedeutsam sein, wodurch die tatsächlich dem Pat. zugeführte Insulindosis unbemerkt stark schwanken kann. Wir untersuchten deshalb in vitro die Insulinadsorption an den Schlauchmaterialien und den Insulinbedarf von Intensivpat. unter Verwendung des Standard-Katheter-Materials Polyethylen (PE) und von Polyurethan (PU) im Vergleich. Material und Methoden: In vitro wurde für 24 Stunden Insulin (Actrapid ${ }^{\circledR}$, Fa. NovoNordisk) mit Hilfe einer Spritzenpumpe durch PE und PU Schläuche appliziert und am Ende der Leitung die austretende Insulinmenge durch eine Proteinbestimmung nach Bradford gemessen. Im zweiten Teil erfolgte nach Zustimmung der Ethikkomission und schriftlicher Einwilligung die kontinuierliche Insulingabe (100 IU Actrapid ${ }^{\circledR}$ in $50 \mathrm{ml} \mathrm{NaCl}$ ) im Rahmen einer intensivierten Insulintherapie bei 10 Pat. einer operativen Intensivstation eines Universitätskrankenhauses prospektiv, doppelblind und im Fall-Kontroll-Design für je 24 Stunden jeweils über einen PE und einen PU-Schlauch. Einschlusskriterien: kontinuierlicher Insulinbedarf $>2 \mathrm{IU} / \mathrm{h}$ in den letzten $24 \mathrm{~h}$. Ausschlusskriterien: Dialysepflichtige Niereninsuffizienz, invasive Intervention im Studienzeitraum, Blutverlust/-substitution $>500 \mathrm{ml} / 24$ Stunden, Kortikoidgabe. Die BZ-Einstellung erfolgte nach Protokoll durch das Pflegepersonal (Zielbereich $80-150 \mathrm{mg} / \mathrm{dl}$ ), welches nicht über das verwendete Schlauchmaterial informiert wurde. Ausgewertet wurden alle gemessenen BZ-Werte sowie das über die Insulin-Spritzenpumpe applizierte Volumen pro 24 Stunden. Ergebnisse: In vitro wurden bei PE am Anfang nur $23 \pm 4 \%$ der errechneten Insulinmenge freigesetzt. Nach $24 \mathrm{~h}$ stieg die Menge auf $39 \pm 2 \%$ an. Unter PU wurden konstant $76 \pm 23 \%$ gemessen. In vivo: Das über die Insulinspritzenpumpen applizierte Volumen (= Insulinmenge) war unter PE $(59 \pm 18 \mathrm{ml} / 24 \mathrm{~h})$ signi- 
fikant höher verglichen mit PU $(44+-15 \mathrm{ml} / 24 \mathrm{~h})(\mathrm{p}=0,0008)$. Die mittleren Blutglukosewerte unterschieden sich nicht: $\mathrm{PE}$ $141 \pm 17 \mathrm{mg} / \mathrm{dl} ; \mathrm{PU} 132 \pm 23 \mathrm{mg} / \mathrm{dl}(\mathrm{p}=0,36)$. An den beiden Studientagen unterschieden sich die Pat. weder im Katecholaminbedarf noch in der $24 \mathrm{~h}$-Bilanz (PE $319 \pm 1326 \mathrm{ml}$; PU $-208 \pm 2155 \mathrm{ml}$ $\mathrm{p}=0,51$ ). Schlussfolgerung: Die für eine strenge BZ-Einstellung zu infundierende Insulinmenge ist bei Verwendung von PE Schläuchen höher als bei PU-Material. Die Ursache ist vermutlich die bekannte höhere Insulinadsorbtion an PE- Schläuchen. Schwankungen der zugeführten Insulinmenge bei Schlauchwechseln sind denkbar. Unter Sicherheitsaspekten sollten daher bei intensivierter Insulintherapie, deren Voraussetzung eine exakte und stabile Insulindosierung ist, bevorzugt PU-Schläuche verwendet werden.

\section{Wie schwierig ist der schwierige Atemweg? Eine Prozessanalyse}

072

St. Eckert, F. Bach, K. Leimkühler, F. Mertzlufft

Klinik für Anästhesiologie, Intensiv-, Notfallmedizin und Schmerztherapie in Bethel Evangelisches Krankenhaus Bielefeld

Einleitung: Der schwierige Atemweg (SAW) ist eine Komplikation mit einer geschätzten Mortalität von etwa 0,1-2 auf $10000 \mathrm{Nar}-$ kosen. Anhand einer Prozessanalyse (PA) des Atemwegsmanagements (AWM) ist es möglich, Schwachstellen aufzudecken und Strategien zur Risikominimierung $\mathrm{zu}$ entwickeln. Material und Methoden 11962 Anästhesien, die zwischen dem 1.1.2005 und 31.3.2006 an einem Klinikum der Maximalversorgung erbracht wurden, kamen zur Auswertung. Die Altersspanne reichte vom 1. Lebenstag bis 90 Jahre, der Body-Mass-Index (BMI, $\left[\mathrm{kg} / \mathrm{m}^{2}\right]$ ) betrug im Mittel 28 (16-58), in 59 Fällen lag der BMI >26. Die Beurteilung nach ASA erfolgte zumeist in die Klassen 2 und 3. 106 Patienten (88\%) standen vor elektiven Eingriffen, 14 (12\%) vor Notfall-OP's. Nach der PA wurden ein Standard zum AWM und eine Checkliste zum präoperativen Erkennen versteckter Intubationshindernisse erstellt. Ergebnisse: 120 Fälle von misslungener oder schwieriger Intubation wurden anhand der Narkoseprotokolle hinsichtlich des dokumentierten Managements analysiert. Die Inzidenz des SAW betrug 1\%. 16 Patienten $(13,4 \%)$ zeigten eine stark eingeschränkte Kopfreklination, 28 (23,4\%) wiesen eine Mundöffnung von $<3 \mathrm{~cm}$ auf. In 11 Fällen $(9,2 \%)$ wurden beide Befunde am selben Patienten erhoben. Die Mundöffnung wurde nach Mallampati zumeist in die Klassen 1 und 2, die Laryngoskopie nach Cormack und Lehane in die Klassen 3 und 4 beurteilt. Insgesamt wurden 9 Techniken in variablen Kombinationen zur Bewältigung des SAW dokumentiert. In 45\% der Fälle wurde in Kombination mit Lagerungsmanövern (Jackson-Position, BURP) die Tubusform mittels Führungsstab modifiziert, gefolgt von Spatellängenwechseln $(19,6 \%)$ und Einsatz des Hebellaryngoskops nach McCoy $(13 \%)$. Eine sekundär fiberoptische Intubation erfolgte in 9,5\%, der Einsatz von Larynxmasken in 6,5\% der Fälle. Einmalig trat eine "cannot ventilate cannot intubate"-Situation auf. Diskussion/Schlussfolgerung: Die Daten des Narkoseprotokolls reichen allein nicht aus, um einen SAW korrekt vorherzusagen (fehlende Korrelation der Mallampati-Klassifikation vs. Cormack \& Lehane) [3]. Die anhand der PA gewonnenen Lösungsansätze (Einführen eines klinikspezifischen Standards AWM) können zur Risikominimierung beitragen. Eine bessere Vorhersage des SAW als bislang soll anhand einer Checkliste ermöglicht werden [3], klare Verhaltensregeln (Standard) und kontinuierliches Training alternativer Techniken können die Mortalität des SAW senken.

Literatur: [1] Airway Management: Leitlinie der deutschen Gesellschaft für Anästhesieologie und Intensivmedizin. Anästh Intensivmed 2004; 45:302-306. [2] Practice Guidelines for Management of the Difficult Airway American Society of Anesthesiologists Task Force on Difficult Airway Management, Oct. 16, 2002. [3] Breitmeier D, Debertin AS et al: Rechtsmedizinische Begutachtung von Behandlungsfehlervorwürfen $\mathrm{zu}$ Todesfällen nach schwieriger Intubation. Rechtsmedizin 2005; 15:363-370
Prozessoptimierung durch den Einsatz von HME Filter im OP

\section{R. Kranabetter}

Institut für Klinikhygiene Medizinische Mikrobiologie u. Klinische Infektiologie Klinikum Nürnberg

Zielsetzung: Kann durch den Einsatz von HME Filtern anstatt einer patientenbezogenen Aufbereitung der Beatmungssysteme in der Anästhesie eine Einsparung von Sach- und Personalkosten erreicht werden. Methode: In einer OP-Abteilung mit 10 Operationssälen wurde eine Prozesskostenanalyse für zwei unterschiedliche Hygieneregime zur Bereitstellung keimfreier Narkoseschlauchsysteme erstellt und seit April 2003 für den gesamten Anästhesiebereich umgesetzt. Der patientenbezogene Wechsel wurde im Vergleich zum Einsatz eines hydrophoben HME-F mit täglichem oder wöchentlichem Schlauchwechsel kalkuliert. Die Kosten wurden real ermittelt und den typischen Einsatzsituationen zugerechnet. Die hygienischen Fakten und die Sicherheit der Verfahren wurden anhand der Literatur diskutiert und mit eigenen Daten zur perioperativen Pneumonierate ergänzt. Ergebnisse: Den Mehrkosten für HME-Filter (Feuchte- und Wärmetauschfilter) stehen Einsparungen für $\mathrm{CO}_{2}$-Leitungen und die Reduktion der Aufbereitungskosten gegenüber, die zu einer Reduktion der Sachaufwendungen führten. Die Arbeitsvorgänge am Narkosegerät wurden erheblich reduziert und vereinfacht, die kalkulatorischen Personalkosten pro Narkose wurden nachdrücklich vermindert. Als Reduktion der Prozesskosten insgesamt können sich bis zu 9,72€ pro Narkose ergeben. Schlussfolgerung: Durch den Einsatz von HME-Filtern kann eine deutliche Einsparung von Kosten erreicht werden. Ein zusätzlicher positiver Aspekt für die Patienten entsteht durch die Filtration der Atemgase für Partikel, Bakterien und Viren und eine verbesserte Klimatisierung der Atemgase. Durch die Infektionserfassung über KISS (Krankenhaus-Infektions-SurveillanceSystem) überwachten und dokumentierten wir die postoperative Pneumonierate auf den nachgeschalteten Intensivstationen ohne dass unser neues Hygieneregime ein erhöhtes Infektionsrisiko aufwies. Mit Einführung dieser Maßnahmen haben wir somit ein Hygienemanagement etabliert, mit dem wir wesentliche Effizienzreserven unseres Hauses mobilisieren konnten.

Workflowunterstützung in der Intensivmedizin Ist die Systemanmeldung an einem PatientenDaten-Management-System ein zuverlässiger Trigger für Erinnerungsfunktionen

M. Meister, M. Sedlmayr ${ }^{1}$,Th. Rose ${ }^{1}$, D. Uphus, A. Michel-Backofen ${ }^{2}$, R. Röhrig

Klinik für Anästhesiologie, Intensivmedizin und Schmerztherapie und ${ }^{1}$ Abteilung für klinische und administrative Datenverarbeitung, Universitätsklinikum Gießen und Marburg, Standort Gießen ${ }^{2}$ Fraunhofer Institut für angewandte Informationstechnologie, St. Augustin

Einleitung: Die Einführung des DRG-Systems und Verpflichtung zur Qualitätssicherung führten zu einer verstärkten Einführung von Standard Operating Procedures (SOPs) [1,2]. In dem DFGgeförderten Projekt „OLGA“ (Online Guideline Assist) wird untersucht, ob die Indikationsstellung, Auswahl und Einhaltung von SOPs mit einem wissensbasierten System in einem Patienten-Daten-Management-System (PDMS) unterstützt werden können. Dieses erfordert eine zielgerichtete Meldung an den Stationsarzt. Neben Meldeverfahren über Handheld-Computer und Pager $[3,4]$ wurde eine zielgerichtete Benachrichtigung von Ärzten an das LogIn in ein PDMS realisiert [5]. Ziel der Arbeit: war die Untersuchung der Zeitintervalle zwischen den Systemanmeldungen der Stationsärzte einer Intensivstation zur Entscheidung ob sich diese als Trigger für Meldungen einer Workflowengine eignen. Methodik: Die Untersuchung erfolgte auf der Operativen Intensivstation des Universitätsklinikums Gießen. Die ärztliche Besetzung erfolgt in 3 Schichten mit je 2 Ärzten, sowie einem Oberarzt im Tag- 
dienst. Die vollständige Dokumentation (Befunde, Verordnungen, Maßnahmen, etc.) erfolgt mit dem PDMS ICUdata (Fa. IMESO $\mathrm{GmbH})$. Nach Zustimmung von Datenschutz und Personalrat, wurden alle Benutzeranmeldungen an dem PDMS über 28 Tage protokolliert, anonymisiert und analysiert. Ergebnisse: Im Beobachtungszeitraum erfolgten 3563 Systemanmeldungen von Stationsärzten. Die durchschnittliche Zeitspanne zwischen zwei Anmeldungen lag bei 11,3 min (SD 14,4), der Median bei 7 min. $75 \%$ der Zeitspannen waren geringer als $14 \mathrm{~min}$, 95\% geringer als $38 \mathrm{~min}$. An 75\% der Beobachtungstage traten Intervalle über 60 min, an $25 \%$ über 90 min auf. Schlussfolgerungen: Die Ergebnisse zeigen, dass Meldungen bei der Systemanmeldung die Einhaltung von SOPs sinnvoll unterstützen könnten. Oniki et al. zeigten, dass selbst eine einmalige Kontrolle pro Schicht das Qualitätsmanagement unterstützt [6]. Meldungen zeitlich kritischer Informationen (z.B. bei neu aufgetretenen erhöhten Herzenzymen) müssen über aktive Verfahren wie z.B. Pager kommuniziert werden [3, 4]. Der Einfluss von Schichtmodellen und Bettenauslastung auf die Anmeldeintervalle muss noch geklärt werden.

Literatur: [1] Martin et al: Anästhesiologie \& Intensivmedizin 2003; 44:871-876. [2] Shea S et al: J Am Med Inform Assoc 1995; 2:58-64. [3] Madhu C et al: J Biomed Inform 2005; 38:229-238. [4] Seggewies C et al: egms 2005: http://www.egms.de/en/meetings/ gmds2005/05gmds468.shtml. [5] Specht M, Rißner F: Projekt „Mecker-Fritze" - nicht publiziert. [6] Oniki et al: J Am Med Inform Assoc. 2003; 10:177-180

A risk score for intensive care patients based on laboratory data

A. Stachon ${ }^{1}$, R. Kempf ${ }^{1}$, T. Holland-Letz ${ }^{2}$, M. Krieg ${ }^{1}$

${ }^{1}$ Inst. für Klinische Chem., Transfus. u. Lab. Med., ${ }^{2}$ Abteilung für

Medizinische Informatik, Biometrie u. Epidemiologie,

Ruhr-Universität Bochum

Introduction: Established risk scores for intensive care patients are usually based on both laboratory parameters and clinical data. Generally, the determination of clinical data is time consuming and depends on the examiner. To estimate the patients prognosis we propose a new risk score for intensive care patients simply by use of common laboratory parameters. Methods: Patients were monitored on the internal medicine intensive care unit and the surgical intensive care unit, respectively. Blood samples were taken within the first 24 hours after admission and the following parameters were analyzed: nucleated red blood cells, hemoglobin, leucocytes, thrombocytes, sodium, potassium, CK, CKMB, C-reactive protein, creatinine, urea, alanine aminotransferase, PT, cholesterol, triglycerides, gender and age. Relevant prognostic parameters were identified by a stepwise logistic regression. Obtained odds ratios served to build the score. Results: 526 internal medicine patients and 284 surgical patients were included in this study, respectively. 86 (internal medicine) and 71 (surgery) of whom died in-hospital, respectively. For calculation of a new risk score for patients of the internal medicine the following parameters were considered: sodium, potassium, thrombocytes, leucocytes, cholesterol, alanine aminotransferase, C-reactive protein and age. The area under curve of this admission score was 0.86 . For calculation of a new risk score for patients of the surgical intensive care unit the following parameters were considered: potassium, leucocytes, CKMB, cholesterol, triglycerides, alanine aminotransferase, C-reactive protein and age. The area under curve of this admission score for surgical patients was 0.84 . Conclusion: We developed a risk score based solely on laboratory parameters helping the physician to identify patients with poor/good prognosis. Currently, analyses were performed to clarify whether the daily monitoring of intensive care patients with a laboratorybased risk score may aid in the early identification of patients at high risk.

\section{Sepsis, SIRS, MOV und Infektionen}

\section{Pathophysiologie I}

Signaling via NOD-Rezeptor, RICK und NF- $\kappa$ B, Zytokinsynthese und T-Zell-Aktivierung durch enterische Gliazellen: Primärer Ausgangspunkt für die intestinale bakterielle Translokation bei Sepsis?

E. Hollenbach, T.M. Maher, M. Zöller, W. Stremmel, M. Neumann, P. Malfertheiner, B. Vallance, A. Rühl und L. Engelmann

Einheit für Multidisziplinäre Intensivmedizin, Universität Leipzig, Harvard Medical School, Dept. of Pharmacology, Cambridge, Massachusetts,

Deutsches Krebsforschungszentrum, Abt. für Tumorimmunologie, Universität Heidelberg,

British Columbia Research Institute, University of British

Columbia, Vancouver

Die intestinale bakterielle Translokation spielt in der Pathogenese der Sepsis eine grosse Rolle. NF- $\kappa \mathrm{B}$-induzierte Zytokine führen zur Darmepithelschädigung und Permeabilitätssteigerung und fördern damit die bakterielle Translokation. Es ist bislang nicht geklärt, welche Zellart im Darm zuerst mit bakteriellen Antigenen in Kontakt tritt und Lymphozyten/Makrophagen aktiviert und welche molekularen Prozesse zu einer intestinalen Entzündung führen. Enterische Gliazellen (EGZ) wurden in ihrer immunologischen Funktion geprüft, da sie die höchste MHC-II-Rezeptordichte für die Antigenpräsentation und T-Zell-Aktivierung aufweisen, EGZ direkten Einfluss auf die intestinale Permeabilität haben und zur IL-6/TNF- $\alpha$-Synthese fähig sind. Deshalb wurde die weltweit erste stabile EGZ-Primärzellinie etabliert. RT-PCR- und FACS-Analysen konnten zeigen, dass EGZ neben MHC II auch das wichtige T-Zell-ko-stimulatorische ICAM-1 exprimieren. Die EGZ-Phagozytosefähigkeit wurde nach Inkubation mit FITC-gekoppelten Dextran (Laserscanningmikroskopie) nachgewiesen. Um die biologische Funktionalität des MHC-II/ICAM-I-Komplexes zu zeigen, wurden EGZ mit Ovalbumin (OVA) inkubiert (72 h). Diese wurden mit syngenetischen, aus mesenterialen Lymphknoten isolierten T-Zellen von OVA-immunisierten Ratten ko-inkubiert ( $72 \mathrm{~h})$. Ein massiver Anstieg der lymphozytären [3H]-Thymidininkorporation bewies die T-Zellproliferation $(\mathrm{p}<0,0001)$, die durch MHC-II- oder ICAMI-Ak blockiert wurde. Kontrollexperimente zeigen keine T-Zell-Proliferation. Neue Mikroskopiertechniken wiesen einen direkten (!) Kontakt der EGZ mit dem Darmepithel in vivo nach. Für die Aufklärung molekularer Grundlagen der EGZ-Aktivierung wurde ein $\mathrm{He}-$ licobacter pylori (HP)-Modell entwickelt. Der HP-Wildtyp (HPWT) transloziert selektiv Peptidoglykane (PGN) als bakterielles Hauptantigen in die EGZ und stimuliert damit den NOD-Rezeptor als Teil der angeborenen Immunität. Ein isogener HP-Stamm (HPMUT) ist dazu nicht in der Lage. Western-Blots und elektrophoretische Shiftanalysen zeigen, dass nur HP-WT eine NF- $\kappa$ B-Aktivierung (IkBa-Degradation, nukleäres p65) induziert. HP-WT, aber nicht HP-MUT kann die proinflammatorischen MAP-Kinasen p38 und ERK aktivieren. Ebenso ist die RICK-Kinase als direkter Interaktionspartner mit dem NOD-Rezeptor, der zur NF- $\kappa$ B-Aktivierung führt, nur nach HP-WT-Stimulation aktiviert (in vitro Kinaseassay). Die NF- $\kappa$ B-Aktivierung war durch den RICK-Inhibitor SB203580 hemmbar. Diese Ergebnisse sind in vivo in der Maus reproduzierbar. NF- $\kappa \mathrm{B}$ ist hier durch PGN PRIMÄR in EGZ und erst SEKUNDÄR in Lymphozyten/Makrophagen aktiviert. Zusammenfassung: EGZ sind neben Epithelien und Lymphozyten//Makrophagen Teil der angeborenen Immunantwort via NOD-Rezeptoraktivierung. Sie sind als Bindeglied zwischen intestinaler bakterieller Translokation und einer Darmwandschädigung in vitro und in vivo hiermit erstmalig nachgewiesen. Damit sind EGZ als therapeutische Zielzelle der bakteriellen Translokation bei Sepsis identifiziert. 
Bedeutung von AP-1, JNK und PPAR-g in enterischen

Gliazellen bei der intestinalen bakteriellen

Translokation bei Sepsis

E. Hollenbach, S. Kavuri, M. Leverkus, M. Neumann,

\section{Engelmann, A. Rühl}

Einheit für Multidisziplinäre Intensivmedizin, Universität Leipzig, D; Institut für Humanbiologie, Technische Universität, München, $D$ NF- $\kappa$ B, die MAP-Kinase JNK und activator protein-1 (AP-1) sind die Haupttranskriptionsfaktoren, die eine pro-inflammatorische Immunantwort induzieren. Eine PPAR-g Rezeptoraktivierung blockiert die NF- $\kappa$ B-Aktivierung bei entzündlichen Darmwandveränderungen. Unsere früheren Arbeiten wiesen die wichtige Rolle von enterischen Gliazellen (EGZ) bei der Immunantwort in der Darmwand via NOD-Rezeptor und NF- $\kappa \mathrm{B}$ nach, die sekundär zu einem Epithelschaden, einer Permeabilitätssteigerung der Darmwand und dies wiederum zur intestinalen bakteriellen Translokation, einem wesentlichen Faktor in der Sepsispathogenese, führt. Deshalb wurde untersucht, ob bei der NF- $\kappa$ B Aktivierung durch bakterielle Peptidoglycane (PGN) in einer selbst etablierten EGZPrimärzellinie AP-1 und der PPAR-g Rezeptor als potentielle therapeutische Zielmoleküle involviert sind. PGN sind der Hauptstimulus bei entzündlichen Darmveränderungen und binden an den NOD-Rezeptor als Teil der angeborenen Immunantwort in EGZ Dafür wurde ein Helicobacter pylori (HP)-Modell entwickelt, dessen HP-Wildtyp (HP-WT) selektiv PGN als bakterielles Hauptantigen in die EGZ transloziert und damit den NOD-Rezeptor und sekundär NF- $\kappa$ B stimuliert. Ein isogener HP-Stamm (HP-MUT) ist dazu nicht in der Lage. Per radioaktivem in-vitro Kinaseassay konnte mit GST-c-jun als Substrat eine JNK-Aktivierung 45 min nach Exposition der EGZ nur mit HP-WT, jedoch nicht mit HPMUT gezeigt werden. Die JNK-Aktivierung wurde durch Präinkubation mit dem JNK-Inhibitor SP600125 geblockt und durch einen AP-1 elektrophoretischen Mobilitätsshiftassay (EMSA) bestätigt worden. Supershift-Analysen zeigten, dass AP-1 vor allem aus c-jun und marginal aus c-fos besteht. Western Blots zeigen eine deutlich reduzierte Degradation des NF- $\kappa$ B-Inhibitors IkBa bei einer Vorbehandlung mit SP600125. Die signifikant geringere NF$\kappa \mathrm{B}$-Aktivierung wurde durch EMSA bestätigt. Die Expression von PPAR-g war $8 \mathrm{~h}$ nach Infektion mit HP-WT signifikant hochreguliert (FACS-Analyse), während HP-MUT nur schwach PPAR-g induzierte. 45 min nach Exposition der EGZ mit HP-WT konnte eine deutliche IkBa-Degradation und nukleäre p65-Translokation als Maß für die NF- $\kappa \mathrm{B}$-Aktivierung nachgewiesen werden, während dies durch eine 30 min Präinkubation der EGC mit dem PPAR-g-Agonisten Ciglitazone $(15 \mu \mathrm{M})$ oder des intestinalen endogenen Liganden 15d-PGJ2 $(20 \mu \mathrm{M})$ signifikant reduziert wurde. Dies wurde durch EMSA-Analysen bestätigt. Zusammenfassung: Die Ergebnisse zeigen die Bedeutung von EGZ als immunregulatorische Zelle, die nach bakterieller PGN-Stimulation mit einer massiven Induktion von pro-inflammatorischen NF- $\kappa \mathrm{B}$, JNK und $\mathrm{AP}-1$ reagiert. Die Aktivierung von NF- $\kappa \mathrm{B}$ kann durch PPARg-Agonisten inhibiert werden. JNK, AP-1 und v.a. der PPAR-g-Rezeptor sind nach diesen Ergebnissen therapeuti-sche Zielmoleküle gegen die bakterielle Translokation bei Sepsis. Die bereits in der Diabetestherapie eingesetzten PPAR-g-Agonisten (z.B. Ciglitazone) sind eine vielversprechende Substanzklasse.
ATP release from activated neutrophils occurs via connexin 43 and modulates adenosine-dependent endothelial cell function

H.K. Eltzschig, T. Eckle, A. Mager, N. Küper, C. Karcher, T. Weissmüller, K. Boengler, R. Schulz, S. C. Robson, S. P. Colgan Department of Anesthesiology and Intensive Care Medicine, Tübingen, Institut für Pathophysiologie, Universitätsklinikum Essen, Harvard Medical School, Boston

Extracellular ATP liberated during hypoxia and inflammation can either signal directly on purinergic receptors, or can activate adenosine receptors following phosphohydrolysis to adenosine. Given the association of polymorphonuclear leukocytes (PMN) with adenine nucleotide/nucleoside signaling in the inflammatory milieu, we hypothesized that PMN are a source of extracellular ATP. Initial studies revealed that PMN release ATP through activation-dependent pathways. In vitro models of PMN-endothelial crosstalk indicated that PMN-derived ATP signals through endothelial adenosine receptors, thereby promoting endothelial barrier function and attenuating PMN-endothelial adhesion. Metabolism of extracellular ATP to adenosine required PMN, and studies addressing these metabolic steps revealed that PMN express surface ectoapyrase (CD39). Such observations were verified in PMN from cd39-/-mice. Additional studies revealed that PMN ATP release occurs through connexin $43(\mathrm{Cx} 43)$ hemichannels in a proteinphosphatase-A dependent manner. These findings in human PMN were confirmed in PMN derived from induced $\mathrm{cx} 43-/-$ mice, whereby activated PMN release less than $15 \%$ of ATP relative to littermate controls, while $\mathrm{cx} 43$ heterozygote PMN were intermediate in their capacity for ATP release. Taken together, our results identify a previously unappreciated role for $\mathrm{Cx} 43$ in activated PMN ATP release, therein contributing to the innate metabolic control of the inflammatory milieu.

Inhibition von MMP-9 mit Doxycyclin verringert das Ausmaß von Pankreatitis-assoziierten Lungenschäden

M. Sochor, V. Friebe, S. Hempel, U. Adam, U.T. Hopt, T. Keck Abteilung für Allgemein- und Viszeralchirurgie, Chirurgische Universitätsklinik Freiburg, Freiburg im Breisgau

Hintergrund: Bei der Pathogenese pulmonaler Komplikationen als Folge einer Pankreatitis spielt, wie wir im Vorfeld zeigen konnten, das proteolytische Enzym Matrix Metalloproteinase 9 (MMP-9) eine zentrale Rolle für die Leukozyteninfiltration ins Lungengewebe und Ausbildung des Pankreatitis-assoziierten Lungenschaden (PALI). Doxyclycin ist ein potenter Inhibitor von MMP-9. Wir haben in vivo und in vitro untersucht, wie sehr sich das Ausmaß des PALI durch die Inhibition von MMP-9 mit Doxycyclin verringern lässt. Methoden: In vitro: Die Evaluation der Transmigration von isolierten humanan Leukozyten durch Basalmembran unter Chemoattraktion $(10 \mu \mathrm{g} / \mathrm{ml}$ TNF- $\alpha$ ) erfolgte in Biocoat Matrigel Invasionkammern in (1) Kontrollen und (2) unter Inhibition mit Doxycyclin $(2 \mu \mathrm{g} / \mathrm{ml})$. Transmigrierte Leukozyten auf der Unterseite der Matrigel Membran wurden angefärbt und ausgezählt. In vivo: 3 Gruppen mit je 6 Ratten (1) Pankreatitis ohne Behandlung, (2) Pankreatitis mit Doxycylin ( $30 \mathrm{mg} / \mathrm{kgKG}$ s.c.) und (3) Kontrolle wurden unterschieden. Die Induktion der schweren nekrotisierenden Pankreatitis erfolgte durch intraduktale Applikation von Glycodesoxycholsäure (GDOC; $10 \mathrm{mmol} / \mathrm{l}$ ) und einer intravenösen Ceruleininfusion $(5 \mu \mathrm{g} / \mathrm{kg} / \mathrm{h})$ über $6 \mathrm{~h}$. Die subkutane Doxycylinapplikation erfolgt direkt nach der Pankreatitisinduktion zum Zeitpunkt $t=0$. Serumanalysen zeigten einen effektiven Wirkspiegel im Blut nach $3 \mathrm{~h}$. Nach $9 \mathrm{~h}$ erfolgte die Bestimmung der Leukozyteninfiltration in die Lunge an histologischen Schnitten mit Naphtol-AS-D-Chloroacetatesterase (NACE) in $10 \mathrm{Ge}$ sichtsfeldern. Zum Zeitpunkt $\mathrm{t}=3 \mathrm{~h}$ und $\mathrm{t}=9 \mathrm{~h}$ erfolgte die Messung des Doxycyclinserumspiegels. Der Mittelwert betrug $2 \mu \mathrm{g} / \mathrm{ml}$. Ergebnisse: In vitro: Doxycyclin im Transmigrationsversuch hemmte signifikant die Transmigration von Granulozyten durch 
Inhibition proteolytischen MMP-9 im Vergleich zur Kontrollgruppe $(12,6 \pm 2,6$ vs. $20,1 \pm 3,9 ; p<0,05)$. In vivo verringerte die Doxycyclinbehandlung die Anzahl pulmonal infiltrierter Granulozyten $(21,2 \pm 5,1$ vs. $32,5 \pm 6,8 ; p<0,05)$, Kontrollgruppe $(14,2 \pm 4,0)$ und histologische Lungenschäden nach Induktion der schweren Pankreatitis. Schlussfolgerung: Doxycyclin hemmt die inflammationsgesteuerte Leukozytentransmigration durch die Basalmembran in vitro, sowie die Leukozyteninfiltration ins Lungengewebe nach Pankreatitisinduktion in vivo und stellt somit eine Therapieoption dar, das Ausmaß des Pankreatitis-assozierten Lungenschadens zu verringern oder dessen Ausbildung zu verhindern.

Neutrophil derived gut barrier dysfunction in rat severe acute pancreatitis - matrix metalloproteinase-9 as regulator of gut barrier integrity

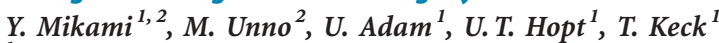

${ }^{1}$ Department of General and Visceral Surgery, University Hospital Freiburg

${ }^{2}$ Division of Hepato-Biliary Pancreatic Surgery, Tohoku University Sendai, Japan School of Medicine, Tohoku University, Sendai, Japan

The role of polymorphonuclear neutrophil (PMN) derived protease, matrix metalloproteinase (MMP), for the gut barrier dysfunction in severe acute pancreatitis (SAP) has not yet been clarified. MMP-9 from PMN plays a key role in basement membrane injury after SAP in lung tissue and pancreatitis-associated lung injury. The aim of this study was to evaluate the effect of MMP inhibitor (BB-94) on gut bacterial translocation (BT) and gut barrier integrity in rat severe acute pancreatitis. Methods: SAP was induced by the intraductal injection of $5 \%$ sodium taurocholate. BB-94 was administrated at 24 hours and just before the induction of acute pancreatitis. Twenty-four hours after the induction of acute pancreatitis, the gut barrier dysfunction was investigated by histology. Leukozyte infiltration and destructive potential were evaluated by myeloperoxidase (MPO) activity and reactive oxygen species (ROS) formation in the gut. MMP-9 levels in the gut were determined by zymography. Bacterial translocation into mesenteric lymph nodes (MLN), pancreas and blood was evaluated in aerobic and anaerobic bacterial cultures at $24 \mathrm{~h}$. Controls, SAP and SAP with treatment of BB-94 were distinguished. Results: The mucosal injury and the infiltration of PMN (1.6 \pm 0.2 vs. $1.2 \pm 0.15$ MPO U/mg protein; $\mathrm{p}<0.05$ ) in the gut of rats with BB-94 were significantly more severe than those of rats without BB-94 treatment. The levels of MMP-9 and ROS as mediators of PMN injury (ROS $4.1 \pm 0.2 \pm 2.9 \pm 0.1 \mu \mathrm{M} / \mathrm{min} / \mathrm{mg}$ protein; $\mathrm{p}<0.05$ ) were significantly decreased in the treatment group. Pre-treatment of BB-94 significantly reduced the occurrence of $\mathrm{BT}$ in the rat severe acute pancreatitis (MLN $12 / 12$ vs. 10/12; p < 0.05). Conclusion: MMP-9 is an important chemical mediators of bacterial translocation in severe acute pancreatitis. MMP inhibitor, BB-94, reduces local injury to the mucosal barrier, reduces PMN infiltration to the gut and PMN injury by oxygen radicals and protease MMP-9 to the gut in severe acute pancreatitis.

Veränderungen der Mikrozirkulation durch selektive

029 Serotoninrezeptorblockade bei experimenteller Endotoxinämie

E. Petri, C. Peter, E. Martin, A. Walther

Klinik für Anästhesiologie, Universitätsklinikum Heidelberg

Einleitung: Auch heute noch wird die hohe Sterblichkeitsrate in der Sepsis maßgeblich vom Endothelschaden bestimmt [1]. Neuere Untersuchungen mit dem GP IIb/IIIa Antagonisten Abciximab lassen auf eine dominierende Rolle der Thrombozyten als Mediatoren des Endothelschadens während Endotoxinämie schließen [2]. Serotonin beeinflusst die Thrombozytenaktivierung und damit die Thrombozten-Zell-Interaktionen [3]. Ziel dieser Arbeit war es, mögliche Effekte der Serotonin(5-HT)-Rezeptorantagonisten Methysergide und Ketanserin auf Veränderungen der Mikrozirkulation während Endotoxinämie zu untersuchen. Material und Methoden: Bei männlichen Wistar Ratten wurde mittels Intravitalmikroskopie an postkapillären Venolen des Mesenteriums Wandscherrate (WSR), Leukozytenadhärenz (LA) und Plasmaextravasation $(\mathrm{PE}) \mathrm{zu} t=0,60$ und 120 Minuten nach Beginn einer kontinuierlichen Endotoxininfusion bestimmt. Die Experimente wurden in eine Pre- und Post-Treatment Block unterteilt. Die Tiere in den Behandlungsgruppen erhielten entweder Methysergid (MSG) oder Ketanserin (KET). Der Vergleichsgruppe wurde nur Endotoxin (ETX) appliziert, Tiere der Kontrollgruppe erhielten äquivalentes Volumen physiologischer Kochsalzlösung $(\mathrm{NaCl})$. Ergebnisse: Pre-Treatment: WSR: Während des Beobachtungszeitraums finden sich keine Unterschiede zwischen Behandlungs- und Vergleichsgruppen. LA: Nur Tiere der ETX-Gruppe zeigen einen signifikanten Anstieg der LA; bei $\mathrm{t}=60 \mathrm{~min}$ und $120 \mathrm{~min}$ liegen die Werte signifikant über den Werten der Kontrolltiere, der MSGund der KET-behandelten Tiere (Mittelwert $120 \mathrm{~min}$ [n/qmm]: ETX: 428; MSG: 161; KET: 138; NaCl: 129). PE: In allen Gruppen findet sich ein signifikanter Anstieg der PE. In der ETX-Gruppe sind die Werte bei $\mathrm{t}=120 \mathrm{~min}$ signifikant gegenüber den anderen Gruppen erhöht (Mittelwert 120 min: ETX: 0,73; MSG: 0,39; KET: 0,31; NaCl: 0,36). Post-Treatment: WSR: Während des Beobachtungszeitraums finden sich keine Unterschiede zwischen Behandlungs- und Vergleichsgruppen. LA: Nur Tiere der ETX-Gruppe zeigen einen signifikanten Anstieg der LA; die Werte sind bei $\mathrm{t}=120 \mathrm{~min}$ signifikant gegenüber den anderen Gruppen erhöht (Mittelwert $120 \mathrm{~min}$ [n/qmm]: ETX: 434; MSG: 161; KET: 191; NaCl: 129). PE: In allen Gruppen findet sich ein signifikanter Anstieg der PE. In der ETX-Gruppe sind die Werte bei $t=120 \mathrm{~min}$ signifikant gegenüber den anderen Gruppen erhöht (Mittelwert 120 min: ETX: 0,77; MSG: 0,29; KET: 0,35; NaCl: 0,34). Schlussfolgerung: Die Modifikation der Thrombozytenaktivierung und -interaktion mit MSG oder KET bewirkt in einem Pre-Treatment und einem Post-Treatment Experiment eine Verringerung die Leukozytenadhärenz und der Extravasation bei Endotoxinämie und stellt damit eine neue, viel versprechende Therapiemöglichkeit zur Verbesserung der Mikrozirkulation während Endotoxinämie dar.

Literatur: [1] Peters K et al: Cardiovasc Res 2003; 60:49-57. [2] Walther A et al: Microcirculation 2004; 11:79-88. [3] Cerrito F, et. al. Life Sci 1993; 53:209-215

Endothelial ATP-loss is attenuated by phosphorylation 063 of serine 368 and HIF-2 dependent repression of connexin 43 by hypoxia

\section{J. Seessle, M. Faigle, H.K. Eltzschig}

Universitätsklinik für Anästhesiologie und Intensivmedizin, Universitätsklinikum, Hoppe-Seyler-Str. 3, D-72076 Tübingen, Germany

Connexins form gap-junctional channels that connect the cytoplasm of adjacent cells directly and allow the exchange of small molecules. In addition, connexins are also integrated into single plasma membranes where they serve as extracellular transport channel for small molecules, such as nucleotides or amino acids. Based on our initial observation that endothelial ATP release is attenuated by hypoxia, we employed real-time RT-PCR analysis to screen endothelial connexins for transcriptional modulation by hypoxia. In fact, we found that connexin $43(\mathrm{Cx} 43)$ mRNA and protein are selectively repressed by hypoxia. Studies in endothelia with loss of hypoxia inducible factor-2 (HIF-2) function suggested a role of HIF-2 in hypoxia repression of $\mathrm{Cx} 43$. In addition, hypoxia exposure of endothelia resulted in immediate phosphorylation of the Serine 368 residue of $\mathrm{Cx} 43$, which is known to functionally switch the channel from an open into a closed state. Moreover, pharmacological inhibition of endothelial $\mathrm{Cx} 43$ or $\mathrm{Cx} 43$-siRNA knockdown attenuated endothelial ATP release. Taken together, 
these results reveal a previously unappreciated role of endothelial Cx43 as ATP release channel and suggest HIF-2-dependent repression and phosphorylation of $\mathrm{Cx} 43$ as innate adaptation of the vascular endothelium to dampen endothelial ATP-loss during hypoxia.

Ein neues Modell zur Untersuchung des Darmödems 064 der isoliert perfundierte Dünndarm der Ratte

S. Kuchenbecker, I. Frerichs, J. Scholz, S. Uhlig, N. Weiler Klinik für Anästhesiologie und Operative Intensivmedizin, UKSH Schleswig-Holstein, Campus Kiel

Einleitung: Das septische Multiorganversagen stellt einen lebensbedrohlichen Zustand für den Intensivpatienten dar. Intestinale Malperfusion und ein daraus resultierendes Darmödem mit gestörter Permeabilität und bakterieller Translokation kann Auslöser für die Entwicklung einer Sepsis sein. Experimentelle Modelle zur systematischen Untersuchung der molekularen Mechanismen des Darmödems und möglicher therapeutischer Ansätze sind daher dringend erforderlich. Deshalb entwickelten wir ein neues ExSitu-Modell des isoliert perfundierten Ratten-Dünndarms zur Untersuchung des Darmödems. Methodik: Bei weiblichen Wistar Ratten (Gewicht: 230-250 g) wurde der Dünndarm freipräpariert. In das proximale und distale Darmlumen sowie in die A. mesenterica superior und die V. portae wurden Perfusionskanülen platziert. Der isolierte Dünndarm wurde dann in eine speziell angefertigte, temperatur- und luftfeuchtigkeitskontrollierte Kammer mit integrierter Organwaage verbracht. Da kein Organbad verwendet wurde, konnte das Gewicht des Dünndarms kontinuierlich aufgezeichnet werden. Modifiziertes DMEM Zellkulturmedium mit Tetramethylrhodamine Isothiocyanat (TRITC) Albumin wurde für die luminale Darmperfusion verwendet. Die vaskuläre Perfusion erfolgte wahlweise druck- oder flusskonstant mit oxygeniertem artifiziellem Plasma, welches zusätzlich Fluorescein Isothiocyanat (FITC) Albumin enthielt. Als Sauerstoffträger wurden humane Erythrozyten verwendet. Vaskuläre und intraluminale Drücke und Flüsse wurden kontinuierlich aufgezeichnet. Die Lymphgefäße wurden eröffnet und die Lymphflüssigkeit aufgefangen, um eine Quantifizierung des Lymphflusses vorzunehmen. Die fluoreszenzmarkierten Albumine, die zum vaskulären und intraluminalen Perfusionsmedium zugesetzt wurden, erlaubten eine Charakterisierung der Flüsssigkeitsverschiebungen zwischen den verschiedenen Kompartimenten. Ergebnisse: Dieses neue Modell erlaubt die simultane Aufzeichung von vaskulärem, luminalem und lymphatischem Fluss, arteriellem, venösem und intraluminalem Druck sowie des Darmgewichts. Erste Ergebnisse zeigen, dass das Organ unter den gegebenen Bedingungen für mindestens 3 Stunden vital und stabil bleibt. Dies wurde durch histologische Untersuchungen, die Messung von Sauerstoff- und Glukoseverbrauch, Ermittlung des Laktat/Pyruvat-Verhältnisses und Messung der Laktatdehydrogenase (LDH) nachgewiesen. Erste Versuche mit Gabe des Sepsismediators Plättchen-aggregierender-Faktor (PAF) zeigten eine Vasokonstriktion und eine Darmödementwicklung. Schlussfolgerung. Das vorgestellte Modell des isoliert perfundierten Dünndarms erlaubt eine Trennung von vaskulärem, luminalem, extrazellulärem und lymphatischem Kompartiment. Dieses Modell wird zukünftig die Untersuchung der molekularen Mechanismen des Darmödems ermöglichen. Gefördert von der Medizinischen $\mathrm{Fa}$ kultät der Christian-Albrechts-Universität in Kiel, Förder-Nr. F346020.
Zerebrale Effekte von Volumentherapie und Vasopressortherapie bei Schädel-Hirn-Trauma und Sepsis im Schaf

H.D. Stubbe, C. Greiner ${ }^{*}$ C. Hucklenbruch, M. Westphal, F. Hinder

Klinik und Poliklinik für Anästhesiologie und operative Intensivmedizin, ${ }^{*}$ Klinik und Poliklinik für Neurochirurgie, Universitätsklinikum Münster

Fragestellung: Das im Rahmen eines Polytraumas entstehende Schädel-Hirn-Trauma (SHT) ist aufgrund massiver Gewebsschädigung häufig bereits initial begleitet von einer systemischen Entzündungsreaktion (SIRS); im weiteren Verlauf kann eine Sepsis den klinischen Verlauf komplizieren. Im Schafmodell des geschlossenen SHT war eine Endotoxin-induzierte Sepsis nicht nur mit systemischer Hypotonie und Abfall des zerebralen Perfusionsdrucks (CPP), sondern gleichzeitig mit cerebraler Vasodilatation und Hyperperfusion als Ausdruck einer zerebralen Vasoregulationsstörung assoziiert. In diesem Modell wurden nun die zerebralen Effekte einer Vasopressortherapie und Volumentherapie zur Aufrechterhaltung des CPP verglichen. Es wurde angenommen, dass die Volumentherapie den zerebralen Blutfluss und den ICP weiter erhöhen und die zerebrale Gewebsoxygenierung $\left(\mathrm{PbrO}_{2}\right)$ beeinträchtigen würde. Methodik: 12 Schafe wurden über den Messzeitraum von 13 Stunden anästhesiert und mechanisch ventiliert. Das Schädel-Hirn-Trauma wurde in allen Tieren durch ein nicht-penetrierendes Bolzenschussgerät induziert. Alle Tiere erhielten dann eine kontinuierliche Endotoxin-Infusion (S. typhi, $\left.10 \mathrm{ng} \cdot \mathrm{kg} \cdot \mathrm{min}^{-1}\right)$. Nach 10 Stunden wurde der CPP in der ersten Gruppe (HAES, $n=6$ ) mit Hydroxyethylstärke (HAES) 6\% auf 60-70 $\mathrm{mmHg}$ angehoben. Die zweite Gruppe (NA, $\mathrm{n}=6$ ) bekam Noradrenalin zur Aufrechterhaltung des CPP von $60-70 \mathrm{mmHg}$ zugeführt. Zusätzlich wurde in der NA-Gruppe Blut isovolämisch gegen HAES ausgetauscht, um vergleichbare Hämatokritwerte in beiden Gruppen zu erzielen. Ergebnisse: Das SHT war in beiden Gruppen mit einem signifikanten Anstieg des intrakraniellen Drucks (ICP) und einem Abfall der $\mathrm{PbrO}_{2}$ assoziiert. Neuropathologisch fanden sich bitemporale intrakranielle Blutungen und ein diffuses Hirnödem. Die Endotoxinämie induzierte eine hyperdyname Kreislaufreaktion mit signifikant erhöhtem Blutfluss in der A. carotis interna (ICBF) bei gleichzeitigem Abfall des mittleren arteriellen Blutdrucks (MAD) und CPP. Die Infusion von HAES erhöhte sowohl den ICBF signifikant von $247 \pm 26(10 \mathrm{~h})$ auf $342 \pm 42 \mathrm{~mL} \cdot \mathrm{min}^{-1}(13 \mathrm{~h})$, als auch den ICP signifikant von $20 \pm 4(10 \mathrm{~h})$ auf ein Maximum von $25 \pm 3 \mathrm{mmHg}(12 \mathrm{~h})$. Der $\mathrm{PbrO}_{2}$, die sinus-venöse Sauerstoffsättigung $\left(\mathrm{SsvO}_{2}\right)$ und die Sauerstoffextraktionsfraktion (OEF) wurden nicht signifikant beeinflusst. Noradrenalin erhöhte den ICBF von $268 \pm 19$ to $342 \pm 58 \mathrm{~mL} \cdot \mathrm{min}^{-1}$ (10 vs. $\left.13 \mathrm{~h}\right)$, ließ den ICP aber statistisch unbeeinflusst. Hierunter stieg die $\mathrm{SsvO}_{2}$ im Gegensatz zur HAESGruppe signfikant von $49 \pm 4(10 \mathrm{~h})$ auf ein Maximum von $59 \pm 6 \mathrm{mmHg}(12 \mathrm{~h})$, während die OEF signifikant abnahm. Der hierunter zu beobachtende Anstieg der $\mathrm{PbrO}_{2}$ war nicht signifikant. Schlussfolgerungen: Wir schließen aus diesen Ergebnissen, dass in diesem Modell trotz identischer globaler zerebraler Blutflüsse nur die Anhebung des CPP mit Noradrenalin das Sauerstoffdefizit des zerebralen Gewebes reduziert. Die Infusion von HAES ist im Gegensatz dazu mit erhöhtem ICP und möglicherweise verstärkten Hirnödem. 
Thorakale Epiduralanästhesie vermindert die intestinale 086 Permeabilität und Stickstoffmonoxidproduktion in der Endotoxinämie

\section{J. Schäper, A. Elster, F. Enigk, B. Brell, H. Habazettl, M. Schäfer, M. Welte \\ Klinik für Anästhesiologie und operative Intensivmedizin, Charité-Universitätsmedizin Berlin, Campus Benjamin Franklin}

Fragestellung: In der Endotoxinämie kommt es vor allem in monozytären Zellen zu einer Aktivierung der induzierbaren NO-Synthetase mit exzessiver Produktion von Stickstoffmonoxid (NO) [1]. Im Darm führt eine stark erhöhte intestinale NO-Gewebekonzentration zur mukosalen Permeabilitätserhöhung mit vermehrter bakterieller Translokation [2]. In endotoxämischen Kaninchen führte eine regionale Sympathikolyse mittels thorakaler Epiduralanästhesie (TEA) zu einer Permeabilitätsabnahme der Darmmukosa [3]. Die Hypothese unserer Studie war, dass eine Verbesserung der intestinalen epithelialen Barrierefunktion durch TEA mit einer Verminderung der NO-Gewebekonzentration assoziiert ist. Methodik: Bei 28 Ratten wurde unter Allgemeinanästhesie ein thorakaler Epiduralkatheter (Spitze bei Th7) implantiert. Durch intravenöse Bolusgabe von $50 \mathrm{mg} / \mathrm{kg}$ Lipopolysaccharid wurde eine normotensive Endotoxinämie induziert, Kontrolltiere erhielten eine entsprechende Menge an $\mathrm{NaCl} 0,9 \%$. Zeitgleich erfolgte eine epidurale Injektion von $30 \mu \mathrm{l}$ Lidocain $1 \%$ oder $\mathrm{NaCl} 0,9 \%$, gefolgt von einer kontinuierlichen Infusion über 5 Stunden $(30 \mu \mathrm{l} / \mathrm{h})$. Nach $5 \mathrm{~h}$ Endotoxinämie wurde mittels ligierter Ileumschleife der transepitheliale Übertritt von niedermolekularem fluoreszenzmarkiertem Dextran quantifiziert. Zudem wurde die Konzentration von Stickstoffmonoxid-Metaboliten im Darm bestimmt. Ergebnisse: Endotoxämische Tiere ohne TEA wiesen im Vergleich zu gesunden Kontrolltieren eine signifikant $(p<0,05)$ erhöhte portalvenöse Dextran-Konzentration auf $(657$ [579/1106] vs. 311 [255/375], Median [25./75.Perzentile]). Die portalvenöse DextranKonzentration bei endotoxämischen Tieren mit TEA war hingegen nur gering verändert (460 [314/497]). Ebenso nahm die Konzentration von NO-Metaboliten im intestinalen Gewebe endotoxämischer Tiere ohne TEA stark zu 133 [96/207] vs. 60 [47/67]). Endotoxämische Tiere mit TEA wiesen signifikant $(\mathrm{p}<0,05)$ niedrigere Werte auf (103 [88/114]). Interpretation: Regionale Sympathikolyse mittels TEA verhindert die Endotoxin-bedingte epitheliale Permeabilitätserhöhung im Ileum von Ratten. Dies ist assoziiert mit einer verminderten intestinalen Konzentration von Stickstoffmonoxid-Metaboliten. Da monozytäre Zellen eine zentrale Rolle in der LPS-induzierten Immunantwort spielen und maßgeblich zur exzessiven NO-Produktion in verschiedenen Geweben beitragen, ist eine Beeinflussung dieser Zellen durch die TEA wahrscheinlich.

Literatur: [1] Alican I und Kubes P: A critical role for nitric oxide in intestinal barrier function and dysfunction. Am J Physiol 1998; 270:G225-237. [2] Mishima S et al: The relationships among nitric oxide production, bacterial translocation, and intestinal injury after endotoxin challenge in vivo. J Trauma 1998; 44(1): 175-182. [3] Kosugi $S$ et al: Epidural analgesia prevents endotoxin-induced gut mucosal injury in rabbits. Anesth Analg 2005; 101:265-272

Hypoxie als Auslöser von Sepsis-relevanten Veränderungen der intestinal-epithelialen Tight-junction Architektur

P. Born, H. Wohlburg, K. Jaeckel, H. E. Eltzschig, J. Karhausen Abteilung für Anästhesiologie und Intensivmedizin, Universitätsklinikum Tübingen, Eberhard Karls Universität Tübingen

Fragestellung: Im septischen Multiorganversagen ist die Sauerstoffversorgung der Darmschleimhaut durch Erhöhung des metabolischen Umsatzes und Minderung der intestinalen Durchblutung kompromittiert. Die resultierende Gewebehypoxie führt zu Barrierestörungen, welche über bakterielle Translokation und un- kontrollierte Stimulation des Darm-assoziierten-Immunsystems den Krankheitsprozess weiter antreiben können. Obwohl klinische wie experimentelle funktionelle Daten ein stimmiges Bild der Barrierestörung geben, bleiben die Veränderungen in Hypoxie auf zellulärer Ebene weiterhin schlecht charakterisiert. Methoden und Ergebnisse: Immunolokalisationsstudien mit dem Hypoxiemarker EF5 zeigten die spezifische Prädisposition der Darmschleimhaut für zellulären Sauerstoffmangel im murinen Sepsismodell („,caecal ligation puncture“). Die Folgen einer solchen hypoxischen Barrierestörung untersuchten wir im murinen Ganzkörper HypoxieModell ( $4 \mathrm{~h}$ bei $8 \% \mathrm{O}_{2}$ ). Hier zeigte sich eine Zunahme der endogenen bakteriellen Translokation (5,9 $\pm 0,2$ fache Erhöhung SerumLPS Spiegels, $\mathrm{p}<0,05)$, sowie als Ausdruck Hypoxie-bedingter Entzündungsreaktion eine Erhöhung des Darm-MPO Gehaltes $(2,10 \pm 0,05$ vs. $1,37 \pm 0,09 \mathrm{U} / \mathrm{mg}$ Gewebe, $\mathrm{p}<0,05)$. Da diese Veränderungen mit einer Störung der parazellulären Permeabilität einhergingen $(9,3 \pm 1,2$ fache Zunahme des Fluxes des Permeabilitätmarkers FITC-dextran, $\mathrm{p}<0,05)$, untersuchten wir in vitro Hypoxie-bedingten Veränderungen der tight-junctionalen Architektur. Auffallend war zunächst in Freeze-Fracture Untersuchungen eine deutliche Disorganisation der Tight-junction nach hypoxischer Exposition. Dieses fand sein Korrelat in der Herabgegulation von Tight-junction-Proteinen Zonula occludens (ZO)-2, ZO-3, Claudin-1,-2 und -3, Occludin und JAM-3 auf Protein- bzw. transkriptioneller Ebene. Interessanterweise war ZO-1 mRNA in Hypoxie $2,5 \pm 0,7$ fach induziert $(p<0,05)$, einhergehend mit einer Zunahme funktionellen ZO-1 Proteins . Die isolierte Hochregulation von ZO-1 ist mit einer Verbesserung der Barrierefunktion (Resistenzmessung mittels Voltmeter) assoziiert und ist weiterhin in zellulären Regulationsprozessen von Differenzierung und Zellzyklusprogression beteiligt. Zusammenfassung: Unsere Untersuchung charakterisiert Hypoxie-induzierte Veränderungen an der intestinal-epithelialen Tight-junction, deren uneingeschränkte Funktion für die Kompartimentalisierung potentiell schädlicher Xenobiotika im Darmlumen eine entscheidende Rolle einnimmt. Hierbei zeigt sich in Hypoxie eine Veränderung der Tight-junction Architektur und Zusammensetzung. Wir sehen in der isolierten Hochregulation von ZO-1 einen möglichen endogenen protektiven Mechanismus, der auch unter hypoxischen Bedingungen zur Aufrechterhaltung der intestinalen Barriere beträgt. Weitere Studien sind notwendig, um die Relevanz und Regulation eines solchen Mechanismus weiter zu untersuchen.

Identification of Vasodilator phosphostimulated protein 106 as a central Hif - regulated tissue permeability factor during hypoxia

P. Rosenberger, A.M. Robinson, T. Kong, J. Khoury, S.P. Colgan Brigham and Women's Hospital/Harvard Medical School Boston MA, USA und Department of Anesthesiology and Intensive Care Medicine, Tübingen University Hospital, Eberhard-Karls-University, Tübingen, Germany

Introduction: Vasodilator phosphostimulated protein (VASP) is a cytoplasmic actin binding protein regulating cytoskeletal dynamics. Previous work has shown that phosphorylation of VASP plays an important role in modulating endothelial permeability. Since vascular leak during hypoxia is linked to an increased endothelial cell permeability and stress fiber formation, we investigated possible regulation of VASP in hypoxia and its importance on vascular leak syndrome. Methods: Human Microvascular Endothelial Cells (HMEC) were grown on polystyrene permeable transwells and exposed to hypoxia $\left(\mathrm{pO}_{2} 20\right.$ torr) up to $48 \mathrm{~h}$. Transendothelial permeability as measured with $70 \mathrm{KD}$ Fitc-Dextran. Protein and mRNA was analyzed at the termination of the experiment. The VASP $5^{\prime}$ flanking region and promoter were cloned by PCR and ligated into a PGL3 luciferase reporter vector. Wild type (Wt) mice and animals conditionally lacking intestinal epithelial hypoxia-inducible factor (hif $1 \alpha-/-$ ) were exposed to $8 \mathrm{~h}$ of normobaric hypoxia (8\%). Mice were gavaged $4 \mathrm{kd}$ FITC- 
Dextran immediately prior to the start of the experiment. VASP mRNA and protein expression was determined in mucosal scrapings of the colon, lungs, kidneys and liver of all mice. Human saphenous vein was obtained from patients undergoing aorta-coronary bypass surgery and exposed to $8 \mathrm{~h} 1 \%$ hypoxia and mRNA extracted. Results: Hypoxia increased transendothelial Fitc-Dextran flux by more than $30 \%(24 \mathrm{~h})$ and by nearly $40 \%$ after $48 \mathrm{~h}$ hypoxia. This increase coincided with a decrease of VASP protein and mRNA by nearly $60 \%$ after $4 \mathrm{~h}$ that only partially recovered after $48 \mathrm{~h}$ in HMEC. In T84 intestinal epithelial cells, a similar reduction of VASP mRNA was observed. VASP siRNA transfected HMEC monolayers demonstrated increased permeability to passive flux. Similarly, transfection of HeLa cells with the VASP-PGL3 reporter plasmid showed a nearly $60 \%$ decrease in luciferase activity compared to normoxic controls. With regard to in vivo determinants, Wt mice exposed to hypoxia demonstrated increased serum FITC-Dextran fluorescence, reflective of increased epithelial permeability, whereas conditional hifl $a-I-$ mice showed no increase in plasma fluorescence in hypoxia. VASP mRNA was significantly reduced in Wt-mice exposed to hypoxia, whereas the hif1 $a-/-$ mice demonstrated no significant change in VASP mRNA level in the intestinal mucosa. In human saphenous vein VASP mRNA were reduced by more than $50 \%$ after $8 \mathrm{~h}$ hypoxia. Conclusion: Hypoxia significantly influences VASP protein and mRNA levels in cell culture and animals exposed to hypoxia. This hypoxia-induced loss of VASP is linked to increased tissue permeability during acute hypoxia, and is likely regulated by HIF-1 $a$. We conclude that VASP may participate in tissue permeability changes associated with hypoxia.

\section{Pathophysiologie II}

\author{
Verstärkt mobilisierte endotheliale Progenitorzellen \\ in der Sepsis \\ C. Hanusch ${ }^{1}$, N. Rafat ${ }^{1}$, P. Brinkkoetter ${ }^{2}$, J. Schulte ${ }^{2}$, \\ F.J. van der Woude ${ }^{2}$, B.A. Yard $^{2}$, K. van Ackern ${ }^{1}$, G.C. Beck ${ }^{1}$ \\ ${ }^{1}$ Klinik für Anästhesiologie und operative Intensivmedizin, \\ ${ }^{2}$ V. Medizinische Klinik, Universitätsklinikum Mannheim
}

Fragestellung: Das Ausmaß der mikrovaskulären Schädigung spielt in der Pathogenese der Sepsis durch Veränderungen der Endothelzellfunktion und des endothelialen Phänotypes eine zentrale Rolle. Diese endothelialen Mikropartikel und zirkulierenden Endothelzellen wurden bei Sepsis vermehrt im Blut nachgewiesen. Eine Reparatur des geschädigten Endothels könnte einen vorteilhaften Einfluss auf den Verlauf einer Sepsis haben. Es gibt zunehmend Hinweise, dass endotheliale Vorläuferzellen (EPC), welche durch eine Reihe von Mediatoren (,vascular endothelial growth factor (VEGF), „granulocyte-macrophage colony-stimulatingfactor (GM-CSF) und Erythropoetin (EPO)) aus dem Knochenmark freigesetzt werden, eine Reendothelialisierung bewirken können. Unter verschiedenen Bedingungen endothelialer Schädigung wurde bereits eine signifikant erhöhte Zahl zirkulierender EPC's (cEPC) gegenüber Gesunden dokumentiert und therapeutische Effekte nachgewiesen. Mittels dieser Studie sollte untersucht werden, ob auch bei Sepsispatienten eine erhöhte Anzahl cEPCs im Blut nachzuweisen ist und ob diese Zahl mit der Mediatorproduktion, der Schwere und der Mortalität der Sepsis korreliert. Methodik: Die cEPC-Konzentration wurde bei 32 Sepsispatienten, 15 Intensivpatienten ohne Sepsis und 15 gesunden Probanden bestimmt. Aus dem Vollblut wurden periphere mononukleäre Blutzellen (PBMC) über die Ficoll-Dichtezentrifugation isoliert, mit Antikörpern gegen EPC-Oberflächenmarker CD133, CD34, VEGF-R2 und AnnexinV inkubiert und mittels FACS-Analyse ausgezählt. Die Serumkonzentrationen von VEGF, GM-CSF und EPO wurden mittels ELISA bestimmt. Der Schweregrad der Sepsis wurde mittels APACHE II Score eingestuft. Ergebnisse: Die Konzent- ration cEPC's war bei Patienten mit Sepsis signifikant gegenüber nicht-septischen und gesunden Kontrollen erhöht und änderte sich im Verlauf der Sepsis nicht. Die hohen EPC-Konzentrationen bei Sepsis korrelierten mit einer besseren Überlebensrate. Die EPC-Konzentration war bei den Überlebenden $(0,170 \pm 0,06 \%)$ signifikant höher als bei den verstorbenen Patienten $(0,06 \pm 0,028 \%)$. Der EPC-Anteil der Letzteren war signifikant höher als bei den Gesunden. Eine Korrelation gab es zwischen der EPC-Konzentration und den APACHE II-Werten, je höher die EPC-Konzentrationen umso geringer die APACHE-Scores. Ebenfalls wurde eine Korrelation zwischen den EPC-Konzentrationen und den Serumspiegeln von VEGF $(\mathrm{r} 2=0,54 ; \mathrm{p}<0,0001), \mathrm{GM}-\mathrm{CSF} \quad(\mathrm{r} 2=0,47$; $\mathrm{p}<0,0001)$ und Epo(r2=0,41; $\mathrm{p}<0,002)$ gefunden, welche bei Sepsis gegenüber der Kontrollen signifikant erhöht waren. Interpretation: Diese Studie unterstützt die Hypothese, dass mit Beginn einer Sepsis endotheliale Reparaturmechanismen in Form der Mediator-induzierten vermehrten Freisetzung endothelialer Vorläuferzellen aus dem Knochenmark initiiert werden. Die gemessenen cEPC-Konzentrationen korrelierten mit der Mortalität und könnten als diagnostischer oder prognostischer Parameter des Gefäßschadens und der Schwere der Sepsis dienen.

Spermin-NONOate und Endothelschaden

bei experimenteller Endotoxinämie

Rolle von Spermin: Vehicle or tool?

C. Peter, C. Barth, E. Martin, A. Walther

Klinik für Anästhesiologie; Universitätsklinikum Heidelberg

Einleitung: Beim frühen Leukozytenunabhängigen Endothelschaden in der Endotoxinämie spielen Thrombozyten (PLT) eine wesentliche Rolle. Vorangegangene Untersuchungen haben gezeigt, dass der frühe Endothelschaden durch Glykoprotein (GP) IIb/IIIa Antagonisten und Stickstoffmonoxid (NO) inhibiert werden kann [1-2]. Diazenolat-NO-Donatoren wie Spermin NONOate nutzen Spermin (SP) als Trägersubstanz. SP kann jedoch in-vitro die PLT-Aggregation und die GP IIb/IIIa Aktivität inhibieren [3]. Ziel dieser Studie war es, die Wirkung von Spermin auf den Endothelschaden bei Endotoxinämie zu untersuchen. Material und Methodik: Bei männlichen Wistar-Ratten wurde mittels Intravitalmikroskopie an postkapillären Venolen des Mesenterium die Plasmaextravasation $(\mathrm{PE}) \mathrm{zu}$ den Zeitpunkten $\mathrm{t}=0,60$ und 120 Minuten nach Start des Experiments gemessen. Die Experimente wurden in einen Leukozyten-unabhängigen (WBC(-)) und einen Leukozyten-abhängigen $(\mathrm{WBC}(+))$ Teil separiert. WBC(-)-Versuchsbedingungen wurden durch die Gabe des L-Selektininhibitors Fucoidin erzielt (Gruppen A-D). Tiere der Gruppe A erhielten SperminNONOate, Tiere der Gruppe B die Trägersubstanz Spermin, Tiere der Vergleichsgruppe (Gruppe C) erhielten eine äquivalente Menge $\mathrm{NaCl} 0,9 \%$, jeweils gefolgt von einer kontinuierlichen Endotoxin [ETX]-Infusion nach der Ausgangsmessung. Eine unbehandelte NaCl-Gruppe (Gruppe D) diente als Kontrolle. Im Leukozytenabhängigen Versuchssetting erhielten die Tiere der Gruppe X wiederum eine kontinuierliche ETX-Infusion, die Tiere der Gruppe Y zusätzlich Spermin. Eine unbehandelte NaCl-Gruppe (Gruppe Z) diente als Kontrolle. Statistik: Zweifaktorielle Varianzanalyse (ANOVA-Test) mit anschließender Bonferroni-Korrektur. Signifikanzniveau: $\mathrm{p}<0,05$. Ergebnis: WBC(-): Die PE zeigte in allen Gruppen eine signifikante Zunahme vom Ausgangswert ( $0 \mathrm{~min}$ : A: 0,05; B: 0,04; C: 0,11; D: 0,12) zum Endpunkt $t=120$ min. $\mathrm{Zu}$ dem Zeitpunkt $\mathrm{t}=20 \mathrm{~min}$ war die PE der Gruppe $\mathrm{C}$ signifikant gegenüber den Gruppen A, B und D erhöht. Zwischen Gruppe A, B vs. Gruppe $\mathrm{D}$ fanden sich keine signifikanten Unterschiede (120 min: A: 0,21; B: 0,11; C: 0,53; D: 0,28). WBC(+): Die PE zeigte in allen Gruppen eine signifikante Zunahme vom Ausgangswert $(0$ min: $\mathrm{X}: 0,15$; Y: 0,08; $\mathrm{Z}: 0,11)$ zum Endpunkt $\mathrm{t}=120 \mathrm{~min} . \mathrm{Zu}$ dem Zeitpunkt $t=120$ min bestand eine signifikant erniedrigte PE in der Kontrollgruppe. Zwischen den mit ETX behandelten Tieren ergaben sich keine signifikanten Unterschiede (120 min: X: 0,51; Y: 0,43; Z: 0,28). Schlussfolgerung: Nur nach Unterbindung der 
Leukozyten-Endothel Interaktion mit Fucoidin kann mit Spermin die PE bei Endotoxinämie vergleichbar den Effekten durch Spermin-NONOate reduziert werden. Die alleinige Gabe von Spermin oder Fucoidin führt zu keiner Beeinflussung des Endothelschadens bei Endotoxinämie.

Literatur: [1] Walther A et al: Shock, 2003; 20:286-291. [2] Walther A et al: Microcirculation 2004; 11:79-88. [3] Coronade-la-Pena N et al: J Cardiovasc Pharmacol 2005; 46:216-221

\section{Trapanal supprimiert die Lipopolysaccharid-induzierte}

172

\section{Tissue Factor Expression}

\section{S. Ozlügedik, J. Peters, M. Hartmann}

Klinik für Anästhesiologie und Intensivmedizin;

Universitätsklinikum Essen

Einleitung: Gram-negative Sepsis führt zu einer Aktivierung des Immunsystems, vermittelt durch die Freisetzung von Lipopolysaccharid (LPS) und die Stimulation von Toll-like Rezeptor 4. Wichtige Konsequenz dieser Aktivierung ist die Expression von Tissue Factor auf der Oberfläche von Monozyten. Diese kann zu mikrovaskulärer Thrombenbildung (DIC), Organischämie und -dysfunktion führen. Thiopental hemmt die Immunantwort verschiedener Zelltypen, allerdings ist der Wirkmechanismus unklar [1-3]. Aufgrund der immunmodulatorischen Wirkung könnte Thiopental auch die Tissue Factor Expression von Monozyten bei Gram-negativer Sepsis beeinflussen. Wir testeten die These, dass Thiopental die LPS-induzierte Tissue Factor Expression von humanen Vollblutproben supprimiert. Methodik: Nach Rücksprache mit der Ethikkommission wurden Zitrat-Vollblutproben von Probanden mit verschiedenen Thiopentalmengen im therapeutischen Konzentrationsbereich $(0-1 \mathrm{mg} / \mathrm{ml}, 1 \mathrm{~h})$ versetzt. Danach wurde die Tissue Factor Expression durch Inkubation mit LPS $(100 \mu \mathrm{g} /$ $\mathrm{ml}, 4 \mathrm{~h}, 37^{\circ} \mathrm{C}$ ) induziert. Die Bestimmung der Tissue Factor-Aktivität erfolgte durch Messung der Clotting Time mittels Thrombelastographie [4]. Mögliche Interaktionen von Barbiturat und Tissue Factor-Molekül wurden untersucht, indem der Einfluss von Thiopental auf die Aktivität von Tissue Factor-Standards bestimmt wurde. Zur Abschätzung der durch LPS-induzierten Tissue Factor Konzentration wurde eine Dosis-Wirkungskurve für Tissue Factor mittels eines Standards erstellt. ASIS und Cycloheximide wurden verwendet, um die Bedeutung von Tissue Factor bzw. Proteinsynthese für den koagulatorischen LPS-Effekt nachzuweisen. Statistik: Wilcoxon-Test. Ergebnisse: Lipopolysaccharid $(100 \mu \mathrm{g} /$ $\mathrm{ml}$ ) führt $\mathrm{zu}$ einer deutlichen Verkürzung der Clotting Time von $760 \pm 18 s$ auf $228 \pm 30 s(n=4 ; p<0,05)$, Versuche mit dem Proteinsynthesehemmer Cycloheximide $(35 \mu \mathrm{M})$ und den TissueFactor-Inhibitor ASIS $(50 \mathrm{mM})$ zeigen, dass dieser Effekt durch die Expression von Tissue Factor hervorgerufen wird. Thiopental hemmt den koagulatorischen LPS-Effekt signifikant: In Gegenwart des Barbiturats wurde die Clotting Time von $782 \pm 85 \mathrm{~s}$ auf lediglich $425 \pm 45 \mathrm{~s}$ verkürzt $(\mathrm{n}=4)$. Ein direkter Einfluss von Thiopental auf Aktivität von Tissue Factor wurde ausgeschlossen: Die Clotting Time in Gegenwart eines exogenen Tissue Factor Standards wurde durch Thiopental nicht beeinflusst $(259 \pm 11 \mathrm{~s}$ vs. $265 \pm 37 \mathrm{~s} ; \mathrm{n}=4$; n.s.). Anhand der Dosis-Wirkungskurve für den exogenen Tissue Factor Standard konnte zudem gezeigt werden, dass Thiopental die Tissue Factor Expression um $90 \%$ hemmt. Zusammenfassung: Thiopental hemmt in klinisch relevanten Konzentrationen die durch LPS induzierte Tissue Factor Expression im Vollblut.

Literatur: [1] Salman et al: Life Sci 1998; 63:2221-2226. [2] Correa-Sales C et al: Int J Immunopharmacol 1997; 19:117128. [3] Matjaz Humar,1 et al: J Pharmacol Exp Ther 2004; 311: 1232-1240. [4] Zacharowski et al: Thromb Haemost 2006; 95:557-561
Heat Shock hemmt die Lipopolysaccharid-induzierte Aktivierung der Blutgerinnung

\section{Sucker, V. Stoldt, M. Hartmann}

Klinik für Anästhesiologie und Intensivmedizin; Universitätsklinikum Essen

Fragestellung: Tissue Factor, der prinzipielle Initiator der Blutgerinnung in vivo, ist unter physiologischen Bedingungen im Blutstrom nicht nachweisbar. Während einer Gram-negativen Sepsis induziert Lipopolysaccharid jedoch die Expression von Tissue Factor auf Monozyten, die Folgen sind disseminierte intravasale Coagulopathie, Ischämie und Organdysfunktion $[1,2]$. Da zahlreiche Studien den Einfluss der Temperatur auf das Immunsystem zeigen, untersuchten wir den Einfluss von Hyperthermie auf die LPS-induzierte Tissue Factor-Aktivität im Vollblut und von isolierten Leukozyten. Methoden: Vollblut wurde nach Rücksprache mit der Ethikkommission von Probanden entnommen, Leukozyten wurden durch Differentialzentrifugation isoliert. In der Kontrollgruppe wurde Vollblut $5 \mathrm{~h}$ lang bei $37 \mathrm{Grad} \mathrm{C}$ inkubiert, in der Hyperthermiegruppe erfolgte zunächst eine Inkubation bei $43^{\circ} \mathrm{C}$ $(2 \mathrm{~h})$ gefolgt von einer Inkubation bei $37^{\circ} \mathrm{C}(3 \mathrm{~h})$. Anschließend wurde die clotting time nach Rekalzifizierung der Proben bestimmt (Amelung KC4 Coagulometer). Analoge Versuche wurden mit Leukozytensuspensionen durchgeführt. Der Mechanismus der LPS-induzierten Gerinnungsaktivierung wurde mit dem Tissue Factor-Inhibitor ASIS und dem Proteinsyntheseinhibitor Cycloheximide eruiert. Kontrollexperimente (Trypan Blau, FACS) wurden zum Nachweis der Zellintegrität durchgeführt. Ergebnisse: Inkubation von Vollblut mit LPS $(100 \mu \mathrm{g} / \mathrm{ml})$ bei $37^{\circ} \mathrm{C}(5 \mathrm{~h})$ führte $\mathrm{zu}$ einer deutlichen Reduktion der Clotting Time von $357 \pm 108 \mathrm{~s}$ auf $82 \pm 8 \mathrm{~s} \quad(\mathrm{n}=12 ; \mathrm{p}<0,05)$. Wurden Vollblutproben jedoch $2 \mathrm{~h}$ lang bei $43^{\circ} \mathrm{C}$ und dann $3 \mathrm{~h}$ bei $37^{\circ} \mathrm{C}$ inkubiert, so zeigte LPS keinerlei Einfluss auf die clotting time, die $312 \pm 66$ s bzw. $277 \pm 65 \mathrm{~s}$ betrug $(n=12 ; n . s$.$) . Die LPS-induzierte Gerinnungsaktivierung$ wird durch die de novo Synthese von Tissue Factor vermittelt wie durch Versuche mit ASIS und Cycloheximide gezeigten werden konnte. Auch in Leukozytensuspensionen hemmt Hyperthermie die LPS-induzierte Tissue Factor-Aktivität. Der Anteil defekter Leukozyten, der mit Hilfe des Trypan Blau Ausschlusses bestimmt wurde, war in Hyperthermie- und Kontrollgruppe identisch. Zudem zeigte die FACS-Analyse, dass Hyperthermie die Integrität der Fluoreszenzmarkierten Monocyten nicht beeinflusst. Schlussfolgerung: Hyperthermie inhibiert die LPS-induzierte Tissue Factor-Aktivität in humanen Vollblutproben, der Einfluss von Fieber auf die Tissue Factor-Aktivität bei Sepsis bedarf weiterer Untersuchungen.

Literatur: [1] Opal SM: Interactions between coagulation and inflammation. Scand J Infect Dis 2003; 35:545-554. [2] Dempfle CE: Coagulopathy of sepsis. Thromb Haemost 2004; 91:213-224

Intakte, aber verzögerte koronare Autoregulation 186 als mögliche Ursache für einen verminderten Ischämie/ Reperfusionsschaden am septischen Herzen

\section{Y. A. Zausig, L. Binder, E.K. Löffler, B. Sinner, W. Zink,}

B.M. Graf

Zentrum für Anästhesiologie, Rettungs- und Intensivmedizin, Zentrum Innere Medizin, Abt. Klinische Chemie, Universität Göttingen

Septische Herzen $(\mathrm{SH})$ zeigen nach Ischämie einen geringeren mechanischen und morphologischen Reperfusionsschaden. Auffällig ist eine deutliche Diskrepanz der koronarvaskulären Reaktion beim Vergleich von SH und gesunden Kontrollherzen (KH) in der Reperfusionsphase. Wir postulierten eine intakte koronare Autoregulation als mögliche Ursache für den verminderten Reperfusionsschaden in SH. Gesunde $(\mathrm{N}=7)$ und septische $(24 \mathrm{~h}$ nach Induktion durch CLP, $\mathrm{N}=9$ ) Rattenherzen (Wistar, 230-260 Gramm) wurden im isolierten Modell nach Langendorff mit 350 Schlägen pro Minute stimuliert, und retrograd unter konstanten Fluss perfundiert. Nach einer Stabilisierungsphase ( $t=15$ Min.) wurden die 
Herzen einer normothermen No-Flow-Ischämie ( $t=40$ Min.), gefolgt von einer Reperfusion ( $t=40$ Min.) ausgesetzt. Dabei wurden elektrophysiologische, mechanische (z.B. Kontraktilität) und metabolische (z.B. koronarer Fluss, Sauerstoffverbrauch) Parameter gemessen. Creatininkinase, Laktatdehydrogenase und Troponin $\mathrm{T}$ wurden als Ausdruck des zellulären Schadens bestimmt. Als Nachweis der Funktionstüchtigkeit des koronaren Systems wurde $0,2 \mathrm{ml}$ Adenosin $(200 \mu \mathrm{M})$ einmalig als Bolus vor und nach der Ischämie intrakoronar verabreicht. Die statistische Auswertung erfolgte mittels T-Test. Alle Daten sind als Mittelwerte \pm Standardfehler dargestellt. $\mathrm{P}<0,05$ wurde als signifikant betrachtet. In der präischämischen Phase fallen die $\mathrm{SH}$ im Vergleich $\mathrm{zu}$ den $\mathrm{KH}$ durch eine signifikante Reduktion des linksventrikulären Drucks (LVP, SH: $63,4 \pm 5,1$ vs. KH: $114,6 \pm 4,5 \mathrm{mmHg}$ ) und der Kontraktilität (+dp/dt, SH:1711,9 $\pm 153,9$ vs. KH: $3003,3 \pm 89,9 \mathrm{mmHg} / \mathrm{s}$ ) auf. In der Reperfusionsphase kommt es bei den $\mathrm{SH}$ im Gegensatz zu den $\mathrm{KH}$ nur $\mathrm{zu}$ einem marginalen Verminderung des LVP $(58,3 \pm 11,8 \mathrm{mmHg})$ und $\mathrm{der}+\mathrm{dp} / \mathrm{dt}(1538,8 \pm 340,0 \mathrm{mmHg} / \mathrm{s}) \mathrm{im}$ Vergleich zu den präischämischen Werten. Begleitend zeigt sich postischämisch eine signifikante Zunahme des koronarvaskulären Widerstandes bei den $\mathrm{KH}(73,9 \pm 5,4 \mathrm{mmHg})$ gegenüber den präischämischen Ausgangswerten $(48,7 \pm 1,1 \mathrm{mmHg})$, und gegenüber den $\mathrm{SH}(53,9 \pm 2,9$ vs. $57,8 \pm 2,6 \mathrm{mmHg})$. Jedoch zeigt sich nach 40 Minuten Reperfusion eine Angleichung der koronaren Perfusionsdrücke (SH: $65,2 \pm 2,9$ vs. $\mathrm{KH}: 67,6 \pm 6,3 \mathrm{mmHg}$ ). $\mathrm{SH}$ zeigen nach Adenosingabe sowohl prä- als auch postischämisch eine Zunahme des kororonaren Flusses $(+49,4$ bzw. $+42,1 \%)$. Hingegen zeigt sich bei den $\mathrm{KH}$ nur in der präischämischen Phase eine intakte koronare Regulation (präischämisch: $+27,9 \%$, postischämisch $+1,2 \%$ ). Nach einer Ischämie von 40 Minuten zeigt sich am septischen Herzen ein signifikant geringerer mechanischer Reperfusionsschaden ausgedrückt in einem nahezu unveränderten LVP und $+\mathrm{dp} /$ dt. Die begleitende verzögerte Zunahme des koronarvaskulären Widerstandes in der Reperfusionsphase und eine (nach Adeonsingabe) erhaltene Koronarregulation in der septischen Gruppe weist auf eine intakte, aber verzögert reagierende kororonarvaskuläre Autoregulation als mögliche Ursache für das kardioprotektive Phänomen in der Sepsis hin.

Stressregulierte Signaltransduktion und physiologische Parameter: Eine Evaluation des murinen Sepsismodells

H. V. Groesdonk, F. Wagner, B. Hoffarth, S. Heydrich, S. Schlottmann, U. Senftleben

Universitätsklinikum Ulm, Abtl. Anästhesiologie und Intensivmedizin

Einleitung: Das Modell der "cecal ligation and puncture (CLP)“ ist ein klinisch relevantes Modell und eignet sich zur Untersuchung lokaler und systemischer Entzündungsreaktionen. Trotz zahlreicher Studien existieren bis heute keine Daten bzgl. der Aktivierungskinetik stressregulierter Signaltransduktionswege und der begleitenden Pathophysiologie im Verlauf von verschiedenen Schweregraden dieses Modells. Ziel dieser Untersuchung war es, inflammatorische und pysiologische Veränderungen in einem CLP-Sepsis Modell mit steigender Letalität zu beschreiben. Methodik: Weibliche C57BL/6J-Mäuse erhielten entweder eine CLP-Operation mit steigender Nadelgröße (G26, G22 und G18) oder eine Sham-Operation. Bis zu 9 Tagen danach wurden die Organe Lunge und Milz entnommen und zelluläre Extrakte generiert. Die DNA-Bindungsaktivität von stressregulierten Transkriptionsfaktoren wurde mittels Gelshift Analysen untersucht. TUNEL-Assay's dienten der Ermittlung von Apoptose-Raten. Zusätzlich wurden 2 Wochen vor der jeweiligen Intervention (CLP/Sham) Minitransmitter (Fa. DSI) subkutan implantiert. Nachfolgend wurden Herzfrequenz (HF), Temperatur und Aktivität der Tiere für die Dauer von 10 Tagen aufgezeichnet. Mortalitäts-Raten und ein muriner „Sepsis Severity Score“ wurden bis zu 10 Tagen bestimmt. Ergebnisse: Erwartungsgemäß zeigten die Mortalitätsraten eine signifikante Korrelation zwischen Nadelstärke und Mortalität $(26 \mathrm{G}=$
$10 \% / 22 \mathrm{G}=30 \% / 18 \mathrm{G}=100 \%$ ). Interessanterweise war der Sepsis Severity Score nicht prediktiv im Hinblick auf die Mortalität. Die NF- $\kappa$ B DNA-Bindungsaktivität in Lunge und Milz Sham operierter Mäuse zeigte einen charakteristischen biphasischen Verlauf. Im Gegensatz dazu war die NF- $\kappa$ B DNA-Bindungsaktivität in beiden Organen der CLP (G18)-Mäuse nach $6 \mathrm{~h}$ deutlich reduziert und zeigte im weiteren Verlauf keinen erneuten Anstieg. Die G22 operierte Gruppe zeigte ebenfalls einen biphasischen Verlauf mit einem verstärkten 2 Aktivierungs-Peak. Im Vergleich zu Lungenund Milz-Apoptose-Raten der Sham-Mäuse war die Apoptose-Rate und die Organdestruktion beider Organe der CLP-Mäuse drastisch erhöht und korrelierte positiv mit der Nadelstärke. Die Herzfrequenz der Sham-Tiere zeigte einen charakteristischen Tag/ Nacht-Rhythmus, wohingegen die CLP-Operation zur Abnahme der HF und zu einem Verlust dieses Rhythmus führte. Zunahme der allgemeinen Aktivität und der Köpertemperatur korrelierten negativ mit der Schwere der Operation. Schlussfolgerung: Unsere Untersuchungen zeigen deutlich, dass mit zunehmendem Schweregrad der Sepsis physiologische Parameter suppremiert werden, es zu einem Verlust des Tag/Nacht-Rhythmus und zu einer zunehmenden Organdestruktion kommt. Dies korreliert positiv mit der Morbidität und Mortaliät in diesem Modell. Interessanterweise ist die NF- $\kappa$ B-DNA-Bindungsaktivität in schwer septischen Mäusen stark reduziert, was vermuten lässt, dass eine adäquate Aktivierbarkeit dieses Signalweges für den Organismus zur Erholung von dieser Erkrankung nötig ist.

Mortalitätsreduktion durch verstärkte lymphozytäre NF- $\kappa$ B Aktivität im schwerern murinen Sepsismodell

H.V. Groesdonk ${ }^{1}$, B. Hoffarth ${ }^{2}$, S. Heydrich ${ }^{2}$, S. Schlottmann ${ }^{2}$, U. Senftleben ${ }^{2}$

${ }^{1}$ Universität Leipzig, Herzzentrum, Abtl. Anästhesiologie und Intensivmedizin II, ${ }^{2}$ Universitätsklinikum Ulm, Abtl. Anästhesiologie und Intensivmedizin

Fragestellung: Immunologische Dysregulationen sowie Mortalität sind im Verlauf der schweren Sepsis oftmals mit einer erhöhten lymphozytären Apoptose-Rate assoziiert. Trotz der Erkenntnis, dass Prävention der Lymphozyten-Apoptose das Überleben im septischen Mausmodell verbessert, ist nur wenig über die zugrundeliegenden molekularen Mechanismen bekannt. Es gibt Hinweise, dass eine intakte Funktion des IKK/NF- $\kappa$ B-Signalweges in diesem Zusammenhang eine wichtige regulatorische Rolle spielt. In dieser Studie erarbeiteten wir den Zusammenhang zwischen sepsisbedingter lymphozytärer Apoptose und der lymphozytären NF- $\kappa \mathrm{B}$ DNA-Bindungsaktivität im murinen Sepsismodell. Weiterhin wurden die Auswirkungen einer verstärkten lymphoyzytären NF- $\kappa \mathrm{B}$ DNA-Bindungsaktivität in diesem Modell untersucht. Methodik: Weibliche C57BL/6J-Mäuse erhielten entweder eine CLP [20G] oder eine Sham-Operation. Bis zu $48 \mathrm{~h}$ danach wurden die Organe Thymus und Milz entnommen und zelluläre Extrakte generiert. Die NF- $\kappa$ B DNA-Bindungsaktivität wurde mittels Gelshift Analysen untersucht. Apoptose-Raten wurden durch TUNEL-Assay's ermittelt und histologische Untersuchungen zusätzlich durchgeführt. Mortalitätsraten wurden bis $\mathrm{zu} 48 \mathrm{~h}$ bestimmt. Um die Auswirkungen einer verstärkten lymphozytären NF- $\kappa$ B-Aktivität zu untersuchen, wurden Ikba-/- oder wild-typ (wt) Leberstammzellen in zuvor subletal bestrahlte Rag1-/- Mäuse transplantiert und die oben beschriebenen Untersuchungen nach 6 wöchiger Rekonstitution durchgeführt. Ergebnisse: Die NF- $\kappa$ B DNA-Bindungsaktivität in Thymus und Milz Sham operierter C57BL/6J-Mäuse zeigt einen charakteristischen biphasischen Verlauf mit einem deutlichen Anstieg nach $6 \mathrm{~h}$ und einem zweiten Aktivierungs-Peak nach $24 \mathrm{~h}$. Im Gegensatz dazu war die NF- $\kappa$ B DNA-Bindungsaktivität in beiden Organen der Sepsis-Mäuse nach $6 \mathrm{~h}$ deutlich reduziert und zeigte im weiteren Verlauf keinen erneuten Anstieg. Im Vergleich zu Thymus- und Milz-Apoptose-Raten der Kontrollmäuse war die Apoptose-Rate beider Organe der CLP-Mäuse nach $12 \mathrm{~h}$ und $24 \mathrm{~h}$ drastisch erhöht. Ergänzend zeigten die histologi- 
schen Untersuchungen eine verstärkte Organdestruktion. Erstaunlicherweise zeigte die NF- $\kappa$ B DNA-Bindungsaktivität im Thymus und in der Milz der CLP-operierten Rag1Ikba-/- Mäuse einen mit der Bindungsaktivität Sham operierter C57BL/6J-Mäuse vergleichbaren biphasischen Verlauf. Thymus- und Milz-ApoptoseRaten der Rag1Ikba-/- CLP-Mäuse glichen ebenfalls denen der C57BL/6J-Sham-Mäuse. Zuletzt zeigten die Rag1Ikba-/- CLPMäuse eine deutlich verbesserte Überlebensrate nach $48 \mathrm{~h}$ schwerer Sepsis (0\% C57BL/6J-CLP vs. $40 \% \operatorname{Rag} 1 \mathrm{Ikb} a-/-)$. Diskussion: Es zeigte sich, dass eine verminderte lymphozytäre NF- $\kappa \mathrm{B}$ DNABindungsaktivität mit gesteigerten Apoptoseraten in Thymus und Milz assoziiert ist. Andererseits konnte in dieser Studie erstmals gezeigt werden, dass verstärkte lymphozytäre NF- $\kappa$ B-DNA-Bindungsaktivität diese Apoptosevorgänge verhindert und das Überleben im murinen Modell der schweren Sepsis verbessert.

\section{Tyrosine phosphorylation modulates vascular response to lipopolysaccharide}

192

D. Pavlovic, H. Erber, M. Manthey, T. Wenzel, A. Stier, M. Wendt, Ch. Lehmann

Klinik und Poliklinik für Anästhesiologie und Intensivmedizin, Ernst-Moritz-Arndt-Universität Greifswald

In a model of vascular hypo-reactivity of sepsis, rings of rat aorta (RA) with or without endothelium ( \pm ENDO), or human mesenteric artery (HMA), were incubated in vitro $(6 \mathrm{~h}, \mathrm{n}=8-10$ per group) with lipopolysaccharide from escherichia coli (LPS, 10-3 or $10-4 \mathrm{~g} / \mathrm{l})$, treated $(10 \mathrm{~min})$ with various agents and response to phenylephrine (PE) examined. The nitric oxide production inhibitor L-NAME $(5 \times 10-4)$ and cGMP inhibitor ODQ $(5 \times 10-5)$ abolished LPS-induced hypo-reactivity. Genistein (tyrosine kinase inhibitor) attenuated maximal tension $\left(\mathrm{T}_{\max }\right)$ while sodium orthovanadate $(\mathrm{OV})$ increased response to $\mathrm{PE} ; \mathrm{T}_{\max }(\mathrm{kg} / \mathrm{g}$, dry muscle): control vs. OV, RA(-ENDO): $0.87 \pm 0.19$ vs $1.42 \pm 0.23$ (10-7); $1.56 \pm 0.28(10-6)$ and $2.33 \pm 0.69(10-5) ; \mathrm{RA}(+\mathrm{ENDO}): 0.88 \pm 0.21$ vs. $1.53 \pm 0.35(10-7) ; 1.35 \pm 0.30(10-6)$ and $2.55 \pm 0.68(10-5)$, and HMA(+ENDO): $1.12 \pm 0.23$ vs. $0,37 \pm 0.14(10-7) ; 2.06 \pm 0.21$ $(10-6)$ and $3.00 \pm 0.07(10-5)$. Tyrosine phosphorylation pathway may play an important role in modulation of the LPS induced vascular hypo-reactivity.

Die kardiale Kontraktion nach Verbrennungstrauma 197

\section{"Crosstalk" zwischen Komplementsystem} und toll-like-Rezeptor-System?

\section{A.D. Niederbichler, L.M. Hoesel, K. R. Ipaktchi, J.V. Sarma,}

P. Kolokythas, H.A. Adams, S.C. Wang, M.R. Hemmila, P. M. Vogt Medizinische Hochschule Hannover, Klinik u. Poliklinik $f$.

Plastische Hand- und Wiederherstellungschirurgie,

Brandverletztenzentrum Niedersachsen

Einleitung: Experimentelle Ansätze deuten auf eine Interaktion zwischen den Rezeptoren des Komplementsystems und toll-likeRezeptoren (TLRs) hin. Die Mechanismen sind jedoch noch unklar. Kardiomyozyten exprimieren den C5a-Rezeptor und den TLR4-Rezeptor, beide spielen eine zentrale Rolle bei der Signaltransduktion der verbrennungsinduzierten Kardiodepression. In dieser Studie untersuchten wir im standardisierten Verbrennungsmodell den Effekt von C5a-Blockade und nachfolgender bakterieller Endotoxinexposition auf die kardiale Kontraktion in vivo und in vitro. Methoden: Ratten $(\sim 300 \mathrm{~g})$ wurde eine $3 .^{\circ}$ Verbrennungsverletzung von $30 \%$ KOF zugefügt. Kontrolltiere erhielten keine Verbrennungsverletzung. Sofort nach Trauma wurde ein eigens hergestellter spezifischer C5a-Antikörper oder IgG (Kontrolle) i.v. injiziert. 1, 6, 12 und $24 \mathrm{~h}$ nach dem Trauma wurden Kardiomyozyten mittels Langendorff-Perfusion isoliert, mit $10 \mathrm{~g}$ Endotoxin (E. coli Serotyp 011:B4) als second-hit inkubiert, und auf Sarkomerkontraktilität mit dem MyoCam ${ }^{\circledR}$ System untersucht. Zum Zeitpunkt $1 \mathrm{~h}$ und $24 \mathrm{~h}$ nach dem Trauma wurden kardiale Funk- tionsparameter mittels linksventrikulärer Mikrokatheterisierung aufgezeichnet. Statistik: Analysis of Variance (ANOVA) und Tukey's post-hoc Tests. Ergebnisse: Nach Verbrennungstrauma zeigte sich eine signifikante Depression der kardialen Kontraktilität, sowohl auf Sarkomerebene als auch auf Organebene. Die niedrigsten Werte wurden zum Zeitpunkt $\mathrm{t}=1 \mathrm{~h}$ nach Trauma gemessen. C5a-Blockade nach Verbrennungstrauma verbesserte die kardiale Kontraktilität signifikant. Am deutlichsten war dieser Effekt zum Zeitpunkt $t=24 \mathrm{~h}$ nach Trauma messbar ( $\mathrm{p}<0,05$, ANOVA). Endotoxinexposition isolierter Kardiomyozyten führte zu einer additiven Depression der Sarkomerkontraktion von Kardiomyozyten, die von verbrannten Tieren isoliert wurden. Herzmuskelzellen aus Kontrolltieren zeigten hingegen eine geringer beeinträchtigte Sarkomerkontraktilität nach Endotoxinexposition. Schlussfolgerungen: Diese Ergebnisse zeigen, dass bei der Signaltransduktion der kardialen Kontraktilität kreuzreaktive Verbindungen des Komplement- und TLR-Rezeptorsystems existieren. Die Entwicklung der therapeutischen C5a-Blockade bei verbrennungsinduzierter Kardiodepression scheint aufgrund unserer Ergebnisse sinnvoll. Auch bei Brandverletzung mit bakterieller Superinfektion (Endotoxämie) könnte die klinische Erprobung unseres Antikörpersystems zur Erweiterung der Behandlungsoptionen der verbrennungsinduzierten kardialen Dysfunktion beitragen.

Einfluss von aktiviertem Protein C und Antithrombin III 227 auf die LPS-induzierte Zytokinfreisetzung aus PBMC's gesunder Probanden und Patienten nach chirurgischem Eingriff

M. Burggraf, H. Trentzsch, S. Zedler, J.N. Hoffmann, E. Faist Chirurgische Klinik und Poliklinik Großhadern, Klinikum der Universität München

Aktiviertes Protein C (APC) und Antithrombin III (ATIII) sind nicht nur physiologische Inhibitoren der Koagulation sondern regulieren offenbar inflammatorische Prozesse. Ziel unserer Studie war die Bewertung von ATIII und APC als Modulatoren der LPSinduzierten Entzündungsantwort auf humane Immunzellen bei gesunden Probanden und die Auswirkungen auf Zellen, die durch operatives Gewebstrauma in Ihrer Funktion beeinflusst sind. Wir haben an mononukleären Zellen des peripheren Blutes (PBMCs) von gesunden Probanden $(n=8)$ bzw. Patienten mit abdominalchirurgischen Eingriff $(n=8)$ präoperativ $(-1)$, sowie an den postoperativen Tagen 1, 3 und 7 den Einfluss physiologischer (APC $4 \mu \mathrm{g} / \mathrm{ml}$, ATIII $1 \mathrm{IU} / \mathrm{ml}$ ) und supraphysiologischer (APC $100 \mu \mathrm{g} / \mathrm{ml}$, ATIII $20 \mathrm{IU} / \mathrm{ml}$ ) Konzentrationen dieser Gerinnungsinhibitoren auf die LPS-induzierte Freisetzung von Tumor Nekrose Faktor $a$ (TNF) und Interleukin 10 (IL10) untersucht. PBMCs wurden aus venösem Blut mittels Ficoll-Trennung isoliert, in serumfreiem Spezialmedium AIMV kultiviert und für 60 Min mit APC oder ATIII präkubiert. Der entzündliche Stimulus erfolgte mit bakteriellem Lipopolysaccharid (Dosis: $1 \mu \mathrm{g} / \mathrm{ml}$ ). Zellkulturen wurden dann mit oder ohne Stimulus für 20 Stunden inkubiert. Messung der Zytokinspiegel im Bioplex Suspensions Assay System. Ohne Stimulation mit LPS zeigte sich kein relevanter Anstieg der Zytokinproduktion in den jeweiligen Proben. Bei gesunden Probanden konnten wir unter LPS-Stimulation in Gegenwart von ATIII (20 IU/ml) signifikante Abfälle sowohl von TNF als auch IL10 beobachten $(\mathrm{p}<0,05)$. Im Patientenkollektiv zeigte sich unter Stimulation mit LPS bei ATIII $(20 \mathrm{IU} / \mathrm{ml})$ der gleiche Effekt mit einem Trend zu reduzierten TNF-Spiegeln, die an Tag 1 und 7 ein signifikante Niveau erreichen $(p<0,05)$. Für IL10 zeigt sich ebenfalls der bei gesunden Probanden beobachtete Abfall der LPS-induzierten IL10-Spiegel, jedoch ohne statistische Signifikanz. Ein Effekt von ATIII in der Konzentration $1 \mathrm{IU} / \mathrm{ml}$ konnte nicht gezeigt werden. Für APC konnte kein Einfluss auf die Immunantwort von PBMC's unter serumfreien Bedingungen nachgewiesen werden. Unsere Ergebnisse zeigen für ATIII in supraphysiologischer Konzentration sowohl eine Reduktion von pro-(TNF) als auch antiinflammatorischen (IL-10) Zytokinen bei gesunden Pro- 
banden. Nach OP bleibt dieser Effekt erhalten. Der zugrundeliegende Mechanismus bedarf weiterer Klärung. Darüber hinaus zeigte sich in unserem Modell kein Effekt von APC auf die Zytokinfreisetzung. Es bleibt zu klären, ob hierfür ein noch nicht bekannter Co-Faktor benötigt wird. Die in-vivo beobachtete antiinflammatorische Potenz von APC scheint aber im serumfreien Ansatz wohl nicht auf einer Modulation der Immunantwort durch die Substanz selbst zu beruhen.

Hochdosis-Antithrombin-Wirkung auf die mikrovaskuläre Thrombose bei Sepsis

H. Sorg ${ }^{1}$, J.N. Hoffmann ${ }^{2}$, M.D. Menger ${ }^{3}$, N. Lindenblatt ${ }^{1}$, B. Vollmar ${ }^{1}$

${ }^{1}$ Abt. f. Exp. Chirurgie, Universität Rostock, ${ }^{2}$ Chirurg. Klinik, Klinikum Großhadern, München, ${ }^{3}$ Abt. f. Klin.-Exp. Chirurgie, Universität des Saarlandes Homburg-Saar

Einleitung: Im Rahmen der KyberSept-Studie konnte die Therapie septischer Patienten mit Antithrombin (AT) keinen Überlebensvorteil gegenüber Plazebo-behandelten Patienten aufzeigen. Bei Betrachtung einer prospektiv definierten Subgruppe an Patienten, die kein gleichzeitiges Heparin zur Thromboseprophylaxe erhielten, wurde jedoch eine signifikante Reduktion der 90-Tage Mortalität beobachtet. Die vorliegende Studie untersuchte deshalb erstmals die AT Wirkung auf die mikrovaskuläre Thrombusbildung bei i.v. Endotoxinämie in einem tierexperimentellen Ansatz, als Voraussetzung für die Vermeidung einer gleichzeitigen Heparingabe unter klinischen Bedingungen. Material und Methode: Im Modell der phototoxisch-induzierten Thrombose am Ohr der haarlosen SKH-1 Maus (Ketamin/Xylazin-Anästhesie $90 \mathrm{mg} /$ $25 \mathrm{mg} / \mathrm{kg}$ ip) wurde die Kinetik der Thrombusbildung in Arteriolen und Venolen in 49 nicht-septischen sowie 20 septischen Tieren mittels intravitaler Fluoreszenzmikroskopie quantitativ erfasst und die subaquale Blutungszeit bestimmt. Zur Induktion der Sepsis wurde E. coli LPS (3 mg/kg; Serotyp 0128:B12) $1 \mathrm{~h}$ vor Thrombose-Induktion iv verabreicht. Unmittelbar vor ThromboseInduktion erhielten die Tiere jeweils eine einmalige i.v.-Bolusgabe von AT $(25,50,100$ oder $250 \mathrm{U} / \mathrm{kg})$, Heparin (100 U/kg) bzw. Hirudin $(1 \mathrm{mg} / \mathrm{kg})$. Kontrolltiere erhielten äquivalente Mengen an $0,9 \%$ Kochsalzlösung $(10 \mathrm{ml} / \mathrm{kg})$. MW \pm SEM; ANOVA und posthoc Paarvergleich; Signifikanzniveau $\mathrm{p}<0,05$. Ergebnisse: In nicht septischen und septischen Kontrolltieren führte die Lichtexposition in Kombination mit hochmolekularem Fluoreszenzfarbstoff in Arteriolen nach etwa $800 \mathrm{~s}$ bzw. $440 \mathrm{~s}$ und in Venolen nach etwa $400 \mathrm{~s}$ bzw. $290 \mathrm{~s}$ zur Ausbildung einer Thrombose. Heparin und Hirudin verzögerten die mikrovaskuläre Thrombusbildung um mehr als 50\%. Interessanterweise konnte die AT-Gabe die Mikrothrombose in nicht-septischen wie auch septischen Tieren dosisabhängig noch deutlich prolongieren und war insbesondere in $\mathrm{Ar}$ teriolen sehr wirksam. Unter nicht septischen Bedingungen konnte schon AT-100 die Okklusion aller untersuchten Arteriolen verhindern, während in der AT-250-Gruppe zusätzlich in den Venolen im Beobach-tungszeitraum von $1500 \mathrm{~s}$ keine Thrombosierung erreicht werden konnte. Nach E. coli-Vorbehandlung war ein kompletter Gefäßverschluss bei $100 \mathrm{U}$ AT in Arteriolen nach $843 \pm 167 \mathrm{~s}$ und Venolen nach $528 \pm 13 \mathrm{~s}$ festzustellen $(\mathrm{p}<0,01$ vs. $\mathrm{NaCl} \&$ Heparin für Venolen). Bei der Höchstdosis mit 250U AT waren Arteriolen nach $1336 \pm 106 \mathrm{~s}$ und Venolen nach $1078 \pm 144 \mathrm{~s}$ durch einen Thrombus komplett okkludiert ( $p<0,05 \mathrm{vs.} \mathrm{NaCl} \& \mathrm{He}-$ parin). In den Kontrollgruppen beider Versuchsansätze konnte eine deutlich kürzere subaquale Blutungszeit gegenüber Heparin und Hirudin festgestellt werden. Hochdosis-AT wies somit eine deutlich verlängerte Blutungszeit im Sinne besserer Antikoagulation auf $(\mathrm{p}<0,05)$. Zusammenfassung In der vorliegenden Arbeit konnte erstmals bei i.v. Endotoxinämie eindrucksvoll gezeigt werden, dass unter Bedingungen der experimentellen Endotoxinämie AT die mikrovaskuläre Thrombosierung hocheffektiv verhindern kann.
Arginin reduziert die Leukozyten-Endothelzell Interaktion bei Endotoxinämie - die kapillare Perfusion wird nicht verbessert

J.M. Fertmann ${ }^{1}$, J.N. Hoffmann ${ }^{1}$, M. Laschke ${ }^{2}$, B. Vollmar ${ }^{3}$, M.D. Menger ${ }^{2}$

${ }^{1}$ Chirurgische Klinik, Klinikum Großhadern, München, ${ }^{2}$ Abt. f. Klin.-Exp. Chirurgie, Universität des Saarlandes Homburg-Saar, ${ }^{3}$ Abt. f. Exp. Chirurgie, Universität Rostock,

Hintergrund: Sepsis und septisches Multiorganversagen sind nach wie vor mit einer hohen Mortalität assoziiert. Aktuelle Studien zur Pathophysiologie zeigen eine Verarmung der semi-essentiellen Aminosäure Arginin bei Sepsis. Die Auswirkung einer hochdosierten Supplementierung mit L-Arginin auf die Mikrozirkulation bei Sepsis wurde bisher nicht untersucht. Die vorliegende Studie charakterisiert deshalb den Einfluss der i.v. L-Arginin Applikation auf die Mikrozirkulation in einem etablierten Modell der normotensiven Endotoxinämie. Methoden: In einer Hautkammerpräparation bei männlichen syrischen Goldhamstern wurde durch i.v. Gabe von $2 \mathrm{mg} / \mathrm{kgKG}$ LPS (E. coli) eine normotensive Endotoxinämie induziert. Vor LPS-Gabe, nach $30 \mathrm{~min}, 3,8$ und $24 \mathrm{~h}$ wurden arterioläres und venuläres Leukozytenrollen und -adhäsion mittels Intravitalmikroskopie quantifiziert sowie die funktionelle Kapillardichte als Mass des mikrovaskulären Perfusionsschadens. L-Arginin Tieren (L-Arg, $\mathrm{n}=5$ ) wurde $15 \mathrm{~min}$ vor LPS-Gabe intravenös L-Arg ( $50 \mathrm{mg} / \mathrm{kgKG})$ verabreicht. Als Kontrolle dienten Tiere, die das Stereoisomer D-Arginin (D-Arg, $\mathrm{n}=4,50 \mathrm{mg} / \mathrm{kgKG}$ ) erhielten und mit $\mathrm{NaCl}(0,9 \%)$ behandelte Tiere. Ergebnisse: Die Gabe von LPS führte zu einer hochsignifikanten Zunahme von Leukozytenrolling und -adhärenz gegenüber den Ausgangswerten bei den Kontrolltieren ( $p<0,01$ vs. Baseline). L-Arg induzierte eine signifikante Reduktion des Leukozyten-Rolling $(\mathrm{p}<0,05)$ sowie der Adhärenz $(p<0,01)$ in postkapillären Venolen, während D-Arg keine signifikanten Unterschiede verglichen mit den unbehandelten Kontrolltieren erbrachte. Interessanterweise war die kapillare Perfusion trotz der Wirkung auf die Leukozyten-Endothelinteraktion durch L-Arg nicht verbessert. Schlussfolgerungen: Die Supplementation von L-Arginin resultierte in vivo in einer deutlichen Reduktion der LPS-induzierten Leukozyten-Endothelzellinteraktion in diesem Mikrozirkulationsmodell (Rückenhautmuskel). Die fehlende Verbesserung der kapillaren Perfusion muss in weiteren Untersuchungen näher charakterisiert werden.

\section{Sepsismonitoring I}

Human $\beta$-Defensin Gen Kopie Polymorphismen haben keinen Einfluss auf die Inzidenz der schweren Sepsis

M. Book, Q.X. Chen, L.E. Lehmann, J.-C. Schewe, S. Weber,

M. Lüpertz, A. Hoeft, F. Stüber

Klinik und Poliklinik für Anästhesiologie und Operative Intensivmedizin, Rheinische-Friedrich-Wilhelm Universität Bonn

Einleitung: Human Defensine zeigen antimikrobielle and immunomodulatorische Eigenschaften. Daher werden sie dem innaten Immunsystem zugerechnet. Die induzierbaren humanen $\beta$-Defensine 2 (hBD2), hBD3 and hBD4 werden von epithelialen, aber auch von peripheren weißen Blutzellen exprimiert. Die Bedeutung der leukozytären Defensine für Patienten mit inflammatorischen oder infektiösen Erkrankungen ist unklar. Die hBD2, hBD3 and hBD4 Gene auf Chromosom 8 sind polymorph hinsichtlich unterschiedlicher Gen Kopie Anzahlen. Bis zu 12 Gen Kopien wurden nachgewiesen. Eigene Untersuchungen zeigten eine Korrelation der induzierbaren hBD2 Expression und der Gen Kopie Zahl. Die vorliegende Untersuchung testet die Assoziation der hBD2, hBD3 und hBD4 Gen Kopie Polymorphismen mit der Inzidenz der schweren Sepsis. Methoden: Nach Genehmigung durch die lokale Ethikkommission wurden bislang 70 Patienten mit schwerer Sep- 
sis und 70 gesunde Probanden in die Studie eingeschlossen. Die Defensin Gen Kopie wurden mit einer Echt-Zeit PCR quantifiziert. Ergebnisse: Es zeigten sich keine Unterschied der hBD2, hBD3 oder hBD4 Gen Kopie Anzahl zwischen gesunden Probanden und Patienten mit schwerer Sepsis. Schlussfolgerung: Eine Korrelation der Kopie Anzahl und der Inzidenz der schweren Sepsis ist in diesem ersten Kollektiv nicht darstellbar. Die Bedeutung der Defensin Gen Wiederholungen für Patienten mit schwerer Sepsis ist unklar. Funktionelle Untersuchungen der mRNA und Protein Expression in Abhängigkeit der Kopie Anzahl haben begonnen.

The study of relationship among COX-2 and p38MAPK and PAF-AH on peripheral blood mononuclear cells in patients with systemic inflammatory response syndrome and multiple organ dysfunction syndrome

H. Tang, Z. Ma, Y. Liang, H. Ye

The first hospital of Sun Yan-sen University

Objective: To measure the content of Cyclooxygenase-2 (COX-2), p38 mitogen-activated protein kinase (p38MAPK) and platelet-activating factor acetylhydrolase (PAF-AH) in patients with systemic inflammatory response syndrome (SIRS) and multiple organ dysfunction syndrome (MODS); to measure the quantity of the expression of COX-2 mRNA, p38MAPK mRNA and PAF-AH mRNA on PBMCs in patients with SIRS and MODS, and to find out their relation with prognosis and the clinical significance. Methods: 28 adult patients who accorded with the American college of chest Physicians/Society of Critical Care Medicine criteria for SIRS and MODS were selected. 13 of these patients were selected in SIRS group ( 8 males and 5 females), the average age $-62.77 \pm 6.22$ among 21 to 71 year old. 15 of these patients were selected in MODS group (8 males and 7 females), the average age $-61.40 \pm 5.42$ among 26 to 90 year old. The normal control group consisted of 11 healthy volunteers ( 6 males and 5 females) whose age (44.91 \pm 4.58 , range from 23 to 72 year old) and sexuality corresponded with the patient's groups. PBMCs were isolated from peripheral samples by centrifugation and Ficoll-Hypaque sedimentation. The enzyme immunometric assay (EIA) method was used to measure the content of COX-2 and p38 MAPK, the activity of PAF-AH of PBMCs. The reverse transcription-polymerase chain reaction (RT-PCR) method was used to measure the COX-2 mRNA, p38MAPK mRNA and PAF-AH mRNA expression of PBMCs. $\beta$-actin was used as internal control to calculate relative ratio of optical density. Results: The content of COX-2 and p38MAPK and the activity of PAF-AH of PBMCs and the expression of their mRNA in MODS group were higher than those in SIRS group and control group $(\mathrm{P}<0.05)$. The content of COX-2 and $\mathrm{p} 38 \mathrm{MAPK}$ and the activity of PAF-AH and the expression of their mRNA of PBMCs in dead patients were higher than those in survival patients $(\mathrm{P}<0.05)$. In the control group and SIRS group and MODS group, positive correlation is indicated between the COX-2 content and p38MAPK content $(\mathrm{r} 1=0.663, \mathrm{P} 1<0.01)$, COX-2 content and PAF-AH activity ( $22=0.329, \quad \mathrm{P} 2<0.05)$, p38MAPK content and PAF-AH activity $(\mathrm{r} 3=0.366, \mathrm{P} 3<0.05)$. Conclusion: COX-2 p38MAPK and PAF-AH all take part in the occurrence of MODS and they can probably be reference figures to judge the prognosis of SIRS and MODS. The diagnostic standard of SIRS is maybe too loose to be special. It is necessary to find out the clinical significance of inflammatory cytokines in the pathophysiologic mechanisms and the treatment of SIRS and MODS.
Standardisierte Quantifizierung der NF- $\kappa$ B-

Aktivierungskapazität im peripheren Blut bei kritischer Erkrankung

F. Wagner, J. Cordes, B. Stahl, M. Georgieff, U. Senftleben

Klinik für Anästhesiologie, Universität Ulm

Einleitung: Der NF- $\kappa$ B-Signalweg ist für die Aktivierung proinflammatorischer Zellen essentiell wichtig. Bei Sepsispatienten besteht ein positiver Zusammenhang zwischen einer verstärkten Aktivität von NF- $\kappa$ B in mononukleären Zellen (MNZ) und dem Apache-II-Score sowie der Letalität. NF- $\kappa \mathrm{B}$ hat also prädiktiven Wert für den Verlauf der Sepsis. Der zugrundegelegte Assay, der "Electrophoretic mobility shift assay" (EMSA), stellt zwar den goldenen Standard zur Quantifizierung der NF- $\kappa$ B-Aktivität dar, ist jedoch semi-quantitativ. Dazu kommt, dass die Zusammensetzung der peripheren MNZ aus zellulären Subpopulationen während Sepsis sehr heterogen sein kann. Dies lässt eher inkonstante Werte für die Aktivität von NF- $\kappa$ B erwarten, zumal NF- $\kappa$ B in Lymphozyten und Monozyten unterschiedlich aktiviert wird. Demzufolge ist die einfache Bestimmung der Aktivität von NF- $\kappa$ B in MNZ mittels EMSA während Sepsis kritisch zu bewerten und hilft nur bedingt, die Rolle dieses Signalweges während kritischen Erkrankungen aufzuklären. Für diese Fragestellung ist eine standardisierter quantitativer Assay notwendig. Methoden: MNZ wurden aus peripherem Blut von 10 gesunden Probanden durch standardisierte Ficoll-Präparation gewonnen. Daraus wurden T-Lymphozyten nach CD3-Markierung durch magnetische Separation und positiver Selektion isoliert. Der Reinheitsgrad dieser Population wurde flusszytometrisch durch Nachweis von CD4- bzw. CD8-Expression bestimmt und war regelhaft 90-95\%. 4×10e6 MNZ bzw. T-Lymphozyten wurden dann mit 10ng TNF $a$ für 30 Minuten stimuliert. Nach Proteinextraktion erfolgte die Bestimmung der NF- $\kappa$ B-Aktivität mittels EMSA. Auf gleiche Weise (EMSA) wurde die DNABindungsaktivität von „Nuclear factor-1“ (NF-1) zur Normalisierung bestimmt. Es folgte eine densitometrische Analyse der autoradiographischen Banden. Die relative Induktion von NF- $\kappa \mathrm{B}$ wurde berechnet: (NF- $\kappa$ B stimuliert/NF-1 stimuliert)/(NF- $\kappa$ B Kontrolle/NF-1 Kontrolle). Ergebnis: Relative NF- $\kappa$ B Induktion nach TNFa Stimulation ex vivo \pm SEM: MNZ 6.39 \pm 4.31 (Median 3.87), T- Lymphozyten $3.73 \pm 1.00$ (Median 3.36). NF- $\kappa \mathrm{B}$ ist in MNZ und T-Lymphozyten deutlich induzierbar. Verglichen mit T-Lymphozyten zeigt die Aktivierungskapazität in MNZ eine höhere Streuung. Die hohe Streubreite der NF- $\kappa$ B-Induktion in MNZ verglichen mit T-Lymphozyten bei gesunden Probanden weist darauf hin, dass die NF- $\kappa$ B Aktivierung in Monozyten heterogenen Mechanismen unterliegt. Diskussion: Ein Differentialblutbild ist nötig um die NF- $\kappa$ B-Aktivität in MNZ zu interpretieren. Abhängig von der Reinheit der untersuchten Zellpopulation ist dieser Assay gut geeignet die NF- $\kappa$ B-Aktivierungskapaziät nach ex vivo Stimulation von humanen peripheren Blutzellen standardisiert zu quantifizieren. Weitere Studien müssen zeigen, ob die NF- $\kappa$ B-Aktivierungskapazität in MNZ z.B. bereits präoperativ das Ausmaß einer späteren systemischen Hyperinflammation vorhersagen kann.

Plasma concentrations of body weight regulating hormones in critical ill patients with sepsis

B. Perras ${ }^{1}$, K. Lütjens ${ }^{2}$, B. Schaaf ${ }^{2}$, C. Dodt ${ }^{1}$

${ }^{1}$ Department of Internal Medicine I and ${ }^{2}$ Department of Internal Medicine III, University of Lübeck

Introduction: Energy balance is disturbed in critical ill patients with systemic inflammatory response. Regulation of body weight and energy metabolism in patients on the ICU is complex and not fully understood. In concert with other hormones the recently discovered peptides Ghrelin and Adiponectin may be involved. Methods: Here we report on plasma ghrelin and adiponectin concentrations (RIA, Linco, St. Charles, USA and ELISA, Biovendor, Modrice, Czech Republic, respectively) in septic patients admitted to a medical ICU. Hormone levels were contrasted to patient's 
characteristics and the APACHE score as assessed on admission and on day 7. Results: 16 consecutive patients with sepsis (7 females, 9 males, mean age $67.5 \mathrm{yr}$, range $38-82 \mathrm{yr}, 9$ patients with pneumonia, 3 with urosepsis, 2 with menigitis) were investigated. On admission Ghrelin and Adiponectin plasma concentrations amounted to $971.2 \pm 225.4 \mathrm{pg} / \mathrm{ml}$ and $17.19 \pm 3.0$ (mean $\pm \mathrm{SEM}) \mu \mathrm{g} /$ $\mathrm{ml}$, respectively. The APACHE score was rated $33.4 \pm 1.9$ (mean \pm SEM, range 19-45). Within the next 7 days 3 patients died, the other recovered (APACHE score mean \pm SEM $22.5 \pm 2.3$, range 8 $38, \mathrm{p}<0.02$ in comparison with admission). Ghrelin levels remained unchanged (mean \pm SEM: $1033.2 \pm 186.3 \mathrm{pg} / \mathrm{ml}$ ), while Adiponectin concentrations significantly increased (23.13 \pm 4.07 $\mathrm{p}<0.02$ ). Plasma concentrations of other weight regulating hormones like leptin and peptide PYY will also be reported. Conclusion: Ghrelin and Adiponectin plasma concentrations in severely ill patients on a medical ICU were slightly elevated. During the next 7 days Ghrelin levels remained stable while Adiponectin plasma concentrations further increased in the course of the disease. Thus Ghrelin and plasma levels may reflect the disturbance of the body's weight regulating system in critically ill humans.

\section{Light and darkness do not regulate melatonin secretion in critically ill patients}

\section{B. Perras, M. Meier, C. Dodt \\ Department of Internal Medicine I, University of Lübeck}

Introduction: The secretion of the pineal hormone melatonin in ICU patients is severly disturbed. Little is known so far about the physiological regulation of pineal function by light and darkness in critically ill patients. Under normal circumstances, we would expect darkness to stimulate pineal activity while light would be a potent inhibitor of melatonin secretion. Methods: Here we report on melatonin plasma concentrations (RIA, DPC Biermann, Germany) in critically ill patients admitted to a medical ICU. Patients were subjected to total darkness ( $>1$ lux) for 1 hour. Thereafter ambient illumination was set to $>10000$ lux for one hour. Melatonin plasma concentrations were measured in close intervals. Results: 14 critcally ill patients (median (range): age: 63.3 (33-83) years; SAPS: 38 (19-62), TISS-28: 13 (range 10-21)) were investigated. During baseline (ambient illumination $\sim 10$ lux) melatonin plasma concentrations in 12 patiens were low $(\mathrm{MW} \pm$ SEM: $5.3 \pm 1.2 \mathrm{pg} / \mathrm{ml})$ and in 2 patients high (118 and $80 \mathrm{pg} / \mathrm{ml}$, respectively). Following darkness in only one patient a significant rise of melatonin plasma levels were observed, in all other patients melatonin plasma concentrations were unchanged. After one hour of illumination with 10000 lux, none ot the patients showed a substantial inhibition of melatonin secretion. Conclusion: In critically ill patients, physiological regulation of melatonin secretion by light and darkness is severely disturbed.

Wirkspiegel von Caspofungin bei Intensivpatienten über einen Behandlungszeitraum von 10 Tagen

\section{T. Kunz, S. Kloesel, B. Scheller, T. Bingold, B. Zwissler,}

\section{H. Wissing}

Klinik für Anästhesiologie, Intensivmedizin und Schmerztherapie, Klinikum der Johann-Wolfgang-Goethe-Universität Frankfurt am Main

Mit der Dosisempfehlung für das Antimykotikum Caspofungin soll vom ersten Behandlungstag an eine minimale Wirkspiegelkonzentration von $>1$ Mikrogramm/ml (1) und damit die minimalen Hemmkonzentrationen (MHK90) klinisch relevanter Pilze (Candida spp., Aspergillus spp.) erreicht werden (2). Daten für die Standarddosierung wurden an gesunden Probanden erhoben (1), Daten bei operativen Intensivpatienten liegen bislang nicht vor Mittels einer in unserem Labor adaptierten HPLC-Methode (3) wurden Talspiegelkonzentrationen von Caspofungin bei 6 septischen chirurgischen Intensivpatienten während eines 10-tägigen Behandlungszeitraums bestimmt. Die Patienten waren beatmet, benötigten Katecholamine und kontinuierliche Nierenersatztherapie (veno-venöse Haemodiafiltration). Material und Methoden: Die zuständige Ethikkommission sowie die gesetzlichen Patientenvertreter genehmigten die Messreihen. Laut Standarddosierungsempfehlung wurden initial $70 \mathrm{mg}$ Caspofungin, folgend $50 \mathrm{mg}$ täglich gegeben. Talspiegel wurden unmittelbar vor erneuter Gabe abgenommen. Ergebnisse: Nach dem 1. Behandlungstag erreichten nur 2 Patienten die minimale Wirkspiegelkonzentration von größer 1 Mikrogramm/ml, der Mittelwert (MW) lag bei 0,78 Mikrogramm/ml (Standardabweichung (STAW) $\pm 0,23$ ). An der Hälfte der Behandlungstage lag der MW unter 1 Mikrogramm/ml. Die höchsten MW wurden am Tag 4 (MW 1,43 Mikrogramm/ml, STAW $\pm 0,85)$ und Tag $8(\mathrm{MW} 1,41 \mathrm{Mikrogramm} / \mathrm{ml}$, STAW $\pm 1,03)$ gemessen. 2 Patienten erreichten zu keinem Zeitpunkt die Zielkonzentration von 1 Mikrogramm/ml. Schlussfolgerung: Anders als in den bisher veröffentlichten Daten konnten keine konstanten Talspiegelkonzentrationen von größer 1 Mikrogramm/ml nachgewiesen werden. Auch kumulierte Caspofungin im Behandlungszeitraum nicht. $\mathrm{Ob}$ die niedrigen Talspiegel durch die Nierenersatztherapie oder durch ein verändertes pharmakokinetisches Verhalten im Rahmen der schweren Grunderkrankungen bedingt waren, muss durch weitere Studien in diesem Patientengut auch hinsichtlich der Wirksamkeit geklärt werden.

Literatur: [1] Stone JA et al: Antimicrob Agents Chemother 2002; 46:739-745. [2] Bartizal K et al: Antimicrob Agents Chemother 1997; 41:2326-2332. [3] Schwartz M et al: Analytica Chimica Acta $1997 ; 352: 299-307$

Changes of cerebral proteom expression in septic rats

\section{A. Peterka, J. Hinkelbein, C. Schubert, A. Kalenka}

University Clinic for Anesthesiology and Intensive Care Medicine, University Hospital Mannheim, Germany

Introduction: Sepsis and septic shock are often complicated by septic encephalopathy. The cause of brain dysfunction in sepsis is not fully understood so far. Proteom analysis is a tool to detect previously unknown protein alterations. The aim of the present study was to explore whether sepsis leads to alterations in the cerebral proteom profile 12,24 , and 48 hours after the onset of sepsis in a common rat model. Material and methods: After approval of the local committee for animal research, 72 male wistar rats were investigated. To induce sepsis, 46 rats unterwent coecal ligation and puncture (CLP) and were investigated $12 \mathrm{~h}(\mathrm{n}=6), 24 \mathrm{~h}$ $(\mathrm{n}=9)$ or $48 \mathrm{~h}(\mathrm{n}=4)$ after CLP. $\mathrm{N}=16$ rats $(12 \mathrm{~h}: \mathrm{n}=6 ; 24 \mathrm{~h}: \mathrm{n}=4$; $48 \mathrm{~h}: \mathrm{n}=6$ ) were assigned to the control group (sham operation). Two- dimensional gel electrophoresis and mass spectrometry were used to identify changes in the protein expression of the whole brain lysat. Results: $\mathrm{N}=27(59 \%)$ rats of the sepsis group died before analysis; no rat of the control group died. Per gel more than 1600 protein spots could be discriminated. Protein expression changes of at least twofold were stated as statistic significant. After 12 hours, six differentially expressed proteins (upregulated/ UR vs downregulated/DR) were associated with functions in cell structure (1DR), metabolism (2UR), respiratory chain (1DR), and signaling (1DR, 1UR). After 24 hours, 29 expressed proteins were associated with functions in cell structure (4DR), gene expression and protein synthesis (5DR), metabolism (9DR), respiratory chain (4DR), signaling (3DR), and secretion (4DR). After 48 hours, six expressed proteins were associated with functions in metabolism (3DR), signaling (2UR), and secretion (1DR). Conclusions: We provide the first study of brain lysat proteom in septic rats. In early sepsis (12 hours) cell integrity seems to be reduced, whereas metabolism is activated. After 24 and 48 hours, mainly all identified proteins were downregulated (e.g. metabolism and secretion). Proteom analysis is a useful tool to detect previously unknown alterations in brain lysat after induction of severe sepsis (mortality $59 \%)$. Further analysis of the function of the differentially expressed proteins in septic encephalopathy is needed. 
Rat Serum Proteom Alterations in Severe Sepsis

C. Schubert, A. Kalenka, A. Peterka, J. Hinkelbein

University Clinic for Anesthesiology and Intensive Care Medicine, University Hospital Mannheim, Germany

Introduction: Sepsis and septic shock have still a high mortality rate. Protein markers for sepsis may be present in the serum and may thus be identified with proteom analyis. The aim of the present study was to explore whether sepsis induced by a common septic rat model may lead to serum proteom disturbances after 12, 24, and 48 hours. Material and methods: After approval of the local committee for animal research, 72 male wistar rats were investigated and assigned to a control group (sham operation) and three sepsis groups $(12 \mathrm{~h}: \mathrm{n}=6$ vs $24 \mathrm{~h}: \mathrm{n}=9$ vs $48 \mathrm{~h}: \mathrm{n}=4)$. To induce sepsis 46 rats unterwent coecal ligation and puncture (CLP), 16 rats were assigned to the control group sham-operated. Blood samples were collected at the killing-time of each group and serum was used for proteomics. Two-dimensional gel electrophoresis and mass spectrometry were used to identify changes in the protein expression between septic and non-septic serum samples with and without albumin removal. Results: $\mathrm{N}=27$ rats of the sepsis group died (mortality 59\%); no rat of the control group died. In the serum with albumin depletion, after 12 hours nine differentially expressed proteins (upregulated/UR vs downregulated/ DR) were associated with functions in cell structure (1DR), transport (4DR), metabolism (1DR), secretion (1DR), proteolysis (1DR), and complement system (1UR). After 24 hours five differentially expressed proteins were associated with functions in transport (2DR), metabolism (1DR), proteolysis (1DR), and other functions (1DR). After 48 hours 17 differentiallly expressed proteins were associated with functions in cell structure (1DR, 1UR), transport (7DR, 2UR), secretion (2DR), proteolysis (1DR), and other functions (2DR, 1UR). Without albumin removal the following functions were changed: At 12 hours: cell structure (1DR, 2UR), metabolism (1DR), and transport (8DR, 4UR). At 24 hours: cell structure (2DR, 1UR), metabolism (1DR), transport (7DR, 2UR), and other proteins (1DR). At 48 hours: cell structure (2UR), protein synthesis (2UR), and metabolism (2UR). Conclusions: Severe sepsis in this animal model induced significant alterations in the serum proteom after 12,24 , and 48 hours after onset of the sepsis in rats. Mainly metabolism, transport, and secretion proteins were upregulated, whereas the expression the other proteins were downregulated.

Einfluss der Saugerblutseparation während

koronarchirurgischer Eingriffe mit HLM

auf die Ausschüttung inflammatorischer Zytokine

\section{A.A. Peivandi, S. Sperling, N. Kayhan, D. Peetz, M. Dahm,}

\section{H. Vahl}

Klinik und Poliklinik der Herz-, Thorax- und Gefäßchirurgie, Institut für Klinische Chemie

Fragestellung: Um die Gabe homologer Blutprodukte in der Herzchirurgie zu minimieren, werden verschiedene Maßnahmen zur Wiederverwertung von Eigenblut angewendet. Hierunter fällt die routinemäßige Retransfusion des im Kardiotomiereservoir innerhalb der Operation gesammelten mediastinalen Blutes (Saugerblut). Verschiedene Studien konnten zeigen, dass dieses Blut extrem hohe Konzentrationen an Zytokinen und anderen Mediatoren enthält. Diese Substanzen können möglicherweise zu einer klinisch relevanten nicht selten auftretenden SIRS sowie Organdysfunktionen führen. In einer prospektiven klinischen Studie wurde der Einfluss der Saugerblutseparation auf die Ausschüttung inflammatorischer Zytokine untersucht. Material und Methodik: In die Studie eingeschlossen wurden 2 Gruppen von insgesamt 102 Patienten mit koronarer Herzkrankheit, die sich einer elektiven Koronarrevaskularisation unterzogen. In Gruppe I $(n=52$, Alter: $65.2 \pm 8,3$ Jahren, $13 \mathrm{w} / 39 \mathrm{~m}$ ) wurde Saugerblut in einem Kardiotomiereservoir gesammelt, gewaschen und anschließend als auto- loger Erythrozytenkonzentrat retransfundiert. In Gruppe II $(\mathrm{n}=50$, Alter: $63,1 \pm 9,4$ Jahren, $14 \mathrm{w} / 36 \mathrm{~m})$ wurde das Saugerblut ohne Separationsvorgang gefiltert dem Herzlungenmaschinenkreislauf wieder zugeführt. Bei allen Patienten wurden Enzymverläufe der pro- und antiinflammatorischen Zytokine IL-6, IL-8, IL-10, TNF- $\alpha$ sowie C-reaktives Protein (CRP) und Leukozytenzahl engmaschig prä-, intra- und postoperativ bestimmt. Ergebnisse: In beiden Gruppen war IL-6 im Serum präoperativ nicht nachweisbar bzw. im Normbereich und stieg intra- und postoperativ signifikant an. Dieser Anstieg war in der Gruppe II jedoch $\mathrm{zu}$ allen untersuchten Zeiten statistisch signifikant höher als in der Gruppe I. IL-6 erreichte seinen medianen Gipfel in der Gruppe I schon nach $2 \mathrm{~h}(62,79 \mathrm{pg} / \mathrm{ml},[\mathrm{Q} 1=43,15, \mathrm{Q} 3=79,38]$ und in der Gruppe II erst nach $4 \mathrm{~h} \quad(55,94 \mathrm{pg} / \mathrm{ml}$, [Q1 $1=39,23$, $\mathrm{Q} 3=110,02]$. Für IL-8 konnten weder signifikante Unterschiede in den einzelnen Gruppen, noch ein bedeutender Anstieg der Enzymkonzentration nachgewiesen werden. In der Gruppe II wurden $\mathrm{zu}$ entsprechenden Zeiten höhere Konzentrationen von TNF- $\alpha$ erreicht. Diese Ergebnisse waren allerdings nicht statistisch signifikant. Als antiinflammatorisches Zytokin war IL-10 nur in der Gruppe I in messbaren Konzentrationen nachweisbar. Die Peaks für die Konzentration von CRP in beiden Gruppen waren $48 \mathrm{~h}$ postoperativ. Signifikante Unterschiede zwischen den beiden Gruppen gab es allerdings nicht. Schlussfolgerung: Die Unterschiede im postoperativen Anstieg der Enzymkonzentrationen von IL-6 und TNF- $\alpha$ nach Transfusion von gewaschenem und ungewaschenem Saugerblut weisen auf die Effektivität der Saugerblutseparation hin. Ob diese Ergebnisse hinsichtlich der Reduktion eines SIRS klinisch relevant sind und damit die Saugerblutseparation als Routineverfahren eingesetzt werden sollte, muss in zukünftigen Untersuchungen näher geklärt werden.

Effect of Drotrecogin alfa (activated) on soluble CD40 ligand levels in septic patients

U. Hoffmann, D. Klank, T. Holzinger, M. Brueckmann, A. Suska, C. Liebetrau, S. Lang, M. Borggrefe

1st Department of Medicine, Faculty of Clinical Medicine Mannheim, University of Heidelberg, Germany

Background: CD40 Ligand (CD40L) a member of the TNF superfamily, is a multifunctional ligand. CD40L stimulates B-Cell secretion of immunoglobulin isotypes in the presence of cytokines. Ligation of monocyte CD40 by the T cell-derived CD40 ligand can trigger the production of various mediators, the transcription and activation of enzymes, and the upregulation of costimulatory molecules involved in the pathogenesis of sepsis. Proteolytic cleavage can also produce soluble forms of CD40L (sCD40L). Both the membrane-bound and soluble forms of CD40L are active. We investigate levels of soluble CD40 ligand in human blood mononuclear cells of septic patients treated with and without Drotrecogin alfa activated in vivo and in vitro. Patients and Methods: Levels of sCD40L were determined in the supernatant of fresh isolated blood mononuclear cells (PMC's) of septic patients treated with and without Drotrecogin alfa (activated) on day 2 and in a healthy control. SCD40L was detected in PMC's of a healthy control in vitro after addition of Drotrecogin alfa (activated) in concentrations of 50,500 and $5000 \mathrm{ng} / \mathrm{ml}$ for $6 \mathrm{~h}$ and in a septic patient treated with and without Drotregocin alfa (activated). SCD40L supernatant levels were measured with ELISA-method. Statistical analysis with ANOVA were performed. Results: Levels of sCD40L in the supernatant of the control were decreased after incubation with Drotregocin alfa (activated) in concentrations of 50,500 and $5000 \mathrm{ng} / \mathrm{ml}$ in vitro. Soluble CD40L levels were lower in the septic patient treated with Drotrecogin alfa on day 2 compared to the patient without treatment. Levels of sCD40L in the supernatant of septic patient treated with Drotregocin alfa (activated) were lower after incubation with Drotregocin alfa (activated) in concentrations of 50,500 and $5000 \mathrm{ng} / \mathrm{ml}$ in vitro compared to the patient without treatment. Conclusions: The ability 
of Drotregocin alfa (activated) to down-regulate sCD40L production in human blood mononuclear cells may represent a new molecular mechanism, by which Drotregocin alfa (activated) controls mediators of systemic inflammation and sepsis, possibly reflecting the anti-inflammatory properties of these drug. Further in vitro and in vivo investigations are needed to confirm these results.

\section{Sepsismonitoring II, Infektionen und Outcome}

Einfluss einer akuten Erhöhung des systemischen Gefäßwiderstandes auf Messwerte der transpulmonalen Thermodilution: Ein Vergleich mittels 2D-Echokardiographie

\section{J. Kozieras, O. Thümer, C. Schelenz, S. Sakka}

Friedrich-Schiller-Universität Jena; Klinik für Anästhesie und Intensivmedizin

Einleitung: Die transpulmonale Thermodilution ermöglicht die Erfassung einer Reihe hämodynamischer Parameter, z.B. Herzindex (HI), globaler Ejektionsfraktion (GEF), intrathorakalem Blutvolumenindex (ITBVI), globalem enddiastolischem Volumenindex (GEDVI) und extravasalem Lungenwasserindex (EVLWI). In der vorliegenden Studie wurden die Effekte einer akuten Erhöhung des systemischen Gefäßwiderstandes (SVRI) auf diese Kreislaufvariablen untersucht und bei einer Subpopulation gleichzeitig eine 2D-transösophageale Echokardiographie zum Vergleich durchgeführt. Patienten und Methoden: Mit Zustimmung der lokalen Ethik-Kommission untersuchten wir 24 maschinell beatmete kritisch kranke Patienten (16今, 8ㅇ, 26-71 Jahre), die aufgrund klinischer Indikation mittels transpulmonaler Thermodilution erweitert kreislaufüberwacht wurden $\left(\mathrm{PiCCO}^{\circledR}, \mathrm{Fa}\right.$. Pulsion Medical Systems, München) und zur Kreislaufunterstützung kontinuierlich Noradrenalin erhielten. Es erfolgte eine Anhebung des mittleren arteriellen Drucks (MAP) durch eine kurzfristige Erhöhung des Noradrenalins und es wurden die Auswirkungen auf diese Kreislaufparameter und den zentralen Venendruck (ZVD) erfasst. Der Volumenstatus und die Respiratoreinstellungen blieben während der Untersuchung unverändert. An jedem der drei Messpunkte (MP) wurden jeweils dreimal $15 \mathrm{ml}$ NaCl-Lösung $0,9 \%$ ig $\left(<8{ }^{\circ} \mathrm{C}\right)$ zentralvenös injiziert. Bei einer Subpopulation von 7 Patienten wurde an den drei Messpunkten zusätzlich die linksventrikuläre enddiastolische Fläche (LVEDF pro $\mathrm{m}^{2}$ Körperoberfläche) mittels transösophagealer 2D - Echokardiographie (TEE) bestimmt. Die statistische Auswertung erfolgte anhand einer Varianzanalyse (ANOVA). Ergebnisse: Während Herzfrequenz (HF), HI, EVLWI und GEF unverändert blieben, zeigte sich eine signifikante Zunahme für GEDVI, ITBVI und LVEDF.

\begin{tabular}{lccc} 
Parameter & MP 1 & MP 2 & MP 3 \\
\hline HF [1/min] & $96 \pm 19$ & $94 \pm 18$ & $94 \pm 18$ \\
MAP [mmHg] & $68 \pm 9$ & $93 \pm 9^{*}$ & $70 \pm 11$ \\
ZVD [mmHg] & $11 \pm 5$ & $12 \pm 6$ & $11 \pm 5$ \\
HI [l/min $\left./ \mathrm{m}^{2}\right]$ & $3,5 \pm 0,9$ & $3,6 \pm 0,9$ & $3,5 \pm 0,9$ \\
SVRI [dyn·s $\left.\cdot \mathrm{cm}^{-5} \cdot \mathrm{m}^{2}\right]$ & $1348 \pm 389$ & $2115 \pm 736^{*}$ & $1406 \pm 415$ \\
GEF [\%] & $23 \pm 6$ & $23 \pm 6$ & $24 \pm 7$ \\
GEDVI $\left[\mathrm{ml} / \mathrm{m}^{2}\right]$ & $701 \pm 158$ & $745 \pm 157^{*}$ & $698 \pm 152$ \\
ITBVI $\left[\mathrm{ml} / \mathrm{m}^{2}\right]$ & $829 \pm 182$ & $881 \pm 178^{*}$ & $828 \pm 184$ \\
EVLWI $[\mathrm{ml} / \mathrm{kg}]$ & $7,9 \pm 2,7$ & $7,5 \pm 2,8$ & $7,5 \pm 2,9$ \\
LVEDF $\left[\mathrm{cm}^{2} / \mathrm{m}^{2}\right]$ & $9,8 \pm 4,4$ & $11,6 \pm 3,8^{*}$ & $7,7 \pm 3,4$
\end{tabular}

Mittelwert \pm Standardabweichung. ${ }^{*} p<0,05$ (MP1 vs. MP2, MP2 vs. MP3)
Zusammenfassung: Eine Erhöhung des systemischen Gefäßwiderstandes mittels Noradrenalin führt $\mathrm{zu}$ einem reversiblen Anstieg zentraler Blutvolumina (ITBVI, GEDVI). Die Zunahme der linksventrikulären enddiastolischen Fläche stützt diese Volumenveränderungen und bestätigt somit die Ergebnisse der transpulmonalen Thermodilution.

Das Überleben, nicht aber die Prävalenz, der schweren Sepsis ist mit dem MIF Promotor MIF-173C/7-CATT assoziiert

L.E. Lehmann, W. Hartmann, M. Book, S. U. Weber, S. Klaschik, J.-C. Schewe, A. Hoeft, F. Stüber

Klinik und Poliklinik für Anästhesie und operative Intensivmedizin, Universitätsklinikum Bonn

Fragestellung: Der Makrophagen inhibierende Factor (MIF) ist ein Zytokin, dass eine wichtige regulatorische Rolle bei der generalisierten Entzündungsreaktion spielt. Im Promotor des MIF-Gens sind zwei funktionell relevante Polymorphismen bekannt: ein G nach C Einzelbasenaustausch an Position-173 und ein (CATT)5-8 Wiederholungsmotiv an Position-793. Das Ziel dieser Studie war es hieraus Promotor Haplotypen zu identifizieren und eine mögliche Assoziation dieser Haplotypen zwischen der Prävlenz bzw. Überleben einer schweren Sepsis zu analysieren. Material, Methoden: Nach Genehmigung durch die Ethikkommission wurden 169 Patienten mit schwerer Sepsis untersucht. Als Kontrolle dienten 277 Individuen, die keine Zeichen einer Sepsis aufwiesen. Die Genotypisierung erfolgte nach Echtzeit-PCR durch Schmelzkurvenanalyse mit genotypspezifischen Hybridisierungssonden, sowie durch DNA Sequenzierung. Die Haplotypenanalyse erfolgte durch EH Software (Rockefeller University, New York). Ergebnisse: Es konnten zunächst sechs Promotor Haplotypen identifiziert werden. Anschließende Kopplungsanalysen zeigten ein signifikant gehäuftes Auftreten der drei Haplotypen: 173G/5-CATT, 173G/6-CATT und 173C/7-GATT. Die Haplotypenverteilung und das Kopplungsungleichgeweicht zwischen den Patienten mit schwerer Sepsis und der Kontrollgruppe waren vergleichbar. Das Auftreten einer schweren Sepsis war mit keinem Haplotyp assoziiert. Demgegenüber zeigten Nichtüberlebende einer schweren Sepsis eine signifikant höhere Frequenz des 173C/7-GATT Haplotyp als Überlebende $(0,21$ gegen 0,$09 ; \mathrm{p}<0,05)$. Das Risiko an einer schweren Sepsis zu versterben war in der 173C/7-GATT-Gruppe signifikant erhöht $(\mathrm{OR}=2,1 ; \mathrm{p}<0,05)$. Diskussion: Der MIF173G/7-GATT-Haplotyp ist kein Prädispositionsmarker für das Auftreten einer schweren Sepsis. Allerdings könnte der MIF173G/7-GATT-Haplotyp als genomischer Marker bei Patienten mit schwerer Sepsis dienen, und Patienten identifizieren, die eine Prädisposition für letale Verläufe einer schweren Sepsis aufweisen.

Vermehrte Apoptose geht in der Sepsis mit der Deaktivierung von Monozyten einher

S. Weber, J. C. Schewe, L.E. Lehmann, M. Book, A. Hoeft, F. Stüber

Klinik für Anästhesiologie und Operative Intensivmedizin, Universitätsklinikum Bonn

Hintergrund: Bei Deaktivierung von Monozyten in der schweren Sepsis wird HLA-DR herunterreguliert, wodurch eine Immunparalyse induziert wird [1]. In jüngster Zeit wird außerdem der progrediente Verlust von immunkompetenten Zellen durch Apoptose, den programmierten Zelltod, als Mitursache für die Immunsupression in der Sepsis diskutiert [2]. Ziel: Ziel der aktuellen Untersuchung war es deswegen zu klären, ob bei Monozyten in der schweren Sepsis die vermehrte Apoptose mit einer Herunterregulation von HLA-DR einhergeht. Methoden: Nach dem Einverständnis der lokalen Ethikkommission wurden 16 Patienten mit schwerer Sepsis (SAPS II Score 60), 10 Patienten mit einem milden systemischen Inflammationssyndrom (SIRS, SAPS II Score 26) und 
11 gesunde Spender in die Studie eingeschlossen. HLA-DR-Expression auf Monozyten [1], Phosphatidylserin (PS)-Externalisierung, Kaspase-3-Aktivierung und Bcl-2-Expression wurden mittels Durchflusszytometrie untersucht. Ergebnisse: Die HLA-DR-Expression auf Monozyten war bei Patienten mit schwerer Sepsis im Vergleich zu Patienten mit SIRS signifikant reduziert (9190 Rezeptoren pro Zelle vs. 23000 bei SIRS, $\mathrm{p}<0,01$ ). In der schweren Sepsis fand sich ein signifikant erhöhter Anteil von Monozyten mit aktivierter Kaspase-3 $(\mathrm{p}<0,05)$, dem Schlüsselenzym der Apoptose. Apoptotische Zellen exprimieren PS auf der Oberfläche. Bei Patienten mit schwerer Sepsis war die Externalisierung von PS signifikant vermehrt $(\mathrm{p}<0,01)$. PS-Externalisierung korrelierte mit einer verminderten HLA-DR-Expression $(\mathrm{p}<0,05, \mathrm{r} 2=0,2)$. Der mitochondriale Weg der Apoptose wird durch das antiapoptotische Protein Bcl-2 reguliert. Die Bcl-2-Expression war in der schweren Sepsis auf Monozyten deutlich vermindert $(p<0,001)$. Außerdem ging eine niedrige Bcl-2-Expression mit einer niedrigen HLA-DR-Expression einher $(\mathrm{p}<0,001, \mathrm{r} 2=0,42)$. Schlussfolgerungen: Die Deaktivierung von Monozyten in der schweren Sepsis geht mit einer erhöhten monozytären Apoptoserate einher. Es ist davon auszugehen, dass sowohl Deaktivierung von Monozyten als auch deren Apoptose zur Immunsupression in der Sepsis beitragen. Möglicherweise können apoptotische Zellen eine Herunterregulation von HLA- DR induzieren [3].

Literatur: [1] Hotchkiss RS, Karl E: N Engl J Med 2003; 348:138-150. [2] Döcke WD, Höflich C, Davis KA, Röttgers K, Meisel C, Kiefer P, Weber SU et al: Clinical Chemistry 2005; 51:2341-2347. [3] Verbovetski I, Bychkov H, Trahtemberg U, Shapira I, Hareuveni M, Ben-Tal O, Kutikov I, Gill O, Mevorach D: J Exp Med 196:1553-1561

\section{Monitoring of surgical intensive care patients} with daily measurement of nucleated red blood cells in the peripheral blood

\section{A. Stachon ${ }^{1}$, R. Kempf ${ }^{1}$, A. Becker ${ }^{1}$, T. Holland-Letz ${ }^{2}$, J. Friese ${ }^{1}$, M. Krieg ${ }^{1}$}

${ }^{1}$ BG Kliniken Bergmannsheil - Universitätsklinik -; ${ }^{2}$ Abteilung für Medizinische Informatik, Biometrie u. Epidemiologie; Ruhr-Universität Bochum

Background: The appearance of nucleated red blood cells (NRBC) in the peripheral blood is associated with a variety of severe diseases. When NRBC are detected in the blood this is generally associated with increased mortality. Methods: In a prospective study the detection of NRBC was analyzed with regard to the clinical circumstances and the course of laboratory parameters of organ injury. NRBC were daily measured in the peripheral blood of surgical intensive care patients $(n=284)$. Results: NRBC were found at least once in $32.0 \%$ of all patients. The mortality of NRBC-positive patients was $44.0 \%(40 / 91)$; this was significantly higher $(\mathrm{P}<0.001)$ than the mortality of NRBC-negative patients $(4.2 \%, 8 /$ 193). With regard to intensive care mortality, NRBC in blood showed sensitivity and specificity of $83.3 \%$ and $78.9 \%$, respectively. The area under curve (C-statistic) was 0.86 . Mortality increased with increasing NRBC concentration. All patients with more than $2000 \mathrm{NRBC} / \mu \mathrm{l}$ died. Moreover, mortality increased with increasing frequency of occurrence. When after first detection of NRBC in blood, during the further course of intensive care treatment the NRBC have disappeared from the circulation, the mortality again decreased to values of NRBC-negative patients. NRBC were detected for the first time, on average, 9 days (median 5 days) before death. Multiple logistic regression analysis under consideration of several other clinical and laboratory risk indicators revealed a significant association between NRBC and increased mortality, the odds ratio being 1.95 (95\% confidence interval $1.35-2.82 ; \mathrm{P}<0.001)$ for each increase in the NRBC category $(0 / \mu \mathrm{l} ; 1-40 / \mu \mathrm{l} ; 41-80 / \mu \mathrm{l} ; 81-240 / \mu \mathrm{l},>240 / \mu \mathrm{l})$. After the initial detection of NRBC in blood there were no significant increases regarding the creatinine concentration and the alanine aminotrans- ferase activity, respectively. However, the appearance of NRBC coincided with increasing C-reactive protein and thrombocyte concentrations, respectively. Conclusion: The detection of NRBC in blood of surgical intensive care patients is of prognostic power with regard to the patients' mortality. This prognostic significance of NRBC was independent of some clinical and other laboratory risk parameters. The appearance of NRBC in blood was not associated with a failure/lesion of the kidney and the liver, respectively.

Response to yeast to exemplify immune defence 210 in critically ill patients

\section{G.P. Otto, R.A. Claus, F.M. Brunkhorst, F. Bloos, V. Oberle,} M. Bauer

Department for Anaesthesiology and Intensive Care Therapy, Division for Experimental Anaesthesiology, University Hospital, Friedrich-Schiller-University Jena

Generalized yeast infections are associated with a high morbidity and mortality, while diagnostic procedures are time consuming and associated with difficulties regarding sensitivity and specificity. So far, the unspecific immune response has been considered to be essential for the host response to yeast. However, recent data provided some evidence that also the specific immune system, especially CD8+ and CD4+ lymphocytes, may play a crucial role in the yeast-host interaction. After institutional approval 35 patients with sepsis, severe sepsis or septic shock who were admitted to a university intensive care unit were included in the study. At first diagnosis, samples of heparinised blood were obtained in between 6 hours and at intervals of 48 hours up to day 7. Blood samples were incubated with different yeast subspecies. Staphylococcus enterotoxin B was used as positive control. The cellular response was analysed by determining the intracellular concentration of interferon gamma (INF $\gamma$ ) as principal cytokine of the specific immune response and CD69 expression as surface activation marker. Data were compared to ICU-controls (20 CABG Patients, $24 \mathrm{~h}$ after cardio- pulmonary-bypass) and 20 healthy individuals. Statistical analyses were performed using the MannWhitney-U-Test and Wilcoxon testing, $\mathrm{p}<0.05$ was considered to be statistically significant. On admission the basal as well as yeast-stimulated CD8+ lymphocyte interferon gamma levels in patients were not different from those measured in healthy controls. During the treatment on ICU 17 of the 35 patients died within the first 28 days. The initial CD4 interferon and CD69 level of the surviving septic patients were significant lower then of healthy controls. CABG patients were also significant depressed. By stimulation with SEB the CD8 und CD4 cells of healthy volunteers achieved higher levels of Interferon and CD69 than septic patients. Over time the basal lymphocyte cytokine levels in surviving and non-surviving septic patients remained unchanged. However, the yeast-induced INF $\gamma$ response in CD8+ showed a significant decline with a maximum at day 5 followed by a partial recovery until day 7. The levels of CD69, especially of the nonsurviving patient, were decline during day 7. Furthermore the CD4+ cell of septic patients produced less INF $\gamma$ at the beginning but exceed both control groups until day 7. Our data demonstrate a time-dependent decline in the specific immune response in surviving and non surviving septic patients as measured by changes in the yeast-induced INF $\gamma$ production in CD8+ lymphocytes, which is in clear contrast to the CD4+ cell which don't be remained responsive in the first 7th day after onset of sepsis. In the light of the maintained SEB-stimulation, the depression of CD8+ cells to mount an IFN $\gamma$ response seems to be a consequence of a pathogen specific immune response or a modulation by the pathogen, rather than the consequence of a general immune suppression. 
Spontanruptur eines Leberhämatomes mit akutem Nierenversagen als Folge einer Hantavirusinfektion

Th. Meyer, B. Meyer ${ }^{*}$ E. Schröpfer, A. Thiede

Chirurgische Universitätsklinik und Polikinik; Klinik für Anästhesiologie*, Zentrum Operative Medizin, Julius-Maximilians-Universität Würzburg, Deutschland

Einleitung: Hantavirusinfektionen, früher als Unterform des hämorrhargischen Fiebers in Mitteleuropa kaum bekannt, haben in den letzten Jahren zunehmend im klinischen Alltag an Bedeutung gewonnen. Hantaviren gehören zu der Familie der Bunyaviridae und werden v.a. durch wildlebende Nager übertragen. Das klinische Spektrum kann - in Abhängigkeit vom Erregertyp - von einer leichten Infektion mit grippeähnlichen Symptomen bis hin zu schweren Hämorrhargien mit protrahiertem Lungen- und Nierenversagen reichen. Fallbeschreibung: Aufgrund rezidivierender abdomineller Beschwerden war eine 23-jährige Patientin in einem peripheren Krankenhaus aufgenommen worden. Anamnestisch klagte die Patientin über grippeähnliche Symptome, die seit fünf Tagen bestanden hätten. Trotz der mehrfachen Einnahme von ASS habe die vormals gesunde Patientin, Kopf- und Gliederschmerzen, sowie Temperaturenspitzen bis $40^{\circ} \mathrm{C}$ gehabt. In der durchgeführten Sonographie wurde, neben freier abdomineller Flüssigkeit, eine unklare Raumforderung im Bereich des linken Leberlappens beschrieben. Nach Explorativlaparoskopie, die eine subcapsuläre, ca. 2-3 cm durchmessende Blutung im linken Leberlappen zeigte, erfolgte eine mediane Oberbauchlaparotomie zur Hämatomausräumung und Blutstillung. Bei zunehmender intraoperativer Blutungsneigung wurde die Leber jedoch mit zwei Bauchtüchern tamponiert und die junge Frau in unsere Klinik verlegt. Bei anhaltender intraabdomineller Blutung wurde nach Aufnahe die Indikation zur Re-Laparotomie gestellt. Als Blutungsquelle konnte dabei ein ca. 1,5 bis $2 \mathrm{~cm}$ großes rupturiertes Hämatom (DD: Hämangiom) im Übergangsbereich der Lebersegmente 2 und 3 eruiert werden. Das übrige Abdomen zeigte keine pathologischen $\mathrm{Be}$ funde. Bei anhaltender starker Blutungsneigung (39000 Thrombozyten/ $\mu$ l; PTT 60,1s; AT III: 47\%; Quick-Wert: 71\%) erfolgte zunächst die erneute Anlage einer Lebertamponade, die nach Stabilisierung der Gerinnungssituation am Folgetag erfolgreich entfernt werden konnte. Bei ausreichender Flüssigkeits- und Elektrolytzufuhr entwickelte die Patientin trotz symptomatischer Therapie nach initialer Oligurie am vierten postoperativen Tag ein akutes Nierenversagen unklarer Genese, das erfolgreich über $48 \mathrm{~h}$ mittels kontinuierlicher veno-venöser Hämofiltration behandelt werden konnte. Aufgrund des raschen und schweren Krankheitsverlaufes wurde - unabhängig von der Blutungssituation im Übergangsbereich der Lebersegmente 2 und 3 - die Verdachtsdiagnose einer Hantavirusinfektion gestellt, die serologisch und immunologisch bestätigt werden konnte (Serotyp: Puumala). Im weiteren Verlauf kam es dann unter rein symptomatischer Therapie zu einer Normalisierung aller pathologischen Befunde. Schlussfolgerung: Der vorliegende Fallbericht zeigt, dass bei einem akuten Nierenversagen unbekannter Ätiologie, welches mit abdominellen Beschwerden einhergeht, auch eine Hantavirus-Infektion in die Differentialdiagnose mit einbezogen werden sollte.

\section{Erfolgreiche Behandlung eines schweren Streptokokken-assoziierten Toxic-Shock-Syndrom}

\section{Th. Schrauzer, S. John, S. Popp, K. U. Eckhardt}

Medizinische Klinik 4 (Nephrologie und Hypertensiologie),

Universität Erlangen - Nürnberg, Klinikum Nürnberg Süd

Das „streptococcal toxic shock syndrome“ (STSS) stellt ein seltenes, durch invasive toxinbildende $\beta$-hämolysierende Streptokkoken der Gruppe A verursachtes Krankheitsbild mit steigender Inzidenz und nach wie vor hoher Letalität dar. Ein 35-jähriger Patient wurde nach 20-stündiger intensivmedizinischer Behandlung mit septischem Schock und Multiorganversagen aus einer externen Klinik übernommen. Der akuten Verschlechterung waren über eine Woche grippeähnliche Symptome vorausgegangen. An Vorerkrankungen bestanden eine arterielle Hypertonie und eine Nephrektomie rechts vor Jahren bei Refluxnephropathie. Bei Aufnahme war der adipöse Patient (BMI $\left.38 \mathrm{~kg} / \mathrm{m}^{2}\right)$ analgosediert und kontrolliert BIBAP-beatmet (RR $80 / 40 \mathrm{mmHg}$, MAP $50 \mathrm{mmHg}$; Sinustachykardie 200/min; AF 30/min; Rektaltemperatur $41,3^{\circ} \mathrm{C}$ ). Neben einer Akrozyanose bestand an beiden Unterschenkeln eine ausgeprägte Livedo retikularis. Im Verlauf kam es zu einer massiven Desquamation der Haut an den Händen und Unterschenkeln. Es bestand ein Multiorganversagen bei septischem Schock mit Leber- und Nierenversagen, Verbrauchskoagulopathie, Rhabdomyolyse und ARDS. Das anfängliche hämodynamische Monitoring (PiCCO) unter Therapie mit Noradrenalin (bis $15 \mathrm{mg} / \mathrm{h}$ ) und Dobutamin (bis $20 \mathrm{mg} / \mathrm{h}$ ) ergab einen deutlich erniedrigten systemischen Widerstandsindex (SVRI $699 \mathrm{DS} \mathrm{m}^{2} / \mathrm{cm} 5$ ) bei erhöhtem Herzindex (HI $5,26 \mathrm{l} / \mathrm{min} / \mathrm{m}^{2}$ ). Unter aggressiver Flüssigkeitsgabe von $50 \mathrm{l}$ Kristalloiden innerhalb der ersten $72 \mathrm{~h}$ (Anstieg des Körpergewichts von 110 auf $160 \mathrm{~kg}$ ), hochdosierter Katecholamintherapie, systemischer Kühlung mittels kontinuierlicher venovenöser Hämofiltration (CVVHF), körpergewichts-adaptierter Therapie mit aktiviertem Protein $C$ über $96 \mathrm{~h}$ und antibiotischer Therapie mit Meropenem und Moxifloxacin (gemäß den Leitlinien der Deutschen Sepsis-Gesellschaft) gelang eine Stabilisierung. Die Katecholamintherapie konnte am Tag 6 beendet werden. Ab Tag 7 erfolgte bereits eine negative Flüssigkeitsbilanzierung. In den Blutkulturen fanden sich Streptokokken der Gruppe A (M-Antigen 3.1, T-Antigen 3). Die Antibiose wurde antibiogrammgemäß um Penicillin G erweitert. Mittels PCR konnte die Expression der Superantigene SpeA (ETA), SpeF und Ssa gezeigt werden. Entsprechend den Kriterien der „Working Group on Severe Streptococcal Infections" bestand ein STSS. Eine Eintrittspforte konnte nicht nachgewiesen werden. Nach Extubation am Tag $17 \mathrm{kam}$ es ab Tag 29 auch zu einer Erholung der Leber- und Nierenfunktion. Amputationen konnten vermieden werden. Der Patient konnte am Tag 41 in eine Rehabilitationsklinik verlegt werden. Der Flüssigkeitsbedarf von Patienten mit STSS kann aufgrund der massiven Endotheldysfunktion anfänglich erheblich sein und ist, wie im dargestellten Fall, nur durch aggressive hochdosierte Flüssigkeitssubstitution beherrschbar. Ein zusätzlicher therapeutischer Nutzen durch Gabe von aktiviertem Protein C scheint möglich.

Moxifloxacin bei ausgedehntem dentogenem Abszess 140 mit Mediastinitis und Sepsis - ein Fallbericht

\section{J. Mayer, S. N. Piper, J. Boldt}

Klinik für Anästhesiologie und operative Intensivmedizin, Klinikum Ludwigshafen, Ludwigshafen

Kasuistik: Wir berichten über einen 65-jährigen Patienten, der zur Sanierung einer abszedierenden Entzündung eines vierwurzligen Zahnes vorgestellt wurde. Anamnestisch waren zahlreiche einschränkende Vorerkrankungen bekannt: terminale Niereninsuffizienz, Z.n. Myokardinfarkt bei generalisierter AVK und Diabetes mellitus Typ II, COPD bei schwerem Nikotinabusus. Nach Spaltung des ausgedehnten, auf den Mundboden übergreifenden Abszesses am Aufnahmetag wurden ein C-reaktives Protein von $18,1 \mathrm{mg} / \mathrm{dl}$, Leukozyten von $14 / \mathrm{nl}$ sowie von $123 / \mathrm{nl}$ auf $28 / \mathrm{nl}$ fallende Thrombozyten gemessen. Es bestand eine katecholaminpflichtige Kreislaufinsuffizienz und die initiale kalkulierte Antibiose wurde mit Levofloxacin, Clindamycin und Vancomycin begonnen. Ein bei zunehmender Kreislaufinstabilität und Sepsiszeichen durchgeführtes Kontrastmittel-CT des Mediastinums zeigt am 3. postoperativen Tag eine diffuse Signalanreicherung vom Mundboden über die Halsweichteile bis ins Mediastinum. Die noch am selben Tag durchgeführte Mediastinoskopie bestätigt die Diagnose einer Mediastinitis. Daher wurde der Patient mit Thorax- und Mediastinaldrainagen sowie einem chirurgischen Tracheostoma nach großflächiger Abszessspaltung der Halsweichteile versorgt. Mikrobiologisch fanden sich massenhaft Staphylococcus capitis sowohl im Mundboden- als auch im Mediastinalabstrich. Die An- 
tibiose wurde nach Antibiogramm auf Moxifloxacin $400 \mathrm{mg} / \mathrm{Tag}$ umgesetzt; der Patient befand sich zu diesem Zeitpunkt im Vollbild einer Sepsis. Die große Wundfläche des Halses musste mehrmals debridiert werden und wurde mit einem Madenkissen versorgt. Moxifloxacin wurde über 21 Tage intravenös verabreicht, worunter sich die Infektsituation stetig besserte. Am 14. Tag nach Aufnahme war der Patient katecholaminfrei. Die laborchemischen Entzündungsparameter bestätigten den Verlauf, so dass der $\mathrm{Pa}$ tient am 42. postoperativen Tag auf die Normalstation verlegt werden konnte. Die deszendierende Mediastinitis ist eine seltene Komplikation oropharyngealer Infektionen mit einer Letalitätsrate von $20-40 \%$ [1]. Neben radikaler chirurgischer Sanierung spielt die kalkulierte Antibiotikatherapie eine entscheidende Rolle, wobei Moxifloxacin das Erregerspektrum odontogener Infektionen sehr gut erfasst. Erste Berichte über eine gute Gewebegängigkeit von Moxifloxacin sind beschrieben [2] und wurden im vorliegenden Fall durch den erfolgreichen Einsatz bei deszendierender Mediastinitis mit Sepsis bestätigt.

Literatur: [1] Sandner A et al: HNO 2006; Apr 20 [Epub ahead of print]. [2] Keating GM et al: Drugs 2004; 64:2347-2377

Hat die Ernährungstherapie einen Einfluss

073 auf die Letalität septischer Patienten Ergebnisse der SepNet-Prävalenzstudie

G. Schmitz, D. Schädler, C. Engel, H. Bogatsch, I. Frerichs, M. Ragaller, F.M. Brunkhorst, K. Reinhart, J. Scholz, N. Weiler, das deutsche Kompetenznetzwerk Sepsis (SepNet)

Klinik für Anästhesiologie und Operative Intensivmedizin, Universitätsklinikum Schleswig-Holstein, Campus Kiel

Einleitung: Die enterale Ernährung stellt die bevorzugte Form der Ernährung bei kritisch kranken Patienten dar und wird auch bei Patienten mit Sepsis empfohlen [1]. Im Vergleich zur parenteralen Ernährung ist die enterale Zufuhr mit einer geringeren Komplikationsrate assoziiert [2]. Ein Einfluss auf die Sterblichkeit konnte bislang jedoch durch kein bestimmtes Ernährungsregime gezeigt werden. Methoden: Im Rahmen der Studie „Prävalenz der schweren Sepsis und des septischen Schocks auf Intensivstationen in Deutschland“, einer prospektiven, querschnittlichen, multizentrischen Beobachtungsstudie, wurden Daten auf 454 Intensivstationen in 310 randomisiert ausgewählten Krankenhäusern durch speziell trainierte Ärzte an einem zufällig ausgewählten Stichtag erhoben. Diese Daten wurden im Hinblick auf die Ernährungstherapie und deren Einfluss auf die Letalität bei septischen Patienten ausgewertet. Ergebnisse: Von insgesamt 415 Patienten mit schwerer Sepsis/septischem Schock wurden 19,3\% rein enteral, 33,7\% rein parenteral sowie 33,3\% kombiniert enteral/parenteral ernährt. 9,9\% der Patienten erhielten keine Ernährung. Für 374 Patienten konnten valide Informationen über die Letalität ausgewertet werden. Diese war in der Gruppe der Patienten mit rein parenteraler (62,3\%) bzw. kombinierter Ernährung $(57,1 \%)$ höher als bei den Patienten, die rein enteral ernährt wurden $(38,9 \%)$. Patienten ohne Ernährung hatten eine Sterblichkeit von 51,3\%. Der APACHEII-Score war bei den Patienten mit rein parenteraler $(20,7)$ bzw. keiner Ernährung $(22,6)$ signifikant höher als in der Gruppe „rein enteral“ $(18,3)$ bzw. „kombinierte Ernährung" $(18,5) \quad(p<0,005)$. In der multivariaten Analyse zeigte sich, dass ein parenterales Ernährungsregime (Odds Ratio $(\mathrm{OR})=2,05,95 \%$ Konfidenzintervall $(\mathrm{CI})=1,26-3,34)$ neben dem APACHE-II-Score $(\mathrm{OR}=1,06,95 \%$ $\mathrm{CI}=1,02-1,09)$ und der renalen Dysfunktion (OR $=2,11,95 \%$ $\mathrm{CI}=1,33-3,36)$ einen unabhängigen Risikofaktor hinsichtlich der Letalität darstellt. Schlussfolgerung: Die rein parenterale bzw. kombinierte Ernährung sind die am häufigsten verwendeten Formen der Ernährung bei Patienten mit schwerer Sepsis/septischem Schock in Deutschland. Diese klinische Praxis widerspricht der gegenwärtigen Evidenz, die eine enterale Ernährung aufgrund geringerer Morbidität empfiehlt [2]. Darüber hinaus zeigen die Ergebnisse, dass trotz der unterschiedlichen APACHE-II-Scores der Einsatz eines parenteralen Ernährungsregimes (entweder exklusiv oder als kombinierte Therapie) mit einem erhöhten Letalitätsrisiko assoziiert ist.

Literatur: [1] Reinhart et al: Internist 2006; 47:356-373. [2] Gramlich et al: Nutrition 2004; 20:843-848. Gefördert vom Bundesministerium für Bildung und Forschung (BMBF), FörderNr. 01 KI 0106 und Lilly Deutschland GmbH

Die Compliance der Lunge ist ein unabhängiger

Risikofaktor für die Sterblichkeit von septischen

Patienten - Ergebnisse der SepNet Prävalenzstudie

D. Schädler, G. Schmitz, C. Engel, H. Bogatsch, I. Frerichs,

R. Kuhlen, M. Quintel, R. Rossaint, J. Scholz, F. M. Brunkhorst,

M. Loeffler, K. Reinhart, N. Weiler für das Kompetenznetzwerk Sepsis (SepNet)

Klinik für Anästhesiologie und Operative Intensivmedizin, Universitätsklinikum Schleswig-Holstein, Campus Kiel

Einleitung: Die künstliche Beatmung spielt in der Therapie von Patienten mit schwerer Sepsis und septischen Schock eine wichtige Rolle, da die Beatmung per se eine Schädigung der Lunge hervorrufen kann [1]. Es konnte gezeigt werden, dass eine lungenprotektive Beatmung mit einem Atemzugvolumen von $6 \mathrm{ml} / \mathrm{kg}$ Körpergewicht die Sterblichkeit bei Patienten mit akutem Lungenversagen senken kann [2]. Desweiteren ist anzunehmen, dass ein lungenprotektives Beatmungsregime mit frühzeitiger assistierter Spontanatmung und Spitzendrücken unter $35 \mathrm{cmH}_{2} \mathrm{O}$ die Schädigungen der Lunge reduzieren kann. Ziel dieser Untersuchung ist es zu überprüfen, ob diese Erkenntnisse in die tägliche Praxis umgesetzt werden. Material und Methodiken: Im Rahmen der Studie „Prävalenz der schweren Sepsis und des septischen Schocks auf Intensivstationen in Deutschland“, einer prospektiven, querschnittlichen, multizentrischen Beobachtungsstudie, wurden Daten auf 454 Intensivstationen in 310 zufällig ausgewählten Krankenhäusern durch speziell trainierte Ärzte an einem zufällig ausgewählten Stichtag erhoben. Folgende Daten wurden analysiert: Art der Beatmung, der Beatmungsmodus, das normierte Atemzugvolumen, der maximale Spitzendruck, APACHE-2-Score, die Intensivstations- und Krankenhaussterblichkeitsrate. Ergebnisse: Von 415 Patienten waren $16,4 \%$ nicht beatmungspflichtig, 3,6\% wurden nicht invasiv per Maske beatmet, 50,4\% intubiert, 25,8\% tracheotomiert, bei 3,6\% wurden keine Angaben gemacht. Ein druckkontrollierter Beatmungsmodus wurde bei $69,9 \%$ benutzt, $7,6 \%$ der Patienten wurden volumenkontrolliert beatmet, $21,5 \%$ atmeten assistiert spontan, bei $0,9 \%$ wurden keine Angaben gemacht. Das normierte Atemzugvolumen betrug $10 \pm 2,5 \mathrm{ml} / \mathrm{kg} \mathrm{KG}$, der maximale Spitzendruck 27,9 $\pm 6,7 \mathrm{cmH}_{2} \mathrm{O}$. Die Sterblichkeitsrate der beatmeten Patienten betrug $46,5 \%$ auf der Intensivstation und 52,3\% im Krankenhaus. Der APACHE-2-Score und die Compliance der Lunge erwiesen sich in einer logistischen Regressionsanalyse als unabhängige Risikofaktoren für die 28-Tage-Sterblichkeit. Schlussfolgerungen: In Deutschland werden die neuen Erkenntnisse zur lungenprotektiven Beatmung nur teilweise in die klinische Praxis umgesetzt. Einerseits wird der überwiegende Teil der Patienten druckkontrolliert oder assistiert mit tolerablen Spitzendrücken beatmet, andererseits wird dabei ein hohes Atemzugvolumen akzeptiert. Im Gegensatz $\mathrm{zu}$ den Ergebnissen anderer Studien zeigen die vorliegenden Ergebnisse, dass die Compliance der Lunge ein entscheidender prognostischer Faktor für das Überleben der Patienten darstellt. Diese Beobachtung muss in weiteren Studien untersucht werden.

Literatur: [1] Ricard et al: Curr Opin Crit Care 2002; 8:12-20. [2] The ARDS Network N Engl J Med 2000; 342:1301-1388. Gefördert vom Bundesministerium für Bildung und Forschung (BMBF) Förder-Nr. 01 KI 0106 und Lilly Deutschland GmbH 
Die Rolle der Intermediate Care Unit

\section{in der interdisziplinären postoperativen Versorgung}

\section{N. Khalaf, F. Graupe, D. Lorenz}

Abteilung Allgemein- und Viszeralchirurgie; HSK, Dr. Horst Schmidt Klinik Wiesbaden

Die chirurgische Intermediate Care Unit (IMC) der Dr. Horst Schmidt Klinik Wiesbaden dient primär der postoperativen $\mathrm{Be}$ handlung von Patienten aller chirurgischen Fachrichtungen nach ausgedehnten Operationen (große abdominale/thoraxchirurgische/ urologische/gynäkologische Tumorchirurgie, Neurochirurgie, Traumata, Gefäßchirurgie, HNO), die lückenlos mit invasivem/ nicht-invasivem Monitoring (EKG, arterielles Monitoring, zentralvenöser Druck, automatische Messung von Blutdruck, Sauerstoffsättigung, Atemfrequenz und Körpertemperatur) überwacht werden müssen, aber nicht die Zeichen einer unmittelbaren Bedrohung der Lebensfunktionen aufweisen oder von Beatmungsgeräten und Organersatzverfahren (z.B. Blutwäsche) abhängig sind. Nach den gleichen Kriterien werden auch postoperative Komplikationen auf der IMC behandelt. Zusätzlich ist die IMC Zwischenstation und Bindeglied zwischen Aufenthalt auf der Intensivstation und der Normalstation. $\mathrm{Zu}$ den wichtigen Aufgaben gehört nicht zuletzt die Schmerztherapie-Einstellung in der akuten postoperativen Phase mit hochpotenten Analgetika, die ein Monitoring der Vitalfunktionen voraussetzen. Oftmals ist nach einer Operation nur eine kurzzeitige Überwachung, manchmal aber auch ein Aufenthalt von mehreren Tagen zur Sicherheit des Patienten notwendig. Im Jahr 2005 wurden insgesamt 2104 Patienten auf IMC behandelt. Es ergab sich folgende Verteilung: Abdominalchirurgie $\mathrm{n}=667(31,7 \%)$; Thoraxchirurgie $\mathrm{n}=546(26,0 \%)$; Unfallchirurgie $\mathrm{n}=255(12,1 \%)$; Urologie $\mathrm{n}=212(10,1 \%) ;$ Gefäßchirurgie $\mathrm{n}=189$ $(9,0 \%)$; Neurochirurgie $n=186(8,8 \%)$; Gynäkologie $n=38(1,8 \%)$; HNO $n=11(0,5 \%)$. Die Sterberate lag bei 0,57\% $(n=12)$. Wegen Beatmungsnotwendigkeit oder unmittelbarer vitaler Bedrohung mussten 71 Patienten (3,4\%) im Verlauf auf die Intensivstation verlegt werden. Insgesamt stellt die IMC einen gleichwertigen Partner der Intensivstation in der interdisziplinären postoperativen Versorgung dar und spielt eine wichtige Rolle in der straffen Zeitplanung größerer Eingriffe der verschiedenen operativen Fächer, bei zunehmenden Patientenzahlen an einem Krankenhaus der Maximalversorgung mit onkologischem Schwerpunkt (high volume) und immer mehr Eingriffen an multimorbiden Patienten. Diese Tendenz ist noch deutlicher zu belegen mit einem Anstieg der Aufnahmen auf der IMC in den ersten 5 Monaten des Jahres 2006 um durchschnittlich $6 \%$.

\section{Rettungs- und Notfallmedizin}

\section{Notarzt- und Rettungsdienst I}

\author{
Stable Wide QRS-Complex Tachycardia in Prehospital \\ Emergency-Medicine: Results of the NAVT-Trial \\ M.-A. Ohlow ${ }^{1}$, A. Beierlein ${ }^{2}$, S. Müller ${ }^{1}$, H. von Korn ${ }^{1}$, \\ J.-C. Geller ${ }^{1}$, J. $\mathrm{Yu}^{1}$ B. Lauer ${ }^{1}$ \\ ${ }^{1}$ Abteilung für Kardiologie, Zentralklinik Bad Berka, Bad Berka, \\ Germany; \\ ${ }^{2}$ Städtisches Krankenhaus Forchheim, Forchheim, Germany
}

Background: Differential diagnosis of wide QRS-complex tachycardia (WCT) remains a diagnostic challenge, and treatment of supraventricular tachycardia with aberrant conduction (SVT) differs considerably from the therapy of a ventricular tachycardia (VT). During the last 20 years, a number of ECG criteria have been established to discriminate VT from SVT. Aim of this study was to determine whether board-certified emergency-physicians (BCEP) are able to differentiate VT from SVT in a high percentage (>90\%). Methods: 8 ECGs with WCT (4 with electrophysiologically proven diagnosis of VT and SVT, respectively) were evaluated in a blinded fashion by 68 BCEP's. Initially, the diagnosis had to be made without any further information. To evaluate the impact of clinical information on the diagnosis, the same ECGs were presented again with additional information (AI) such as left ventricular function, coronary angiogram, clinical presentation, and age of the patient. Results: $53.6 \%$ of the physicians were able to establish the correct diagnosis by evaluating the ECG only. Providing $\mathrm{AI}$, the number of correct diagnoses increased to $60.6 \%$. These results were roughly similar in all subgroups (anaesthesia: $43.8 \% / 60.0 \%$, internal medicine: $53.6 \% / 55.8 \%$, surgery: $59.4 \% /$ $62.5 \%$ ), only the subgroup of cardiologists showed a trend to better results with correct diagnoses in $66.7 \%$ without and $71.8 \%$ with AI, respectively. None of the subgroups reached the prespecified cut-off of $>90 \%$. The specialist-status as well as the experience in emergency medicine ( $<1$ year, $1-5$ years, $>5$ years) had no significant influence on the results. Conclusion: although a number of ECG criteria have been published in the past two decades, in case of stable WCT the sole evaluation of ECG in prehospital emergency-medicine leads to unsatisfying results. The diagnosis in WCT can be improved by using AI. Because of the overall low diagnostic accuracy, the differential diagnosis of stable WCT in preclinical emergency-medicine cannot be recommended, and any stable WCT should still be managed as if it were VT unless proven otherwise.

Organisation und Spektrum des betrieblichen Rettungsdienstes an einem dezentralen Großklinikum

M. Conradi, S. Heigenmooser, G. Seelemann, R. Schalk, C. Byhahn Klinik für Anästhesiologie, Intensivmedizin und Schmerztherapie, Klinikum der J.-W.-Goethe-Universität Frankfurt am Main

Während an vielen Großkliniken ein Notfall- und Reanimationsdienst für stationäre Patienten fest etabliert wurde, ist die Versorgung von Notfällen außerhalb der bettenführenden Bereiche zumeist ungeregelt. Aufgrund der dezentralen Struktur unseres Klinikums wurde 1999 ein betrieblicher Rettungsdienst (bRTD) etabliert, der mit Ausnahme der Stationen des zentralen Bettengebäudes auf dem gesamten Klinikgelände zum Einsatz kommt. Dieses umfaßt eine Fläche von $460000 \mathrm{~m}^{2}$ mit $27 \mathrm{~km}$ Wegen und Straßen sowie 60 Gebäuden, darunter 17 bettenführende mit insgesamt 78 Krankenstationen. Die restlichen Gebäude beherbergen Versorgungseinrichtungen, Verwaltung und wissenschaftliche Einrichtungen. Das Klinikum wird täglich von über 17000 Personen frequentiert, hinzu kommen 3000 Kraftfahrzeuge. Darüber hinaus befinden sich auf dem Gelände seit mehreren Jahren verschiedene Großbaustellen. Aufgrund der hohen Akeptanz dieses Systems mit Einsatzfrequenzen um 110/Jahr erfolgte 2004 eine Veränderung der personellen Struktur des bRTD. Seitdem wird der Notarzt durch das Personal der anästhesiologisch-operativen Intensivstation gestellt und durch eine Intensivpflegekraft der Station unterstützt. Die Pflegekräfte besitzen überwiegend die Fachweiterbildung „Anästhesie und Intensivmedizin“; Ausbildung und Training für das Pflegepersonal erfolgen in turnusmäßigen Einweisungen auf Material und Logistik des bRTD. Alle eingesetzten Ärzte verfügen über die Zusatzbezeichnung „Notfallmedizin“. Ein Mitarbeiter des hausinternen Patiententransportes, häufig mit rettungsdienstlicher Qualifikation, ergänzt das Team. Die Aufgabe des bRTD ist die notärztliche Versorgung von Patienten, Besuchern und Mitarbeitern auf dem gesamten Betriebsgelände und in den Gebäuden des Klinikums mit Ausnahme der Krankenstationen des Zentralgebäudes, die über separate Reanimationsteams betreut werden. Die Ausrüstung besteht aus einem Notfallrucksack, einer Zusatztasche für pädiatrische Notfälle sowie einem halbautomatischen Defibrillator. Das medizinische Personal ver- 
sieht seinen regulären Dienst auf der Intensivstation und wird per Funkmelder alarmiert, um dann vom Transportdienst für die Fahrt zum Einsatzort abgeholt zu werden. Dort erfolgt eine bedarfsgerechte Versorgung und bei Bedarf die Transportbegleitung in eine weiterbehandelnde Funktionseinheit. Im Jahr 2005 erfolgten 104 Alarmierungen (Fehlalarme: $n=17$ ). In etwa 50\% wurden Patienten mit einem NACA-Score IV-VII behandelt. Die hohe Fehlalarmierungsquote resultierte häufig aus Einsätzen bei Notfällen in dezentralen Bettengebäuden, die vom diensthabenden Stationsarzt mangels notfallmedizinischer Erfahrung nicht adäquat versorgt werden konnten. Die Einsatzzahlen sowie der Anteil von etwa 50\% NACA IV-VII-Patienten am Einsatzaufkommen unterstreichen den Stellenwert des bRTD an einem dezentralen Großklinikum, zeigen aber auch den trotz stetiger innerbetrieblicher Fortbildungen bestehenden Schulungsbedarf des Klinikpersonals in der Notfallmedizin auf.

Der Notarzteinsatz im Pflegeheim: Missbrauch des Rettungsdienstes oder Notwendigkeit?

D. Flaschar, J. Bathe, D. Müller, J. Breckwoldt, S. Poloczek, R. Kreutz, H.-R. Arntz Charité, Campus Benjamin Franklin

Hintergrund: Der Rettungsdienst ist, gerade für die Schwächsten der Gesellschaft, oft der einzig verfügbare Weg zur Hilfe in medizinischer und auch in sozialer Hinsicht. Vor allem großstädtische Rettungsdienste beklagen wachsende Einsatzzahlen mit „weichen“ Indikationen. Dies ist $\mathrm{z}$. T. durch missbräuchliche Inanspruchnahme z.T. aber auch durch fehlende Betreuungsangebote erklärbar. Die Betreuung von chronisch kranken Patienten (Pat.) in Altenund Pflegeheimen erweist sich als besonders problematisch. Die Pat. werden von niedergelassenen Ärzten zu festen Terminen versorgt, in Notfällen sind diese i.d.R. jedoch nicht verfügbar. Da uns steigende Einsatzzahlen bei chronisch pflegebedürftigen Pat. auffielen, haben wir in einer prospektiven Studie Einsatzindikationen sowie strukturelle und praktische Versorgungsqualität bei Notarztalarmierungen zu dieser Patientengruppe untersucht. Ergebnisse: Insgesamt wurden 351 Einsätze registriert, davon 39,6\% in Pflegeheimen, $24,8 \%$ in Seniorenheimen, $8,6 \%$ bei ambulanter Hauskrankenpflege, der Rest in sonstigen Einrichtungen. Das Durchschnittsalter der Pat. betrug 87 Jahre (31-108 Jahre), 79\% waren weiblich. $3 \%$ der Pat. waren der Pflegestufe 0 zuzuordnen, $17 \%$ Stufe 1, 33\% Stufe 2, 23\% Stufe 3 und 4\% waren Härtefälle, bei $20 \%$ war eine Zuordnung nicht möglich. $45 \%$ der Pat. waren dement, $23 \%$ chronisch bettlägerig. $47 \%$ der Pat. erhielten mindestens 5 Medikamente als Dauermedikation. Die akuten Erkrankungen waren: $16 \%$ cerebro-vaskuläre Ereignisse, $7 \%$ Krampfanfälle, $8 \%$ akute Herzinsuffizienz, 9\% akutes Koronarsyndrom, Hypoglykämie $10 \%$, Synkopen $7 \%$, die übrigen verteilten sich auf ein weites Spektrum. Die Erkrankungsschwere wurde bei $67 \%$ der Einsätze als potentiell lebensbedrohlich eingeschätzt. 154 Pat. wurde nach Erstversorgung in ein Krankenhaus eingewiesen. In $2 / 3$ der Fälle war eine examinierte Pflegekraft bei dem Pat., in $22 \%$ eine Pflegehelferin, in $10 \%$ nur eine ungelernte Kraft. Häufig notwendige Erstmaßnahmen waren spezielle Lagerung, RR-Messung, BZ-Bestimmung, Absaugen und Reanimation, die in $<50 \%$ richtig durchgeführt wurden. Geräte und Einrichtungen, wie z.B. Sauerstoff, Absauggeräte, Beatmungsbeutel waren bei gegebener Indikation nur bei etwa $1 / 3$ der Fälle verfügbar. In $22 \%$ der Fälle war die Dokumentation der Krankengeschichte, in 5\% die Pflegedokumentation sowie Angaben zur Medikation unzureichend. Strukturierte Anweisungen für Notfälle gab es nur für $23 \%$ der Pat., in nur $18 \%$ existierten „DNR“-Absprachen oder Patientenverfügungen. Schlussfolgerung: Unsere Untersuchung belegt ein weites Spektrum von Mängeln in der Struktur und Prozessqualität bei der Versorgung chronisch pflegebedürftiger Pat. Mängelschwerpunkte sind die Qualifikation des Pflegepersonals, Fehlen von Geräten und von strukturierten Anweisungen für Notfälle. Dem gegenüber steht eine häufig kaum nachvollziehbare oft „,ag- gressive" medikamentöse Behandlung der meist multimorbiden, hochbetagten, häufig dementen und vielfach bettlägrigen Patienten.

Entwicklung der Ausstattung Notarzt-besetzter Rettungsmittel zur Behandlung von Patienten mit ACS: Vergleich baden-württembergischer Umfragedaten von 2001 und 2005

\section{P. Lessing ${ }^{1}$ H. Genzwürker ${ }^{2} J_{\text {. Hehl }}{ }^{1}$ J. Hinkelbein ${ }^{2}$ K. Ellinger ${ }^{1}$} 1 Klinik für Anästhesie, Oberschwabenklinik KH St.-Elisabeth, Ravensburg

${ }^{2}$ Klinik für Anästhesiologie, Universitätsklinikum Mannheim

Hintergrund: 2001 wurde eine landessweite Umfrage zur Ausstattung von Notarzt-besetzten Rettungsmitteln in Baden-Württemberg durchgeführt [1]. Der Grad der Umsetzung der aktuellen Empfehlungen des European Resuscitation Council aus 2005 [2], welche die Ableitung eines 12-Kanal-EKG bei Patienten mit akutem Koronarsyndrom (ACS) als Schlüsseluntersuchung einstufen, um Patienten mit akutem Myokardinfarkt zu identifizieren und einer frühen Reperfusionstherapie zuzuführen, wurde durch Wiederholung der Umfrage im Herbst 2005 eruiert. Material und Methoden: Alle dem Sozialministerium gemeldeten Notarztstandorte in Baden-Württemberg erhielten einen Fragebogen, in dem die Verfügbarkeit von technischer und medikamentöser Ausstattung (hier: 12-Kanal-EKG und Fibrinolytika) abgefragt wurde. Für die Befragung im Juni 2001 standen 127 Standorte zur Verfügung, 2005 waren in Baden-Württemberg 120 Notarztstandorte gemeldet. Ergebnisse: Bei der Umfrage 2001 antworteten 116 Standorte (91,3\%), 2005 beteiligten sich 107 Standorte (89,2\%). Die Zahl der Notarzteinsätze je Standort betrug 2001 im Median 1269 (60 bis 4821) und 20051754 (80 bis 4312). Ein 12-Kanal-EKG stand 2001 auf $52,6 \%(n=61)$ und 2005 auf $89,7 \%(n=96)$ der Notarzt-besetzten Rettungsmittel zur Verfügung. 6,5\% $(n=7)$ der 2005 befragten Standorte planten innerhalb 6 Monaten, $1,9 \%(n=2)$ innerhalb 12 Monaten die Beschaffung eines 12-Kanal-EKG. An zwei Standorten $(1,9 \%)$ war keine Beschaffung eines 12-Kanal-EKGs geplant. An 19 (17,8\% von 107) der 96 Standorte mit 12-KanalEKG bestand die Möglichkeit zur Übertragung des EKG von der Einsatzstelle in die Klinik. Fibrinolytika wurden 2001 in 12,9\% $(n=15)$ und 2005 in $59,8 \%(n=64)$ mitgeführt. Eine präklinische Thrombolyse war im Oktober 2005 in 40,2\% $(n=43)$ aller Notarzt-besetzten Rettungsmittel nicht möglich, an $6,5 \%(n=7)$ der Standorte war keine Einführung der Prähospitallyse vorgesehen. Eine Beschaffung von Thrombolytika war innerhalb 6 Monaten bei $16,8 \%(n=18)$, innerhalb 12 Monaten bei $13,1 \%(n=14)$ und innerhalb 24 Monaten bei $0,9 \%(\mathrm{n}=1)$ geplant. An den Standorten, die 2005 über Fibrinolytika verfügten $(59,5 \% / \mathrm{n}=64)$, wurden Metalyse in 79,6\% $(n=51)$, Actilyse in 10,9\% $(n=7)$ und Rapilysin in $9,4 \%(n=6)$ mitgeführt. Zusammenfassung: Bei der Ausstattung Notarzt-besetzter Rettungsmittel in Baden-Württemberg zur Versorgung von Patienten mit akutem Koronarsyndrom lässt sich ein eindeutiger Unterschied zwischen 2001 und 2005 nachweisen. Trotz deutlicher Verbesserungen können die Notärzte die aktuellen ERC-Empfehlungen nicht flächendeckend umsetzen, sodass Optimierungsbedarf besteht.

Literatur: [1] Genzwürker H, Isovic H, Finteis T, Hinkelbein J, Denz C, Ellinger K. Ausstattung von Notarzt-besetzten Rettungsmitteln in Baden-Württemberg. Anästhesist 2002; 51:367-373. [2] Arntz HR, Bossaert L, Fillipatos G. Initiales Management von Patienten mit akutem Koronarsyndrom. Notfall+Rettungsmedizin 2006; 9:81-89 
Die Berufsgenossenschaftlichen Unfallkliniken auch prähospital präsent?

\section{F. Struck, P. Hilbert, G.O. Hofmann, R. Stuttmann}

Berufsgenossenschaftliche Kliniken Bergmannstrost Halle

Fragestellung: Die Berufsgenossenschaftlichen Unfallkliniken (BGU) leisten vor allem in der klinischen Versorgung von Schwerverletzten einen essentiellen Beitrag für die medizinische Qualitätssicherung in Deutschland. Da die BGU oft von Rettungsmitteln als Zielkliniken aufgesucht werden, stellt sich die Frage ihrer Leistungsfähigkeit in der prähospitalen Rettung und Intensivtransport. Methoden: Mit einer Umfrage an allen neun deutschen BGU wurde deren Einbindung in das öffentlich-rechtliche Rettungswesen analysiert. Dabei wurde die Fachrichtung und Qualifikation der Notärzte, sowie deren Verteilung auf die Rettungsmittel erfasst. Ergebnisse: Alle BGU beteiligen sich am bodengebundenen (NEF/ITW) und/oder luftgebundenen Notarztdienst (RTH/ ITH). Es werden insgesamt 19 Rettungsmittel (8 NEF, 1 ITW und $10 \mathrm{RTH} / \mathrm{ITH}$ ) mit $\mathrm{n}=352$ Ärzten besetzt. Dabei sind an sechs Kliniken NEF stationiert, an einer ein ITW. Zwei BGU stellen Notärzte für externe NEF. An vier BGU sind sowohl bodengebundene Rettung als auch Hubschrauber stationiert. An einem Standort ist lediglich ein RTH stationiert. Eine BGU stellt Personal für vier externe Hubschrauber und zwei NEF (eines davon extern). Eine BGU stellt Notärzte für einen externen ITH. Zwei Drittel $(n=225$, $64 \%)$ der Notärzte werden im bodengebundenen Notarztdienst eingesetzt, davon sind $60 \%$ Fachärzte (davon $69 \%$ FÄ, $8 \%$ FOÄ, 23\% OÄ). Nach Fachrichtung: 43\% Chirurgie, 40\% Anästhesie, $17 \%$ Innere. In der Luftrettung zu 81\% Fachärzte (davon $72 \% \mathrm{FÄ}$ 9\% FOÄ, 19\% OÄ). Nach Fachrichtung: 55\% Unfallchirurgie, 45\% Anästhesie. Regional bestehen deutliche Unterschiede in der Personalpolitik. Die Koordination des Luftrettungsmittels obliegt an 6 von 10 Stationen einem Arzt der BGU (4 Unfallchirurgie, 2 Anästhesie), die Koordination des bodengebundenen Notarztdienstes bei 7 von 9 Standorten (4 Anästhesie, 3 Chirurgie). Schlussfolgerung: Die BGU engagieren sich in großem Maßstab und vielfältiger Weise am Notarztdienst. Das Spektrum reicht von der Besetzung eines bis sechs Rettungsmittel pro Klinik. Mehr als zwei Drittel aller Notärzte erfüllen den Facharztstandart von denen über $33 \%$ oberärztlich tätig sind. Unfallchirurgen und Anästhesisten stellen den Haupanteil der Notärzte. Im Jahr 2005 leisteten die Notärzte der BGU über 22000 Einsätze auf Notarztfahrzeugen und Hubschraubern.

\section{Die Bedeutung der Luftrettung} für ein Brandverletztenzentrum in Mitteldeutschland

\section{F. Struck, J.H. Voelpel, R. Stuttmann, M. Steen}

Berufsgenossenschaftliche Kliniken Bergmannstrost Halle

Die Bedeutung der Luftrettung für ein Brandverletztenzentrum in Mitteldeutschland M. F. Struck, J. H. Voelpel, R. Stuttmann, M. Steen Berufsgenossenschaftliche Kliniken Bergmannstrost Halle Fragestellung: Der Versorgungsauftrag von Brandverletztenzentren (BVZ) ist überregional und Patienten werden häufig über den Luftweg in die Zielklinik transportiert. Ziel der Untersuchung war es, die demographischen Aspekte der Herkunft von Patienten und Luftrettungsmitteln (LRM) näher zu betrachten. Methoden: Wir evaluierten das Patientengut eines BVZ mit acht Erwachsenenbetten hinsichtlich der Herkunft der Patienten, der anfliegenden LRM und der Aufnahmezeiten. Es wurden alle Anflüge mit dem Ziel BVZ in einem Untersuchungszeitraum von sechs Jahren (1/2000-12/2005) ausgewertet. Ergebnisse: BVZ-Patienten wurden in über $80 \%$ der Fälle via Hubschrauber eingeflogen. Vom Gesamtvolumen der Anflüge im Auswertungszeitraum $(n=2085)$ waren dies 12\% $(n=249)$. Die Länder Sachsen-Anhalt $(A)$, Thüringen $(T)$ und Sachsen $(S)$ stellten 95\% $(n=235)$ des Patientengutes. $45 \%(n=112)$ der BVZ-Patienten kam aus A, dem Bundesland der BVZ-Einrichtung. Davon wurden $71 \%$ mit LRM aus A, 19\% aus S und $9 \%$ aus $\mathrm{T}$ eingeflogen. $37 \%(\mathrm{n}=91)$ der Patienten kam aus $\mathrm{T}$, wobei fast alle (95\%) mit thüringischen LRM transportiert wurden. $13 \%(n=32)$ der Patienten waren sächsischer Herkunft bei denen die Hubschrauber zu $81 \%$ aus S und zu $13 \%$ aus T stammten. Patienten aus Niedersachsen $(n=6)$ und Bayern $(n=3)$ wurden in $70 \%$ der Fälle mit LRM aus A und T ins BVZ gebracht. Insgesamt flogen 16 verschiedene Luftrettungszentren Brandverletzte ein. Dabei wurden drei Doppelstandorte jeweils als ein gemeinsames Zentrum gewertet. Christoph Sachsen-Anhalt/Christoph Halle flog 29\% $(n=69)$, gefolgt von Christoph Thüringen $20 \%(n=46)$, Christoph 61/Christoph Leipzig 11\% $(n=27)$, Christoph 36 Magdeburg und Christoph 70 Jena jeweils 9\% der Patienten. Bei den Ankunftzeiten war der Zeitraum von 10:00-17:00 Uhr am häufigsten frequentiert (52\%), ein Drittel (32\%) der Landungen lag $>0,5 \mathrm{~h}$ nach dem jahreszeitenspezifischen Sonnenuntergang. Schlussfolgerung: Das Haupteinzugsgebiet für das BVZ der BG-Halle wird mit einem Radius von 130 Kilometern in den Bundesländern $\mathrm{A}, \mathrm{T}$ und $\mathrm{S}$ definiert. Brandverletzte aus A profitieren von den benachbarten LRM in S und T. Durch die nahezu flächendeckenden Einsatzradien der LRM in Mitteldeutschland ist eine gute Transportkapazität auch nachts gewährleistet. Dabei besteht an fünf Standorten die Möglichkeit der Durchführung von Nachtverlegungen (Halle, Bad Berka, Bautzen, Erfurt und Holzdorf).

Der zerebrale Krampfanfall im Notarztdienst 128 Ätiologie, Therapie, Komplikationen: Eine retrospektive Analyse von 220 Notarzteinsätzen des Jahres 2005 bei Patienten mit zerebralem Krampfanfall

\section{Kulbe, C. Hahn, F. Bach, M. Korth, F. Mertzlufft}

Klinik für Anästhesie, Intensiv- und Notfallmedizin, Schmerztherapie, Evangelisches Krankenhaus Bielefeld-Bethel

Fragestellung: Das notärztliche Vorgehen bei Patienten mit zerebralen Krampfanfällen erfordert eine differenzierte Kenntnis der Ätiologie, Differenzialdiagnose und Therapie. In der Literatur existieren nur wenige Daten zur Häufigkeit der zugrunde liegenden Krankheitsursachen, Inzidenz schwerer Komplikationen (z. B. Status epilepticus), Einsatzverläufen und Behandlungserfolgen. Gegenstand der vorliegenden Untersuchung war es, aus einer großen Einsatzzahl notärztlich-relevante Daten $\mathrm{zu}$ generieren und Konsequenzen für das notärztliche Vorgehen abzuleiten. Methodik: Retrospektiv analysiert wurden 2432 Notarzteinsätze des Jahres 2005 im Hinblick auf biometrische und einsatztaktische Daten, Vorerkrankungen, Komplikationen, Vitalparameter, Therapie und Therapieergebnis. Ergebnisse: Insgesamt wurden 220 Patienten (40\% weiblich, $60 \%$ männlich) mit zerebralem Krampfanfall notärztlich behandelt. Das Durchschnittsalter betrug 44 Jahre (10 Monate bis 90 Jahre). Zum Zeitpunkt der Alarmierung hatten alle Patienten gekrampft. Nach durchschnittlich 6 Minuten Anfahrt lag bei 41 Patienten (19\%) ein Status epilepticus vor, $76 \%$ befanden sich in einem postiktalen Zustand, $4 \%$ waren bewusstlos ohne erkennbare Ursache. Der durchschnittliche NACA-Score als Parameter für den Schweregrad einer Erkrankung betrug 3 $\pm 0,8$ (schwere Erkrankung mit der Notwendigkeit einer stationären Abklärung). Eine akute vitale Bedrohung (NACA-Score 4 und 5) bestand für 56 Patienten (25\%). Die initiale Glasgow-Coma-Scale (GCS) betrug $11 \pm 3,9$. Bei $4 \%$ handelte es sich um die Erstmanifestation eines Krampfanfalls, bei $58 \%$ bestand eine Epilepsie in der Vorgeschichte. Andere Ursachen der Krampfanfälle waren: zerebrale Blutung, Tumor oder Insult bei 6,5\%, Alkoholentzug 5\%, Fieber $1,4 \%$, Hypoglykämie $1,4 \%$, psychogen $0,9 \%$, Elektrolytentgleisung $0,5 \%$, Stromunfall 0,5\%. Bei $26 \%$ war die Ätiologie des Krampfanfalls während der notärztlichen Therapie nicht bekannt. $95 \mathrm{~Pa}-$ tienten $(43 \%)$ wurden medikamentös therapiert. Häufigstes Antikonvulsivum war Midazolam in 85 Fällen mit einer mittleren Dosis von $6 \mathrm{mg}$ i.v.. Midazolam durchbrach den Krampfanfall auch bei 10 Patienten, bei denen andere Antiepileptika erfolglos appliziert worden waren. Bei 6 Patienten wurde eine Intubationsnarkose zur Statustherapie notwendig. 209 Patienten (95\%) mussten 
stationär aufgenommen werden, davon wurden 21 Patienten $(9,5 \%$ aller Patienten) auf einer Intensivstation weiterbehandelt, $11 \mathrm{~Pa}$ tienten (5\%) wurden ambulant behandelt. Schlussfolgerung: Der akute Krampfanfall stellt bei einem hohen Anteil der Patienten eine vitale Bedrohung dar. Er erfordert eine notärztliche Behandlung und meistens eine stationäre Aufnahme, teilweise mit Intensivtherapie. Die Epilepsie ist die häufigste Ursache eines zerebralen Krampfanfalles, wobei andere lebensbedrohliche Ätiologien differenzialdiagnostisch von Bedeutung sind und einer stationären Abklärung bedürfen. Das erfolgreichste und am meisten verwendete Antikonvulsivum war Midazolam.

Konzept für das Atemwegsmanagement durch Rettungsassistenten mit dem Larynx-Tubus: Auswertung eines Zeitraums von 18 Monaten

M. Eitzer ${ }^{1}$ O. Steller ${ }^{1}$ O. Kurbel ${ }^{1}$ M. Rudolph ${ }^{1}$ T. Finteis ${ }^{1,2}$, J. Hinkelbein ${ }^{1,2}$, H. Genzwürker ${ }^{1,2}$

${ }^{1}$ Johanniter-Unfall-Hilfe Mannheim, ${ }^{2}$ Klinik für Anästhesiologie und Operative Intensivmedizin, Universitätsklinikum Mannheim

Hintergrund: Maskenbeatmung und Intubation werden als Maßnahmen betrachtet, die Rettungsassistenten selbständig durchführen sollen. Die aktuellen Empfehlungen des European Resuscitation Council (1) thematisieren neben Problemen der Maskenbeatmung (Magenbeatmung bei fallendem ösophagealem Sphinkterdruck) auch die Problematik, große Helferzahlen adäquat zu qualifizieren, um adäquate Durchführungssicherheit bei der Durchführung der endotrachealen Intubation zu erzielen. Der Larynx-Tubus (VBM Medizintechnik) wird als eine supraglottische Alternative zu Maskenbeatmung und Intubation genannt. Material und Methoden: Ab Einführung des Larynx-Tubus (Einmalvariante LTD - Laryngeal Tube Disposable) auf allen Rettungsmitteln (RTW/KTW) der Johanniter-Unfall-Hilfe Mannheim im Januar 2005 erfolgte die prospektive Dokumentation aller Anwendungen. Alle haupt- und ehrenamtlichen Mitarbeiter des Rettungsdienstes wurden in 2 theoretischen und 2 praktischen Unterrichtseinheiten am Modell in die Anwendung des Larynx-Tubus eingewiesen, eine Ausbildung am Patienten erfolgte nicht. Erfasst wurden demographische Patientendaten, Einsatzindikation, Indikation zur Anwendung des LTD (schwierige Maskenbeatmung und/oder Intubation, primäre Strategie zur Atemwegssicherung), Anwendungsprobleme (Anzahl Versuche, Undichtigkeit) sowie der weitere Verlauf. Nach 18 Monaten erfolgte eine erste Auswertung. Ergebnisse: Im Untersuchungszeitraum wurden 1766 Einsätze in Zusammenarbeit mit einem arztbesetzten Rettungsmittel durchgeführt. Der LTD wurde bei 18 Patienten (5 Frauen, 13 Männer) durch die ersteintreffenden Rettungsassistenten platziert (1,01\%), Alter 74,3 (48-98) Jahre. In 17 Fällen handelte es sich um kardiopulmonale Reanimationen, in einem Fall um ein Schädelhirntrauma. Der LTD kam in 14 Fällen $(77,8 \%)$ als primäre Strategie zur Atemwegssicherung zur Anwendung, in 2 Fällen von schwieriger Maskenbeatmung, einem Fall von schwieriger Intubation und einem Fall mit Schwierigkeiten bei beiden Techniken durch einen pharyngealen Tumor. Die Ventilation gelang bei allen Patienten, wobei in 12 Fällen $(66,7 \%)$ keine Probleme dokumentiert wurden (u.a. im Fall des Patienten mit pharyngealem Tumor). In 3 Fällen $(16,7 \%)$ war ein zweiter Platzierungsversuch notwendig, in 3 Fällen traten Undichtigkeiten auf (orale Leckage, in einem Fall zusätzlich Zeichen von Magenbelüftung). Im Verlauf wurde bei 9 Patienten durch Notärzte die Intubation angestrebt, die in einem Fall scheiterte, in einem Fall 2 und in einem Fall 3 Intubationsversuche notwendig machte. $4 \mathrm{~Pa}$ tienten $(22,2 \%)$ erreichten die Klinik, die übrigen verstarben an der Einsatzstelle. Zusammenfassung: Bei der Anwendung durch Assistenzpersonal ermöglicht der Larynx-Tubus nach Ausbildung am Modell eine hohe Erfolgsrate bezüglich der Atemwegssicherung und der Beatmung auch bei Patienten, bei denen Probleme mit Maskenbeatmung und Intubation auftreten.

Literatur: [1] Nolan JP, Deakin CD, Soar J, Böttiger BW, Smith G. Resuscitation 2005; 67(S1):39-86
Vergleich eines interdisziplinären und eines anästhesiologisch besetzten Notarztstandortes: intubieren Anästhesisten häufiger?

S. Wegener ${ }^{1}$ J. Hinkelbein ${ }^{{ }^{2}}$, F. Hess-Jähnig ${ }^{1}$ W. Segiet ${ }^{3}$ T. Finteis ${ }^{2}$, J. Meinhardt ${ }^{2} H$. Genzwürker ${ }^{2}$

${ }^{1}$ Abt. Innere Medizin III und ${ }^{2}$ Klinik für Anästhesiologie, Universitätsklinikum Mannheim, ${ }^{3}$ Abt. Anästhesiologie Theresienkran-

kenhaus Mannheim,

Hintergrund: Die Intubation zählt zu den Grundfertigkeiten, die jeder Notarzt beherrschen muss. Fachgebiet und Tätigkeitsbereich können Unterschiede des individuellen Trainingszustandes bedingen. Zwei bodengebundene urbane Notarztstandorte (interdisziplinär mit Kollegen aus Innerer Medizin, Chirurgie, Anästhesie und Allgemeinmedizin bzw. rein anästhesiologisch besetzt) werden im Hinblick auf die Intubationshäufigkeit und -inzidenz verglichen. Methoden: Alle Einsätze eines Einjahreszeitraums wurden retrospektiv ausgewertet. Erfasst wurden die Anzahl der beteiligten Notärzte mit Ausbildungsstand (Assistenzarzt/AA, Facharzt/ FA) und Fachgebiet sowie die Anzahl der Einsätze und Intubationen je Notarzt. Bezogen auf den jeweiligen Standort, Ausbildungsstand und Fachgebiet erfolgte die Kalkulation der Intubationshäufigkeit (Intubationen/Notarzt) sowie der Intubationsinzidenz (Intubationen/Einsatz). Ergebnisse: Am interdisziplinär besetzten Standort (S1) wurden im Auswertungszeitraum 2255 Einsätze (6,2 Einsätze/Tag) durchgeführt, am anästhesiologisch besetzten Standort (S2) 3241 (8,9 Einsätze/Tag). Die 27 Notärzte (14 AA, 13 FA) an S1 führten 128 (0-18) Intubationen durch (AA: 71, FA: 57), die 31 Notärzte (22 AA, 9 FA) an S2 206 (0-28) Intubationen (AA: 166, FA: 40). Bezogen auf die Standorte ergibt sich eine Intubationshäufigkeit von 4,74 Intubationen/Jahr für S1 (AA: 5,07, FA: $4,38)$ und von 6,65 für S2 (AA: 7,55, FA: 4,44$)$ sowie eine Intubationsinzidenz von 0,056 oder $1: 17,6$ Einsätzen für S1 (AA: $0,064 / 1: 15,7$, FA: $0,050 / 1: 20,0)$ und 0,064 oder $1: 15,7$ Einsätzen für S2 (AA: 0,072/1:13,8, FA: 0,042/1:23,9). Eine Aufschlüsselung nach Fachgebieten für S1 ergibt eine Intubationshäufigkeit von 5,28 für Notärzte aus der Inneren Medizin (1128 Einsätze, AA: 4,22, FA: 7,20), Chirurgie 4,14 (524 Einsätze, AA: 7,00, FA 2,00), Anästhesie 4,00 (468 Einsätze, AA: 7,00, FA: 3,25) und Allgemeinmedizin 5,00 (135 Einsätze, nur AA). Die Intubationsinzidenz nach Fachgebieten beträgt an S1 für Notärzte aus der Inneren Medizin 0,066 oder $1: 15,2$ (AA: 0,059/1:16,8, FA: 0,074/1:13,6), Chirurgie 0,055 oder $1: 18,1$ (AA: $0,081 / 1: 12,4$, FA $0,030 / 1: 32,9$ ), Anästhesie 0,043 oder $1: 23,4$ (AA: $0,089 / 1: 11,3$, FA: 0,033/1:29,9) und Allgemeinmedizin 0,037 oder 1:27,0 (nur AA). Die Intubationshäufigkeit liegt für Assistenzärzte zwischen 4,22 (Innere Medizin, S1) und 7,55 (S2), die Intubationsinzidenz zwischen 0,089 oder 1:11,3 (Anästhesie, S1) und 0,037 oder 1:27,0 (Allgemeinmedizin, S1). Für Fachärzte findet sich eine Intubationshäufigkeit von 2,00 (Chirurgie, S1) bis 7,20 (Innere, S1) sowie eine Intubationsinzidenz von 0,074 oder $1: 13,6$ (Innere, S1) bis 0,030 oder 1:32,9 (Chirurgie, S1). Schlussfolgerung: Die Intubationshäufigkeit ist vorwiegend von der Einsatzhäufigkeit abhängig. Bezüglich der Intubationsinzidenz lassen sich Unterschiede feststellen, die sich aber in erster Linie zwischen den beiden Standorten und bezogen auf den Ausbildungsstand (AA $>$ FA, Ausnahme: Innere Medizin) finden.

Vergleich verschiedener Strategien zur Beatmung eines eingeklemmten Patienten: eine Untersuchung am Modell

S. Krahl, T. Finteis, J. Hinkelbein, L. Aniset, H. Genzwürker Klinik für Anästhesiologie und Operative Intensivmedizin, Universitätsklinikum Mannheim

Hintergrund: Die Beatmung und die Sicherung des Atemweges zählen zu den Maßnahmen mit höchster Priorität beim Traumapatienten. Bei Patienten, die im Fahrzeug eingeklemmt sind, kann der Zugang erschwert sein, so dass Maskenbeatmung und endo- 
tracheale Intubation erschwert oder unmöglich sind. Supraglottische Atemwege stellen eine Alternativstrategie dar und werden an einem Modell zur Simulation eines eingeklemmten Patienten mit Maskenbeatmung und Intubation verglichen. Material und Methoden: Ein Reanimationsmodell (Ambu Megacode Trainer) mit Cervikalstütze wurde auf dem Fahrersitz eines VW Golf IV in sitzender Position fixiert. Die Beatmung wurde durch Rettungsassistenten und Notärzte jeweils durch das Fenster der Fahrertür und vom Rücksitz mit 3 verschiedenen Techniken versucht: Maskenbeatmung, endotracheale Intubation und supraglottische Atemwege (LMA-Fastrach, Combitube, Easytube und LTS II in randomisierter Reihenfolge nach vorangegangener Einweisung). Die Anzahl der Versuche bis zum ersten Atemzugvolumen $>400 \mathrm{ml}$ (maximal 3 Versuche/maximal 3 Minuten), der Zeitbedarf (Aufnehmen des Hilfsmittels bis erste Beatmung) und Anzeichen von Magenbeatmung wurden erfasst. Ergebnisse: 21 Rettungsassistenten und 17 Notärzte beteiligten sich an der Untersuchung. Die Maskenbeatmung gelang 8 Probanden $(21,1 \%)$ vom Seitenfenster aus (1./2./3. Versuch: 3/4/1) und 21 Probanden $(55,3 \%)$ vom Rücksitz aus (1./2./3. Versuch: 5/5/11). Magenbeatmungen waren in $97,4 \%$ (Seite) und 100,0\% (Rücksitz) nachweisbar. Die endotracheale Intubation durch das Seitenfenster gelang 29 Probanden $(76,3 \%$; 1./2./3. Versuch: 14/9/6), 45,6 (20-74) sec für den erfolgreichen Versuch. Vom Rücksitz konnten 35 Probanden (92,1\%) intubieren (1./2./3. Versuch: 24/6/5), 40,8 (23-93) sec für den erfolgreichen Versuch. Magenbeatmung durch Fehlintubation trat in 55,3\% (Seite) und $39,5 \%$ (Rücksitz) auf. Für die supraglottischen Atemwege fanden sich die folgenden Daten für den Seitenzugang: LMA-Fastrach 1./2. Versuch 97,4/2,6\%, 19,4 (11-45) sec, keine Magenbeatmung; Combitube 1./2./3. Versuch 92,1/5,3/2,6\%, 35,7 (17-100) sec, alle ösophageale Position, keine Magenbeatmung; Easytube 1./2./3. Versuch $92,1 / 5,3 / 2,6 \%, 30,9(18-61) \mathrm{sec}$, alle ösophageale Position, Magenbeatmung 2,6\%; LTS II 1./2. Versuch 94,7/5,3\%, $18,8(11-34) \mathrm{sec}$, Magenbeatmung 2,6\%. Für den Zugang vom Rücksitz ergaben sich die nachstehenden Daten: LMA-Fastrach 1./2. Versuch 97,4/2,6\%, 20,1 (9-9) sec, keine Magenbeatmung; Combitube 1. Versuch $100,0 \%, 29,6$ (15-57) sec, alle ösophageale Position, keine Magenbeatmung; Easytube 1. Versuch 100,0\%, 28,2 (13-52) sec, alle ösophageale Position, keine Magenbeatmung; LTS II 1./2. Versuch 94,7/5,3\%, 20,1 (12-38) sec, keine Magenbeatmung. Zusammenfassung: Am gewählten Modell gelingt erfahrenem Personal mit supraglottischen Atemwegen die Beatmung zuverlässiger und schneller als mit Maskenbeatmung und endotrachealer Intubation. Ausbildung und Vorhaltung geeigneter Alternativen sind unabdingbar.

\section{Notarzt-/Rettungsdienst II}

\section{Die MANV-Planung bei einer Großveranstaltung - Eine prospektive Untersuchung zur Organisation und Kommunikation im Sanitätsdienst \\ T. Finteis, J. Hinkelbein, H. Hippler, H. Genzwürker \\ Klinik für Anästhesiologie und Operative Intensivmedizin, Universitätsklinikum Mannheim}

Hintergrund: Die Planung und Durchführung eines Sanitätsdienstes bei Großveranstaltungen muss medizinische und organisatorische Aspekte berücksichtigen. Anhand einer regelmäßig stattfindenden mobilen Großveranstaltung (Karnevalsumzug mit 40000 Zuschauern) wird nach abgeschlossener Planungsphase prospektiv die praktische Umsetzung der organisatorischen Abläufe im Hinblick auf die Kommunikation aller beteiligten Einsatzkräfte (Führungs- und Sanitätspersonal) untersucht. Methoden: Die Projektplanung wurde nach aufbau- und ablauforganisatorischen Aspekten für den Einsatz erarbeitet. Es wurde ein planungs- und prozessbegleitendes Qualitätsmanagement installiert, von dem eine prospektive Untersuchung zu den Bereichen „Kommunikation“ und „Ablauforganisation“ initialisiert wurde. Hierzu wurde jeder Einsatzkraft ein Fragebogen mit 14 geschlossenen Fragen zu den beiden Themenkomplexen ausgehändigt, der nach Einsatzende bei der Einsatzleitung abzugeben war. Je Frage war nur eine festgelegte Bewertung mit Punktesystem [sehr gut (4), befriedigend (3), ausreichend (2), schlecht (1)] zu markieren. QM-Zielvorgabe: Abweichung bis zu $10 \%$ von der Maximalpunktzahl ist akzeptabel, bei Abweichung von mehr als $10 \%$ ist die Ursache zu ermitteln und $\mathrm{zu}$ bewerten. Vor Einsatzbeginn wurden die Führungskräfte von der Einsatzleitung umfassend instruiert mit der Maßgabe, alle notwendigen Informationen zum Einsatzablauf anhand der vorliegenden Planungsunterlagen an ihre nachgeordneten Einsatzkräfte weiterzugeben. Ergebnisse: Die Rückgabequote der Fragebögen $(\mathrm{n}=69)$ betrug $98 \%$. Von 14 Fragen wiesen 8 Abweichungen unter $10 \%$ aus, 6 Fragen lagen mit Werten zwischen $12,7 \%$ bis $29,6 \%$ über der festgelegten Grenze. Bei diesen Fragen ergaben sich Anhaltspunkte für Kommunikationsdefizite zwischen Führungskräften und nachgeordneten Helfern $\mathrm{zu}$ rein ablauforganisatorischen Aspekten: a) Ablauf vor Einsatzbeginn mit Materialübernahme \& -abgabe, b) Art und Durchführung der Einsatzdokumentation, c) Verhalten beim MANV, d) geplante Standorte von einzurichtenden Verletztenablagen/Unfallhilfestellen beim MANV, e) Betreuung/ Versorgung der eigenen Helfer während des Einsatzes. Schlussfolgerung: Obwohl den Führungskräften alle relevanten Informationen zur Verfügung standen (Planungsunterlagen), ließen sich deutliche Defizite in der Kommunikation zwischen Führungskräften und nachgeordneten Kräften auf allen Ebenen nachweisen. Neben Informationen zum Vorgehen beim MANV, welche eine adäquate Umsetzung der vorliegenden Konzepte in Frage stellten, fanden die persönlichen Belange der Einsatzkräfte (Versorgung) die geringste Berücksichtigung. Eine Verbesserung der Kommunikationsfähigkeit (Sprache, Visualisierung, Nutzung technischer Hilfsmittel, Hand-out) von Führungskräften durch Aus- und Fortbildungsmaßnahmen könnte dazu beitragen, Informationsdefizite zu erkennen und zu beseitigen, so dass detaillierte Kenntnisse zur Einsatzplanung sicher bis zur operativen Ebene gelangen.

Unfallanalyse von Rettungshubschraubern

in Deutschland zwischen 1999 und 2004

\section{J. Hinkelbein ${ }^{1}$ T. Viergutz ${ }^{1}$ M. Dambier ${ }^{2}$ H. Genzwürker ${ }^{1}$}

${ }^{1}$ Universitätsklinik für Anästhesiologie und Operative Intensivmedizin, Universitätsklinikum Mannheim, ${ }^{2}$ DRK Waghäusel

Einleitung: Jährlich werden in Deutschland etwa 80000 Hubschraubereinsätze zur Rettung von schwerkranken oder schwerverletzten Patienten durchgeführt. Im Vergleich zur gewerbsmäßigen Beförderung von Passagieren im Routineflugbetrieb ist das Risiko für Flugunfälle bei Rettungseinsätzen jedoch deutlich erhöht. Ziel der vorliegenden Untersuchung war die deskriptive Analyse von registrierten Unfällen mit Rettungshubschraubern in Deutschland innerhalb der Jahre 1999 bis 2004. Material und Methoden: Die Flugunfälle wurden in den jährlich publizierten Flugunfallberichten der Bundesstelle für Flugunfalluntersuchung (BFU) identifiziert. Rahmendaten der Unfälle und aller beteiligten Träger (ADAC, Team DRF, Bundeswehr, Bundesministerium des Innern und private Träger) wurden zusätzlich im Internet (Unfallberichte, Zeitungsartikel u. a.) recherchiert und durch telefonische Nachfrage bei den Betreibern validiert. Die Unfälle des Untersuchungszeitraums wurden mit den Unfalldaten der Jahre 1973 bis 1994 verglichen [1, 2]. Zur statistischen Analyse wurde der Fisher's exact test genutzt, ein $\mathrm{P}<0,05$ galt als statistisch signifikant. Ergebnisse: In den analysierten sechs Jahren wurden insgesamt $\mathrm{n}=24$ Rettungshubschrauberunfälle identifiziert $(\mathrm{n}=22$ tagsüber, $\mathrm{n}=2$ nachts). 54\% $(\mathrm{n}=13)$ der Unfälle traten bei der Landung auf und 17\% $(\mathrm{n}=4)$ beim Stand auf dem Boden. $29 \%$ $(n=7)$ verteilten sich auf die restlichen Flugabschnitte (Start, Abflug, Reiseflug). Bei fünf Flugunfällen wurden insgesamt sieben Besatzungsmitglieder getötet, drei schwer und zwei leicht verletzt. Eine Person außerhalb des Luftfahrzeugs wurde schwer verletzt 
und zwei weitere leicht verletzt. Patienten wurden nicht verletzt. Die Unfallrate pro 10000 Einsätze sank im Vergleich zu den Daten der Vorjahre [2] $(\mathrm{p}<0,05)$, pro 100000 Flugstunden konnte keine Reduktion nachgewiesen werden (n.s.) [1]. Schlussfolgerungen: Die Mehrzahl der Unfälle ereignet sich tagsüber und bei der Landung. Im Vergleich mit älteren Daten wurde eine geringere Unfallrate pro 10.000 Einsätze gefunden. Bei der Analyse der Daten pro 100.000 Flugstunden konnte diese Tendenz nicht statistisch belegt werden.

Literatur: [1] Rhee KJ et al: Aviat Space Environ Med 1990; 61:750-752. [2] Lippay C. Rettungsdienst 1996; 19:14-18

\section{Accuracy and precision of three different methods}

to determine $\mathrm{PCO}_{2}\left(\mathrm{PaCO}_{2}\right.$ vs $\mathrm{PetCO}_{2}$ vs $\left.\mathrm{PtcCO}_{2}\right)$

during interhospital ground transport of critically ill and ventilated adults

J. Hinkelbein ${ }^{1}$, F. Floss ${ }^{1}$, C. Denz ${ }^{1}$, H. Krieter ${ }^{1,2}$

${ }^{1}$ University Clinic for Anesthesiology, University Hospital

Mannheim, ${ }^{2}$ Clinic for Anesthesiology, Klinikum Saarbruecken

Introduction: Transportation of critically ill and mechanically ventilated patients represents a common, yet difficult, problem. Three different methods to determine $\mathrm{PCO}_{2}$ during interhospital transport (IHT) are available: arterial blood gas analysis $\left(\mathrm{PaCO}_{2}\right)$, end-tidal $\left(\mathrm{PetCO}_{2}\right)$ and transcutaneous $\left(\mathrm{PtcCO}_{2}\right)$ measurement. The aim of the present study was to compare accuracy and precision of these methods simultaneously in critically ill and ventilated adults during IHT. Material and Methods: Patients requiring IHT were investigated after informed consent in a prospective study. $\mathrm{PCO}_{2}$ was determined five times in each patient during the transport simultaneously by 1$)$ arterial blood gas analysis $\left(\mathrm{PaCO}_{2}\right.$ (IRMA), IRMA ${ }^{\circledR}$, Diametrics Inc, St. Paul, USA), 2) end-tidal (Pet$\mathrm{CO}_{2}$, Propaq 106 EL Monitor, Protocol Systems Inc., Beaverton, USA) and 3) transcutaneous measurements $\left(\mathrm{PtcCO}_{2}\right.$, Radiometer Copenhagen, Brønshøj, Denmark). The data obtained during transportation were compared to an in-hospital reference arterial blood-gas-analysis (ABL 625, Radiometer, Copenhagen, DK). For statistical analysis the Bland-Altman method was used. A $\mathrm{P}<0.05$ was considered statistically significant. Results: 170 data sets $\left(2 \mathrm{xaCO}_{2}, \mathrm{PetCO}_{2}, \mathrm{PtcCO}_{2}\right)$ were obtained in 34 patients $(61 \pm 16$ years old; 19 male, 15 female). The mean $\mathrm{PaCO}_{2}$ (ABL625) was $43.2 \pm 8.8 \mathrm{mmHg}$ ranging from 24.9 to $72.4 \mathrm{mmHg}$. Bland-Altman analysis revealed a bias and precision of $-0.6 \pm 2.5 \mathrm{mmHg}$ for the IRMA device $(\mathrm{P}>0.05)$ and $-0.6 \pm 7.5 \mathrm{mmHg}$ for the transcutaneous measurement $(\mathrm{P}>0.05)$. The end-tidal bias and precision $(-$ $5.3 \pm 6.1 \mathrm{mmHg})$ differed significantly $(\mathrm{P}<0.003)$ when compared to the reference measurement. Conclusions: During IHT $\mathrm{PaCO}_{2}$ (IRMA) and $\mathrm{PtcCO}_{2}$ provide the best accuracy when compared to the reference measurement. For patients who either require a tight control of $\mathrm{PCO}_{2}$ or endured lengthy transportation, caponography alongside arterial blood gases or the transcutaneous measurement of $\mathrm{PCO}_{2}$ could be useful.

\section{Versorgung des akuten ST-Hebungsinfarktes \\ im klinischen Alltag; klinische Realität und Optimierung der leitlinienkonformen Behandlung}

T. Pohl, D. Leistner, S. Mittenzwei, M. Greif, R. Wakili,

P. Boekstegers, A. Knez

Medizinische Klinik I, Klinikum Großhadern, Ludwig-MaximilianUniversität München

Hintergrund: Nach den aktuellen Leitlinien ist die primäre perkutane Angioplastie die anzustrebende Therapie bei Patienten mit einem ST-Hebungsinfarkt, wenn diese innerhalb von 90 Minuten nach Eintreffen in ein Krankenhaus durchgeführt wird. Ziel unserer Untersuchung war es $\mathrm{zu}$ analysieren, wie die Versorgungssituation bei Patienten in unserer Klinik mit städtischem Einzugsgebiet und städtischem Umland ist. Methoden: Es wurden retro- spektiv 193 Patienten im Jahr 2005 mit einem ST-Hebungsinfarkt bezüglich des zeitlichen Ablauf der Versorgung, der medikamentösen Therapie und des klinischen Verlaufs mit 9 Monats Follow-up analysiert. Ergebnisse: $40 \%$ der Patienten wurden primär in unserer Klinik aufgenommen, 3/4 über den Notarzt. 60\% der Patienten kamen über ein Primärkrankenhaus ohne interventionelle kardiologische Abteilung. 30\% der Patienten im Primärkrankenhaus erhielten eine Lysetherapie. Die Dauer vom Schmerzbeginn bis zum ersten Arztkontakt betrug $79 \pm 143$ Minuten. Wurde der Rettungsdienst primär verständigt verkürzte sich diese Dauer auf $66 \pm 137$ Minuten, stellten sich die Patienten selbständig in der Notaufnahme vor, vergingen $159 \pm 214$ Minuten. Wurden Patienten aus einem Primärkrankenhaus in unsere Klinik verlegt, betrug die mittlere Dauer von Aufnahme im Primärkrankenhaus bis zur invasiven Untersuchung 3,4 \pm 14 Stunden. Die durchschnittliche „Door-to-Balloon-Zeit" in unserer Klinik betrug $106 \pm 167$ Minuten. Bei Patienten die nicht lysiert waren, die weiterhin Beschwerden oder ST-Hebungen hatten, betrug die mittlere „Door-to-Balloon-Zeit" $64 \pm 49$ Minuten. $70 \%$ aller Patienten wurden innerhalb einer „Door-to-Balloon-Zeit" von 90 Minuten invasiv untersucht. Nicht lysierte Patienten mit Beschwerden oder STHebungen wurden $\mathrm{zu} 86 \%$ innerhalb von 90 Minuten invasiv untersucht. Die Mortalität im Krankenhaus betrug 7,3\%, die 9 Monats-Mortailität 14,6\%. Die „Door-to-Ballon-Zeit" war bei Patienten, die verstarben signifikant kürzer als bei den restlichen $\mathrm{Pa}-$ tienten (50 \pm 8 Minuten vs. $100 \pm 14$ Minuten). Diskussion: Die Zeit vom Schmerzbeginn bis zum ersten Arztkontakt ist trotz aller Bemühungen der letzten Jahre mit fast 80 Minuten noch immer zu lange, dies gilt für Patienten mit Erstereignis und bekannter KHK. Eine leitlinienkonforme Reperfusionstherapie von Patienten mit ST-Hebungsinfarkten wird derzeit in einer Minderheit der Patienten durchgeführt. $86 \%$ der Patienten, die primär in unserer Klinik aufgenommen werden, werden innerhalb von 90 Minuten invasiv untersucht und behandelt. Bei Aufnahme in ein Primärkrankenhaus ohne Herzkatheterabteilung ist eine leitlinienkonforme Therapie nicht umgesetzt. Nur 30\% der Patienten ohne zeitgerechte invasive Diagnostik werden lysiert. Ziel ist es durch eine Optimierung der Patienteninformation und eine strukturelle Optimierung die Zeiten bis zur invasiven Untersuchung zu verkürzen, resp. Patienten ohne realistische Chance der zeitnahen Katheterdiagnostik durch einen Fibrinolyse zu therapieren.

Präklinische und klinische Versorgung

des akuten Koronarsyndrom ohne ST-Hebung mit früher invasiver Strategie

T. Pohl, D. Leistner, M. Greif, R. Wakili, C. Kupatt, P. Boekstegers, A. Knez

Medizinische Klinik I, Klinikum Großhadern, Ludwig-MaximilianUniversität München

Hintergrund: Eine leitliniengetreue Behandlung des akuten Koronarsyndroms ohne ST-Hebung beinhaltet eine frühe Risikostratifizierung mit der Festlegung eines invasiven Koronarinterventionszeitpunktes und eine effektive medikamentöse Therapie. Methoden: Es wurden retrospektiv 187 Patienten analysiert, die 2005 mit einem akuten Koronarsyndrom ohne ST-Hebung behandelt wurden. Anhand der Notarztprotokolle, Herzkatheterberichte und der Verlaufsdokumentation wurde der zeitliche Ablauf der Versorgung, die medikamentöse Therapie und der klinische Verlauf analysiert. Alle Patienten wurden nach 9 Monaten angeschrieben und der klinische Verlauf wurde durch einen Fragebogen erhoben. Ergebnis: $27 \%$ der Patienten wurden primär in unserer Klinik aufgenommen, 1/3 dieser Patienten hatte sich vorher beim Hausarzt vorgestellt. Die restlichen Patienten wurden über ein Primärkrankenhaus ohne interventionelle kardiologische Abteilung überwiesen. Vom Schmerzbeginn bis zur Vorstellung vergingen bei den Patienten 19,9 $\pm 39,6$ Stunden. Im Primärkrankenhaus verblieben die Patienten durchschnittlich $13,5 \pm 10,5$ Stunden, in unserer Klinik dauerte es im Schnitt 8,8 $\pm 9,5$ Stunden bis zur Koronarangio- 
graphie. 92\% der Patienten sind aufgrund einer bekannten KHK, eines Diabetes, dynamischer EKG-Veränderungen mit ST-Senkungen oder hämodynamischer- oder Rhythmusinstabilität nach den Leitlinien für das akute Koronarsyndrom als Hoch-Risiko-Patient einzustufen. $89 \%$ aller Patienten waren präklinisch mit ASS versorgt, $26 \%$ hatten ASS als Dauermedikation. $82 \%$ erhielten im Primärkrankenhaus Clopidogrel, $75 \%$ in einer Dosis von bis $\mathrm{zu}$ $300 \mathrm{mg}$ und nur 7\% bekamen $600 \mathrm{mg}$ Clopidogrel. 46,6\% wurden präklinisch mit Heparin behandelt, kein Patient mit niedermolekularem Heparin. Im Primärkrankenhaus wurden 12\% mit einem GP-IIbIIIa-Rezeptor-Antagonisten behandelt. Die Patienten verbrachten im Durchschnitt 2,6 $\pm 3,2$ Tage auf der Intensivstation, die gesamte Krankenhausaufenthaltsdauer betrug 12 $\pm 6,8$ Tage. Bei 9\% der Patienten wurde nach der diagnostischen Herzkatheteruntersuchung die Indikation zur chirurgischen Revaskularisation gestellt, $86 \%$ wurden durch eine PTCA und Stentimplantation behandelt. Ein erneutes Koronarsyndrom oder einen Myokardinfarkt erlitten 5,9\% der Patienten, $2 \%$ wurden im weiteren Verlauf mit einer Bypass-Operation versorgt. Die 9-Monats-Mortalität betrug 6,4\%, Diskussion: Patienten mit akutem Koronarsyndrom ohne ST-Hebung werden bezüglich des Zeitmanagements leitliniengetreu behandelt. Präklinisch ist die Versorgung mit ASS fast bei allen Patienten gewährleistet, allerdings werden nur ca. die Hälfte der Patienten mit Heparin versorgt. Niedermolkulares Heparin findet keine Anwendung, trotz positiver Studienlage. Ebenso werden Clopidiogrel und GP-IIbIIIa-Rezeptor-Antagonisten präklinisch nicht angewandt. Der Einsatz im Primärkrankenhaus bei Hoch-Risiko-Patienten ist gekennzeichnet von einer häufigen Clopidogrel-Unterdosierung und einer zurückhaltenden Anwendung von GP-IIbIIIa-Antagonisten.

\section{Diagnosesicherheit in der präklinischen notärztlichen Versorgung}

J.-C. Schewe, N. Laabs, S. Weber, A. Hoeft, U. Heister, F. Stüber Klinik für Anästhesiologie und Operative Intensivmedizin, Universitätsklinikum Bonn

Einleitung: Trotz zahlreicher Bemühungen zur Verbesserung des Qualitätsmanagements [1] und der Ergebnisqualität von Notarzteinsätzen sind verlässliche Aussagen zur Diagnosesicherheit in der präklinischen Versorgung strukturell bedingt weiterhin schwierig. Vorhandene Publikationen betrachten nur Teilaspekte oder untersuchen nur das Outcome einzelner Diagnosen [2]. Aktuelle vergleichende Untersuchungen an einem größeren Patientenkollektiv fehlen. Fragestellung: Ziel der Untersuchung war die Beurteilung der Diagnosesicherheit im Routinealltag unter Berücksichtigung von notärztlichen Haupt- und Nebendiagnosen und der Krankenhausentlassungsberichte in der Bundesstadt Bonn anhand aller Notarzteinsätze eines Jahres. Methodik: Grundlage waren eine PC-gestützte Einsatzdokumentation mit dem MedlinqEasy System der Primärnotarzteinsätze im Jahr 2004 und die Krankenhausentlassungsberichte der eingewiesenen Patienten. Zur Beurteilung der Routinetätigkeit erfolgte bewusst eine retrospektive Datenanalyse. Unter Berücksichtigung einer freien Haupt- und Nebendiagnose des Notarztes wurden diese mit den Krankenhausentlassungsberichten verglichen und eine fallbezogene Einschätzung der Diagnosequalität durchgeführt. Ergebnisse: Von insgesamt 5483 Primärnotarzteinsätzen im Jahr 2004 kam es in 4444 Fällen zu einer Krankenhauseinweisung. Davon konnten 996 Krankenhausentlassungsberichte ausgewertet werden. Bezogen auf die Einsatzklassifizierung (NACA Score) der eingewiesenen $\mathrm{Pa}$ tienten lag die Rücklaufquote der auswertbaren Epikrisen somit bei 31,4\% (NACA 4-6) bzw. bei 24,1\% (NACA 3-6). Die Ergebnisqualität mit $87 \%$ korrekten Diagnosen war Ausdruck eines hohen Qualitätsniveaus. Die häufigsten diagnostischen Irrtümer waren das Übersehen oder Fehleinschätzen eines Akuten Koronarsyndroms. Nur in $1,7 \%$ der Fälle wurde eine gravierende Diagnose übersehen oder falsch eingeschätzt. Unter Berücksichtigung der Einsatzzeiten nahm die Fehlerquote der durch den Notarzt gestell- ten Diagnosen in den Nachtstunden zu. Ebenso war bei älteren Patienten eine deutlich erhöhte Fehlerquote zu beobachten. Diskussion: Als ein Baustein des Qualitätsmanagements in der Notfallmedizin war das Ziel der vorliegenden Untersuchung, die $\mathrm{Zu}-$ verlässigkeit von Notarztdiagnosen zu überprüfen. Insgesamt ergab sich eine hohe Diagnosesicherheit. Im Hinblick auf die stetig steigenden Einsatzzahlen in der notärztliche Versorgung und die aktuellen Diskussionen um ärztliche Arbeitszeiten mag es nicht verwundern, dass die Fehlerquote in den Nachtstunden dabei deutlich höher lag. Trotz Verbesserungen in der apparativen diagnostischen Ausstattung der Notarztwagen (u.a. 12-Kanal-EKG) wurde am häufigsten die Diagnose des Akuten Koronarsyndroms übersehen bzw. falsch eingeschätzt.

Literatur: [1] Messelken $M$ et al: Ergebnisqualität in der Notfallmedizin. Notfall und Rettungsmedizin 1998; 1:143-149. [2] Carlsson J et al: Kurz- und Langzeitüberleben nach cardiopulmonaler Reanimation. Intensivmedizin 1998; 35:34-41

Die notärztliche Versorgung von Schlaganfallpatienten 194 A. Misera, C. Konrad, J. Stückemann, H.-J. Trappe, W. Frebel, D. Nabavi

Marienhospital Herne, Klinikum der Ruhr-Universität Bochum, Klinik und Poliklinik für Neurologie und Klinik und Poliklinik für Anästhesiologie und operative Intensivmedizin des Universitätsklinikums Münster, ${ }^{4}$ Berufsfeuerwehr der Stadt Münster

Hintergrund und Ziel: Pathophysiologie und moderne Therapieoptionen des Schlaganfalls erfordern eine Behandlung des Schlaganfallpatienten als akuten Notfall. Dabei ist durch die Thrombolyse ein enges Zeitfenster vorgegeben, darüber hinaus steht diese Therapie fast ausschließlich an Zentren mit Stroke Unit zur Verfügung. Ziel der Studie war die Erhebung einer IST-Analyse zum prästationären Notfallmanagement akuter Schlaganfallpatienten im Einzugsgebiet einer überregionalen Stroke Unit. Methodik: Es wurden 3138 Notarzteinsatzprotokolle aus dem Jahr 2000 ausgewertet und Informationen zu Patientencharakteristika, Zeitablauf der Einsätze, der von den Notärzten durchgeführten Diagnostik und Therapie sowie zu den angefahrenen Krankenhäusern analysiert. Die Versorgung der Schlaganfallpatienten $(n=386)$ wurde mit der Versorgung anderer Patientengruppen $(n=2752)$ verglichen, die überwiegend internistische (z.B. kardiovaskuläre und pulmonale) oder chirurgische Krankheitsbilder aufwiesen. Ergebnisse: 1. Epidemiologie: Die Patienten der Schlaganfallgruppe waren im Mittel 73,8 Jahre alt, die der Vergleichsgruppe 56,7 Jahre. In der Schlaganfallgruppe waren jeweils 50\% der Patienten männlich bzw. weiblich, in der Vergleichsgruppe waren 57,2\% männlich und 42,8\% weiblich. 2. Analyse der Zeitabläufe: In der Schlaganfallgruppe betrug die Hilfsfrist im Mittel 5,1 min. Die Dauer des Einsatzes betrug im Mittel 36 min. 3. Auswahl des Zielkrankenhauses: $30,1 \%$ der Patienten der Schlaganfallgruppe wurden in ein Zentrum mit Erfahrung in der Thrombolyse, CT, Neurologie und Neurochirurgie gebracht (Vergleichsgruppe 16,9\%). Weitere $18,4 \%(13,0 \%)$ wurden in Krankenhaus mit Neurologie und CT und $17,1 \%(20,0 \%)$ in ein Krankenhaus mit CT und Neurochirurgie gefahren. 4 . Antikoagulanziengabe ohne CCT: 13 von 368 Patienten erhielten ohne Ausschluss einer Blutung Acetylsalicylsäure oder Heparin vom Notarzt. Schlussfolgerungen: 1. Die Patienten der Schlaganfallgruppe sind signifikant älter als die $\mathrm{Pa}$ tienten der Vergleichsgruppe, die Geschlechterverteilung ist ausgeglichen. 2. Die gesetzlichen Zeitvorgaben im Rettungsdienst werden in über $90 \%$ der Fälle erfüllt. 3. Der Notarzt entscheidet durch die Wahl des Zielkrankenhauses, ob einem Patienten eine Therapie mittels Thrombolyse zur Verfügung steht. Trotz signifikanter Unterschiede in den gewählten Zielkrankenhäusern in der Schlaganfallgruppe und der Vergleichsgruppe werden nur 48,5\% der Patienten aus der Schlaganfallgruppe in Krankenhaus mit neurologischer Abteilung gebracht. 4. Ziel weiterer Bemühungen sollte es sein, (a) die Verabreichung von Antikoagulanzien durch Notärzte bei der Verdachtsdiagnose Schlaganfall zu vermeiden, 
(b) einem wesentlich größeren Teil der Patienten die Behandlungsoption der Thrombolyse durch geeignete Auswahl des Zielkrankenhauses zu eröffnen und (c) die Versorgung in der Prähospitalphase durch gezielte Fortbildungsmaßnahmen des Rettungsdienstes weiter zu verbessern.

Können Ärzte ohne spezielle Vorkenntnisse eine ALS-basierte Echokardiographie innerhalb eines 8-stündigen Kurses erlernen?

H. Ilper ${ }^{1}$, F.H. Seeger ${ }^{2}$, M. Rüsseler ${ }^{3}$, H. Steiger ${ }^{4}$, F. Walcher $^{3}$, E. Mueller ${ }^{5}$, R. Breitkreutz ${ }^{1}$

${ }^{1}$ Anästhesiologie, ${ }^{2}$ Kardiologie, ${ }^{3}$ Unfallchirurgie, Universitätsklinikum, Frankfurt am Main, ${ }^{4}$ Kardiologie, ${ }^{5}$ Inst. f. Notfallmedizin, Klinikum Darmstadt

Kontext: Bei Schock unklarer Genese, Zyanose, PEA und bei kardiopulmonaler Reanimation (CPR) sollte eine transthorakale Echokardiographie (TTE) zur Differentialdiagnose durchgeführt werden. Bei einer CPR fordern die alten und neuen ERC/ILCOR Leitlinien einerseits die Diagnose behandelbarer Ursachen, setzen aber andererseits hierfür enge zeitliche Grenzen [1]. Wir stellten den Algorithmus „Fokussierte Echokardiographische Evaluation bei Reanimation (FEER)" vor, der eine zeitlimitierte, ALS-konforme TTE beinhaltet [2]. Wir überprüften die Hypothese, ob Aerzte ohne spezielle Vorkenntnisse in der TTE innerhalb eines 8-stündigen Kursus [3] das korrekte Einstellen eines subcostalen 4-Kammerblicks in der langen Achse mit Einhalten einer Untersuchungszeit von weniger als 10 Sekunden erlernen können. Methoden: Der Kurs enthielt 4 Unterrichtseinheiten $(1 \mathrm{UE}=45 \mathrm{~min})$ Theorie und 4 UE Praxis zum Basiswissen Echokardiographie in 2 hands-on Trainingseinheiten. Es wurden weiterhin der Ablauf von FEER und einfache pathologische Befunde erlernt sowie Trainingseinheiten zur Einstellung eines „Vier-Kammerblicks“ von subcostal in der langen Achse am gesunden Probanden mit einem portablen Sonographiegerät geübt. Untersuchungssequenzen der TTE der Kursteilnehmer wurden auf DVD aufgenommen und von einem Kardiologen in Hinblick auf die benoetigte Untersuchungszeit und -qualität verblindet ausgewertet. Die Erfolgsrate (ER) definierten wir als das Erreichen einer richtigen Anlotung und Darstellung innerhalb von 30 Sekunden für mindestens 3 Sekunden im Verhältnis zu allen Teilnehmerversuchen. Zur Kontrolle des theoretischen Lernerfolgs wurde ein Multiple-Choice-Test mit 21 Fragen jeweils vor und nach dem Kurs durchgeführt. Insgesamt nahmen bisher 43 Ärzte (AE) und 9 Instruktoren (INSTR, Kardiologen oder Ärzte erfahren in mehr als 200 TTE) an dem Kurs teil. Ergebnisse (Stand 18.6. 2006): Bis zu vier voneinander unabhängige Versuche (A-D) wurden absolviert. AE: (A): 9,9 $\pm 4,6$ (Zeit in Sekunden; $\mathrm{MW} \pm \mathrm{SD}$, ER 55\%, (B): 10,4 $\pm 9,5$, ER 55\%, (C): $8,1 \pm 5,7$, ER $86 \%(+)$, (D): $6,6 \pm 4,3(+)$; ER $83 \%$. INSTR: (A):

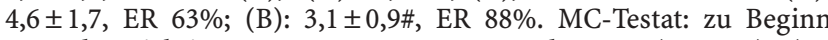
$58 \%$ der richtigen Antworten, zu Kursende $76 \%(\mathrm{p}<0,01)$. (UTest, (+), $\mathrm{p}<0,05$ vs. 1 . und 2. Test, \#: $p<0,05$ für den Unterschied zwischen INSTR und AE). Schlussfolgerungen: Die meisten Ärzte können eine zeitlimitierte, ALS-basierte TTE erlernen und haben dabei eine gute Lernkurve zur Steigerung ihres Basiswissens in Echokardiographie und zum Erkennen einfacher Pathologien. Unser Kurskonzept unterstützt damit die Hypothese.

Literatur: [1] Multiple authors: ERC guid. Resuscitation 2005; 6751:1-185. [2] Breitkreutz et al: The Concept of Focused Echocardiographic Evaluation in Resuscitation management (FEER): an ALS-conformed algorithm. Critical Care Med 2006; in press. [3] Interdisziplinärer Kurs: Sonographie und Echokardiographie in der Intensiv- und Notfallmedizin; www.notfallsonographie.de (Zugriff: 18. 6.2006)
EDV-unterstütztes Ressourcenmanagement im Katastrophenfall

S.-M. Heining ${ }^{1}$, S. Nestler ${ }^{2}$, G. Klinker ${ }^{2}$, P. Hornburger ${ }^{3}$, K.-G. Kanz ${ }^{1}$

${ }^{1}$ Chirurgische Kinik und Poliklinik - Innenstadt, Klinikum der LMU, München, Germany, ${ }^{2}$ CAMP, TU, München, Germany,

${ }^{3}$ Berufsfeuerwehr, Landeshauptstadt, München, Germany,

${ }^{4}$ Chirurgische Klinik und Poliklinik, Klinikum LMU - Innenstadt, München, Germany

Fragestellung: Bei Großschadensereignissen wie Bus- oder Eisenbahnunfällen, Großbränden oder Terroranschlägen kommen auf die Rettungskräfte durch den Massenanfall von Verletzten (MANV) besondere logistische Herausforderungen zu. Die Verletzten müssen von der Einsatzleitstelle auf alle erreichbaren Krankenhäuser entsprechend der dort vorhandenen Kapazitäten und der Schwere der Verletzungen aufgeteilt werden. Nach der Triage durch einen Notarzt oder speziell ausgebildete Rettungsassistenten werden die Verletzen den entsprechenden Krankenhäusern zugeteilt und dorthin zur Weiterversorgung verbracht. Methode: Unter anderem in Hinblick auf die Großveranstaltungen im Rahmen der Fußballweltmeisterschaft 2006 in Deutschland wurden Schwachpunkte im Informationsfluss analysiert und anhand eines prototypischen Systems Vorschläge zur Unterstützung der Einsatzkräfte gemacht. Die Sichtung erfolgte nach dem mSTaRT-Konzept, welches seit 8/2005 im Rettungsdienstbereich München implementiert ist (Kanz 2006). Zum Einsatz kommen PDAs für die Sichtungsteams und Tablet PCs für die mobile Einsatzleitstelle, deren Daten dann zusammen mit den Kapazitätsmeldungen der Krankenhäuser in der Einsatzleitstelle zur Koordination der weiteren Versorgung benutzt werden. Die Patienten werden mittels einer Umhängekarte mit aufgedrucktem Barcode identifiziert, die von den mit Barcodelesern ausgestatteten PDAs ausgelesen werden kann. Hierbei ist insbesondere darauf zu achten, dass die vorhandenen Abläufe nicht behindert oder gar verzögert werden. Ergebnisse: Bei einer Übung zur Vorbereitung der Rettungsdienste in München auf mögliche Großschadensereignisse wurden Sichtungsteams der Berufsfeuerwehr München mit dem PDA-gestützten Triagesystem ausgestattet. Der Ablauf wurde von der Sichtung am Unfallort über die Transportentscheidung bis zur Übergabe und Erstdiagnostik in der Klinik erprobt. Durch die parallele Erfassung der Sichtungsdaten in Echtzeit ist der Leitstelle vor Ort ein schnellere und präzisere Abbildung der Schadenslage möglich. Der LNA kann die Entscheidungen der Sichtungsteams nachvollziehen und ggf. korrigieren, wodurch der Anspruch auf ärztliche Leitung der Triage gewahrt wird. Die Zuordnung der Patienten vom Unfallort bis in die Klinik bleibt nachvollziehbar und die Datenübermittlung von Personendaten an Behörden zur Indentifizierung ist möglich. Zusammenfassung: Die Einführung eines EDV-gestützten Triage- und Identifizierungsystems ermöglicht eine schnelle und inhaltlich präzise Datenerhebung und -übermittlung bei Großschadensereignissen. Die Definition von Standards bzgl. erfasster Triage- und Personendaten ist notwendig und eine zentrale Erfassung sinnvoll um die Identifizierung und das Patiententracking zu optimieren.

Literatur: Kanz KG, Hornburger P, Kay MV, Mutschler W, Schäuble W: mSTaRT-Algorithmus für Sichtung, Behandlung und Transport bei einem Massenanfall von Verletzten. Notfall Rettungsmed 9:264-270 
Überprüfung der Anwendbarkeit des Larynxntubus (LT-S II) und einer modifizierten Einführtechnik für Kleinkinder bei Ärzten ohne spezielle Vorkenntnisse

R. Schalk, W.M. Roßkopf, C. Hofstetter, M. Luijc, C. Byhahn,

\section{R. Breitkreutz}

Klinik für Anästhesiologie, Intensivmedizin und Schmerztherapie, Klinikum der Johann Wolfgang Goehte Universität, Frankfurt am Main

Kontext: Es gibt supraglottische Atemwegshilfen, die für Notfälle mit Kleinkindern zur Verfügung stehen. Eine neue Entwicklung ist der Larynxtubus LT-S II, der für Kinder sämtlicher Altersklassen entwickelt wurde und als einziger supraglottische Atemweg über ein Drainagelumen das Einlegen einer Magensonde auch bei Kleinkindern erlaubt. Um eine breite Anwendung für Notfallsituationen zu ermöglichen sollte die Einführtechnik z.B. bei Elektiveingriffen geübt werden. Daher führten wir eine prospektive Beobachtungsstudie in der Narkoseeinleitung durch, um die Wahrscheinlichkeit der erfolgreichen Anlage durch eine modifizierte Einführtechnik zu untersuchen. Methode: 4 Anästhesisten ohne spezielle Vorkenntnisse in der Anlage eines LT-S II (VBM-Medizintechnik, Sulz (Neckar), Deutschland) wurde eine modifizierte Einführtechnik jeweils unmittelbar nach ausreichender Präoxygenierung und Narkoseeinleitung erklärt und daraufhin der LT-S II (2 Größen, je nach Körpergewicht und -größe, wiederverwendbar) eingelegt sowie nach den Angaben des Herstellers geblockt. Die korrekte Platzierung wurde durch Auskultation, Messung des end-expiratorischen $\mathrm{CO}_{2}$, Spitzendruck bei druckkontrollierter Beatmung und die erfolgreiche Anlage einer Magensonde/Absaugkatheters überprüft. Insgesamt wurden 25 Kleinkinder $(n=2$, weiblich, $\mathrm{n}=23$ männlich, mittleres Lebensalter: 3,8 Jahre, mittleres Körpergewicht/-größe: $18,6 \mathrm{~kg} / 104 \mathrm{~cm}$ in die Untersuchung eingeschlossen. Das Aufklärungsgespräch mit den Eltern im Rahmen der Prämedikationsvisite beinhaltete die Intubationsnarkose und die Angabe von Atemwegsalternativen. Es wurden alle Ereignisse im Zusammenahang mit der Anlage des LT-S II im Hinblick auf Komplikationen und Änderung des Atemwegs im Verlauf der Narkose protokolliert. Ergebnisse: Bei 20/25 Anlagen gelang eine korrekte Positionierung beim erstem Mal. Bei 5/25 Anlagen gelang dies beim zweiten Versuch. Es kam bei 5 Narkosen zu einem Anstieg des Spitzendrucks, so dass der LT-S II ca. $1 \mathrm{~cm}$ weiter in den Ösophagus verschoben und damit das Problem beseitigt wurde. Bei $3 / 25$ Positionierungen war bedingt durch den Eingriff eine Kopfseitlagerung nötig, die zum Abfall des Atemzugvolumens führte, wobei durch ein Esmarchmanöver eine Normalisierung der Ventilation erreicht wurde. Bei 1/25 Anlagen kam es zu einem Laryngospasmus, woraufhin eine Intubation durchgeführt werden musste. Schlussfolgerung: Die vorliegenden Ergebnisse dieser Beobachtungsstudie weisen darauf hin, dass der LT-S II mit einer modifizierten Einführtechnik mit hoher Wahrscheinlichkeit durch einen Ungeübten zu einer erfolgreichen Sicherung des Atemwegs und bei Beachtung und Therapie weniger Komplikationen $\mathrm{zu}$ einer suffizienter Beatmung führen kann. Daher stellt der LT-S II einen interessanten „alternativer Atemweg“ für Notfälle bei Kleinkindern dar.

\section{Reanimation, Lehre}

\section{Schulungskonzept CPR für Pflegepersonal \\ Dönitz, A. Uhl \\ BG-Unfallkrankenhaus - Hamburg}

Einleitung: Die aktuellen Guidelines des ERC machten eine zeitnahe Schulung der Mitarbeiter eines Krankenhauses unabdingbar. Um in einem möglichst kurzen Zeitraum eine große Anzahl von Mitarbeitern bei laufendem Betrieb zu schulen wurde ein modulares Konzept entwickelt. Methode: Zu schulen sind 120 Mitarbeiter der Bereiche Anästhesie und Intensivstationen. Da bekannte, zweitägige Kurse aus mehreren Gründen nicht wahrgenommen werden konnten entwickelten wir ein eigenes Konzept. Vorteilhaft dabei, dass ein Mitarbeiter der Anästhesieabteilung ERC-Instructor ist. Eine Reanimationspuppe ist im Haus vorhanden. Der gesamte Schulungsinhalt wurde in einzelne Pakete verpackt, die stundenweise von den Mitarbeitern besucht werden können. Ergebnis: Das Konzept sieht vor, dass nach einer zweistündigen Basisschulung im eigenen Arbeitsbereich, hierzu bieten sich Abteilungsbesprechungen an, ein Großteil der Mitarbeiter erreicht wird. Darauf aufbauend können dann weitere Unterrichtspakete, die in ein bis zwei Stunden variieren, von Mitarbeitern aller Bereiche die eine Basisschulung absolviert haben besucht werden. Das Komplettpaket endet mit einer Wissensabfrage. Schlussfolgerung: Durch Aufteilung der Unterrichtsinhalte in Stundenpakete ist es einem Großteil der Mitarbeiter möglich, zeitnah an Schulungen zur CPR während der Dienstzeit teilzunehmen. Vorteilhaft wenn Trainingsmaterial vorhanden ist. Durch eigene Trainer entstehen geringe Kosten. Die Unterrichtspakete können jederzeit nach Angebot von allen Mitarbeitern, unter Beachtung einer maximalen Teilnehmerzahl, regelhaft besucht werden.

Die Anwendung des Larynxtubus während der Reanimation zur Verringerung der "No Flow Time"

C.H.R. Wiese, T. Steffen T, U. Bartels, J. Reese, T. Immenroth, J. Bahr, B.M. Graf

Universitätsklinikum Göttingen, Zentrum Anästhesiologie, Rettungs- und Intensivmedizin

Einleitung: Der Larynxtubus gewinnt während der ersten Phase der Reanimation zunehmend an Bedeutung. Dieses ist durch seine einfache Handhabung für trainierte, aber auch für nicht trainierte Anwender begründet. Dadurch lässt sich in der Notfallsituation schnell und effektiv ein sicherer Atemweg etablieren. In der vorliegenden Arbeit wurde untersucht, inwieweit sich die „No Flow Time" (Zeit, in der während einer Reanimation keine Thoraxkompressionen stattfinden) bei der Anwendung des Larynxtubus im Verhältnis zur endobronchialen Intubation unterscheidet. Material und Methoden: Im Rahmen eines eintägigen Reanimationstrainings für Rettungsdienstpersonal wurde ein standardisiertes Reanimationsszenario mit einem „Mega Code Trainer" erstellt. In diesem Szenario wurde die Versorgung eines Patienten mit Kammerflimmern durch ein rettungsdienstliches Team (2 Personen) über sieben Minuten simuliert. Bestandteil der korrekten Versorgung war die Thoraxkompression, die Beatmung, die Defibrillation, die Sicherung der Atemwege und die Applikation der notwendigen Pharmaka entsprechend der neuen ERC Empfehlungen von 2005. Es wurden zwei Untersuchungsgruppen gebildet. In der Versuchsgruppe wurde der Atemweg mittels eines Larynxtubus gesichert, in der Kontrollgruppe erfolgte die konventionelle endotracheale Intubation zur Atemwegssicherung. Alle weiteren Maßnahmen waren identisch. In beide Gruppen wurden jeweils 50 Probanden integriert, die sich in Alter, Berufserfahrung oder Profession nicht unterschieden. In beiden Gruppen wurde die „No Flow Time" sowohl als Gesamtzeit addiert als auch während der einzelnen Handlungen erfasst. Die statistische Auswertung erfolgte mit dem Wilcoxon-Test. Das Signifikanzniveau wurde mit $\mathrm{p}<0,05$ festgelegt. Ergebnisse: Bezüglich der erhobenen Parameter waren die Versuchs- und die Kontrollgruppe miteinander vergleichbar. Der Larynxtubus konnte in $98 \%$ der Szenarien im ersten Versuch erfolgreich platziert werden. Dagegen gelang dieses bei der endotrachealen Intubation nur in $62 \%$ der Fälle. Die Gesamtdauer der Simulation betrug 420 Sekunden. Bei Anwendung des Larynxtubus lag die „No Flow Time“ im Mittel bei 124 Sekunden (30\% der Gesamtzeit). Bei der endotrachealen Intubation betrug die „No Flow Time“ durchschnittlich 196 Sekunden (47\% der Gesamtzeit). Schlussfolgerung: Der Larynxtubus ist ein sicheres und effektives Instrument zur Sicherung des Atemweges während der Reanimation. Bei der hier vorliegenden Untersuchung führt die Anwendung 
des Larynxtubus im Gegensatz zur endotrachealen Intubation zu einer signifikanten Verkürzung der „No Flow Time“ $(\mathrm{p}<0,05)$. Dieses könnte zu einem besseren Outcome bei Patienten nach einer Reanimation beitragen.

Der zeitliche Ablauf eines Reanimationsszenarios über 420 Sekunden - Nutzung des Larynxtubus versus konventioneller endotrachealer Intubation

\section{C.H.R. Wiese, T. Steffen T, U. Bartels, J. Reese, T. Immenroth,}

J. Bahr, B.M. Graf

${ }^{1}$ Universitätsklinikum Göttingen, Zentrum Anästhesiologie, Rettungs- und Intensivmedizin; ${ }^{2}$ Städtisches Klinikum Wolfenbüttel Einleitung: Im November 2005 wurden durch den European Resuscitation Council (ERC) neue Empfehlungen zur Reanimation (CPR) publiziert. Neben Änderungen im Verhältnis der Thoraxkompression zur Beatmung, der Pharmako- und Elektrotherapie sind auch die Empfehlungen für den Ablauf der gesamten Maßnahmen aktualisiert worden. Hierzu zählt unter anderem die Schaffung eines effektiven und sicheren Atemweges. Als Alternative zum Goldstandard der endotrachealen Intubation wird zunehmend auch der Larynxtubus zur Atemwegssicherung etabliert. Wir haben untersucht, welche zeitlichen Unterschiede sich im Ablauf des ERC-Algorithmus zum Kammerflimmern bei der endotrachealen Intubation versus Einsatz des Larynxtubus ergeben. Material und Methoden: In einem Reanimationsszenario wurde die Versorgung eines Patienten mit Kammerflimmern durch ein rettungsdienstliches Team (2 Personen) über sieben Minuten simuliert. Entsprechend der ERC Guidelines umfasste die Versorgung des „Patienten“ die Kontrolle des Bewusstseins, die Thoraxkompression, die Beatmung, die Sicherung der Atemwege, die Defibrillation eines defibrillationswürdigen Rhythmus und die Pharmakotherapie. Hierfür wurden entsprechende Zeitvorgaben gestellt. In der Untersuchungsgruppe erfolgte die Sicherung der Atemwege durch einen Larynxtubus, in der Kontrollgruppe wurden die Atemwege durch die endotracheale Intubation gesichert. Insgesamt wurden pro Gruppe 50 Probanden untersucht, die freiwillig an dem Szenario teilnahmen. Für die Untersuchung wurde ein handelsüblicher "Mega Code Trainer" mit entsprechendem Aufzeichnungsprogramm genutzt. Die statistische Auswertung erfolgte mit dem Wilcoxon-Test. Das Signifikanzniveau wurde entsprechend des $5 \%$ Niveaus bei $\mathrm{p}<0,05$ festgelegt. Ergebnisse: Die Untersuchungs- und die Kontrollgruppe waren bezüglich der demografisch erhobenen Parameter miteinander vergleichbar. Es zeigte sich, dass es in der Untersuchungsgruppe (Einsatz des Larynxtubus) in $94 \%$ der Simulationen gelang, alle Maßnahmen, die seitens des ERC bei der CPR innerhalb der ersten sieben Minuten empfohlen werden, durchzuführen. In der Kontrollgruppe (endotracheale Intubation) gelang dieses nur in $12 \%$ der Fälle. Schlussfolgerung: Der Einsatz des Larynxtubus als Instrument der primären Atemwegssicherung in einer Reanimationsphase ermöglicht es mit signifikantem Unterschied zur endotrachealen Intubation, den zeitlichen Vorgaben der aktuellen ERC Empfehlungen zu entsprechen. Das Verfahren ist vor allem durch eine einfache und effektive Anwendung gekennzeichnet. Dieses könnte zu einer Verbesserung des Patienten-Outcome beitragen.
Airwaymanagement im Rahmen der Reanimation, Eine Subgruppenanalyse aus dem Reanimationsregister der DGAI

J. T. Gräsner, J. Wnent, V. Dörges, R. Franz, T. Rosolski, T. Zeng, J. Scholz

Universitätsklinikum Schleswig-Holstein, Campus Kiel, Klinik für Anästhesiologie und Operative Intensivmedizin

Fragestellung: Im Rahmen der Reanimationsdatenerfassung (RDE) durch das DGAI Reanimationsregister (RR) werden Daten bzgl. Komplikationen wärend der Reanimation, sowie Angaben zum eingesetzten Luftweg gemäß „Utstein-Style“ erfasst. In dieser Auswertung wurden diese Daten im Hinblick auf die Häufigkeit eines schwierigen Luftweges, des eingesetzten alternativen Airway-Tools und der daraus resultierenden Auswirkung auf den Reanimationserfolg betrachtet. Material und Methoden: Es wurden 670 präklinische Reanimationen der Städte Dortmund, Lüneburg und Wismar aus dem Zeitraum vom 1.1.2004 bis zum 5.2. 2006 analysiert. Die Ereignisse wurden mittels Dortmunder Protokoll zur RDE (Version 1.00 und 1.03) auf der Basis des DGAI Reanimationsdatensatzes „Erstversorgung“ erfasst. Ergebnisse: Bei $n=562$ wurden keine Schwierigkeiten bzgl. des Airways dokumentiert. Bei $n=96$ wurde eine schwierige Intubation angegeben, bei weiteren $n=10$ Patienten war eine Intubation nicht möglich. Bei allen betrachteten Reanimationen wurden in $\mathrm{n}=23$ eine Larynxmaske (LMA), bei $n=1$ eine Notfalltracheotomie, bei $n=33$ ein Larynxtubus und bei $n=16$ Fällen eine Intubationslarynxmaske eingesetzt. Ein Combitubus kam nie zum Einsatz. Betrachtet man die Ereignisse, bei denen Intubationsschwierigkeiten angegeben wurden $(n=96)$, so wurden bei $n=5$ Fällen die LMA, bei $n=6$ der Larynxtubus und bei $\mathrm{n}=4$ die Intubationslarynxmaske angewandt. Im Hinblick auf die nicht zu intubierenden Patienten $(n=10)$ wurde jeweils bei $n=1$ Fällen die LMA, die Notfalltracheotomie und der Larynxtubus eingesetzt. $\mathrm{Zu}$ den weiteren $\mathrm{n}=7$ sind keine Angaben in Bezug auf den eingesetzten alternativen Airway gemacht worden. Die Rate des Return of spontaneus circulation (ROSC) liegt im Gesamtkollektiv bei $n=248$. In den Fällen in denen eine schwierige Intubation dokumentiert wurde, erreichten $\mathrm{n}=30$ einen ROSC. Die ROSC-Rate bei den Fällen, die nicht zu intubieren waren liegt bei $n=1$. Schlussfolgerungen: In einer nicht unerheblichen Zahl der Ereignisse wurden eine schwierige Intubation oder eine nicht mögliche Intubation dokumentiert. Ein alternativer Airway kam nur in einem Teil der Fälle zum Einsatz. Diese Tatsache zeigt, dass ein alternatives Airwayverfahren präklinisch noch nicht flächendeckend zur Verfügung steht oder nicht eingesetzt wird. Hier ist es nötig ein größeres Problembewusstsein $\mathrm{zu}$ schaffen, die logistischen Veraussetzungen herzustellen und die ärztlichen Mitarbeiter hinsichtlicher dieser Maßnahmen zu schulen, da nicht zuletzt Komplikationen im Bereich Airway einen wesentlichen negativen Einfluss auf das Outcome haben.

Algorithmus einer ALS-konformen Echokardiographie: Training und Evaluation (OSCE)

M. Rüsseler ${ }^{1}$, S. Bergdold ${ }^{3}$, T. Kunz ${ }^{3}$, H. Ilper ${ }^{2}$, F. Walcher ${ }^{1}$, H. Steiger ${ }^{4}$ R. Breitkreutz ${ }^{2}$

${ }^{1}$ Klinik für Unfallchirurgie, ${ }^{2}$ Klinik für Anästhesiologie, ${ }^{3}$ Klinikum der Johann Wolfgang Goethe Universität, ${ }^{4}$ Kardiologie, Klinikum Darmstadt

Kontext: Die neuen und alten Leitlinien der CPR fordern einerseits die Identifikation behandelbarer Ursachen (Pericardtamponade, Hypovolämie, Herzinsuffizienz, Rechtsherzbelastung) und eine qualitativ hochwertige kardiopulmonale Reanimation (CPR), empfehlen aber andererseits nur kurze Unterbrechungen $[1,2]$. Unsere Arbeitsgruppe entwickelte den ALS-konformen Algorithmus FEER (fokussierte echokardiographische Evaluation bei Reanimation) [3], der auf seine Anwendbarkeit hin überprüft wird. Unsere Frage hier war, ob FEER innerhalb eines 8-stündigen Kurses für Intensivmediziner und Notärzte erlernt werden kann [4]. 
Methodik: Der Kurs enthielt 4 Unterrichtseinheiten (1UE $=45 \mathrm{~min})$ Theorie und 4 UE Praxis zum Basiswissen Echokardiographie in 2 hands-on Trainingseinheiten. 12 Ärzte wurde im 1. hands-on Training $\mathrm{zu}$ einer $10 \mathrm{~min}$ andauernden CPR im standardisierten Megacode hinzugezogen und aufgefordert eine Echokardiographie innerhalb der CPR mit einem portablen Sonograpiegerät an einer Puppe (Resusci-Anne, Laerdal, Norwegen) durchzuführen. Die Praxis für FEER war durch 10 Elemente vordefiniert [3]. Als Ergebnis der Echokardiographie wurde das Vorhandensein von Wandbewegungen standardisiert vorgegeben. Nach dem 1 . handson erhielten die Teilnehmer eine mündliche Unterweisung, eine 10 minütige Präsentation zum genauen Ablauf von FEER sowie die geforderten 10 Elemente in schriftlicher Form und durchliefen im 2. hands-on das gleiche Szenario. Die Tests wurden mit Hilfe des OSCE-Vefahrens (objective structured clinical examination) [5] parallel überprüft (Checkliste) und durch eine post-hoc Konfirmation der erhobenen Daten mit Videoaufzeichnungen überprüft. Ergebnisse: Chronologische Reihenfolge der FEER Elemente und die mittlere Erfolgsrate (in \%) vor und nach Schulung in der hands-on: 1. Teaminfo $(46,100), 2$. Vorbereitung Sonographiegerät und Funktionstest $(100,100)$, 3. Anzählen $(8,92), 4$. Ansage Puls parallen prüfen $(0,70), 5$. Ansage zur Unterbrechung $(100,100), 6$. Positionierung subxyphoidal parallel zu HDM (100, $100), 7$. Durchführung $(100,100)$, 8. Anweisung Fortführen (100, $100), 9$. Befundmitteilung $(62,100), 10$. Angabe von Konsequenzen $(100,100)$. Konklusion: Obgleich die Teilnehmer bereits über relativ hohe praktische Fertigkeiten verfügten, eine ALS-konforme Echokardiographie anzuwenden, waren diese bei einzelnen Elementen noch steigerbar.

Literatur: [1] Nolan JP et al: ERC guidelines for resuscitation, Section 4. AALS. Resuscitation 2005; 67 S1:S39-86. [2] Hazinski MF: Major Changes in the 2005 AHA Guidelines for CPR and ECC. Circulation 2005; 112:IV-206-IV-211. [3] Breitkreutz et al: The Concept of Focused Echocardiographic Evaluation in Resuscitation management (FEER): an ALS conformed Algorithm. Critical Care Medicine 2006; in press. [4] www.notfallsonographie.de (Zugriff 18. 6. 2006). [5] Harden RM et al: Assessment of clinical competence using an objective structured clinical examination (OSCE). Med Educ 1979; 13(1):41-54

Werden nach den neuen Leitlinien zur Reanimation 222 ERC/ILCOR 2005 im Vergleich zu 2000 die Dauer und die Anzahl der No-flow-Intervalle oder der beatmungsfreien Intervalle im 2-Helfer BLS/ALS Trainingsmodell reduziert?

H. Ilper ${ }^{1}$, F.H. Seeger ${ }^{2}$, S. Heigenmooser ${ }^{1}$, V. Wilken ${ }^{4}$, D. Oberndörfer ${ }^{4}$, H. Pfleger ${ }^{4}$, R. Schalk ${ }^{1}$, H.H. Ackermann ${ }^{3}$, R. Breitkreutz

${ }^{1}$ Anästhesiologie, ${ }^{2}$ Kardiologie, ${ }^{3}$ Biostatistik, Universitätsklinikum, ${ }^{4}$ FIRN, Berufsfeuerwehr, Frankfurt am Main

Kontext: Die neuen Leitlinien nach AHA/ERC/ILCOR $2005 \mathrm{zu}$ kardiopulmonaler Reanimation (CPR) empfehlen die Minimierung der Dauer und der Anzahl der „No-flow Intervalle“ (NFI) [1, 2]. Hierzu wurde der Algorithmus zugunsten von früheren und häufigeren Thoraxkompressionen umgestellt [1]. CPR Pausen sollten „kurz" gehalten werden bzw. wurden durch den Terminus „briefly" nicht genau definiert. Es wurde behauptet, dass es dadurch zur Verringerung der Dauer der NFI kommen soll [1, 2], jedoch wurde ein Beweis dazu formal bisher nicht geführt. Daher stellten wir in dieser Untersuchung die Frage, ob und in welchem Ausmass die Änderung des BLS/ALS Trainings (Megacode) eine Reduktion der Dauer und der Anzahl der NFI und auch der beatmungsfreien Intervalle (BFI) erzeugt werden kann. Methoden: Typisches MegaCode-Szenario [3] mit dem Reanimationstrainer Resusci Anne, (Fa. Laerdal, Norwegen) und dem halbautomatischen Defibrillator/EKG-Monitor, (Fa. Zoll Medical, USA). Geschulte Rettungsassistenten führten den MegaCode (MC) im 2-Helfermodell nach den ERC-Leitlinien von $2000(n=19)$ und $2005(n=10)$ durch und reagierten auf Kammerflimmern, Asystolie, Kammerflimmern, pulslose elektrische Aktivität und Sinusrhythmus (Ende der CPR bei tastbarem Puls). DVD-Mitschnitte wurden posthoc auf vordefinierte Phasen, NFI und BFI sowie die CPR-Zyklusdauer und die Anzahl von NFI und BFI innerhalb der Testzeit hin analysiert. Die Dauer der NFI 2000/2005 oder BFI 2000/2005 wurde definiert $\mathrm{ab}$ Beenden der Thoraxkompressionen oder Beatmung bis zu deren Wiederaufhahme. Die Dauer aller NFI oder BFI der jeweiligen MC wurden gepoolt und eine explorative, deskriptive Datenanalyse für die Verteilung durchgeführt und der MannWhitney U-Test für die Gruppenvergleiche angewendet. Ergebnisse (Stand 18.6.2006): Angegeben sind in den Klammern jeweils Paare: Mittelwert der Dauer NFI oder BFI in Sekunden \pm Standardabweichung (SD) und Mittelwert der Anzahl NFI oder BFI pro MC \pm SD. 1) NFI $2000(21,5 \pm 23,1 ; 16 \pm 3)$, 2) NFI 2005 $(9,9 \# \pm 7,8 ; 18 \pm 3), 3)$ BFI $2000(20,1 \pm 17,7 ; 15 \pm 5), 4)$ BFI 2005 , $(16,3 \pm 11,3 ; 36 \# \pm 3) ;{ }^{*}, \mathrm{p}<0,05 ; \#, \mathrm{p}<0,001$, für den jeweiligen Gruppenvergleich. Schlussfolgerung: Nach den neuen ERC Leitlinien 2005 werden im ALS-Training (MegaCode) im Vergleich zu ERC 2000 die NFI global im Mittel um ca. 50\% reduziert, obwohl häufiger Unterbrechungen der CPR vorgenommen werden. Dieses Ergebnis kann für das wichtigste Ziel des BLS/ALS Algorithmus ERC 2005 als formaler Beweis im Modell gewertet werden.

Literatur: [1] Multiple authors: ERC and ILCOR guidelines for resuscitation. Resuscitation 2005; 6751:1-185. [2] de Latorre F et al: ERC Guidelines 2000 for Adult Advanced Life Support. Resuscitation 2001; 48:211-221. [3] Breault R et al: The Mega-Code for training the advanced cardiac life support team. Heart \& Lung $1981 ; 10: 860-865$

Können Ärzte ohne spezielle Vorkenntnisse kurze,

höchstens 5 Sekunden andauernde, pathologische

Echokardiographiebefunde erkennen lernen?

N. Nieuwkamp ${ }^{\star}$, H. Steiger ${ }^{4}$ F. H. Seeger ${ }^{2}$ C. Teupe ${ }^{2}$ F. Walcher ${ }^{3}$ R. Breitkreutz (1)

Kliniken für Anästhesiologie ${ }^{1}$, Kardiologie ${ }^{2}$ und Unfallchirurgie ${ }^{3}$

${ }^{*}$ Klinikum der Johann Wolfgang Goethe Universität, ${ }^{4}$ Kardiologie, Klinikum Darmstadt

Kontext: Die neuen und alten Leitlinien der CPR fordern einerseits die Identifikation behandelbarer Ursachen und eine qualitativ hochwertige kardiopulmonale Reanimation (CPR), empfehlen aber andererseits nur kurze Unterbrechungen $[1,2]$. Unsere Arbeitsgruppe entwickelte den ALS-konformen Algorithmus FEER (Fokussierte Echokardiographische Evaluation bei Reanimation) [3], der auf seine Anwendbarkeit hin überprüft wird. Eine wesentliche Vorraussetzung ist das Erkennen eines normalen oder pathologischen Befundes (Perikarderguß, eingeschränkte LVEF, Rechtsherzbelastungszeichen, pulslose elektrische Aktivität (PEA), Asystolie, Hypovolämie) innerhalb von 5 Sekunden. Unsere Frage hier war, ob Ärzte ohne spezielle Vorkenntnisse innerhalb eines 8-stündigen Kurses für Intensivmediziner und Notärzte erlernen können, einen pathologischen Befund innerhalb dieser kurzen Zeit (wieder-)zuerkennen [4]. Methodik: Es wurde ein interaktives Programm entwickelt (Die Infographin, Frankfurt). Nach einem Probefilm wurden 15 kardiale Befunde mit einer maximalen Filmdauer von 5 Sekunden eingespielt und danach durch zeitlich begrenzte Freitexteingabe und multiple Choice (MC)-Testfragen analysiert, ob der Befund erkannt wurde. Der Kurs [3,4] enthielt 4 Unterrichtseinheiten ( $1 \mathrm{UE}=45 \mathrm{~min})$ Theorie und 4 UE Praxis zum Basiswissen in Echokardiographie durch 2 hands-on Trainingseinheiten. Die UE entsprechen der Intervention. Die Erkennungsraten von 12 Ärzten ohne Vorkenntnisse wurden vor und nach den UE über die pathologischen Befunde in einem Test durch das o.g. Programm geprüft. Die Auswertung erfolgte durch deskriptive Statistik und den gepaarten Wilcoxon-Test, (signed ranks) für verbundene Stichproben. Ein $p<0,05$ im Vergleich der Mittelwerte wurde als signifikanter Unterschied und Lernerfolg angesehen. Ergebnisse: Mittlere Anzahl richtiger Antworten \pm SD 
vor Intervention: $8 \pm 3 \quad(53,3 \% \pm 17,5)$; nach Intervention: $10 \pm 3 \# \#(66 \% \pm 16 \# \#)$. Am häufigsten richtig wurden Pericarderguß, hochgradig reduzierte LVEF und die PEA erkannt. (\#\#; $\mathrm{p}<0,01$ für den Gruppenvergleich). Konklusion: Ärzte ohne spezielle Vorkenntnisse können bestimmte kurze Sequenzen von maximal 5 Sekunden ausgewählter pathologischer Befunde durch Unterricht erkennen lernen. Das vorgestellte Programm eignet sich zur Überprüfung dieser Kenntnisse und des Lernerfolgs. Damit unterstützten die vorliegenden Ergebnisse das Konzept einer ALS-konformen Echokardiographie. Allerdings sollte eine größere Fallzahl die Ergebnisse bestätigen und das Kurskonzept noch modifiziert werden.

Literatur: [1] Nolan JP et al: ERC guidelines for resuscitation. Section 4. AALS. Resuscitation 2005; 67S1:S39-86. [2] Hazinski MF: Major Changes in the 2005 AHA Guidelines for CPR and ECC. Circulation 2005; 112:IV-206-IV-211. [3] Breitkreutz et al: The Concept of Focused Echocardiographic Evaluation in Resuscitation management (FEER): an ALS conformed Algorithm. Critical Care Medicine 2006; in press. [4] www.notfallsonographie.de (Zugriff 18. 6. 2006)

\section{Integration von Notfall und Intensivmedizin} in die Lehre im Dresdner Reform Curriculum( DIPOL ${ }^{\circledR}$ )

M. Ragaller, M. Frank, M. Müller, A. Graupner, S. Weber,

R. Grass *, S Rammelt ${ }^{*}$, T. Koch

Klinik für Anästhesiologie und Intensivtherapie,

* Klinik für Unfall- und Wiederherstellungschirurgie

Einleitung: Im Gegensatz zu ihrer medizinischen und ökonomischen Bedeutung sind die Querschnittsfächer Notfallmedizin und Intensivmedizin in der Studentenausbildung bislang unterrepräsentiert. Zur Verbesserung der studentischen Ausbildung wurden an der Medizinischen Fakultät der TU-Dresden zwei interdisziplinäre Kurse „Akute Notfälle“, „Notfallmedizin - Verletzungen - Intensivmedizin“ (NVI) etabliert. Methoden: Im 4 wö. Kurs Akute Notfälle (6. Sem.) werden die Prinzipien des Basic und Advanced Life Support, vor allem in Form von praktischen Übungen (Skills Training) unter Verwendung von Simulatoren und problemorientierten Falldiskussionen vermittelt. In dem 6 wö. NVIKurs (10 Semester) werden die Prinzipien der Intensivmedizin (Beatmung, Sepsistherapie, etc.) sowie spezielle notfallmedizinische Algorithmen (Polytraumalgorithmus im Krankenhaus) vermittelt. Durch eine interdisziplinäre Verteilung der Verteilung der Referenten wird Bedeutung der interdisziplinären Denk- und Handlungsweise dieser beiden Querschnittsfächer betont. Der NVI-Kurs besteht ebenfalls aus einer Kombination von Vorlesungen, Seminaren, Unterricht am Krankenbett, Praktika und Fall-Tutorien. Die Kurse werden durch eine externe Evaluation begutachtet. Ergebnisse: In der Evaluation lies sich insgesamt bei den Studierenden und Lehrenden eine hohe Akzeptanz des Kurses zeigen, wobei der hohe Anteil von praktischer Ausbildung am Simulator besonders positiv beurteilt wird. Im Kriterium Transfererwartung für Studium und Beruf wurde der NVI-Kurs sehr gut bewertet 4,9 (Skala 1-6). Im fakultätsinternen Ranking der Evalution der Studenten belegen die beiden Kurse seit Jahren die vorderen Plätze. Die Bestehensquote der Abschlussprüfung beträgt in beiden Kursen im Mittel zwischen 90 und 95\%. Schlussfolgerung: Die verstärkte Integration von Notfallmedizin und Intensivmedizin in die studentische Ausbildung wird von den Studierenden äußerst positiv aufgenommen. Die Gesamtkursevaluierung zeigt eine hohe $\mathrm{Zu}-$ friedenheit der Studierenden und der Lehrenden. Durch Analyse der Evaluationsergebnisse können Qualität und Inhalt des Curriculums ständig verbessert werden.
Moderne Diagnostik des akuten Abdomen

M. Gmeiner ${ }^{1}$ J. Pfeifer ${ }^{2}$

Abteilung für Pulmologie, Landeskrankenhaus Graz-West ${ }^{1}$ Klinische Abteilung für Allgemeinchirurgie, Medizinische Universität $\mathrm{Graz}^{2}$

Einleitung: Akutes Abdomen ist die klinische Bezeichnung für eine lebensbedrohliche Erkrankung im Bereich der Bauchhöhle. Für ein erfolgreiches Endergebnis ist eine gezielte Abklärung und Therapie unbedingt erforderlich. Ziel dieser Arbeit ist, einen pragmatischen Ansatz zu finden, das „einzig richtige“ Vorgehen auszuwählen. Methoden: Trotz zahlreicher Untersuchungsmöglichkeiten muss das weitere Vorgehen klinisch abgeschätzt werden. Für das weitere Vorgehen helfen Leitsymptome: Leitsymptom Schmerz: Art, Ausmaß, Dauer und Beginn. Leitsymptom Blutung: Offen, okkult, arteriell oder venös, präklinisch oder klinisch, allgemeine Schockzeichen, Trauma. Leitsymptom Fieber: Beginn, Dauer, Höhe und Bezug zum Alter. Leitsymptom Erbrechen: Art, Ausmaß und Häufigkeit. Ergebnisse: Leitsymptom Schmerz: Als Faustregel gilt, dass ein Schmerz, der länger als 6 Stunden anhält, meist einer chirurgischen Intervention bedarf, ebenso der Vernichtungsschmerz. Leitsymptom Blutung: präklinisch traumatisch offen: Lagerung, Kompressionsverband; präklinisch traumatisch okkult: Schockindex - nach Allgöwer, nur in seltenen Fällen „LOAD and GO“; präklinisch nicht traumatisch: vasoaktive Wirkstoffe (Terlipressin, Vasopressin, Somatostatin und Octreotid). Terlipressin auch wirksam bei Noradrenalin-resistentem Schock! klinisch traumatisch okkult: Abdominalsonografie, KM - CT; klinisch nicht-traumatisch: Endoskopie (Gastroskopie, Koloskopie, Proktoskopie). Cave: $90 \%$ der Blutungen kommen aus dem oberen GI-Trakt, wobei in $70-80 \%$ der Fälle eine primäre endoskopische Blutstillung erreicht werden kann. Leitsymptom Fieber: Kindesalter: rasch bedrohlich („Alles im Bauch“ z. B. Otitis media, Gastroenteritis, Appendizitis, HWI) - Labor, Klinik. Jugend- und junges Erwachsenenalter: entzündliche Darmerkrankungen wie Colitis ulcerosa oder Morbus Crohn; gyn. Differentialdiagnosen (Adnexitis/Salpingitis) auszuschließen - Sonografie. Erwachsenenalter: Cholezystitis, Divertikulitis mit dem Höhepunkt zwischen dem 60.-80. Lebensjahr: Sonografie, CT. Leitsymptom Erbrechen: Ausdruck von stärkstem Schmerz (z.B. Nierenkolik) oder Ausdruck einer GI-Erkrankung (z. B. Enteritis, Hämatemesis). Danach richtet sich das weitere Vorgehen. Zusammenfassung: Trotz modernster Untersuchungstechniken ist die klinische Einschätzung der Schwere des akuten Abdomens unumgänglich. Orientierung an Leitsymptomen kann helfen, rasch, gezielt und kompetent die apparative Diagnostik voranzutreiben.

Basic Life Support und Anwendung eines AED in der Schülerausbildung an einem Gymnasium

\section{W. Prull, C. Perings, H.J. Trappe}

Medizinische Klinik II, Marienhospital Herne, Ruhr-Universität Bochum

Fragestellung: Im Falle eines plötzlichen Herzstillstandes sind der Kenntnisstand über die Reanimation (CPR) und die Bereitschaft zu helfen gering. Eine neue Zielgruppe in der Ausbildung in Basismaßnahmen der CPR (BLS) und die Anwendung halbautomatischer Defibrillatoren (AED) könnten Schüler (Sch) darstellen. Ungeklärt ist die Frage, ab welchem Alter Kinder in BLS und AED geschult werden können und ob sich deren Eltern (E) motivieren lassen, ihre Kenntnisse über CPR aufzufrischen. Methoden: An einem Gymnasium wurden getrennt voneinander eine 5. Klasse (5K) und eine 9. Klasse (9K) in BLS und AED von einem Notfallmediziner geschult. In der 1 . Unterrichtsstunde wurden theoretische Hintergründe und Durchführung des BLS und AED vermittelt. In einer bzw. zwei weiteren Unterrichtsstunden wurden praktische Übungen an CPR-Mannequins (Little Anne, Laerdal, Norwegen) und mit einem AED (Medtronic LIFEPAK CPR-Trainer, USA) vermittelt. 4 Wochen später wurden 3 Fragen zur BLS an al- 
le Sch gestellt. Es wurden Fragen an die E und nach 4 Monaten nochmals gestellt. Ergebnisse: In der 5K $(10,4+0,5$ Jahre) befanden sich 26 Sch (58\% weiblich w). Die 1. Frage (Reihenfolge der einzelnen Schritte des BLS/AED) haben 9 Sch richtig beantwortet (35\%). Frage 2 (Ventilations/Kompressions-Verhältnis) haben 17 Sch korrekt beantwortet (65\%). Frage 3 (Notrufnummer) haben 25 Sch (96\%) korrekt beantwortet. 22 E haben die Fragebögen beantwortet (88\%). An einem Kurs in 1. Hilfe haben $100 \%$ teilgenommen: $7 \mathrm{E} 1 \times(32 \%), 7 \mathrm{E} 2 \times(32 \%), 5 \mathrm{E}>2 \times(23 \%), 3 \mathrm{E}$ regelmäßig (13\%). Interesse bekunden $20 \mathrm{E}(91 \%)$. An einem Kurs in BLS und AED würden $17 \mathrm{E}(77 \%)$ teilnehmen. In der $9 \mathrm{~K}(14,5+$ 0,6 Jahre) befanden sich 27 Sch $(26 \%$ w). Die 1 . Frage haben 10 Sch $(37 \%)$ korrekt beantwortet. Frage 2 haben 27 Sch korrekt beantwortet (100\%), ebenso Frage 3. 10 E (37\%) haben die Fragebögen beantwortet. An einem Kurs in 1. Hilfe haben $100 \%$ teilgenommen: $5 \mathrm{E} 1 \times(50 \%), 3 \mathrm{E} 2 \times(30 \%), 1 \mathrm{E}>2 \times(10 \%), 1 \mathrm{E}$ regelmäßig $10 \%$. Interesse bekunden $7 \mathrm{E}(70 \%)$. An einem Kurs in BLS und AED würden $7 \mathrm{E}(70 \%)$ teilnehmen. In einem Kontrollzeitraum von 4 Monaten hat keiner der befragten $\mathrm{E}$ einen Kurs in BLS/AED besucht. Bei der praktischen Ausbildung benötigten die 10jährigen Sch 1 Stunde länger, bis eine sichere Durchführung von BLS und AED erfolgen konnte (33\% längere Ausbildungsdauer). Konklusion: BLS und die Anwendung eines AED kann Sch ab einem Alter von 10 Jahren beigebracht werden. $5 \mathrm{~K}$ benötigt länger in der praktischen BLS/AED Ausbildung. Die Reihenfolge der einzelnen Schritte innerhalb des BLS und AED wird nur von der Minderheit korrekt wiedergegeben. Bei Anwendung der neuen ERC-Leitlinien 2005 könnten diese Ergebnisse durch Simplifizierung verbessert werden. Freiwillige Schulung scheint keine adäquate Methode zu sein, auch motivierte Erwachsene zu erreichen. Die Unterrichtung der Kinder in BLS stellt eine neue und viel versprechende Möglichkeit dar, Kenntnisse über BLS und AED in der Allgemeinbevölkerung zu verbreiten.

\section{Intoxikationen, Atemwegsmanagement, Schockraummanagement}

Intoxikation mit Isoniazid - eine seltene, aber lebensbedrohliche Situation

J. Graf, W. Radunz, M. Obergföll, J. Cyran, N. Scheffold

SLK Kliniken Heilbronn, Klinikum Am Gesundbrunnen, Innere Medizin I, Schwerpunkt Kardiologie

Hintergrund: Isoniazid, eigentlich Isonicotinsäurehydrazid (INH) ist ein antimykobakterieller Wirkstoff der bei verschiedenen Formen der Tuberkulose therapeutisch eingesetzt wird. Eine Intoxikation mit INH stellt eine Rarität dar. Bereits geringe Mengen (ab ca. 1,5 g) der Substanz können toxisch wirken, in höheren Dosierungen $(80-150 \mathrm{mg} / \mathrm{kg} \mathrm{KG})$ verläuft eine Intoxikation unbehandelt in der Regel letal. Wir beschreiben den seltenen Fall einer Intoxikation mit INH in suizidaler Absicht. Anamnese: Eine 55jährige Frau nahm aufgrund einer familiären Konfliktsituation eine zunächst unklare Menge an INH-Tabletten zusammen mit Alkohol ein. Kurze Zeit nach der Einnahme kam es zu einem cerebralen Krampfanfall, im Beisein des Notarztes dann wiederholt zu Krampfanfällen. Die Patientin wurde respiratorisch- und kreislaufstabil in die Notaufnahme eingewiesen und direkt auf die Intensivstation übernommen. Untersuchungsbefunde: Bei Aufnahme sahen wir eine Patientin in reduziertem Allgemeinzustand mit deutlich reduzierter Vigilanz nach Gabe von Benzodiazepinen im Rahmen der Krampfanfälle, GCS 10. Herzfrequenz 115/min, RR 148/78 $\mathrm{mmHg}$, Atemfrequenz 21/min. Während der Untersuchung trat ein Status epilepticus auf mit konsekutiver respiratorischer Insuffizienz. Es erfolgte die rasche Intubation, da trotz Benzodiazepin-Gabe keine Krampffreiheit erreicht werden konnte. Die Blutgasanalyse direkt nach Intubation zeigte eine ausgeprägte me- tabolische Azidose ( $\mathrm{pH} \mathrm{7,17,} \mathrm{Lactat} \mathrm{11,9} \mathrm{mmol/l,} \mathrm{BE} \mathrm{-13,9} \mathrm{mmol/l,}$ $\mathrm{pO}_{2} 246 \mathrm{mmHg}, \mathrm{pCO}_{2} 38,5 \mathrm{mmHg}$ ). Der Alkoholspiegel lag bei 1,7 Promille. Verlauf: Die Azidose konnte unter Krampffreiheit und Gabe von Natriumbicarbonat rasch korrigiert werden. Als Antidot wurden bei zunächst unbekannter Ingestionsdosis $5 \mathrm{~g}$ Pyridoxin i.v. verabreicht. Weitere Krampfanfälle traten nachfolgend nicht mehr auf. Die hepatotoxische Wirkung des Isoniazid zeigte sich in einer Störung der Gerinnung mit einem Spontan-INR von 1,9 bei Aufnahme. Zudem kam es zu einem leichten Abfall der Cholinesterase und $\mathrm{zu}$ einem Anstieg der Transaminasen auf das 2,7 fache des oberen Normbereiches (NB). Das Bilirubin stieg auf $6,3 \mathrm{mg} / \mathrm{dl}(\mathrm{NB}<1,4)$, die yGT auf $195 \mathrm{U} / \mathrm{l}(\mathrm{NB}<38)$ an. Die CK stieg auf max. $1923 \mathrm{U} / \mathrm{l}$ im Sinne einer moderaten Rhabdomyolyse. Es erfolgte an Tag 2 eine INH-Spiegelbestimmung. Dieser lag mit $109 \mathrm{mg} / \mathrm{l}$ deutlich außerhalb des therapeutischen Bereichs (NB 1-8 mg/l). Die Patientin konnte nachfolgend rasch extubiert und an Tag 5 ohne neurologische Defizite auf Normalstation verlegt werden. Schlussfolgerung: Therapierefraktäre cerebrale Krampfanfälle, metabolische Azidose und Koma sollten differentialdiagnostisch an eine Intoxikation mit INH denken lassen. Die akute Therapie der Krämpfe besteht in der Gabe von Antikonvulsiva. Sobald verfügbar, sollte Pyridoxin als spezifisches Antidot gegeben werden. Die weitere intensivmedizinische Therapie zielt auf die Behandlung der begleitenden metabolischen Azidose und einer möglicherweise auftretenden Rhabdomyolyse.

\section{Fallberichte von Hyperthermie unter Clozapineinnahme bei hohen Außentemperaturen M. Held}

Abteilung Innere Medizin, Missionsärztliche Klinik Würzburg

Wir berichten über 2 Patienten mit Hyperthermie unter Clozapineinnahme bei hohen Außentemperaturen im heißen Sommer 2003: Fall 1: Notarztalarmierung wegen Herzkreislaufstillstand. Bei Eintreffen der Ersthelfer wird ein nicht ansprechbarer 35-jähriger Mann vorgefunden, GCS 3. Es besteht Pulslosigkeit und Apnoe bei Asystolie. Sofortiger Beginn einer mechanischen Reanimation. Bei Notarzteintreffen sofortige Intubation und zusätzliche medikamentöse Reanimation. Infusion kristalloider und kolloidaler Lösungen, Gabe von Suprarenin und später Atropin. Nach ca. 1 Stunde Abbruch der erfolglosen Reanimation. Bei Abbruch der Reanimation wird eine Körperkerntemperatur $>42,1^{\circ} \mathrm{C}$ ermittelt. Die weitere Anamnese ergab: 15 Minuten vor Auffinden durch Kollegen letzter Verbalkontakt zu Kollegen. Seit 5 Stunden körperlich anstrengende Tätigkeit im Freien, stark sonnenexponiert. In der Vorgeschichte psychiatrische Erkrankung. Vormedikation Valproat und Clozapin. Fall 2: Am selben Tage nachmittags Notarztalarmierung wegen Synkope: Bei Eintreffen des Rettungsteams und Notarzt wird eine 41-jährige desorientierte Patientin mit Schwindel, massiv überwärmter Haut und Myoklonien der Extremitätenmuskulatur vorgefunden. GCS 14, Blutdruck 140/80 mmHg, Herzfrequenz 150/min, $\mathrm{SO}_{2} 94 \%$, Blutzucker $307 \mathrm{mg} / \mathrm{dl}$. Vormedikation mit Clozapin bei bekannter schizophrener Psychose. Einleitung einer Infusionstherapie mit kristalloider Lösung und zügige stationäre Einweisung unter dem V.a. drohenden Hitzschlag. Bei stationärer Aufnahme Körpertemperatur $41,2{ }^{\circ} \mathrm{C}$ rektal. Unter Infusionstherapie und symptomatischer Antipyrese Normalisierung von Körpertemperatur, neurologischem Befund und Blutzuckerspiegel. Schlussfolgerung: Bei hohen thermischen Belastungen muss insbesondere bei Patienten, die mit Clozapin vorbehandelt sind mit Hyperthermien und schweren Verläufen gerechnet werden und dies auch bei Anwendung des Präparates bedacht werden. 
Überleben bei Intoxikation mit 100 fach erhöhtem

Serumspiegel von Metamizol und 200fach erhöhtem

Serumspiegel von Doxepin - ein Fallbericht

S. Machmüller, D. A. Vagts

Universität Rostock, Klinik und Poliklinik für Anästhesiologie und Intensivtherapie

Metamizol gehört zu den nichtsauren Analgetika. Bei einem Serumspiegel bis $10 \mu \mathrm{g} / \mathrm{ml}$ wirkt es vornehmlich über die Hemmung der zentralen Cyclooxigenase antipyretisch, spasmolytisch, analgetisch und wenig antiphlogistisch. Bei Überdosierung muss mit Erbrechen, Abdominalbeschwerden, akutem Nierenversagen, selten ZNS-Symptomen, sowie Herzrhythmusstörungen gerechnet werden. Die Therapie der Überdosierung ist symptomatisch mit lebenserhaltenden Maßnahmen, Entfernung nicht resorbierter Substanzen via Magenspülung, Medizinalkohle, forcierter Diurese oder Elimination des Hauptmetaboliten mittels Hämodialyse oder Hämofiltration. Intoxikationen mit Metamizol sind selten. Doxepin ist ein trizyklisches Antidepressivum, dessen Intoxikationshäufigkeit zunehmend ist. Der therapeutische Bereich liegt bei $0,05-0,15 \mu \mathrm{g} / \mathrm{ml}$, als potentiell lethal gelten Serumkonzentrationen von $10-20 \mu \mathrm{g} / \mathrm{ml}$. Die akute Wirkung ist ein sedierender Effekt, bei Überdosierung kommt es zu parasympatholytischen Nebenwirkungen, weiterhin zu einer Senkung der zerebralen Krampfschwelle, Blutdruckabfall, QRS-Verbreiterungen, Blockbildern, Herzrhythmusstörungen und allergischen Reaktionen. Die primäre Detoxifikation sollte über Magenspülung und Medizinalkohle erfolgen. Wir berichten über einen 53jährigen Mann, der hypotherm $\left(24^{\circ} \mathrm{C}\right)$ in einem Auto aufgefunden wurde. Nach Umlagerung in einen RTW trat ein Herzkreislaufstillstand ein. Nach erfolg reicher Reanimation wurde der Patient über ein peripheres Krankenhaus unter Reanimationsbedingungen zur Aufwärmung an der Herz-Lungen-Maschine in unsere Klinik verlegt. Während der Wiedererwärmung an der HLM (bis $32^{\circ} \mathrm{C}$ ) wurde im Screeningverfahren nach Spuren einer Intoxikation gesucht, für die Hinweise vorlagen, und probatorisch medizinische Kohle verabreicht. Die Untersuchungen erbrachten zunächst einen Metamizolserumspiegel von $894 \mu \mathrm{g} / \mathrm{ml}$. Erst $17 \mathrm{~h}$ später zeigte sich ein Doxepinserumspiegel von $17 \mu \mathrm{g} / \mathrm{ml}$ (erste Probe negativ!). Die weitere Therapie erfolgte mit Analgosedierung und einer neuroprotektiven Kühlung nach Reanimation auf $34^{\circ} \mathrm{C}$ Körpertemperatur für weitere $48 \mathrm{~h}$, Alkalisierung mit Natriumhydrogenkarbonat $8,4 \%$ und forcierte Diurese bei Myoglobinwerten $>4300 \mathrm{ng} / \mathrm{ml}$ für 4 Tage. Die ALAT und ASAT-Werte waren maximal auf das 5 fache der Normwerte erhöht, zu einem akuten Nierenversagen kam es nicht. Am 5. Tag konnte der Patient extubiert werden, am 7. Tag wurde er bei weiter bestehender suizidaler Absicht in die Psychiatrie verlegt. Der Patient war orientiert, es bestanden keine grobneurologischen Defizite. Nach unserem Wissen handelt es sich bei diesem Fall um die bisher höchsten in der Literatur beschriebenen Serumspiegel von Metamizol und Doxepin, die nach Intoxikation überlebt wurden. Protektiv dürfte in diesem Fall die Hypothermie des Patienten sowie die lange erhaltene Vita minima des Patienten gewirkt haben. Der Fall zeigt, dass durch entsprechende supportive Maßnahmen auch Megadosen von Metamizol und Doxepin überlebt werden können.

Suizid mit Lackverdünnung, resultierend in beidseitigem Kleinhirninfarkt

M.H.T. Reinges, T. Krings, F.J. Hans

Neurochirurgische Klinik und Abteilung Neuroradiologie, Universitätsklinikum Aachen

Ein 19-jähriger Patient wurde zu Hause komatös aufgefunden, bei Inspektion des Auffindeortes wurde eine fast leere Getränkeflasche mit aromatisch riechender Flüssigkeit in der Nähe des $\mathrm{Pa}$ tienten gefunden. Noch am Auffindeort des Patienten erfolgten Intubation und Beatmung. Das vor Aufnahme auf der Intensivstation angefertigte CCT zeigte ausgedehnte Hypodensitäten in bei- den Kleinhirnhemisphären, vereinbar mit einem beidseitigen Kleinhirninfarkt, sowie einen resultierenden Hydrocephalus occlusus. Es folgten die sofortige Anlage einer frontalen externen Ventrikeldrainage mit pathologisch erhöhtem Eröffnungsdruck und die Entnahme von Liquor zur weiteren Diagnostik. Die im Anschluss durchgeführte CT-Angiographie konnte keinen Basilarisverschluss oder andere Auffälligkeiten des Gefäßsystems nachweisen. Aufgrund des Hinweises des primär behandelnden Notarztes, dass bei dem Patienten eine Flasche mit aromatisch riechendem Inhalt aufgefunden wurde, erfolgte die toxikologische Analyse dieser Flüssigkeit sowie des Liquors des Patienten. Hierbei konnte diese Flüssigkeit als Verdünnungsmittel, am ehesten Lackverdünnung, identifiziert werden. Nachdem sich im Verlaufs-CCT am Folgetag eine weitere Progredienz der Infarkte zeigte, wurde bei infauster Prognose die Therapie minimiert, der Patient verstarb kurz danach. Auch wenn in diesem Fall keine lebensrettende Therapie mehr möglich war, so unterstreicht er doch, wie wichtig eine Inspektion des Auffindeortes des Patienten durch Notarzt und Polizei war. Nur so konnte letztlich die Diagnose „Suicid mit Lackverdünnung" gestellt werden.

Kombinierter Suizidversuch mit dem Rodentizid

Cumatan (Difenacoum/Sulfachinoxalin)

und selbstzugeführten Schnitt- bzw. Stichverletzungen:

Implikationen für Primärversorgung und Intensivtherapie

\section{J. Schmidt, A. Scholler, A. Irouschek, S. Albrecht}

Anästhesiologische Klinik der FAU Erlangen-Nürnberg

Einleitung: Bei Suizidversuchen stellt in Deutschland die Intoxikation $(64 \%)$ die am häufigsten angewandte Methode dar, gefolgt von Schnitt- und Stichverletzungen (16\%) sowie anderen Methoden. Komplexe Suizide, d.h. die Kombination zweier Methoden, sind eher selten [1]. Wir stellen hier die Kasuistik eines sekundär komplexen Suizidversuch mit oraler Einnahme des Rodentizides Cumatan ${ }^{\circledR}$ und sekundärer Selbstbeibringung von diversen Schnitt- und Stichwunden vor. Kasuistik: Der Notarzt wird in die Wohnung eines 27-jährigen Patienten gerufen. Dieser weist als Verletzungsmuster mehrere stark blutende Schnitt- bzw. Stichwunden am Thorax (sternal und präcordial), beiden Oberschenkeln und dem linken Oberarm auf. Er ist somnolent, blass und mit nicht tastbaren peripheren Pulsen und einer Herzfrequenz von 150/min im klinischen Vollbild eines Volumemmangelschocks. Nach Stabilisierung (Druckverbände, Volumentherapie, Intubation) erfolgt der Transport in den Schockraum eines Zentrums der Maximalversorgung. Hier fällt im Blutbild ein Hämoglobin von 2,3 g/dl (Hkt 8\%) und bei der Gerinnung ein Quickwert von $6 \%$ sowie eine PTT von $196 \mathrm{sec}$ auf. Parallel zum Ausschluss von Verletzungen intraabdominaler und intrathorakaler Organe mittels bildgebender Diagnostik (Sono-Abdomen, Thorax-CT) erfolgt die Transfusion von 6 Erythrozytenkonzentraten und 10 Frischplasmen. Nach operativer Blutstillung und Wundversorgung wird der Patient zur weiteren Überwachung auf die operative ICU verlegt, wo er am nächsten Morgen nach Stabilisierung des Hämoglobins bei 8,0 g/dl und der Gerinnung (Quick 45\%, PTT und TZ im Normbereich) extubiert werden kann. Hinsichtlich des Suizidversuchablauf berichtet er über die initiale Einnahme von $400 \mathrm{~g}$ Cumatan ${ }^{\circledR}$-Puder (Difenacoum/Sulfachinoxalin). Nachdem diese Suizidmethode anscheinend erfolglos war, fügte er sich sekundär die Stich- und Schnittwunden zu. Der Patient wurde unter Vitamin K1-Substitution in die Psychiatrie verlegt, wo es innerhalb von 24 Stunden zur erneuten Blutung aus den Wunden bei einem Quickwert von $<10 \%$ (INR 5,6) kam. Erst nach mehrtägiger alternierender Gabe von Frischplasma und Prothrombinkomplex sowie täglicher Gabe von $20 \mathrm{mg} / \mathrm{d}$ Vitamin $\mathrm{K} 1$ gelang es, die Gerinnungssituation zu stabilisieren. Diskussion: Komplexe Suizidversuche können hinsichtlich Diagnostik und Therapie eine besondere Herausforderung für die Primärversorgung und die intensivmedizinische Therapie darstellen. Gerade in Fällen, wo der 
Tathergang offensichtlich und plausibel erscheint (blutende Stichverletzung), kann die zweite Suizidmethode (Intoxikation) verschleiert werden, obwohl sie entscheidend für den weiteren Verlauf ist. Moderne freikäufliche Rodentizide enthalten potente und langwirksame Superwarfarine, die als Antidottherapie die hochdosierte Zufuhr von Vitamin K1 (20-125 mg/d) über einen Zeitraum von bis zu 8 Monate erforderlich machen [2,3].

Literatur: [1] Forensic Sci Int 2006; 157:83-86. [2] Blood 1990; 12:2555-2559. [3]Forensc Sci Int 1996; 78:13-18

Notfall- und intensivmedizinische Besonderheiten des Intensivtransports zur hyperbaren Sauerstofftherapie ( $\mathrm{HBO})$

S. Wiese, S. Beckers, S. Schröder

Klinik für Anästhesiologie, Universitätsklinikum der RheinischWestfälischen Technischen Hochschule (RWTH)

Die frühzeitige hyperbare Oxygenation (HBO) ist für bestimmte Erkrankungen, z. B. bei der arteriellen Gasembolie, beim schweren Tauchunfall oder bei der CO-Vergiftung, eine wesentliche Komponente einer umfassenden Intensivtherapie. In Deutschland gibt es nur wenige Druckkammern mit 24-Stunden-Bereitschaft, die über eine Beatmungsmöglichkeit verfügen, so dass viele Patienten einen Intensivtransport zur HBO benötigen. Dadurch wird manchem Patienten diese Therapieform vorenthalten im Hinblick auf den Aufwand des Transports und dem häufig überschätzten Risiko. Jedoch ist die Anwendung der HBO ein seit 30 Jahren erprobtes Verfahren und kann in interdisziplinärer Zusammenarbeit mit speziell ausgebildeten Druckkammerärzten sicher und zeitgerecht durchgeführt werden. Ist die Indikation zur HBO gestellt, sollte möglichst frühzeitig mit einem geeigneten Druckkammerzentrum Kontakt aufgenommen werden, um die Transport- und Behandlungsvorbereitung $\mathrm{zu}$ koordinieren. Zur Vorbereitung im Krankenhaus gehören z.B. der Ausschluss und/oder ggf. Therapie eines Pneumothorax, die Anlage und Sicherung geeigneter Zugänge sowie ggf. eine angepasste Sedierung. Zuweilen sind auch eine Parazentese und die Einlage eines Paukenröhrchens notwendig. Trotz der insgesamt geringen Komplikationsrate der $\mathrm{HBO}$ sind fundierte Kenntnisse der Überdruckmedizin erforderlich, um bei intensivpflichtigen Patienten eine sichere Therapie zu gewährleisten. Aus diesem Grund sollte entweder die betreuende Klinik oder die jeweilige Druckkammer selbst Ärzte stellen, die in Tauch- und Überdruckmedizin erfahren sind und die den Transport zur HBOTherapieeinrichtung begleiten. Spezifische Aspekte der HBO gliedern sich in: - Allgemeine Besonderheiten der HBO-Therapie, - Komplikationen durch die intensivmedizinischen Interventionen unter Überdruckbedingungen. Besonderheiten der HBO-Therapie umfassen beispielsweise Kompressionsbarotraumen der Ohren, der Sinus, das Barotrauma der Lunge mit Pneumothorax, die $\mathrm{O}_{2}$-Toxizität sowie Dekompressionserkrankungen. Komplikationen durch Interventionen unter HBO-Bedingungen umfassen außer Schwierigkeiten, die sich aus der räumlichen Enge und der Überdruckexposition des Begleiters in der Druckkammer ergeben, die Gasembolie und Besonderheiten der Beatmung unter Überdruckbedingungen. Leitsymptome möglicher HBO-Nebenwirkungen müssen sowohl den begleitenden Rettungsdienstpersonal als auch der weiterbehandelnden Intensivstation bekannt sein, um die seltenen, aber zuweilen lebensbedrohlichen Zwischenfälle adäquat behandeln zu können. Diese können sowohl dem kardiopulmonalen als auch dem neurologischen Formenkreis angehören. Fazit: Unter guter und frühzeitiger Zusammenarbeit zwischen überweisender Intensivstation und behandelndem Druckkammerzentrum kann die HBO-Therapie als sicherer Baustein des therapeutischen Repertoires der Intensivmedizin gelten, auch wenn hierzu ein Intensivtransport nötig ist.
Atemwegsmanagement bei HNO-Tumorpatienten mit akuter Dyspnoe

M. Bücheler, G. Putensen $*$ S. Wirz ${ }^{\star}$, A. Gerstner,

H.-.J. Straehler-Pohl, F. Bootz

Kliniken für HNO-Heilkunde und Klinik für Anästhesie und operative Intensivmedizin*, Universitätsklinikum Bonn

Maligne Tumore des oberen Aerodigestivtraktes können aufgrund ihrer anatomischen Lage oder ihrer Größe zu rasch progredienter Dyspnoe mit sofortigem notfallmedizinischem Interventionsbedarf führen. Die verschiedenen Algorithmen für den schwierigen Atemweg treffen für HNO-Tumorpatienten nur zum Teil zu oder sind klinisch nicht durchführbar, wie zum Beispiel die fiberoptische Wachintubation bei mechanischer Verlegung der Glottis durch große nicht verdrängbare Malignome. Anhand ausgewählter Patientenbeispiele einer HNO-Klinik mit hohem Anteil von Tumorpatienten stellen wir unser interdisziplinäres Vorgehen bei tumorbedingter akuter Dyspnoe dar. Nach Möglichkeit erfolgt vor der Sicherung des Atemweges die flexible transnasale Endoskopie zur Orientierung über die Lage und Größe des Tumors und die begleitende Schleimhautschwellung. Bei darstellbarem Glottislumen führen wird ein primärer oraler Intubationsversuch unternommen. Nach erfolgreicher Intubation können dann ein Tumordebulking zur Erweiterung des Atemweges und weitere diagnostische oder andere therapeutische Maßnahmen vorgenommen werden. Als Alternative zur Intubation hat sich bei kleineren Tumoren auch die laserchirurgische Glottiserweiterung in Jet-Anasthesie bewährt. Gelingt die primäre orale Intubation nicht, oder wird sie aufgrund der Tumorausdehnung als nicht möglich angesehen, führen wir in Tracheotomie-Bereitschaft mit dem starren Oesophaguskop eine direkte Laryngkopie durch. Lässt sich Glottis und Tracheallumen auf diese Weise darstellen, wird dann ein Führungskatheter eingelegt, über den dann nach Entfernen des Oesophaguskops ein Endotrachealtubus platziert wird. Bei raschem Abfall der Sauerstoffsättigung stehen als weitere Notfallmaßnahmen Koniotomie bzw. Tracheotomie zur Verfügung. Als alternatives Verfahren zum oralen oder endoskopisch-kontrollierten Intubationsversuch ist die Tracheotomie in Lokalanästhesie eine weitere Option. Der Patient wird hierzu während des Eingriffs über die Maske beatmet und ggf. sediert. Entscheidend für das erfolgreiche Management der akuten Dyspnoe bei HNO-Tumorpatienten ist eine gut etablierte Kooperation zwischen Anästhesisten und HNO-Chirurg mit fundierter Kenntnis der jeweiligen Möglichkeiten der anderen Fachdisziplin, um eine schnelle Sicherung des Atemweges ohne zusätzliche Gefährdung des Patienten $\mathrm{zu}$ erreichen.

Ausstattung von Notarzt-besetzten Rettungsmitteln 148 für das Atemwegsmanagement

J. Hinkelbein ${ }^{1}$ P. Lessing ${ }^{2}$ J. Hehl ${ }^{2}$ K. Ellinger ${ }^{2}$ H. Genzwürker ${ }^{1}$

${ }^{1}$ Klinik für Anästhesiologie, Universitätsklinikum Mannheim

${ }^{2}$ Klinik für Anästhesie, Oberschwabenklinik KH St. Elisabeth, Ravensburg

Hintergrund: Die Überprüfung der korrekten Tubuslage sowie der Einsatz von Alternativen zu Maskenbeatmung und Intubation mit dem Ziel der Oxygenierung des Patienten sind zentrale Anliegen von Konzepten für das Atemwegsmanagement. Anhand einer Umfrage unter allen baden-württembergischen Notarztstandorten im Herbst 2005 wurden die apparativen Voraussetzungen für eine Leitlinien-konforme Therapie evaluiert und mit den Ergebnissen einer entsprechenden Befragung aus dem Jahre 2001 [1] verglichen. Material und Methoden: Der Fragebogen, der an alle dem Sozialministerium gemeldeten Notarztstandorte in BadenWürttemberg versandt wurde, enthielt Fragen zur Verfügbarkeit von Möglichkeiten zur Kontrolle der Tubuslage (Kapnometrie, Kohlendioxid-Indikatorsysteme, Ösophagusdetektor) und Alternativen $\mathrm{zu}$ Maskenbeatmung und endotrachealer Intubation (supraglottische Alternativen: Combitube, Larynxmaske, Intubations- 
larynxmaske, Larynxtubus, andere; Notkoniotomie). Ergebnisse: 107 von 120 Standorten $(89,2 \%)$ antworteten (2001: 116 von 127 Standorten, 91,3\%). An 79 Standorten (73,8\%) stehen Möglichkeiten zur Überprüfung der Tubuslage zur Verfügung. Dabei handelt es sich in $66,4 \%(n=71)$ um Kapnometer und in 7,5\% $(n=8)$ um Kohlendioxid-Indikatorsysteme, ein Standort verfügt über Kapnometer und Ösophagusdetektor. 2001 verfügten 26,7\% der Standorte (31 von 116) über Tubuslage-Kontrollmöglichkeiten. Die Beschaffung ist an einem Standort binnen 6 Monaten geplant, an einem nicht. 26 Standorte machten keine Angaben. Supraglottische Alternativen werden an 104 Standorten (97,2\%) mitgeführt: 53 Standorte $(49,5 \%)$ Larynxmaske, 49 Standorte $(45,8 \%)$ Combitube, 22 Standorte $(20,6 \%)$ Larynxtubus, jeweils 1 Standort $(0,9 \%)$ Intubationslarynxmaske und Easytube. 24 Standorte (22,4\%) führen 2 Alternativen mit, 1 Standort 3 (Larynxmaske/Combitube/Larynxtubus). 1 Standort plant die Beschaffung binnen $6 \mathrm{Mo}-$ naten, 2 Standorte planen keine Beschaffung. Die Frage nach den verfügbaren Größen der verschiedenen Alternativen (Kinder/Erwachsene) wurde von der Mehrzahl der Standorte nicht beantwortet, sodass eine Auswertung nicht möglich ist. 2001 verfügten $54,3 \%$ der Standorte (63 von 116) über supraglottische Alternativen, wobei Larynxmaske und Combitube an jeweils 32 Standorten vorgehalten wurden, der Larynxtubus an 11 Standorten, die Intubationslarynxmaske an 2 Standorten und der Pharyngotracheale Lumentubus an 1 Standort. Die Notkoniotomie steht 2005 an 75 Standorten $(70,1 \%)$ zur Verfügung; 2001 waren dies 97 von 116 Standorten $(83,6 \%)$. Zusammenfassung: Im Vergleich zu den Umfragedaten von 2001 lässt sich im Bereich Atemwegsmanagement eine positive Entwicklung bei der Ausstattung der Notarzt-besetzten Rettungsmittel nachweisen. Die flächendeckende Umsetzung der Vorhaltung von Möglichkeiten zur Tubuslagekontrolle sollte forciert werden.

Literatur: [1] Genzwürker H, Isovic H, Finteis T, Hinkelbein J, Denz C, Ellinger K: Ausstattung von Notarzt-besetzten Rettungsmitteln in Baden-Württemberg. Anaesthesist 2002; 51:36

Einfluss des Heidelberger Schockraumalgorithmus auf die innerhospitalen Versorgungszeiten und die Mortalität schwerverletzter Patienten

M. Bernhard ${ }^{1}$, T.K. Becker ${ }^{1}$, T. Nowe ${ }^{1}$, M. Mohorovic ${ }^{1}$, M. Sikinger $^{1}$, Th. Brenner ${ }^{1}$, G.M. Richter ${ }^{2}$, B. Radeleff ${ }^{2}$, P.-J. Meeder ${ }^{3}$, M. W. Büchler ${ }^{3}$, B. W. Böttiger ${ }^{1}$, E. Martin ${ }^{1}$, A. Gries ${ }^{1}$

${ }^{1}$ Klinik für Anästhesiologie, ${ }^{2}$ Radiologische Universitätsklinik, ${ }^{3}$ Chirurgische Universitätsklinik, Universitätsklinikum Heidelberg

Hintergrund: Der Zeitfaktor spielt bei der Versorgung schwerverletzter Patienten eine besonders wichtige Rolle. Für das Überleben dieser Patienten sind in der frühen klinischen Phase die schnelle Durchführung einer gezielten bildgebenden Diagnostik und der schnellstmögliche Beginn notwendiger Notfallinterventionen essentiell [1]. Vor diesem Hintergrund wurde an unserer Klinik interdisziplinär ein Schockraum (SR-)Algorithmus mit dem Ziel entwickelt, die Zeitintervalle von Aufnahme der Patienten bis zum Abschluss der bildgebenden Diagnostik und dem Beginn von Notfalloperationen zu reduzieren. Material und Methode: In der prospektiven Untersuchung wurden Patienten vor (Gruppe 1, 01/04-10/04) und nach (Gruppe 2, 01/05-11/05) Einführung des SR-Algorithmus untersucht. Neben Alter, Geschlecht und Verletzungsschwere wurden die Zeitintervall zwischen Aufnahme der Patienten im Schockraum und Abschluss der bildgebenden Diagnostik (Sonographie [Sono], Röntgenthorax [RTX], kraniale Computertomographie [CCT]) sowie zwischen Aufnahme und Beginn ggf. notwendiger Notfalloperationen (Not-OP) evaluiert. Darüber hinaus wurde die Mortalität in Abhängigkeit von der Verletzungsschwere ermittelt. Ergebnisse: Während der jeweiligen Evaluationszeiträume wurde 170 Patienten der Gruppe 1 und 199 Patienten der Gruppe 2 untersucht. Das Durchschnittsalter $(44 \pm 20 \mathrm{vs}$. $42 \pm 23$ Jahre), der Anteil männlicher Patienten (74\% vs. 69\%) und die Verletzungsschwere (ISS: $20 \pm 18$ vs. $19 \pm 15$ ) beider Grup- pen waren vergleichbar. Durch die Einführung des SR-Algorithmus konnten die Zeitintervalle zwischen Patientenaufnahme und dem Vorliegen der radiologischen Befunde signifikant reduziert werden (Sono: $11 \pm 10$ vs. $7 \pm 6 \min [\mathrm{p}<0,05]$, RTX: $21 \pm 12$ vs. $12 \pm 9 \mathrm{~min}$ [p $<0,01]$, CCT: $55 \pm 27$ vs. $32 \pm 14 \mathrm{~min}[\mathrm{p}<0,01])$. Ebenfalls konnte durch die Einführung des SR-Algorithmus das Zeitintervall zwischen Aufnahme und dem Beginn einer Not-OP signifikant reduziert werden (Not-OP: $126 \pm 90$ vs. $51 \pm 10 \mathrm{~min}$ $[\mathrm{p}<0,01])$. Darüber hinaus konnte nach Einführung des SR-Algorithmus für die Gruppe der am schwersten verletzten Patienten (ISS $>24)$ eine signifikante Mortalitätsreduktion nachgewiesen werden $(33,3 \%(17 / 51)$ vs. $16,7 \%(11 / 66)$ [p $<0,05])$. Schlussfolgerung: Die vorliegende Untersuchung zeigt, dass die Einführung eines an die lokalen Gegebenheiten angepassten und interdisziplinär umgesetzten SR-Algorithmus die Zeitintervalle zwischen Patientenaufnahme und dem Abschluss der bildgebenden Diagnostik einerseits sowie dem Beginn ggf. notwendiger Not-OPs andererseits signifikant reduzieren kann. Darüber hinaus hatte die Einführung des SR-Algorithmus einen signifkanten Einfluss auf die Mortalität in der Gruppe der besonders schwer verletzten $\mathrm{Pa}$ tienten (ISS $>24$ ).

Literatur. [1] Clarke et al: J Trauma 2002; 52:420-425

Die 64-multi-slice-CT in der Primärdiagnostik beim Polytraumatisierten

M. Baacke ${ }^{1}$, K. Wiele ${ }^{1}$, M. Schiffer ${ }^{2}$, J. Jeibmann ${ }^{3}$, A. Junge ${ }^{1}$

${ }^{1}$ Klinik für Unfall- und Wiederherstellungschirurgie

${ }^{2}$ Klinik für Intensivmedizin und Anästhesiologie

${ }^{3}$ Abteilung ür Nuklerarmedizin und Radiologie

Fragestellung: Die primäre Röntgenthoraxaufnahme ist neben der Abklärung von Wirbelsäulen- und Beckenverletzungen zentraler Bestandteil aktueller Schockraumprotokolle. Die CT-Diagnostik als evidenzbasierter Goldstandard in der Detektion von Verletzungen nahezu aller Körperregionen war bislang lediglich dem kreislaufstabilen Patienten vorbehalten. Die Einführung eines 64-Zeilen-CT (64-MSCT) in die Schockraumdiagnostik erforderte die Entwicklung eines neuen Algorythmus jenseits evidenzbasierter Behandlungspfade. Unter Verzicht auf eine primäre konventionelle Thoraxaufnahme wurde eine prioritätenorientierte CT-Diagnostik mit standardisierten Indikationskriterien auch beim kreislaufinstabilen Patienten durchgeführt. Ziel dieser prospektiven Beobachtungsstudie war die UUberprüfung dieses neuen Schockraumprotokolls mit dem klinischen Endpunkt: Patientensicherheit. Meßpunkte waren 1. Respiratorische Insuffizienz durch einen Pneumothorax, 2. Schwere Hypotonie während des CT-scans. Methodik: In einer prospektiven Erhebung wurden die Schockraumpatienten von Juni 2005 bis Januar 2006 dokumentiert und alle primär aufgenommenen Traumapatienten mit einem ISS $>16$ zwischen 16 und 70 Jahren in die Studie eingeschlossen. Die Basisdokumentation nutzte den DGU-Polytraumabogen, erweitert um die Beobachtungsfelder „Pneumothorax“ und „Kreislaufinstabilität". 1 . Die arterielle Hypotonie wurde in drei Schweregrade unterteilt: Grad-I = kompensierbar mit Volumensubstiution, Grad-II = kompensierbar mit Volumen und Catecholamin(en), Grad-III = nicht kompensierbar. 2. Die respiratorische Funktion wurde nach Oxigenierungsindex in acute-lung-injury und acute-respiratory-distresssyndrome unterteilt. Ein Pneumothorax als Ursache der resp. Insuffizienz wurde angenommen, wenn nach erfolgreicher Drainage der Oxigenierungsindex um mind. $100 \mathrm{mmHg}$ verbessert werden konnte. 3. Die Wertigkeit der Sonographie von Abdomen und Retroperitoneum wurde anhand der Therapierelevanz der Untersuchungsergebnisse, evaluiert durch die Abdomen-CT beurteilt. Bei den 43 eingeschlossenen Patienten lag der mittlere ISS bei 33 (min 17, max 50). Die mittlere Zeit der Primärversorgung (Eintreffen bis Verlegung aus Schockraum lag bei $65 \mathrm{~min}$ (min 43, $\max 175 \mathrm{~min})$. Eine ALI lag bei 18 Patienten $(43 \%)$ vor, bei $4 \mathrm{~Pa}-$ tienten (9\%) ein ARDS. Ein Pneumothorax war in keinem Fall die Ursache der resp. Insuffizienz. Bei 27 Patienten (62\%) bestand ei- 
ne Hypotonie, davon bei 6 Patienten (14\%) im Schweregrad II ein Patient $(2 \%)$ hatte eine nicht rekompensierbare Hypotonie und verstarb vor Erreichen des OP. Die Scan-Zeit, als einzige Zeit erschwerten Patientenzugangs, hatte keinen Einfluss auf die Behandelbarkeit der Hypotonie. Schlussfolgerung: Die Einbeziehung eines 64-MSCT in die Primärdiagnostik beim Polytrauma anhand eines standardisierten Indikationsprotokolls steigert die diagnostische Aussagekraft erheblich. Gefährdungen der Patientensicherheit wurden durch einen Verzicht auf die primäre Thoraxaufnahme nicht beobachtet.

\section{Polytrauma}

\section{Psychosoziale Belastungen von PatientInnen mit einem Polytrauma \\ C. Petersen, S. Meier, H. Boettcher, P. Ueblacker, U. Koch Universitätsklinikum Hamburg-Eppendorf}

Trotz einer in Deutschland insgesamt rückläufigen Auftretenswahrscheinlichkeit des Polytraumas stellt die Polytrauma-Behandlung weiterhin eine Herausforderung für die medizinische Versorgung dar. Bisherige Forschungsansätze fokussierten vor allem auf die Feststellung von traditionellen Behandlungsergebnissen wie Mortalität oder Morbidität. Einige wenige Forschungsbestrebungen widmen sich der Ermittlung von Langzeitfolgen nach einem Polytrauma. Die Ergebnisse der Studien weisen auf langfristige psychosoziale Beeinträchtigungen trotz zufriedenstellender somatischer Behandlungsergebnisse hin. Zur einer genaueren Analyse der psychosozialen Beeinträchtigungen wurde ein Projekt am Universitätsklinikum Hamburg-Eppendorf (UKE) initiiert, dass darauf abzielt, die psychosozialen Probleme polytraumatisierter Patienten $\mathrm{zu}$ analysieren sowie einen polytrauma-spezifischen Fragebogens und ein Beratungskonzept für die Nachsorgephase zu entwickeln. Bei der von der Wiebke und Wolfgang Boden Stiftung geförderten Studie handelt es sich um ein Kooperationsprojekt der Klinik und Poliklinik für Unfall-, Hand- und Wiederherstellungschirurgie, des Zentrums für Anästhesiologie und Intensivmedizin und des Instituts und Poliklinik für Medizinische Psychologie. Zur Erfassung der psychosozialen Belastung wurden Fokusgruppen und Einzelinterviews mit betroffenen Patienten, Familienangehörigen und Behandlern durchgeführt. Insgesamt nahmen $n=16$ Personen an den Gesprächen teil. Die Teilnehmerinnen sprachen neben körperlichen Folgeerscheinungen und kognitiven Einbußen in Folge einer Kopfverletzung in hohem Umfang psychosoziale Belastungen durch z.B. Autonomie- und Rollenverlust an. Die Belastungen manifestierten sich im psychischen Bereich in Form von Depression, Ängsten und/oder einer posttraumatisches Belastungsreaktion bis hin zu Suizidgedanken. Die Rückkehr in den Beruf stellte sich als eine wichtige Einflussgröße für das psychische Befinden der Betroffenen dar. Das familiäre/soziale Umfeld leidet oft erheblich unter der schweren Verletzung eines Angehörigen. Zum Zeitpunkt der Akutversorgung dominiert bei den Angehörigen die Angst, der Betroffene könne sterben oder nur schwer beeinträchtigt überleben. Im Langzeitverlauf muss oft eine vollständige „Neuorganisation“ familiärer Strukturen erfolgen. Aus diesen Themenbereichen wurden $n=52$ Items für den Patientenfragebogen und $n=32$ Items für den Angehörigenfragebogen generiert, die derzeit im Rahmen einer querschnittlichen Befragung psychometrisch überprüft werden. Hierzu wurde $n=66 \mathrm{An}$ gehörigen und Patienten, die 2003-2005 im UKE wegen einer schweren Mehrfachverletzung behandelt wurden, ein Fragebogen zugeschickt. Mit ersten Ergebnissen der Befragung kann im Juli 06 gerechnet werden. Auf die Ergebnisse aufbauend wird ein Nachsorge- und Beratungskonzept entwickelt.
Gerinnungsmanagement unter klinischen

Gesichtspunkten ohne erweiterte Diagnostik -

Was tun bei chirurgisch nicht stillbaren Blutungen

\section{A. Falthauser, A. Thomas}

Bundeswehrzentralkrankenhaus Koblenz

Hintergrund: Leider besteht in den Einsatzlazaretten der Bundeswehr trotz einem hohem Aufkommen an polytraumatisierten $\mathrm{Pa}$ tienten derzeit keine Möglichkeit zur erweiterten differenzierten Gerinnungsdiagnostik, wie beispielsweise der Thrombelastographie. Daher ist das Gerinnungsmanagement bei Traumapatienten derzeit im Wesentlichen auf die klinische Erfahrung der Therapeuten und die Bestimmung der globalen Gerinnungsparameter gestützt. Ein Algorithmus zur schnellen und effizienten Therapie mit Blutprodukten, Gerinnungsfaktoren und aktivierenden Substanzen ist derzeit mangels klinischer Daten nicht etabliert. Im Jahr 2005 wurden allein im Feldlazarett des Camp Warehouse, Kabul Afghanistan mehr als 80 Patienten mit einem ISS $>20$ versorgt. Material/Methode: Retrospektive Analyse einer kleinen Kohorte von 4 Patienten, die als ultima ratio Therapie kombiniert Fibrinogen (Hemocompletan ${ }^{\circledR}$, Fa. ZLB Behring) und rFVIIa (NovoSeven ${ }^{\circledR}$, Fa. NovoNordisk, off label use) erhalten haben. Ergebnisse: Wegen des Mangels an Erythrozytenkonzentraten und keinerlei Vorrat an Thrombozytenkonzentraten wurde allen vier Patienten bereits binnen $30 \mathrm{~min}$ nach Eintreffen im Schockraum bei stattgehabter oder bestehender massiver Blutung zur Vermeidung einer Hyperfibrinolyse und zur Thrombozytenaktivierung Tramexamsäure und DDAVP appliziert. Während der ersten Phase der chirurgischen Versorgung (damage control) zeigte sich in allen Fällen jeweils unter Transfusion von EK und Lyoplasmen das klinische Bild einer Dilutionskoagulopatie ohne weitere chirurgische Interventionsmöglichkeit. Um einen weiteren Verlust von Thrombozyten zu stoppen erhielten alle Patienten als ultima ratio Therapie $3 \mathrm{~g}$ Fibrinogen und unmittelbar anschließend $120 \mu \mathrm{g} / \mathrm{kg}$ Körpergewicht rFVIIa. In allen vier Fällen konnte die Blutung damit binnen 30 min gestoppt werden. Alle Patienten überlebten im weiteren Verlauf ihre Verletzungen. Diskussion: Unter den Bedingungen der Einsatzmedizin scheint die Anwendung einer Kombination von rFVIIa und Fibrinogen bei einer noch akzeptablen Thrombozytenzahl von etwa $30-50000 / \mathrm{nl}$ bei Traumapatienten ohne chirurgische Option zur Blutstillung eine hilfreiche Therapieoption zu sein. Wir haben bei keinem der Patienten Nebenwirkungen der Therapie weder initial noch im Verlauf beobachtet. Trotzdem wäre vor allem auch unter Kostengesichtspunkten eine differenziertere Therapie mit Gerinnungsfaktoren nach entsprechender Analytik wünschenswert. Diese ist allerdings selbst in Deutschland in vielen gerade kleineren Kliniken ebensowenig schnell verfügbar wie ausreichende Mengen an Blutprodukten, so dass auch in Deutschland unter den genannten Bedingungen eine kombinierte Applikation von Fibrinogen und rFVIIa erwogen werden sollte.

ROTEM-basiertes Gerinnungsmanagement nach Röhren-Überrolltrauma

D. Müller ${ }^{1}$ K. Görlinger ${ }^{2}$ A. Hanke ${ }^{2}$ D. Dirkmann ${ }^{2}$ H. Groeben ${ }^{1}$ ${ }^{1}$ Klinik für Anästhesiologie, Intensiv- und Schmerztherapie, Kliniken Essen Mitte, ${ }^{2}$ Klinik für Anästhesiologie und Intensivmedizin, Universitätsklinikum Essen

Präklinik: Ein 26-jähriger Arbeiter wurde von einer ca. $10 \mathrm{t}$ schweren Stahlröhre (Mannesmann Röhrenwerke) im BeckenBein-Bereich überrollt. Bei klinisch instabilem Becken wurde der Patient vor Ort narkotisiert und intubiert. Trotz Infusion von $250 \mathrm{ml}$ HyperHAES, 1,5 1 HAES 10\% und 3,5 1 kristalliner Lösung waren die Kreislaufparameter auf dem Transport nicht suffizient zu stabilisieren. Schockraum: Der Aufnahmebefund zeigte eine Herzfrequenz von $130 / \mathrm{min}$, einen Blutdruck von $60 / 30 \mathrm{mmHg}$, eine instabile Beckenringfraktur, eine Femurfraktur links mit Ischämie des linken Beines, ein prall gespanntes Abdomen, sowie eine 
diffuse Blutungsneigung. Das Aufnahmelabor zeigte eine massive Hämodilution. In der 35-minütigen Schockraumphase wurden Becken und Oberschenkel mittels Fixateur extern versorgt und die Hämodynamik mit 6 Erythrozytenkonzentraten und einem Adrenalinperfusor stabilisiert. Intraoperatives Gerinnungsmanagement: Der Patient war nach dem Transport in den OP azidotisch und hypotherm. Das ROTEM zeigte eine massive Dilutionskoagulopathie mit einer Nulllinie im FibTEM. Nach Stabilisierung der Rahmenbedingungen wurde die Koagulopathie zunächst mit der Gabe von Aprotinin, Fibrinogen und PPSB therapiert. Aufgrund der weiter bestehenden Verminderung der maximalen Gerinnselfestigkeit im ExTEM und der isolierten Verlängerung der Gerinnungszeit im InTEM wurden zusätzlich noch Thrombozyten und FFP substituiert. 1 Stunde nach Aufnahme in den OP war der $\mathrm{Pa}$ tient hämodynamisch stabil und zeigte keine weitere diffuse Blutungsneigung. Im weiteren Verlauf ließ sich die Körpertemperatur durch ein konsequentes Wärmemanagement wieder normalisieren. Nach einer Operationszeit von insgesamt 8 Stunden (Revaskularisation des linken Beines bei Abriß der Arteria iliaca) konnte der Patient hämodynamisch stabil auf die Intensivstation verlegt werden. In der Operationsphase erhielt der Patient insgesamt 20 EK, 14 FFP, 2 gepoolte Thrombozytenkonzentrate, 2 Mio. KIE Aprotinin, $10 \mathrm{~g}$ Fibrinogen und 2000 IE PPSB. Während der Versorgung des Patienten auf der Intensivstation kam es nicht zu einem Multiorganversagen. Schlussfolgerung: Der Einsatz der Rotationsthrombelastometrie (ROTEM) erlaubt eine schnelle Point-ofCare- Gerinnungsdiagnostik und ermöglicht damit eine zielgerichtete Therapie mit gerinnungsaktiven Komponenten $[1,2,3]$. Daneben kommt der Optimierung der Rahmenbedingungen $(\mathrm{pH}$, Temperatur, Kalzium, $\mathrm{Hb}$ ) eine essentielle Bedeutung $\mathrm{zu}[2,4]$.

Literatur: [1] Goerlinger K: ROTEM-based algorithm for management of acute haemorrhage and coagulation disorders in trauma patients. Eur J Anaesth 2006; 23 Suppl37:84-85. [2] Goerlinger K: Differenzierte Therapie komplexer Gerinnungsstörungen. J Anaesth Intensivbeh 2005; 12(1):120-124. [3] Fries D, Haas T, Velik-Salchner C, Lindner K, Innerhofer P: Gerinnungsmanagement beim Polytrauma. Anaesthesist 2005; 54:137-144. [4] Spahn DR, Rossaint R: Coagulopathy and blood component transfusion in trauma. Br J Anaesth 2005; 95:130-139

Interleukin-6 Serumspiegel $<\mathbf{3 0}$ Minuten nach Trauma 120 vs. Traumascores zur Abschätzung der Prognose beim Polytrauma

M. Sattler ${ }^{1}$, M. van Griensven ${ }^{2}$, T. Gerich ${ }^{1}$, M. Lüpkemann ${ }^{3}$, F. Hildebrand ${ }^{1}$, C. Krettek ${ }^{1}$, C. W. Mueller ${ }^{1}$

${ }^{1}$ Unfallchirurgische Klinik, Medizinische Hochschule Hannover,

${ }^{2}$ Ludwig Boltzmann Institute für experimentelle Traumatologie, Wien ${ }^{3}$ Christoph 4, Johanniter Unfallhilfe, Hannover

Fragestellung: Interleukin 6 (IL-6), ein pro-inflammatorisches $\mathrm{Zy}$ tokin, wird zur Einschätzung der Immunreaktion von Patienten herangezogen. Traumascores wie der Injury Severity Score (ISS) und der Polytraumaschlüssel (PTS) stellen ein Instrument zur Beurteilung der Verletzungsschwere dar. Sie spiegeln jedoch nicht die individuelle Immunreaktion wieder. Wir untersuchten ein Kollektiv von 57 Patienten mit der Fragestellung, ob „präklinisch“ festgestellte IL-6 Werte eine bessere Prognosesicherheit bzgl. Multiorganversagen (MOV) und Überleben erreichen als etablierte Traumascores. Methodik: Bei 57 Verunfallten wurden, bevor eine Therapie eingeleitet wurde, an der Einsatzstelle IL-6-Blutproben entnommen. Transport und Behandlung des Unfallverletzten erfolgte durch ein Rettungshubschrauberteam. IL-6 wurde im weiteren klinischen Verlauf in der zentralen Notaufnahme, sowie auf der Intensivstation dokumentiert. Zusätzlich wurden klinische Scores und andere relevante Parameter (Bakteriämie, MOV, Überleben) bestimmt. Diese wurden mit initialen IL-6 Werten korreliert. Ergebnisse: Der mittlere ISS betrug 16,9 (0-57), der mittlere PTS betrug 20,3. Der Mittelwert für „präklinisches“ IL-6 lag bei 11,7 (0-122), im weiteren Verlauf betrugen die IL-6 Mittelwerte in der ZNA 67,6 (0-939), der Maximumwert im Verlauf betrug 11000 (0-11000), der Mittelwert 631,1 ng/l. MOV trat bei 21 von 57 Patienten auf, 5 Patienten verstarben im weiteren Verlauf. Die Korrelation des „präklinischen“ IL-6 mit MOV $\left(0,255^{\star}\right)$ war niedriger als die Korrelation des MOV mit den Traumascores ISS und PTS $\left(0,681^{* *} / 0,704^{* *}\right)$. IL-6 in der ZNA korrelierte besser mit den beiden untersuchten Polytraumascores $\left(0,681^{* *} / 0,607^{* *}\right)$. Eine Korrelation mit "präklinischem" IL-6 und Überleben fand sich nicht $(0,036){ }^{*}=$ die Korrelation ist auf dem 0,05 Niveau signifikant (einseitig); ${ }^{* *}=$ die Korrelation ist auf dem 0,01 Niveau signifikant (einseitig). Schlussfolgerung: IL-6 zeigt eine nachweisbare Erhöhung innerhalb von Minuten nach Trauma, und korrelierte mit ISS, PTS und späterem Multiorganversagen. Aufgrund einer besseren Korrelation zwischen ISS, PTS und MOV anstelle von IL-6 stellt das „präklinisch“ gewonnene IL-6 keine bessere Prognosesicherheit in Bezug auf MOV und Überleben dar. Weitere Untersuchungen sind erforderlich um darzustellen, in wieweit die individuelle Disposition den Verlauf nach Trauma beeinflusst.

ROTEM-basiertes Gerinnungsmanagement bei diffuser Blutungsneigung nach LKW-Überrolltrauma

173

\section{A. Hanke, K. Görlinger, D. Dirkmann, J. Peters}

Klinik für Anästhesiologie und Intensivmedizin, Universitätsklinikum Essen

Präklinik und Schockraum: Eine 37-jährige Frau wurde von einem LKW erfasst und in Bereich von Becken und Oberschenkeln überrollt. Die Diagnostik ergab eine instabile Beckenringfraktur, grossflächige Decollements und ein ausgedehntes retroperitoneales Hämatom. Die inititalen Laborparameter waren: $\mathrm{Hb} 8,5 \mathrm{~g} / \mathrm{dl}$, Thr 144 /nl, Quick 69\%, PTT 29,3 s. Neben einer Volumentherapie mit 31 Kristalloid wurden der Patientin in den ersten 2 Stunden nach Aufnahme 8 EK, 10 FFP und 1 gepooltes Thrombozytenkonzentrat transfundiert. Das Becken wurde noch im Schockraum mit einem Fixateur externe versorgt und die Patientin nach Röntgen- und CT-Diagnostik - bei chirurgisch nicht angehbarer Blutung - auf die Intensivstation verlegt. Verlauf auf der Intensivstation: Bei persistierender diffuser Blutungsneigung im Bereich des Beckens, des Retroperitoneums und der Decollements zeigte sich weiterhin ein hoher Substitutionsbedarf. Innerhalb der ersten 4 Stunden mussten auf der Intensivstation weitere 13 EK, 16 FFP und 2 gepoolte Thrombozytenkonzentrate transfundiert werden, ohne dass es dadurch zu einer Verbesserung der Blutungsneigung kam. 6 Stunden nach Aufnahme in die Klinik wiesen sowohl die „klassischen“ Laborparameter (Hb 10,3 g/dl; Thr 55 /nl; Quick 80\%; PTT 35,3 s; Fib $149 \mathrm{mg} / \mathrm{dl}$ ), als auch die ROTEM-Parameter Werte im unteren Normbereich auf (MCF-Fib $11 \mathrm{~mm}$, MCF-Ex $45 \mathrm{~mm}$, CT-Ex $35 \mathrm{~s}$ ). Bei weiterhin bestehender diffuser Blutungsneigung und unverändertem Substitutionsbedarf erfolgte die Gabe von 1,5 Mio. KIE Aprotinin, 4 g Fibrinogen, 2000 IE AT III, 2000 IE PPSB und 1 gepoolten Thrombozytenkonzentrat mit dem Ziel, hochnormale Gerinnungswerte - insbesondere bezüglich der Gerinnselfestigkeit - zu erreichen. Dementsprechend stieg die MCFFib auf $21 \mathrm{~mm}$ und die MCF-Ex auf $51 \mathrm{~mm}$ an. Das „klassische“ Labor zeigte ebenfalls hochnormale Quick- (108\%) und Fibrinogenwerte $(406 \mathrm{mg} / \mathrm{dl})$. Außerdem kam es innerhalb von $30 \mathrm{Minu}-$ ten zum Sistieren der Blutungen und der Katecholaminpflichtigkeit der Patientin. Der Substitutionsbedarf während der nächsten 12 Stunden beschränkte sich auf $1 \mathrm{EK}, 1 \mathrm{FFP}$ und 1 gepooltes Thrombozytenkonzentrat. Schlussfolgerung: Bei „chirurgisch nicht angehbaren Blutungen" polytraumatisierter Patienten sind die in der Literatur angegebenen Zielwerte für Gerinnungsparameter [1] - wie z.B. Fibrinogen - häufig nicht ausreichend. Durch die Gabe von Faktorenkonzentraten und Anhebung der Gerinnselfestigkeit auf hochnormale Werte kann allerdings auch in solchen Fällen eine suffiziente Blutstillung - auch ohne den Einsatz von rekombinantem Fakor VIIa - erreicht werden $[2,3]$.

Literatur: [1] Spahn DR, Rossaint R: Coagulopathy and blood component transfusion in trauma. Br J Anaesth 2005; 95:130-139. 
[2] Goerlinger K: Differenzierte Therapie komplexer Gerinnungsstörungen. J Anaesth Intensivbeh 2005; 12(1):120-124. [3] Goerlinger K: ROTEM-based algorithm for management of acute haemorrhage and coagulation disorders in trauma patients. Eur J Anaesth 2006; 23 Suppl37:84-85

Gerinnungsmanagement beim Polytrauma auf der Grundlage eines ROTEM-basierten Algorithmus

K. Görlinger ${ }^{1}$, M. Vorweg ${ }^{2}$, A. Hanke ${ }^{1}$, E. Monaca $^{2}$, F. Wappler ${ }^{2}$, J. Peters ${ }^{1}$

${ }^{1}$ Klinik für Anästhesiologie und Intensivmedizin, Universitätsklinikum Essen

${ }^{2}$ Klinik für Anästhesiologie und operat. Intensivmedizin, Krankenhaus Merheim, Universität Witten Herdecke

Hintergrund: Das Gerinnungsmanagement bei polytraumatisierten Patienten basiert bislang auf klassischen Gerinnungsparametern die allerdings meist erst nach 30 bis 60 Minuten zur Verfügung stehen - und empirischen Regeln. Demgegenüber erlaubt der Einsatz der Rotationsthrombelastometrie (ROTEM) als Point-of-Care(POC)-Methode eine schnelle und zielgerichtete Therapie von Gerinnungsstörungen. Ziel der Untersuchung: Entwicklung eines ROTEM-basierten Algorithmus zum Gerinnungsmanagement beim Polytrauma. Material und Methode: Das Universitätsklinikum Essen und das Krankenhaus Merheim sind Traumazentren der höchsten Versorgungsstufe (Level I) in deren Schockräumen jährlich etwa 800 Patienten versorgt werden. Darunter sind ca. 250 Schwerverletzte mit einem ISS $\geq 16$. Seit Juni 1998 wird in Merheim und seit Januar 2000 in Essen das ROTEM als POC-Methode zum perioperativen Geinnungsmanagement eingesetzt. Auf der Basis der seitdem durchgeführten über 50000 Einzelbestimmungen wurde ein Diagnose- und Therapiealgorithmus zum Gerinnungsmanagement bei polytraumatisierten Patienten erarbeitet. ExTEM, InTEM, FibTEM und ApTEM wurden als Routinetests eingesetzt. Ausgewertet wurden die Coagulation Time (CT), die Maximum Clot Firmness (MCF) und die Maximum Lysis (ML). Ergebnisse: Das ROTEM erlaubt eine sichere Erfassung einer Hyperfibrinolyse $(\mathrm{ML}>15 \%)$. Bei einer Verminderung der Gerinnselfestigkeit (MCF-Ex $<50 \mathrm{~mm}$ ) lässt sich anhand der MCF im FibTEM zwischen einem Fibrinogenmangel (MCF-Fib $<12 \mathrm{~mm}$ ) und einer Thrombozytopenie/pathie (MFC-Fib $>12 \mathrm{~mm}$ ) differenzieren. Eine CT im ExTEM $>80 \mathrm{~s}$ oder eine CT im InTEM $>240 \mathrm{~s}$ weist in Zusammenhang mit einer diffusen Blutungsneigung auf einen therapiebedürftigen Faktorenmangel hin. Dabei kann ein Heparineffekt mittels HepTEM innerhalb von 10 Minuten ausgeschlossen bzw. gesichert werden. Neben der Berücksichtigung der ROTEM-Ergebnisse kommt der Beachtung der Rahmenbedingungen (Hypothermie, Azidose, Hypokalzämie und Anämie) eine entscheidende Rolle zu. Die prophylaktische Gabe eines Fibrinolysehemmers erscheint bei Patienten mit einem ISS $>25$, einer bereits bei Aufnahme stark verminderten Gerinnselfestigkeit (MCF-Ex $<35 \mathrm{~mm}$ ) oder Verlängerung der Coagulation-Time (CT-Ex $>80$ s) gerechtfertigt. Diskussion: Die Rotationsthrombelastometrie erlaubt als POC-Methode eine schnelle und zielgerichtete Gerinnungstherapie. Eine rationale und zielgerichtete Therapie von Blutungen und Gerinnungsstörungen ist aufgrund begrenzter Resourcen an Blutprodukten und hoher Kosten von Gerinnungsfaktorkonzentraten heute mehr denn je von Bedeutung. Schlussfolgerung: Der hier vorgestellte, auf empirischen Daten basierende ROTEM-Algorithmus stellt einen Schritt zur Verbesserung und Rationalisierung des Gerinnungsmanagements bei polytraumatisierten Patienten dar, muss allerdings noch in kontrollierten Studien validiert werden.
Maximalversorung polytraumatisierter Patienten unter DRG-Bedingungen - Eine Falldarstellung unter ökonomischen Gesichtspunkten

M. Blömer, R. Röhrig, V. Mann, M. Meister, S. Jessen ${ }^{1}$, R. Ruwoldt, A. Junger

Klinik für Anästhesiologie und Intensivmedizin und ${ }^{1}$ Medizin-

Controlling des Universitätsklinikum Gießen und Marburg, Standort Gießen

Einleitung: Das DRG-System fasst Fälle gleicher ökonomischer Schwere zusammen, so dass diese in einer Mischkalkulation kostendeckend sind. Ziel dieser Arbeit ist es mit einem Fallbeispiel die Probleme der Mischkalkulation bei polytraumatisierten Patienten darzustellen. Fallschilderung: Ein 18-jähriger PKW Fahrer wurde nach einem Verkehrsunfall polytraumatisiert vom Notarzt intubiert und wegen einer Kreislaufinstablilität in das nächstgelegene Krankenhaus der Regelversorgung gebracht. Die Diagnostik ergab folgende Diagnosen: Aortenbogenruptur, schwere Lungenund Nierenkontusionen, Leber-, Milz-, und Sigmaruptur, Frakturen von LWK 5, Beckenring (instabil), Femur rechts, Unterarm links. Es folgte die sofortige Verlegung in ein Haus der Maximalversorgung und Versorgung mittels Aortenprothese, Milzextirpation, Leber- und Sigmaübernähung, Beckenzwinge, Fixateur extern am rechten Femur und eine Massentransfusion von 71 Erythrozytenkonzentraten. Trotz intensiver Substitution mit Blut- und Gerinnungspräparaten (u.a. Thrombozytenkonzentrate, Fibrinogen, Aktivierter Faktor VII) konnten die diffusen Blutungen nicht gestillt werden. Der Patient verstarb 20 Stunden nach dem Unfall auf der Intensivstation. Methodik: Die Kosten der Verbrauchsmaterialien und des Personalaufwands wurden anhand von Daten aus Patientenakte, Narkose- und OP-Protokolle ermittelt, weitere indirekte Kosten wurden über das Modell der DGU kalkuliert [1]. Ergebnisse: Die Gesamtkosten des Falles betrugen $63667 €$. Davon entfielen unter anderem $24550 €$ auf Personal, 33974,00 $€$ auf Blutprodukte und 5144 auf Materialien. Der Fall erbrachte incl. Zusatzentgelten einen Erlös von 17218,42 €. (DRG W6oZ). Die Deckungslücke betrug 46448,58 €. Schlussfolgerungen: Entgegen der Aussage von Schwermann et al. [2] zeigt sich, dass auch Patienten, die innerhalb der ersten 24 Stunden versterben, nicht kostendeckend behandelt werden können. Wie bereits von anderen Autoren [3] beschrieben, ist die Maximalversorgung schwerstverletzter Patienten mit einem erheblichen betriebswirtschaftlichen Defizit verbunden, da die Abschätzung und Analyse der Varianz der realen Kosten nicht allein durch das Verletzungsmuster, sondern vor allem durch die Behandlungsmöglichkeiten der Klinik verursacht werden. So wäre die Versorgung des vorgestellten $\mathrm{Pa}$ tienten in einem Krankenhaus der Regelversorgung aufgrund beschränkter Behandlungsmöglichkeiten kostengünstiger und somit kostendeckend verlaufen. Es gilt daher Regelungen zu finden, die diese unterschiedliche Strukturqualität adäquat abbilden.

Literatur: [1] Pape et al: Unfallchirurg 2003; 106:348-357. [2] Schwermann et al: Unfallchirurg 2004; 107:563-574. [3] Grotz et al: Unfallchirurg 2004.107:68-76

Der Einfluss des Versorgungszeitpunktes thorakaler 195 und lumbaler Wirbelsäulenverletzungen auf den klinischen Verlauf - eine retrospektive Analyse

C. Schinkel, T. Frangen, S. Ruppert, G. Muhr

Chirurgische Klinik, BG Kliniken Bergmannsheil, Ruhr-Universität Bochum

Fragestellung: Der optimale Versorgungszeitpunkt von mehrfach verletzten Patienten mit thorakalen/lumbalen Wirbelsäulenverletzungen wird kontrovers diskutiert. Insbesondere bei Frakturen der BWS ist das begleitende Thoraxtrauma zu beachten. Um Informationen über den Einfluss des Versorgungszeitpunktes auf den klinischen Verlauf zu erhalten, untersuchten wir retrospektiv ein Kollektiv von mehrfach verletzten Patienten mit operierten Wirbelsäulenfrakturen. Material und Methode: 199 Patienten wur- 
den im Untersuchungszeitraum 01/2000-09/2003 an Frakturen der BWS/LWS operativ stabilisiert, 160 konnten retrospektiv analysiert werden. Es handelte sich dabei um mehrfach verletzte Patienten, der mittlere ISS lag bei 19 Punkten (range 9-66), der SOFA-Score betrug zum Aufnahmezeitpunkt im Median 6, Aufnahme- $\mathrm{Hb}$ 13,0 mg/dl (range 7,0-17,0 mg/dl). Der Altersdurchschnitt des Patientenkollektivs betrug 39 Jahre (range 18-82 Jahre). 57 Patienten (36\%) wurden innerhalb von $72 \mathrm{~h}$ nach Trauma operiert, $103 \mathrm{~Pa}$ tienten $(64 \%)$ wurden nach $72 \mathrm{~h}$ operativ stabilisiert. 154 Patienten $(96 \%)$ wurden einseitig von dorsal instrumentiert. Ergebnisse: Die mittlere OP-Zeit betrug in der früh versorgten Gruppe (Gruppe I, $<72 \mathrm{~h}$ ) $78 \pm 22 \mathrm{~min}$, in der spät versorgten Gruppe (Gruppe II, $>72 \mathrm{~h}$ ) $139 \pm 52 \mathrm{~min}$, der intraoperative Blutverlust in Gruppe I lag bei $0,6 \pm 1,51$, in Gruppe II bei $0,4 \pm 0,9$ l. Der Horowitz-Quotient differierte insbesondere in der Gruppe I nicht signifikant zwischen prä-, intra- und postoperativen Werten. Die mediane Verweildauer der früh operierten Patienten auf der Intensivstation betrug $8 \mathrm{~d}$, bei den später versorgten Patienten $6 \mathrm{~d}$. Die mittlere Gesamtliegezeit der früh versorgten Patienten lag bei $73 \mathrm{~d}$, Patienten mit einer Versorgung nach $72 \mathrm{~h}$ waren im Mittel $110 \mathrm{~d}$ im Krankenhaus. Die Letalität im Gesamtkollektiv betrug 4\% (6 Patienten). Schlussfolgerung: Auch wenn die retrospektive Analyse einen nicht unerheblichen Bias bei der Auswahl des Versorgungszeitpunktes beinhaltet, scheint die frühe Versorgung von thorakalen und lumbalen Wirbelsäulenverletzungen trotz des begleitenden Thoraxtraumas ohne signifikante perioperative Verschlechterung der Lungenfunktion möglich $\mathrm{zu}$ sein. Des Weiteren sind Beatmungszeiten und Liegedauern bei früh versorgten Patienten deutlich verkürzt. Insbesondere schwerer Verletzte scheinen besonders von einer Wirbelsäulenstabilisierung innerhalb von 72 Stunden nach Trauma zu profitieren.

Der Tryptophan Stoffwechsel bei der Entstehung posttraumatischer SIRS: Indikator und therapeutisches Ziel?

M. Scholz, J. Reipen, P. Weidle, W. Linhart, M. Schädel-Höpfner, J. Windolf

Heinrich-Heine Universität

Prädiktive Marker zur posttraumatischen Entstehung von SIRS und Sepsis sowie spezifische präventive Maßnahmen existieren derzeit nicht. Tryptophan und dessen Abbauprodukte könnten eine Schlüsselrolle bei der Steuerung immunologischer Prozesse nach einem Polytrauma spielen. Die durch das Enzym Indoleamin 2,3-Dioxygenase (IDO) entstehenden Abbauprodukte von Tryptophan, z. B. Kynurenin, können zusätzlich direkte Organschädigungen bewirken. Daher sollte die Rolle von IDO, Tryptophan, Kynurenin im Rahmen der posttraumatischen SIRS-Entstehung unter Berücksichtigung der Neutrophilen-Aktivierung (freie Neutrophilen-DNA) untersucht werden. Bei Polytrauma Patienten (ISS $>16$ $\mathrm{n}=10$ ) wurden sequentiell Blutproben über einen Zeitraum von Tag 1 (Schockraum) bis Tag 10 oder länger auf den Gehalt an Tryptophan und Kynurenin mittels HPLC bestimmt. Zur Messung der IDO mRNA in neutrophilen Granulozyten und Lymphozyten wurden die Leukozyten über eine Dichtegradientenzentrifugation getrennt und für die quantitative PCR in Trizol eingefroren. Die erhaltenen Messdaten werden mit den klinischen Daten aus der DGU Polytrauma-Datenbank korreliert. Bei allen Polytrauma Patienten wurde initial nach Einlieferung in den Schockraum zum Normbereich (60-90 micromol/l) verminderte Tryptophan-Konzentrationen (20-50 micromol/l) im Serum gemessen. Am 4.-8. Tag nach dem Trauma stiegen die Tryptophan-Konzentrationen sowie die Kynurenin-Konzentrationen um bis zu 700\% bzw. $400 \%$ an. Die quantitative Bestimmung der IDO mRNA in Lymphozyten und Neutrophilen wird bis zur DIVI Tagung abgeschlossen sein. Die Erhöhung der Tryptophan- und Kynurenin-Konzentrationen sowie die Menge freier DNA im Serum scheint mit einer klinischen Verschlechterung der Patienten zu korrelieren. Unsere Vermutung, dass aktivierte Neutrophile neben der Freisetzung von
DNA verstärkt IDO freisetzen, welches über die Spaltung von Tryptophan wesentliche inflammatorische Prozesse steuert, wird derzeit untersucht.

Intermittant infrarenal endoclamping improves renal

blood flow - an experimantal pig study

A.M. Scheule, T. Walker, M. Mayer, B. Neumann, H.P. Wendel, G. Ziemer

Thorax, Herz- und Gefäßchirurgie, Universitätsklinikum Tübingen

Objectives: Circulatory instability after polytrauma often requires drug therapy at the expense of reduced perfusion of the arterial vessels especially of the abdominal organs. We tested if temporary balloon occlusion of the infrarenal aorta allows instant and reversible increase of blood pressure and/or perfusion in the renal and suprarenal arterial system. Methods: In 7 German landrace pigs $(73 \pm 6 \mathrm{~kg})$ flow probes were fitted around the left renal artery and at the decending aorta. A balloon sheath was placed via the femoral artery into the infrarenal aorta. A period of 20 minutes of infrarenal endoclamping was followed by 10 minutes of unclamped reperfusion. This cycle was repeated four times. The renal and aortic flow as well as the suprarenal arterial pressure were monitored online. Parameters were compared with starting measurements. Results: The flow in the left renal artery increased cascade like (1. clamping $129 \%$ first reperfusion $108 \%$; 2.144 to $128 \%$; 3 . 151 to $138 \%$; 4 . 156 to $152 \%$ ). The suprarenal mean arterial pressure rose after clamping and dropped after declamping to the initial level (1. clamping $130 \%$ first reperfusion $100 \% ; 2.137$ to $100 \%$; 3.139 to $109 \%$; 4 . 146 to $109 \%$ ). The flow in the descending aorta slightly increased (1. clamping $81 \%$ first reperfusion $114 \%$; 4.93 to $115 \%)$. Conclusions: This study could demonstrate a cascade like increase of the renal blood flow and reproducible increase of blood pressure after a cycle of clamping and declamping of the infrarenal aorta. This balloon sheath could be a tool in therapy of patients with circulatory instability after polytrauma and might reduce renal insufficiency in these patients.

Zuverlässige Daten für fundierte Prävention: Die EU Injury Database

T. Krafft ${ }^{1}$, A. Kortevo $\beta^{1}$, A. Siefer ${ }^{2}$, C. Butsch ${ }^{1}$

${ }^{1}$ Geographisches Institut der Universität zu Köln; ${ }^{2}$ Bundesamt für Arbeitsschutz und Arbeitsmedizin

Hintergrund: Die Europäische Union baut im Kontext der europäischen Gesundheitsberichtserstattung (GBE) seit 1986 ein überwiegend krankenhausbasiertes Berichtsystem für Erkrankungen aus dem Bereich der äußeren Ursachen, die „Injury Database IDB“, auf. Eine flächendeckende Implementierung des Systems konnte bislang aus mehreren Gründen nicht erreicht werden. So erscheint die Auswahl der beteiligten Institutionen zufällig und wenig repräsentativ, darüber hinaus bereiligen sich mit Großbritannien und Deutschland zwei der bevölkerungsreichsten EU-Mitgliedsstaaten nicht an diesem System. Die IDB kann in der jetzigen Form daher nur einen eingeschränkten Beitrag zur GBE und zur Entwicklung von Präventionsstrategien leisten. Ziel: Vor dem Hintergrund der Struktur der medizinischen Versorgung in der Bundesrepublik Deutschland wird untersucht, welche Datenquellen dazu geeignet sind, die für die IDB relevanten Informationen $\mathrm{zu}$ generieren. Es wird analysiert, welche Teilbereiche des Verletzungsgeschehens sich kontinuierlich aus Routinedaten abbilden lassen und ob insb. standardisierte Daten der präklinischen Notfallversorgung eine sinnvolle Ergänzung des bisher ausschließlich krankenhausbasierten Erhebungsansatzes darstellen können. Methoden: In einer Pilotstudie wird das Verletzungsgeschehen exemplarisch für einen Landkreis in Baden-Württemberg anhand von Krankenhausdaten und Notfalldaten abgebildet. Ein Schwerpunkt liegt hierbei auf dem Vergleich der räumlichen Muster der Inzidenzraten, die sich den unterschiedlichen Grundgesamtheiten er- 
geben. Darauf aufbauend wird versucht, den Abbildungsumfang und die Abbildungsqualität der einzelnen Datenquellen für das Verletzungsgeschehen innerhalb des definierten Gebietes zu quantifizieren. Ergebnisse: In den Krankenhausdaten werden insgesamt deutlich mehr Verletzungsereignisse erfasst als in den Daten der präklinischen Versorgung. Jedoch bilden die Krankenhausdaten im Gegensatz zu den Daten der präklinischen Versorgung in räumlicher Hinsicht das Verletzungsgeschehen nur für Teilbereiche des Kreisgebietes ab. Die im Krankenhaus erfassten Fallzahlen nehmen mit zunehmender Distanz zum Krankenhausstandort signifikant ab. Diskussion: Die Distanzüberwindung stellt eine räumliche Zugangsbarriere für die Behandlung im Krankenhaus dar, die bei der Notfallversorgung nicht vorhanden ist. Die Daten der präklinischen Versorgung bilden insgesamt einen kleineren Ausschnitt des Verletzungsgeschehens ab, in räumlicher Hinsicht sind diese Daten hingegen vollständiger. Zusätzlich kann festgehalten werden, dass sich standardisierte Notfallprotokolle (basierend auf den Vorgaben der DIVI) für die Erfassung der für die IDB relevanten Variablen besser eignen als die Krankenhausdaten Schlussfolgerungen: Die IDB sollte um weitere Datenquellen ergänzt werden. Insbesondere die Einbeziehung präklinischer Versorgungdaten bietet die Chance, ein zuverlässigeres Bild der gesellschaftlichen Belastungen durch Verletzungen zu erhalten.

\section{Neurologische und Neurochirurgische Intensivmedizin}

\section{Experimentelle Herpes-simplex-Virus-Enzephalitis: Expressionsmuster von Aquaporin-4, Alpha-Syntrophin und KIR 4.1 unter verschiedenen Therapiestrategien \\ F.-J. Martinez-Torres, N. Dörner, S. Nielsen, A. Krick, U. Meyding-Lamadé \\ Neurologische Klinik, Universitätsklinikum Heidelberg}

Trotz frühzeitiger antiviraler Therapie ist die Herpes-Simplex-Virus-Enzephalitis (HSVE) weiterhin mit hoher Mortalität und Morbidität verbunden. Aciclovir hemmt die Vermehrung des HerpesSimplex-Virus-1 (HSV-1), neueste Untersuchungen deuten aber auf sekundäre pathologische Mechanismen hin, die die Langzeitbeeinträchtigungen möglicherweise mitverantworten. AQP4 ist ein wichtiges Wasser-Transport-Protein im Hirngewebe. Eine Schlüsselrolle in der Modulation der Bluthirnschrankenpermeabilität wird für AQP4 im Zusammenhang mit anderen TransportMolekülen wie KIR 4.1 und strukturellen Ankerproteinen wie $a$ Syntrophin ( $a$-Syn), vermutet. Ein verändertes Expressionsmuster, oder ein verändertes Verteilungsmuster von AQP4 und KIR 4.1 an der Zellmembran der Astrozyten könnte zur Entstehung eines zytotoxischen Ödems beitragen. Ziel unserer Arbeit war, im Langzeitverlauf der experimentellen HSVE mit verschiedenen Therapieansätzen die mRNA Expression von AQP4, KIR 4.1 und $a$-Syn mit Real-time-PCR in-vivo zu untersuchen. Wir haben 39 Mäuse untersucht, davon wurden 33 mit je $2 \times 10^{5}$ plaque forming units (pfu) HSV-1 intranasal inokuliert und in 4 Gruppen eingeteilt: untherapierte Tiere; Aciclovir behandelte Tiere; Prednisolon behandelte Tiere und Kombinationstherapiegruppe (Aciclovir + Prednisolon). Die virale Transkriptionsaktivität wurde parallel mittels Nested-PCR gegen das virale Glykoprotein-D untersucht. Sechs Monate nach Inokulation wurde aus den Gehirnen der einzelnen Gruppen mRNA isoliert und die Expression der jeweiligen Gene normiert gegen GAPDH untersucht. Die statistische Analyse erfolgte mit ANOVA und Bonferroni post-hoc. Die Ergebnisse zeigten eine signifikante Erhöhung von AQP-4-(2,3fach), $a$-Syn(2,9fach), und KIR 4.1-(1,8fach) mRNA Expression jeweils in der Gruppe der untherapierten Tiere gegenüber den nicht infizierten Kontrolltieren. Interessanterweise wurde die Regulation von $a$-Syn und KIR 4.1 in der Kombinationstherapiegruppe (Aciclovir +
Prednisolon) auf dem Niveau der Kontrolltiere gehalten, nicht jedoch in den beiden Monotherapiegruppen. Bei der Expression von AQP4 innerhalb der Gruppen erkrankter Tiere ließen sich keine signifikanten Unterschiede feststellen, wohl aber eine Tendenz der supprimierten Expression unter kombinierter Aciclovir + Prednisolon Therapie. Diese Arbeit zeigt, dass das Expressionsmuster von AQP4, dem Strukturprotein $a$-Syntrophin, sowie dem Kaliumkanal KIR 4.1 von einer kombinierten Therapiestrategie mit Aciclovir und Prednisolon beeinflusst wird. Die pharmakologische Beeinflussung der AQP4-Expression könnte eine Therapieoption bei dem in der HSVE auftretenden Hirnödem darstellen.

GACHE: German Trial of Acyclovir and Corticosteroids 103 in Herpes-simplex-Encephalitis: Studienprotokoll

U. Meyding-Lamadé, F. Martinez-Torres, M. Pritsch, S. Luntz, E. Jenetzky, N. Dörner, P. Beck, N. Victor, W. Hacke Neurologische Klinik, Universitätsklinikum Heidelberg

GACHE ist eine multizentrische, doppelblinde, randomisierte und prospektive Studie zur Beurteilung des Effektes von adjuvantem Dexamethason bei Patienten mit Herpesenzephalitis. Die Herpesenzephalitis ist eine lebensbedrohliche Hirnentzündung, die durch das Herpesvirus verursacht wird. Trotz der gegenwärtigen Behandlung mit Aciclovir sterben noch immer rund 20\% der $\mathrm{Pa}$ tienten. Von den Überlebenden ist nur jeder fünfte in der Lage, in seinen früheren Beruf zurückzukehren. Neben direkt virusvermittelten Gewebeschäden spielen möglicherweise auch sekundäre Autoimmunmechanismen eine Rolle für die Schwere dieser lebensbedrohlichen Hirnentzündung. Dexamethason könnte diese Autoimmunphänomene unterdrücken und ist dadurch wirksam in der Behandlung der Herpesenzephalitis. Da es hierzu bisher keine prospektiven Studien gibt, kann dies erst abschließend nach einer multizentrischen Studie beantwortet werden. Hauptfragestellung: Effekt von adjuvantem Dexamethason auf Folgeschäden bei Patienten mit Herpesenzephalitis. Hypothese: Unter einer kombinierten Therapie mit Acyclovir und Dexamethason ist die Rate von Patienten mit schlechtem Outcome geringer als unter alleiniger Therapie mit Aciclovir. Der primäre Endpunkt ist ein dichotomes Outcome: Modified Rankin Scale (mRS) nach 6 Monaten. Ein mRS von 3 bis 6 wird als schlechtes Outcome betrachtet. Sekundäre Endpunkte: Mortalität nach 6 Monaten, Mortalität nach 12 Monaten, Funktionelles Outcome (Glasgow Outcome Scale $=$ GOS und EuroQol 5D) nach 6 Monaten, Funktionelles Outcome nach 12 Monaten (mRS, GOS, EuroQol 5D), Neuropsychologische Testungen nach 6 Monaten, Befunde im kranialen MRT nach 6 Monaten. Die teilnehmenden Studienzentren sind 33 Neurologische Abteilungen von Universitätskliniken und großen städtischen Häusern in Deutschland, Österreich und Holland. Die bisher teilnehmenden Kliniken werden namentlich während der Präsentation veröffentlicht, wobei eine Initiierung neuer Zentren - falls sie die Voraussetzungen erfüllen - jederzeit möglich ist. GACHE ist ein „Investigator Initiated Trial (IIT)“ und wird vom Projektträger des DLR (Deutsches Zentrum für Luft- und Raumfahrt e.V.) und vom Bundesministerium für Bildung und Forschung (BMBF) gefördert. Das DLR-BMBF Förderkennzeichen lautet 01KG0504. EudraCT Nummer: 2005-003201-81.

Angioneurotisches Ödem nach systemischer Thrombolyse des Hirninfarkts mit Alteplase (rt-PA)

W. Dietrich, M. Röbke, I. Bär, F.J. Erbguth

Neurologische Klinik, Klinikum Nürnberg

Während intrakranielle Blutungen mit einer Rate von etwa $6 \% \mathrm{zu}$ den definierten Risiken der systemischen Thrombolyse mit Actily$\mathrm{se}^{\circledR}$ (Alteplase; rt-PA) zählen, sind anaphylaktoide Reaktionen oder Angioödeme selten. Ein Alteplase-assoziiertes Angioödem tritt beim Myokardinfarkt in $<0,02 \%$, beim Hirninfarkt in $1,9 \%$ auf. Im Vergleich dazu sind Angioödeme unter ACE-Hemmertherapie seltener $(0,2 \%)$. Eine 76 -jährige Frau wurde mit einer hoch- 
gradigen Hemiparese links, Blickwendung nach rechts, Somnolenz und Dysarthrie aufgenommen. Der NIHSS Score betrug 18. Aufgrund eines langjährigen Hypertonus bestand eine Therapie mit Quinalapril, allergische Reaktionen waren in der Vorgeschichte nicht aufgetreten. Nach Ausschluss einer intrakraniellen Blutung erfolgte innerhalb des Zeitfensters nach 130 Minuten eine systemische Thrombolyse mit $70 \mathrm{mg}$ Alteplase, eine Begleitmedikation wurde nicht appliziert. 40 Minuten nach Infusionsende entwickelte die Patientin eine symmetrische Zungenschwellung ohne Lippenbeteiligung oder systemische allergische Symptome. Sie wurde sofort mit Adrenalin und Methylprednisolon i.v. behandelt, musste jedoch aufgrund einer zunehmenden Schwellung von Epi-/Hypopharynx und Larynx fiberoptisch intubiert werden. Das CT der Halsweichteile zeigte eine rechtsbetontes Ödem von Epi-, Oround Hypopharynx und Larynx, aber nicht, wie zunächst differentialdiagnostisch vermutet, eine lokale Einblutung. Aktivität und Konzentration des C1-Esterase-Inhibitors waren ebenso wie die Komplementfaktoren $\mathrm{C} 3$ und $\mathrm{C} 4$ normal. Im Verlauf demarkierte sich ein großer rechtsseitiger Mediateilinfarkt. Als Hirninfarktursache wurde bei intermittierendem Vorhofflimmern eine kardiogene Embolie angenommen. Das Angioödem bildete sich unter antihistaminerger und antiödematöser Therapie nur langsam zurück, so dass eine Tracheotomie und längerfristige Beatmung notwendig war. Eine nicht-hereditäres angioneurotisches Ödem nach Thrombolysetherapie des akuten Hirninfarktes mit rt-PA ist mehrfach kasuistisch beschrieben, meist mild und kontralateral zur Infarktseite. Durch Aktivierung von Plasminogen zu Fibrin abbauendem Plasmin wirkt rt-PA thrombolytisch und Ischämie limitierend. Gleichzeitig wird durch Plasmin 1) Kininogen zu Bradykinin umgewandelt oder 2) das Komplementsystem aktiviert und so bei entsprechender Disposition ein Angioödem verursacht. Eine vorbestehende ACE-Hemmer-Therapie kann dabei durch simultane Hemmung des Bradykininabbaus begünstigend wirken (Hemmung der Kininase II $=$ ACE und gleichzeitige Aktivierung der Kininase I als minor pathway mit nur unter ACE-Inhibition relevanter Bildung des aktiven Metaboliten des-Arg-Bradykinin). Das Risiko für die Angioödementstehung ist bei Einnahme eines ACE-Hemmers ( $R R=$ relatives Risiko 13,6$)$ und bei Ischämiefrühzeichen im CCT im frontalen oder insulären Kortex (RR 9,1) erhöht. Eine routinemäßige Inspektion der Zunge und des Rachenraumes zur Erfassung dieser seltenen Lysekomplikation sollte 30 bis 45 Minuten nach Lysetherapie erfolgen.

Protrahiertes Koma nach einmalig niedrig dosierter Haloperidolgabe - eine Kasuistik

\section{Stövesand, A. Schumacher, D.A. Vagts}

Universität Rostock, Klinik und Poliklinik für Anästhesiologie und Intensivtherapie

Ca. 5-10\% der Krankenhauseinweisungen sind auf unerwünschte Arzneimittelwirkungen zurückzuführen. Umso wichtiger ist die Kenntnis der pharmakologischen Eigenschaften von Medikamenten unter Berücksichtigung der pharmakokinetischen und -dynamischen Beziehungen, um das Risiko unerwünschter Reaktionen zu minimieren. Für Haloperidol als Vertreter der hochpotenten Neuroleptika vom Butyrophenontyp ist eine Gesamthäufigkeit der Nebenwirkungen mit $89 \%$ beschrieben. Hier ist mit $17 \%$ insbesondere der Parkinsonismus zu nennen. Wir berichten über eine seltene komatöse Bewusstseinsstörung einer 64-jährigen $\mathrm{Pa}$ tientin nach einmaliger Haloperidolgabe. Kasuistik: Die Patientin $\left(64 \mathrm{y}, 110 \mathrm{~kg}\right.$, BMI $40,4 \mathrm{~kg} / \mathrm{m}^{2}$, ASA 3) wurde nach thorakaler Stentgraft-Implantation aufgrund einer thorakoabdominalen Aortendissektion postinterventionell intubiert und beatmet auf unsere Intensivstation übernommen. Aus der Eigenanamnese der adipösen Patientin sind neben einem arteriellen Hypertonus eine intermittierende absolute Arrhythmie bei Vorhofflimmern bekannt. Auf unserer Station entwickelte die bereits extubierte Patientin ein postoperatives Delir, das durch Desorientiertheit, Aggressivität und optische Halluzinationen gekennzeichnet war. Daraufhin ver- abreichten wir einmalig(!) 2,5 mg Haloperidol i.v. Zwei Stunden nach der Neuroleptikagabe war die Patientin komatös (GCS 3) und musste reintubiert werden. Morphologische Ursachen konnten im CCT und CT-Thorax ausgeschlossen werden. Auffällig war jedoch ein noch $72 \mathrm{~h}$ nach Neuroleptikagabe im unteren therapeutischen Bereich liegender Haloperidolserumspiegel, woraus sich die Diagnose einer haloperidolbedingten Bewusstseinsstörung ergab. Typische Symptome eines malignen neuroleptischen Syndroms fehlten. Probatorisch erhielt die Patientin Biperiden (anticholinerg wirkendes Antiparkinsonmittel). Unmittelbar danach war sie wach und bewegte alle Extremitäten spontan. Trotz mehrerer folgender Biperidengaben trübte die Patientin erneut ein (GCS 4). Im weiteren Verlauf klarte die Patientin langsam auf, so dass sie $99 \mathrm{~h}$ nach Haloperidolgabe extubiert werden konnte. Im Anschluss war sie neurologisch und psychiatrisch unauffällig und konnte am 9. Behandlungstag in die kardiologische Klinik verlegt werden. Schlussfolgerung: Bisher sind für Haloperidol vor allem extrapyramidale Nebenwirkungen, wie der Parkinsonismus bekannt. Sedierende Effekte sind nur sehr selten beschrieben und treten vorrangig bei der Therapie mit niedrigpotenten Neuroleptika auf. Unsere Kasuistik zeigt, dass nach nur einmaliger Haloperidolgabe schwerste Nebenwirkungen mit Akinesie und Koma auftreten können. Die kurzzeitig erfolgreiche Gabe von Biperiden lässt einen Zusammenhang mit dem bekannten beschriebenen Parkinsonismus nach Neuroleptikagabe vermuten. Der protrahierte Verlauf steht im Zusammenhang mit dem noch nach 3 Tagen vorhandenen therapeutischen Haloperidolserumspiegel. Pharmakokinetische Beziehungen müssen hierfür verantwortlich gemacht werden.

Erweitertes Neuromonitoring - Möglichkeiten der Darstellung CPP-unabhängiger Veränderungen der regionalen cerebralen Perfusion im Tierexperiment und bei Patienten mit neuronaler Schädigung

J. Soukup, C. Holz, L. Sanchin, I. Bramsiepe, N. Nemeth, I. Miko, M. Menzel

Klinik für Anästhesiologie und Operative Intensivmedizin und Universitätsklinik für Neurochirurgie der MLU Halle, Dept. Surgical Research, Debrecen, Klinik für Anästhesie, Intensiv- und Rettungsmedizin, Städtisches Klinikum Wolfsburg

Mit der Überwachung des cerebralen Perfusionsdruckes (CPP) ist es möglich, das cerebrale Ischämierisiko zu vermindern und über den Mechanismus der cerebrovaskulären Autoregulation den ICP günstig zu beeinflussen. Limitiert wird das Konzept durch die fehlende Möglichkeit, den Einfluss CPP-unabhängiger Parameter auf Veränderungen der cerebralen Perfusion, z. B. unter den Bedingungen einer kontrollierten Hyperventilation, abzuschätzen. Ziel dieser Analyse war die Überprüfung der Veränderungen des regionalen Blutflusses (rCBF) und der Hirngewebeoxygenierung $\left(\mathrm{ptiO}_{2}\right)$ während kontrollierter Hyperventilation im nicht geschädigtem Gehirn (Tierversuch) und bei Patienten mit neuronaler Schädigung. Methode: Bei insgesamt 9 Schweinen (S) und 10 neurochirurgischen Patienten $(P)$ wurde ein erweitertes Neuromonitoring durchgeführt. Dieses beinhaltete eine kombinierte ICPTemperaturmessung Neurovent $\mathrm{PT}^{\circledR}, \mathrm{Fa}$. Rehau), eine miniaturisierte Multiparametersonde zur Messung der Hirngewebeoxygenierung $\left(\mathrm{ptiO}_{2}\right)$, des Hirngewebe- $\mathrm{CO}_{2}\left(\mathrm{ptiCO}_{2}\right)$ sowie des Hirngewebe-pH (pHti), und eine Thermodiffusionssonde zur Messung des regionalen CBF ( $\mathrm{rCBF}$ ). Nach einer 2-stündigen Stabilisierungsphase erfolgte zunächst die Normoventilation und im Verlauf unter Kontrolle des endexpiratorischen $\mathrm{CO}_{2}$ eine kurzzeitige Hyperventilation bis entweder der rCBF oder der $\mathrm{ptiCO}_{2}$ deutliche Veränderungen zeigten. Ergebnisse: Im Tierexperiment konnte durch Hyperventilation von $\mathrm{paCO}_{2} \quad 45,0 \pm 2,3 \mathrm{mmHg}$ auf $24,0 \pm 2,3 \mathrm{mmHg}$ eine signifikante Reduktion des $\mathrm{rCBF}$ von $33 \pm 2,6 \mathrm{ml} / 100 \mathrm{~g} / \mathrm{min}$ auf $21 \pm 3,2 \mathrm{ml} / 100 \mathrm{~g} / \mathrm{min} \quad(\mathrm{p}<0,0001)$ als auch des $\mathrm{ptiO}_{2}$ von $20,3 \pm 7,5 \mathrm{mmHg}$ auf $10,5 \pm 4,5 \mathrm{mmHg}$ (p $<0,0001)$ erreicht werden. Bei neurochirurgischen Patienten führte 
die kontrollierter Hyperventilation $\left(\mathrm{paCO}_{2} \quad 37,5 \pm 3,8 \mathrm{mmHg}\right.$ auf $29,3 \pm 4,5 \mathrm{mmHg}$ ) zu einer deutliche Reduktion des rCBF von $34 \pm 4 \mathrm{ml} / 100 \mathrm{~g} / \mathrm{min}$ auf $20 \pm 6 \mathrm{ml} / 100 \mathrm{~g} / \mathrm{min}(\mathrm{p}<0,0001)$. Entsprechend den theoretischen Erwartungen reduzierte sich darunter auch der $\mathrm{ptiO}_{2}$ von $33 \pm 13 \mathrm{mmHg}$ auf $11 \pm 7 \mathrm{mmHg}(\mathrm{p}<0,001)$. Schlussfolgerung: Sowohl im Tierexperiment als auch bei neurochirurgischen Patienten konnte mittels erweitertem Neuromonitoring individuelle $\mathrm{CPP}$-unabhängige Veränderungen der cerebralen Perfusion und Oxygenierung sicher bestimmt werden. Nach kontrollierter Hyperventilation wurde für den $\mathrm{rCBF}$ ein zugehöriger unterer Schwellenwert bei ca. $20 \mathrm{ml} / 100 \mathrm{~g} / \mathrm{min}$ und für den ptiO bei ca. $20 \mathrm{mmHg}(\mathrm{T})$ bzw. $11 \mathrm{mmHg}(\mathrm{P})$ festgestellt. Bei Vorhandensein einer neuronalen Schädigung ist das Ausmaß der $\mathrm{CO}_{2}$-Reduktion zum Erreichen dieser Veränderungen deutlich geringer.

Epilepsiechirurgische Eingriffe im Kleinkindesalter 091 Anaesthesiologisches Management

J. Sommer ${ }^{1}$, U. Brandt ${ }^{1}$, H. Pannek ${ }^{2}$, F. Mertzlufft ${ }^{1}$, F. Bach ${ }^{1}$ ${ }^{1}$ Klinik für Anästhesiologie und operative Intensivmedizin, ${ }^{2}$ Klinik für Neurochirurgie; Evangelisches Krankenhaus Bielefeld, Bielefeld-Bethel

Fragestellung: Trotz großer Fortschritte der pharmakologischen Epilepsietherapie, gibt es im Kleinkindesalter therapierefraktäre Verläufe mit langfristig schlechtem neuropsychologischem Ergebnis. Aufgrund guter Erfolge epilepsiechirurgischer (E-Chir) Eingriffe bei Erwachsenen, wird das Indikationsspektrum zur E-Chir auch auf Kleinkinder ausgedehnt [1]. Demgegenüber bedeutet solch komplexer und mit erheblichen Volumenverschiebungen einhergehender Eingriff bei den oft mit Komorbiditäten behafteten Kindern ein nicht unerhebliches perioperatives Risiko, das ein besonderes anästhesiologisches Management verlangt. Die retrospektive Erhebung soll spezifische perioperative Risiken dieser Eingriffe herausarbeiten und das klinikeigene Protokoll diskutieren. Methoden: Es wurden im Zeitraum von 01/2001 bis $05 / 2004$ 53 Säuglinge und Kleinkinder (0-5 Jahre; mittleres Gewicht $13,15 \mathrm{~kg}$, max: $30,0 \mathrm{~kg}$, min: 5,1 kg) E-Chir operiert. Die Kinder wurden nach Narkoseeinleitung $(5 \mathrm{mg} / \mathrm{kg}$ Trapanal, $2-4 \mu \mathrm{g} / \mathrm{kg}$ Fentanyl, 0,1 mg/kg Pankuronium) instrumentiert (art. Kanüle, 2-Lumen-ZVK, Blasenkatheter, Magensonde). Die Narkose wurde als balancierte Anästhesie mit Isofluran und Fentanyl-Bolusgaben bedarfsadaptiert aufrechterhalten. Evaluiert wurden Indikationsstellung, anästhesiologisches Management sowie der postoperative Verlauf. Ergebnisse: Bei allen Kindern lagen konservativ nicht beherrschbare Epilepsieverläufe vor. Der intraoperative Transfusionsbedarf war mit 52,4 $\pm 45,0 \mathrm{ml} / \mathrm{kg}$ Erythrozytenkonzentrat (EK) und $38,45 \pm 37,0 \mathrm{ml} / \mathrm{kg}$ Plasma (FFP) sehr hoch, der kristalloide Volumenersatz betrug intraoperativ $16,59 \pm 5,92 \mathrm{ml} / \mathrm{kg} / \mathrm{h}$. Die OpZeit lag bei $6,25 \pm 1,20 \mathrm{~h}$, die Kinder wurden auf der Intensivstation $4,14 \mathrm{~h} \pm 3,18$ beatmet und nach $1 \pm 1,01$ Tag verlegt. Die mittlere Krankenhausverweildauer betrug 15 Tage $\pm 3,53$, ohne dass im Beobachtungszeitraum ein Todesfall auftrat. Dargestellt sind jeweils $M W \pm S D$. Schlussfolgerung: Die Daten verdeutlichen den sehr hohen intraoperativen Blutverlust. Zur Stabilisierung von Hämodynamik und Hämostase ist eine differenzierte Substitution von EK, FFP und Volumenersatz, ggf. ergänzt durch die Gabe von spezifischen Gerinnungsfaktoren, zwingend. Bei intraoperativer Hirnschwellung wird eine Osmotherapie (Mannitol 20\%) mit begleitender milder Hyperventilation $\left(\mathrm{paCO}_{2}: 30-35 \mathrm{mmHg}\right.$ ) durchgeführt. Unsere Ergebnisse zeigen, dass die komplexen und nicht risikoarmen E-Chir-Eingriffe im Kleinkindesalter bei strenger Indikationsstellung, enger Kooperation zwischen Chirurg, Anaesthesist und Intensivmediziner sowie fundierter Erfahrung des gesamten Teams bei sonst refraktärer juveniler Epilepsie erfolgreich durchzuführen sind.

Literatur: [1] Brodie M J et al: Epilepsia 1997; 38:1245-1250
Assessment of neuroendocrine parameters in the acute phase of aneurysmal subarachnoid hemorrhagen (SAH) - first prospective data

A. Boström, U. Bürgel, P. Reinacher, J.M. Gilsbach, J. Brockmann, I. Kreitschmann-Andermahr

Neurochirurgische Klinik, Universitätsklinikum der RWTH Aachen

Introduction: Anterior pituitary hormone abnormalities have been reported in up to $55 \%$ of aneurysmal SAH survivors investigated between 3 months up to several years after the acute event. The frequency and extent of pituitary dysfunction in the acute phase of aneurysmal SAH is, however, unknown. Objectives: The aim of this study is to evaluate the prevalence of anterior and posterior pituitary dysfunction within the first 3 weeks of SAH (t0-2). So far, the parameters of the first 21 consecutive patients who were admitted to the neurosurgical unit were studied in the immediate post injury phase Methods: In this prospective study baseline thyroid function (fT3, fT4), TSH, cortisol, ACTH, IGF-1, FSH, LH, testosterone or estradiol, prolactin, plasma and urine osmolalities were assessed within 24-hours (t0) (before surgical or endovascular treatment) and the neuroendocrine parameters again after 7 (t 1) and 14-21 (t2) days. An ACTH-test was performed in all patients before discharge in order to rule out primary adrenal insufficiency as a consequence of the critical illness itself. Results: Within the first 24 hours after SAH in all patients thyroid function was normal, but low T3/fT4 values were observed within the following 2 weeks in 10/21 patients. TSH was decreased in 2 patients at $\mathrm{t} 1$ and $\mathrm{t} 2$. Abnormally low IGF- 1 values were initially seen in 6 patients, returned to normal in 3 and was increased in 3 patients at $\mathrm{t} 2$. Abnormally low cortisol (below $170 \mathrm{nmol} / \mathrm{l}$ ) was only observed in 1 patient at t 0 and 1 patient at $t 2$, whereas normal to elevated cortisol levels were measured in all other patients at all other time points. The circadian rhythm was, however, disturbed in all but 1 patient throughout the entire investigation period. ACTH levels were increased in 2 patients at $t 0$, in 6 at $t 1$ and 2 at $\mathrm{t} 2$. Gonadotropins and/or sex steroids were decreased in all patients at some point of time during the investigation period. Prolactin levels were increased in 7/21 patients on day one and in $10 / 21$ at $\mathrm{t} 1$ and $\mathrm{t} 2$. Plasma osmolality was decreased in 7 patients at $\mathrm{t} 0,10$ at $\mathrm{t} 1$, and in 6 at $\mathrm{t} 2$. The ACTH-stimulation test demonstrated normal adrenal function in all patients. Conclusion: Our data show that neuroendocrine changes do occur in the acute phase of aneurysmal SAH which resemble hormonal changes which are also seen during other severe and life-threatening illnesses. However, hypocortisolism which would have significant implications for the early course of the disease was not observed in our small cohort.

Equine-related injuries: the neurosurgeons perspective 204 M.F. Oertel ${ }^{1}$, M.H.T. Reinges ${ }^{1}$, F.-J. Hans ${ }^{1}$, J.M. Gilsbach ${ }^{1}$, E. $U h l^{2}$

${ }^{1}$ Department of Neurosurgery, RWTH Aachen University, Aachen

${ }^{2}$ Department of Neurosurgery, LMU University, Munich

Objective: Trauma specialists are frequently faced with the diagnosis and treatment of head and spine injuries in horse-related traumatized patients. The aim of the present study was to evaluate the data and outcome of patients with equine-related neurotrauma (ERNT). Methods: The data of 161 consecutive patients admitted to the University Hospital Aachen with head and spinal injuries due to horse-related accidents between 1993 and 2002 were evaluated retrospectively. 112/161 patients had minor ERNT, whereas 49/161 patients suffered from serious injuries necessitating further neurosurgical treatment. Long-term clinical outcome (180 \pm 187 weeks posttraumatic) was available of all patients. The reference point of the study was the date of accident. The patients were assessed according to the Glasgow Outcome Score (GOS). Results: $112 / 161$ patients presented with minor head or spinal trauma including skull contusion (42/161), cerebral concussion 
(64/161), cervical distorsion (4/161), minor spinal contusion (1/ 161 ), and stable lumbar fracture (1/161). 49/161 patients were admitted with severe ERNT (age $34.2 \pm 18.9$ years). The median Glasgow Coma Score (GCS) on scene and on admission was 13 and 14 , respectively. The majority of injuries were caused by a fall from the horse (40/49). 5/49 patients were kicked by the horse, 3/ 49 patients fell from the horse and got a kick, and 1/49 patient fell from a coach. 38/49 patients suffered from severe head injuries including epidural $(8 / 49)$, subdural $(8 / 49)$, and subarachnoid haematoma (28/49), combined intracranial haemorrhages (7/49), cerebral contusion (17/49), and skull fractures (29/49), requiring operative treatment in $26 / 38$ cases. $11 / 49$ patients had severe spinal injuries including 4 unstable fractures treated by spinal instrumentation. 19/49 patients were managed conservatively. The duration of hospital stay was $10.4 \pm 10.2$ days. $42 / 49$ patients required intensive care therapy (duration 5.4 \pm 9.8 days). 5/49 patients died because of intractable brain swelling. GOS at discharge was 1: 5/49, 2: 0/49, 3: 7/49, 4: 6/49, and 5: $31 / 49$ compared to long-term GOS of 1: 5/49, 2: 0/49, 3: 2/49, 4: 1/49, and 5: 41/49, respectively. Conclusions: $70 \%$ of patients with ERNT admitted to hospital after horse-related accidents have minor ERNT, whereas major ERNT occur in 30\% of patients. $89 \%$ of patients with ERNT suffer head injury, $11 \%$ endure spinal trauma. $65 \%$ of spinal injuries are major and $24 \%$ have to be treated surgically. $26 \%$ of head injuries caused by horse-related accidents are severe and require urgent neurosurgical intervention in $68 \%$ of the patients. From the neurosurgeons view horse back riding is a dangerous sport with a mortality rate of $13 \%$ in patients with severe head injuries. Although in the majority of patients with ERNT a good recovery can be expected, the risk of fatal injury in this kind of sports-related accidents is high.

Kleiner metallischer Fremdkörper Erhebliche Probleme!

A. Faltlhauser ${ }^{1}$, M. Braun ${ }^{1}$, C. Schulz ${ }^{2}$, H. Räkers ${ }^{2}$, U. Wörner ${ }^{2}$, A. Thomas ${ }^{2}$

Bundeswehrzentralkrankenhaus Koblenz

Abt. für Anästhesie ${ }^{1}$, Abt. für Neurochirurgie ${ }^{2}$

Hintergrund: Das Feldlazarett (role 2+) der Deutschen ISAF Truppen in Camp Warehouse Kabul, Afghanistan verfügt nicht über eine neurochirurgische Einrichtung. Leider weisen ein Großteil der Opfer von Minen- und IED (Improvised Explosive Devices)-Explosionen craniofaciale und nicht selten auch intracerebrale Fremdkörpereinsprengungen auf. Dieser Fallbericht soll dieses häufige Problem und eine unkonventionelle Lösung beschreiben. Anamnese: Während eines Massenanfalls von 7 teils schwerstverletzten Patienten stellte sich ein türkischer Zivilmitarbeiter mit einem Kopfverband in der Notaufnahme vor. Er berichtete von einer IED-Explosion, die seinen Fahrer tötete vor etwa 8 h. Eine Bewußtlosigkeit könne er nicht erinnern. Der Patient wurde daher bei GCS 15 in die Gruppe "Grün“ triagiert. Nach ca. 1 h wurde eine konventinelle Schädelröntgenaufnahme in zwei Ebenen gefertigt und der hochgrandige Verdacht auf einen temporooccipital liegenden intracraniellen Fremdkörper gestellt, der sich im unmittelbar darauf durchgeführten CCT erhärtete. Des Weiteren wurde ein kombiniertes subdural/epidurales Hämatom an der Eintrittspforte und ein ausgeprägtes lokales Hirnödem diagnostiziert. Problem: Einerseits ist die Entfernung eines intracerebralen Fremdkörpers unter Feldbedingungen selbst für einen erfahrenen Neurochirurgen eine schwierige und überaus gefährliche Prozedur, auf der anderen Seite ist unter diesen Bedingungen eine strategische Luftevakuierung nach Deutschland (ca. 8000 km Dauer etwa $12 \mathrm{~h}$ ) auf Grund des kombinierten sub/epiduralen Hämatoms, fortschreitenden lokalen Oedems und der intracerebralen freien Luft in höchstem Maße gefährlich. Darüber hinaus ist bekannt, dass das Outcome bei diesem Verletzungsmuster ohne initiale Versorgung der Blutung nach $4-6 \mathrm{~h}$ extrem schlecht ist. Prozedere: Sofort nach Diagnosestellung wurde der Patient intubiert und beatmet und eine cerebroprotektive Therapie begonnen. Eine strategische Luftevakuierung wurde mit der höchsten Dringlichkeit in die Wege geleitet. Eine telemedizinische Konsultation mit Neurochirurgen, Radiologen und Anästhesisten in der Heimatklinik favorisierte ein zweizeitiges Vorgehen. $4 \mathrm{~h}$ nach Eintreffen des Patienten erfolgte nach genau festgelegtem Procedere die Entlastung des kombiniert sub/epiduralen Hämatoms durch einen afghanischen Neurochirurgen zusammen mit einem deutschen HNO-Arzt mittels osteoklastischer Trepanation. In Ermangelung von geeignetem Drainagematerial wurde um einen Druckausgleich während des Fluges zu ermöglichen ein $16 \mathrm{Ch}$ Foley-Katheter platziert. Der Erfolg der Massnahme wurde nach CCT-Kontrolle telemedizinisch gesichert. Unmittelbar danach erfolgte die strategische Luftevakuierung, so dass der Patient die Heimatklinik $23 \mathrm{~h}$ nach Eintreffen im Feldlazarett erreichte. Der Fremdkörper wurde am Folgetag mittels Neuronavigation entfernt. Der weitere Verlauf gestaltete sich komplikationslos. Der Patient wurde am 12. Tag nach Trauma ohne neurologisches Defizit nach Istambul ausgeflogen.

\section{Pädiatrische Intensivmedizin}

Clinical use of hypertonic saline bolus infusions

in severe pediatric head injury

A. Joeris ${ }^{1}$, A. Fichtner ${ }^{1}$, B. Wagner $^{2}$, S. Berger ${ }^{1}$

${ }^{1}$ Kinderchirurgische Klinik und Poliklinik, ${ }^{2}$ Med. Kinderklinik,

Pädiatrische Intensivstation, Universitätsklinik, Inselspital, Bern, Schweiz

Introduction: Continuous infusions of hypertonic saline (HS) solutions have been used successfully in pediatric head injury for reduction of elevated intracranial pressure (ICP). In adults however, HS is usually administered by bolus infusion. So far, effects of bolus infusions of HS were reported only anecdotically in children. Material and methods: The data of 13 children (6-15 y, mean age $10.8 \mathrm{y}$ ) treated for severe isolated head injury (initial GCS 3-8, mean 4.8) from 1998-2003 were analysed retrospectively. All children had ICP-monitors and arterial blood pressure monitoring, 11 had monitoring of oxygen saturation in the bulbus v. jugularis $\left(\mathrm{SvjO}_{2}\right)$. All children received several bolus infusions of HS during the first 3 days. Patient charts were analysed as to all physiological parameters, CT scans, laboratory parameters and outcome data (Glasgow outcome score, GOS) recorded. Results: The children were admitted after a mean rescue and transport time of $1 \pm 0.08 \mathrm{~h}$ with normal blood pressure (116.6 $\pm 4.6 /$ $65.0 \pm 5.0 \mathrm{mmHg}$ systolic/diastolic) and normal hemoglobin concentration $(11.9 \pm 0.4 \mathrm{~g} / \mathrm{dl})$. The mean intubation period was $5.6 \pm 0.6 \mathrm{~d}$, the mean ICU time was $6.9 \pm 0.9 \mathrm{~d}$. HS bolus infusions reduced ICP $(\mathrm{p}=0.0001)$ and increased cerebral perfusion pressure $(\mathrm{CPP})(\mathrm{p}=0.004)$ while increasing $\mathrm{SvjO}_{2}(\mathrm{p}=0.01)$ significantly. Only moderate increases of serum sodium concentration were found, even after multiple infusions $\mathrm{Na}+$ remained below $155 \mathrm{mosmol} / \mathrm{l} .4$ of $13(30.8 \%)$ patients died (GOS 1$), 3$ on day 4 and 1 on day 6 . Outcome was good in 3 (GOS 4), 5 were moderately disabled (GOS 3) and 1 was severely disabled (GOS 2) upon discharge from the hospital. Discussion: In a subgroup of children with most severe head injury and detailed cerebro-hemodynamic monitoring, bolus administration of HS proved effective and safe for improvement of $\mathrm{CPP}$ and $\mathrm{SvjO}_{2}$ with reduced sodium dosage and side effects as compared to continuous infusion protocols. These preliminary results with comparatively good outcome data justify prospective studies that compare continuous and bolus infusions of HS. 
Assoziation von persistierender rechter Umbilikalvene

081 und komplexem Vitium cordis

T. Hoehn, K. Schmidt, J. Schaper, E. Mayatepek

Neonatologie und Pädiatrische Intensivmedizin, Klinik

für Allgemeine Pädiatrie, Universitätsklinikum Düsseldorf

Einleitung: Die Kanülierung der Nabelvene ist ein etablierter venöser Notfallzugang im Rahmen der postnatalen Versorgung von kranken Früh- und Neugeborenen. Obwohl es theoretisch möglich ist, während der Katheterisierung Arterie und Vene zu unterscheiden, gelingt dies in der Praxis nicht immer mit ausreichender Sicherheit. Dabei ist - eine normale Anatomie mit zwe Nabelarterien und einer Nabelvene vorausgesetzt - das unvorteilhafteste, was dem Neonatologen passieren kann, die Kanülierung von zwei Nabelarterien. Im Folgenden berichten wir über ein Frühgeborenes, das unmittelbar postnatal mit ausgeprägter Zyanose auffiel und Beatmung und einen zeitnahen vaskulären $\mathrm{Zu}$ gang benötigte. Fallbericht: Die Mutter hatte einen unauffälligen Schwangerschaftsverlauf einschließlich der fetalen Biometrie, chronische Erkrankungen hätten nicht vorgelegen. Nach spontaner, vaginaler Geburt nach $35+2$ Schwangerschaftswochen war der Nabelschnur-pH 7.21, der APGAR 4/5/6. Wegen persistierender Zyanose wurden die Neonatologen sofort nach Geburt zum Kind gerufen, die klinische Untersuchung zeigte zunächst keinerlei Auffälligkeiten oder dysmorphe Stigmata, die Nabelschnur besaß drei Gefäße. Nach Beginn einer Beatmung und weiterhin persistierender Zyanose wurde ein zyanotisches Vitium cordis vermutet. Nach der Katheterisierung der Nabelgefäße und einer initialen Stabilisierung wurde das Kind zur weiteren interdisziplinären Betreuung ins Tertiärzentrum verlegt. Radiologisch ließen sich zwei Katheter identifizieren, die beide von der Eintrittsstelle am Nabelgrund nach kranial in Richtung des Zwerchfells führten. Beide Katheter waren gut aspirierbar und die gemessenen Blutgase aus beiden Kathetern waren identisch und mit zentralvenösen Blutgasen kompatibel. Echokardiografisch wurde ein Double outlet right ventricle mit Mitralatresie und eine levo-atriale Kardinalvene, die den linken Vorhof in die Vena azygos drainierte, diagnostiziert. Das Foramen ovale war verschlossen und eine konventionelle Ballonatrioseptostomie konnte nicht durchgeführt werden. Mehrfache Versuche einer transseptalen Punktion und einer Schaffung eines Defekts auf Vorhofebene waren nicht erfolgreich und das Kind verstarb schließlich. Eine persistierende rechte Umbilikalvene (PRUV) ist viel häufiger als es der gegenwärtigen Wahrnehmung entspricht. Die Inzidenz in einem Kokollektiv mit niedrigem Risiko ist auf 1:526 geschätzt worden. Es gibt eine beschriebene Assoziation mit angeborenen Herzfehlern, das Vorhandensein einer PRUV kann pränatal der einzige Hinweis auf das Vorhandensein derartiger Fehlbildungen sein. Andere assoziierte Erkrankungen sind abnormale systemische venöse Gefäßverbindungen und Noonan's Syndrom. Schlussfolgerung: PRUV kann pränatal diagnostiziert werden sofern spezifisch danach gesucht wird. Die postnatale Diagnose eines PRUV kann eine Echokardiografie und eine kinderkardiologische Untersuchung auch in der Abwesenheit einer Zyanose rechtfertigen.

Anwendung von Protein C bei einem 10 Jahre alten Mädchen mit Meningokokkensepsis

M. Reetz, G. Laske, K.-U. Schunck, U. Brosch

Vivantes Klinikum im Friedrichshain, Klinik für Kinder und Jugendmedizin

Meningokokkenerkrankungen werden durch das Bakterium Neisseria meningitidis hervorgerufen. Trotz frühzeitigem Einsatz effektiver Antibiotika sind schwere Verlaufsformen und Spätschäden nicht immer vermeidbar. Weltweit werden 300-500 Meningokokkenerkrankungen pro Jahr beobachtet. Die Letalität der Meningokokkeninfektion beträgt ca. $10 \%$, die der Meningokokkensepsis 5-25\%. Bei dem Waterhouse Friderichsen Syndrom ist, abhängig vom Erkrankungsalter und klinischem Zustand des $\mathrm{Pa}$ - tienten, eine Letalität von 60 bis zu 95\% zu erwarten. Wir behandelten im Frühjahr 2006 ein Kind mit einer Sepsis mit Protein C, sowie Dexamethason und Cefotaxim bei bestehender Meningokokken-Meningitis. Der Verlauf der erstaunlich rasanten Besserung nach Therapiebeginn, sowie die Darstellung der Gerinnungswerte sollen besonders hervorgehoben werden. Bei der Verabreichung des Protein $\mathrm{C}$ orientierten wir uns in der Dosierung mit 100 Internationale Einheiten/kilogramm Körpergewicht als Kurzinfusion. Im Verlauf haben wir zur Dokumentation die Gerinnungsparameter engmaschig untersucht und konnten folgende Dynamik erkennen: Nach Verabreichung des Protein C über eine Stunde stieg dessen Wert von initial 53\% auf $156 \%$ an. Bereits drei Stunden nach Gabe des Protein $C$ kam es zu einem Abfall auf 137\%, 7 Stunden nach Gabe auf 116\%. Die nächste Kontrolle erfolgte 16 Sunden nach Gabe mit einem Wert von $92 \%$ und lag nach 28 Stunden bei einem Wert von 85\%. Die Werte für Quick, PTT und Fibrinogen lagen nach 16 Stunden im Bereich der bestimmten Initialwerte. Das maximal nachweisbare CrP von 146,4 Gramm/Liter war nach 16 Stunden erreicht, und die Patientin reagierte, wenn auch noch leicht verzögert, adäquat. Nebenwirkungen traten während und nach Therapie nicht auf. Erstaunlich ist aus unserer Sicht die rasche Stabilisierung des Kindes nach Anwendung von Protein $\mathrm{C}$ in Kombination mit antimikrobieller und antiinflammatorischer Therapie. Dies könnte durch den frühzeitigen Therapiebeginn bedingt sein. Insgesamt wäre zu postulieren, dass der frühzeitige Einsatz des Medikamentes nach Diagnosestellung in Kombination mit antimikrobieller und antiinflammatorischer Therapie zu einem positivem Heilungsprozeß geführt hat.

Transport von Neugeborenen mit akutem Lungenversagen zu ECMO und unter ECMO (ExtrakorporaleMembranoxygenierung)

\section{F. Loersch, St. Hien, M. Kratz, S. Demirakca, Ch. V. Buch, T. Schaible Universitätskinderklinik Mannheim}

Hintergrund: Trotz Optimierung der Beatmungsstrategien und Verbesserung der pränatalen und postnatalen Versorgung von Neugeborenen bleibt die ECMO-Therapie ein Standbein der neonatologischen Intensivmedizin beim akuten Lungenversagen. Im ECMO-Zentrum Mannheim werden pro Jahr etwa 25 neonatale ECMOs durchgeführt, wobei 50-60 Kinder zur Option ECMO stationär sind. Bei einigen Neugeborenen mit schwerem Lungenversagen ist der Zustand zu kritisch um einen konventionellen Transport mit HFOV oder iNO in ein ECMO-Zentrum durchzuführen. Hier schient der Transport unter ECMO als Rescue-Therapie die letzte Möglichkeit zu sein. In zahlreichen Ländern wie USA oder Schweden haben sich Abholteams für den Transport von Patienten unter ECMO etabliert. In Deutschland gibt es lediglich Berichte von Einzelfällen für diese Art der Verlegung. Eigene Erfahrungen: In Mannheim wurden in den letzten fünf Jahren 141 Neugeborene zur Option ECMO zuverlegt, weitere 132 Neugeborene wurden auf Grund eines pränatalen Befundes in Mannheim entbunden. 91 Neugeborene wurden durch die zuständige zu verlegende Klinik gebracht. Bei 50 Neugeborenen wurde eine Abholung durch das Mannheimer Team durchgeführt, da der Zustand der Kinder sehr kritisch war, 52\% wurden mit iNO, 24\% mit HFO transportiert, $78 \%$ wurden mit dem Hubschrauber und $22 \%$ mit dem INUVER verlegt. Zusätzlich wurden in den letzten fünf Jahren neun Transporte unter ECMO durchgeführt $(6 \times \mathrm{CDH}$, $1 \times$ PPHN, $1 \times$ MAS, $1 \times$ Herzfehler). Die ersten Transporte wurden mit einer konventionellen ECMO-Pumpe (HL15, Jostra) durchgeführt. Der Patient lag auf einem offenen Wärmebett, die Pumpe stand daneben. Hierfür wurde ein Intensivbus mit Hebebühne benötig, der eine Vorlaufzeit von ca. 6 Stunden hatte. Ziel war es nun eine kompakte und voll funktionsfähige Transport-ECMO Einheit zusammenzustellen, die schnell einsatzbereit ist. Hierfür wurde eine transportable Rollerpumpe (NovaCirc, Jostra) auf eine Standart Ferrno Trage montiert. Diese kann mit jedem DIN-RTW 
bzw. Hubschrauber befördert werden. Zusätzlich ist ein Beatmungsgerät (Stephanie oder Stephan), eine Wärmepumpe (Aquatherm 660) sowie 12 Perfusoren auf der Einheit montiert. Die Stromversorgung ist anhand einer USV für den Transport zum RTW und auf Station sichergestellt. Während der Fahrt wird die Stromversorgung über den $220 \mathrm{~V}$ Stromwandler des Fahrzeuges gewährleistet. Eine solche Einheit ist in etwa einer Stunde einsatzbereit. Grundsätzlich ist auch der Transport mit einem luftgebundenen Fahrzeug möglich. Diskussion: Die, wenn möglich pränatale bzw. frühe postnatale, Verlegung in ein ECMO-Zentrum sollte der Standard bei Kindern mit schwerem Lungenversagen bleiben. Speziell geschultes Personal und spezielle Ausrüstung sind für den Transport von kritisch kranken Kindern dringend notwendig. Der Einsatz von Transport-ECMO ist jedoch nach reiflicher Risiko-Nutzen Abwägung eine Möglichkeit bei Neugeborenen als Rescue Therapie. Eine Kanülierung muss dann vor Ort von den mitgebrachten Kinderchirurgen erfolgen.

Kardiopulmonale Veränderungen durch Laparoskopie bei gesunden und pulmonal geschädigten Schweinen

C. Matthes, T. Huckstadt, S. Richter, T. Ploenes, F. Schier Klinik für Kinderchirurgie, Universitätsklinik Mainz

Hintergrund: Laparoskopie ist ein Routineverfahren in vielen operativen Fachbereichen. Auch in der Neugeborenenchirurgie hat sie sich etabliert. Diese Patienten sind oft in der respiratorischen Funktion beeinträchtigt. Ziele: Diese Untersuchung wurde durchgeführt, um Veränderungen durch erhöhten intraabdominellen Druck bei gesunden und pulmonal vorgeschädigten neugeborenen Schweinen zu quantifizieren. Methode: Es wurden 28 neugeborene Schweine im Alter von 2 bis 3 Wochen in einer tierexperimentellen Studie (Tierversuchsnummer 1.5 177-07/051-6) randomisiert zwei Gruppen zugeordnet. Eine Gruppe wurde einer pulmonalen Lavage unterzogen bis ein arterieller Sauerstoffpartialdruck von weniger als $200 \mathrm{mmHg}$ erreicht war. Die anderen Tiere dienten als Referenzgruppe. Die Tiere wurden intramuskulär analgosediert. Die Narkose wurde intravenös mit Pentobarbital, Fentanyl und Pancuronium fortgeführt. Bei Untersuchungsbeginn bestand Normokapnie $\left(\mathrm{paCO}_{2} 35-40 \mathrm{mmHg}\right)$. Ventiliert wurden alle Tiere bei einem $\mathrm{FiO}_{2}$ von 1,0 mit einer Inspirationsdauer von $0,5 \mathrm{~s}$, einer Exspirationszeit von $2,5 \mathrm{~s}$ und einem PEEP von $4 \mathrm{~cm} \mathrm{H} \mathrm{H}_{2} \mathrm{O}$ am Babylog 1 (Dräger). Die Normokapnie wurde durch Adaptation des Beatmungsdruckes beibehalten. Messparameter waren mittlerer arterieller Blutdruck (MAP) in der Arteria carotis, mittlerer pulmonalarterieller Druck (PAP) und das Tidalvolumen (VT). Der Oxygenierungsindex wurde errechnet. Nach Registrierung der Ausgangswerte erfolgte eine Erhöhung des abdominellen Druckes in $6 \mathrm{mmHg}$-Schritten auf $24 \mathrm{mmHg}$. Jede Druckstufe wurde für jeweils 20 Minuten belassen und die Werte registriert. Abschließend erfolgte die Desufflation. Ergebnisse: Das Tidalvolumen blieb durch Anpassung des Beatmungsdruckes in beiden Gruppen unverändert. Bei intraabdomineller Druckerhöhung stieg im Median bei den gesunden Tieren der MAP von 52 auf $72 \mathrm{mmHg}$ und der PAP von 13 auf $18 \mathrm{mmHg}$ und bei den pulmonal geschädigten Tieren der MAP von 48 auf $57 \mathrm{mmHg}$ und der PAP von 21 auf $32 \mathrm{mmHg}$. Die Parameter erreichten bei beiden Gruppen nach Desufflation wieder die Ausgangslage (gesunde Gruppe MAP 55 und PAP $14 \mathrm{mmHg}$; pulmonal geschädigte Gruppe MAP 45 und PAP $22 \mathrm{mmHg}$ ). Die größten Veränderungen waren bei der ersten Drucksteigerung von 0 auf $6 \mathrm{mmHg}$ zu verzeichnen. Der Oxygenierungsindex zeigte in der gesunden Gruppe nur einen geringen Anstieg von 0,9 auf 2,1 und in der pulmonal geschädigten Gruppe einen Anstieg von 8,3 auf 14,7. Auch dieser Parameter erreichte wieder die Ausgangslage $(0,9$ und 8,6). Schlussfolgerung: Durch Laparoskopie bedingte erhöhte intraabdominelle Drücke werden sowohl von gesunden als auch von pulmonal geschädigten neugeborenen Schweinen toleriert. Die kardiopulmonalen Veränderungen waren nach Ablassen des Kapnoperitoneums und Wiederherstellung der physiologischen Bedingungen vollständig reversibel.

\section{Transplantation}

Indikationsstellung zur Tracheotomie

nach Lebertransplantation - wird die epithelialisierte

Tracheotomie eine Renaissance erleben?

J. Swol-Ben ${ }^{1}$, Th. Bein ${ }^{2}$, A. Obed, H.J. Schlitt

1 Klinik und Poliklinik für Chirurgie der Universität Regensburg

${ }^{2}$ Klinik für Anästhesiologie der Universität Regensburg

Einleitung: Die zunehmende Diskrepanz zwischen Organbedarf und Spenderangebot, sowie das Eurotransplant Meldeverfahren führen dazu, dass der größte Anteil der Empfänger erst im fortgeschrittenen Stadium der Erkrankung in einem reduzierten Allgemeinzustand transplantiert werden. Bedingt durch das labile Gleichgewicht zwischen der eigenen Immunabwehr und der Immunsuppression, kommt es nach nach einer Lebertransplantation (LTx) häufig zu systemischen Inflammationsreaktionen. Damit assoziierte pulmonale Komplikationen erfordern eine differenzierte antibiotische und intensivmedizinische Therapie, bei Langzeitbeatmung häufig eine Tracheotomie. Methode: Retrospektive Erfassung aller LTx an der Klinik für Chirurgie der Universität Regensburg im Zeitraum 05/03 bis 05/06 mit der Fragestellung nach Indikation zur Tracheotomie im Hinblick auf die angewandte Methode (dilatativ vs. offen). Ergebnisse: Von 100 Patienten (105 LTx, davon 5 HU ReLTx) sind 57 unmittelbar postoperativ, spätestens jedoch nach 12 Std Beatmung, extubiert worden. In 32 Fällen traten schwere Pneumonien auf. Bei 19 Patienten war aus diesem Grund eine Reintubation unvermeidbar, davon wurden 12 im Verlauf tracheotomiert. In besonders fulminanten Verläufen wurde die Tracheotomie ohne vorausgegangener Extubation (12 Fälle) durchgeführt. Insgesamt sind 24 Patienten tracheotomiert worden (14 dilatativ nach Griggs, 1 nach Fantoni und 9 offen chirurgisch). In 2 Fällen kam es zu einer revisionspflichtigen Nachblutung bei offen chirurgisch angelegtem Tracheostoma. Neben den pulmonalen Vorerkrankungen (COPD, Pleuraverwachsungen) spielt die Konstitution des Patienten bei der Indikation zur Tracheotomie eine wesentliche Rolle. Fast ein Drittel (7 von 24) der tracheotomierten Patienten war mit einem BMI von über 25 adipös. Allerdings mussten auch 5 Patienten die sich in einem kachektischem Zustand $(\mathrm{BMI}<20)$ als Folge der dekompensierten Leberzirrhose befanden, tracheotomiert werden. Die Dauer des Verbleibens der Trachealkanüle betrug in Median 27 Tage. Schlussfolgerungen: Wir sehen bei Lebertransplantierten eine rasche Entscheidung zur Tracheotomie als indiziert, wenn eine kurzfristige Entwöhnung von der Beatmung unwahrscheinlich ist. Dies zeigt sich insbesondere bei Patienten im kachektischen oder adipösen Zustand, der die Atemmechanik negativ beeinflusst oder in den Fällen, in denen die Lungenschädigung einen prolongierten Weaningsprozess vermuten lässt. Bei der Auswahl der Tracheotomiemethode (offen vs. dilatativ) ist neben den bislang anerkannten Kontraindikationen, die zu erwartende Dauer des Intensivaufenthaltes oder Rehabilitation mit der Gefahr der Trachealkanülendislokation zu berücksichtigen. Nach einer offen chirurgisch durchgeführten Tracheotomie ist ein permanenter Zugang zur Trachea für die Replatzierung der Kanüle angelegt, was bei einem langwierigen und kostenintensiven Verlauf nach einer LTx einen Weg zu Vermeidung von Komplikationen wie z. B. einer Reintubation darstellt. 
Comparison of two methods for measuring cardiac output (pulse contour analysis vs. thermodilution) during human orthotopic liver transplantations

\section{A.F. Poisel, B.H. Hetz, C.C.-G. Krenn}

Ludwig Boltzmann Institut for Anaesthesiology and Intensive Care, Wien

Introduction: Cardiac output (CO, 1/min) and Cardiac Index (CI, 1/ $\mathrm{min} / \mathrm{m}^{2}$ body surface area) measurement is often required for hemodynamic management. Bolus thermodilution $\mathrm{CO}$ and accordingly $\mathrm{CI}$ (ICO, ICI) and continuous $\mathrm{CO}$ and accordingly $\mathrm{CI}(\mathrm{CCO}$, CCI) measurements via pulmonary artery catheters (PAC) are the most commonly used methods for this purpose. In order to avoid its risks $[1,2]$ a less invasive $\mathrm{CO}$ and $\mathrm{CI}$ respectively measuring method, deriving $\mathrm{CO}$ or CI via pulse contour analysis (arterial pressure cardiac output, APCO, arterial pressure cardiac index, APCI), has been introduced into clinical practice (FloTrac ${ }^{\mathrm{TM}}$ sensor, Vigileo $^{\mathrm{TM}}$, Edwards Lifesciences ${ }^{\mathrm{TM}}$ ). It only needs access to a standard peripheral artery catheter. Recently a close correlation between APCO and ICO or CCO in patients whose CO is within the normal range (of 4-7 1/min) was shown [3]. Hypothesis: Aim of this study was to evaluate the correlation between APCI and ICI or CCI in patients undergoing orthotopic liver transplantations (OLT), a procedure with great fluid shifts and varying CI states. Patients/Methods: We compared the two methods during 8 OLTs. ICI and CCI were measured with PAC and Vigilence ${ }^{\mathrm{TM}}$ from Edwards Lifesciences ${ }^{\mathrm{TM}}$. CCI and APCI were automatically monitored every 20 seconds by a computer, connected to Vigileo ${ }^{\mathrm{TM}}$ Monitor and Vigilence ${ }^{\mathrm{TM}}$. ICI was measured at five specific time points: in preparation phase, 10 minutes before cross clamp of vena cava inferior, 10 minutes after cross clamp, 10 minutes before reperfusion and 10 minutes after reperfusion by bolus thermodilution. A total of 80 data points was evaluated. Measurements of ICI, CCI and APCI were analysed for bias and correlation via Bland-Altmann analysis. Results: Average age was $56 \pm 4,2$, all of them were male. Bland-Altmann analyses of the differences between CCI and APCI and between ICI and APCI were conducted. The analysis yielded a mean bias of $-11 / \mathrm{min} / \mathrm{m}^{2}$. The correlation was, following the formula $Y=1,603+0,252 \cdot X, R^{2}=0,33$. Conclusion: Our results for measurements obtained during OLT showed a poor correlation. Weather these results are of clinical relevance has to be evaluated by further investigations.

References: [1] Abreu AR, Campos MA, Krieger BP: Pulmonary artery rupture induced by a pulmonary artery catheter: a case report and review of the literature. J. Intensive Care Med 2004; 19: 291-296. [2] Huang L, Elsharydah A, Nawadi A, et al: Entrapment of pulmonary artery catheter in a suture at the inferior vena cava cannuation site. J Clin Anesth 2004; 16:557-559. [3] Mc Gee W, Horswell J, Janvier G: Validation of a continuous cardiac output measurement using arterial pressure waveforms. Critical Care 2005; 9(Suppl 1)

ROTEM-basierter Algorithmus zum Point-of-Care-(POC)Gerinnungsmanagement bei Lebertransplantationen Erfahrungen aufgrund von 666 Patienten mit LTX

K. Görlinger, D. Dirkmann, M. Adamzik, A. Hanke, F. Dusse, L. Bergmann, J. Peters

Klinik für Anästhesisiologie und Intensivmedizin, Universitätsklinikum Essen

Hintergrund: Eine Vielzahl von Patienten entwickelt im Rahmen von Lebertransplantationen Gerinnungsstörungen und Blutungskomplikationen. Andererseits kommt es bei einigen Patienten auch zu zum Teil tödlichen thromboembolischen Ereignissen. Dies macht perioperativ ein Gerinnungsmanagement erforderlich, welches eine zeitnahe und zielgerichtete Diagnostik und Therapie von Koagulopathien ermöglicht. Ziel der Untersuchung: Entwicklung eines ROTEM-basierten Algorithmus zum perioperativen $\mathrm{Ge}$ rinnungsmanagement bei Lebertransplantationen. Material und
Methode: Von Januar 2000 bis März 2006 wurden am Universitätsklinikum Essen 666 Lebertransplantationen mit Point-of-Care(POC)-Monitoring mittels ROTEM durchgeführt. Die Messungen erfolgten zu definierten Zeitpunkten. Die Ansätze ExTEM, InTEM, FibTEM und ApTEM wurden als Routinetests eingesetzt. Bei Hinweisen auf einen Heparineffekt wurde zusätzlich ein HepTEM durchgeführt. Als Variable wurden die Coagulation Time (CT), die Maximum Clot Firmness (MCF), die Maximum Lysis (ML) und der Clot Lysis Index (CLI) ausgewertet. Ergebnisse: Eine prophylaktische Gabe eines Antifibrinolytikums wurde bei Patienten mit einem fulminanten Leberversagen und bei Patienten mit einer schon zu OP-Beginn stark reduzierten Gerinnselfestigkeit (MCF-Ex $\leq 35 \mathrm{~mm}$ ) durchgeführt. Bei den übrigen Patienten konnte in $60 \%$ der Transplantationen eine Hyperfibrinolyse (ML $>15 \%)$ nachgewiesen werden. Allerdings war eine Therapie der Hyperfibrinolyse nur bei $2 / 3$ dieser Patienten erforderlich, da sie in $1 / 3$ der Fälle nach Reperfusion selbstlimitierend war. Bei einer Verminderung der Gerinnselfestigkeit (MCF-Ex $<45 \mathrm{~mm}$ ) lässt sich anhand der MCF im FibTEM zwischen einem Fibrinogenmangel (MCF-Fib $<8 \mathrm{~mm}$ ) und einer Thrombozytopenie/pathie (MFC-Fib $>8 \mathrm{~mm}$ ) differenzieren. Eine CT $>80 \mathrm{~s}$ im ExTEM oder eine CT $>240 \mathrm{~s}$ im InTEM weist auf einen Faktorenmangel hin. Dabei kann ein Heparineffekt mittels HepTEM innerhalb von 10 Minuten ausgeschlossen bzw. gesichert werden. Eine Therapie aufgrund dieser ROTEM-Ergebnisse erfolgte selbstverständlich nur bei gleichzeitigem Vorliegen einer diffusen Blutungsneigung. Neben der Berücksichtigung der ROTEM-Ergebnisse kommt der Beachtung der Rahmenbedingungen (Hypothermie, Azidose, Hypokalzämie und Anämie) eine entscheidende Rolle zu. Diskussion: Die Rotationsthrombelastometrie erlaubt als POC-Methode - im Gegensatz zu den klassischen Gerinnungsparametern - eine schnelle und zielgerichtete Gerinnungstherapie. Eine rationale und zielgerichtete Therapie von Blutungen und Gerinnungsstörungen ist aufgrund begrenzter Resourcen an Blutprodukten und hoher Kosten von Gerinnungsfaktorkonzentraten heute mehr denn je von Bedeutung. Schlussfolgerung: Der hier vorgestellte, auf empirischen Daten basierende ROTEM-Algorithmus ist ein Schritt zur Verbesserung und Rationalisierung des perioperativen Gerinnungsmanagements bei Lebertransplantationen, muss allerdings noch in kontrollierten Studien validiert werden.

Chronisches Nierenversagen durch Immunsuppression 233 Untersuchungen von renaler, hepatischer und peripherer Mikrozirkulation bei freiwilligen Probanden

\section{K.E. Wirsching, M. Mauer, J.M. Fertmann, K. W. Jauch,} J.N. Hoffmann

Chirurgische Klinik, Klinikum Großhadern, München

Hintergrund: Immunsupressiva haben das Langzeitüberleben transplantierter Organe wesentlich verbessert. Jedoch werden gerade den klassischen Calcineurininhibitoren negative Effekte auf die Transplantatfunktion nachgesagt, die zum chronischen Transplantatversagen beitragen können. Hierbei sollen insbesondere rezidivierende Mikrozirkulationssstörungen im Transplantat eine Rolle spielen. Diese Studie untersucht folglich die renale Mikrozirkulation, die Mundschleimhautmikrozirkulation sowie die periphere Vasoreaktivität nach Gabe verschiedener Immunsuppressiva. Methodik: Nach Genehmigung durch die Ethikkommission werden vier Gruppen ( $n=5$ freiwillige, gesunde Probanden) mit jeweils einmaliger Einnahme von Cyclosporin A, Mycophenolat moffetil (MMF), Tacrolimus (FK 506), oder Sirolimus in diesem Protokoll untersucht. Mikrozirkulationsmessungen werden vor Einnahme, 60 Min., 120 Min., 180 Min. sowie nach 240 Min. durchgeführt. $\mathrm{Zu}$ diesen Zeitpunkten erfolgt die venöse Blutentnahme (Blutbild, Medikamentenspiegel, Gerinnung, vasoaktive Faktoren) sowie die Messung der Makrohämodynamik mittels vollautomatischer Blutdruck und Herzfrequenzmessung. Die Quantifizierung der Mundschleimhautperfusion erfolgt mittels 
OPS ${ }^{\circledR}$ (Orthogonal Polarization Spectral) Imaging. Gleichzeitig werden durch Laserdopplerflowmetrie $\left(\mathrm{O}_{2} \mathrm{C}=\right.$ Oxygen too see) die $\mathrm{Hb}$-Konzentration, Hb-Sättigung, Flow und Velocity quantitativ erfasst sowie die periphere Vasoreaktivität nach 2-minütiger Ischämie des Unterarms. Zusätzlich werden per Duplexsonographie in beiden Nieren arterio-kapsuläre Abstände am oberen und unteren Nierenpol und an der Leberkapsel als Maß für die Organperfusion bestimmt. Ergebnisse: Zum jetzigen Zeitpunkt haben sich insgesamt 10 Probanden der Messung unterzogen, wobei sich keine makrohämodynamischen Veränderungen nach einmaliger Einnahme von Immunsuppressiva zeigten. Erste $\mathrm{O}_{2} \mathrm{C}$ Ergebnisse zeigen eine positive Korrelation der Akrendurchblutung mit $\mathrm{Hb}$ Sättigung, Flow und Velocity. Schlussfolgerung: Die in der Studie angewandten Methoden der Mikrozirkulationsmessung (OPS und $\mathrm{O}_{2} \mathrm{C}$ ) eignen sich um periphere als auch die zentrale Mikrozirkulation parallel nicht invasiv und ohne Belastung für Probanden und Patienten zu untersuchen. Die Ergebnisse der Studie werden zeigen, ob die in vivo Messung von Mikrozirkulationsveränderungen eventuell zur Modifikation der immunsuppressiven Therapie zur Vermeidung von Nebenwirkungen und chronischem Transplantatversagen eingesetzt werden können. 